\title{
Al-Quantum Phases on Chern-Simons Topological Geometrics for the Generation of A Ligand Targeting COVID-19-SARS-COV-2 SPIKE D614G Binding Sites.
}

loannis Grigoriadis ( $\nabla$ jgrigoriadis@biogenea.gr )

Aristotle University of Thessaloniki

\section{Research Article}

Keywords: COVID19, SARS-COV-2 SPIKE D614G, Chern-Simons Topological, Al-Quantum computing, Quantum-Inspired Evolutionary Algorithm Predictive toxicology, QSAR quantum gates,cheminfomratics artificial intelligence, phase data mining, machine learning, learning, cheminformatics

Posted Date: January 25th, 2021

DOI: https://doi.org/10.21203/rs.3.rs-140391/v1

License: (a) (1) This work is licensed under a Creative Commons Attribution 4.0 International License.

Read Full License 


\title{
AI-Quantum Phases on Chern-Simons Topological Geometrics for the generation of a ligand targeting COVID-19-SARS-COV-2 SPIKE D614G binding sites.
}

\author{
1. Department of BiogenetoligandorolQMMIDDD/QPRPICA/MACHNOT/
}

QIICDNNDCA ADMET/QIICDNNDCA Stations, Aristotle University of Thessaloniki,

\author{
Biogenea Pharmaceuticals Ltd - Greece.
}

Grigoriadis Ioannis* email: jgrigoriadis@biogenea.gr

tel:+306936592686 ORCID iD: https://orcid.org/0000-0002-8443-1777

Keywords: COVID19, SARS-COV-2 SPIKE D614G, Chern-Simons Topological, AI-Quantum computing, QuantumInspired Evolutionary Algorithm Predictive toxicology, QSAR quantum gates, cheminfomratics artificial intelligence, phase data mining, machine learning, learning, cheminformatics

Abstract

SARS coronavirus 2 (SARS-CoV-2) in the viral spike (S) encoding a SARS-COV-2 SPIKE D614G mutation protein predominate over time in locales revealing the dynamic aspects of its key viral processes where it is found, implying that this change enhances viral transmission. It has also been observed that retroviruses infected ACE2-expressing cells pseudotyped with SG614 that is presently affecting a growing number of countries markedly more efficiently than those with SD614. The availability of newer powerful computational resources, molecular modeling techniques, and cheminformatics quality data have made it feasible to generate reliable algebraic calculations to design new chemical entities, merging chemicals, fragmentizing natural products, and a lot of other substances fuelling further development and growth of this AI-quantum based drug design field to balance the trade-off between the structural complexity and the quality of such biophysics predictions that cannot be obtained by any other method. In this paper, we strongly combine topology geometric methods targeting at the atomistic level the protein apparatus of the SARS-COV-2 virus that are simple in machine learning anti-viral characteristics, to propose computer-aided rational drug design strategies efficient in computing docking usage, and powerful enough to achieve very high accuracy levels for this in-silico effort for the generation of the AI-Quantum designed molecule the RoccustyrnaTM small molecule, a multi-targeting druggable scaffold (1S,2R,3S)-2-(\{[(1S,2S,4S,5R)-4-ethenyl-4-sulfonylbicyclo[3.2.0] heptan-2-yl]oxy\}amino)-3[(2R,5R)-5-(2-methyl-6-methylidene-6,9-dihydro-3H-purin-9-yl)-3-methylideneoxolan-2-yl]phosphirane-1-carbonitrile targeting the COVID-19-SARS-COV-2 SPIKE D614G mutation using Chern-Simons Topology Euclidean Geometric in a Lindenbaum-Tarski generated QSAR automating modeling and Artificial Intelligence-Driven Predictive Neural Networks.

\section{Introduction}

$(1,2,3)$ The COVID-19 disease which emerged in China at the end of 2019 was declared on March 2020 a pandemic by the World Health Organization (WHO) and is accountable for a large number of fatal cases. $(2,4,5)$ On January 202, WHO committee declared a global health emergency $(3,4-6)$ based on the rate of increasing spread of the infection (4,5-7) with a reproductive number (RN) in the range 2.0-6.5, 4 higher than SARS and MERS, (8) with more than 85,000 casualties and fatality rate of about 4\%.(1-4) Collaborative efforts for Genomic characterization, (5,6,7-9) Evolution, phylogeny, high contagion rates, molecular epidemiology, of SARS coronavirus and epidemiology from scientists worldwide are underway to understand the rapid spread of the novel coronavirus (CoVs), and to develop effective interventions for control and prevention of the disease. (1-10) Coronaviruses are positive-single stranded, enveloped large RNA viruses that infect humans and a wide range of animals. $(7,8-11)$ Tyrell and Bonne reported the first coronavirus in 1966, (11) who cultivated the viruses from the patients suffering with common cold. (4,6,7-10) In Latin, Corona means —crown \|| based on their shapes. As a megadiverse country, Brazil accounts for $10-20 \%$ of known living species in the world. However, a major part of the biological and chemical biodiversity in Brazil remains unexplored (17). (2-13,14,15,16,17) Molecular structure were determined in heterodox interpretations (22) by solving the time-independent (21-22) Schrödinger equation: QM methods, vertex prizes and edge costs including ab initio 
Density Filed Theories (DFT) (23) and semi-empirical in place (24) of the quantum processor and energy among other observables,(25) under simulated sampling error as well as to reposition drugs about bonding may represent the similarities (26) and dissimilarities(27) between drugs and repurposed viral proteins respectively. (28) However, the Schrödinger equation cannot actually be solved for any but a one- data-driven (29) electron system methods (the hydrogen atom), (30) and approximations need to be made. According to QM, $(2-19,23)$ an electron bound that converges quickly and reliably to an atom cannot possess any $(2-17,21,22,23,24,25,26,2728)$ arbitrary energy to produce the desired distribution by analyzing pharma- cological data or occupy any position in space using statistical and machine $(23,24-37,38)$ learning concepts. The Lindenbaum-Tarski algebra logical spaces and vectorial representations of the eigenvalue statements were used in this project and has been previously introduced as a 3D logical subspace allowing a well-defined position for each fragmented pharmacophore. This shows the application to quantum computing through the example of three coupled harmonic oscillators allows pure mechanical computation both for generating rules and inferences. $(25,27,28)$ Since in previous studies It has been shown that Path selection for quantum repeater networks can be geometrically represented by Chern-Simons logical spaces and subspaces I decided to implement supersymmetric solutions and borel Singularities for $\mathrm{N}=2$ allowing a quantum repeater based vectorial Supersymmetric Chern-Simons representation in this drug design project . (20,26,27,28-31) In general, the notions of Lindenbaum matrix and Lindenbaum-Tarski algebra have paved a way to further algebraization of logic, which had been begun by George Boole in the 19th century, as well as to a new branch of logic, model theory. Philosophical interpretations of QM were conditioned by ideals of what an explanatory theory should be. (Minkowski-type, waveedge, etc), (20,27,32-33) as well as probabilistic transformations Algebraic multi-metrics (Triangle area, Bond-angle, etc) and the associated axiomatic formulations (AQFT) treat observables rather than states as foundational for the interaction information extraction. $(20,33,34,35)$ In this project, we show an original strategy and an application to quantum computing through the example of two coupled harmonic black-hole oscillators obtained by molecular modeling and simulations as orthogonical coordinates applied for the design of a novel multi-chemo-structure against the crystal structure of COVID-19 protein targets in a Lindenbaum-Tarski generated QSAR automating modeling lead compound design approach. $(29,35,36)$ A meta server and a Kappa-Symmetry C algebra of local observables were incorporated for the docking FDA approved small molecules, peptide-mimetic and humanized antibodies against potential targets of COVID-19 via a generalized procedure of Quantization of classical fields which were fused together with QSAR automating modeling to lead the commutation and anticommutation relations. $(37,39,41,42)$ Dynamic niching and flexible heuristic genetic algorithmic states for automatic molecule recoring and fragmentation were applied to fragment and recore a database of 20,000+ molecules for use with the group contribution model Universal Quasichemical Functional Group Activity Coefficients (UNIFAC) against the structure and functions of SARS-CoV-2 as linear functional on the algebra of free energy docking observables. $(37,38,40)$ Hybrid quantum repeater via a robust creation of entanglement between remote memory qubits were implemented for predicting drug targets and for multitarget and multi-site based virtual screening against COVID-19 (41,42) Flexible Topology Euclidean Geometric, were used to fragment molecules automatically in this molecular modeling and drug designing project on several parameters while keeping the definition of the groups as simple as possible. Artificial Intelligence-Driven Predictive Neural Networks and Quantum- Inspired frameworks of parallel-Docking interactions were employed for supercritical entanglements introducing an advanced quantum mechanical inverse docking algorithm providing further insight to confirm the practicality of docking energy predictions for wild type and selected mutations for Nsp3 (papain-like, PLpro domain), Nsp5 Nsp15 (NendoU), (Mpro, 3CLpro), Nsp12 (RdRp), N protein and Spike in understanding the key element functions of SARS-CoV-2 protein pathways and in designing possible novel antiviral agents, from both an quantum algebraic and a cheminformatic perspective.

\section{Materials and Methods}

\section{Preparation of the protein structures}

The National Center (NCBI) database for Biotechnology Information was used to retrieve the S glycoprotein proteins of SARS-CoV-2. For the N protein, we aligned the clustered 31 conformations $(1,2,35)$ from the 1731 full-length SARS-CoV-2 sequences and stored as a FASTA format file for analysis with Glu174 and Glu166 present in the opened RBD down conformation out of a total of 40 states COVID19 in the NMR-derived COVID19 associated protein structure (PDB codes, 6xs6,1xak,2g9t, 3fqq, 2ghv,6yb7) $(1,3,4)$ to select a small subset representative of the protein flexibility downloaded from NCBI (30 April 202, txid2697049, NC_045512.2 with minimum length $=29,000$ bp) as a 
coordinate reference and aligned using the MAFFT tool. $(2-5,6)$ The BioEdit v7.2.3 sequence alignment editor was deployed to identify the conserved binding sites and short linear peptide region among the aligned sequences through multiple-sequence alignment (MSA) with ClustalW. The conserved alignment was visually generated, inspected and curated by preserving all nucleotides using Genbank NC_045512.2 as a coordinate reference in genomes such as the ball python genome, and further proceeded with the RSFIEDLLFNKV, e.g. KNFIDLLLAGF short linear motifs as identified between the Wuhan isolate beyond the limit of serious detection of the reptile shingle back for spike protein nidovirus 1 model construction by utilizing again the NC_045512.2 and annotated (ORFs) Open Reading Frames plus additional ORFs. We then provided to the DockThor-VS online docking platforms the protein structures in PDB format files of the SARS-CoV-2 (PDB codes, 6xs6,1 xak,2g9t,3fqq,2ghv,6yb7) (1,3-10) as potential therapeutic drug targets $(5-11,12)$ for the design of our new druggable scaffold named Roccustyrna. $(13,14,15)$ For this main purpose, we initially selected the Nsp3, Nsp5 non-structural proteins (PLpro domain), Nsp15 (endoribonuclease), Nsp12 $(\mathrm{RdRp})(5-16,17)$ and the structural proteins Spike and nucleocapsid protein (N protein). $(9,10-21))$ We then clustered the opened conformation states (31 alligned out of 40 conserved states) $(9,17,22)$ using the Conformer Cluster web server tool $(6,14,16)$ according to the position of the residues $(5,7,8) \operatorname{Arg} 102$, Glu166 and Tyr109 using the weighted sum of the centroid distances as the single linkage method. $(7,8-12,22)$ Finally, the nearest to the pair group centroid structure per cluster was selected as the representative conformation of each group to be available at

BiogenetoligandorolTM. In this article, we effectively use a AI-decision tree and an optimum quantum walk number of small chemical active chemical features from a collection of hundreds of them utilizing neural network and jointly docking free energy cumulative features and ranking method with input toxicity values taking both network decision tree parameters into account. In this work, we prepared the protein structures using the Protein Preparation Wizard from the BiogenetoligandorolTM (BiogenetoligandorolTM, SynthocureTM, Thessaloniki, Biogenea Pharmaceuticals Ltd-GR, 2020). $(8,13,17,22)$ hydrogen-bond optimization and Protonation assignment were applied by using the PROPKA and the ProtAssign publicly available software at the reported experimental $\mathrm{pH}(2-11,17)$ considering when available the presence of the bound small molecule $(8-12,15,19)$.

\section{Screening NuBEE Phyto-library and COVID2019 targets.}

Virtual screening of the final proposed model and high throughput molecular docking based on existing literature were implemented to a collection of 9591 drugs including 2037 chemical structures of FDA-approved small molecule drugs and $(2,3-14,17)$ over 6000 herbals and phytical extracts from the NuBBEDB updated database to get an insight into the potential inhibitors and to uncover chemical and biological druggable information from Brazilian biodiversity $(4-6,17)$. Drugs selected for the docking studies with an ideal number of non-hydrogen and metal atoms not related with macromolecules, organic and inorganic active fragments (e.g., Pt, Fe, Hg, etc.) below 5 or above 10, phytical elements and drugs having Molecular Weight $>120$, and already approved drugs that are consisted of original pharmacophoric elements of approved drugs into specific subcategories of prototropic tautomer, separate enantiomer, and protomer fingerprint alternatives. $(7,8,17,18)$ Parallel Virtual screening technique for molecular docking was deployed at the center of the X: 228.75, Y: 190.82, and Z: 304.15. on its .pdbqt converted libraries of small molecules for the motif binding target sites using standard Web technologies such as CSS, HTML and JavaScript (AJAX) including graphics, text-based, and spectral files. (9,13-17) When more than one form of the screened drugs and phytical elements for the cross-validation (e.g., more than one protomer, more than one enantiomer, etc.) were screened, as suitable druggable candidates for recoring and fragmentating the forms with the highest GP and Docking Energy values were considered in the ranking. $(14,16,17)$ Finally, drugs and selected NuBBEDB phytical extracts were docked according to the descending GP docking and binding energy values and only the screened hit candidates generating the highest binding energy values were considered for fragmenting and re-merging into the Roccustyrna small molecule using the BiogenetligandorolTM cluster of algorithms from the below. 


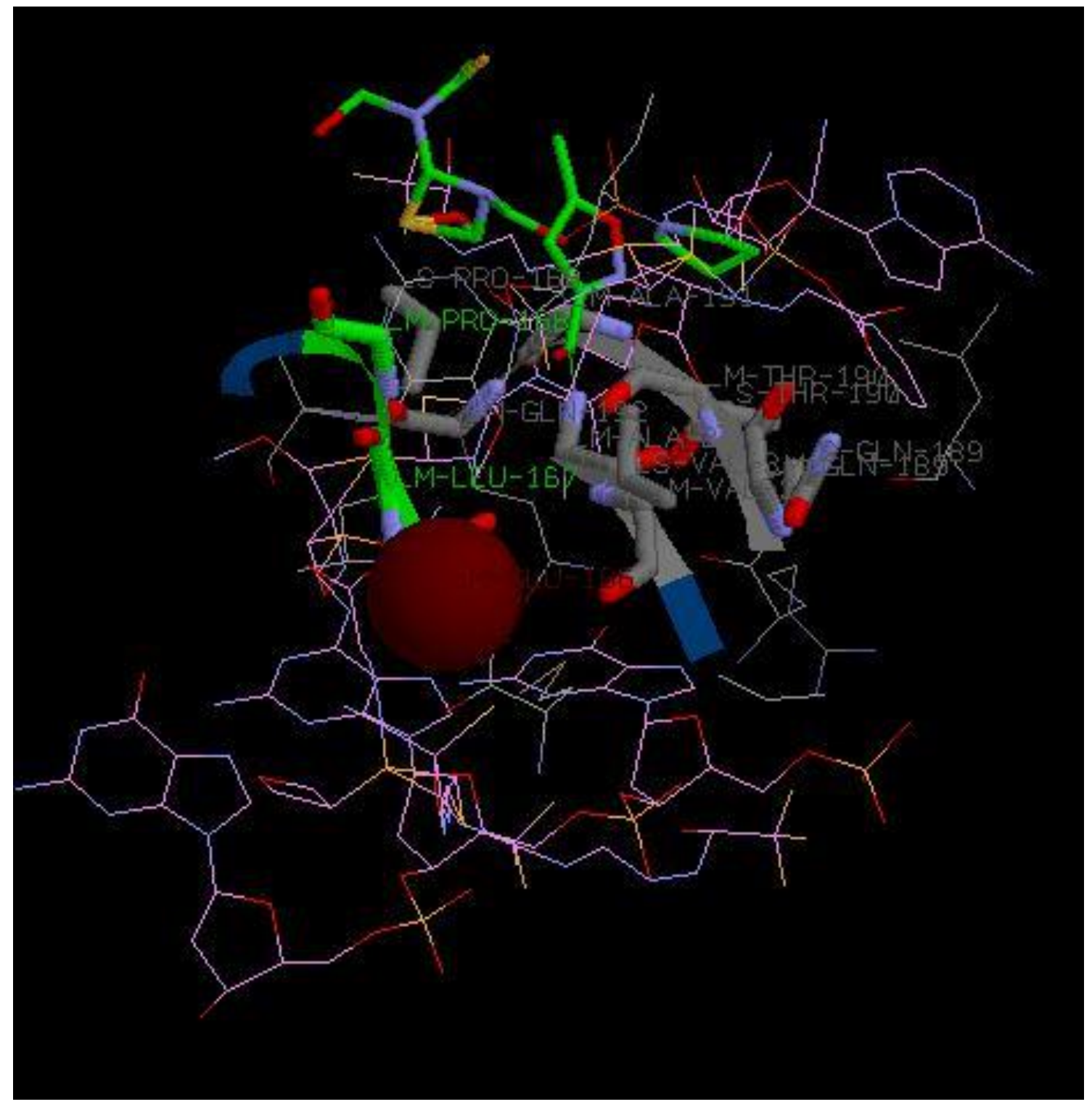

Figure 1a. 3D Docking interactions of the selected NuBEE phytical elements inside the PDB:6XS6, SARS-CoV-2 Spike SARS-COV-2 SPIKE D614G variant, minus RBD.

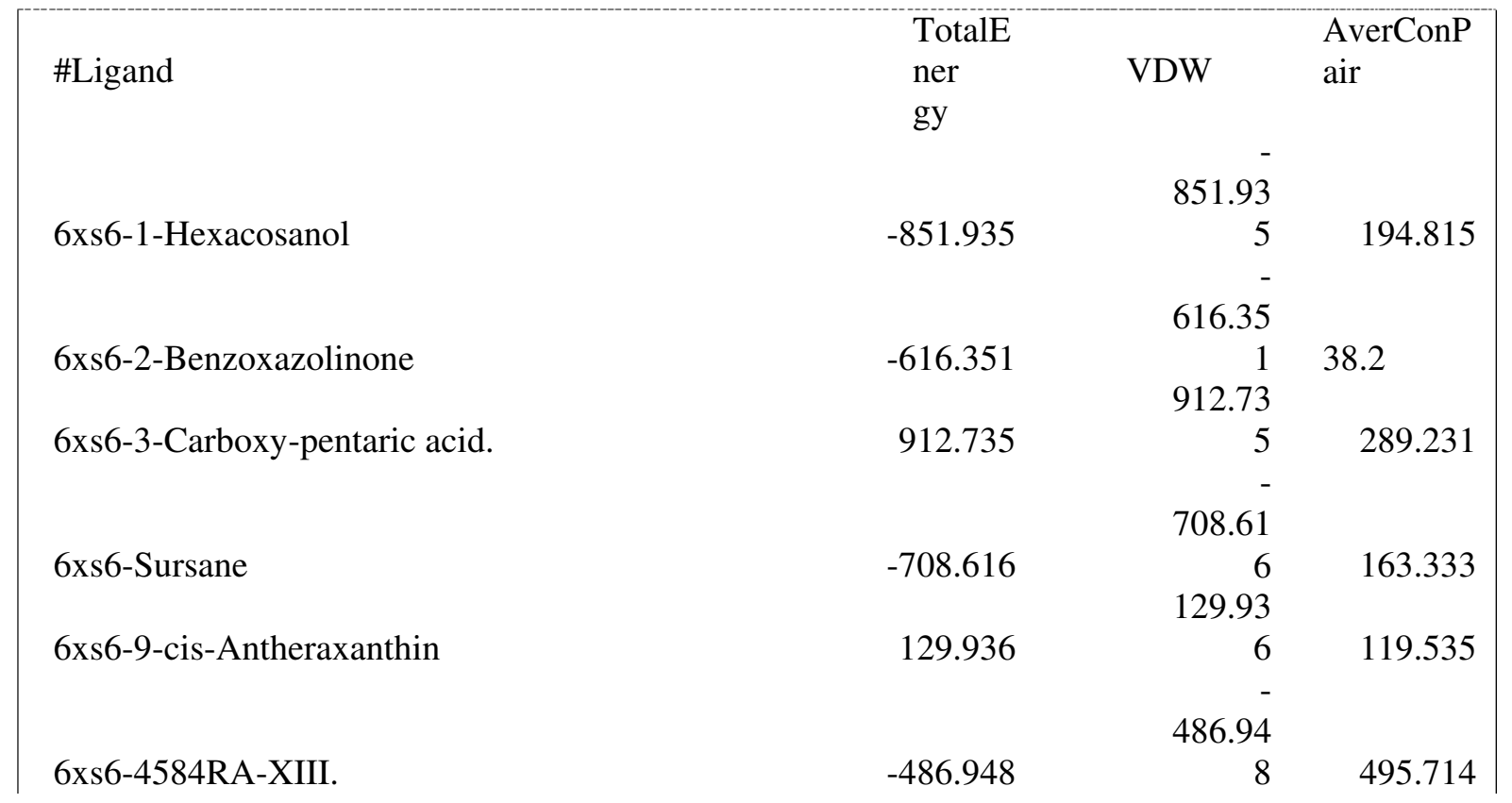




\begin{tabular}{|c|c|c|c|}
\hline \multirow{3}{*}{$\begin{array}{l}\text { 6xs6-crotonate. } \\
\text { 6xs6-abyssinica }\end{array}$} & \multicolumn{3}{|c|}{399.07} \\
\hline & -399.075 & 5 & 37.5 \\
\hline & 72.329 & 72.329 & 230.606 \\
\hline \multirow{3}{*}{ 6xs6-acacia } & & $943.23^{-}$ & \\
\hline & -943.239 & 9 & 200.303 \\
\hline & & 721.68 & \\
\hline \multirow[t]{2}{*}{ 6xs6-acetovanillone } & -721.685 & 5 & 409.167 \\
\hline & & 412.80 & \\
\hline \multirow[t]{3}{*}{ 6xs6-acteoside } & 412.805 & 5 & 227.955 \\
\hline & & - & \\
\hline & & 722.89 & \\
\hline \multirow[t]{3}{*}{ 6xs6-adenosin } & -722.898 & 8 & 259.474 \\
\hline & & - & \\
\hline & & 249.94 & \\
\hline \multirow[t]{3}{*}{ 6xs6-africanal } & -249.945 & 5 & 142.222 \\
\hline & & - & \\
\hline & & 561.75 & \\
\hline \multirow{2}{*}{ 6xs6-agarin } & -561.753 & 3 & 42.875 \\
\hline & & 147.81 & \\
\hline 6xs6-aloe-emodin & 147.811 & 1 & 18.2 \\
\hline
\end{tabular}


6xs6-alpha-L-Rhamnose
6xs6-alpha-Tocopherol
6xs6-alpha-Turmerone
6xs6-ammonium glycyrrhizate
6xs6-anemone blue anthocyanin

6xs6-aniline

6xs6-annona

6xs6-antitrypsin

6xs6-arachidonic Acid

6xs6-aristolochiac acid

6xs6-aristolochic acid

6xs6-asparagusate

6xs6-aspartic acid

6xs6-astragalin

6xs6-atraric acid

6xs6-atrazine

6xs6-avicine

6xs6-azadirachtin

6xs6-baicalein-7-methyl ether

6xs6-balanitin 3106. $\begin{array}{rrr}-54.989 & 54.989 & 303.63 \\ & & 6 \\ 238.62 & 238.62 & 210.64 \\ & & 5\end{array}$

5

$-658.863$

658.86

723.806

264.373

324.875

723.80

$\begin{array}{ll}6 & 259.83\end{array}$

1

3878.02

2

520.15

$-520.154$

$4 \quad 442.85$

215.21

$\begin{array}{lll}215.215 & 5 & 206.81\end{array}$

8

931.26

$-931.264 \quad 4 \quad 150.95$

2

759.69

$-759.696$

$6 \quad 211.81$

441.07

$\begin{array}{lrr}441.074 & 4 & 138.33\end{array}$

8

3

905.71

$\begin{array}{lll}-905.713 & 3 & 26.44\end{array}$

508.31

$-508.316$

$6 \quad 36.875$

109.24

$\begin{array}{lll}-109.242 \quad 2 & 192.22\end{array}$

$\begin{array}{lll}300.38 & 300.38 \quad 200.31\end{array}$

733.87

$-733.872$

757.60

$\begin{array}{rrr}-757.602 & 2 & 36.5\end{array}$

145.17

$\begin{array}{lll}145.172 & 2 & 21.32\end{array}$

$\begin{array}{lll}171.47 & 171.47 & 130.78\end{array}$

$\begin{array}{lll}-68.083 & 68.083 \quad 191.42\end{array}$

663.53

$\begin{array}{lll}663.539 & 9 & 13.871\end{array}$ 
6xs6-balanitin 4107.

6xs6-balanitin 5108.

6xs6-balanitin 7
258.27

$-258.278$

$\begin{array}{ll}8 & 19.589\end{array}$

$\begin{array}{lll}-87.321 & 87.321 & 994.44\end{array}$

887.16

$-887.161$

193.662 


\begin{tabular}{|c|c|c|c|}
\hline 6xs6-baueronol & -707.698 & $\begin{array}{r}707 . \\
69 \\
8\end{array}$ & 16.129 \\
\hline 6xs6-b-Chlorogenin & -707.493 & $\begin{array}{r}707 . \\
49 \\
3\end{array}$ & 171.613 \\
\hline 6xs6-behenic acid & -746.006 & $\begin{array}{r}746 . \\
00 \\
6\end{array}$ & 213.333 \\
\hline 6xs6-benzo(c)phenanthridine & -713.002 & $\begin{array}{r}713 . \\
00 \\
2\end{array}$ & 321.429 \\
\hline 6xs6-Benzyl alcohol & -47.602 & $\begin{array}{r}47.60 \\
2 \\
907.5\end{array}$ & 31.875 \\
\hline 6xs6-benzyl isothiocyanate (BITC) & 907.592 & $\begin{array}{l}9 \\
2\end{array}$ & 24.1 \\
\hline 6xs6-benzylic amines & -788.472 & $\begin{array}{r}788 . \\
47 \\
2 \\
111.5\end{array}$ & 8.625 \\
\hline 6xs6-berberine alkaloids & 111.594 & $\begin{array}{r}9 \\
4\end{array}$ & 15.44 \\
\hline
\end{tabular}

Table1a. Docking energy rankings of the selected phytical hit compounds when docked onto the SARS-COV-2 protein targets of the (pdb:6xs6). 
The whole set of molecules able to interact with the SARS-CoV PLpro enzyme were extracted after extensive machine learning similarity studies, retrieving only those small molecule ligands with absolute IC50 values. $(2-17,34)$ This druggable .sdf set consisted of 11 PLpro approved inhibitors. Depending on the cross docking energy activities and fitness scoring analysis threshold, out of 11 compounds, 300 small molecule compounds with proven anti-viral properties were characterized as Actives and 153 molecules were categorized as Inactives. Physarum-prize-collective for molecular docking algorithm, Schrodinger-inspired Neural Matrix Factorization and a drug repositioning scoring analysis were implemented to a hybrid collection of the Natural Products of the Chemistry Institute of UNESP, Araraquara/SP and NuBBEDB phytical extracts (9-17,35) . (10-17,36) Protein-molecule complexes, (4,5,7,8-17) followed by structural relaxation were generated through $(8,13-117)$ flexible-ligand rigid-receptor molecular docking $(7,9,13,16-17)$ in these local energy minimization to optimize protein-ligand interactions capping the $\mathrm{C}$ - and $\mathrm{N}$-terminal for each active druggable fragment with i-GEMDOCK $(3-14,17)$ through cycles in all keeping conserved amino-acids within $4 \AA$ of all the docked ligands of each cation arrangement as considered for each relaxed chemical structure free of local energy and geometry minimizations. $(1,2,5-17,34,35)$ Virtual screening experiments generated with KNIME pipelined DockThor Virtual Screening tool for the NuBBEDB and e-Drug3D dataset and for the ChEMBL database, at the reference $\mathrm{pH}$ (6.6 to 7.4) for all SARS-CoV-2 drug targets including the contig maps (a set of overlapping DNA segments) as were assembled with the use of CLC Genomics software, version 4.6.1 (CLC Bio)publicly and are available so far. (BetaCoV/Wuhan/IVDC-HB-01/2020|EPI_ISL_402119), (e.g., N-terminal S1 subunit, PLpro, NendoU, (residue 14-685) in Spike Mpro,RdRp, and complete genome sequences of the three novel coronaviruses were submitted to GISAID (BetaCoV/Wuhan/IVDC-HB-01/2019, accession ID: EPI_ISL_402119; BetaCoV/Wuhan/IVDC-HB-04/2020, accession ID: EPI_ISL_402120; BetaCoV/Wuhan/IVDC-HB-05/2019, accession ID: EPI_ISL_402121) and a C-terminal S2 region N protein) were prepared for GEMDOCK docking experiments using the wild isogenic type of the transcriptomic variants and 10 best matching phytical and chemical small molecule compounds (Table1a) after exhaustive virtual screening analysis were obtained accordingly, Colchicine, Raltegravir, Hexacosanol, Benzoxazolinon, Carboxy-Pentaric acid, Ursane, Antheraxanthin, RA-XIII, Crotonate and Byrsonima Coccolobifolia against the SARS-COV-2 protein targets of the (pdb:1xak), (pdb:6xs6) and (pdb:6lu7). (6,7-17,36) For each target, all crucial amino-acids involved virtually in this project of the cut-out parallel docking system when linked together with hydrogens were then collected and the hit candidates who energetically favored within $8 \AA$ of any docked molecule onto the hydrophobic side chain were then used to build a reduced phase docking system to be in contact with water where the -o C[C@]1(C[C@H] ([C@H]2 N1CN2)ONN1C[P@]1(C[C@H]1O[C@H](C[C\| subscript. In the first smiles term in the spaces of targeted sequences refered to the difference of the free energy calculated using the protein-ligand (PL), protein (P) and ligand (L) sequence of amino acid conformations bonded evaluations for dispersion/repulsion, hydrogen bonding, electrostatics, and desolvation to the central atom of each screened compound. $(10,17,37)$ (Figure1a).The GQM (X) refers to the energy of the cluster of the phytical small molecule known as the $\mathrm{R}$ group from the docked complex, in the free unbound state the fourth term corresponds to the change in conformational entropy, which were generated and the second and third unbound states of ligand side-chain conformations are calculated through local energy minimization as $\Delta \mathrm{GQMconf}(\mathrm{X})=\mathrm{GQMo}(\mathrm{X})-\mathrm{GQM}(\mathrm{X}),(\mathrm{X}=\mathrm{L}, \mathrm{P})(2)$ where GQMo $(\mathrm{X})$ is the energy of the isolated active fragments in the conformation of the docked PL on both protein and molecule complex Inhibitors and conformations when applied Bioactivity-Guided Fractionations for the cluster of the selected compounds of the Colchicine, Raltegravir, Hexacosanol, Benzoxazolinon, Carboxy-Pentaric acid, Ursane, Antheraxanthin, RA-XIII, Crotonate and Byrsonima coccolobifolia Leaves and Stems to be fragmented, re-cored and accordingly merged into the RoccustyrnaTM small hyperactive druggable scaffold $(3,4,17,36)$. The acknowledgement of the binding of the selected 8 phytical compounds to their full-genome evolutionary novel coronavirus (2019-nCoV) protein targets was accomplished using Molinspiration (http://www.molinspiration.com/cgi-bin/properties) and DrugBank $(1,11,12,13,15,17,34)$. 


\section{Pharmacophoric-ODEs fragmentating, merging and recoring : Biogenetoligandorol AI-heuristic algorithm.}

The patterns of this Biogenetoligandorol fragmentation scheme are sorted into the working inputs of the Galilean transformation by examining the -extended\| Galilean transformation based on a set of heuristically determined descriptors moving with relative acceleration to a rigid system to a nonrotating geometric observer having an arbitrary time-dependent acceleration $(20,21,27,28)$. $(25,29)$ These descriptors as an applied nonconcurrent to both translate and rotatecan be, for example, the number of atoms describing the pattern of a force system which can both translate and rotate and be determined by the substitution as a polynomial in ip $(r, t)=e i J\{r, t)\left(p\left(r^{\prime}, t\right)\right.$. V'ip $=\left(V^{\prime} i p+i V\right.$ 'f) eif, $\rho-s Q F I \approx \sum i=01 \operatorname{Re}(\rho i 12) 2$ $(1 \rho i 11+1 \rho \mathrm{i} 22)+(\rho \mathrm{i} 11-\rho \mathrm{i} 22) 2 \rho \mathrm{i} 11+\rho \mathrm{i} 22, \rho 1 \mathrm{ij}=\langle\varphi \mathrm{i}\langle 1 \rho \mathrm{\rho s}(0) \varphi \mathrm{j}\rangle \quad 1\rangle \rho 0 \mathrm{ij}=\langle\varphi \mathrm{i}\langle 0 \rho s(0) \varphi \mathrm{j}\rangle \quad 0\rangle \quad 0\rangle$

1) $\left.\left.\left.\mathrm{H} 1=\gamma \mathrm{B} 0 \cdot \mathrm{S}^{\wedge} 1 \mathrm{Re}(\rho \mathrm{1} 12) \rho \mathrm{i} 12 \rho \mathrm{s}(0) \rho^{-} \mathrm{s} \mathrm{S}\right\rangle=12(\rangle-01\right\rangle\right) 30 \% \rho s(0) \rho^{-} \mathrm{s}=\mathrm{H} \otimes \mathrm{m} 0 \mathrm{~V}^{\prime} 2 \mathrm{ip}=\left\{\mathrm{V}^{\prime} 2 \mathrm{ip}+2 \mathrm{iV} \mathrm{f}^{\prime}-\mathrm{V}^{\prime}\left(\mathrm{p}+\left(\mathrm{pV} \mathrm{V}^{\prime} \mathrm{f}+\mathrm{W}\right.\right.\right.$

$\left.\left.\leftarrow \mathrm{S}(\mathrm{I} \otimes \mathrm{H}) \mathrm{M} 1 ;<\mathrm{p}\left(\mathrm{V}^{\prime} \mathrm{f}\right) 2\right) \mathrm{e}_{\mathrm{f}} \mathrm{f}, \mathrm{i}\right\rangle=\langle\Phi|\mathrm{MT}(\mathrm{I} 3-\mathrm{NT})-2(\rho \otimes \mathrm{I} 2)| \Phi\rangle$ (f) + if $\langle\mathrm{p})$ eif, and the Schrödinger equation becomes n 22

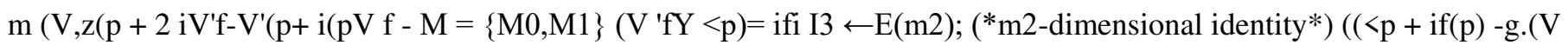
' $\left(p+i\left(p v^{\prime} f\right)\right)$ where $p+2$ are the the number of bonds predicted or the number of double bonds. $(20,25)$

\section{Structure}<smiles>[NH3+]C1=NC(=O)C2=NC=NC2N1</smiles><smiles>[NH3+]C1NC(O)C2NC=NC2N1</smiles>

ID

Mass

152.0566862

6

158.1036364
SMILES

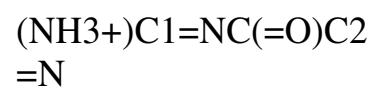

$(\mathrm{NH} 3+) \mathrm{C} 1 \mathrm{NC} 2 \mathrm{~N}=\mathrm{CNC}$ $2 \mathrm{C}$ 


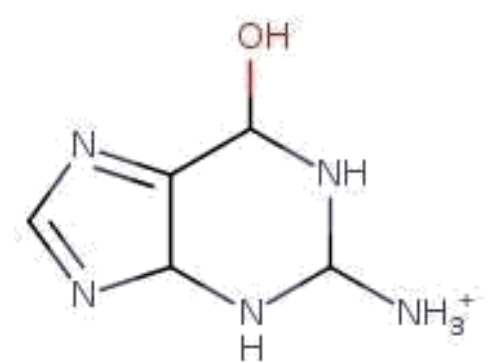

No

Ima

ge<smiles>N=C1N=CC2=NC=NC2=N1</smiles><smiles>[OH+]=C1N=CN=C2N=CN=C12</smiles>

156.0879864

$(\mathrm{NH} 3+) \mathrm{C} 1 \mathrm{NC} 2 \mathrm{~N}=\mathrm{CN}=\mathrm{C}$

\section{2}

8

154.0723363

$(\mathrm{NH} 3+) \mathrm{C} 1 \mathrm{NC}(=\mathrm{O}) \mathrm{C} 2=\mathrm{N}$
$\mathrm{C}$

9

134.0461215

$(\mathrm{NH} 2+)=\mathrm{C} 1 \mathrm{~N}=\mathrm{CC} 2=\mathrm{NC}$

$$
=
$$

10

135.0301371

$(\mathrm{OH}+)=\mathrm{C} 1 \mathrm{~N}=\mathrm{CN}=\mathrm{C} 2 \mathrm{~N}=$ 
<smiles>NC1=NC(=O)C(=[NH2+])C=N1</smiles><smiles></smiles><smiles>C=C1N=CN=C1C=O</smiles>

12

13<smiles>NC=[NH2+]</smiles>

14 43.02907452 $\mathrm{HN}=\mathrm{NH}_{2}$
135.0301371

45.04472458

$\mathrm{NC}=(\mathrm{NH} 2+)$ C1

$\mathrm{NC} 1=\mathrm{NC}(=\mathrm{O}) \mathrm{C}(=(\mathrm{NH} 2$ $+)$

$(\mathrm{NH}+) \# \mathrm{CN}=\mathrm{C} 1 \mathrm{~N}=\mathrm{CN}=$ 

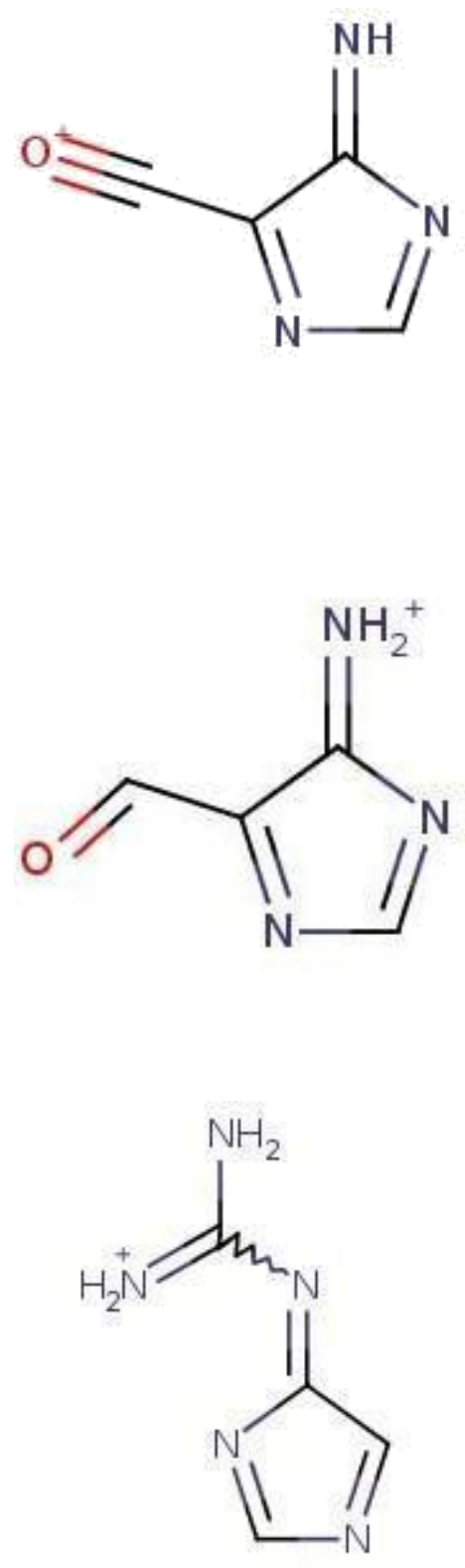

17 $\mathrm{NC}(=(\mathrm{NH} 2+)) \mathrm{N}=\mathrm{C} 1 \mathrm{C}=$ $\mathrm{NC}$<smiles>N=C1N=CN=C1C([NH3+])=O</smiles> 


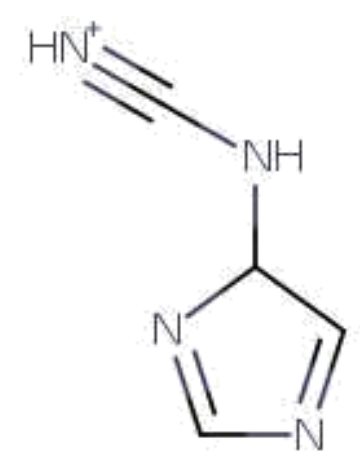

19
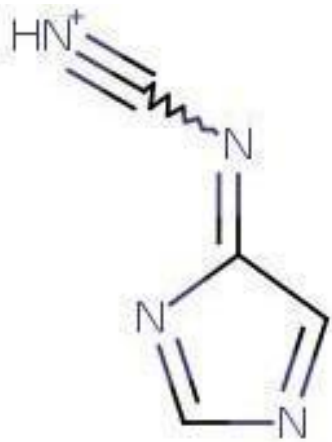

20

107.0352225

21

82.03997355

$(\mathrm{NH} 2+)=\mathrm{C} 1 \mathrm{C}=\mathrm{NC}=\mathrm{N} 1$<smiles>[NH3+]C1=NC(=O)C2=NC=NC2=N1</smiles> 


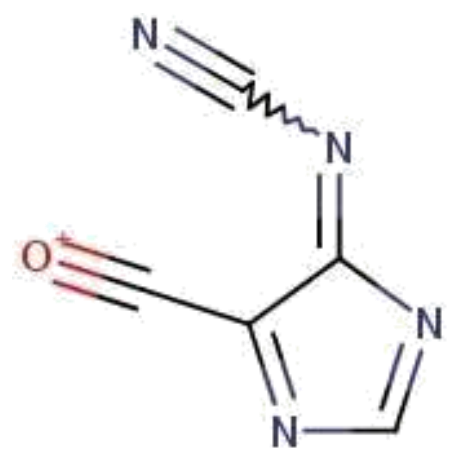

\section{$23 \quad 133.0144871$ $\mathrm{N} \# \mathrm{CN}=\mathrm{C} 1 \mathrm{~N}=\mathrm{CN}=\mathrm{C} 1 \mathrm{C}$ \#(}

$\mathrm{N} \equiv \mathrm{C}^{+}$

$\mathrm{CH}_{3}^{+}$

95 55.05422664

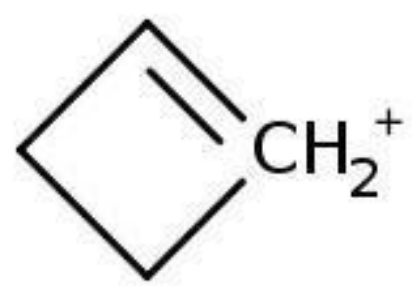



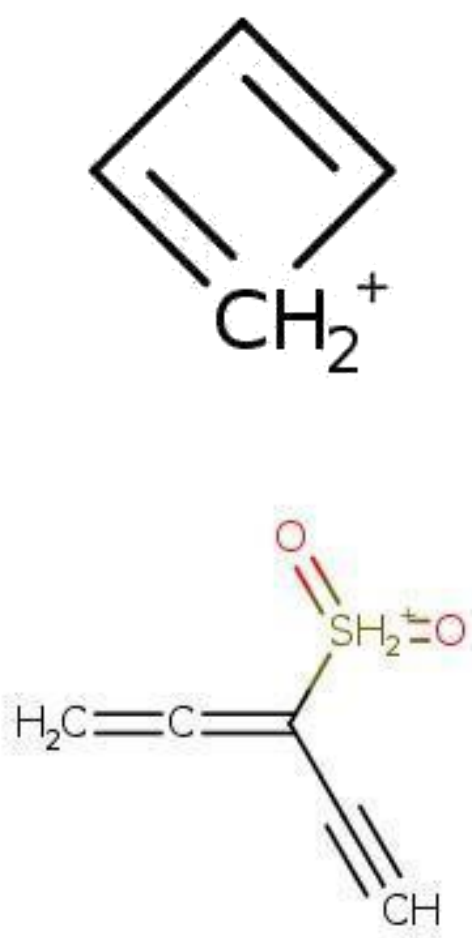
$\mathrm{O})=\mathrm{O}$
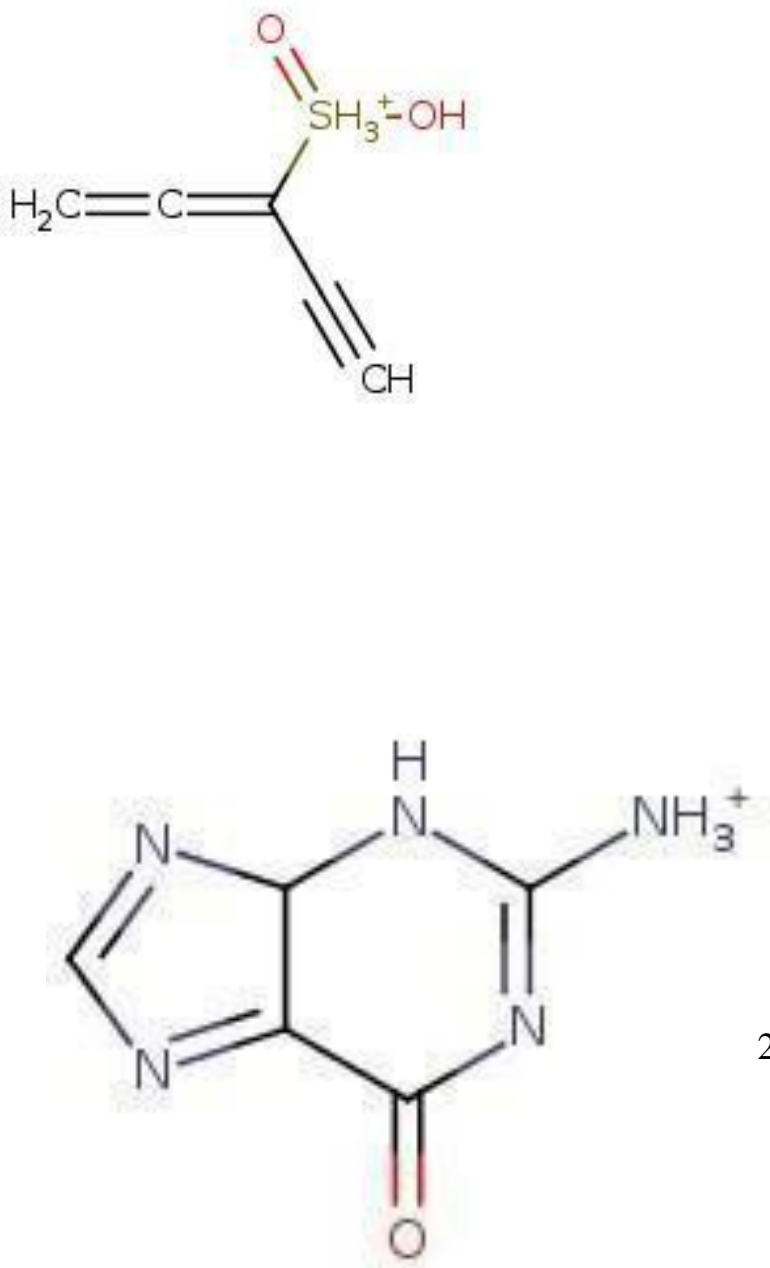

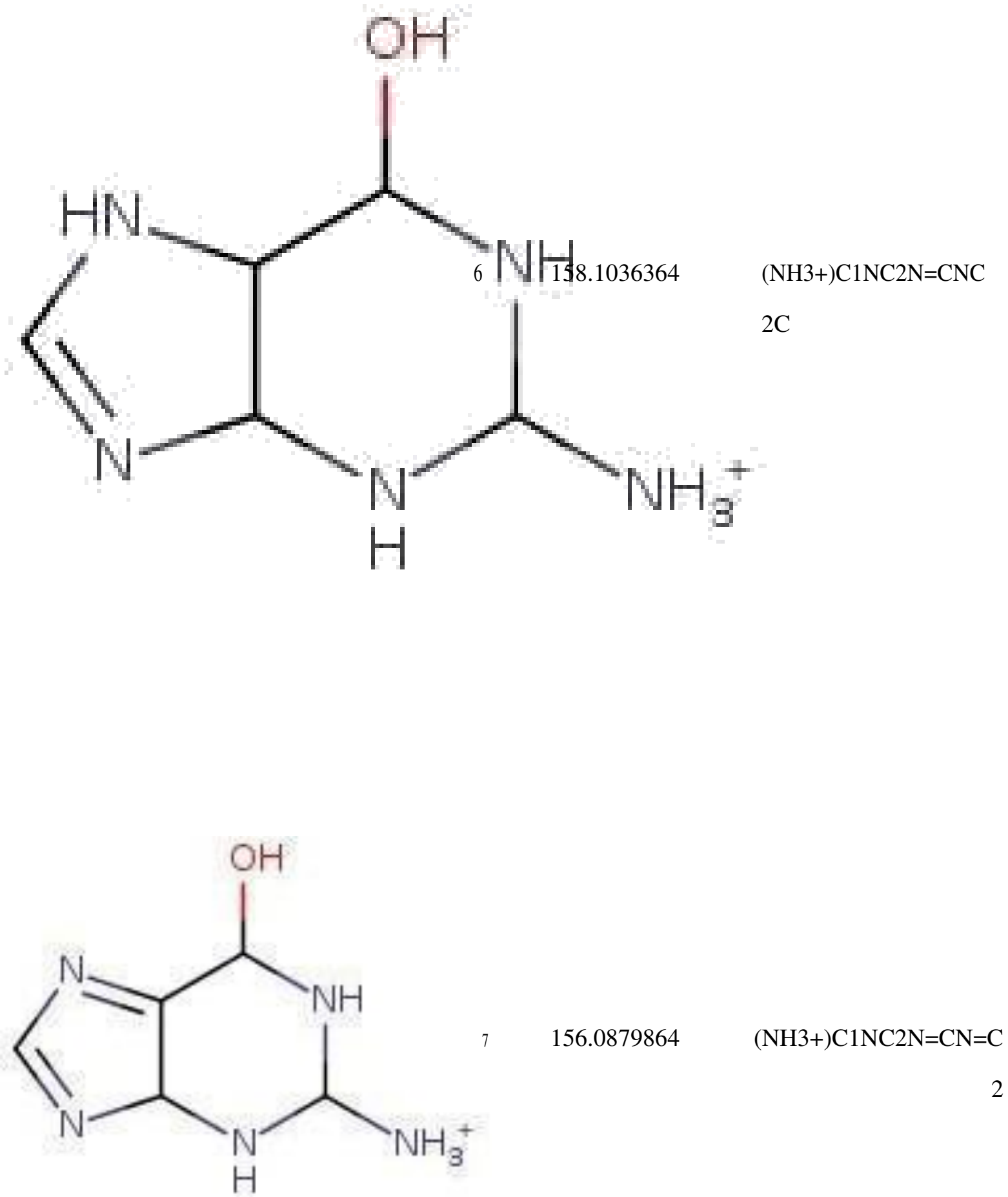


$$
\text { No }
$$

8

Image

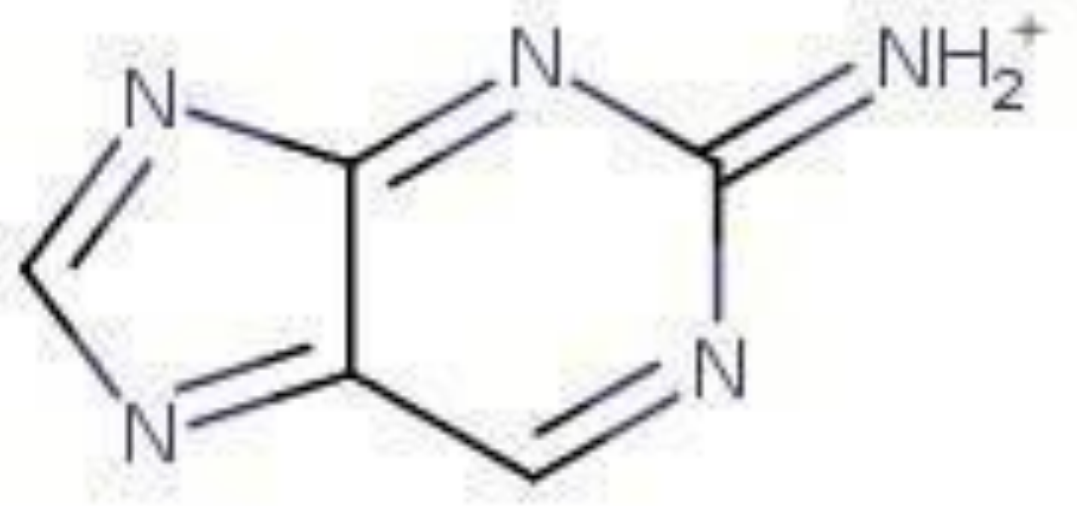

$9 \quad 134.0461215$

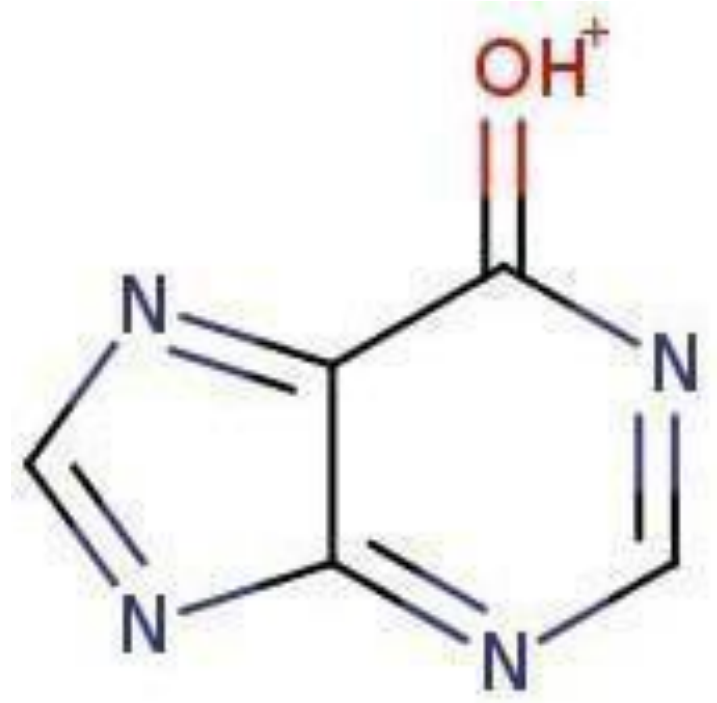

154.0723363

$(\mathrm{NH} 3+) \mathrm{C} 1 \mathrm{NC}(=\mathrm{O}) \mathrm{C} 2=\mathrm{N}$
$(\mathrm{NH} 2+)=\mathrm{C} 1 \mathrm{~N}=\mathrm{CC} 2=\mathrm{NC}$

$$
=
$$
$\mathrm{C}$ 

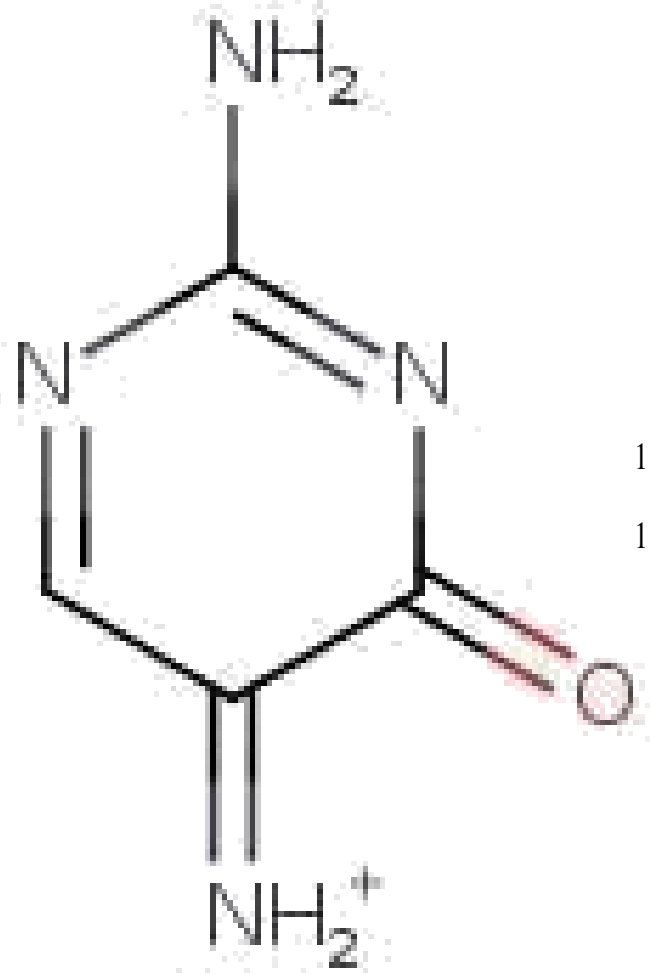

$125.0457872 \quad \mathrm{NC} 1=\mathrm{NC}(=\mathrm{O}) \mathrm{C}(=(\mathrm{NH} 2$

$+)$

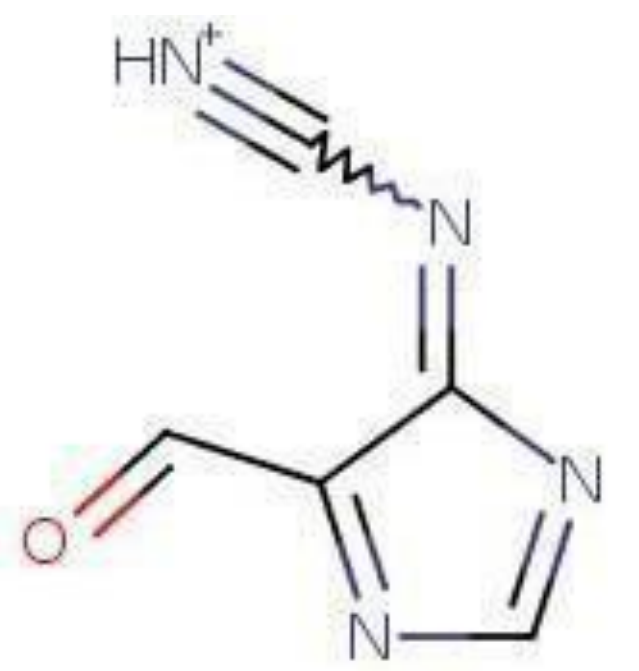

135.0301371

$(\mathrm{NH}+) \# \mathrm{CN}=\mathrm{C} 1 \mathrm{~N}=\mathrm{CN}=$ C1

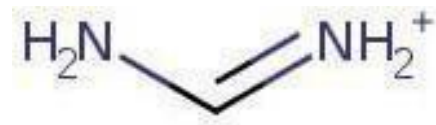




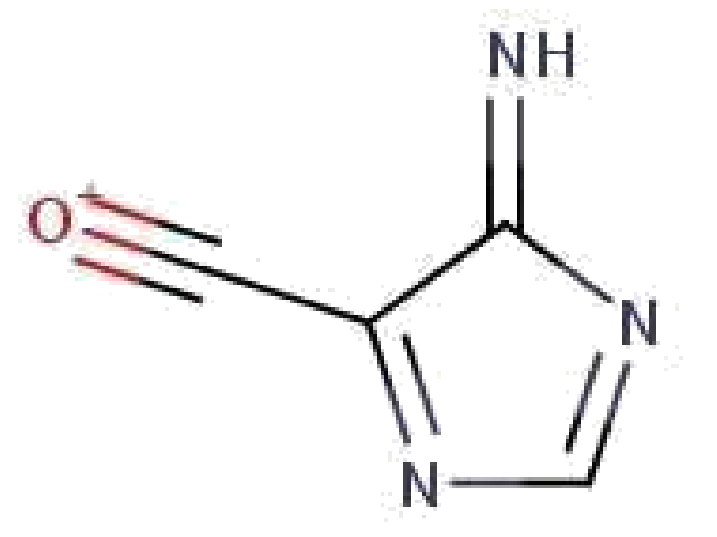

$+)$

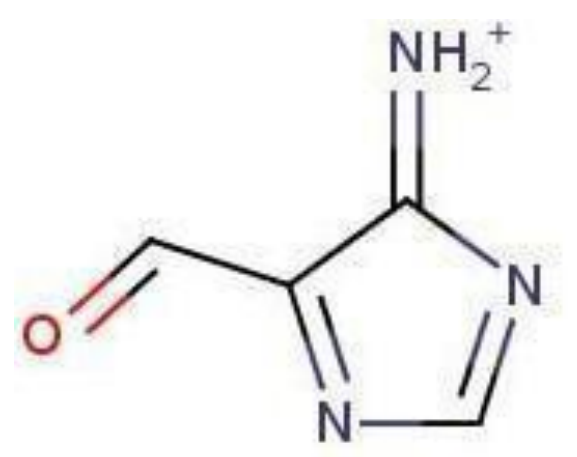

110.0348882

$(\mathrm{NH} 2+)=\mathrm{C} 1 \mathrm{~N}=\mathrm{CN}=\mathrm{C} 1$

$\mathrm{C}=$ 

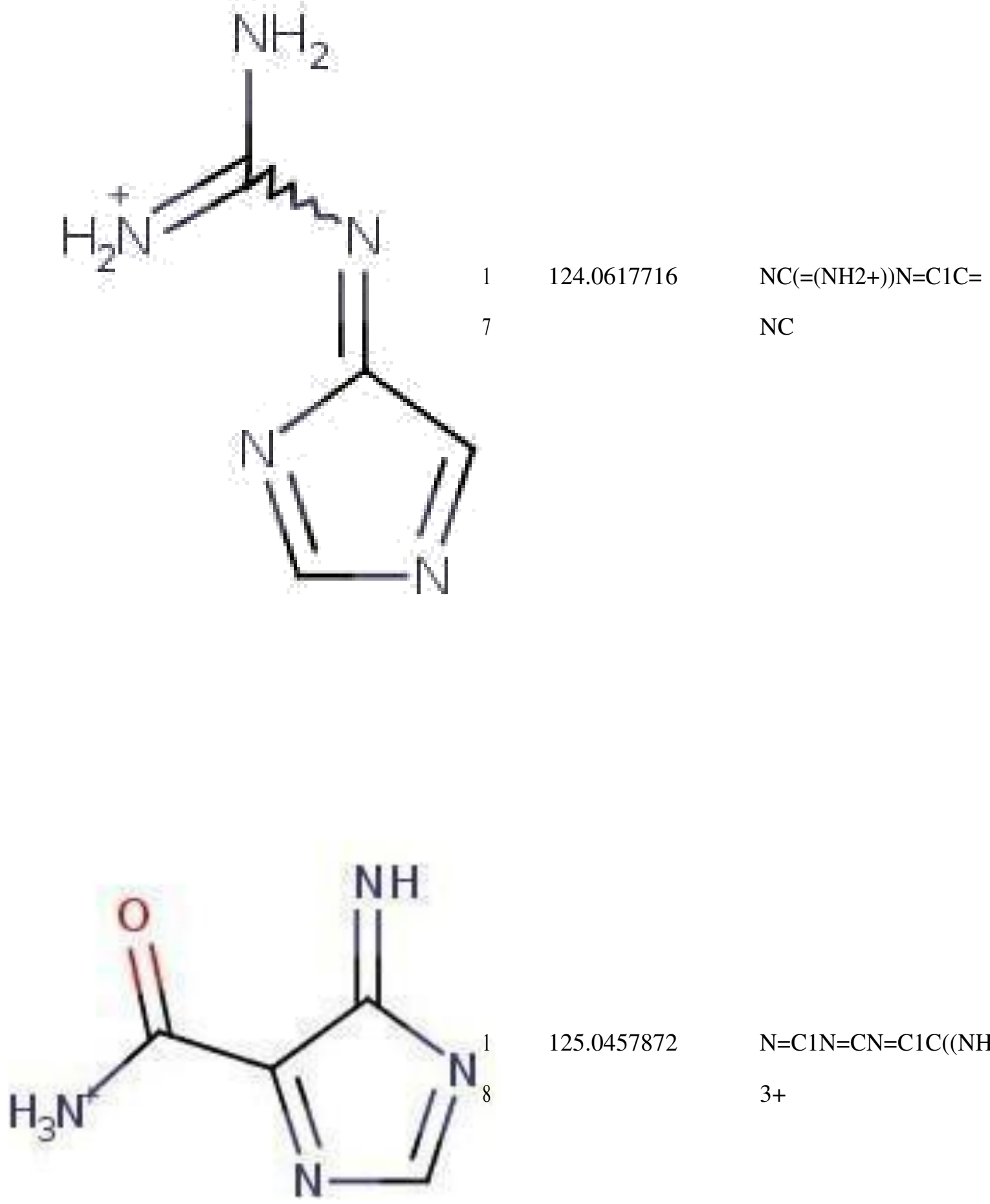

125.0457872

$\mathrm{N}=\mathrm{C} 1 \mathrm{~N}=\mathrm{CN}=\mathrm{C} 1 \mathrm{C}((\mathrm{NH}$ $3+$ 

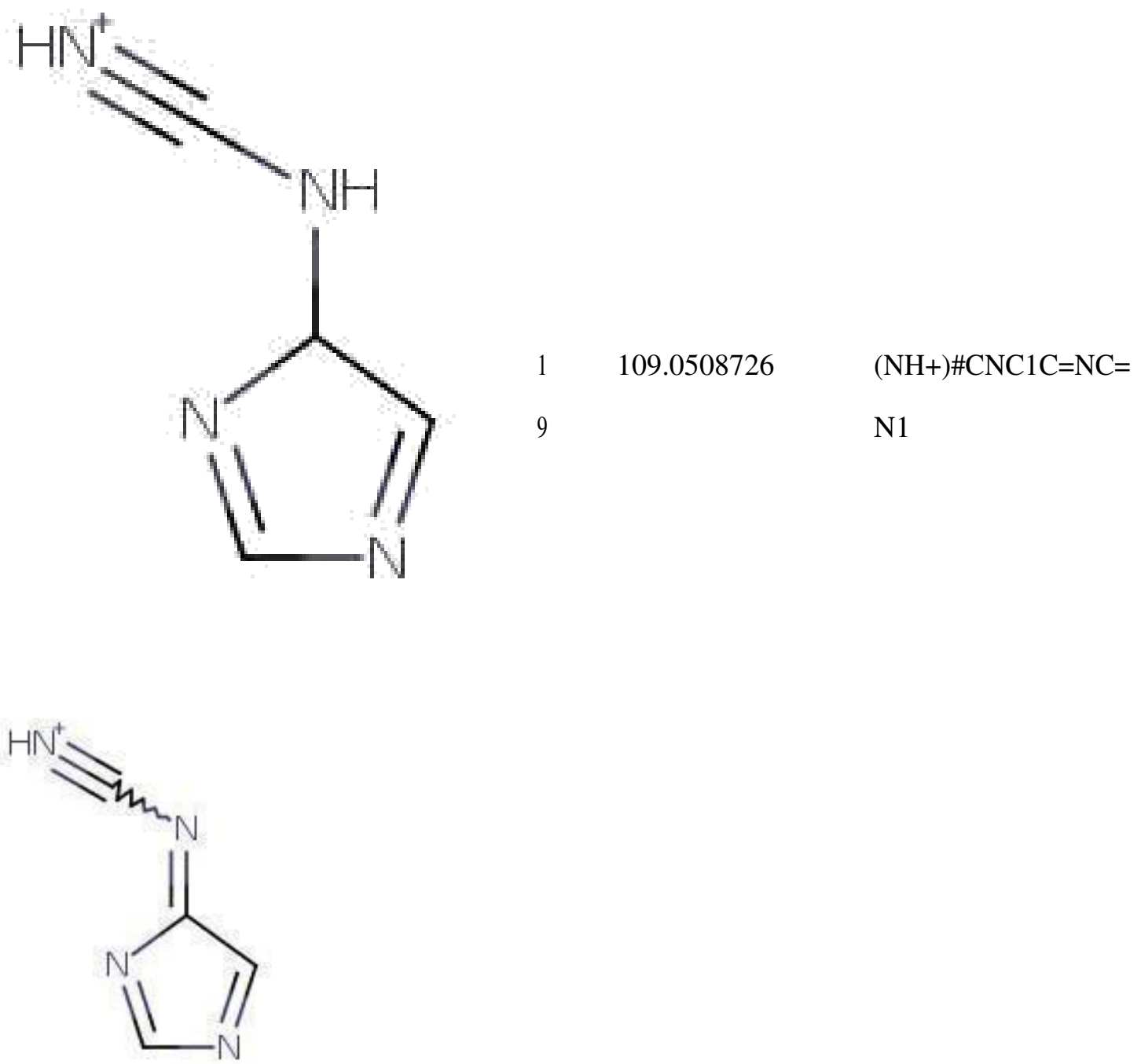

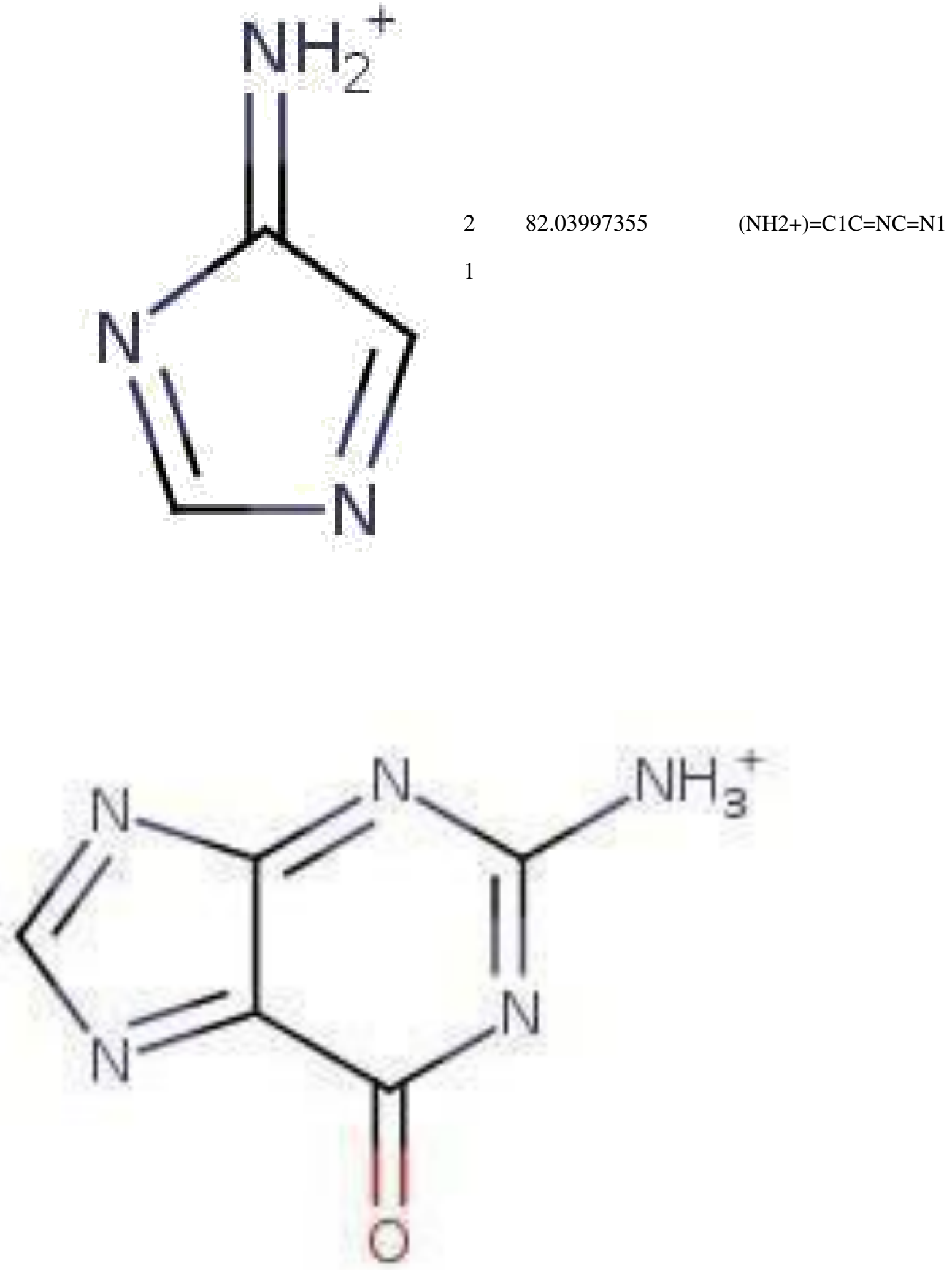

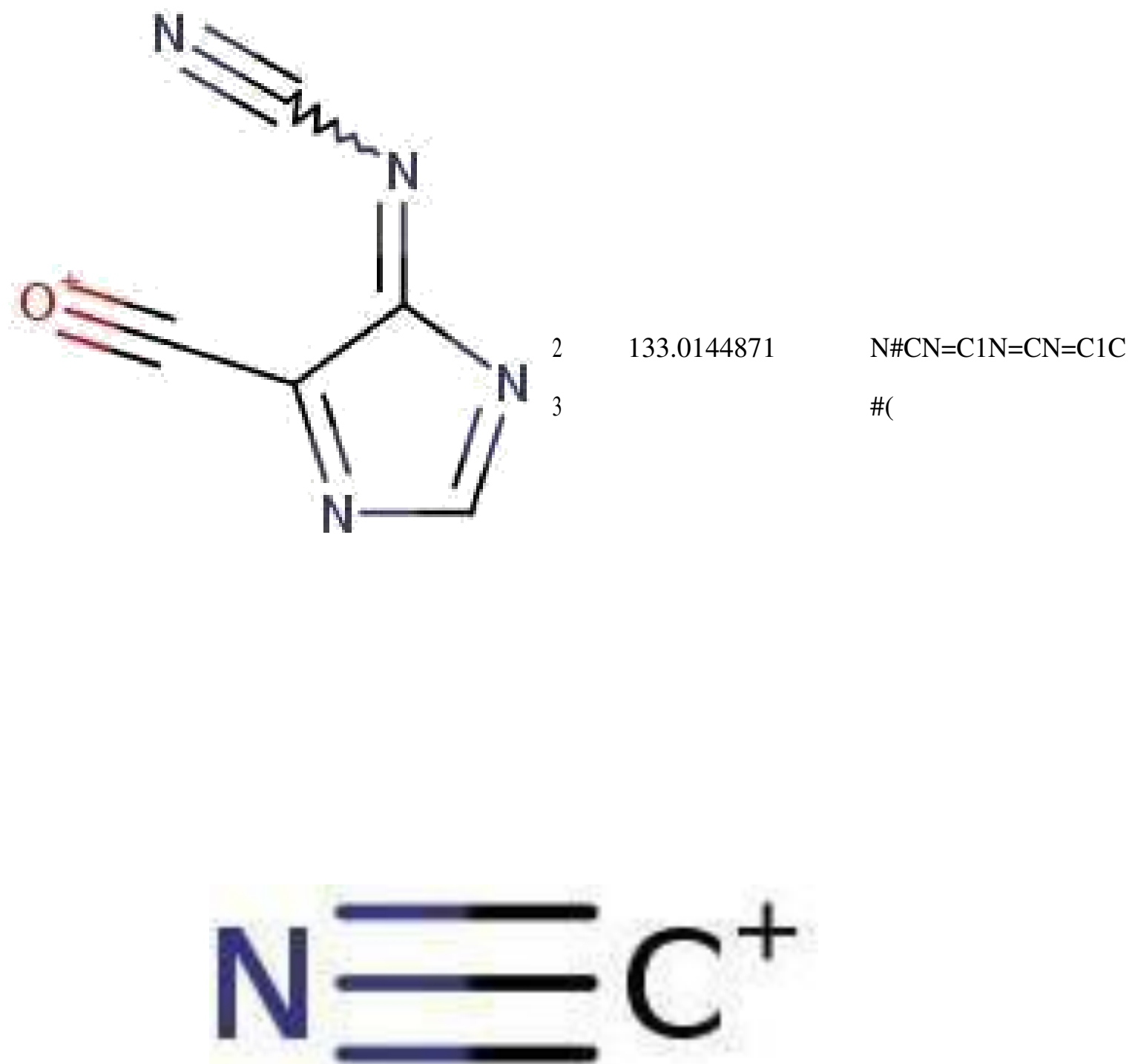

$95 \quad 55.05422664$

$\mathrm{C} 1=[\mathrm{CH} 2+] \mathrm{CC} 1$

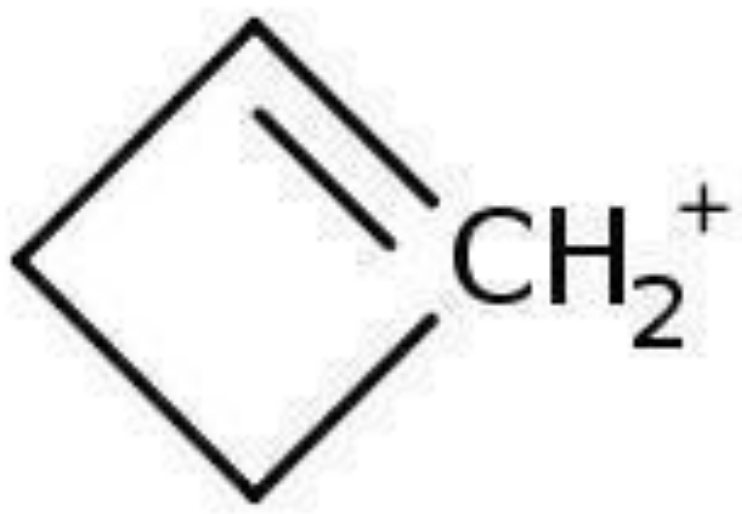



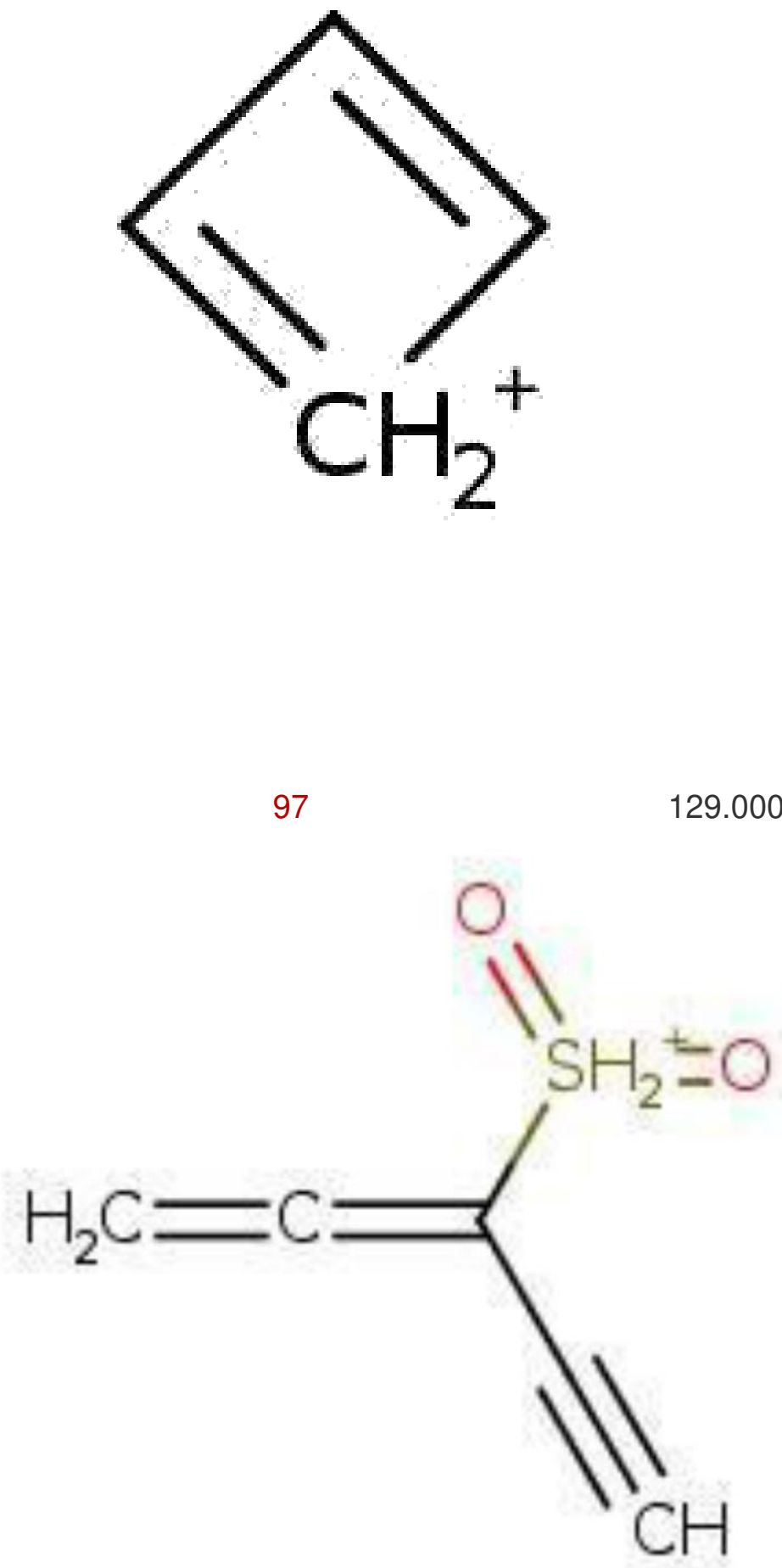

98

131.0161269 $\mathrm{C \# CC}(=\mathrm{C}=\mathrm{C})[\mathrm{SH} 3+](=\mathrm{O}) \mathrm{O}$

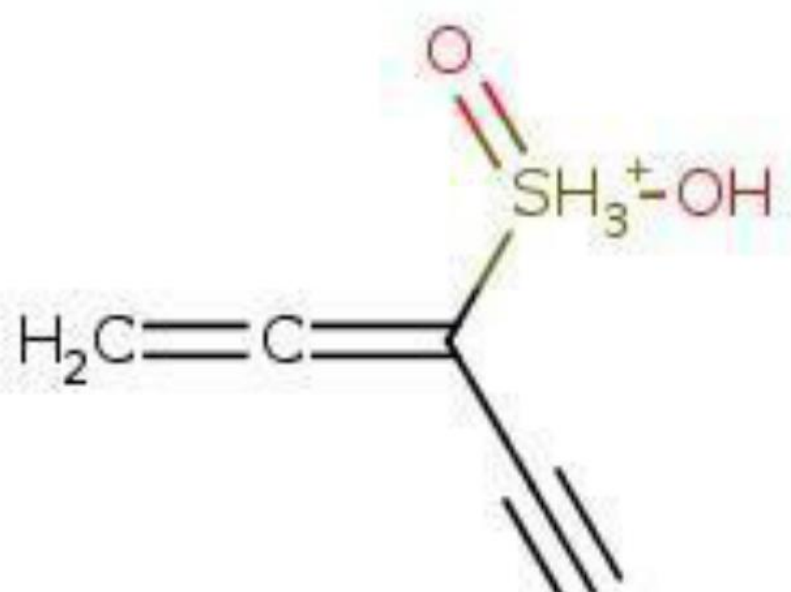



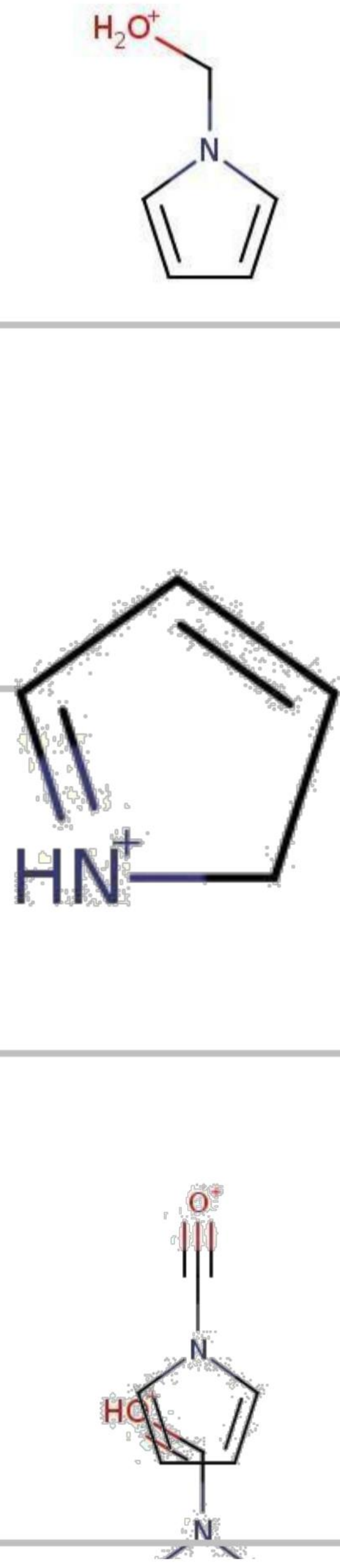

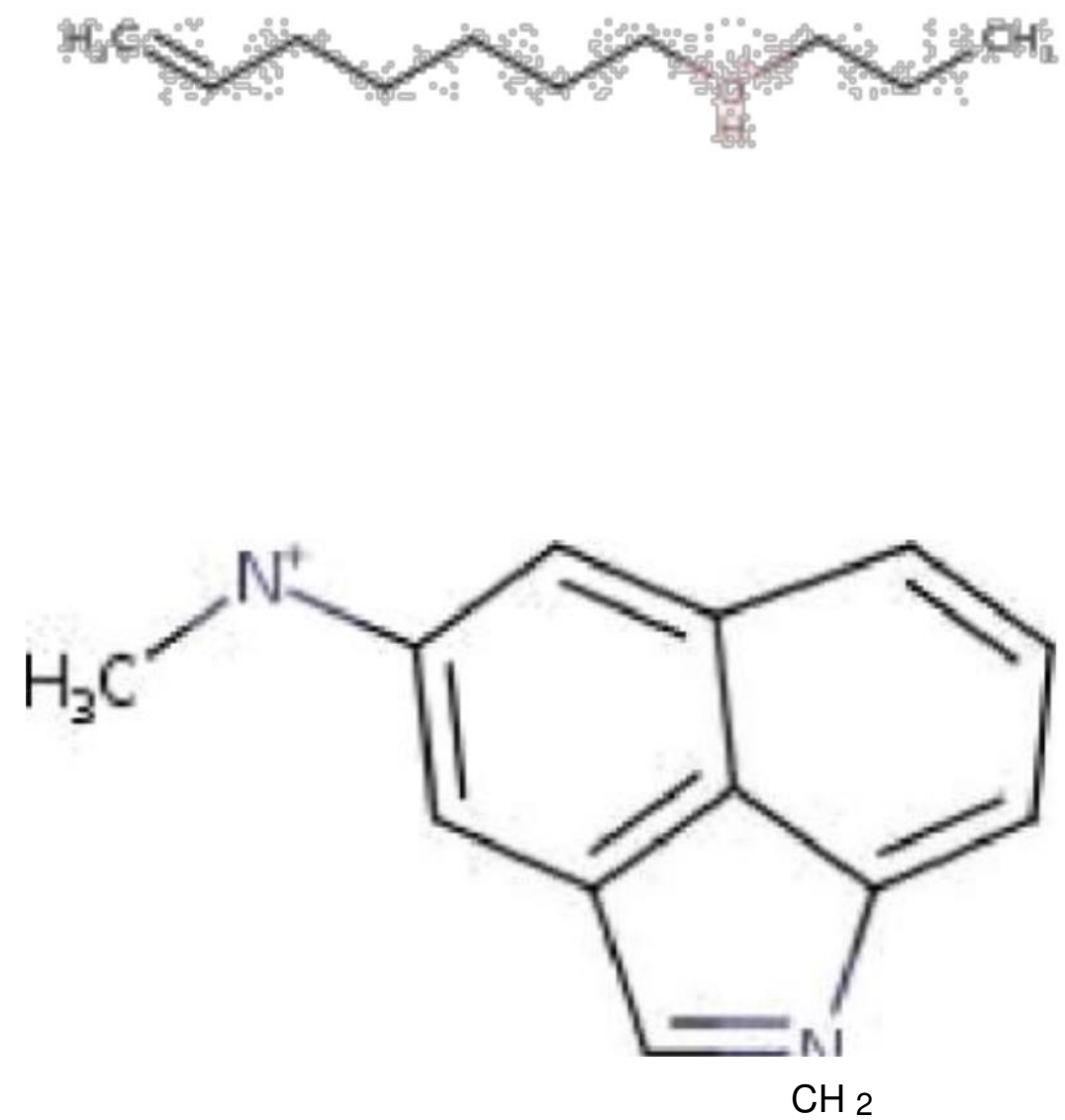

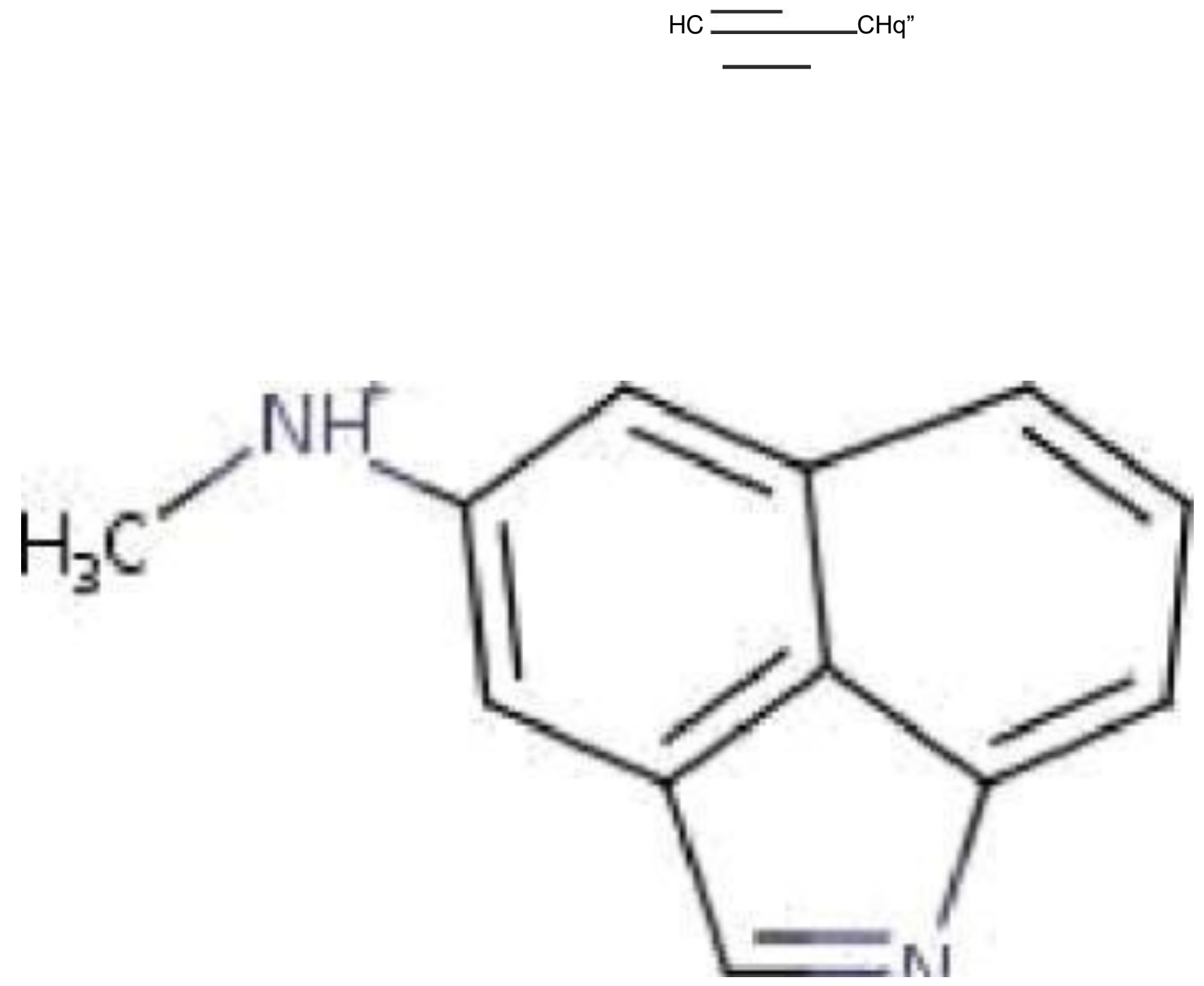


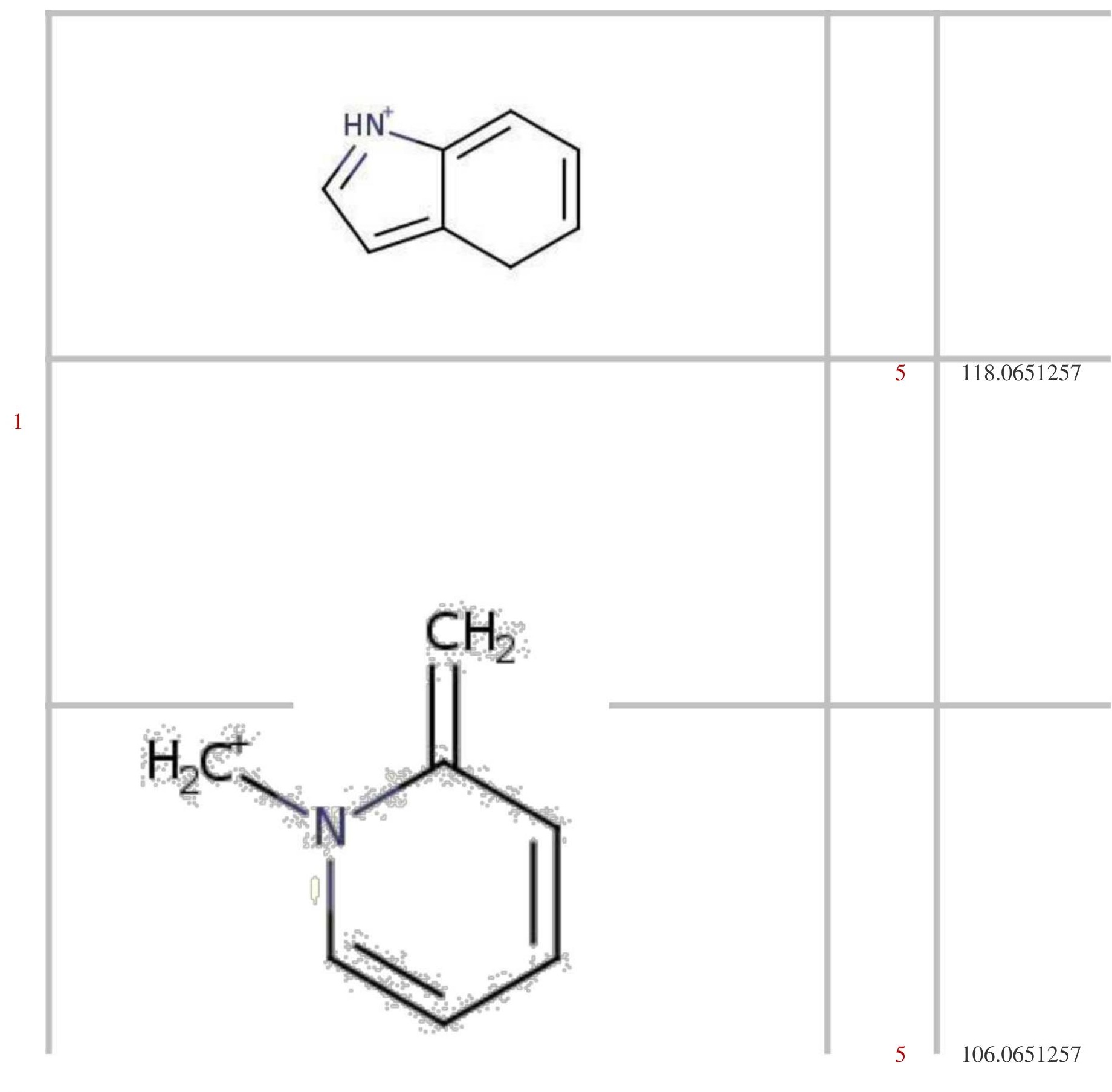




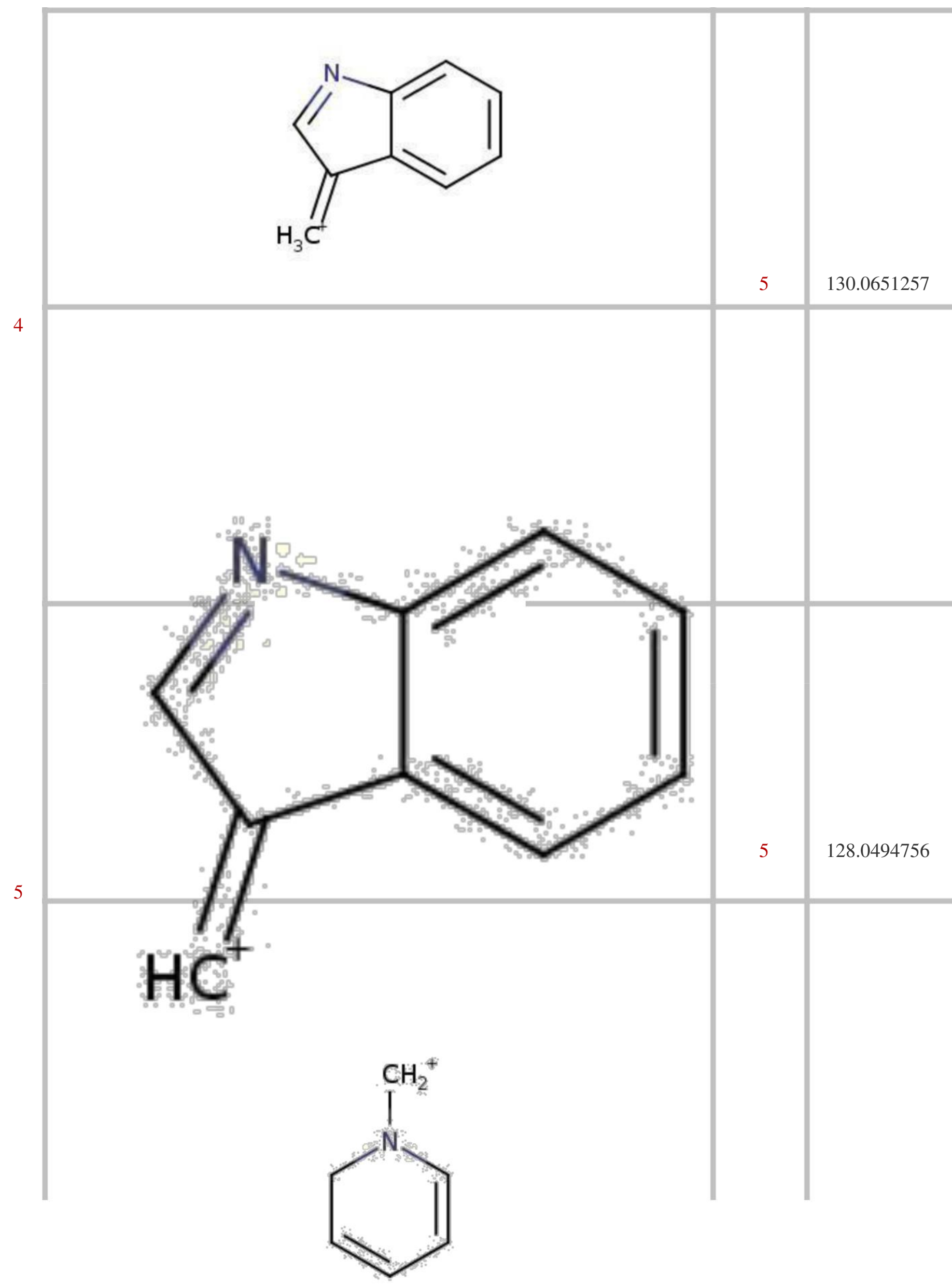



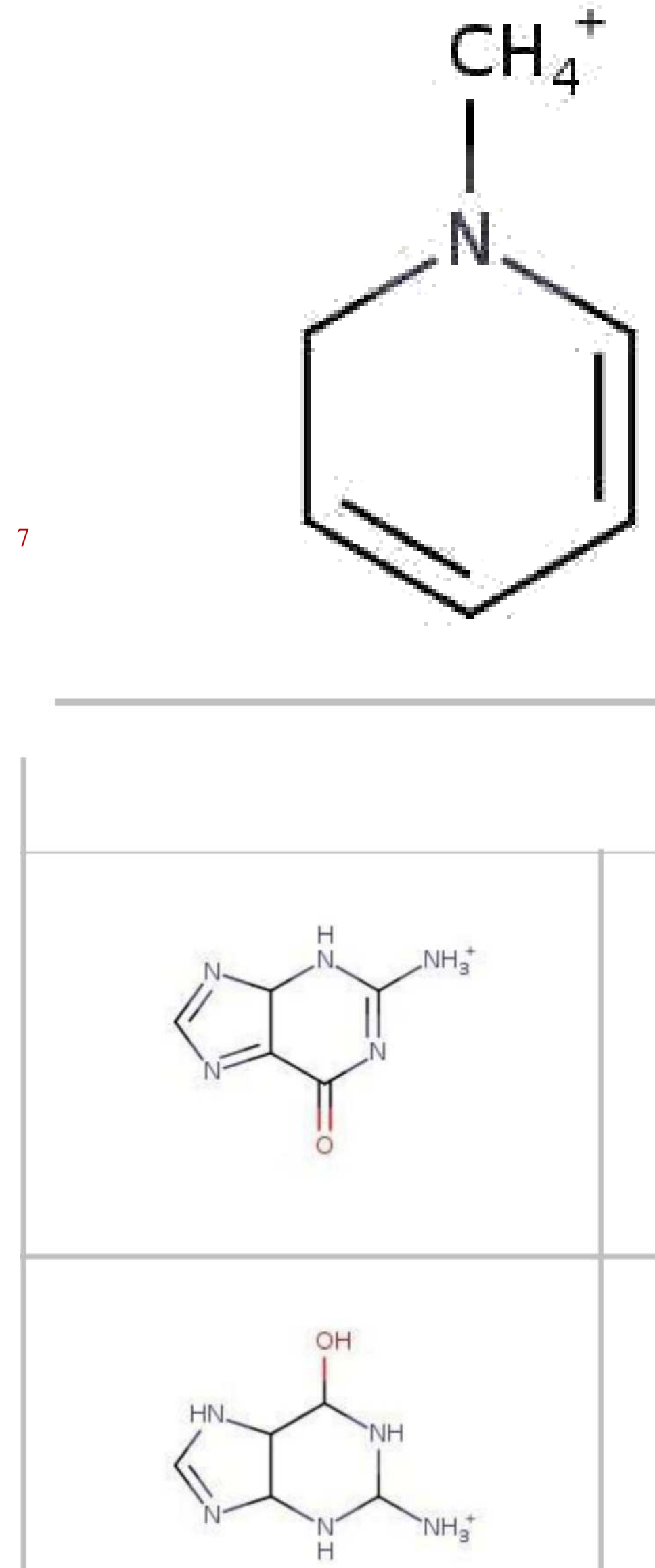


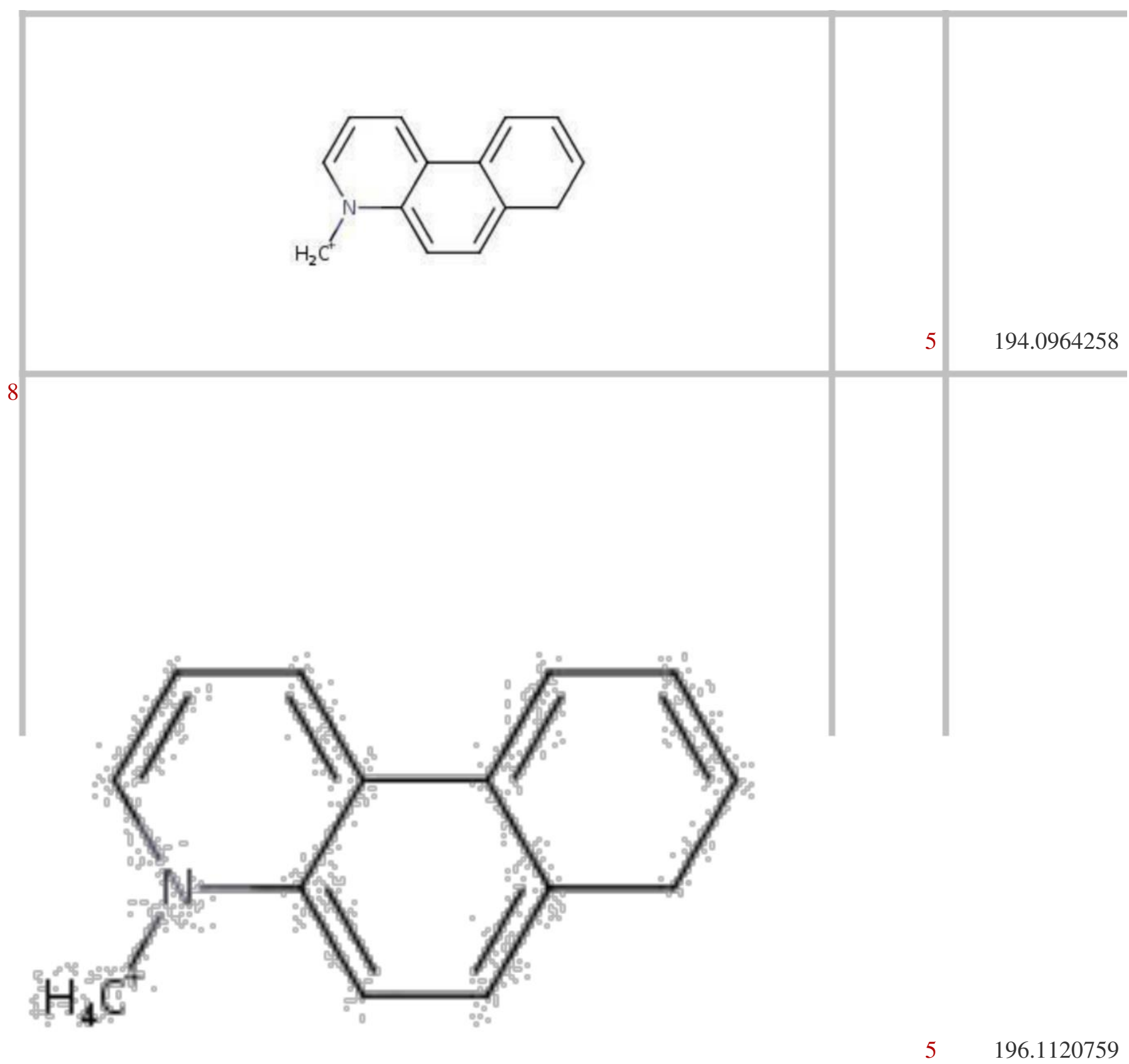

9 


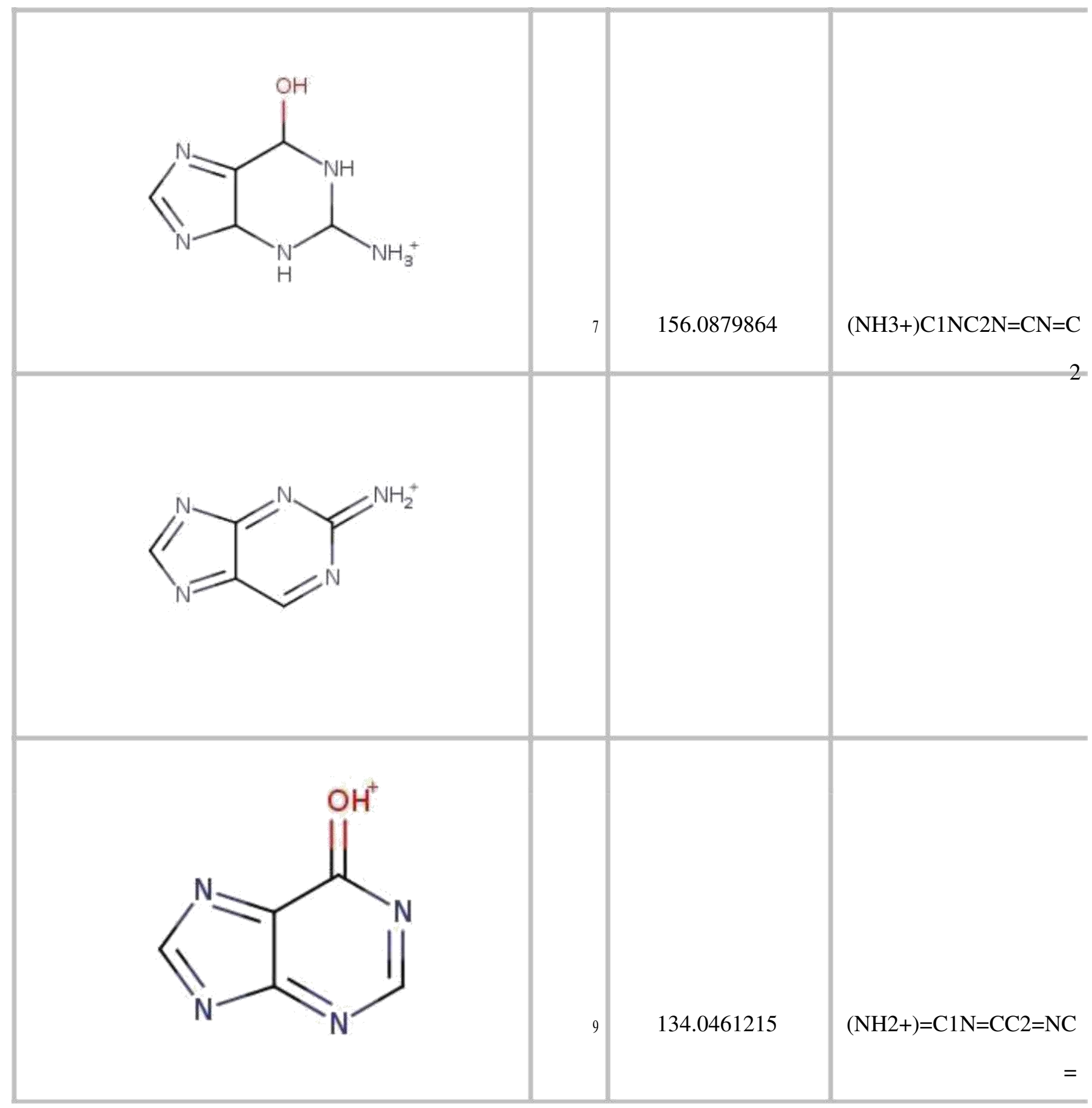




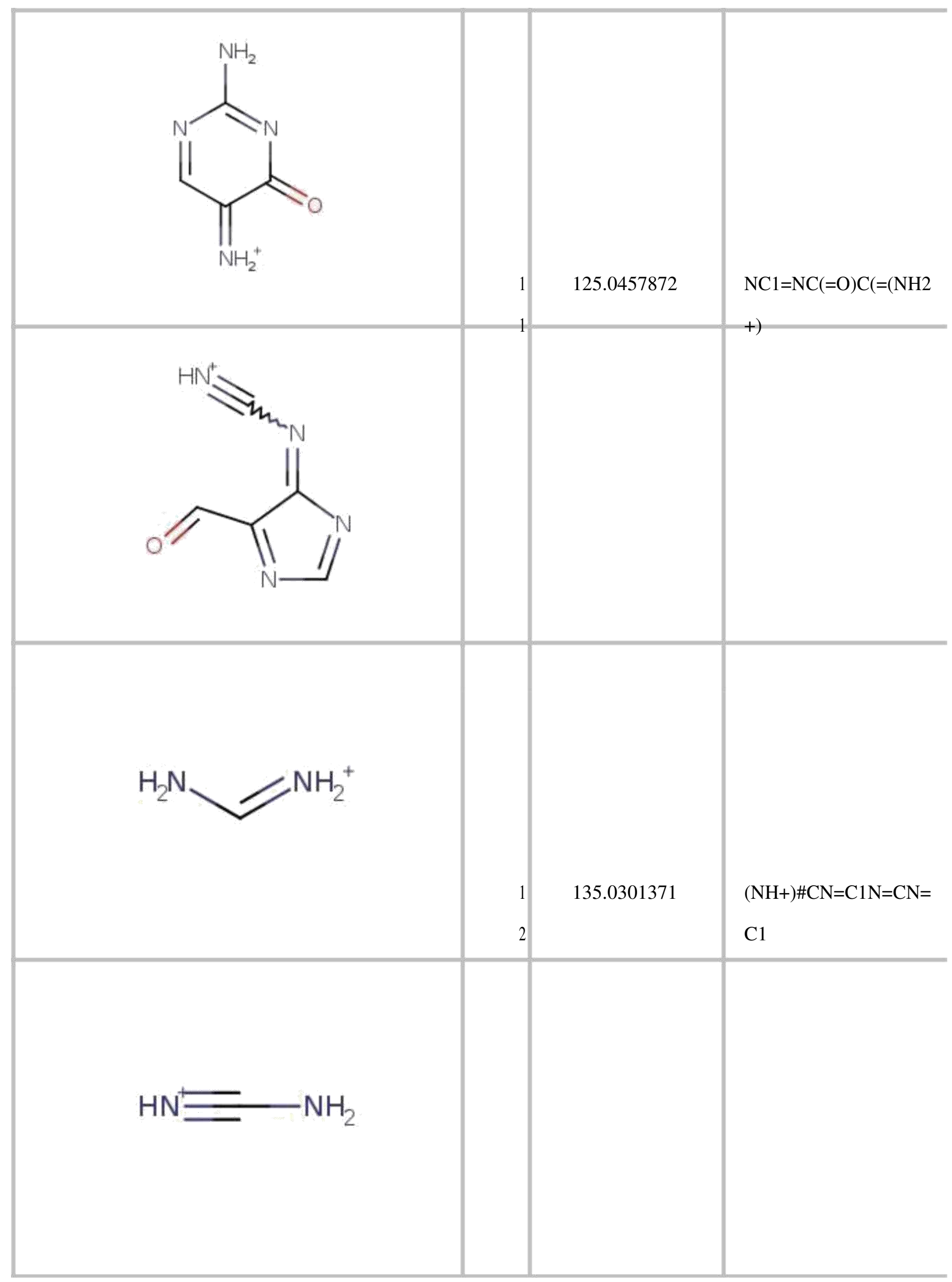




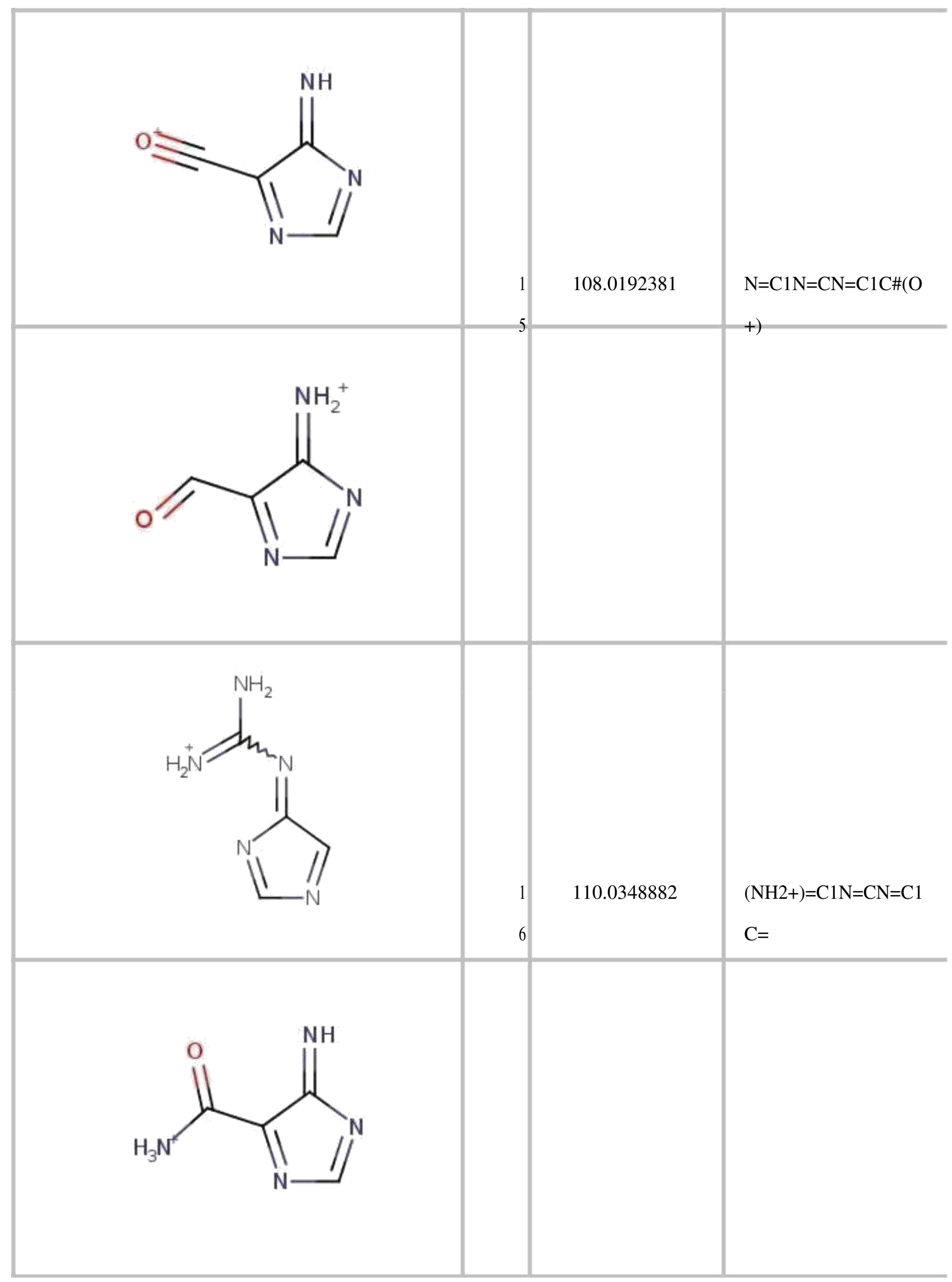




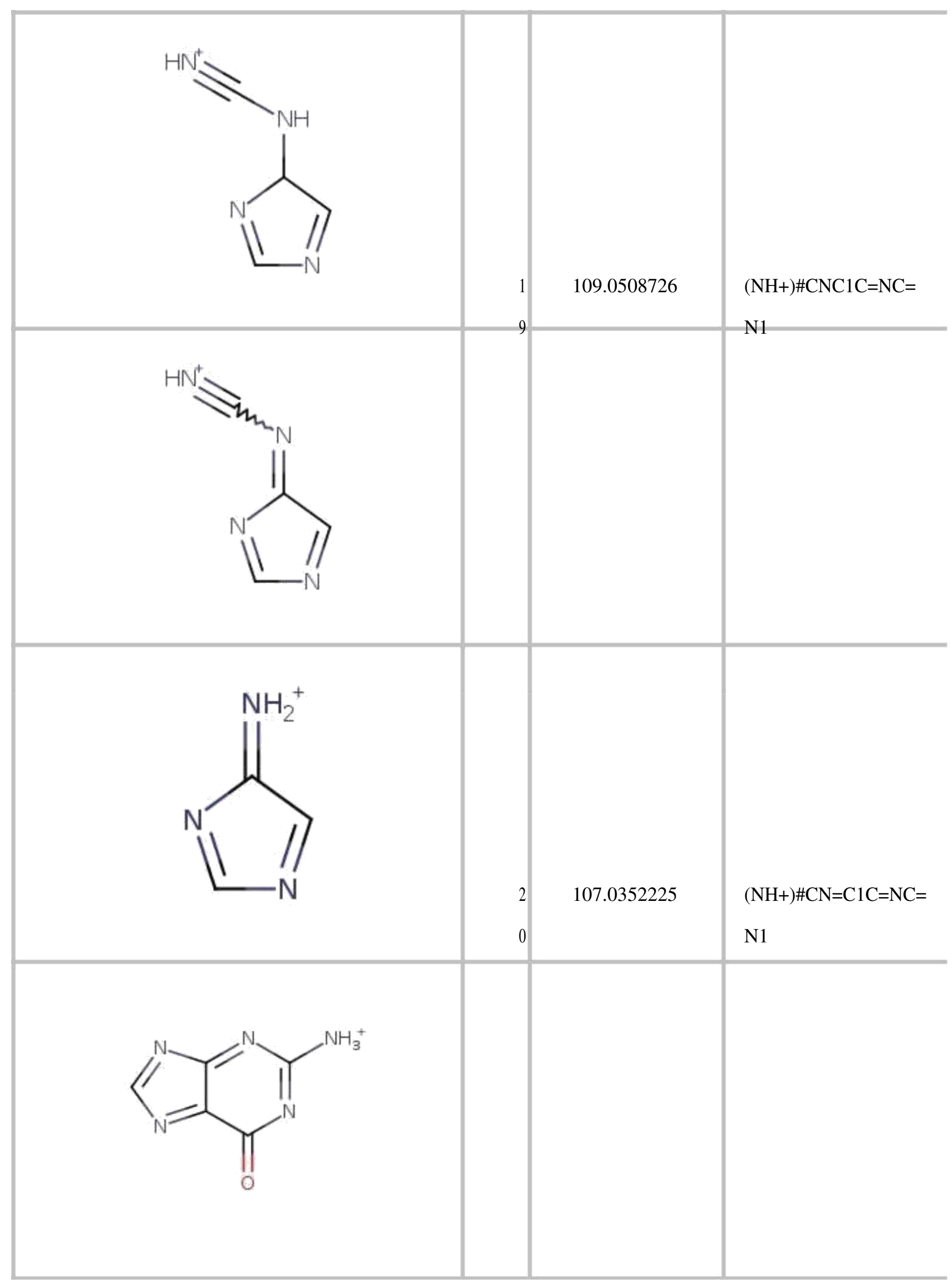




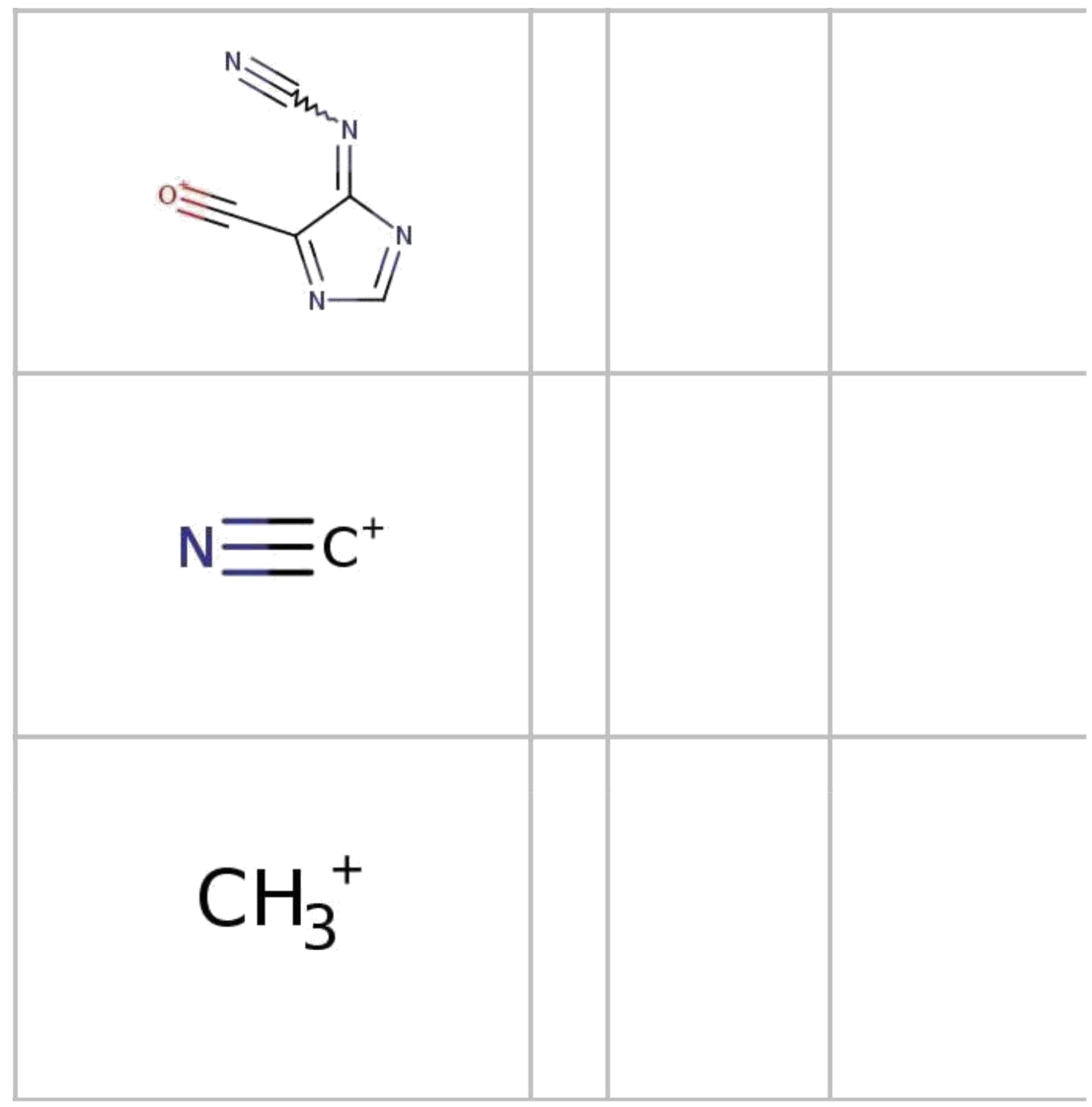

Table1b. List of the Roccustyrna fragmented recored active compounds.

The complete pharmacophoric fragmentation scheme was analyzed to compare similar series of chemical patterns that are contained in the chemical phase structures as exctraced from within the selected 10 hit compounds of the Colchicine, Raltegravir, Hexacosanol, Benzoxazolinon, Carboxy-Pentaric acid, Ursane, Antheraxanthin, RA-XIII, Crotonate and Byrsonima coccolobifolia. (Table1a), (20,35) Whenever searching for a specific pattern, if the group has such a parent pattern, the parent pattern is searched first eliminate the terms in $V^{\prime}\left(p\right.$, which gives $f=-\%-r^{\prime}+g(t)$. Then one can choose $n$ $\mathrm{g}\{\mathrm{t})$ such as to eliminate the purely time-dependent terms, and one finally arrives at, $=\left(2 \mathrm{mV}{ }^{\prime} 2\left(\mathrm{p}+\mathrm{mf} ; \mathbf{m} \mathrm{r}^{\prime}(\mathrm{p}=\mathrm{ih}(\mathrm{p}\right.\right.$, ipir, $\mathrm{t})$ $=-$ ea $\mathrm{h} \mathrm{J}$ (pir',t). $(20,25,26)$ of the strong equivalence principle in quantum theory. After that, the child pharmacophoric pattern is searched in an inertial repeated merged system $\mathrm{S}$ asip $=\%(\mathrm{ml} 5 \mathrm{r}, \mathrm{t})+\mathrm{ip} 2 \mathrm{im} 2, \mathrm{r}, \mathrm{t}) .(25,26,37)$ Then assume that one fragmented pharrmacophore can describe the same superposition in an accelerating to a larger ligand-receptor system $S^{\prime}$ that obeys (20), with $\S=£(r), £(0)=£(7)=$, so that the system S' performs a closed quantum circuit and coincides with the chemical structure system the $S$ at times $t=0$ and $t=T$, such that $r$ ' $i T)=r(7)$. (25-34,37) To avoid $q\{h \mathrm{q} ;\} q$ reg $q(3)$; creg c(3);reset q(0);reset q(1);reset q(2);h q(0);u2(pi/2,pi/2) q(1); incomplete 
(1Z)-2- $\{((2 S, 3 S, 5 R)-5$-(2-amino-6-oxo- 6,9-dihydro-3H-purin-9-yl)-3-hydroxyoxolan-2-yl) methylidene\}-2-cyano$1-(\{((2 S, 4 R, 5 R)-2$ group assignments, through two hydrogen-bonding interactions whenever a part into the $S 2$ subsite of the structure relative to the complex between the cyano-1-( $(((2 S, 4 R, 5 R)-2$-methyl- 2- (methylamino)-

1,6-diazabicyclo with heptan-4-yl)oxyjimino)-1lambda5,2 lambda5-azaphosphiridin-1-ylium chemical groups of the cyclohexyl methyl is already recored and fragmented, the subsequent carbonyl oxygen matches have to be adjacent to the from the main-interacting chain amide of the residue Glu166 amino acid already occupied the space normally by the canonical S4- cyano-1-(\{((2S,4R,5R)-2-methyl-2- (methylamino)-1,6-diazabicyclo(3.2.0) heptan-4-yl) (1S,2R,3S)-2-([[(1S,2S,4S,5R)- 4- ethenyl-4-sulfonylbicyclo[3.2.0]heptan-2-yl] oxy\}amino)-3-

[(2R,5R)-5-(2-methyl-6-methylidene-6,9-dihydro-3H-purin-9-yl)-3-methylideneoxolan-2-yl]phosphirane-1-carbonitrile -oxy\}imino)-1 lambda5, 2lambda5-azaphosphiridin-1-ylium binding site of the PDB:6LU7 main protease. (26,31-39) As a first step, our Chern-Simons oriented fragmentation algorithm performs a quick fragmentation scheme search for extracting different chemical groups formed by the nucleophilic attack of the catalytic domains of the target proteases onto the $\alpha$-carbon of my new Roccustyrna small molecule by applying the heuristic quantum phases for group prioritization when performing parent-child group prioritization as described above. (29,32-39) This topology geometric for pharmacophoric search and design indicating the canonical binding pockets moieties of a new small sized prototype that goes sequentially through a sorted fragmentation scheme, adding groups that are found in its active conformation phases and do not overlap with the cyano-1-(\{((2S,4R,5R)-2-methyl-2- (methylamino)-1,6diazabicyclo(3.2.0)heptan-4-yl) oxylimino)- 1lambda5,2lambda5- azaphosphiridin-1-ylium chemical groups that were already screened. In case it successfully estimates a valid fragmentation, scheme this is taken as the phase solution relating to how one clustered pharmacophoric element occupies the space normally filled by the protease "s substrate "s interacting main chain; would describe the same phase in an alterantive XYZ coordinate smile system. (33,35-42) (27-

38) This Lindenbaum-Tarski algebraic algorithm was implemented as a recursive algorithm that leads to enhancement of my novel small molecule 's catalytic activity against the $3 \mathrm{C}$ protease-like domains I and II and performs a complete decision tree search of all possible combinations of fragmentation, merging and pharmacophoric recoring systems targeting in the in SARS-CoV Mpro. $(12,13,28-39)$

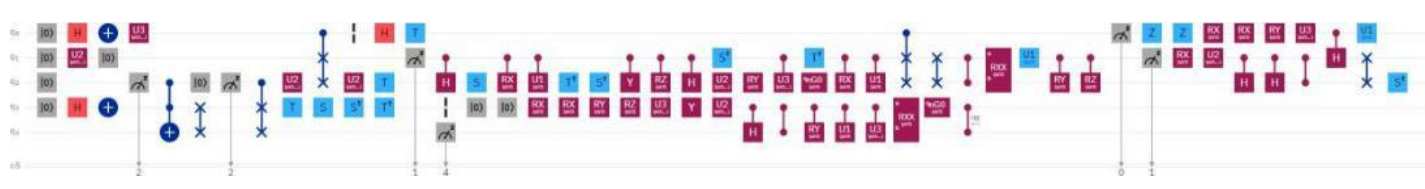

Figure1b. Quantum Circuit of the BiogenetoligandoirolTM Cluster for the Roccustyrna small molecule Merging and Hope-Re-coring algorithms. Gate nG0 ( param ) q \{ h q; \}qreg q(3);creg $\mathrm{c}(3)$;reset q(0);reset q(1);reset q(2);hq(0);u2(pi/2,pi/2) q(1);measure q(2) -> c(2);x q(0);reset $\mathrm{q}(1) ; \operatorname{resetq}(2) ; \mathrm{u} 3(\mathrm{pi} / 2, \mathrm{pi} / 2, \mathrm{pi} / 2) \mathrm{q}(0) ;$ measure $\mathrm{q}(2)->\mathrm{c}(2) ; \mathrm{u} 2(\mathrm{pi} / 2, \mathrm{pi} / 2) \mathrm{q}(2) ;$ cswap $\mathrm{q}(0), \mathrm{q}(1), \mathrm{q}(2) ;$ barrier $\mathrm{q}(0) ; \mathrm{u} 2(\mathrm{pi} / 2, \mathrm{pi} / 2) \mathrm{q}(2) ; \mathrm{h} \mathrm{q}(0) ; \mathrm{t} \mathrm{q}(2) ; \mathrm{t} \mathrm{q}(0) ;$ measure $\mathrm{q}(1)$-> c(1);ch q(1),q(2); q(2);crx(pi/2) q(1),q(2);cu1(pi/2) q(1),q(2);tdg q(2);sdg q(2);cy $\mathrm{q}(1), \mathrm{q}(2) ; \operatorname{crz}(\mathrm{pi} / 2) \mathrm{q}(1), \mathrm{q}(2) ; \mathrm{ch} \mathrm{q}(1), \mathrm{q}(2) ; \mathrm{sdg} \mathrm{q}(1) ; \mathrm{u} 2(\mathrm{pi} / 2, \mathrm{pi} / 2) \mathrm{q}(2) ; \mathrm{ry}(\mathrm{pi} / 2)$ $\mathrm{q}(2) ; \mathrm{cu} 3(\mathrm{pi} / 2, \mathrm{pi} / 2, \mathrm{pi} / 2) \mathrm{q}(1), \mathrm{q}(2) ; \operatorname{tdg} \mathrm{q}(1) ; \mathrm{nG} 0(\mathrm{pi} / 2) \mathrm{q}(2) ; \operatorname{crx}(\mathrm{pi} / 2) \mathrm{q}(1), \mathrm{q}(2) ; \mathrm{cu} 1(\mathrm{pi} / 2)$ $\mathrm{q}(1), \mathrm{q}(2) ; \operatorname{cswap} \mathrm{q}(0), \mathrm{q}(1), \mathrm{q}(2)$;swap q(1),q(2);cz q(1),q(2);rxx(pi/2) $\mathrm{q}(1), \mathrm{q}(2) ; \mathrm{u} 1$ (pi/2) $\mathrm{q}(1) ; \operatorname{cry}($ pi/2) $\mathrm{q}(1), \mathrm{q}(2) ; \operatorname{crz}(\mathrm{pi} / 2) \mathrm{q}(1), \mathrm{q}(2) ;$ measure $\mathrm{q}(0)$-> c(0);z q(0);measure $\mathrm{q}(1)$ - 
$>\mathrm{c}(1) ; \mathrm{z} \mathrm{q}(0) ; \mathrm{rx}(\mathrm{pi} / 2) \mathrm{q}(1) ; \mathrm{rx}(\mathrm{pi} / 2) \mathrm{q}(0) ; \mathrm{u} 2(\mathrm{pi} / 2, \mathrm{pi} / 2) \mathrm{q}(1) ; \mathrm{rx}(\mathrm{pi} / 2) \mathrm{q}(0) ; \mathrm{ch} \mathrm{q}(1), \mathrm{q}(2) ; \mathrm{ry}(\mathrm{pi} / 2) \mathrm{q}(0) ; \mathrm{ch}$ $\mathrm{q}(1), \mathrm{q}(2) ; \mathrm{u} 3(\mathrm{pi} / 2, \mathrm{pi} / 2, \mathrm{pi} / 2) \mathrm{q}(0) ; \mathrm{cz} \mathrm{q}(1), \mathrm{q}(2) ; \mathrm{ch} \mathrm{q}(0), \mathrm{q}(1) ; \mathrm{u} 1$ (pi/2) q(0);swap q(1),q(2);sdg q(2);

Therefore, from a mathematical perspective, a TQFT that recovers the above quantum Hilbert spaces may be called -Chern-Simons theory. We present here potential condensed eigenvalues applications for the positive spectrum and finite-dimensional eigenspaces: $S=14 \pi \int M 3(\mathbf{a}+\mathfrak{l}) \mathbf{t a}+\mathfrak{\ell}-\mathbf{1} A \wedge d A k+13(\mathbf{a}+\mathfrak{l}) \mathbf{t a}+\mathfrak{\ell}-\mathbf{1} A \wedge[\mathrm{A} \wedge \mathrm{A}] \mathrm{k}(\bmod 2 \pi) 1 \rightarrow \mathrm{U}(1) \rightarrow \mathrm{LG} \sim$ $\rightarrow L G \rightarrow 1$ of the free loop group $L G=(\mathbf{a}+\mathfrak{l}) \mathbf{t a}+\mathfrak{l}-\mathbf{1}(\mathbf{a}+\mathfrak{l}) \mathbf{t a}+\mathfrak{\ell}-\mathbf{1}(\mathrm{S} 1, \mathrm{G})[1]$ is a functional of $\mathrm{G}$ bundles with connections over compact 3 manifolds. Here, $A$ is the connection form, $(\mathbf{a}+\mathfrak{l}) \mathbf{t a}+\boldsymbol{\ell}-\mathbf{1}(\mathbf{a}+\mathfrak{l}) \mathbf{t a}+\boldsymbol{\ell}-\mathbf{1} \wedge(\mathbf{a}+\boldsymbol{\ell}) \mathbf{t a}+\boldsymbol{\ell}-\mathbf{1 F})$

2zEGk: 2zEG $\otimes \mathbf{2 z E G} \rightarrow \mathbb{R}$ is a certain metric constructed and the integral from the level, is taken over a global

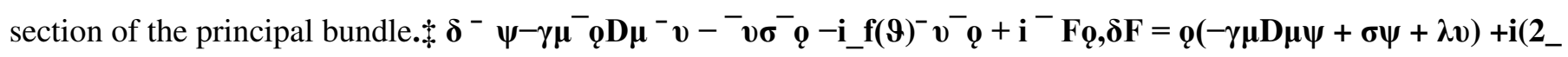

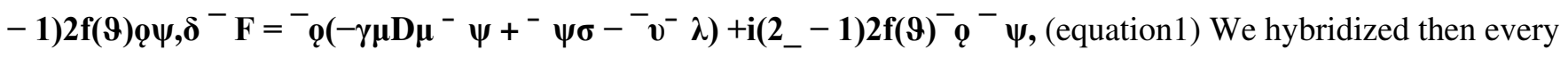
level $\mathrm{k} \in \mathrm{H} 4(\mathrm{BG}, \mathbb{Z})$ that yields the quantum field theory with those levels $\mathrm{k}$ that satisfy the following positivity condition: Chern-Simons theory equation of the: $\left(\int \operatorname{tr}(+23) . S=k 4 \pi \int M \operatorname{tr}\right)(3 \mathrm{~d} N=2 \mathrm{CSA} \wedge \mathrm{dA}+23 \mathrm{~A} \wedge \mathrm{A} \wedge \mathrm{A})($ equation2). $\mathrm{A}$ (equation 1)(ie. considering $\mathrm{S}$ as a functional of $\mathrm{A}$ ). associated to a connected Lie group a $\mathrm{S}$ a simple current Roccustryna XYZ chemical extension of the tensor product of the $d / d x\left(33833 y^{\wedge} n \times 1152 N^{\wedge} 2 x^{\wedge} 2 k^{\wedge} 4\right)=3 d N 2 C S$ $77951232 \mathrm{k}^{\wedge} 4 \mathrm{~N}^{\wedge} 2 \mathrm{x} \mathrm{y}^{\wedge} \mathrm{n}$ associated to an even lattice and $38975616 \mathrm{k}^{\wedge} 4 \mathrm{~N}^{\wedge} 2 \mathrm{x}^{\wedge} 2 \mathrm{y}^{\wedge} \mathrm{n}=\operatorname{sum}_{-}(\mathrm{v}=0)^{\wedge} \infty\left(\mathrm{n}^{\wedge} \mathrm{v} 38975616\left\{\mathrm{x} \sin ^{\wedge}(-1)(\operatorname{sqrt}(3)), \mathrm{x}\right.\right.$ $\sin ^{\wedge}(-1)\left(\operatorname{sqrt}(779) \operatorname{sqrt}\left(\theta^{\wedge} 3\right)\right), x \sin ^{\wedge}(-1)(2 \operatorname{sqrt}(114109)),\left(\operatorname{sqrt}(1-\operatorname{sqrt}(456457) \operatorname{sqrt}(x)) x^{\wedge}(1 / 4)(2 \operatorname{sqrt}(456457) \operatorname{sqrt}(x)+3)\right) /(8$ $\left.456457^{\wedge}(3 / 4)\right)+(x-3 / 3651656) \sin ^{\wedge}(-1)\left(456457^{\wedge}(1 / 4) x^{\wedge}(1 / 4)\right), 1 / 2 \operatorname{sqrt}\left(x / 456456754-x^{\wedge} 2\right)+(x-1 / 912913508)$ $\sin ^{\wedge}(-1)(\operatorname{sqrt}(456456754) \operatorname{sqrt}(x)), x \sin ^{\wedge}(-1)\left(2^{\wedge}(3 / 4) 57057^{\wedge}(1 / 4)\right), x \sin ^{\wedge}(-1)(\operatorname{sqrt}(\operatorname{sum} 444546 \theta)), x \sin ^{\wedge}(-$ 1) $\left.\left.\left(2^{\wedge}(3 / 4) 431683182057^{\wedge}(1 / 4) \operatorname{sqrt}\left(\operatorname{sqrt}(\theta) /\left(\sin ^{\wedge}(-1)(44545545)\right)\right)\right)\right\}+\operatorname{constant}\left(\mathrm{k}^{\wedge} 4 \mathrm{~N}^{\wedge} 2 \mathrm{x}^{\wedge} 2 \log \mathrm{v}(\mathrm{y})\right)\right) /(\mathrm{v} !)$ 9equation3) associated to a simply connected Lie group classical equations of motion obtained via $i$ and ii are known to be equivalent by combining the works of Finkelberg obtained in this way are: $\quad 1=0 \mathrm{k} 2 \pi \mathrm{F}=03 \mathrm{~d} \mathrm{~N}=2 \mathrm{CS}$

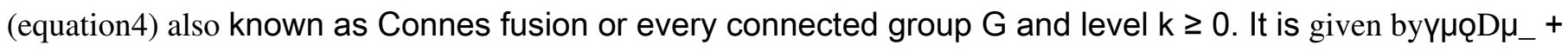
$\mathrm{Q}(\rho \cdot \sigma)_{-}+\mathrm{i}_{-} \mathrm{f}(\vartheta) \mathrm{Q}_{-}=\mathrm{M} Q_{-}$, (equation 5) where $\mathrm{M}$ is the eigenvalue given by $\mathrm{M}=\mathrm{Mm}, \mathrm{n}=\rho \cdot \sigma+\mathrm{i}_{-} \mathrm{mb}+\mathrm{nb}-1$ $+Q 2$ _ $m, n 2 Z \_0, Q=b+b-1$. (equation 6) The product of the eigenvalues roughly gives -denominator\| of the one-loop determinant., where the right action on $\mathrm{H}$ uses the isomorphism induced by reflection along the horizontal axis. (41) For every simple simply connected Lie group $G$ with the quantum Hilbert space CSG,k(S1 and every level $k \geq 0$, a tensor category $A \wedge A, k$ whose Drinfel'd center $Z(d A, A)$ associated to a Riemann surface $\Sigma$ can and has been defined at a mathematical level of precision and is equivalent to the the VectkG[G] Vectk[G])CSG,k(pt) d/dx(33833 $\left.y^{\wedge} n \times 1152 \mathrm{~N}^{\wedge} 2 \mathrm{x}^{\wedge} 2 \mathrm{k}^{\wedge} 4\right)=77951232 \mathrm{k}^{\wedge} 4 \mathrm{~N}^{\wedge} 2 \mathrm{x} \mathrm{y}^{\wedge} \mathrm{n}$ (equation7) categories of the $\sin (\mathrm{a}+\ell)$ ta $+\ell-1 \operatorname{Vectk}((\mathrm{a}+\ell)$ ta $+\ell-1+(\mathrm{a}+\ell)$ ta $+\ell-1 \wedge(\mathrm{a}+\ell)$ ta $+\ell-1 \mathrm{~F}) \mathrm{k}^{\wedge} 4 \mathrm{x}^{\wedge} 4 \mathrm{y}^{\wedge} \mathrm{n} \sin ^{\wedge}(-1)(\mathrm{n})^{\wedge} 338335429 \mathrm{~N}^{\wedge} 2$ (newtons squared) - 1/3 $\mathrm{x}^{\wedge} 6\left(\mathrm{k}^{\wedge} 4 \mathrm{y}^{\wedge} \mathrm{n} \sin ^{\wedge}(-1)(\mathrm{n})^{\wedge} 338335429 \mathrm{~N}^{\wedge} 2\right.$ (newtons squared) $)+2 / 45 \mathrm{k}^{\wedge} 4 \mathrm{x}^{\wedge} 8 \mathrm{y}^{\wedge} \mathrm{n} \sin ^{\wedge}(-$ 1)(n)^33833 $5429 \mathrm{~N}^{\wedge} 2$ (newtons squared) $+\mathrm{O}\left(\mathrm{x}^{\wedge}\right.$ ) (equation8) of canonical quantization, it is the geometric quantization of $\operatorname{LocG}(\Sigma)$ with respect tothe natural symplectic Roccustyrna structure coming from the Chern-Simons Lagrangian. We explain below that, at leastalong the direction $\theta$ is defined by $S \theta P(g)=Z 1$ ei $\theta 0 d t e-\operatorname{tg} B P(t)$, (1)where $\mathrm{BP}(\mathrm{t})$ is analytic continuation of the formal Borel transformation $\mathrm{P} 1 \ell=0 \mathrm{c} \ell(\mathrm{a}+\ell)$ ta $+\ell-1$. whenG $432 \pi$ $k^{\wedge} 4 N^{\wedge} 2 x^{\wedge} 2 \sin ^{\wedge}(-1)(n)^{\wedge} 33833(2 x-\sin (2 x)) y^{\wedge}(\wedge n)$ for Vectk[G] dCSG,k(pt) (equation9)is simply connected with 
the the Reshetikhin-Turaev TQFT associated to the modular tensor category Repk(LG) of positive energy representations of the loop group at level $\mathrm{k}$ has that property $14 \pi \int \mathrm{M} 3(\mathrm{a}+\ell) \operatorname{ta}+\ell-1 \mathrm{~A} \wedge \mathrm{dA} \mathbf{2 z E G Z c l}(\sigma)=$

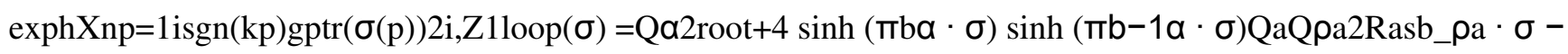

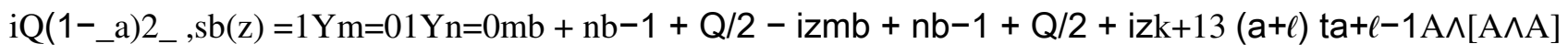
(equation10) the coordinate $(\mathrm{x} 1, \mathrm{x} 2, \mathrm{x} 3, \mathrm{x} 4)=(\cos \vartheta \cos \phi, \cos \vartheta \sin \phi, \sin \vartheta \cos \mathrm{X}, \sin \vartheta \sin \mathrm{X})$ and $\mathrm{f}(\vartheta)=\mathrm{pb}-2 \sin 2 \vartheta$ $+\mathrm{b} 2 \cos 2 \vartheta \cdot \mathrm{k}(\bmod 2 \pi)[1]+\sin (\mathrm{a}+\ell) \operatorname{ta}+\ell-1 \mathrm{Vectk}((\mathrm{a}+\ell) \operatorname{ta}+\ell-1+(\mathrm{a}+\ell) \operatorname{ta}+\ell-1 \wedge(\mathrm{a}+\ell) \operatorname{ta}+\ell-1 \mathrm{~F}) 14 \pi \int \mathrm{M} 3(\mathrm{a}+\ell)$ ta $+\ell-1 \mathrm{~A} \wedge d \mathrm{~A} 2 \mathrm{zEGk}+13(\mathrm{a}+\ell)$ ta $+\ell-1 \mathrm{~A} \wedge[\mathrm{A} \wedge \mathrm{A}] \mathbf{2 z E G k}(\bmod 2 \pi)[1] \wedge \sin (\mathrm{a}+\ell)$ ta $+\ell-1 \operatorname{Vectk}((\mathrm{a}+\ell) \operatorname{ta}+\ell-1+(\mathrm{a}+\ell)$

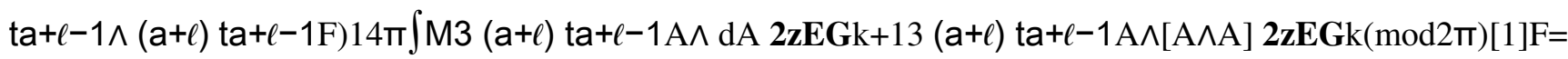
$\mathrm{dA}+\mathrm{A} \wedge \mathrm{A} \operatorname{Repk}(\mathrm{dA}+\mathrm{A} \wedge \mathrm{A} A G A)$ by $[28] \mathrm{F} \mu \mathrm{v}=0, \sigma=$ const., $\mathrm{D}=-\sigma \mathrm{f}(\vartheta), \lambda={ }^{-} \lambda=0$. (4)Rep ${ }^{\mathrm{ss}}\left(\mathrm{F}_{\mathrm{q}} \mathbf{2 z E G}\right)\left[\operatorname{or}_{\operatorname{Rep}}{ }^{\mathrm{k}}(\mathrm{AF}\right.$ 2zEG) $]$ and $\operatorname{Rep}(\operatorname{Dk}(\mathbb{C}[G]))=\operatorname{Rep} p_{f}\left((a+\ell)\right.$ ta $\left.+\ell-1_{G, k}\right)($ equation11) are equivalent as balanced tensor 
category Repk(dA+A^AAGA) of positive energy representations of the loop group at the:

$$
\begin{aligned}
& \sin ^{-1}(n)^{33833} y^{n \wedge}\left(\int t d t\right) \sum g\left(\int \begin{array}{l|l|l|l|l|}
\begin{array}{l}
\text { cumulative } \\
\text { distribution } \\
\text { function }
\end{array} & \text { normal distribution } & \text { mean } & \mu=0 \\
\cline { 3 - 4 } & \text { standard deviation } & \sigma=1
\end{array}\right] \\
& \left(\int 1152 N^{2} \sin ^{2}(x) k^{4} \pi d x\right) \int\left(\int 3 d x\right) d x
\end{aligned}
$$

INPUT: $\left(432 \mathrm{k}^{\wedge} 4 \mathrm{~N}^{\wedge} 2 \pi \mathrm{t}^{\wedge} 2 \mathrm{y}^{\wedge}(\mathrm{n} \Lambda)\left\{\theta \sin ^{\wedge}(-1)(\operatorname{sqrt}(3)), \theta \sin ^{\wedge}(-1)(\operatorname{sqrt}(779)\right.\right.$ $\left.\operatorname{sqrt}\left(\theta^{\wedge} 3\right)\right)-3 / 5 \operatorname{sqrt}(779) \theta \operatorname{sqrt}\left(\theta^{\wedge} 3\right) 2 \mathrm{~F} 1\left(1 / 2,5 / 6,11 / 6,779 \theta^{\wedge} 3\right), 1 / 6 \operatorname{sqrt}(1 / 49394-$ $9 \theta) \operatorname{sqrt}(\theta)+(\theta-1 / 889092) \sin ^{\wedge}(-1)(3 \operatorname{sqrt}(49394) \operatorname{sqrt}(\theta)), \theta \sin ^{\wedge}(-1)\left(\operatorname{sqrt}\left(\sin ^{\wedge}(-\right.\right.$ 1) $(44545545)))\}+$ constant $\left.\sin ^{\wedge}(-1)(n)^{\wedge} 33833 \operatorname{sum~g~} x^{\wedge} 4+\mathrm{O}\left((1 / \mathrm{x})^{\wedge} 6\right)\right)+\sin (2 \mathrm{x})(-$ $\left.216\left(k^{\wedge} 4 N^{\wedge} 2 \pi t^{\wedge} 2 y^{\wedge}(n \Lambda) \sin ^{\wedge}(-1)(n)^{\wedge} 33833 \operatorname{sum} g\right) x^{\wedge} 3+O\left((1 / x)^{\wedge} 6\right)\right)+e^{\wedge}\left(-x^{\wedge} 2 / 2\right)$ $\sin (2 \mathrm{x})\left(-108\left(\mathrm{k}^{\wedge} 4 \mathrm{~N}^{\wedge} 2 \operatorname{sqrt}(2 \pi) \mathrm{t}^{\wedge} 2 \mathrm{y}^{\wedge}(\mathrm{n} \Lambda) \sin ^{\wedge}(-1)(\mathrm{n})^{\wedge} 33833 \operatorname{sum} \mathrm{g}\right) \mathrm{x}^{\wedge} 2+\right.$ $\left.\mathrm{O}\left((1 / \mathrm{x})^{\wedge} 6\right)\right)+\mathrm{e}^{\wedge}\left(-\mathrm{x}^{\wedge} 2 / 2\right) \sin (2 \mathrm{x})\left(108 \mathrm{k}^{\wedge} 4 \mathrm{~N}^{\wedge} 2 \operatorname{sqrt}(2 \pi) \mathrm{t}^{\wedge} 2 \mathrm{y}^{\wedge}(\mathrm{n} \Lambda) \sin ^{\wedge}(-\right.$ 1)(n) $333833 \operatorname{sum} \mathrm{g} \mathrm{x}{ }^{\wedge} 2-108\left(\mathrm{k}^{\wedge} 4 \mathrm{~N}^{\wedge} 2 \operatorname{sqrt}(2 \pi) \mathrm{t}^{\wedge} 2 \mathrm{y}^{\wedge}(\mathrm{n} \Lambda) \sin ^{\wedge}(-1)(\mathrm{n})^{\wedge} 33833 \mathrm{sum} \mathrm{g}\right)+$ $\left(324 \mathrm{k}^{\wedge} 4 \mathrm{~N}^{\wedge} 2 \operatorname{sqrt}(2 \pi) \mathrm{t}^{\wedge} 2 \mathrm{y}^{\wedge}(\mathrm{n} \Lambda) \sin ^{\wedge}(-1)(\mathrm{n})^{\wedge} 33833 \operatorname{sum} \mathrm{g}\right) / \mathrm{x}^{\wedge} 2-\left(1620\left(\mathrm{k}^{\wedge} 4 \mathrm{~N}^{\wedge} 2\right.\right.$ $\left.\left.\left.\operatorname{sqrt}(2 \pi) \mathrm{t}^{\wedge} 2 \mathrm{y}^{\wedge}(\mathrm{n} \Lambda) \sin ^{\wedge}(-1)(\mathrm{n})^{\wedge} 33833 \mathrm{sum} \mathrm{g}\right)\right) / \mathrm{x}^{\wedge} 4+\mathrm{O}\left((1 / \mathrm{x})^{\wedge} 6\right)\right)+\mathrm{e}^{\wedge}\left(-\mathrm{x}^{\wedge} 2 / 2\right)\left(216 \mathrm{k}^{\wedge} 4\right.$ $\left.\mathrm{N}^{\wedge} 2 \operatorname{sqrt}(2 \pi) \mathrm{t}^{\wedge} 2 \mathrm{y}^{\wedge}(\mathrm{n} \Lambda) \sin ^{\wedge}(-1)(\mathrm{n})^{\wedge} 33833 \operatorname{sum} \mathrm{g} \mathrm{x} \mathrm{x}^{\wedge} 3+\mathrm{O}\left((1 / \mathrm{x})^{\wedge} 6\right)\right)+\mathrm{e}^{\wedge}\left(-\mathrm{x}^{\wedge} 2 / 2\right)(-216$ $\left(\mathrm{k}^{\wedge} 4 \mathrm{~N}^{\wedge} 2 \operatorname{sqrt}(2 \pi) \mathrm{t}^{\wedge} 2 \mathrm{y}^{\wedge}(\mathrm{n} \Lambda) \sin ^{\wedge}(-1)(\mathrm{n})^{\wedge} 33833 \operatorname{sum} \mathrm{g}\right) \mathrm{x}^{\wedge} 3+216 \mathrm{k}^{\wedge} 4 \mathrm{~N}^{\wedge} 2 \operatorname{sqrt}(2 \pi)$ $\mathrm{t}^{\wedge} 2 \mathrm{y}^{\wedge}(\mathrm{n} \Lambda) \sin ^{\wedge}(-1)(\mathrm{n})^{\wedge} 33833 \operatorname{sum} \mathrm{g} \mathrm{x}-\left(648\left(\mathrm{k}^{\wedge} 4 \mathrm{~N}^{\wedge} 2 \operatorname{sqrt}(2 \pi) \mathrm{t}^{\wedge} 2 \mathrm{y}^{\wedge}(\mathrm{n} \Lambda) \sin ^{\wedge}(-\right.\right.$ 1)(n)^33833 sum g) $) / \mathrm{x}+\left(3240 \mathrm{k}^{\wedge} 4 \mathrm{~N}^{\wedge} 2 \operatorname{sqrt}(2 \pi) \mathrm{t}^{\wedge} 2 \mathrm{y}^{\wedge}(\mathrm{n} \Lambda) \sin ^{\wedge}(-\right.$ 1)(n $)^{\wedge} 33833$ sum $\left.g\right) / x^{\wedge} 3-\left(22680\left(k^{\wedge} 4 N^{\wedge} 2 \operatorname{sqrt}(2 \pi) t^{\wedge} 2 y^{\wedge}(n \Lambda) \sin ^{\wedge}(-1)(n)^{\wedge} 33833\right.\right.$ sum $\left.g)) / x^{\wedge} 5+O\left((1 / x)^{\wedge} 6\right)\right)$

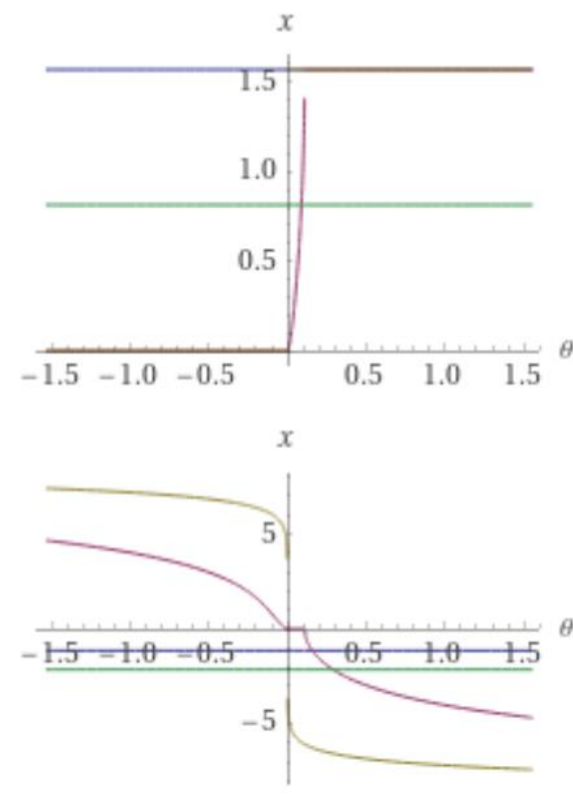

$(\theta$ from -1.5 to 1.5$)$

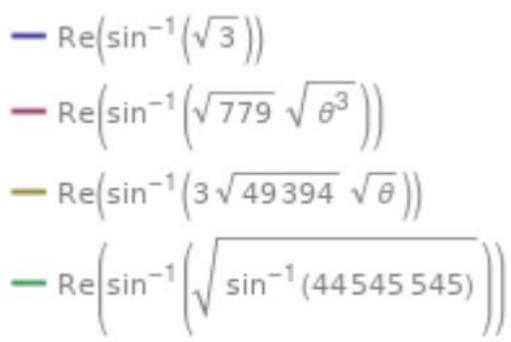

( $\theta$ from -1.5 to 1.5$)$

$-\operatorname{Im}\left(\sin ^{-1}(\sqrt{3})\right)$

$-\operatorname{lm}\left(\sin ^{-1}\left(\sqrt{779} \sqrt{\theta^{3}}\right)\right)$

$-\operatorname{lm}\left(\sin ^{-1}(3 \sqrt{49394} \sqrt{\theta})\right)$

$-\operatorname{lm}\left(\sin ^{-1}\left(\sqrt{\sin ^{-1}(44545545)}\right)\right)$

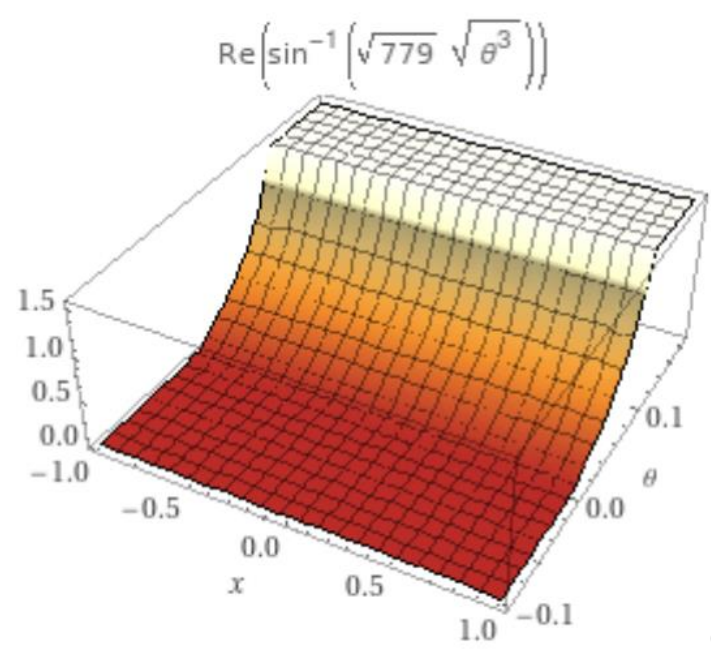

(equation1) 


$$
\begin{aligned}
& \left(432 k^{4} N^{2} \pi t^{2} y^{\pi \Lambda} \sin ^{-1}(n)^{33833} \sum g x^{4}+O\left(\left(\frac{1}{x}\right)^{6}\right)\right)+ \\
& \sin (2 x)\left(-216\left(k^{4} N^{2} \pi t^{2} y^{n} \sin ^{-1}(n)^{33} 833 \cdot g\right) x^{3}+O\left(\left(\frac{1}{x}\right)^{6}\right)\right)+ \\
& e^{-\dot{x}^{2} / 2} \sin (2 x)\left(-108\left(k^{4} N^{2} \sqrt{2 \pi} t^{2} y^{n \pi} \sin ^{-1}(n)^{33833} \sum g\right) x^{2}+o\left(\left(\frac{1}{x}\right)^{6}\right)\right)+ \\
& e^{-\dot{x}^{2} / 2} \sin (2 x)\left(108 k^{4} N^{2} \sqrt{2 \pi} t^{2} y^{n \cdot N} \sin ^{-1}(n)\right)^{33833} \sum g x^{2}= \\
& 108\left(k^{4} N^{2} \sqrt{2 \pi} t^{2} y^{n \lambda} \sin ^{-1}(n)^{33833} \sum g\right)+ \\
& \frac{324 k^{4} \cdot N^{2} \sqrt{2 \pi} t^{2} y^{n \Lambda} \sin ^{-1}(n)^{333833} \sum g}{x^{2}}= \\
& \left.\frac{1620\left(k^{4} \cdot N^{2} \cdot \sqrt{2 \pi} t^{2} \cdot y^{n \Lambda} \sin ^{-1}(n)^{33833} \sum g\right)}{x^{4}}+O\left(\left(\frac{1}{x}\right)^{6}\right)\right)+ \\
& e^{-x^{2} / 2}\left(216 k^{4} N^{2} \cdot \sqrt{2 \pi} \cdot t^{2} \cdot y^{n \dot{\lambda}} \sin ^{-1}(n)^{33833} \sum g x^{3}+o\left(\left(\frac{1}{x}\right)^{6}\right)\right)+ \\
& e^{-\dot{x}^{2} / 2}\left(-216\left(k^{4} N^{2} \cdot \sqrt{2 \pi} \cdot t^{2} \cdot y^{n \dot{\lambda}} \sin ^{-1}(n)^{33833} \sum g\right) x^{3}+\right. \\
& 216 k^{4} N^{2} \sqrt{2 \pi} t^{2} y^{n \pi} \sin ^{-1}(n)^{33833} \sum g x- \\
& \frac{648\left(k^{4} N^{2} \sqrt{2 \pi} t^{2} y^{n !} \sin ^{-1}(n)^{33833} \sum g\right)}{x}+ \\
& \frac{3240 k^{4} N^{2} \sqrt{2 \pi} t^{2} y^{n} \sin ^{-1}(n)^{33833} \sum g}{x^{3}}= \\
& \frac{22680\left(k^{4} \cdot N^{2} \cdot \sqrt{2 \pi} t^{2} \cdot y^{n} \sin ^{-1}(n)^{33833} \sum g\right)}{x^{5}}+O\left(\left(\frac{1}{x}\right)^{6}\right)
\end{aligned}
$$

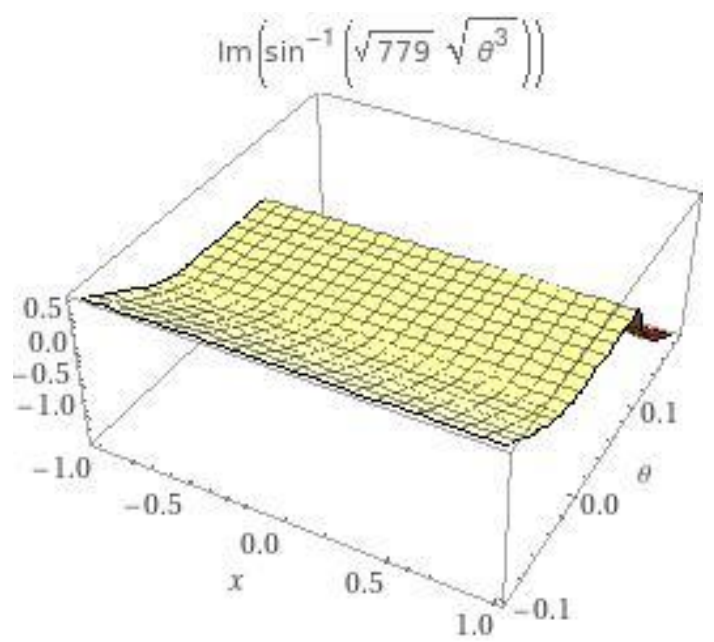

(equation2) 


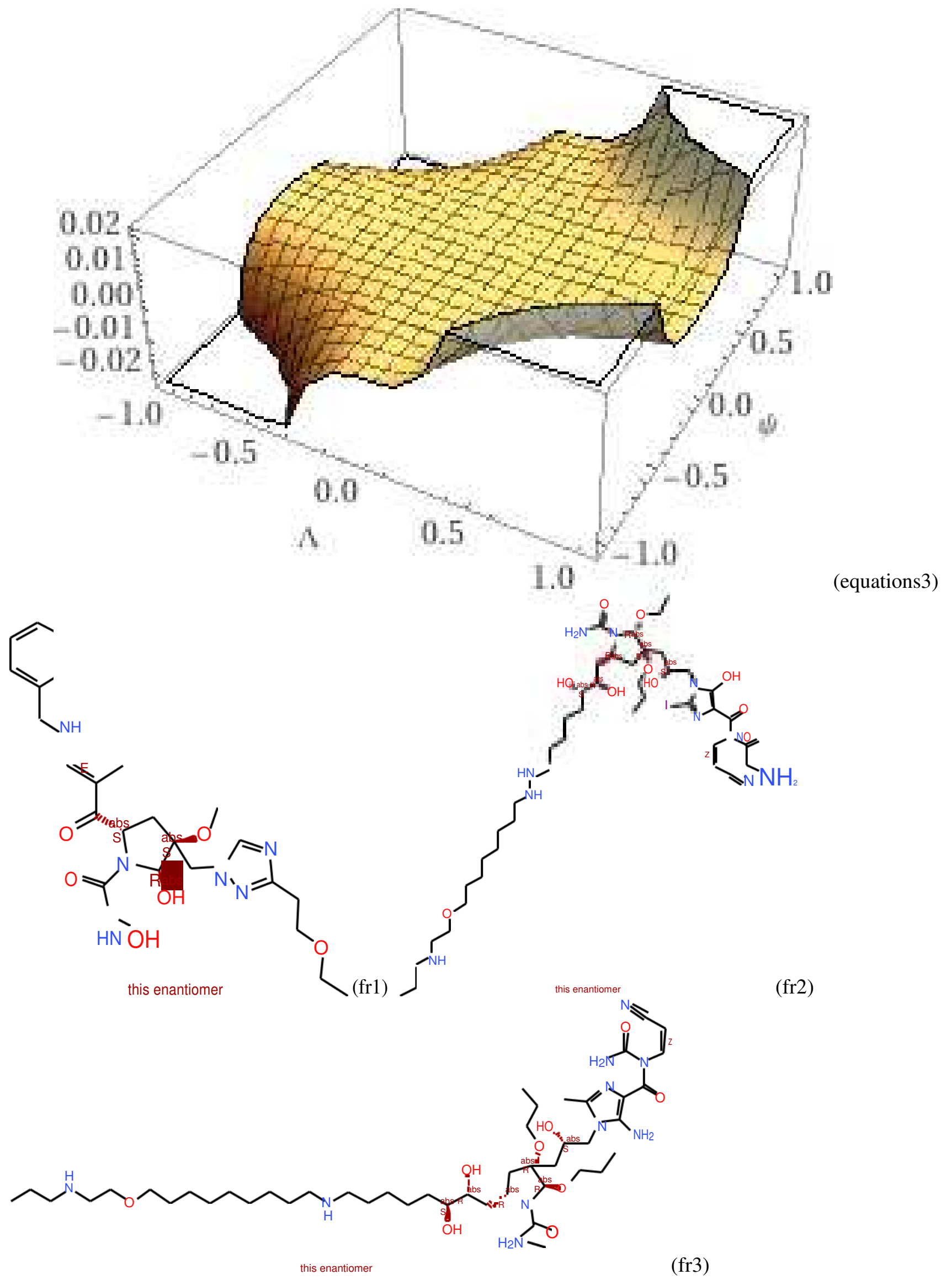

Figure1c. Chern-Simons topology geometrics of the 3 selected hit fragmented candidates (fr1), (fr2), (fr3). 
OUTPUT:Roccustyrna XYZ Cartesian coordinates

\begin{tabular}{|c|c|c|c|}
\hline $\mathrm{C}$ & $\begin{array}{c}7.2670 \\
0\end{array}$ & 1.33000 & $\begin{array}{l}- \\
5.0970 \\
0\end{array}$ \\
\hline $\mathrm{O}$ & $\begin{array}{c}6.9480 \\
0\end{array}$ & $\begin{array}{c}- \\
2.7070 \\
0\end{array}$ & $\begin{array}{l}- \\
5.3570 \\
0\end{array}$ \\
\hline C & $\begin{array}{c}8.0880 \\
0\end{array}$ & $\begin{array}{c}- \\
3.53600\end{array}$ & $\begin{array}{l}- \\
5.1060 \\
0\end{array}$ \\
\hline C & $\begin{array}{c}9.1170 \\
0\end{array}$ & $\begin{array}{c}- \\
2.68100\end{array}$ & $\begin{array}{l}- \\
4.3420 \\
0\end{array}$ \\
\hline $\mathrm{C}$ & $\begin{array}{c}8.7810 \\
0\end{array}$ & $\begin{array}{c}- \\
1.24900\end{array}$ & $\begin{array}{l}- \\
4.7710 \\
0\end{array}$ \\
\hline $\mathrm{O}$ & $\begin{array}{c}9.0740 \\
0\end{array}$ & $\begin{array}{c}- \\
0.3070 \\
0\end{array}$ & $\begin{array}{l}- \\
3.7400 \\
0\end{array}$ \\
\hline $\mathrm{N}$ & $\begin{array}{c}8.6100 \\
0\end{array}$ & $\begin{array}{c}- \\
4.0880 \\
0\end{array}$ & $\begin{array}{l}- \\
6.3290 \\
0\end{array}$ \\
\hline C & $\begin{array}{c}8.6210 \\
0\end{array}$ & $\begin{array}{c}- \\
3.51000\end{array}$ & $\begin{array}{l}- \\
7.5550 \\
0\end{array}$ \\
\hline $\mathrm{N}$ & $\begin{array}{c}9.1640 \\
0\end{array}$ & $\begin{array}{c}- \\
4.3170 \\
0\end{array}$ & $\begin{array}{l}- \\
8.4870 \\
0\end{array}$ \\
\hline C & $\begin{array}{c}9.5170 \\
0\end{array}$ & 5.42800 & $\begin{array}{l}- \\
7.8280 \\
0\end{array}$ \\
\hline & $\begin{array}{c}10.128 \\
00\end{array}$ & 6.61300 & $\begin{array}{l}- \\
8.2660 \\
0\end{array}$ \\
\hline
\end{tabular}




\begin{tabular}{|c|c|c|c|}
\hline $\mathrm{O}$ & $\begin{array}{l}10.4230 \\
0\end{array}$ & -6.79100 & -9.46800 \\
\hline $\mathrm{N}$ & $\begin{array}{l}10.4040 \\
0\end{array}$ & -7.57300 & -7.34100 \\
\hline $\mathrm{C}$ & $\begin{array}{l}10.0990 \\
0\end{array}$ & $\begin{array}{c}- \\
7.41200\end{array}$ & -6.02100 \\
\hline $\mathrm{N}$ & $\begin{array}{l}10.4270 \\
0\end{array}$ & -8.37500 & -5.14600 \\
\hline $\mathrm{N}$ & $\begin{array}{l}9.4790 \\
0\end{array}$ & $\begin{array}{c}- \\
6.27600\end{array}$ & $\begin{array}{l}- \\
5.5920 \\
0\end{array}$ \\
\hline $\mathrm{C}$ & $\begin{array}{l}9.1920 \\
0\end{array}$ & $\begin{array}{c}- \\
5.29100\end{array}$ & $\begin{array}{l}- \\
6.4830 \\
0\end{array}$ \\
\hline $\mathrm{C}$ & $\begin{array}{l}6.8360 \\
0\end{array}$ & $\begin{array}{c}- \\
0.50700\end{array}$ & $\begin{array}{l}- \\
6.2150 \\
0\end{array}$ \\
\hline $\mathrm{P}$ & 5.31500 & -0.46900 & $\begin{array}{l}- \\
6.6920 \\
0\end{array}$ \\
\hline $\mathrm{N}$ & $\begin{array}{l}4.4570 \\
0\end{array}$ & 0.67200 & $\begin{array}{l}- \\
6.1220 \\
0\end{array}$ \\
\hline $\mathrm{C}$ & $\begin{array}{l}4.7980 \\
0\end{array}$ & $\begin{array}{c}1.0370 \\
0\end{array}$ & $\begin{array}{l}- \\
7.3780 \\
0\end{array}$ \\
\hline $\mathrm{C}$ & $\begin{array}{l}4.5480 \\
0\end{array}$ & $\begin{array}{c}- \\
1.74200\end{array}$ & $\begin{array}{l}- \\
7.1820 \\
0\end{array}$ \\
\hline $\mathrm{N}$ & $\begin{array}{l}3.9910 \\
0\end{array}$ & $\begin{array}{c}- \\
2.68300\end{array}$ & $\begin{array}{l}- \\
7.5320 \\
0\end{array}$ \\
\hline $\mathrm{N}$ & $\begin{array}{l}3.8360 \\
0\end{array}$ & 1.04900 & $\begin{array}{l}- \\
5.1380 \\
0\end{array}$ \\
\hline $\mathrm{O}$ & $\begin{array}{l}3.7490 \\
0\end{array}$ & 0.35300 & $\begin{array}{l}- \\
4.0670 \\
0\end{array}$ \\
\hline $\mathrm{C}$ & $\begin{array}{l}2.9890 \\
0\end{array}$ & $\begin{array}{c}1.0510 \\
0\end{array}$ & $\begin{array}{l}- \\
3.0600 \\
0\end{array}$ \\
\hline $\mathrm{C}$ & $\begin{array}{l}3.0440 \\
0\end{array}$ & $\begin{array}{c}0.2750 \\
0\end{array}$ & $\begin{array}{l}- \\
1.7300 \\
0\end{array}$ \\
\hline $\mathrm{C}$ & $\begin{array}{l}1.8160 \\
0\end{array}$ & $\begin{array}{c}- \\
0.68000\end{array}$ & $\begin{array}{l}- \\
1.7610 \\
0\end{array}$ \\
\hline $\mathrm{N}$ & $\begin{array}{l}0.7770 \\
0\end{array}$ & 0.04700 & $\begin{array}{l}- \\
2.5630 \\
0\end{array}$ \\
\hline
\end{tabular}




$\begin{array}{llcl}\mathrm{C} & 1.4700 & 1.0750 & - \\ & 0 & 0 & 3.3660 \\ & & & 0 \\ \mathrm{C} & 0.0280 & 1.1560 & - \\ & 0 & 0 & 1.9120 \\ & & & 0 \\ \mathrm{~N} & 0.7330 & 2.16400 & - \\ & 0 & & 2.7060 \\ & & & 0 \\ \mathrm{~N} & 1.2670 & - & - \\ & 0 & 0.97500 & 0.4020 \\ & & & 0 \\ \mathrm{C} & 2.1360 & - & - \\ & 0 & 2.02000 & 2.4980 \\ & & & 0 \\ \mathrm{C} & 2.1340 & - & 0.6120 \\ & 0 & 1.61200 & 0 \\ \mathrm{H} & 8.7920 & 0.56000 & - \\ & 0 & & 4.1010 \\ & & & 0 \\ \mathrm{H} & 6.6660 & - & - \\ & 0 & 0.95800 & 4.2670 \\ & & & 0\end{array}$


$\begin{array}{llll}\mathrm{H} & 7.76800 & - & - \\ & & 4.41900 & 4.5530 \\ & & 0\end{array}$

$\begin{array}{lcll}\mathrm{H} & 8.90800 & - & - \\ & & 2.77000 & 3.2760 \\ & & & 0\end{array}$

$\begin{array}{llll}\mathrm{H} & 10.1090 & - & - \\ & 0 & 2.92200 & 4.7250 \\ & & & 0\end{array}$

$\begin{array}{llcl}\mathrm{H} & 9.40400 & - & - \\ & & 0.84800 & 5.5700\end{array}$

0

$\begin{array}{llcl}\mathrm{H} & 8.23800 & - & - \\ & & 2.51200 & 7.7670\end{array}$

0

$\begin{array}{llll}\mathrm{H} & 9.72300 & - & - \\ & & 9.01900 & 4.8160\end{array}$

0

$\begin{array}{llll}\mathrm{H} & 11.3780 & - & - \\ & 0 & 8.45500 & 4.8160 \\ & & & 0\end{array}$

$\begin{array}{llll}\mathrm{H} & 9.23500 & - & - \\ & & 6.16400 & 4.6180\end{array}$

0

$\begin{array}{cccl}\mathrm{H} & 7.63400 & 0.0680 & - \\ & & 0 & 6.6850\end{array}$

0

$\begin{array}{cccc}\mathrm{H} & 3.98400 & 0.9950 & - \\ & & 0 & 8.1030\end{array}$

0

$\begin{array}{cccl}\mathrm{H} & 5.54000 & 1.8320 & - \\ & & 0 & 7.4380\end{array}$

0

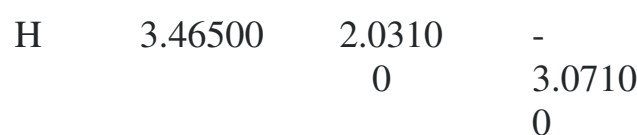

$\mathrm{H} \quad 2.88700 \quad 0.9940 \quad-$

$0 \quad 0.9250$

0

$\begin{array}{llll}\mathrm{H} & 3.94400 & - & - \\ & & 0.33800 & 1.7450\end{array}$

0

$\begin{array}{cccl}\mathrm{H} & 1.41600 & 1.0520 & - \\ & & 0 & 4.4540\end{array}$

0

$\begin{array}{cccl}\mathrm{H} & 0.30800 & 1.2410 & - \\ & & 0 & 0.8620\end{array}$

0

$\begin{array}{llcl}\mathrm{H} & - & 1.1230 & - \\ & 1.02400 & 0 & 2.1950\end{array}$ 


$\begin{array}{lccl}\mathrm{H} & 0.71800 & 3.1720 & - \\ & & 0 & 2.7710 \\ & & & 0 \\ \mathrm{H} & 0.31200 & - & - \\ & & 0.74500 & 0.1690 \\ & & & 0 \\ \mathrm{H} & 2.32300 & - & - \\ & & 2.80300 & 1.7630 \\ & & & 0 \\ \mathrm{H} & 1.28800 & - & - \\ & & 2.30300 & 3.1220 \\ & & & 0 \\ \mathrm{H} & 3.02000 & - & - \\ & & 1.88900 & 3.1220 \\ & & & 0 \\ \mathrm{H} & 1.78000 & - & 1.6090 \\ & & 1.35200 & 0 \\ \mathrm{H} & 2.10500 & - & 0.4870 \\ & & 2.69500 & 0 \\ \mathrm{H} & 3.15800 & - & 0.4870 \\ & & 1.26000 & 0\end{array}$

To reduce the fragmentation space for the generation of salt-bridge interaction between the Glu290 and the new ligand that needs to be searched, the algorithm of the two independent Chern-Simons $S=14 \pi \int M 3$ A level $k \in H 4(B G, Z)$ $(\mathbf{a}+\mathbf{l})$ ta $+\boldsymbol{\ell}-1 \mathrm{~A} \wedge \mathrm{dA} \mathbf{2 z E G k}+13(\mathbf{a}+\boldsymbol{l}) \mathbf{t a}+\boldsymbol{\ell}-1 \mathrm{~A} \wedge[\mathrm{A} \wedge \mathrm{A}] \mathbf{2 z E G} \mathrm{k}(\bmod 2 \pi)$ actions with loop group at level $\mathrm{k}$ has that property $\left(14 \pi \int \mathrm{M} 3(\mathrm{a}+\ell)\right.$ ta $+\ell-1 \mathrm{~A} \wedge \mathrm{dA} \quad 2 \mathrm{zEGk}+1 \quad 3(\mathrm{a}+\ell)$ ta $+\ell-1 \mathrm{~A} \wedge[\mathrm{A} \wedge \mathrm{A}] \quad 2 \mathrm{zEG} \mathbf{k}(\bmod 2 \pi)[1]+\sin (\mathrm{a}+\ell)$

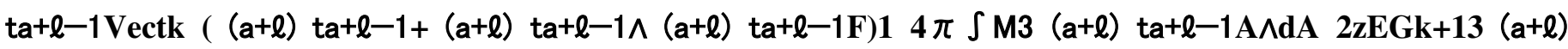
$\operatorname{ta}+\ell-1 \mathrm{~A} \wedge[\mathrm{A} \wedge \mathrm{A}] 2 \mathrm{zEG} k(\bmod 2 \pi)[1] \wedge \sin (a+l)$ ta $+l-1 \operatorname{Vectk}((a+l)$ ta $+l-1+(a+l)$ ta $+l-1 \wedge(a+l)$ ta+l-1F) $14 \pi \int$ M3 $(a+\ell)$ ta $+\ell-1 A \wedge d A 2 z E G k+13(a+\ell)$ ta $+\ell-1 A \wedge[A \wedge A] 2 z E G k(\bmod 2 \pi)[1] F=d A+A \wedge A \operatorname{Repk}(d A+A \wedge A$ AGA) $\operatorname{Repss}(F q 2 z E G)[o r \operatorname{Repk}(A F) 2 z E G)]$ and $\operatorname{Rep}(D k(\mathbb{G}[G]))=\operatorname{Repf}((a+\ell)$ ta $+\ell-1 G, k))$ equation $(13)$ on the heuristic level of path integrals ( $(a+l)$ ta $+\mathbf{l}-1 \mathrm{~A}$ is the potential 11-form and $\mathrm{F}$ is the field strength 224 -form) where the partition function for the Chern-Simons theory to gather eigenvalues of the matrix models when associated with knot theory is the modulus square of the poles and zeroes in the one-loop determinant partition function. 


$$
\begin{aligned}
& -\log \left(\sqrt{1+14692375882461045811756348944\left(e^{-i X}-e^{i X}\right)^{2} x(\tau)^{2}}=\right. \\
& \left.121212111121212\left(e^{-i x}-e^{i X}\right) x(\tau)\right) \\
& \log \int \sqrt{ } 1+x(\tau)^{2} x^{\prime}(22 \tau)^{488} \log ^{2} \int \sqrt{\left(1-\frac{1}{4 y(\tau)} x^{\prime}\right.} \\
& 22)^{576431275750959675405783039290773810744762062805850371259715614902742679552} \\
& +\frac{1}{2 \sqrt{y(\tau)}} i x \\
& \int y^{\prime}(
\end{aligned}
$$

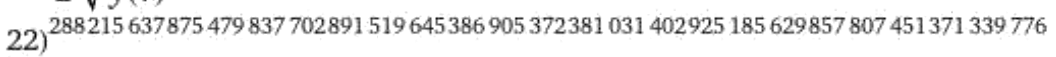

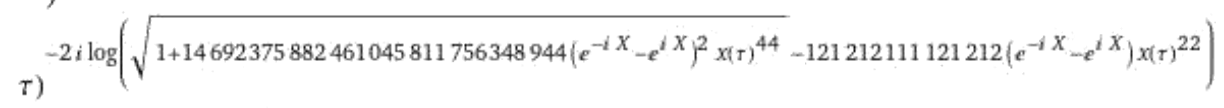

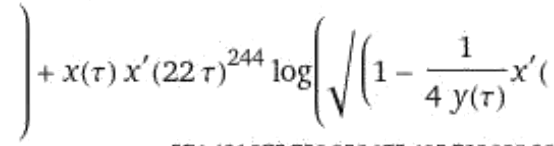

$$
\begin{aligned}
& 22)^{576431275750959675405783039290773810744762062805850371259715614902742679552} \\
& +\frac{1}{2 \sqrt{y(t)}} i x^{\prime}( \\
& \int y^{\prime}( \\
& 22)^{28821563787547983770289151964538695372381031.402925185629857807451371339776}
\end{aligned}
$$

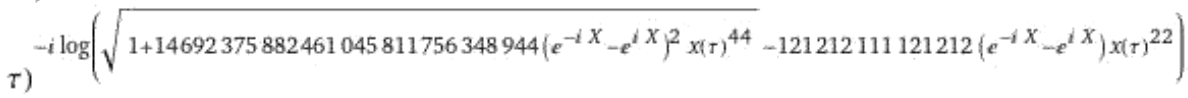

$$
\begin{aligned}
& \begin{array}{l}
\frac{\partial}{\partial n}\left(-\frac{n^{33834} k^{-\left(k^{4} n^{33835} N^{2}\right)} N^{213}}{33834432 k^{4}}\right)= \\
\quad \underline{n^{33833} N^{213} k^{-k^{4} n^{33835} N^{2}-4}\left(33835 k^{4} n^{33835} N^{2} \log (k)-33834\right)}
\end{array} \\
& 33834432 \\
& \int-\frac{k^{-4-k^{4} n^{33835} N^{2}} n^{33834} N^{213}}{33834432} d n=\frac{N^{211} k^{-k^{4} n^{33835} N^{2}-8}}{1144788006720 \log (k)}+\text { constant }
\end{aligned}
$$

$(22,27,28,35)$ This In Silico approach keeps track of the solutions already found of the selected group of the selected hit candidates which were fragmented, recored and superposed in a non-relativistic quantum mechanics environment and finally led us to the complete Roccustyrna chemical structure. (Table1a), $(20,28,29,30)$ If several chemical space solutions were found in the phase end, the combined theory has a sequence of similar such function spaces of finite but arbitrarily large selected dimension, where the partition dimension increases with the finite resolution of relative knot space measurements to the first dihydro-3H-purin-9-yl)- system when exctracted from the 10 hit selected small molecules as the possible chemical bimodules over a hyperfinite 1 factor solutions which were sorted by the number of different SARS-CoV Mpro, PDB entry 6lu7 receptor patterns. 


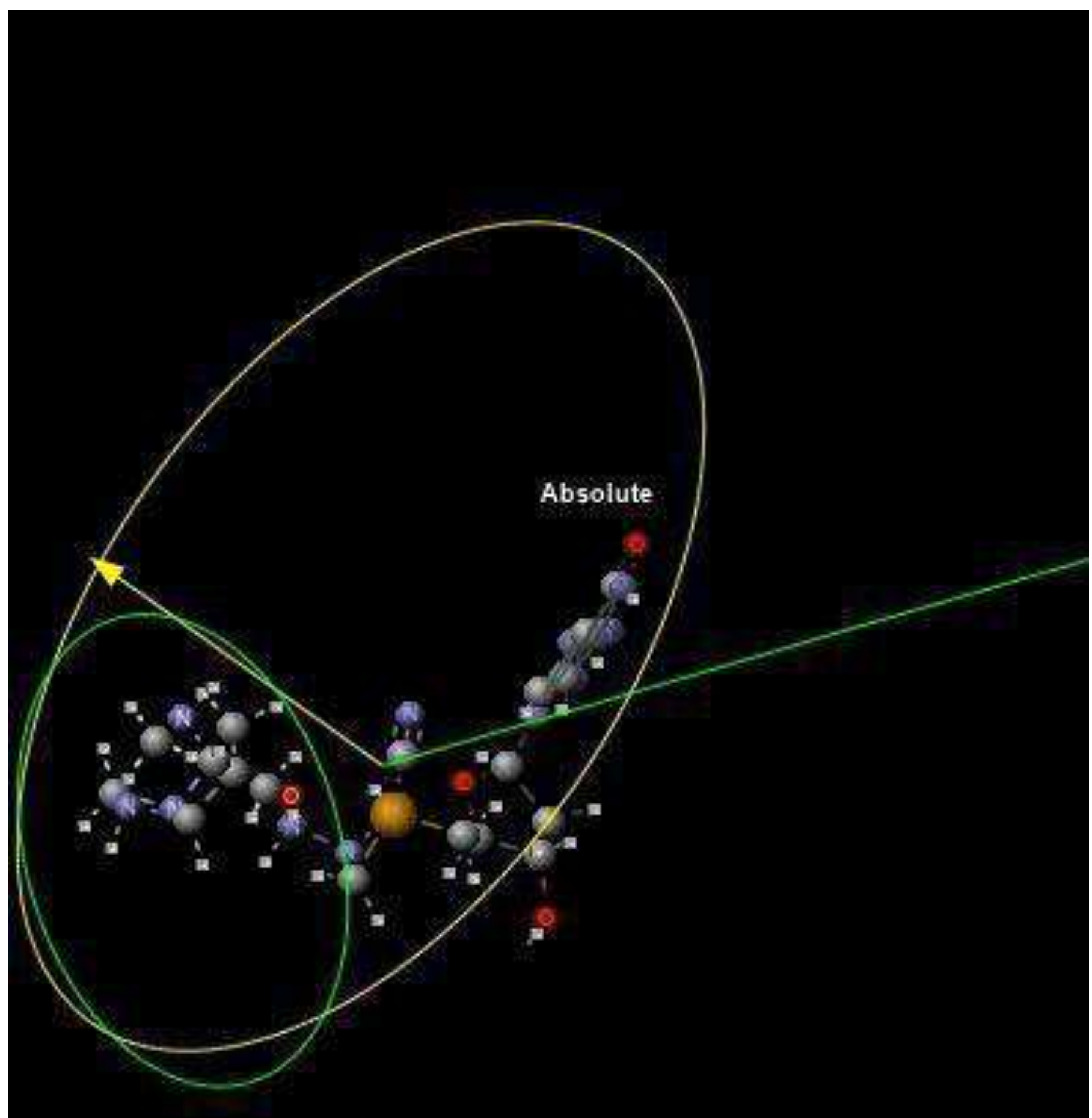

\section{RoccustyrnaTM}
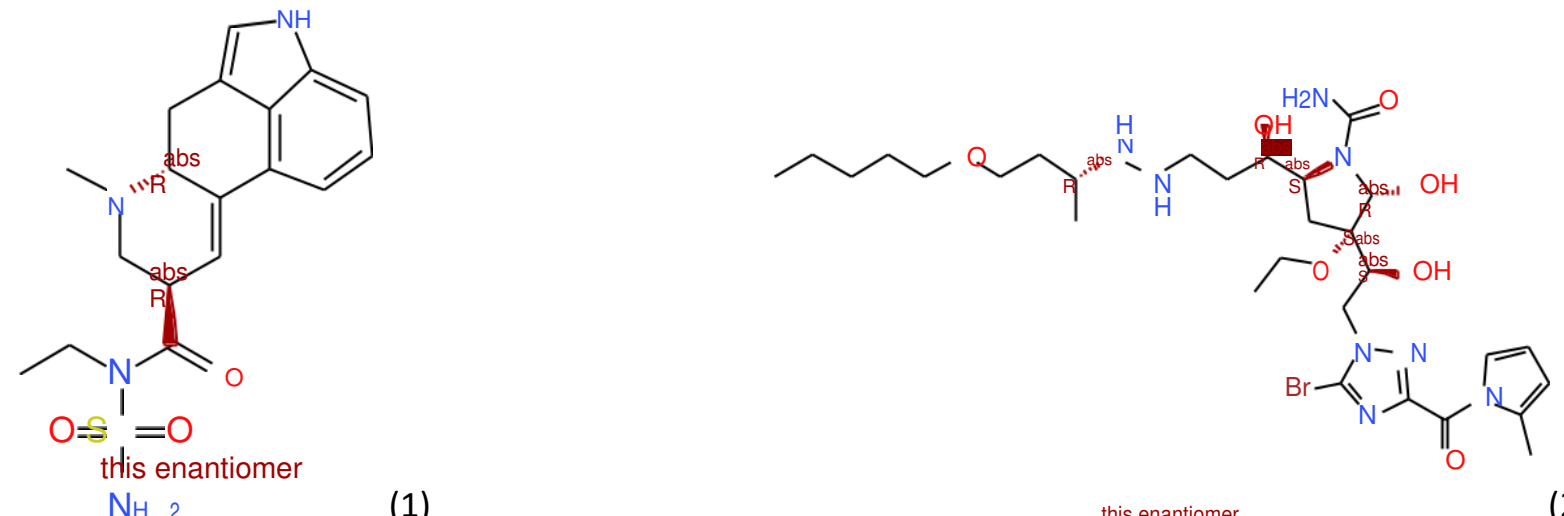

(1) 
Figure2a. Geometrical descriptors of the Roccustyrna small molecule. Dreiding energy $=287,17 \mathrm{kcal} / \mathrm{mol} \mathrm{MMFF94}$ energy $=234,59 \mathrm{kcal} / \mathrm{mol}$, Minimal projection area $=63,36$, Maximal projection area $=133,25$, Minimal projection radius $=6,25$, Maximal projection radius $=9,68$, Length perpendicular to the max area $=8,32$, Length perpendicular to the $\min$ area $=19,36$, van der Waals volume $=440,91$

The Roccustyrna small molecule is the fusion product of such chemical space representations of negative energy selected representations as merged into
the connection form, $(\mathbf{a}+\ell)$ ta $+\boldsymbol{\ell}-\mathbf{1}$ 2zEGk: $\mathbf{2 z E G} \otimes \mathbf{2 z E G} \rightarrow$ 践 of the 6,9-dihydro-3H-purin loop group.

COMPND C:IUsersluserlDownloadslRoccustyrna.pdb

AUTHOR GENERATED BY OPEN BABEL 2.4.1

HETATM 1 C UNK $0 \quad 7.267-1.330-5.0971 .000 .00 \quad$ C

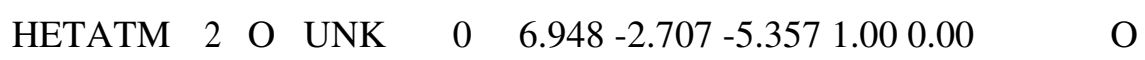

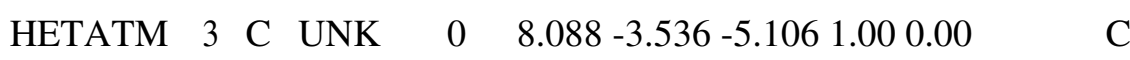

$\begin{array}{llllllllll}\text { HETATM } \quad 4 & \mathrm{C} & \mathrm{UNK} & 0 & 9.117 & -2.681 & -4.342 & 1.00 & 0.00 & \mathrm{C}\end{array}$

$\begin{array}{lllllllll}\text { HETATM } \quad 5 & \mathrm{C} & \mathrm{UNK} & 0 & 8.781-1.249-4.771 & 1.00 & 0.00 & \mathrm{C}\end{array}$

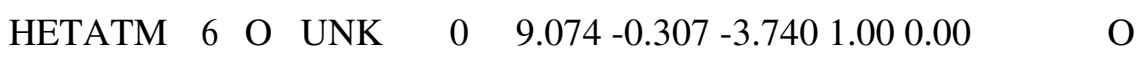

$\begin{array}{lllllllll}\text { HETATM } & 7 & \mathrm{~N} & \mathrm{UNK} & 0 & 8.610-4.088 & -6.329 & 1.00 & 0.00 \quad \mathrm{~N}\end{array}$

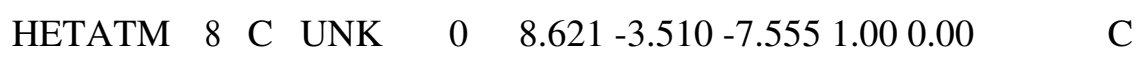

$\begin{array}{llllllllll}\text { HETATM } & 9 & \mathrm{~N} & \mathrm{UNK} & 0 & 9.164 & -4.317-8.487 & 1.00 & 0.00 \quad \mathrm{~N}\end{array}$

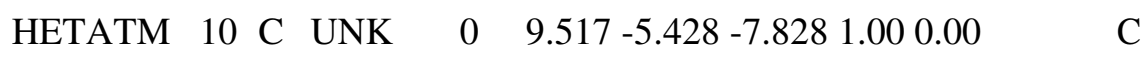

HETATM 11 C UNK $\quad 0 \quad 10.128-6.613-8.2661 .000 .00 \quad$ C

HETATM 12 O UNK $\quad 0 \quad 10.423-6.791-9.4681 .000 .00 \quad$ O

$\begin{array}{llllllllll}\text { HETATM } 13 & \mathrm{~N} & \mathrm{UNK} & 0 & 10.404-7.573-7.341 & 1.00 & 0.00 \quad \mathrm{~N}\end{array}$

HETATM 14 C UNK $0 \quad 10.099-7.412-6.0211 .000 .00 \quad$ C

$\begin{array}{lllllllll}\text { HETATM } & 15 & \mathrm{~N} & \mathrm{UNK} & 0 & 10.427-8.375 & -5.146 & 1.00 & 0.00 \quad \mathrm{~N}\end{array}$

HETATM $16 \mathrm{~N}$ UNK $0 \quad 9.479-6.276-5.5921 .000 .00 \quad \mathrm{~N}$

HETATM 17 C UNK $\quad 0 \quad 9.192-5.291-6.4831 .000 .00 \quad$ C

HETATM 18 C UNK $0 \quad 6.836-0.507-6.2151 .000 .00 \quad$ C

$\begin{array}{llllllll}\text { HETATM } 19 & \mathrm{P} & \mathrm{UNK} & 0 & 5.315-0.469-6.692 & 1.00 & 0.00 & \mathrm{P}\end{array}$

$\begin{array}{llllllllll}\text { HETATM } 20 \mathrm{~N} & \text { UNK } & 0 & 4.457 & 0.672 & -6.122 & 1.00 & 0.00 & \mathrm{~N}\end{array}$

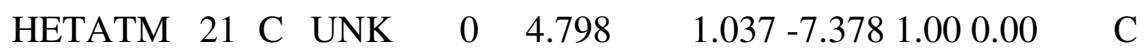

HETATM 22 C UNK $0 \quad 4.548-1.742-7.1821 .000 .00 \quad$ C

$\begin{array}{llllllll}\text { HETATM } 23 \mathrm{~N} & \text { UNK } & 0 & 3.991 & -2.683 & -7.532 & 1.00 & 0.00 \quad \mathrm{~N}\end{array}$ 


\begin{tabular}{|c|c|c|c|c|c|c|}
\hline HETATM & $24 \mathrm{~N}$ & UNK & 0 & 3.836 & 1.049 & -5.1381 .000 .00 \\
\hline HETATM & $25 \mathrm{O}$ & UNK & 0 & 3.749 & 0.353 & -4.0671 .000 .00 \\
\hline HETATM & $26 \mathrm{C}$ & UNK & 0 & 2.989 & 1.051 & -3.0601 .000 .00 \\
\hline HETATM & $27 \mathrm{C}$ & UNK & 0 & 3.044 & 0.275 & -1.7301 .000 .00 \\
\hline HETATM & $28 \mathrm{C}$ & UNK & 0 & 1.816 & -0.680 & $0-1.7611 .000 .00$ \\
\hline HETATM & $29 \mathrm{~N}$ & UNK & 0 & 0.777 & 0.047 & -2.5631 .000 .00 \\
\hline HETATM & $30 \mathrm{C}$ & UNK & 0 & 1.470 & 1.075 & -3.3661 .000 .00 \\
\hline HETATM & $31 \mathrm{C}$ & UNK & 0 & 0.028 & 1.156 & $5-1.9121 .000 .00$ \\
\hline HETATM & $32 \mathrm{~N}$ & UNK & 0 & 0.733 & 2.164 & -2.7061 .000 .00 \\
\hline HETATM & $33 \mathrm{~N}$ & UNK & 0 & 1.267 & -0.975 & $5-0.4021 .000 .00$ \\
\hline HETATM & $34 \mathrm{C}$ & UNK & 0 & 2.136 & -2.020 & - 2.4981 .000 .00 \\
\hline HETATM & $35 \mathrm{C}$ & UNK & 0 & 2.134 & -1.612 & 0.6121 .000 .00 \\
\hline HETATM & $36 \mathrm{H}$ & UNK & 0 & 8.792 & 0.560 & -4.1011 .000 .00 \\
\hline HETATM & $37 \mathrm{H}$ & UNK & 0 & 6.666 & -0.958 & $8-4.2671 .000 .00$ \\
\hline HETATM & $38 \mathrm{H}$ & UNK & 0 & 7.768 & -4.419 & $9-4.5531 .000 .00$ \\
\hline HETATM & $39 \mathrm{H}$ & UNK & 0 & 8.908 & -2.770 & $0-3.2761 .000 .00$ \\
\hline HETATM & $40 \mathrm{H}$ & UNK & 0 & 10.109 & $-2.922-4$ & .7251 .000 .00 \\
\hline HETATM & $41 \mathrm{H}$ & UNK & 0 & 9.404 & -0.848 & $8-5.5701 .000 .00$ \\
\hline HETATM & $42 \mathrm{H}$ & UNK & 0 & 8.238 & -2.512 & $2-7.7671 .000 .00$ \\
\hline HETATM & $43 \mathrm{H}$ & UNK & 0 & 9.723 & -9.019 & $9-4.8161 .000 .00$ \\
\hline HETATM & $44 \mathrm{H}$ & UNK & 0 & 11.378 & $-8.455-4$ & 8161.000 .00 \\
\hline HETATM & $45 \mathrm{H}$ & UNK & 0 & 9.235 & -6.164 & $4-4.6181 .000 .00$ \\
\hline HETATM & $46 \mathrm{H}$ & UNK & 0 & 7.634 & 0.068 & -6.6851 .000 .00 \\
\hline HETATM & $47 \mathrm{H}$ & UNK & 0 & 3.984 & 0.995 & -8.1031 .000 .00 \\
\hline HETATM & $48 \mathrm{H}$ & UNK & 0 & 5.540 & 1.832 & -7.4381 .000 .00 \\
\hline HETATM & $49 \mathrm{H}$ & UNK & 0 & 3.465 & 2.031 & -3.0711 .000 .00 \\
\hline HETATM & $50 \mathrm{H}$ & UNK & 0 & 2.887 & 0.994 & -0.9251 .000 .00 \\
\hline HETATM & $51 \mathrm{H}$ & UNK & 0 & 3.944 & -0.338 & $8-1.7451 .000 .00$ \\
\hline HETATM & $52 \mathrm{H}$ & UNK & 0 & 1.416 & 1.052 & -4.4541 .000 .00 \\
\hline HETATM & $53 \mathrm{H}$ & UNK & 0 & 0.308 & 1.241 & $\begin{array}{l}-0.8621 .00 \\
0.00\end{array}$ \\
\hline HETATM & $54 \mathrm{H}$ & UNK & 0 & -1.024 & 1.12 & 1951.000 .00 \\
\hline
\end{tabular}




$\begin{array}{llllrl}\text { HETATM } & 55 \mathrm{H} \text { UNK } & 0 & 0.718 & 3.172-2.7711 .000 .00 & \mathrm{H} \\ \text { HETATM } & 56 \mathrm{H} \mathrm{UNK} & 0 & 0.312-0.745-0.1691 .000 .00 & \mathrm{H} \\ \text { HETATM } & 57 \mathrm{H} \mathrm{UNK} & 0 & 2.323-2.803-1.7631 .000 .00 & \mathrm{H} \\ \text { HETATM } & 58 \mathrm{H} \mathrm{UNK} & 0 & 1.288-2.303-3.1221 .000 .00 & \mathrm{H} \\ \text { HETATM } & 59 \mathrm{H} \mathrm{UNK} & 0 & 3.020-1.889-3.1221 .000 .00 & \mathrm{H} \\ \text { HETATM } & 60 \mathrm{H} \mathrm{UNK} & 0 & 1.780-1.352 & 1.6091 .000 .00 & \mathrm{H} \\ \text { HETATM } & 61 \mathrm{H} \mathrm{UNK} & 0 & 2.105-2.695 & 0.4871 .000 .00 & \mathrm{H} \\ \text { HETATM } & 62 \mathrm{H} \mathrm{UNK} & 0 & 3.158-1.260 & 0.4871 .000 .00 & \mathrm{H}\end{array}$

CONECT 1182537

$\begin{array}{llll}\text { CONECT } & 2 & 3 & 1\end{array}$

$\begin{array}{llll}\text { CONECT } & 3 & 7 & 2384\end{array}$

CONECT $4 \quad 3 \quad 54039$

CONECT 541146

$\begin{array}{llll}\text { CONECT } & 6 & 5 & 36\end{array}$

$\begin{array}{llll}\text { CONECT } & 7 & 8 & 173\end{array}$

$\begin{array}{llll}\text { CONECT } & 8 & 9 & 427\end{array}$

$\begin{array}{llll}\text { CONECT } & 9 & 10 & 8\end{array}$

$\begin{array}{lllll}\text { CONECT } & 10 & 9 & 11 & 17\end{array}$

$\begin{array}{lllll}\text { CONECT } & 11 & 12 & 10 & 13\end{array}$

CONECT $12 \quad 11$

$\begin{array}{llll}\text { CONECT } & 13 & 11 & 14\end{array}$

$\begin{array}{lllll}\text { CONECT } & 14 & 13 & 16 & 15\end{array}$

$\begin{array}{lllll}\text { CONECT } & 15 & 14 & 43 & 44\end{array}$

$\begin{array}{lllll}\text { CONECT } & 16 & 17 & 14 & 45\end{array}$

$\begin{array}{lllll}\text { CONECT } & 17 & 10 & 7 & 16\end{array}$

$\begin{array}{lllll}\text { CONECT } & 18 & 19 & 46 & 1\end{array}$

$\begin{array}{llllll}\text { CONECT } & 19 & 21 & 22 & 18 & 20\end{array}$

$\begin{array}{lllll}\text { CONECT } & 20 & 21 & 19 & 24\end{array}$

$\begin{array}{llllll}\text { CONECT } & 21 & 47 & 48 & 19 & 20\end{array}$

$\begin{array}{llll}\text { CONECT } & 22 & 23 & 19\end{array}$

CONECT $23 \quad 22$ 
$\begin{array}{llll}\text { CONECT } & 24 & 20 & 25\end{array}$

$\begin{array}{llll}\text { CONECT } & 25 & 24 & 26\end{array}$

$\begin{array}{llllll}\text { CONECT } & 26 & 25 & 30 & 49 & 27\end{array}$

$\begin{array}{llllll}\text { CONECT } & 27 & 26 & 28 & 51 & 50\end{array}$

$\begin{array}{llllll}\text { CONECT } & 28 & 29 & 34 & 27 & 33\end{array}$

$\begin{array}{lllll}\text { CONECT } & 29 & 30 & 31 & 28\end{array}$

$\begin{array}{llllll}\text { CONECT } & 30 & 52 & 26 & 32 & 29\end{array}$

$\begin{array}{llllll}\text { CONECT } & 31 & 32 & 29 & 54 & 53\end{array}$

$\begin{array}{lllll}\text { CONECT } & 32 & 30 & 55 & 31\end{array}$

$\begin{array}{lllll}\text { CONECT } & 33 & 28 & 56 & 35\end{array}$

$\begin{array}{llllll}\text { CONECT } & 34 & 58 & 59 & 57 & 28\end{array}$

$\begin{array}{llllll}\text { CONECT } & 35 & 33 & 61 & 62 & 60\end{array}$

CONECT $36 \quad 6$

$\begin{array}{lll}\text { CONECT } & 37 & 1\end{array}$

CONECT $38 \quad 3$

CONECT $39 \quad 4$

CONECT $40 \quad 4$

CONECT $41 \quad 5$

CONECT $42 \quad 8$

CONECT $43 \quad 15$

CONECT $44 \quad 15$

CONECT $45 \quad 16$

CONECT $46 \quad 18$

CONECT $47 \quad 21$

CONECT $48 \quad 21$

CONECT $49 \quad 26$

CONECT $50 \quad 27$

CONECT $51 \quad 27$

CONECT $52 \quad 30$

CONECT $53 \quad 31$

CONECT 5431 
CONECT 5532

CONECT $56 \quad 33$

CONECT $57 \quad 34$

CONECT $\quad 58 \quad 34$

CONECT 5934

CONECT $60 \quad 35$

CONECT $61 \quad 35$

CONECT $62 \quad 35$

$\begin{array}{lllllllllllll}\text { MASTER } & 0 & 0 & 0 & 0 & 0 & 0 & 0 & 0 & 62 & 0 & 62 & 0\end{array}$

END

Figure2b. RoccustyrnaTM small molecule's PDB file 


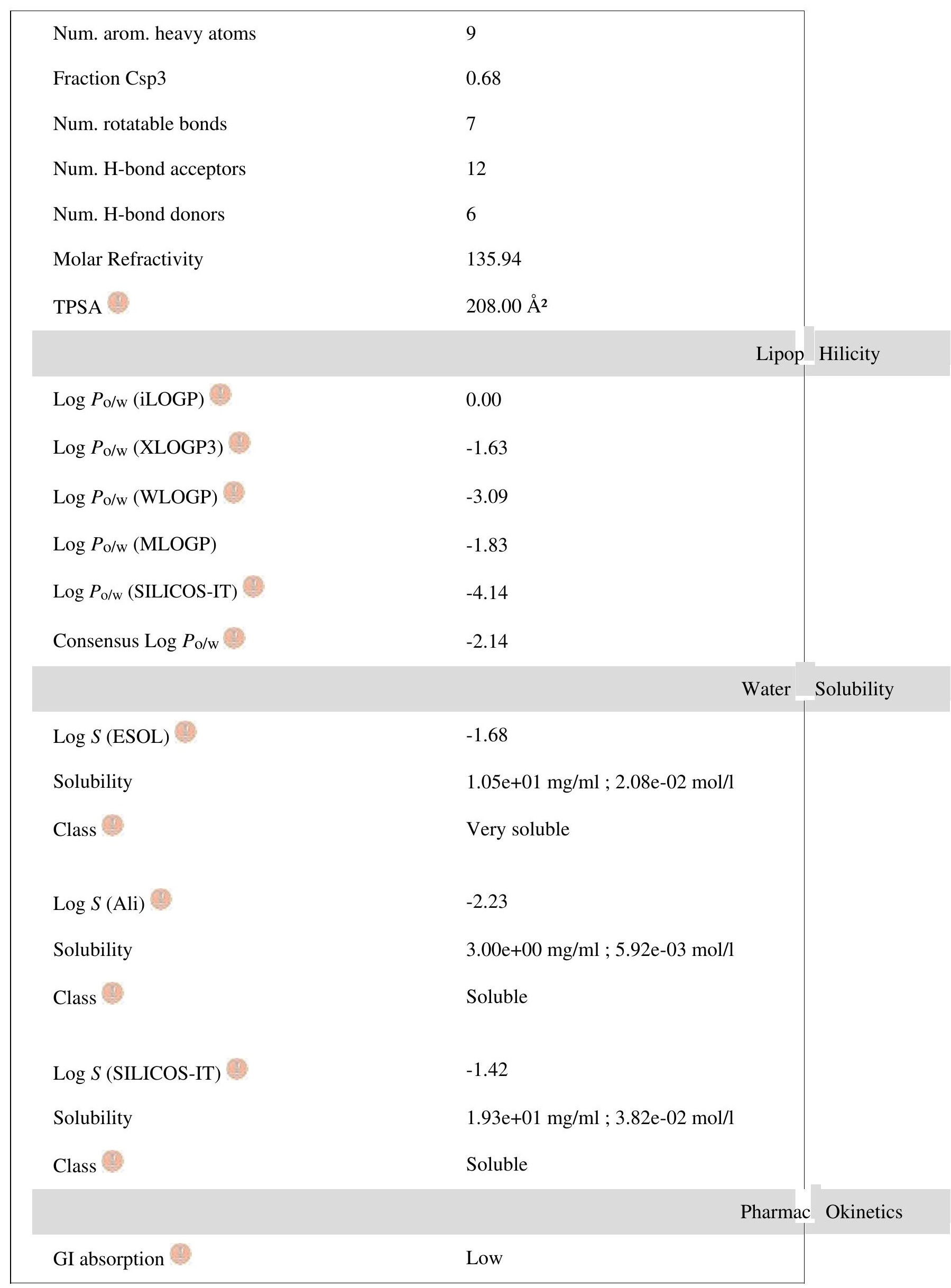




\begin{tabular}{|c|c|c|c|}
\hline BBB permeant $\odot$ P-gp & No & & \\
\hline substrate CYP1A2 & Yes & & \\
\hline inhibitor $\mathrm{CYP} 2 \mathrm{C} 19$ & No & & \\
\hline inhibitor 1 CYP2C9 & No & & \\
\hline inhibitor $\odot$ CYP2D6 & No & & \\
\hline inhibitor CYP3A4 & No & & \\
\hline inhibitor 9 & No & & \\
\hline \multirow[t]{2}{*}{$\log K_{\mathrm{p}}($ skin permeation $)$} & $-10.55 \mathrm{~cm} / \mathrm{s}$ & & \\
\hline & & Medicinal & Chemistry \\
\hline PAINS & 0 alert & & \\
\hline Brenk 9 & $\begin{array}{l}3 \text { alerts: Three- } \\
\text { membered_heterocycle, oxy }\end{array}$ & & $\begin{array}{l}\text { gen- } \\
\text { nitrogen_sinin }\end{array}$ \\
\hline Leadlikeness & No; 1 violation: $M W>350$ & & \\
\hline Synthetic accessibility & 6.25 & & \\
\hline
\end{tabular}

Figure2c.RoccustyrnaTM_Preferred_IUPACName=(1Z) - $2-\{((2 S, 3 S, 5 R)-5-(2-$ amino $-6-$ oxo-6,9-dihydro- $1 \mathrm{H}-$ purin -9 -yl $)-3-$ hydrox yoxolan-2-yl)methylidene $\}$-2-cyano-1-(\{((2S,4R,5R)-2-methyl-2-(methylamino)-1,6-diazabicyclo(3.2.0)heptan-4-yl)oxy $\}$ imino) 1lambda5,2lambda5-azaphosphiridin-1-ylium. 


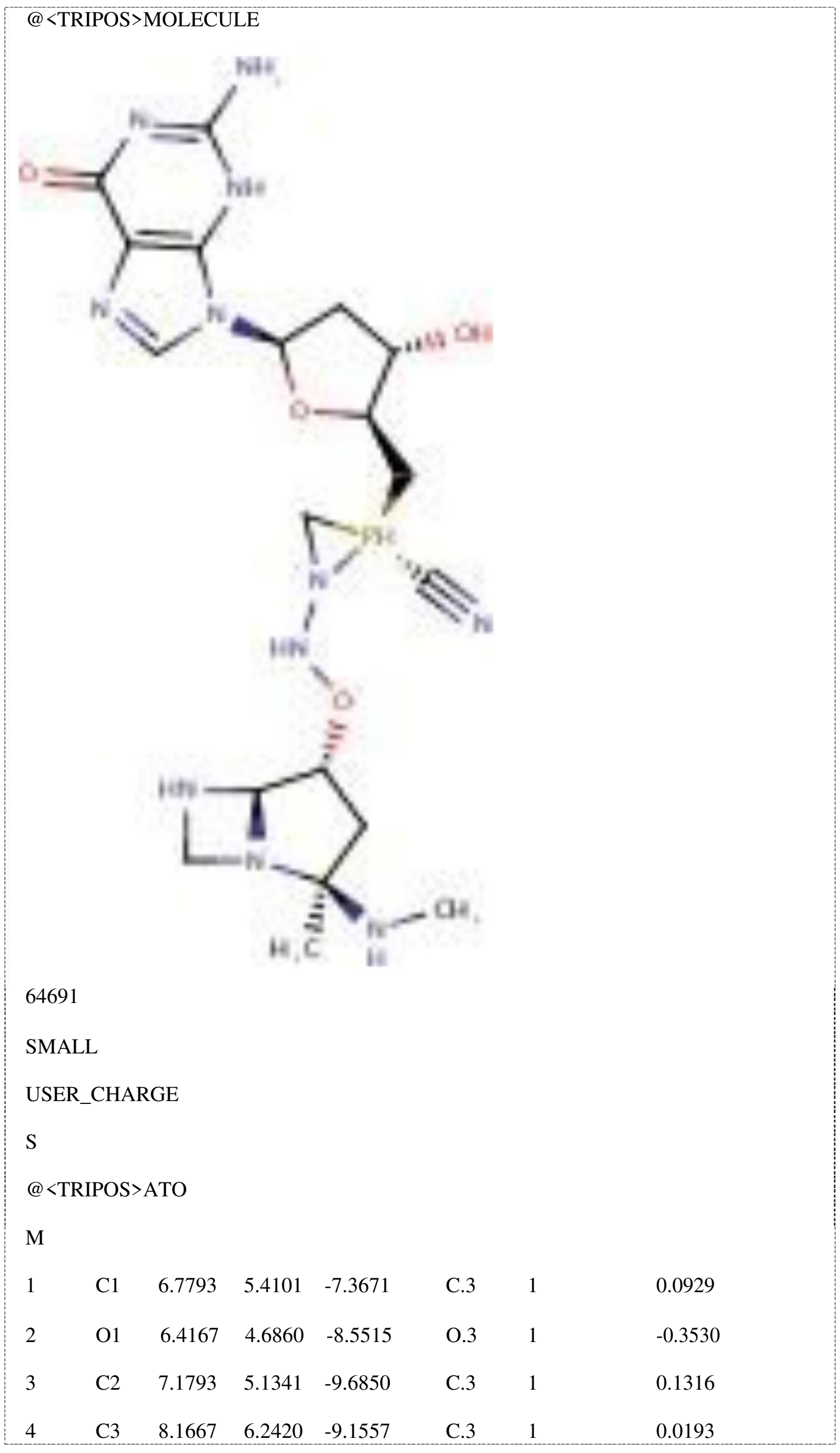




\begin{tabular}{|cccccccc|}
\hline 5 & $\mathrm{C} 4$ & 7.4664 & 6.7292 & -7.8136 & $\mathrm{C} .3$ & 1 & 0.0850 \\
6 & $\mathrm{O} 2$ & 8.3312 & 7.2161 & -6.7537 & $\mathrm{O} .3$ & 1 & -0.3901 \\
7 & $\mathrm{~N} 1$ & 6.2816 & 5.6652 & -10.6793 & $\mathrm{~N} .2$ & 1 & 0.0158 \\
8 & $\mathrm{C} 5$ & 5.0344 & $6.1629-10.4967$ & $\mathrm{C} .2$ & 1 & -0.0334 \\
9 & $\mathrm{~N} 2$ & 4.4579 & 6.5206 & -11.6609 & $\mathrm{~N} .2$ & 1 & -0.2093 \\
10 & $\mathrm{C} 6$ & 5.3716 & 6.2562 & -12.6024 & $\mathrm{C} .2$ & 1 & 0.0617 \\
11 & $\mathrm{C} 7$ & 5.3381 & $6.4013-13.9991$ & $\mathrm{C} .2$ & 1 & 0.1449 \\
12 & $\mathrm{O} 3$ & 4.3223 & 6.8295 & -14.5891 & $\mathrm{O} .2$ & 1 & -0.2466 \\
13 & $\mathrm{~N} 3$ & 6.4475 & 6.0473 & -14.7039 & $\mathrm{~N} .2$ & 1 & -0.1575 \\
14 & $\mathrm{C} 8$ & 7.5681 & 5.5602 & -14.0975 & $\mathrm{C} .2$ & 1 & -0.0476 \\
15 & $\mathrm{~N} 4$ & 8.6368 & 5.2334 & -14.8398 & $\mathrm{~N} .3$ & 1 & -0.1336 \\
16 & $\mathrm{~N} 5$ & 7.6009 & 5.4035 & -12.7434 & $\mathrm{~N} .2$ & 1 & 0.0739 \\
17 & $\mathrm{C} 9$ & 6.5147 & 5.7408 & -12.0019 & $\mathrm{C} .2$ & 1 & 0.0095 \\
18 & $\mathrm{C} 10$ & 5.5391 & 5.6450 & -6.4567 & $\mathrm{C} .3$ & 1 & 0.0181 \\
19 & $\mathrm{P} 1$ & 4.4871 & 4.2358 & -6.1084 & $\mathrm{P} .3$ & 1 & -0.1484 \\
\hline & & & & & & & \\
\hline & & & & & 1 & \\
\hline & & & & & 1 & \\
\hline
\end{tabular}




\begin{tabular}{|c|c|c|c|c|c|c|c|}
\hline 20 & N6 & 4.4157 & 2.6297 & -5.5202 & N.3 & 1 & -0.0518 \\
\hline 21 & $\mathrm{C} 11$ & 5.5025 & 3.3359 & -5.0335 & C. 3 & 1 & 0.0624 \\
\hline 22 & N7 & 3.4152 & 2.4619 & -4.5388 & N.3 & 1 & -0.0934 \\
\hline 23 & $\mathrm{O} 4$ & 3.5802 & 1.3462 & -3.7641 & 0.3 & 1 & -0.3178 \\
\hline 24 & $\mathrm{C} 12$ & 2.7318 & 1.4614 & -2.5999 & C. 3 & 1 & 0.1125 \\
\hline 25 & $\mathrm{C} 13$ & 3.0058 & 0.3420 & -1.5704 & C.3 & 1 & 0.0100 \\
\hline 26 & $\mathrm{C} 14$ & \multicolumn{3}{|c|}{$1.8142-0.6553-1.6624$} & C. 3 & 1 & 0.0734 \\
\hline 27 & N8 & 0.6767 & 0.1212 & -2.2390 & N.3 & 1 & -0.2544 \\
\hline 28 & $\mathrm{C} 15$ & 1.2258 & 1.2977 & -2.9316 & C.3 & 1 & 0.0925 \\
\hline 29 & N9 & 0.3485 & 2.2044 & -2.1731 & N.3 & 1 & -0.2864 \\
\hline 30 & $\mathrm{C} 16$ & -0.0886 & 1.0558 & -1.3757 & C. 3 & 1 & 0.0528 \\
\hline 31 & N10 & \multicolumn{3}{|c|}{$1.3728-1.2053-0.3452$} & N.3 & 1 & -0.3022 \\
\hline 32 & $\mathrm{C} 17$ & 2.3014 & -2.0166 & 0.4626 & C. 3 & 1 & -0.0147 \\
\hline 33 & $\mathrm{C} 18$ & \multicolumn{3}{|c|}{$2.1137-1.8150-2.6582$} & C. 3 & 1 & -0.0330 \\
\hline 34 & $\mathrm{C} 19$ & 3.1251 & 4.9597 & -5.6402 & C. 1 & 1 & 0.0718 \\
\hline 35 & N11 & 2.1558 & 5.4787 & -5.3100 & N.1 & 1 & -0.1788 \\
\hline 36 & H1 & 7.4770 & 4.7777 & -6.8180 & $\mathrm{H}$ & 1 & 0.0637 \\
\hline 37 & $\mathrm{H} 2$ & 7.6610 & 4.2815 & -10.1636 & $\mathrm{H}$ & 1 & 0.0824 \\
\hline 38 & $\mathrm{H} 3$ & 9.1078 & 5.7581 & -8.8944 & $\mathrm{H}$ & 1 & 0.0338 \\
\hline 39 & $\mathrm{H} 4$ & 8.1578 & 7.0689 & -9.8659 & $\mathrm{H}$ & 1 & 0.0338 \\
\hline 40 & H5 & 6.8657 & 7.6318 & -7.9260 & $\mathrm{H}$ & 1 & 0.0629 \\
\hline 41 & H6 & 8.7137 & 6.4708 & -6.2850 & $\mathrm{H}$ & 1 & 0.2105 \\
\hline 42 & $\mathrm{H} 7$ & 4.5506 & 6.2639 & -9.5252 & $\mathrm{H}$ & 1 & 0.1016 \\
\hline 43 & $\mathrm{H} 8$ & 8.7834 & 4.2740 & -15.1192 & $\mathrm{H}$ & 1 & 0.1257 \\
\hline 44 & H9 & 9.2948 & 5.9469 & -15.1192 & $\mathrm{H}$ & 1 & 0.1257 \\
\hline 45 & $\mathrm{H} 10$ & 8.4270 & 5.038 & $7-12.2910$ & $\mathrm{H}$ & 1 & 0.0676 \\
\hline 46 & H11 & 6.0012 & 5.9571 & -5.5201 & $\mathrm{H}$ & 1 & 0.0379 \\
\hline 47 & H12 & 4.9596 & 6.3583 & -7.0427 & $\mathrm{H}$ & 1 & 0.0379 \\
\hline
\end{tabular}




\begin{tabular}{|c|c|c|c|c|c|c|c|}
\hline 48 & H13 & 5.4208 & 3.5873 & -3.9760 & $\mathrm{H}$ & 1 & 0.0539 \\
\hline 49 & H14 & 6.4567 & 3.0042 & -5.4427 & $\mathrm{H}$ & 1 & 0.0539 \\
\hline 50 & H15 & 2.6390 & 3.0933 & -4.4012 & $\mathrm{H}$ & 1 & 0.1759 \\
\hline 51 & H16 & 3.0064 & 2.4621 & -2.2662 & $\mathrm{H}$ & 1 & 0.0657 \\
\hline 52 & $\mathrm{H} 17$ & 3.9007 & -0.1865 & -1.8986 & $\mathrm{H}$ & 1 & 0.0327 \\
\hline 53 & H18 & 2.9745 & 0.7986 & -0.5811 & $\mathrm{H}$ & 1 & 0.0327 \\
\hline 54 & H19 & 1.1772 & 1.3623 & -4.0186 & $\mathrm{H}$ & 1 & 0.0667 \\
\hline 55 & $\mathrm{H} 20$ & 0.1370 & 3.1919 & -2.1918 & $\mathrm{H}$ & 1 & 0.1251 \\
\hline 56 & $\mathrm{H} 21$ & 0.3474 & 1.1003 & -0.3777 & $\mathrm{H}$ & 1 & 0.0601 \\
\hline 57 & $\mathrm{H} 22$ & -1.1605 & 0.8980 & -1.4956 & $\mathrm{H}$ & 1 & 0.0601 \\
\hline 58 & $\mathrm{H} 23$ & 0.4409 & -1.0275 & 0.0013 & $\mathrm{H}$ & 1 & 0.1237 \\
\hline 59 & $\mathrm{H} 24$ & 2.1062 & -1.8461 & 1.5213 & $\mathrm{H}$ & 1 & 0.0392 \\
\hline 60 & $\mathrm{H} 25$ & 2.1567 & -3.0721 & 0.2319 & $\mathrm{H}$ & 1 & 0.0392 \\
\hline 61 & $\mathrm{H} 26$ & 3.3279 & -1.7317 & 0.2319 & $\mathrm{H}$ & 1 & 0.0392 \\
\hline 62 & $\mathrm{H} 27$ & 2.3479 & -2.7217 & -2.1003 & $\mathrm{H}$ & 1 & 0.0263 \\
\hline 63 & $\mathrm{H} 28$ & 1.2397 & -1.9900 & -3.2856 & $\mathrm{H}$ & 1 & 0.0263 \\
\hline 64 & $\mathrm{H} 29$ & 2.9632 & -1.5450 & -3.2856 & $\mathrm{H}$ & 1 & 0.0263 \\
\hline \multicolumn{8}{|c|}{ @< <TRIPOS>BOND } \\
\hline 1 & 1 & 2 & 1 & & & & \\
\hline 2 & 2 & 3 & 1 & & & & \\
\hline 3 & 3 & 4 & 1 & & & & \\
\hline 4 & 4 & 5 & 1 & & & & \\
\hline 5 & 1 & 5 & 1 & & & & \\
\hline 6 & 5 & 6 & 1 & & & & \\
\hline 7 & 3 & 7 & 1 & & & & \\
\hline 8 & 7 & 8 & ar & & & & \\
\hline 9 & 8 & 9 & ar & & & & \\
\hline 10 & 9 & 10 & ar & & & & \\
\hline
\end{tabular}




$$
\begin{aligned}
& 11 \quad 10 \quad 11 \quad \text { ar } \\
& \begin{array}{llll}
12 & 11 & 12 & 2
\end{array} \\
& \begin{array}{llll}
13 & 11 & 13 & \text { ar }
\end{array} \\
& \begin{array}{llll}
14 & 13 & 14 & \text { ar }
\end{array} \\
& \begin{array}{llll}
15 & 14 & 15 & 1
\end{array} \\
& \begin{array}{llll}
16 & 14 & 16 & \text { ar }
\end{array} \\
& \begin{array}{llll}
17 & 16 & 17 & \text { ar }
\end{array} \\
& \begin{array}{llll}
18 & 7 & 17 & \text { ar }
\end{array} \\
& \begin{array}{llll}
19 & 10 & 17 & \text { ar }
\end{array} \\
& \begin{array}{llll}
20 & 1 & 18 & 1
\end{array} \\
& \begin{array}{llll}
21 & 18 & 19 & 1
\end{array} \\
& \begin{array}{llll}
22 & 19 & 20 & 1
\end{array} \\
& \begin{array}{llll}
23 & 20 & 21 & 1
\end{array} \\
& \begin{array}{llll}
24 & 19 & 21 & 1
\end{array} \\
& \begin{array}{llll}
25 & 20 & 22 & 1
\end{array} \\
& \begin{array}{llll}
26 & 22 & 23 & 1
\end{array} \\
& \begin{array}{llll}
27 & 23 & 24 & 1
\end{array} \\
& \begin{array}{llll}
28 & 24 & 25 & 1
\end{array} \\
& \begin{array}{llll}
29 & 25 & 26 & 1
\end{array} \\
& \begin{array}{llll}
30 & 26 & 27 & 1
\end{array} \\
& \begin{array}{llll}
31 & 27 & 28 & 1
\end{array} \\
& \begin{array}{llll}
32 & 24 & 28 & 1
\end{array} \\
& \begin{array}{llll}
33 & 28 & 29 & 1
\end{array} \\
& \begin{array}{llll}
34 & 29 & 30 & 1
\end{array} \\
& \begin{array}{llll}
35 & 27 & 30 & 1
\end{array} \\
& \begin{array}{llll}
36 & 26 & 31 & 1
\end{array} \\
& \begin{array}{llll}
37 & 31 & 32 & 1
\end{array} \\
& \begin{array}{llll}
38 & 26 & 33 & 1
\end{array}
\end{aligned}
$$




\begin{tabular}{|c|c|c|}
\hline 39 & 19 & 34 \\
\hline 40 & 34 & 35 \\
\hline 41 & 1 & 36 \\
\hline 42 & 3 & 37 \\
\hline 43 & 4 & 38 \\
\hline 44 & 4 & 39 \\
\hline 45 & 5 & 40 \\
\hline 46 & 6 & 41 \\
\hline 47 & 8 & 42 \\
\hline 48 & 15 & 43 \\
\hline 49 & 15 & 44 \\
\hline 50 & 16 & 45 \\
\hline 51 & 18 & 46 \\
\hline 52 & 18 & 47 \\
\hline 53 & 21 & 48 \\
\hline 54 & 21 & 49 \\
\hline 55 & 22 & 50 \\
\hline 56 & 24 & 51 \\
\hline 57 & 25 & 52 \\
\hline 58 & 25 & 53 \\
\hline 59 & 28 & 54 \\
\hline 60 & 29 & 55 \\
\hline 61 & 30 & 56 \\
\hline 62 & 30 & 57 \\
\hline 63 & 31 & 58 \\
\hline 64 & 32 & 59 \\
\hline 65 & 32 & 60 \\
\hline 66 & 32 & 61 \\
\hline
\end{tabular}




$\begin{array}{llll}67 & 33 & 62 & 1 \\ 68 & 33 & 63 & 1 \\ 69 & 33 & 64 & 1\end{array}$
@ $<$ TRIPOS $>$ SUBSTRUCTU
RE
Table1c. RoccustyrnaTM MOL2 file.

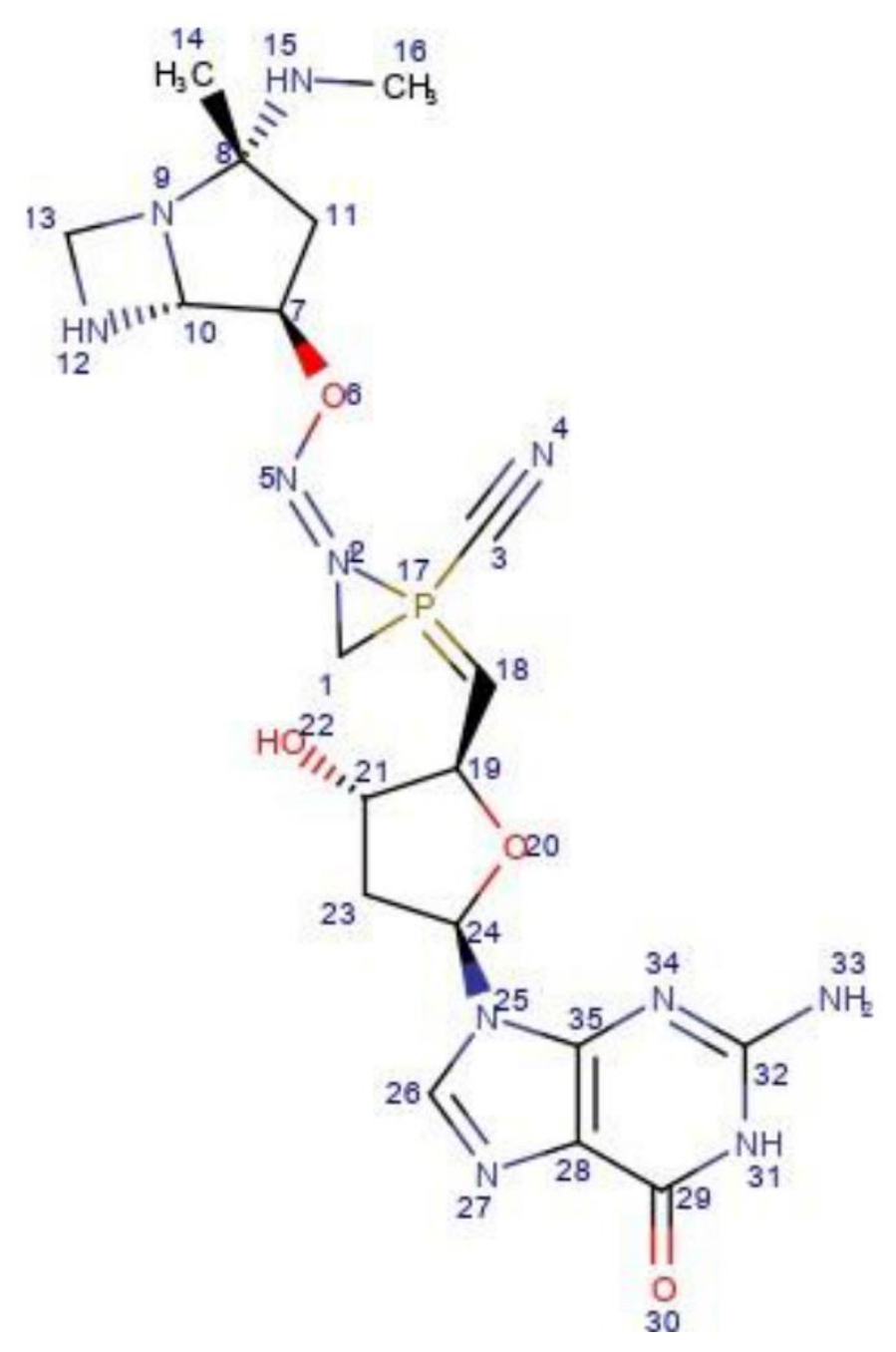

Figure2d.RoccustyrnaTM_Preferred_IUPACName $=(1 Z)-2-\{((2 S, 3 S, 5 R)-5-(2-$ amino $-6-$ oxo- $6,9-$ dih ydro-1H-purin-9-yl)-3-hydroxyoxolan-2-yl)methylidene $\}$-2-cyano-1-(\{((2S,4R,5R)-2-methyl-2-(methylamino)1,6-diazabicyclo(3.2.0)heptan-4-yl)oxy\}imino)-1lambda5,2lambda5-azaphosphiridin-1-ylium.Forinstance,a generalized coordinate is commonly used in the study of tunnelling effects and may be a bound length, a bound angle, or a dihedral angle in solid state systems. 2d Structure Map of the RoccustyrnaTM Atoms Geometrical Descriptors of the RoccustyrnaTM small molecule: Dreiding energy $=305,20 \mathrm{kcal} / \mathrm{mol}$, MMFF94 energy $=35,06 \mathrm{kcal} / \mathrm{mol}$, Minimal projection area $=66,49$, Maximal projection area $=123,65$ Minimal projection radius $=5,71$ Maximal projection radius $=9,24$ Length perpendicular to the max area $=10,29$ Length perpendicular to the min area $=19,04$ van der Waals volume $=409,41$ Donor count $=5$ Donor sites $=6$ Acceptor count 11 Acceptor sites $=14127.9718331$

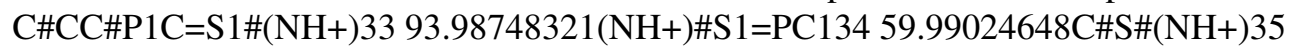




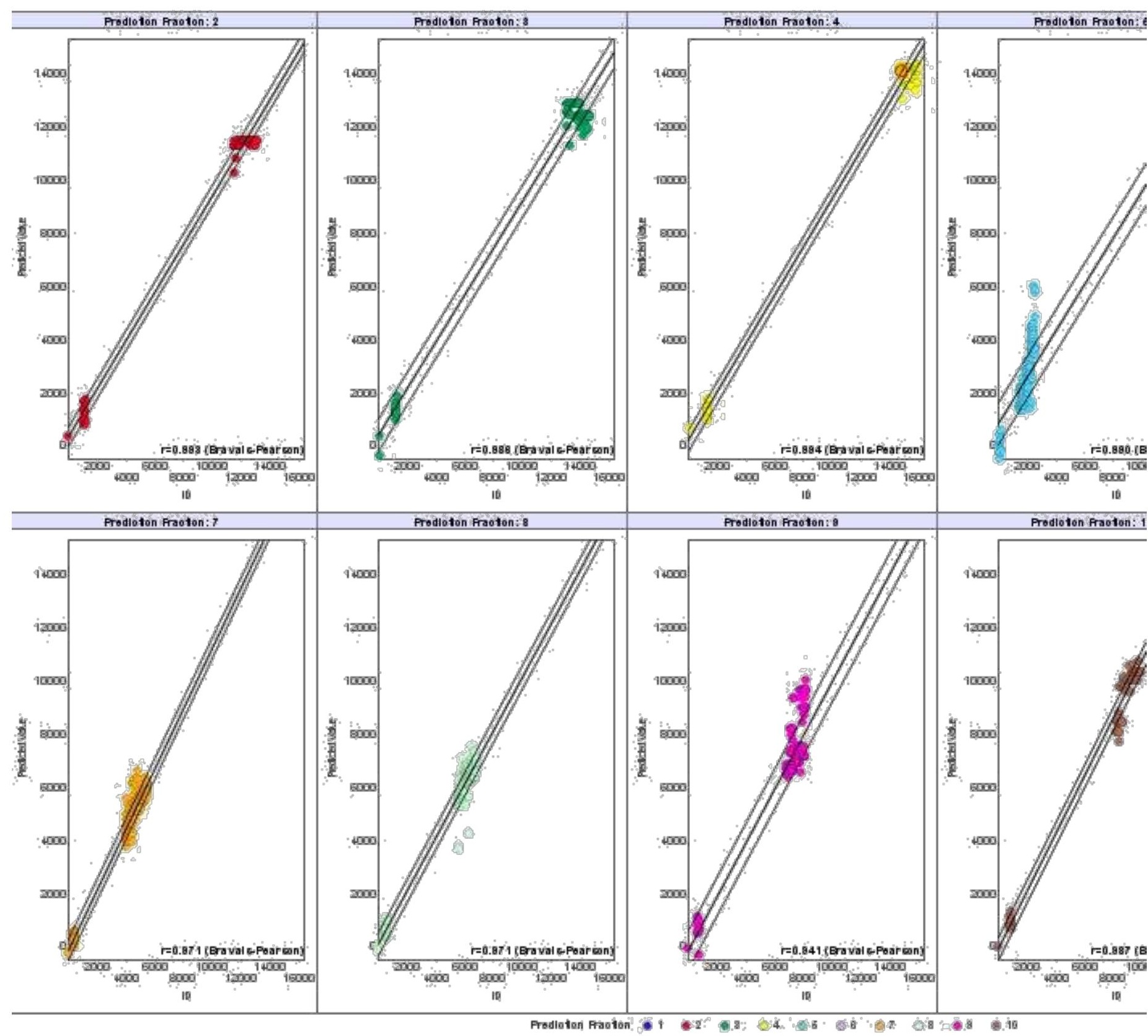

Figure3. Roccustyrna active fragments smiles prediction correletaion analysis.

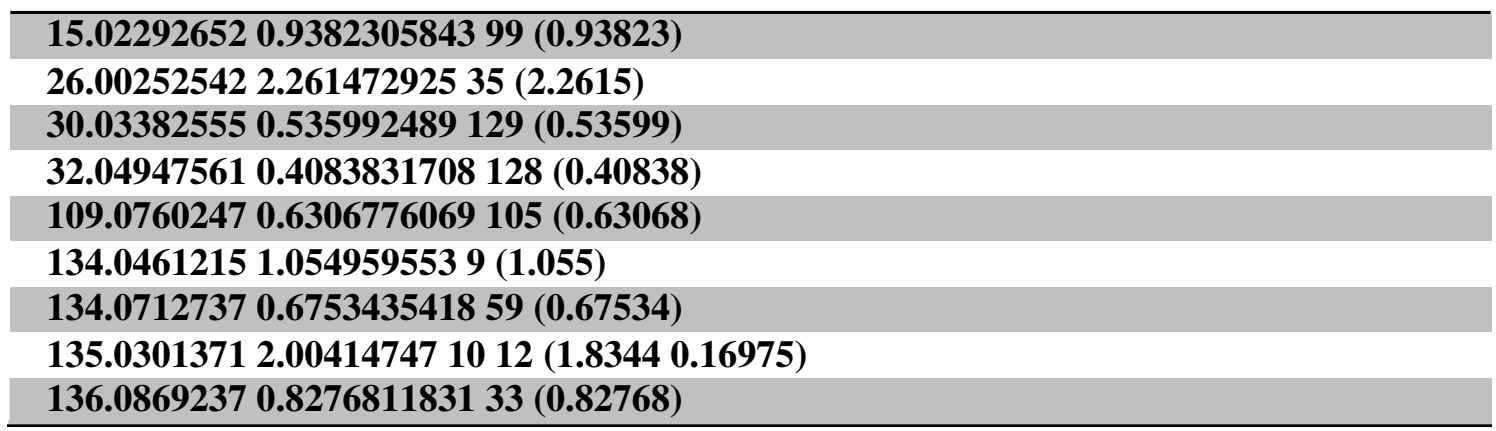




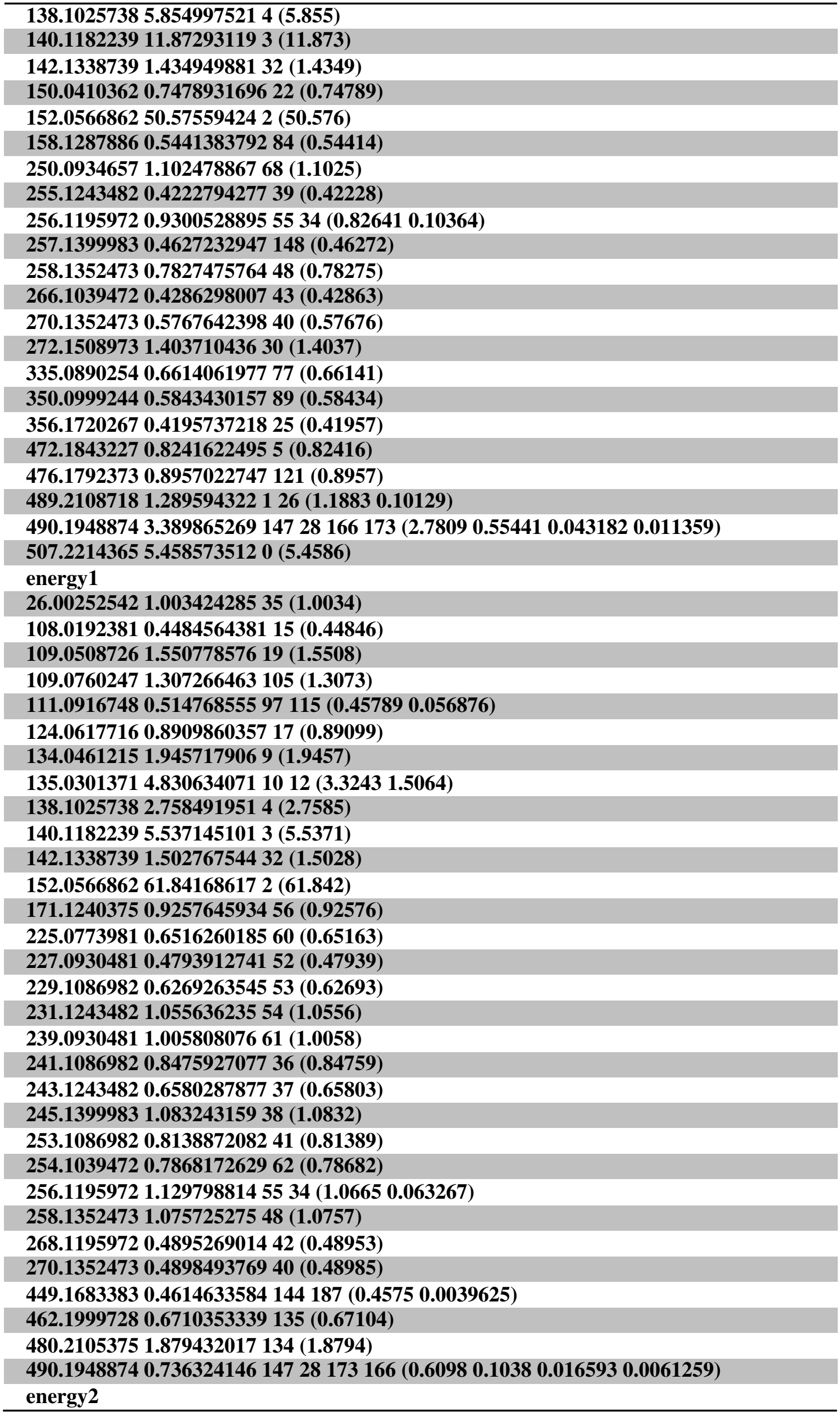


$15.022926520 .99502318199(0.99502)$

26.002525421 .02409142135 (1.0241)

$45.044724580 .951804931313(0.9518)$

$79.029074520 .7948376336118(0.79484)$

80.049475613 .661453795119 (3.6615)

81.044724581 .362092912109 (1.3621)

82.039973551 .63094242921 (1.6309)

82.065125680 .9001924745113 (0.90019)

83.060374641 .196365366101 (1.1964)

84.080775742 .268763628103 (2.2688)

85.076024712 .328821417102 (2.3288)

86.0964258 1.087814221 104 (1.0878)

$107.03522253 .06712183620(3.0671)$

107.06037461 .010133785116 (1.0101)

108.0192381 2.161146957 15 (2.1611)

109.0508726 4.111770418 19 (4.1118)

109.07602474 .299628128105 (4.2996)

110.0348882 4.444814629 16 (4.4448)

111.09167481 .71550952597115 (1.662 0.053524$)$

$124.08692370 .6660839118106(0.66608)$

$125.04578721 .4900988981118(0.977210 .51289)$

126.10257381 .95890532100 (1.9589)

$134.04612155 .9963755449(5.9964)$

135.0301371 15.39694322 1210 (10.757 4.6396)

136.08692371 .90263054933 (1.9026)

138.10257385 .0835008484 (5.0835)

$140.11822395 .7294836133(5.7295)$

$142.13387391 .74882147432(1.7488)$

$150.04103621 .32031485322(1.3203)$

152.056686218 .903737232 (18.904)

356.17202670 .790775848325 (0.79078)

0507.2214365

CNC1(C)CC(ONN2C(P)2(C\#N)CC2OC(n3enc4c3(nH)c((NH3+))nc4=O)CC2O)C2N

CN 21

1489.2108718

$\mathrm{CNC1}(\mathrm{C}) \mathrm{C}=\mathrm{C}(\mathrm{ONN} 2 \mathrm{C}(\mathrm{P}) 2(\mathrm{C}=\mathrm{C} 2 \mathrm{CC}=\mathrm{C}(\mathrm{N} 3 \mathrm{C}=\mathrm{NC} 4=\mathrm{C}(\mathrm{O}) \mathrm{N}=\mathrm{C}((\mathrm{NH} 3+)) \mathrm{N}=\mathrm{C} 43) \mathrm{O} 2) \mathrm{C}$

N) C2NCN21

$2152.0566862(\mathrm{NH} 3+) \mathrm{C} 1=\mathrm{NC}(=\mathrm{O}) \mathrm{C} 2=\mathrm{NC}=\mathrm{NC} 2 \mathrm{~N} 1$

$3140.1182239 \mathrm{C}(\mathrm{NH} 2+) \mathrm{C} 1(\mathrm{C}) \mathrm{C}=\mathrm{CC} 2 \mathrm{NCN} 21$

$4138.1025738 \mathrm{C}(\mathrm{NH} 2+) \mathrm{C} 1(\mathrm{C}) \mathrm{C}=\mathrm{CC} 2=\mathrm{NCN} 21$

5472.1843227

$\mathrm{CNC1}(\mathrm{C}) \mathrm{C}=\mathrm{C}(\mathrm{ONN} 2 \mathrm{C}(\mathrm{P}) 2(\mathrm{C}) \mathrm{C}=\mathrm{C} 2 \mathrm{CC}=\mathrm{C}(\mathrm{N} 3 \mathrm{C}=\mathrm{NC} 4=\mathrm{C}(\mathrm{O}) \mathrm{N}=\mathrm{C}((\mathrm{NH3}+)) \mathrm{N}=\mathrm{C43}) \mathrm{O2}) \mathrm{C}$

$2=\mathrm{NCN} 21$

$6158.1036364(\mathrm{NH} 3+) \mathrm{C} 1 \mathrm{NC} 2 \mathrm{~N}=\mathrm{CNC} 2 \mathrm{C}(\mathrm{O}) \mathrm{N} 1$

$7156.0879864(\mathrm{NH3}+) \mathrm{C} 1 \mathrm{NC} 2 \mathrm{~N}=\mathrm{CN}=\mathrm{C} 2 \mathrm{C}(\mathrm{O}) \mathrm{N} 1$

$8154.0723363(\mathrm{NH} 3+) \mathrm{C} 1 \mathrm{NC}(=\mathrm{O}) \mathrm{C} 2=\mathrm{NC}=\mathrm{NC} 2 \mathrm{~N} 1$

$9134.0461215(\mathrm{NH} 2+)=\mathrm{C} 1 \mathrm{~N}=\mathrm{CC} 2=\mathrm{NC}=\mathrm{NC} 2=\mathrm{N} 1$

$10135.0301371(\mathrm{OH}+)=\mathrm{C} 1 \mathrm{~N}=\mathrm{CN}=\mathrm{C} 2 \mathrm{~N}=\mathrm{CN}=\mathrm{C} 21$

11 125.0457872 $\mathrm{NC} 1=\mathrm{NC}(=\mathrm{O}) \mathrm{C}(=(\mathrm{NH} 2+)) \mathrm{C}=\mathrm{N} 1$

$12135.0301371(\mathrm{NH}+) \# \mathrm{CN}=\mathrm{C} 1 \mathrm{~N}=\mathrm{CN}=\mathrm{C} 1 \mathrm{C}=\mathrm{O}$

$1345.04472458 \mathrm{NC}=(\mathbf{N H} 2+)$

14 43.02907452 (NH+)\#CN

$15108.0192381 \mathrm{~N}=\mathrm{C} 1 \mathrm{~N}=\mathrm{CN}=\mathrm{C} 1 \mathrm{C} \#(\mathrm{O}+)$

$16110.0348882(\mathrm{NH} 2+)=\mathrm{C} 1 \mathrm{~N}=\mathrm{CN}=\mathrm{C} 1 \mathrm{C}=\mathrm{O}$ 
$17124.0617716 \mathrm{NC}(=(\mathrm{NH} 2+)) \mathrm{N}=\mathrm{C} 1 \mathrm{C}=\mathrm{NC}=\mathrm{N} 1$

$18125.0457872 \mathrm{~N}=\mathrm{C} 1 \mathrm{~N}=\mathrm{CN}=\mathrm{C} 1 \mathrm{C}((\mathrm{NH} 3+))=\mathrm{O}$

$19109.0508726(\mathrm{NH}+) \# \mathrm{CNC} 1 \mathrm{C}=\mathrm{NC}=\mathrm{N} 1$

$20107.0352225(\mathrm{NH}+) \# \mathrm{CN}=\mathrm{C} 1 \mathrm{C}=\mathrm{NC}=\mathrm{N} 1$

$2182.03997355(\mathrm{NH} 2+)=\mathrm{C} 1 \mathrm{C}=\mathrm{NC}=\mathrm{N} 1$

$22150.0410362(\mathrm{NH} 3+) \mathrm{C} 1=\mathrm{NC}(=\mathrm{O}) \mathrm{C} 2=\mathrm{NC}=\mathrm{NC} 2=\mathrm{N} 1$

23 133.0144871 $\mathrm{N} \# \mathrm{CN}=\mathrm{C} 1 \mathrm{~N}=\mathrm{CN}=\mathrm{C} 1 \mathrm{C} \#(\mathrm{O}+)$

$24354.1563766 \mathrm{CNC} 1(\mathrm{C}) \mathrm{C}=\mathrm{C}(\mathrm{O}(\mathrm{NH} 2+) \mathrm{N} 2 \mathrm{C}(\mathrm{P}) 2(\mathrm{C}=\mathrm{C} 2 \mathrm{OC}=\mathrm{CC} 2=\mathrm{O}) \mathrm{CN}) \mathrm{C} 2 \mathrm{NCN} 21$

$25356.1720267 \mathrm{CNC1}(\mathrm{C}) \mathrm{CC}(\mathrm{O}(\mathrm{NH} 2+) \mathrm{N} 2 \mathrm{C}(\mathrm{P}) 2(\mathrm{C}=\mathrm{C} 2 \mathrm{OC}=\mathrm{CC} 2=\mathrm{O}) \mathrm{CN}) \mathrm{C} 2 \mathrm{NCN} 21$

26489.2108718

$\mathrm{CNC} 1(\mathrm{C}) \mathrm{CC}(\mathrm{ONN} 2 \mathrm{C}(\mathrm{P}) 2(\mathrm{C}=\mathrm{C} 2 \mathrm{OC}(\mathrm{N} 3 \mathrm{C}=\mathrm{NC} 4=\mathrm{CN}=\mathrm{C}((\mathrm{NH} 3+)) \mathrm{N}=\mathrm{C} 43)=\mathrm{CC} 2=\mathrm{O}) \mathrm{CN})$

C 2NCN21

$27136.0617716(\mathrm{NH} 2+)=\mathrm{C} 1 \mathrm{~N}=\mathrm{CC} 2=\mathrm{NC}=\mathrm{NC} 2 \mathrm{~N} 1$

28490.1948874

$\mathrm{CNC1}(\mathrm{C}) \mathrm{CC}(\mathrm{O}(\mathrm{NH} 2+) \mathrm{N} 2 \mathrm{C}(\mathrm{P}) 2(\mathrm{C}=\mathrm{C} 2 \mathrm{OC}(\mathrm{N} 3 \mathrm{C}=\mathrm{NC} 4=\mathrm{C}(\mathrm{O}) \mathrm{N}=\mathrm{CN}=\mathrm{C} 43)=\mathrm{CC} 2=\mathrm{O}) \mathrm{CN}) \mathrm{C}$

2NCN21

$29274.1665474 \mathrm{CNC1}(\mathrm{C}) \mathrm{C}=\mathrm{C}(\mathrm{O}(\mathrm{NH} 2+) \mathrm{N} 2 \mathrm{C}(\mathrm{P}) 2(\mathrm{C}) \mathrm{CN}) \mathrm{C} 2 \mathrm{NCN} 21$

$30272.1508973 \mathrm{CNC} 1(\mathrm{C}) \mathrm{C}=\mathrm{C}(\mathrm{O}(\mathrm{NH} 2+) \mathrm{N} 2 \mathrm{C}(\mathrm{P}) 2(\mathrm{C}) \mathrm{CN}) \mathrm{C} 2=\mathrm{NCN} 21$

$31102.0341363 \mathrm{C}(\mathrm{P}) 1(\mathrm{C}=(\mathrm{NH} 2+)) \mathrm{C}=\mathrm{N} 1$

$32142.1338739 \mathrm{C}(\mathrm{NH} 2+) \mathrm{C} 1(\mathrm{C}) \mathrm{CCC} 2 \mathrm{NCN} 21$

$33136.0869237 \mathrm{C}=(\mathrm{NH}+) \mathrm{C} 1(\mathrm{C}) \mathrm{C}=\mathrm{CC} 2=\mathrm{NCN} 21$

34 256.1195972 $\mathrm{CC} 1(\mathrm{~N}) \mathrm{C}=\mathrm{C}(\mathrm{O}(\mathrm{NH} 2+) \mathrm{N} 2 \mathrm{C}(\mathrm{P}) 2(\mathrm{C}) \mathrm{C}=\mathrm{N}) \mathrm{C} 2=\mathrm{NCN} 21$

$3526.00252542(\mathrm{C}+\#)$

$36241.1086982 \mathrm{C}=(\mathrm{P}) 1 \mathrm{CN} 1(\mathrm{NH} 2+) \mathrm{OC} 1=\mathrm{CC}(\mathrm{C})(\mathrm{NC}) \mathrm{N} 2 \mathrm{CN}=\mathrm{C} 12$

37 243.1243482 $\mathrm{C}=(\mathrm{P}) 1 \mathrm{CN} 1(\mathrm{NH} 2+) \mathrm{OC} 1=\mathrm{CC}(\mathrm{C})(\mathrm{NC}) \mathbf{N} 2 \mathrm{CNC12}$

$38245.1399983 \mathrm{C}=(\mathrm{P}) 1 \mathrm{CN} 1(\mathrm{NH} 2+) \mathrm{OC} 1 \mathrm{CC}(\mathrm{C})(\mathrm{NC}) \mathrm{N} 2 \mathrm{CNC} 12$

$39255.1243482 \mathrm{C}=\mathrm{NC1}(\mathrm{C}) \mathrm{C}=\mathrm{C}(\mathrm{O}(\mathrm{NH} 2+) \mathrm{N} 2 \mathrm{C}(\mathrm{P}) 2(\mathrm{C}) \mathrm{C}) \mathrm{C} 2=\mathrm{NCN} 21$

$40270.1352473 \mathrm{C}=\mathrm{NC} 1(\mathrm{C}) \mathrm{C}=\mathrm{C}(\mathrm{O}(\mathrm{NH} 2+) \mathrm{N} 2 \mathrm{C}(\mathrm{P}) 2(\mathrm{C}) \mathrm{CN}) \mathrm{C} 2=\mathrm{NCN} 21$

$41253.1086982 \mathrm{C} \#(\mathrm{~N}+) \mathrm{C} 1(\mathrm{C}) \mathrm{C}=\mathrm{C}(\mathrm{ONN} 2 \mathrm{C}(\mathrm{P}) 2(\mathrm{C}) \mathrm{C}) \mathrm{C} 2=\mathrm{NCN} 21$

$42268.1195972 \mathrm{C}=\mathrm{NC} 1(\mathrm{C}) \mathrm{C}=\mathrm{C}(\mathrm{O}(\mathrm{NH} 2+) \mathrm{N} 2 \mathrm{C}(\mathrm{P}) 2(\mathrm{C}) \mathrm{C}=\mathrm{N}) \mathrm{C} 2=\mathrm{NCN} 21$

$43266.1039472 \mathrm{C}=\mathrm{NC} 1(\mathrm{C}) \mathrm{C}=\mathrm{C}(\mathrm{O}(\mathrm{NH} 2+) \mathrm{N} 2 \mathrm{C}(\mathrm{P}) 2(\mathrm{C}) \mathrm{C \# N}) \mathrm{C} 2=\mathrm{NCN} 21$

$44264.0882971 \mathrm{C \#}(\mathrm{N}+) \mathrm{C} 1(\mathrm{C}) \mathrm{C}=\mathrm{C}(\mathrm{ONN} 2 \mathrm{C}(\mathrm{P}) 2(\mathrm{C}) \mathrm{C \# N}) \mathrm{C} 2=\mathrm{NCN} 21$

$45234.0621655(\mathrm{NH3}+) \mathrm{C} 1=\mathrm{NC}(\mathrm{O})=\mathrm{C} 2 \mathrm{~N}=\mathrm{CN}(\mathrm{C} 3=\mathrm{CC}(=\mathrm{O}) \mathrm{CO3}) \mathrm{C} 2=\mathrm{N} 1$

$46236.0778156(\mathrm{NH} 3+) \mathrm{C} 1=\mathrm{NC}(\mathrm{O})=\mathrm{C} 2 \mathrm{~N}=\mathrm{CN}(\mathrm{C} 3=\mathrm{CC}(=\mathrm{O}) \mathrm{CO3}) \mathrm{C} 2 \mathrm{~N} 1$

$47262.1665474 \mathrm{CNC1}(\mathrm{C}) \mathrm{CC}(\mathrm{O}(\mathrm{NH} 2+) \mathrm{N} 2 \mathrm{C}(\mathrm{PH}) 2 \mathrm{CN}) \mathrm{C} 2 \mathrm{NCN} 21$

$48258.1352473 \mathrm{CNC1}(\mathrm{C}) \mathrm{C}=\mathrm{C}(\mathrm{O}(\mathrm{NH} 2+) \mathrm{N} 2 \mathrm{C}(\mathrm{PH}) 2 \mathrm{CN}) \mathrm{C} 2=\mathrm{NCN} 21$

$4986.00283615(\mathrm{NH}+) \# \mathrm{C}(\mathrm{P}) 1=\mathrm{NC} 1$

$5088.01848621(\mathrm{NH} 2+)=\mathrm{C}(\mathrm{P}) 1=\mathrm{NC} 1$

$51242.1039472 \mathrm{CC1}(\mathrm{N}) \mathrm{C}=\mathrm{C}(\mathrm{O}(\mathrm{NH} 2+) \mathrm{N} 2 \mathrm{C}(\mathrm{P}) 2=\mathrm{CN}) \mathrm{C} 2=\mathrm{NCN} 21$

$52227.0930481 \mathrm{C}=\mathrm{NC} 1(\mathrm{C}) \mathrm{C}=\mathrm{C}(\mathrm{O}(\mathrm{NH} 2+) \mathrm{N} 2 \mathrm{C}(\mathrm{PH} 2) 2) \mathrm{C} 2=\mathrm{NCN} 21$

$53229.1086982 \mathrm{CNC1}(\mathrm{C}) \mathrm{C}=\mathrm{C}(\mathrm{O}(\mathrm{NH} 2+) \mathrm{N} 2 \mathrm{C}(\mathrm{PH} 2) 2) \mathrm{C} 2=\mathrm{NCN} 21$

$54231.1243482 \mathrm{CNC1}(\mathrm{C}) \mathrm{C}=\mathrm{C}(\mathrm{O}(\mathrm{NH} 2+) \mathrm{N} 2 \mathrm{C}(\mathrm{PH} 2) 2) \mathrm{C} 2 \mathrm{NCN} 21$

$55256.1195972 \mathrm{C}=\mathrm{NC} 1(\mathrm{C}) \mathrm{C}=\mathrm{C}(\mathrm{O}(\mathrm{NH} 2+) \mathrm{N} 2 \mathrm{C}(\mathrm{PH}) 2 \mathrm{CN}) \mathrm{C} 2=\mathrm{NCN} 21$

56 171.1240375 $\mathrm{CNC} 1(\mathrm{C}) \mathrm{C}=\mathrm{C}(\mathrm{O}(\mathrm{NH} 3+)) \mathrm{C} 2 \mathrm{NCN} 21$

$57156.1131385 \mathrm{C}(\mathrm{NH} 2+) \mathrm{C1}(\mathrm{C}) \mathrm{CC}(=\mathrm{O}) \mathrm{C} 2 \mathrm{NCN} 21$

$58101.0137352 \mathrm{N \# C(P)1=CN1(NH3+)}$

$59134.0712737 \mathrm{C \#}(\mathrm{N}+) \mathrm{C} 1(\mathrm{C}) \mathrm{C}=\mathrm{CC} 2=\mathrm{NCN} 21$

$60225.0773981 \mathrm{C}=\mathrm{C} 1 \mathrm{C}=\mathrm{C}(\mathrm{O}(\mathrm{NH} 2+) \mathrm{N} 2 \mathrm{C}(\mathrm{P}) 2=\mathrm{CN}) \mathrm{C} 2=\mathrm{NCN} 21$

$61239.0930481 \mathrm{C}=\mathrm{NC1}(\mathrm{C}) \mathrm{C}=\mathrm{C}(\mathrm{O}(\mathrm{NH} 2+) \mathrm{N} 2 \mathrm{C}(\mathrm{P}) 2=\mathrm{C}) \mathrm{C} 2=\mathrm{NCN} 21$

$62254.1039472 \mathrm{C}=\mathrm{NC} 1(\mathrm{C}) \mathrm{C}=\mathrm{C}(\mathrm{O}(\mathrm{NH} 2+) \mathrm{N} 2 \mathrm{C}(\mathrm{P}) 2=\mathrm{CN}) \mathrm{C} 2=\mathrm{NCN} 21$

$63237.0773981 \mathrm{C \#}(\mathrm{N}+) \mathrm{C} 1(\mathrm{C}) \mathrm{C}=\mathrm{C}(\mathrm{ONN} 2 \mathrm{C}(\mathrm{P}) 2=\mathrm{C}) \mathrm{C} 2=\mathrm{NCN} 21$

$64252.0882971 \mathrm{C}=\mathrm{NC} 1(\mathrm{C}) \mathrm{C}=\mathrm{C}(\mathrm{O}(\mathrm{NH} 2+) \mathrm{N} 2 \mathrm{C}(\mathrm{P}) 2=\mathrm{C}=\mathrm{N}) \mathrm{C} 2=\mathrm{NCN} 21$

$65250.072647 \mathrm{C} \#(\mathrm{~N}+) \mathrm{C1}(\mathrm{C}) \mathrm{C}=\mathrm{C}(\mathrm{ONN} 2 \mathrm{C}(\mathrm{P}) 2=\mathrm{C}=\mathrm{N}) \mathrm{C} 2=\mathrm{NCN} 21$

$66246.0621655 \mathrm{C}=\mathrm{C} 1 \mathrm{OC}(\mathrm{N} 2 \mathrm{C}=\mathrm{NC} 3=\mathrm{C}(\mathrm{O}) \mathrm{N}=\mathrm{C}((\mathrm{NH} 3+)) \mathrm{N}=\mathrm{C} 32)=\mathrm{CC} 1=\mathrm{O}$

$67248.0778156 \mathrm{CC1OC}(\mathrm{N} 2 \mathrm{C}=\mathrm{NC} 3=\mathrm{C}(\mathrm{O}) \mathrm{N}=\mathrm{C}((\mathrm{NH3}+)) \mathrm{N}=\mathrm{C32})=\mathrm{CC} 1=\mathrm{O}$ 
$68250.0934657 \mathrm{CC} 1 \mathrm{OC}(\mathrm{N} 2 \mathrm{C}=\mathrm{NC} 3=\mathrm{C}(\mathrm{O}) \mathrm{N}=\mathrm{C}((\mathrm{NH} 3+)) \mathrm{NC} 32)=\mathrm{CC} 1=\mathrm{O}$

69232.082901 $\mathrm{C}=\mathrm{C} 1 \mathrm{CC}=\mathrm{C}(\mathrm{N} 2 \mathrm{C}=\mathrm{NC3}=\mathrm{C}(\mathrm{O}) \mathrm{N}=\mathrm{C}((\mathrm{NH3}+)) \mathrm{N}=\mathrm{C32}) \mathrm{O1}$

70252.1091157 $\mathrm{CC1OC}(\mathrm{N} 2 \mathrm{C}=\mathrm{NC} 3=\mathrm{C}(\mathrm{O}) \mathrm{NC}((\mathrm{NH} 3+)) \mathrm{NC32})=\mathrm{CC} 1=\mathrm{O}$

71254.1247658 CC1OC $(\mathrm{N} 2 \mathrm{C}=\mathrm{NC3C} 2 \mathrm{NC}((\mathrm{NH3}+)) \mathrm{NC3O})=\mathrm{CC} 1=\mathrm{O}$

72256.1404159 $\mathrm{CC1OC}(\mathrm{N} 2 \mathrm{CNC} 3 \mathrm{C} 2 \mathrm{NC}((\mathrm{NH} 3+)) \mathrm{NC} 3 \mathrm{O})=\mathrm{CC} 1=\mathrm{O}$

73258.1560659 CC1OC $(\mathbf{N} 2 \mathrm{CNC3C} 2 \mathrm{NC}((\mathrm{NH} 3+)) \mathrm{NC3O})=\mathrm{CC} 10$

74173.1396876 CNC1 $(\mathrm{C}) \mathrm{CC}(\mathrm{O}(\mathrm{NH} 3+)) \mathrm{C} 2 \mathrm{NCN} 21$

75140.0818384 $\mathrm{CC} 1 \mathrm{C}=\mathrm{C}(\mathrm{O}(\mathrm{NH} 3+)) \mathrm{C} 2=\mathrm{NCN} 21$

76167.0927374 $\mathrm{C}=\mathrm{NC} 1(\mathrm{C}) \mathrm{C}=\mathrm{C}(\mathrm{O}(\mathrm{NH} 3+)) \mathrm{C} 2=\mathrm{NCN} 21$

77335.0890254 NC $(\mathrm{P}) 1(\mathrm{C}=\mathrm{C} 2 \mathrm{OC}(\mathrm{N} 3 \mathrm{C}=\mathrm{NC} 4=\mathrm{C}(\mathrm{O}) \mathrm{N}=\mathrm{C}((\mathrm{NH} 3+)) \mathrm{N}=\mathrm{C} 43)=\mathrm{CC} 2=\mathrm{O}) \mathrm{CN} 1$ 78100.0184862 C(P)1(C\#(NH+))C=N1

79318.0624763 $\mathrm{C}(\mathrm{P}) 1(\mathrm{C}=\mathrm{C} 2 \mathrm{OC}(\mathrm{N} 3 \mathrm{C}=\mathrm{NC} 4=\mathrm{C}(\mathrm{O}) \mathrm{N}=\mathrm{C}((\mathrm{NH3}+)) \mathrm{N}=\mathrm{C} 43)=\mathrm{CC} 2=\mathrm{O}) \mathrm{C}=\mathrm{N} 1$ $80337.1046754 \mathrm{NC}(\mathrm{P}) 1(\mathrm{CC} 2 \mathrm{OC}(\mathrm{N} 3 \mathrm{C}=\mathrm{NC} 4=\mathrm{C}(\mathrm{O}) \mathrm{N}=\mathrm{C}((\mathrm{NH} 3+)) \mathrm{N}=\mathrm{C} 43)=\mathrm{CC} 2=\mathrm{O}) \mathrm{CN} 1$ $81339.1203255 \mathrm{NC}(\mathrm{P}) 1(\mathrm{CC} 2 \mathrm{OC}(\mathrm{N} 3 \mathrm{C}=\mathrm{NC} 4=\mathrm{C}(\mathrm{O}) \mathrm{N}=\mathrm{C}((\mathrm{NH} 3+)) \mathrm{NC43})=\mathrm{CC} 2=\mathrm{O}) \mathrm{CN} 1$ 82341.1359756 NC(P) $1(\mathrm{CC2OC}(\mathrm{N3C}=\mathrm{NC} 4=\mathrm{C}(\mathrm{O}) \mathrm{NC}((\mathrm{NH3}+)) \mathrm{NC} 43)=\mathrm{CC} 2=\mathrm{O}) \mathrm{CN1}$ $83343.1516256 \mathrm{NC}(\mathrm{P}) 1(\mathrm{CC} 2 \mathrm{OC}(\mathrm{N} 3 \mathrm{C}=\mathrm{NC} 4 \mathrm{C} 3 \mathrm{NC}((\mathrm{NH} 3+)) \mathrm{NC} 4 \mathrm{O})=\mathrm{CC} 2=\mathrm{O}) \mathrm{CN} 1$ 84158.1287886 C(NH2+)C1(C)CC(O)C2NCN21

85142.0974884 CC1 $((\mathrm{NH} 3+)) \mathrm{CC}(=\mathrm{O}) \mathrm{C} 2 \mathrm{NCN} 21$

86125.0709393 $\mathrm{CC} 1=\mathrm{CC}(=(\mathrm{OH}+)) \mathrm{C} 2 \mathrm{NCN} 12$

87152.0818384 $\mathrm{C}=(\mathrm{NH}+) \mathrm{C} 1(\mathrm{C}) \mathrm{CC}(=\mathrm{O}) \mathrm{C} 2=\mathrm{NCN} 21$

$88150.0661883 \mathrm{C} \#(\mathrm{~N}+) \mathrm{C} 1(\mathrm{C}) \mathrm{CC}(=\mathrm{O}) \mathrm{C} 2=\mathrm{NCN} 21$

89350.0999244

$\mathrm{NC}(\mathrm{P}) 1(\mathrm{C}=\mathrm{C} 2 \mathrm{OC}(\mathrm{N} 3 \mathrm{C}=\mathrm{NC} 4=\mathrm{C}(\mathrm{O}) \mathrm{N}=\mathrm{C}((\mathrm{NH} 3+)) \mathrm{N}=\mathrm{C43})=\mathrm{CC} 2=\mathrm{O}) \mathrm{CN} 1 \mathrm{~N}$

$90332.0893597 \mathrm{N \# C}(\mathrm{P}) 1(\mathrm{C}=\mathrm{C} 2 \mathrm{CC}=\mathrm{C}(\mathrm{N} 3 \mathrm{C}=\mathrm{NC} 4=\mathrm{C}(\mathrm{O}) \mathrm{N}=\mathrm{C}((\mathrm{NH} 3+)) \mathrm{N}=\mathrm{C} 43) \mathrm{O} 2) \mathrm{CN} 1 \mathrm{~N}$

$91115.0293852(\mathrm{CH} 2+)(\mathrm{P}) 1(\mathrm{C} \# \mathrm{~N}) \mathrm{CN} 1 \mathrm{~N}$

92 333.0733753 C $(\mathrm{P}) 1((\mathrm{C}+)=\mathrm{C} 2 \mathrm{OC}(\mathrm{N} 3 \mathrm{C}=\mathrm{NC} 4=\mathrm{C}(\mathrm{O}) \mathrm{N}=\mathrm{C}(\mathrm{N}) \mathrm{N}=\mathrm{C} 43)=\mathrm{CC} 2=\mathrm{O}) \mathrm{CN} 1 \mathrm{~N}$

93352.1155745

$\mathrm{NC}(\mathrm{P}) 1(\mathrm{CC} 2 \mathrm{OC}(\mathrm{N} 3 \mathrm{C}=\mathrm{NC} 4=\mathrm{C}(\mathrm{O}) \mathrm{N}=\mathrm{C}((\mathrm{NH} 3+)) \mathrm{N}=\mathrm{C} 43)=\mathrm{CC} 2=\mathrm{O}) \mathrm{CN} 1 \mathrm{~N}$

$94354.1312245 \mathrm{NC}(\mathrm{P}) 1(\mathrm{CC} 2 \mathrm{OC}(\mathrm{N} 3 \mathrm{C}=\mathrm{NC} 4=\mathrm{C}(\mathrm{O}) \mathrm{N}=\mathrm{C}((\mathrm{NH} 3+)) \mathrm{NC43})=\mathrm{CC} 2=\mathrm{O}) \mathrm{CN} 1 \mathrm{~N}$

$95356.1468746 \mathrm{NC}(\mathrm{P}) 1(\mathrm{CC2OC}(\mathrm{N3C}=\mathrm{NC} 4=\mathrm{C}(\mathrm{O}) \mathrm{NC}((\mathrm{NH3}+)) \mathrm{NC43})=\mathrm{CC} 2=\mathrm{O}) \mathrm{CN} 1 \mathrm{~N}$

$96358.1625246 \mathrm{NC}(\mathrm{P}) 1(\mathrm{CC} 2 \mathrm{OC}(\mathrm{N} 3 \mathrm{C}=\mathrm{NC} 4 \mathrm{C} 3 \mathrm{NC}((\mathrm{NH} 3+)) \mathrm{NC} 4 \mathrm{O})=\mathrm{CC} 2=\mathrm{O}) \mathrm{CN} 1 \mathrm{~N}$

$97111.0916748(\mathrm{CH} 4+) \mathrm{C} 1 \mathrm{C}=\mathrm{CC} 2 \mathrm{NCN} 21$

$98113.1073248(\mathrm{CH} 4+)$ C1CCC2NCN21

$9915.02292652(\mathrm{CH} 3+)$

$100126.1025738 \mathrm{CC} 1((\mathrm{NH} 3+)) \mathrm{C}=\mathrm{CC} 2 \mathrm{NCN} 21$

$10183.06037464 \mathrm{C \#}(\mathrm{CH}+) \mathrm{C} 1 \mathrm{NCN1}$

$10285.07602471 \mathrm{C}=(\mathrm{CH} 2+) \mathrm{C} 1 \mathrm{NCN} 1$

$10384.08077574 \mathrm{C \# CC}(\mathrm{C})(\mathrm{NH} 2+) \mathrm{C}$

$10486.0964258 \mathrm{C}=\mathrm{CC}(\mathrm{C})(\mathrm{NH} 2+) \mathrm{C}$

$105109.0760247(\mathrm{CH} 4+) \mathrm{C} 1 \mathrm{C}=\mathrm{CC} 2=\mathrm{NCN} 21$

$106124.0869237 \mathrm{C}(\mathrm{NH} 2+) \mathrm{C} 1 \mathrm{C}=\mathrm{CC} 2=\mathrm{NCN} 21$

10760.08077574 CC(NH2+)C

$10856.04947561 \mathrm{C}=\mathrm{C}=(\mathrm{NH}+) \mathrm{C}$

$10981.04472458 \mathrm{C \#}(\mathrm{CH}+) \mathrm{C} 1=\mathrm{NCN} 1$

$11087.09167477 \mathrm{C}(\mathrm{CH} 3+) \mathrm{C} 1 \mathrm{NCN} 1$

$111112.0869237 \mathrm{C}(\mathrm{NH}+)=\mathrm{C}(\mathrm{C}) \mathrm{N1C}=\mathrm{NC1}$

11259.06037464 C1NC(NH2+)1

$11382.06512568 \mathrm{C} \# \mathrm{CC}(\mathrm{C})=(\mathrm{NH}+) \mathrm{C}$

$114113.1073248 \mathrm{C}(\mathrm{NH} 2+) \mathrm{C} 1(\mathrm{C}) \mathrm{C}=\mathrm{CCN} 1$

$115111.0916748 \mathrm{C}(\mathrm{NH} 2+) \mathrm{C} 1(\mathrm{C}) \mathrm{C}=\mathrm{CC}=\mathrm{N} 1$

$116107.0603746(\mathrm{CH} 3+)=\mathrm{C} 1 \mathrm{C}=\mathrm{CC} 2=\mathrm{NCN} 21$

$117122.0712737(\mathrm{CH} 2+) \mathrm{C} 1(\mathrm{~N}) \mathrm{C}=\mathrm{CC} 2=\mathrm{NCN} 21$

$11879.02907452 \mathrm{C} \#(\mathrm{CH}+) \mathrm{C} 1=\mathrm{NC}=\mathrm{N} 1$

$11980.04947561 \mathrm{C \# CC}(\mathrm{C})=(\mathrm{N}+)=\mathrm{C}$

120368.1104891

$\mathrm{NC}(\mathrm{P}) 1(\mathrm{CC} 2 \mathrm{OC}(\mathrm{N} 3 \mathrm{C}=\mathrm{NC} 4=\mathrm{C}(\mathrm{O}) \mathrm{N}=\mathrm{C}((\mathrm{NH} 3+)) \mathrm{N}=\mathrm{C} 43)=\mathrm{CC} 2=\mathrm{O}) \mathrm{CN} 1 \mathrm{NO}$ 
121476.1792373

$\operatorname{CC1CC}(\mathrm{ONN} 2 \mathrm{C}(\mathrm{P}) 2(\mathrm{C}=\mathrm{C} 2 \mathrm{OC}(\mathrm{N3C}=\mathrm{NC} 4=\mathrm{C}(\mathrm{O}) \mathrm{N}=\mathrm{C}((\mathrm{NH} 3+)) \mathrm{N}=\mathrm{C} 43)=\mathrm{CC} 2=\mathrm{O}) \mathrm{CN}) \mathrm{C}$ 2 NCN21

122458.1686726

$\mathrm{CC1C}=\mathrm{C}(\mathrm{ONN} 2 \mathrm{C}(\mathrm{P}) 2(\mathrm{C}=\mathrm{C} 2 \mathrm{CC}=\mathrm{C}(\mathrm{N} 3 \mathrm{C}=\mathrm{NC} 4=\mathrm{C}(\mathrm{O}) \mathrm{N}=\mathrm{C}((\mathrm{NH} 3+)) \mathrm{N}=\mathrm{C} 43) \mathrm{O} 2) \mathrm{CN}) \mathrm{C} 2=$

NCN21

$123241.1086982 \mathrm{C}=\mathrm{C} 1 \mathrm{C}=\mathrm{C}(\mathrm{O}(\mathrm{NH} 2+) \mathrm{N} 2 \mathrm{C}(\mathrm{P}) 2(\mathrm{C}) \mathrm{CN}) \mathrm{C} 2=\mathrm{NCN} 21$

124459.1526882

$\mathrm{CC1C}=\mathrm{C}(\mathrm{ONN} 2 \mathrm{C}(\mathrm{P}) 2(\mathrm{C}) \mathrm{C}=\mathrm{C} 2 \mathrm{OC}(\mathrm{N3C}=\mathrm{NC} 4=\mathrm{C}(\mathrm{O}) \mathrm{N}=\mathrm{C}((\mathrm{NH} 3+)) \mathrm{N}=\mathrm{C} 43)=\mathrm{CC} 2=\mathrm{O}) \mathrm{C} 2$

NCN21

125480.2105375

$\mathrm{CC1CC}(\mathrm{ONN} 2 \mathrm{C}(\mathrm{P}) 2(\mathrm{CN}) \mathrm{CC} 2 \mathrm{OC}(\mathrm{N3C}=\mathrm{NC} 4=\mathrm{C}(\mathrm{O}) \mathrm{N}=\mathrm{C}((\mathrm{NH} 3+)) \mathrm{NC43})=\mathrm{CC} 2=\mathrm{O}) \mathrm{C} 2$

NC N21

126491.1901364

$\mathrm{CC1}(\mathrm{N}) \mathrm{CC}(\mathrm{ONN} 2 \mathrm{C}(\mathrm{P}) 2(\mathrm{C}=\mathrm{C} 2 \mathrm{OC}(\mathrm{N3C}=\mathrm{NC} 4=\mathrm{C}(\mathrm{O}) \mathrm{N}=\mathrm{C}((\mathrm{NH} 3+)) \mathrm{N}=\mathrm{C} 43)=\mathrm{CC} 2=\mathrm{O}) \mathrm{CN})$

C2NCN21

127491.1901364

$\mathrm{CNC1CC}(\mathrm{ONN} 2 \mathrm{C}(\mathrm{P}) 2(\mathrm{C}=\mathrm{C} 2 \mathrm{OC}(\mathrm{N} 3 \mathrm{C}=\mathrm{NC} 4=\mathrm{C}(\mathrm{O}) \mathrm{N}=\mathrm{C}((\mathrm{NH3}+)) \mathrm{N}=\mathrm{C} 43)=\mathrm{CC} 2=\mathrm{O}) \mathrm{CN}) \mathrm{C}$

2NCN21

$12832.04947561 \mathrm{C}(\mathrm{NH} 3+)$

$12930.03382555 \mathrm{C}=(\mathrm{NH} 2+)$

130476.1792373

$\mathrm{CNC} 1(\mathrm{C}) \mathrm{CC}(\mathrm{ONN} 2 \mathrm{C}(\mathrm{PH}) 2 \mathrm{C}=\mathrm{C} 2 \mathrm{OC}(\mathrm{N} 3 \mathrm{C}=\mathrm{NC} 4=\mathrm{C}(\mathrm{O}) \mathrm{N}=\mathrm{C}((\mathrm{NH} 3+)) \mathrm{N}=\mathrm{C} 43)=\mathrm{CC} 2=\mathrm{O}) \mathrm{C}$

2NCN21

131458.1686726

$\mathrm{CNC1}(\mathrm{C}) \mathrm{C}=\mathrm{C}(\mathrm{ONN} 2 \mathrm{C}(\mathrm{P}) 2=\mathrm{C}=\mathrm{C} 2 \mathrm{CC}=\mathrm{C}(\mathrm{N3C}=\mathrm{NC} 4=\mathrm{C}(\mathrm{O}) \mathrm{N}=\mathrm{C}((\mathrm{NH} 3+)) \mathrm{N}=\mathrm{C43}) \mathrm{O2})$

C2 NCN21

132478.1948874

$\mathrm{CNC1}(\mathrm{C}) \mathrm{CC}(\mathrm{ONN} 2 \mathrm{C}(\mathrm{PH}) 2 \mathrm{CC} 2 \mathrm{OC}(\mathrm{N3C}=\mathrm{NC} 4=\mathrm{C}(\mathrm{O}) \mathrm{N}=\mathrm{C}((\mathrm{NH} 3+)) \mathrm{N}=\mathrm{C} 43)=\mathrm{CC} 2=\mathrm{O}) \mathrm{C}$ 2 NCN21

133460.1843227

$\mathrm{CNC1}(\mathrm{C}) \mathrm{CC}(\mathrm{ONN} 2 \mathrm{C}(\mathrm{P}) 2=\mathrm{C}=\mathrm{C} 2 \mathrm{CC}=\mathrm{C}(\mathrm{N3C}=\mathrm{NC} 4=\mathrm{C}(\mathrm{O}) \mathrm{N}=\mathrm{C}((\mathrm{NH3}+)) \mathrm{N}=\mathrm{C} 43) \mathrm{O} 2) \mathrm{C}$

2N CN21

134480.2105375

$\mathrm{CNC} 1(\mathrm{C}) \mathrm{CC}(\mathrm{ONN} 2 \mathrm{C}(\mathrm{PH}) 2 \mathrm{CC} 2 \mathrm{OC}(\mathrm{N3C}=\mathrm{NC} 4=\mathrm{C}(\mathrm{O}) \mathrm{N}=\mathrm{C}((\mathrm{NH} 3+)) \mathrm{NC} 43)=\mathrm{CC} 2=\mathrm{O}) \mathrm{C} 2 \mathrm{~N}$

CN21

135462.1999728

$\mathrm{CNC} 1(\mathrm{C}) \mathrm{CC}(\mathrm{ONN} 2 \mathrm{C}(\mathrm{PH}) 2 \mathrm{C}=\mathrm{C} 2 \mathrm{CC}=\mathrm{C}(\mathrm{N3C}=\mathrm{NC} 4=\mathrm{C}(\mathrm{O}) \mathrm{N}=\mathrm{C}((\mathrm{NH}+)) \mathrm{N}=\mathrm{C} 43) \mathrm{O} 2) \mathrm{C}$

2N CN21

136247.1556484 CNC1(C)CC(O(NH2+)N2C(PH)2C)C2NCN21

137 233.1399983 CNC1(C)CC(O(NH2+)N2C(PH2)2)C2NCN21

$138308.0781263(\mathrm{NH} 3+) \mathrm{C} 1=\mathrm{NC}(\mathrm{O})=\mathrm{C} 2 \mathrm{~N}=\mathrm{CN}(\mathrm{C} 3=\mathrm{CC}(=\mathrm{O}) \mathrm{C}(\mathrm{C}(\mathrm{PH}) 4 \mathrm{CN} 4) \mathrm{O3}) \mathrm{C} 2=\mathrm{N} 1$

$139310.0937764(\mathrm{NH3}+) \mathrm{C} 1=\mathrm{NC}(\mathrm{O})=\mathrm{C} 2 \mathrm{~N}=\mathrm{CN}(\mathrm{C3}=\mathrm{CC}(=\mathrm{O}) \mathrm{C}(\mathrm{C}(\mathrm{PH}) 4 \mathrm{CN} 4) 03) \mathrm{C} 2 \mathrm{~N} 1$

$140312.1094265(\mathrm{NH} 3+) \mathrm{C} 1 \mathrm{NC}(\mathrm{O})=\mathrm{C} 2 \mathrm{~N}=\mathrm{CN}(\mathrm{C} 3=\mathrm{CC}(=\mathrm{O}) \mathrm{C}(\mathrm{C}(\mathrm{PH}) 4 \mathrm{CN} 4) 03) \mathrm{C} 2 \mathrm{~N} 1$

$141323.0890254 N N 1 C(P H) 1 C C 1 O C(N 2 C=N C 3=C(O) N=C((N H 3+)) N=C 32)=C C 1=0$

$142325.1046754 \mathrm{NN} 1 \mathrm{C}(\mathrm{PH}) 1 \mathrm{CC} 1 \mathrm{OC}(\mathrm{N} 2 \mathrm{C}=\mathrm{NC} 3=\mathrm{C}(\mathrm{O}) \mathrm{N}=\mathrm{C}((\mathrm{NH}+)) \mathrm{NC} 32)=\mathrm{CC} 1=\mathrm{O}$

$143327.1203255 \mathrm{NN} 1 \mathrm{C}(\mathrm{PH}) 1 \mathrm{CC} 1 \mathrm{OC}(\mathrm{N} 2 \mathrm{C}=\mathrm{NC} 3=\mathrm{C}(\mathrm{O}) \mathrm{NC}((\mathrm{NH} 3+)) \mathrm{NC32})=\mathrm{CC} 1=\mathrm{O}$

144449.1683383

$\mathrm{CC} 1 \mathrm{CC}(\mathrm{ONN} 2 \mathrm{C}(\mathrm{PH}) 2 \mathrm{CC} 2 \mathrm{OC}(\mathrm{N} 3 \mathrm{C}=\mathrm{NC} 4=\mathrm{C}(\mathrm{O}) \mathrm{N}=\mathrm{C}((\mathrm{NH} 3+)) \mathrm{N}=\mathrm{C} 43)=\mathrm{CC} 2=\mathrm{O}) \mathrm{C} 2 \mathrm{NC}$

N 21

$145186.1349366 \mathrm{CNC1}(\mathrm{C}) \mathrm{CC}(\mathrm{ON}=(\mathrm{NH} 2+)) \mathrm{C} 2 \mathrm{NCN} 21$

$146281.0672273(\mathrm{NH} 3+) \mathrm{C} 1=\mathrm{NC}(\mathrm{O})=\mathrm{C} 2 \mathrm{~N}=\mathrm{CN}(\mathrm{C} 3=\mathrm{CC}(=\mathrm{O}) \mathrm{C}(\mathrm{C}(\mathrm{PH} 3)) \mathrm{O3}) \mathrm{C} 2=\mathrm{N} 1$

147490.1948874

$\mathrm{CNC1}(\mathrm{C}) \mathrm{CC}(\mathrm{ONN} 2 \mathrm{C}(\mathrm{P}) 2(\mathrm{C}) \mathrm{C}=\mathrm{C} 2 \mathrm{OC}(\mathrm{N3C}=\mathrm{NC} 4=\mathrm{C}(\mathrm{O}) \mathrm{N}=\mathrm{C}((\mathrm{NH} 3+)) \mathrm{N}=\mathrm{C43})=\mathrm{CC} 2=\mathrm{O})$ C2NCN21 


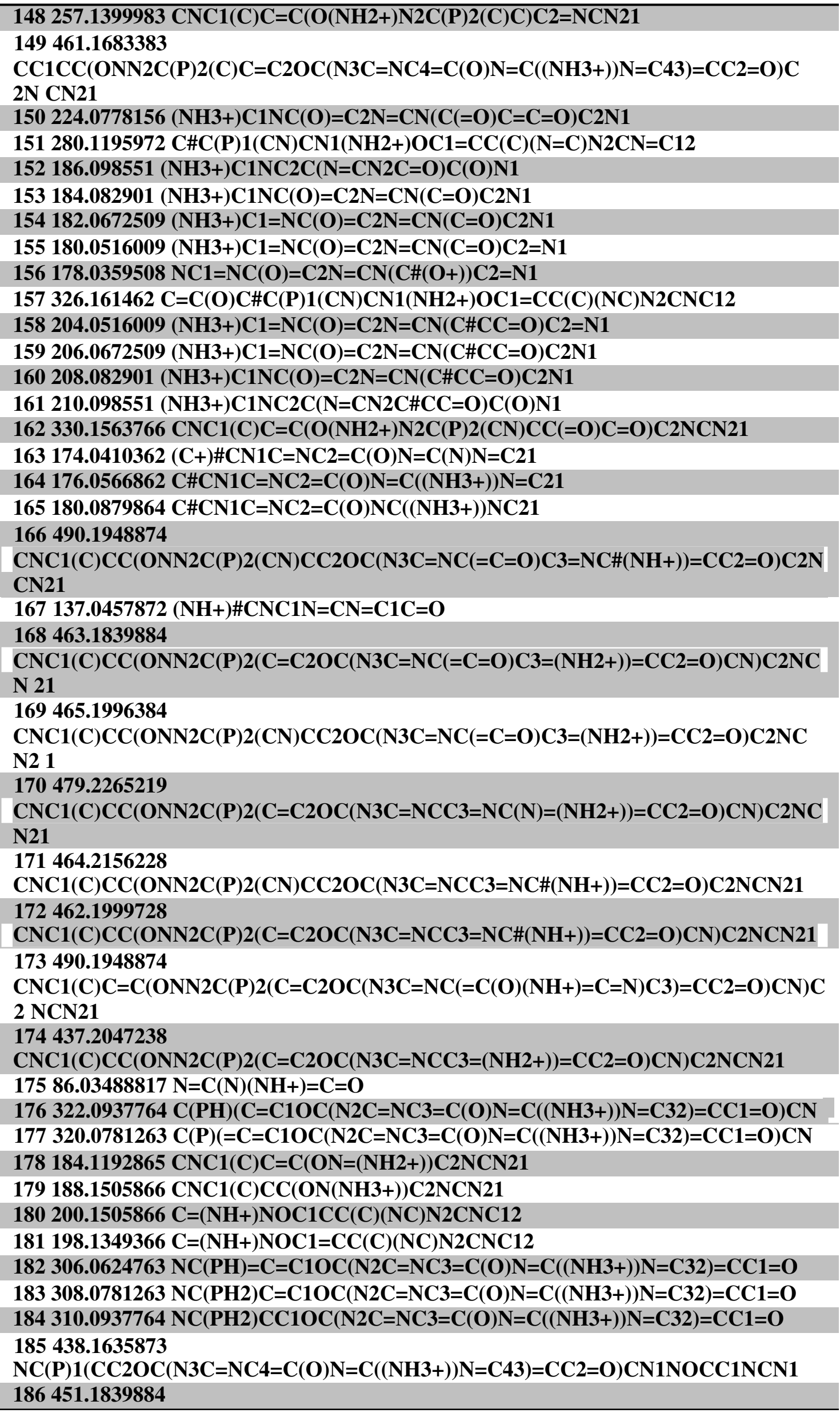




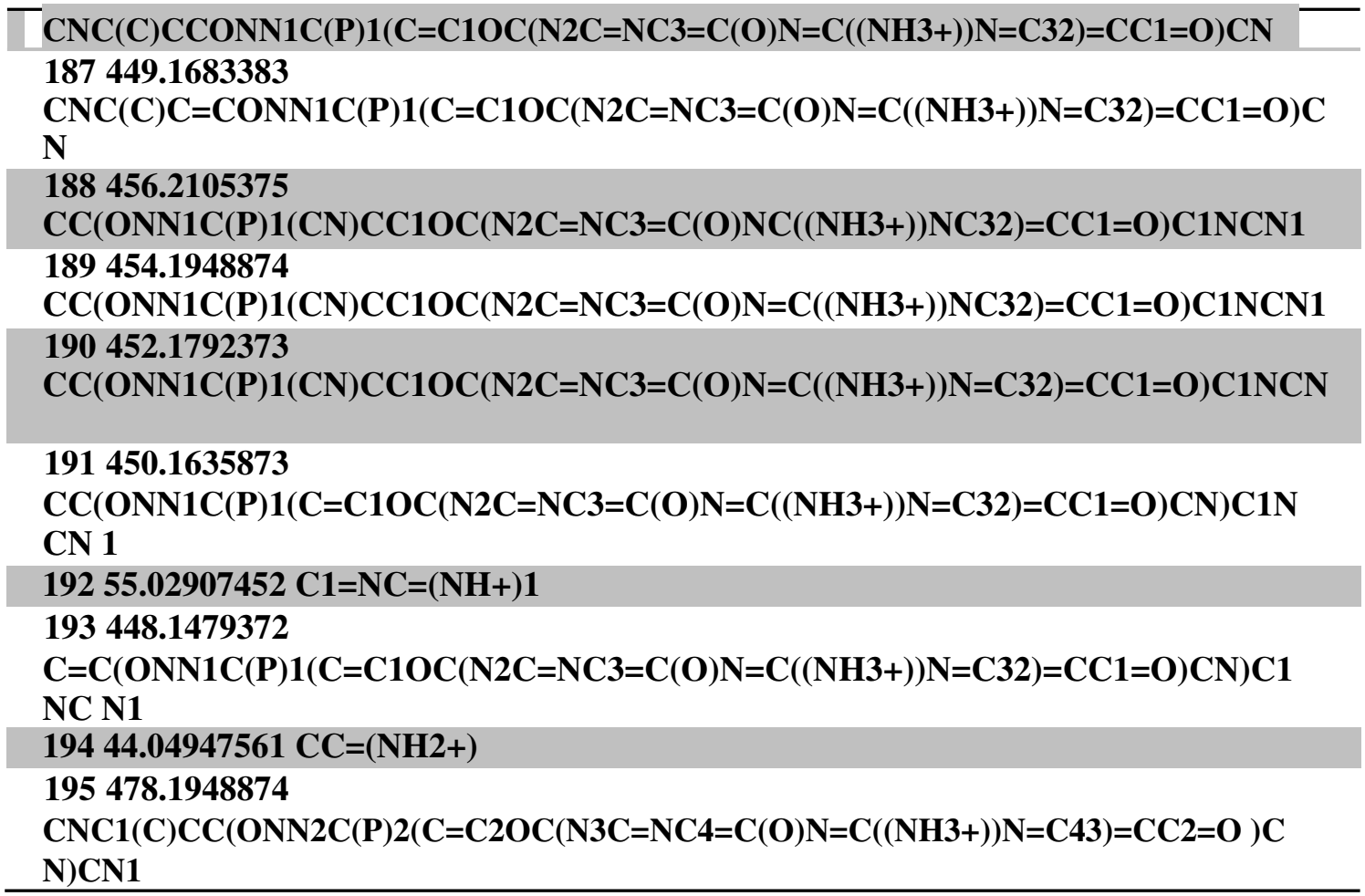

Table2a. List of smiles of the Roccustyrna active fragments.

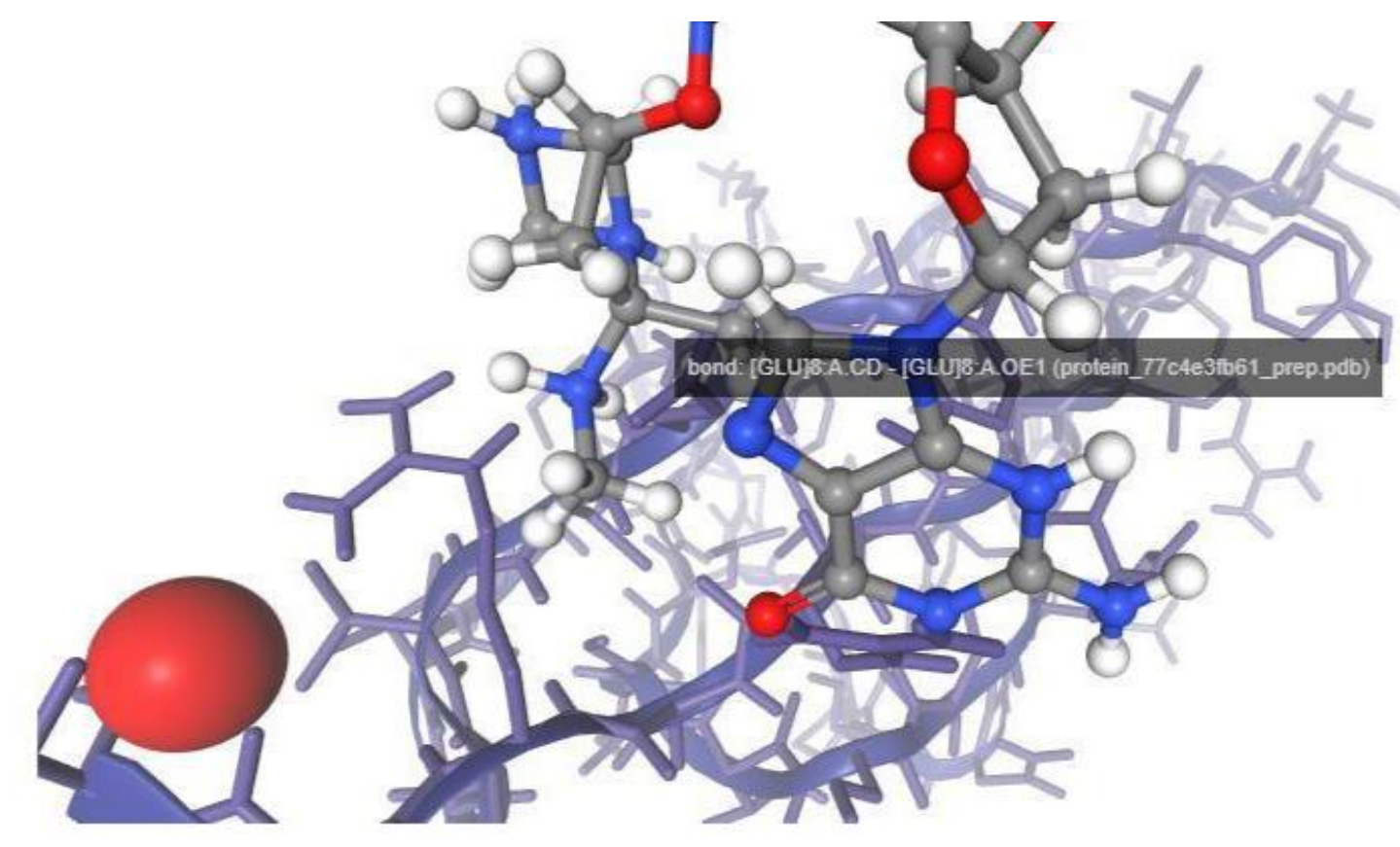

\begin{tabular}{l}
\hline File Model T.Energy I.Energy vdW Coul NumRotors RMSD Score \\
\hline ligand_67d2edfaf0_1_run_16.log 1 -19.625 $-35.4837 .633-43.11670 .000-5.813$ \\
ligand_67d2edfaf0_1_run_8.log 1 -19.117 -36.858 5.372 -42.230 7 2.445 -5.804 \\
ligand_67d2edfaf0_1_run_4.log 4 -16.689 -32.913 4.288 -37.201 72.159 -5.863 \\
\hline
\end{tabular}

Figure4a. Binding patterns, CoMFA contour map and aminoacid interactions of the steric regions (blue, favored; yellow; disfavored) around Roccustyrna scaffold (Ser682, Thy456, and Arg624 with a distance of $1.224,2.445$, and $2.159 \AA$, respectively); Blue regions are favored by positively charged groups and red regions favored by negatively charged groups inside the SARS-COV-2 protein targets (pdb:1xak). EDO:A:506 (EDO) - SMALLMOLECULE

Interacting chain(s): A 


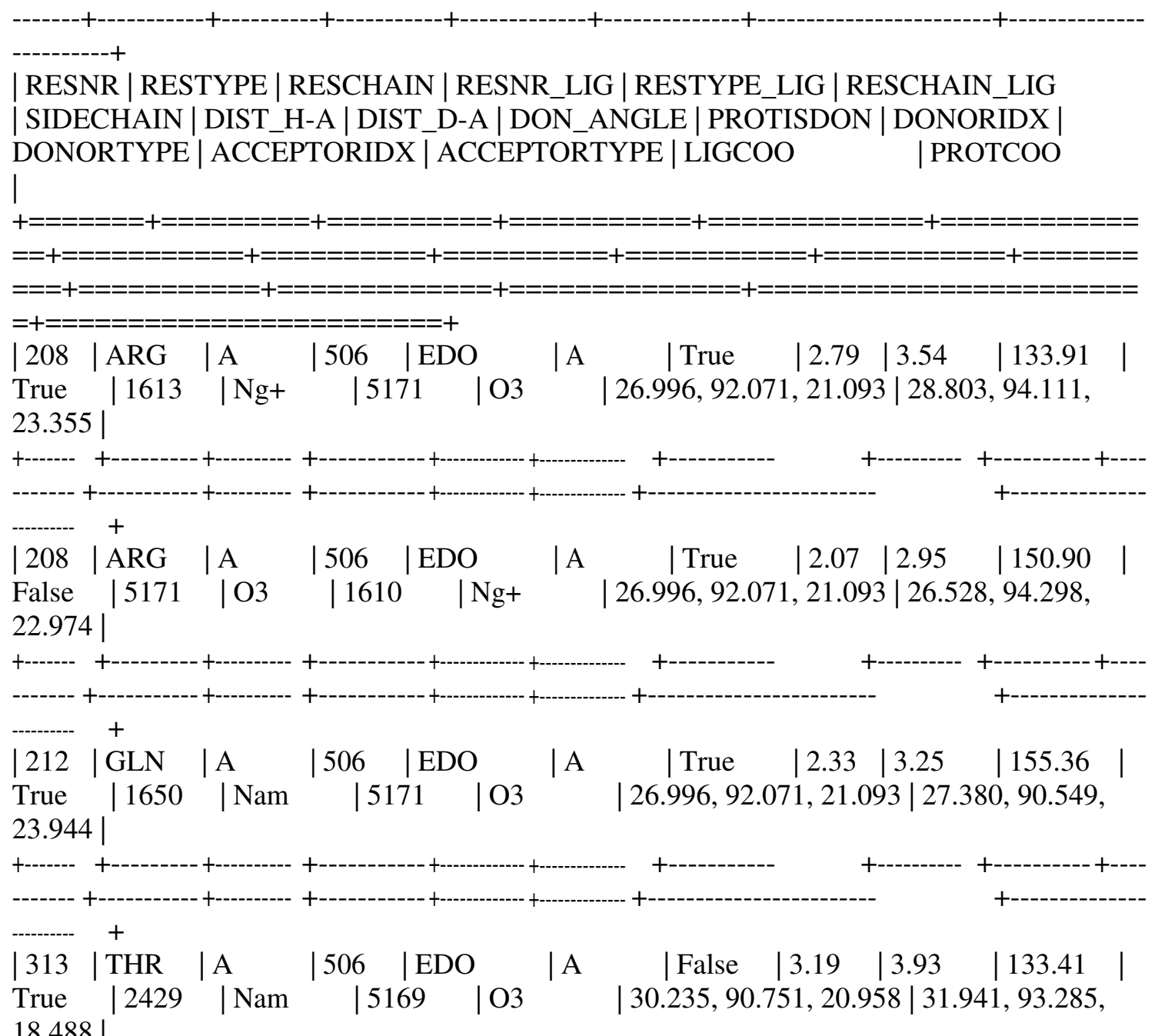

18.488

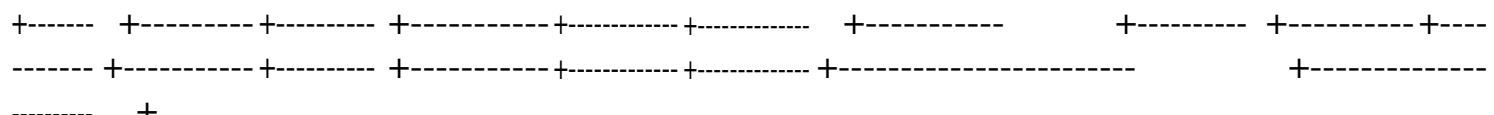

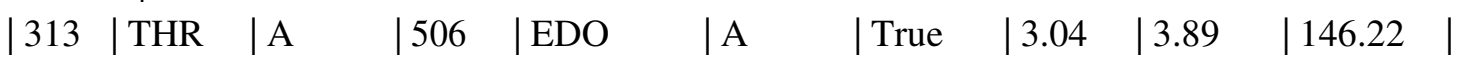
False $|5169 \quad| \mathrm{O} 3 \quad|2434 \quad| \mathrm{O} 3 \quad|30.235,90.751,20.958| 32.771,90.620$, $18.014 \mid$

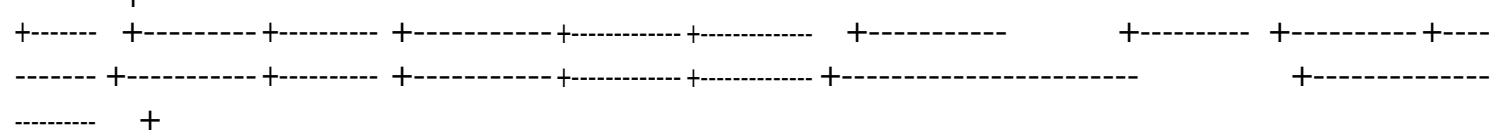

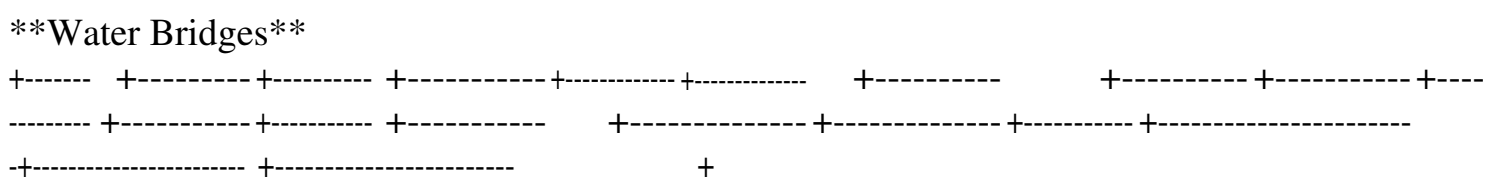
| RESNR | RESTYPE | RESCHAIN | RESNR_LIG | RESTYPE_LIG | RESCHAIN_LIG | DIST_A-W | DIST_D-W | DON_ANGLE | WATER_ANGLE | PROTISDON | DONOR_IDX | DONORTYPE | ACCEPTOR_IDX | ACCEPTORTYPE | WATER_IDX | LIGCOO PROTCOO |WATERCOO

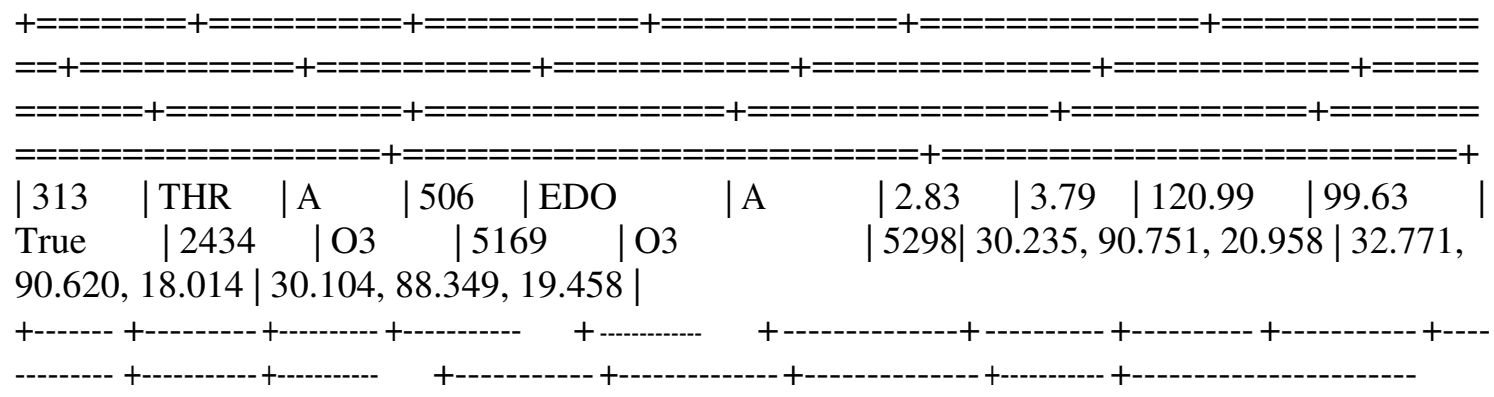


EDO:B:405 (EDO) - SMALLMOLECULE

Interacting chain(s): B

**Hydrogen Bonds**

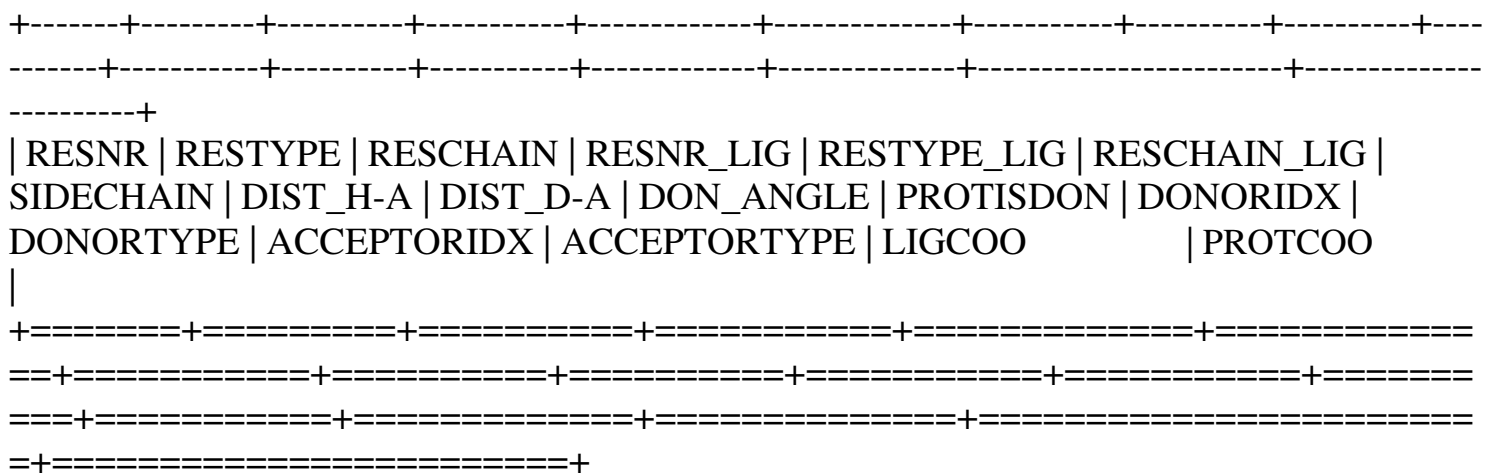

$=+=====================+$

| 208 | ARG | B | | 405 | EDO |B $\quad$ | True | $2.98 \quad 3.84 \quad$ | 146.58 |

True $\quad|4186| \mathrm{Ng}+\quad|5191 \quad| \mathrm{O} 3 \quad|-1.831,92.150,31.712|-0.507,90.101$,

28.744 |

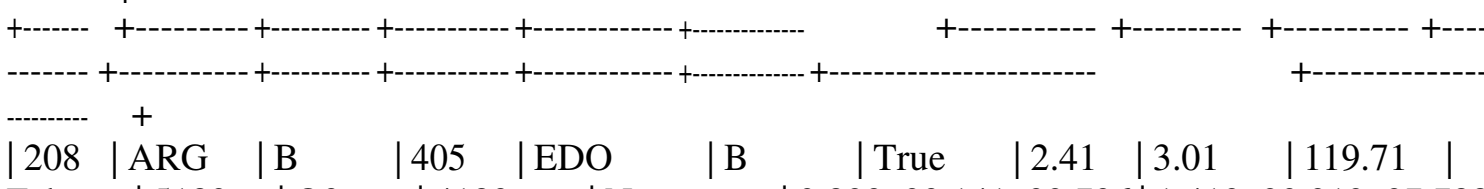

False $|5189 \quad| \mathrm{O} 3 \quad|4183 \quad| \mathrm{Ng}_{+} \quad|0.823,93.141,29.736| 1.412,90.919,27.788$

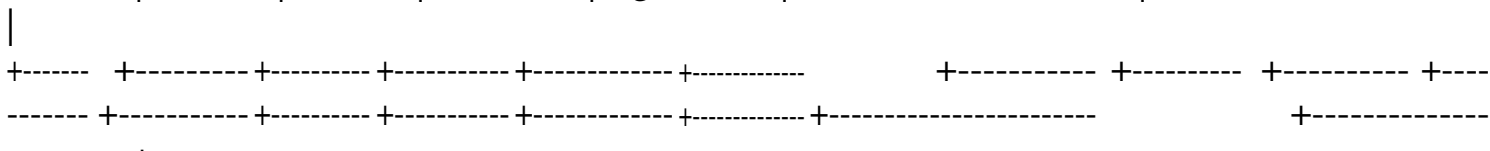

| $212 \mid$ GLN | B |405 |EDO |B | | True |2.11 |3.08 |169.77 |

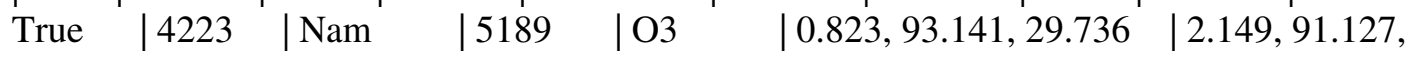

31.655

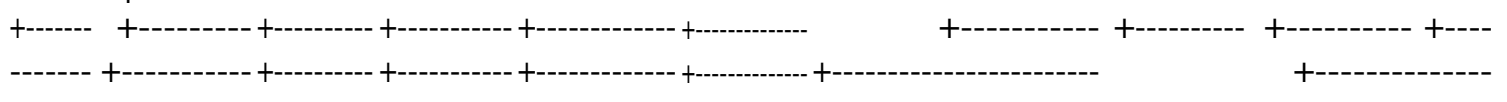

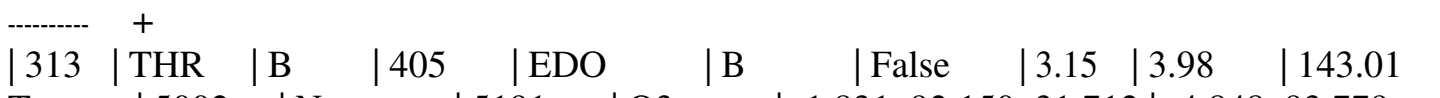

True $|5002 \quad|$ Nam $\quad \mid 5191 \quad$ O O3 $\quad|-1.831,92.150,31.712|-4.848,93.779$, $29.689 \mid$

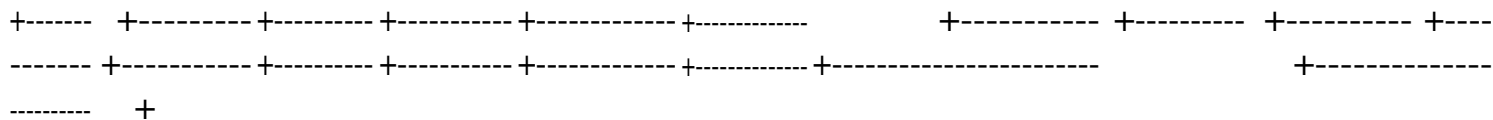

**Water Bridges**

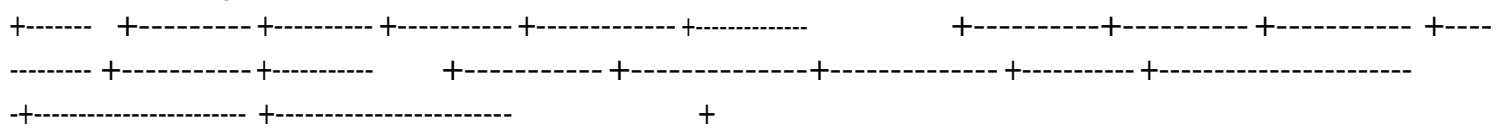

| RESNR | RESTYPE | RESCHAIN | RESNR_LIG | RESTYPE_LIG | RESCHAIN_LIG | DIST_A-W | DIST_D-W | DON_ANGLE | WATER_ANGLE | PROTISDON | DONOR_IDX | DONORTYPE | ACCEPTOR_IDX | ACCEPTORTYPE | WATER_IDX | LIGCOO

PROTCOO 


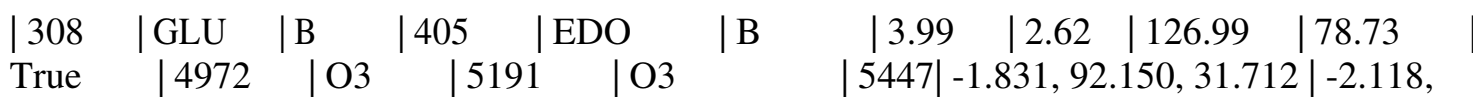
$87.833,29.305|-4.031,88.984,30.667|$

MG:A:505 (MG) - ION

Interacting chain(s): A,B

**Metal Complexes**

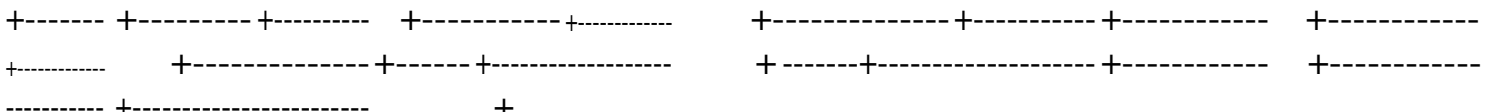

| RESNR | RESTYPE | RESCHAIN | RESNR_LIG | RESTYPE_LIG | RESCHAIN_LIG |

METAL_IDX | METAL_TYPE | TARGET_IDX | TARGET_TYPE | COORDINATION | DIST

| LOCATION | RMS | GEOMETRY | COMPLEXNUM | METALCOO | TARGETCOO |

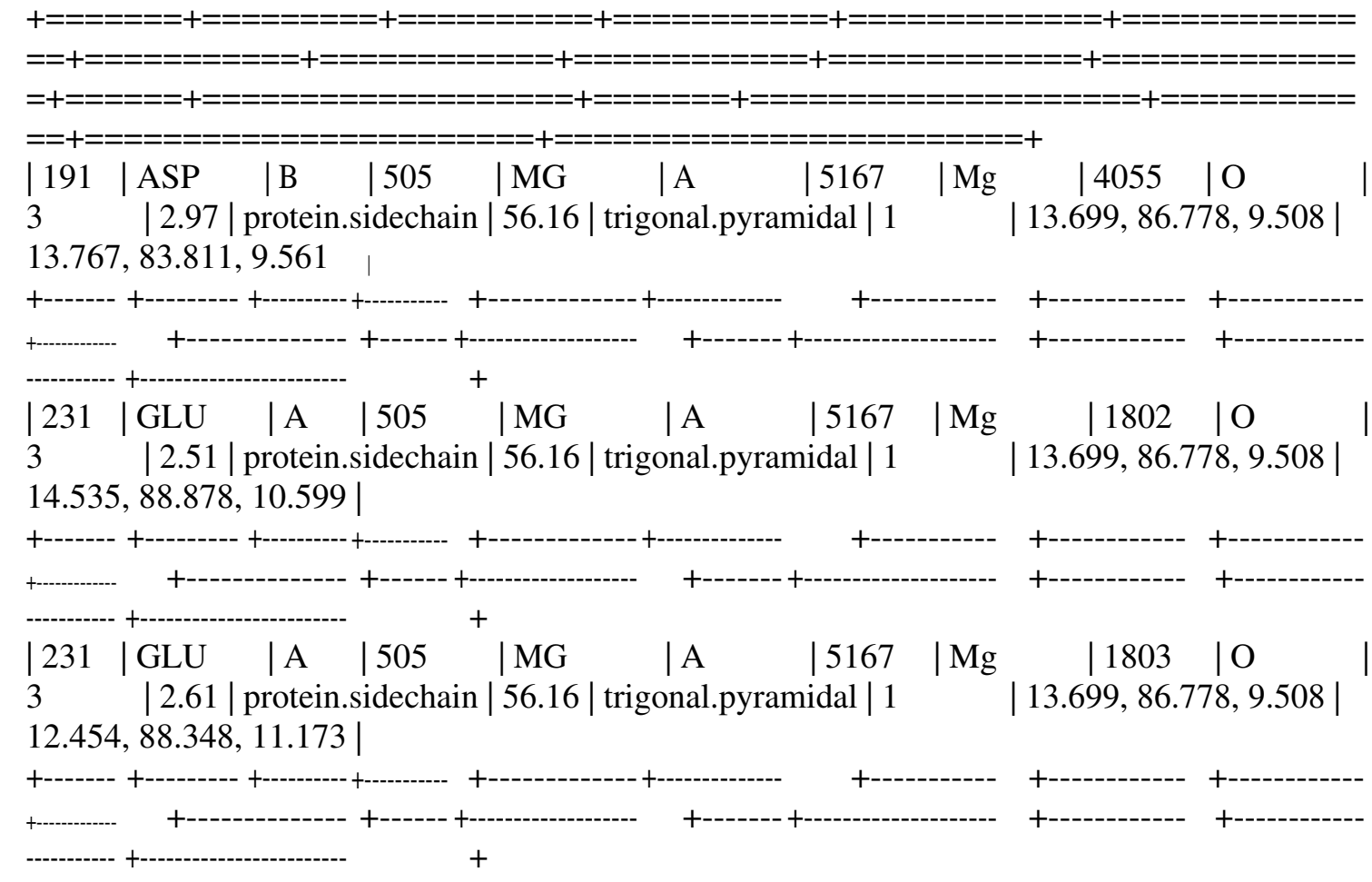

MG:B:404 (MG) - ION

Interacting chain(s): B

**Metal Complexes** 

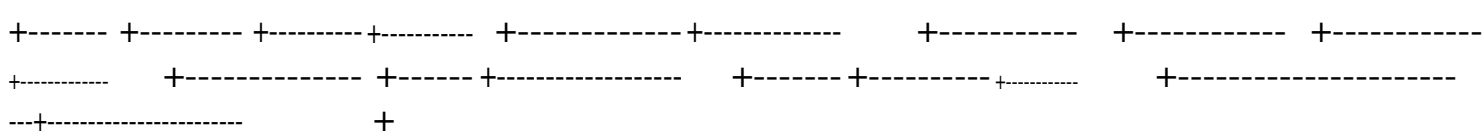

| RESNR | RESTYPE | RESCHAIN | RESNR_LIG | RESTYPE_LIG | RESCHAIN_LIG | METAL_IDX | METAL_TYPE | TARGET_IDX | TARGET_TYPE | COORDINATION | DIST | LOCATION $\quad \mid$ RMS $\mid$ GEOMETRY $\mid$ COMPLEXNUM $\mid$ METALCOO 


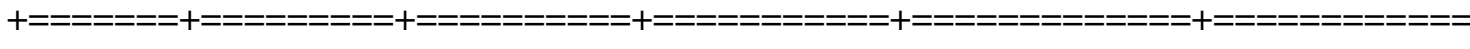

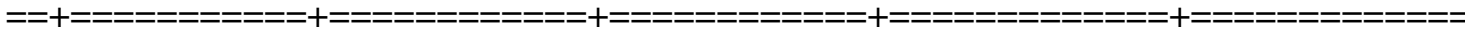

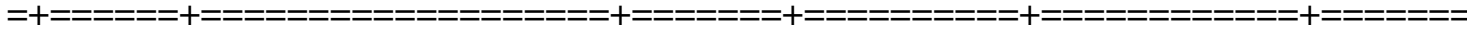
ニニニニニニニニニニニニニニニニニニ+ニニニニニニニニニニニニニニニニニニニニニニニニ+

| 227 | GLU |B | $404 \quad$ |MG |B $\quad$ | $15187 \quad|\mathrm{Mg}| 4344 \quad$ |O | $2 \quad|2.24|$ protein.sidechain $|98.07|$ linear $\quad 1 \quad|10.436,108.864,27.911| 9.354$, $107.168,28.892$

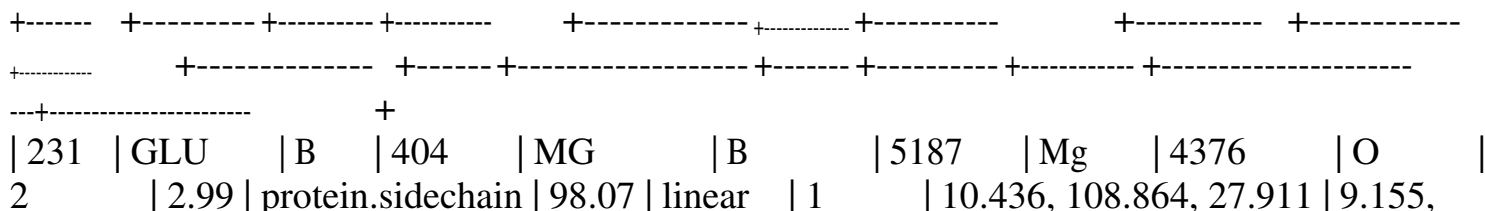

$107.715,25.469 \mid$

SO4:A:501 (SO4) - SMALLMOLECULE

Interacting chain(s): A,B

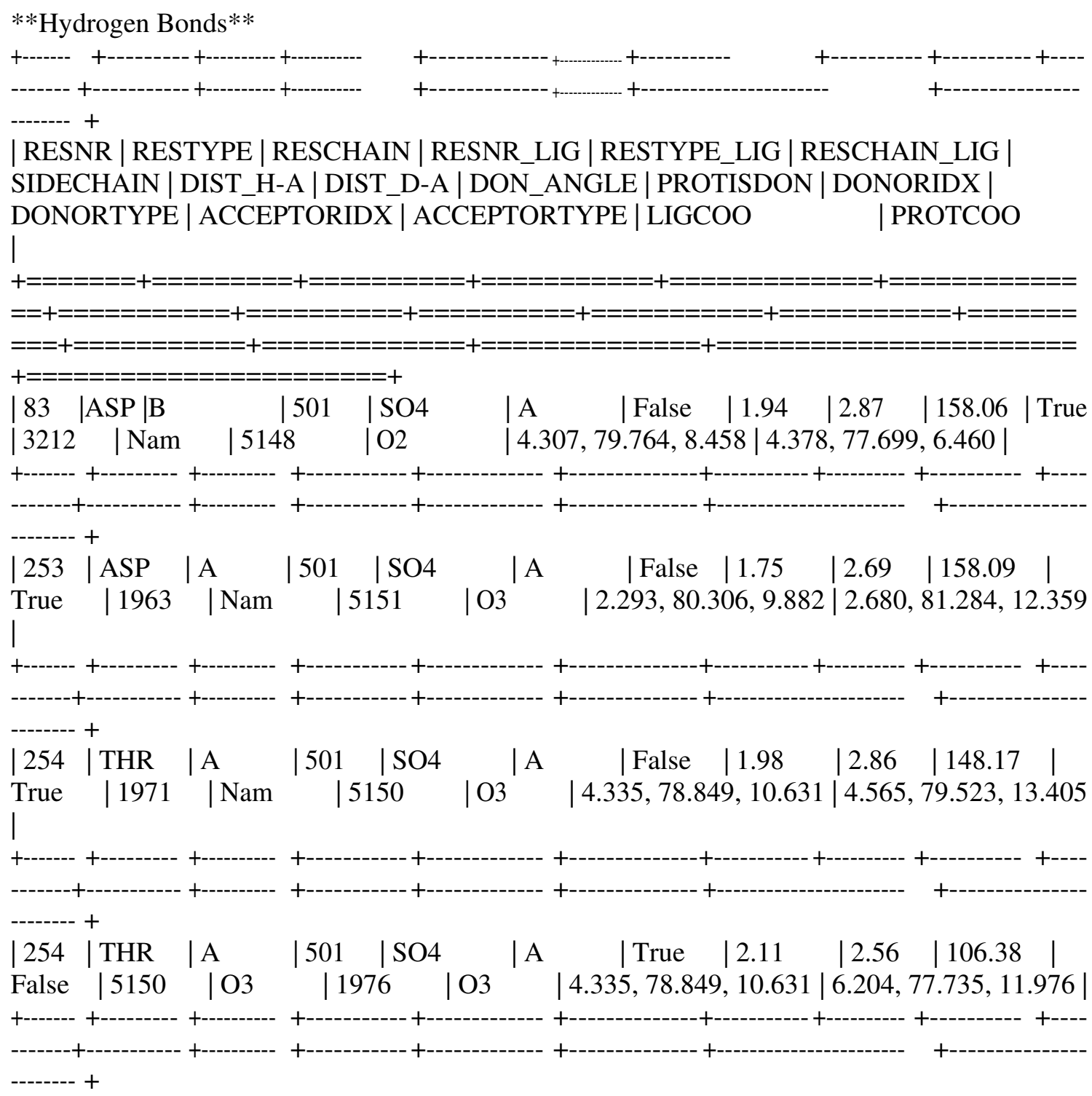


SO4:A:502 (SO4) - SMALLMOLECULE

Interacting chain(s): A

**Hydrogen Bonds**

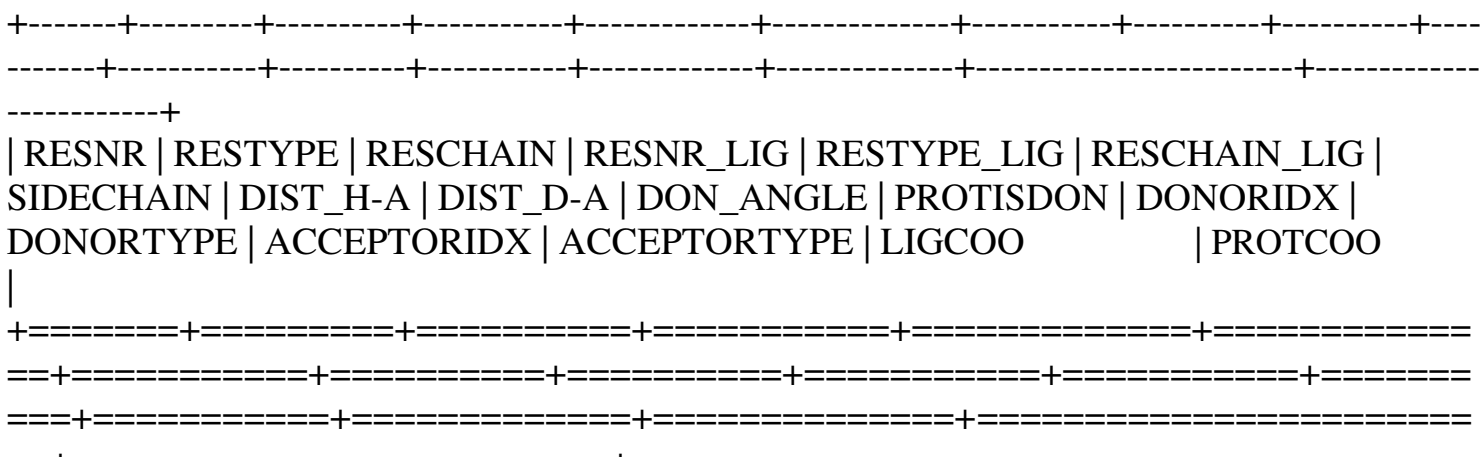

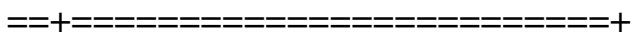

\begin{tabular}{|l|l|l|l|l|l|l|l|l|l|}
51 & GLY & A & | 502 & SO4 & |A & False & 3.66 & 4.05 & 106.38
\end{tabular} True $|381 \quad|$ Nam $|5156 \quad|$ O3 $\quad|27.732,124.421,22.168| 28.453,127.892$, 20.214 |

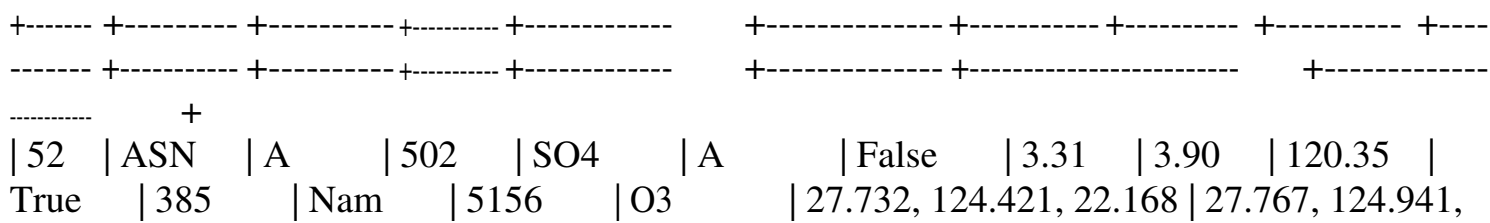
18.305

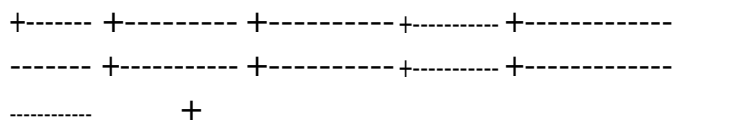

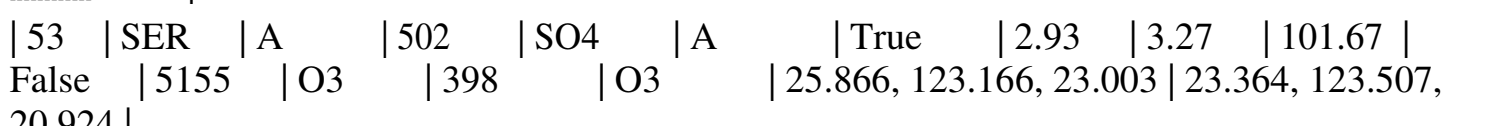

$20.924 \mid$

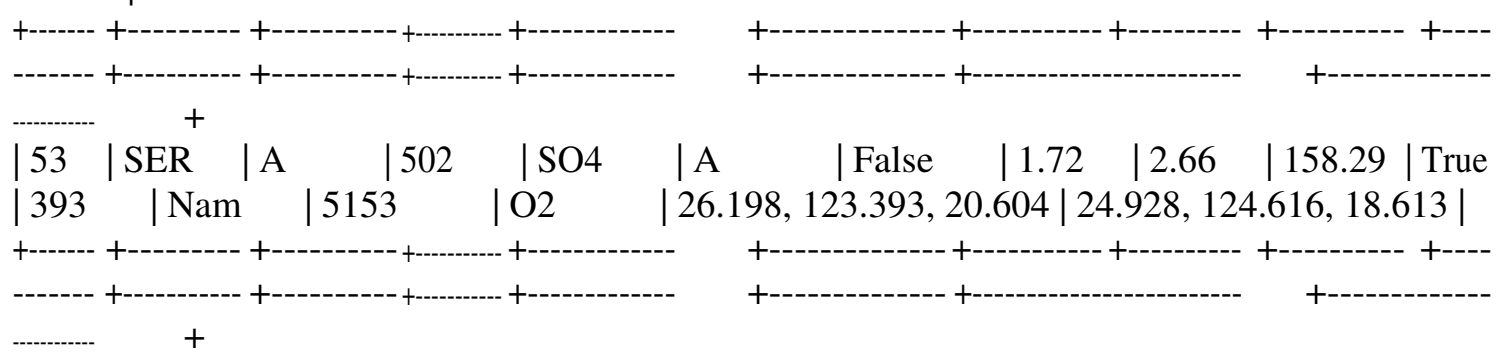

**Water Bridges**

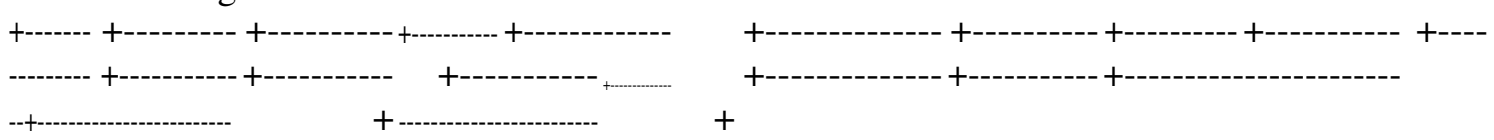

| RESNR | RESTYPE | RESCHAIN | RESNR_LIG | RESTYPE_LIG | RESCHAIN_LIG | DIST_A-W | DIST_D-W |DON_ANGLE | WATER_ANGLE | PROTISDON | DONOR_IDX | DONORTYPE | ACCEPTOR_IDX | ACCEPTORTYPE | WATER_IDX | LIGCOO PROTCOO | WATERCOO

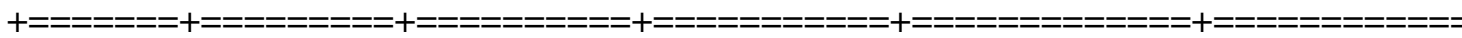

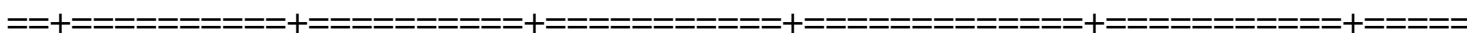

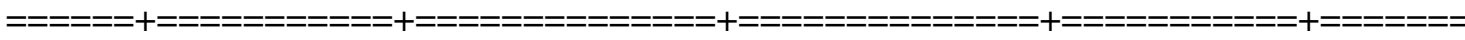


\begin{tabular}{l|l|l|l|l|l|l|} 
True $\quad \mid 370$ & $\mid \mathrm{Ng}+$ & $|5154 \quad| \mathrm{O} 2$ & 5194 & $|27.729,122.064,21.789| 27.192$,
\end{tabular} $123.946,26.322|29.651,124.050,23.379|$

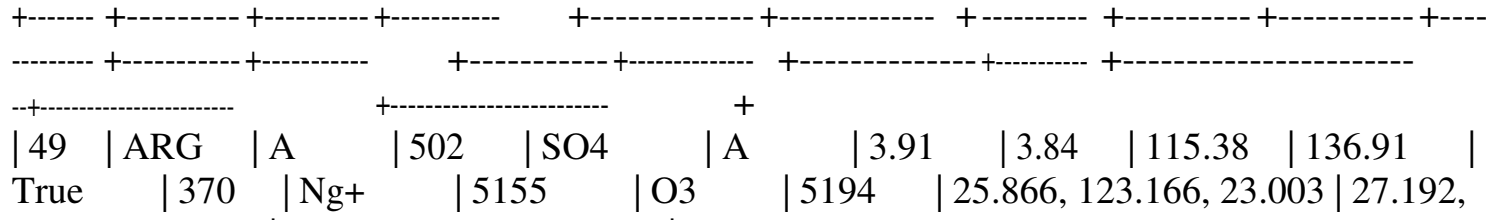
\begin{tabular}{l|l|l|} 
True & $|370| \mathrm{Ng}+$ & $|5155 \quad| \mathrm{O} 3$ \\
$123.946,26.322|29.651,124.050,23.379|$
\end{tabular}

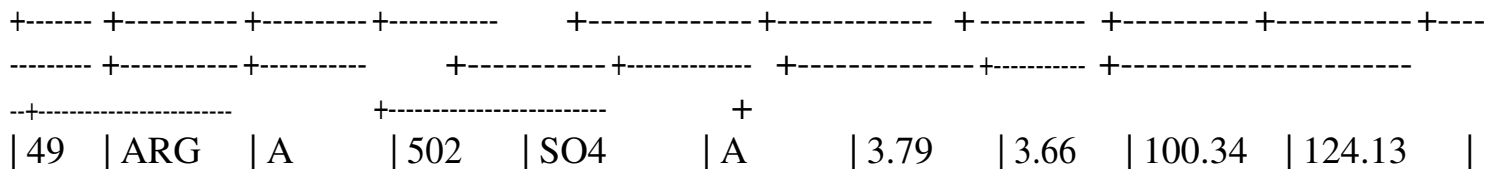
True $\quad 370\left|\mathrm{Ng}_{+} \quad\right| 5156 \quad|\mathrm{O} 3 \quad| 5360 \quad|27.732,124.421,22.168| 27.192$, $123.946,26.322|25.238,126.306,24.311|$

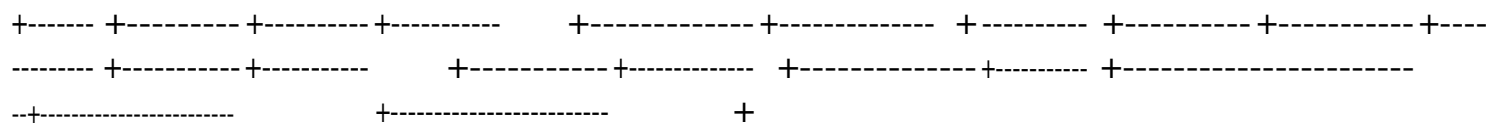

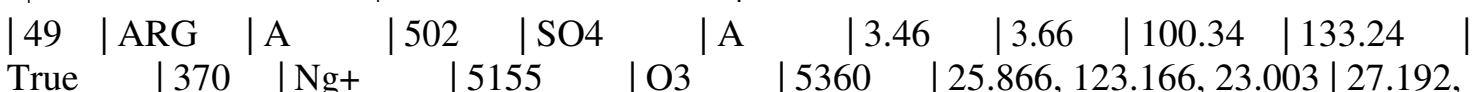
123.946, 26.322|25.238, 126.306, 24.311| |5360 |25.866, 123.166, 23.003|27.192, $123.946,26.322|25.238,126.306,24.311|$

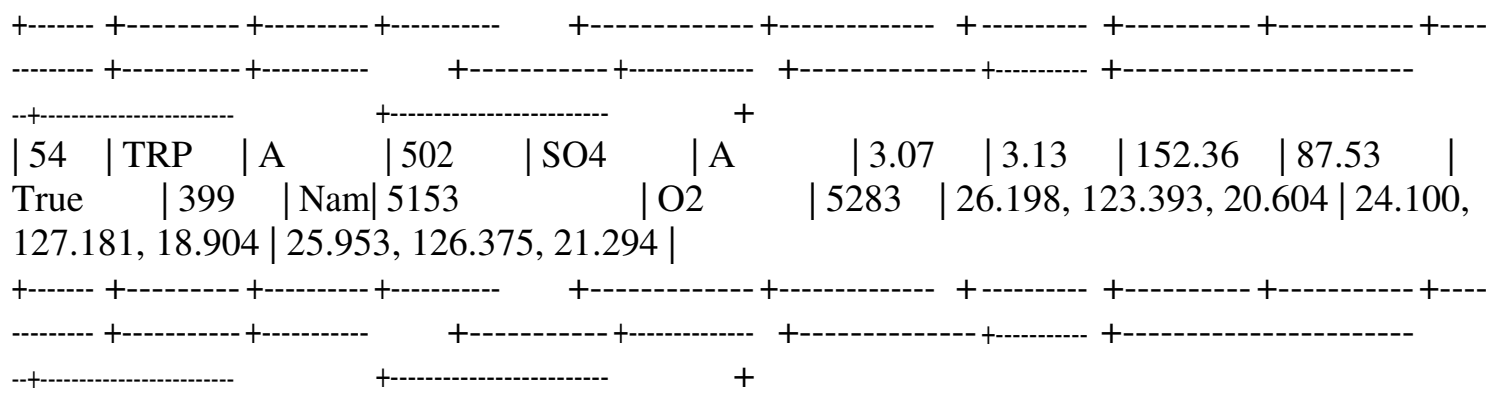

**Salt Bridges**

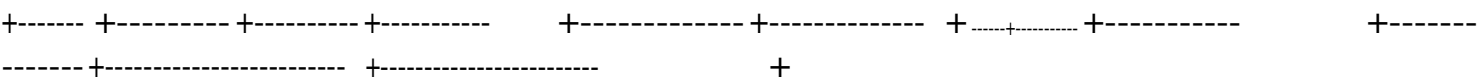

| RESNR | RESTYPE | RESCHAIN | RESNR_LIG | RESTYPE_LIG | RESCHAIN_LIG | DIST | PROTISPOS | LIG_GROUP | LIG_IDX_LIST | LIGCOO

| PROTCOO

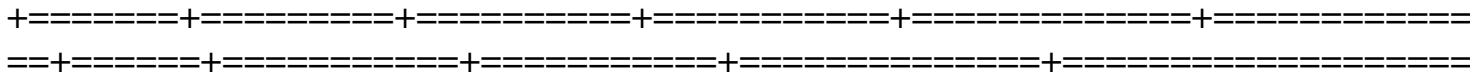

$=====+=======================++$

| 49 | ARG |A | | $502 \quad$ |SO4 |A | $15.14 \mid$ True $\quad$ Sulfate | 5152,5152 |

$26.868,123.261,21.907|28.246,124.697,26.644|$

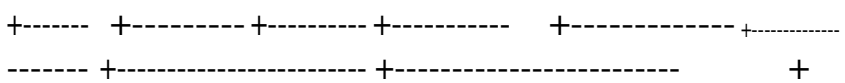

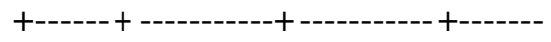

SO4:A:503 (SO4) - SMALLMOLECULE

Interacting chain(s): A

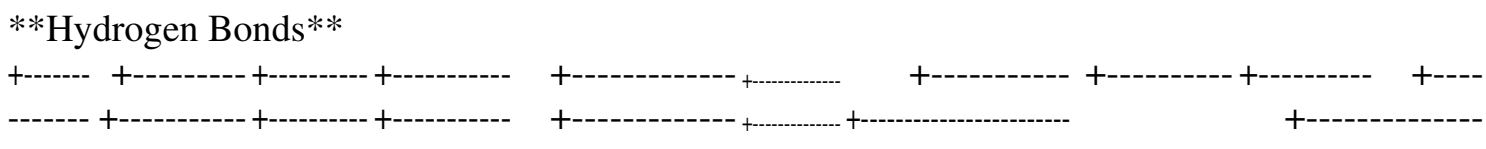

| RESNR | RESTYPE | RESCHAIN | RESNR_LIG | RESTYPE_LIG | RESCHAIN_LIG

| SIDECHAIN | DIST_H-A | DIST_D-A | DON_ANGLE | PROTISDON | DONORIDX | 
DONORTYPE | ACCEPTORIDX | ACCEPTORTYPE | LIGCOO | PROTCOO | 


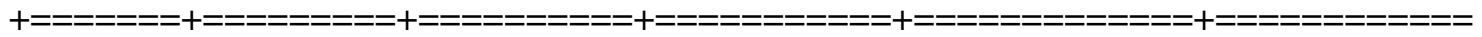

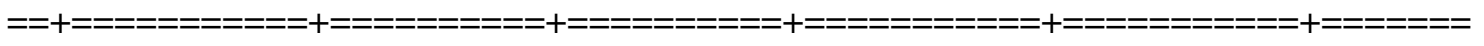

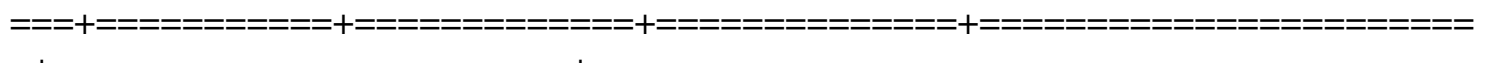

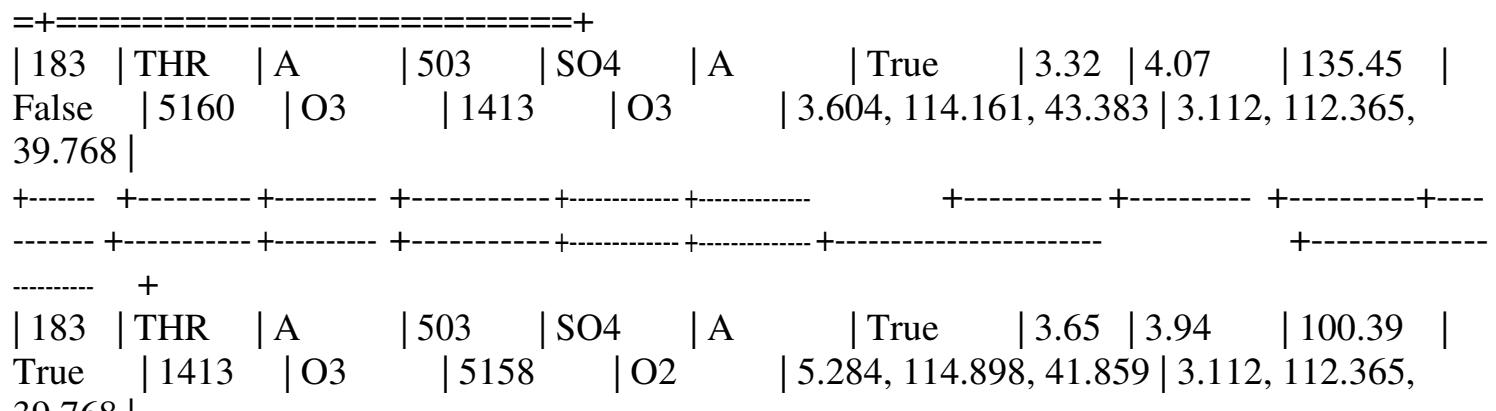
39.768

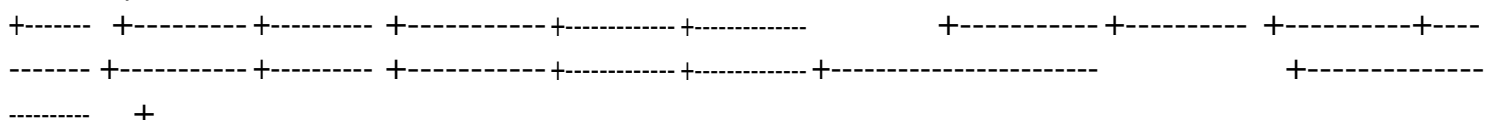

| 184 | PHE |A | $503 \quad$ |SO4 |A $\quad$ |False | $2.95 \quad$ |3.31 | $102.62 \mid$ True $|1415|$ Nam $\mid 5158 \quad$ |O2 $\quad$ |5.284, 114.898, 41.859| 5.002, 115.141, 38.575 |

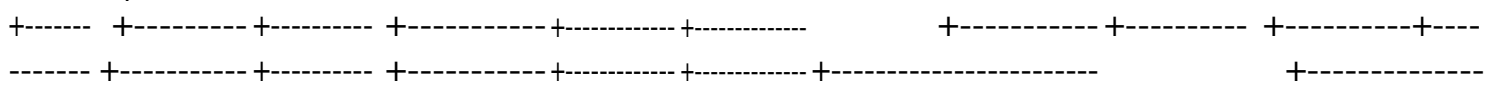

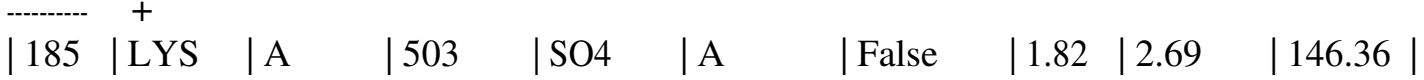
True $|1426|$ Nam $\quad \mid 5158 \quad$ O $2 \quad$ | 5.284, 114.898, 41.859|6.495, 113.312, 40.049

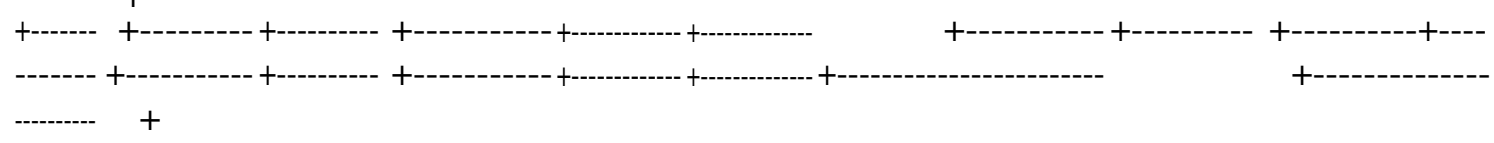

**Water Bridges**

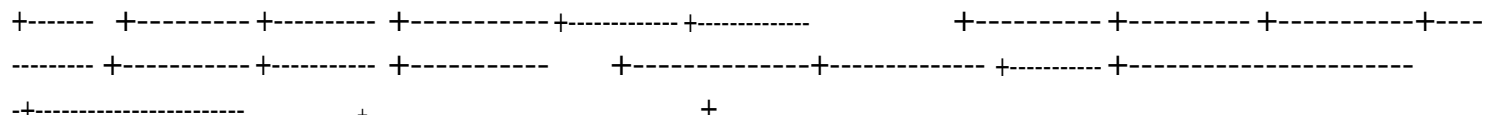

| RESNR | RESTYPE | RESCHAIN | RESNR_LIG | RESTYPE_LIG | RESCHAIN_LIG | DIST_A-W | DIST_D-W | DON_ANGLE | WATER_ANGLE | PROTISDON | DONOR_IDX | DONORTYPE | ACCEPTOR_IDX | ACCEPTORTYPE | WATER_IDX | LIGCOO PROTCOO

| WATERCOO

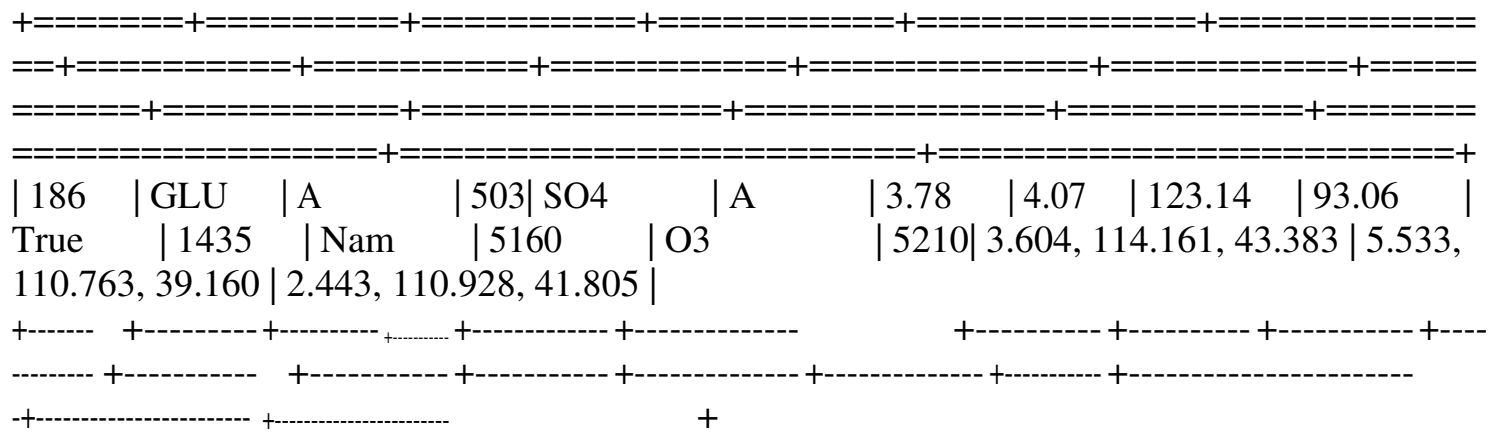

SO4:A:504 (SO4) - SMALLMOLECULE

Interacting chain(s): A 
| RESNR | RESTYPE | RESCHAIN | RESNR_LIG | RESTYPE_LIG | RESCHAIN_LIG | SIDECHAIN | DIST_H-A | DIST_D-A | DON_ANGLE | PROTISDON | DONORIDX | DONORTYPE | ACCEPTORIDX | ACCEPTORTYPE | LIGCOO | PROTCOO |

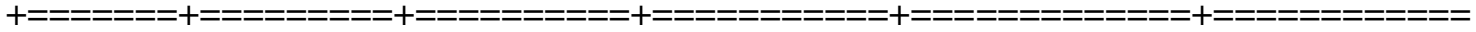

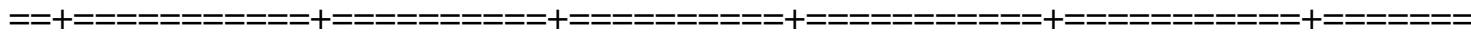

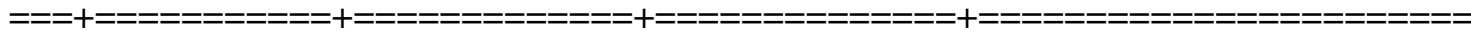
=ニ+ニニニニニニニニニニニニニニニニニニニニニニニニニ+

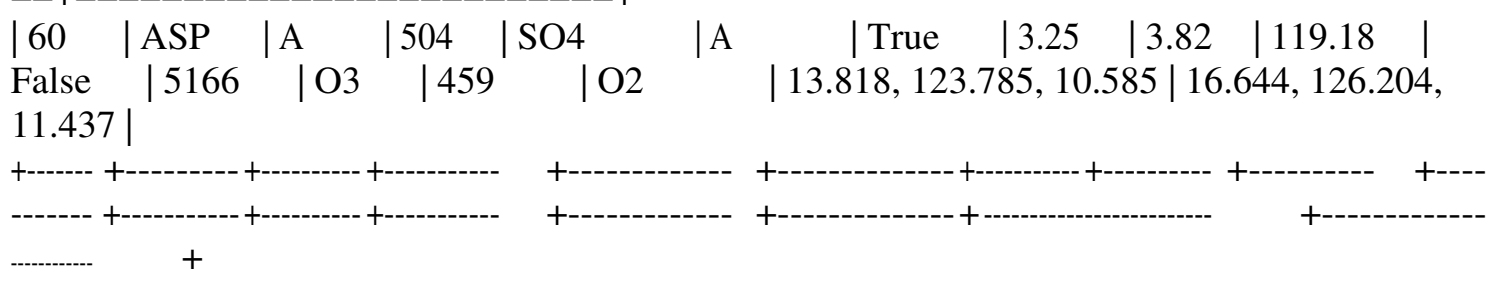

**Salt Bridges**

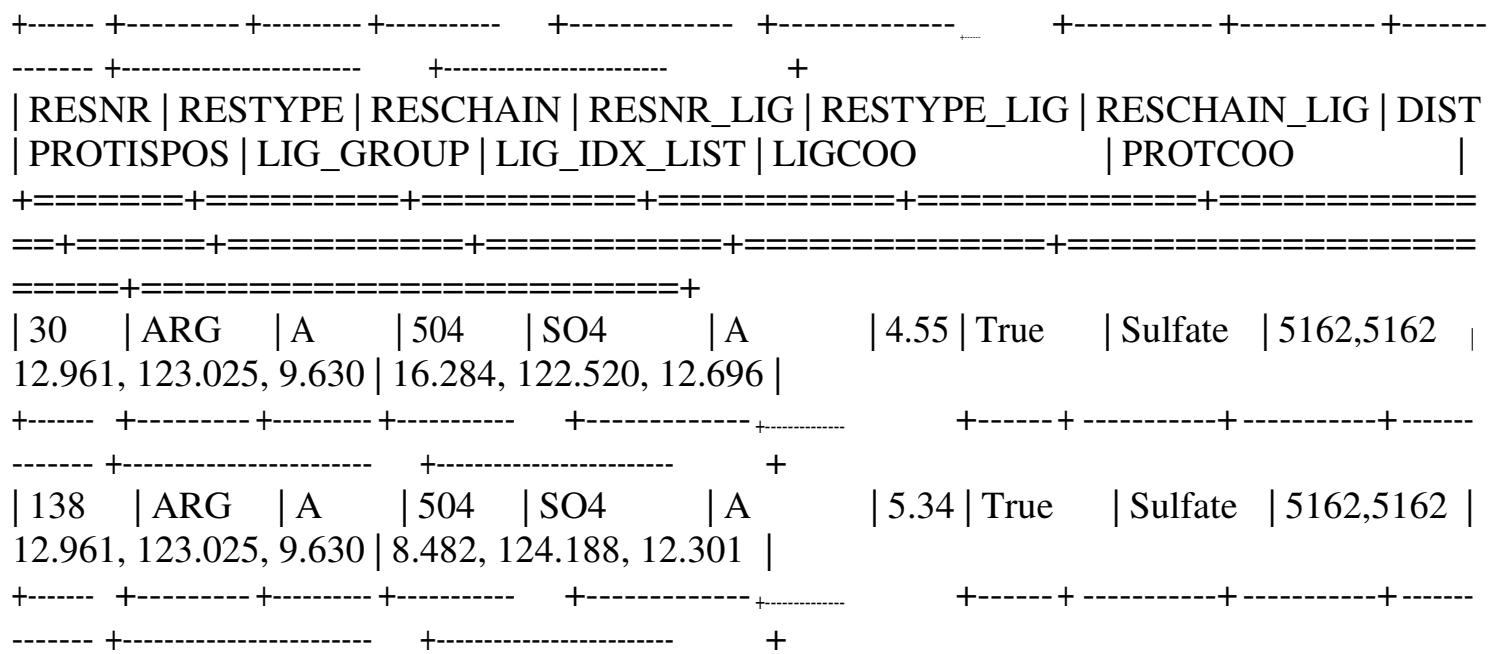

SO4:B:401 (SO4) - SMALLMOLECULE

Interacting chain(s): B

**Hydrogen Bonds**

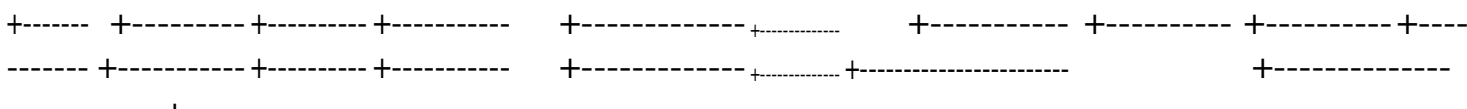

| RESNR | RESTYPE | RESCHAIN | RESNR_LIG | RESTYPE_LIG | RESCHAIN_LIG | SIDECHAIN | DIST_H-A | DIST_D-A | DON_ANGLE | PROTISDON | DONORIDX | DONORTYPE | ACCEPTORIDX | ACCEPTORTYPE | LIGCOO

| PROTCOO

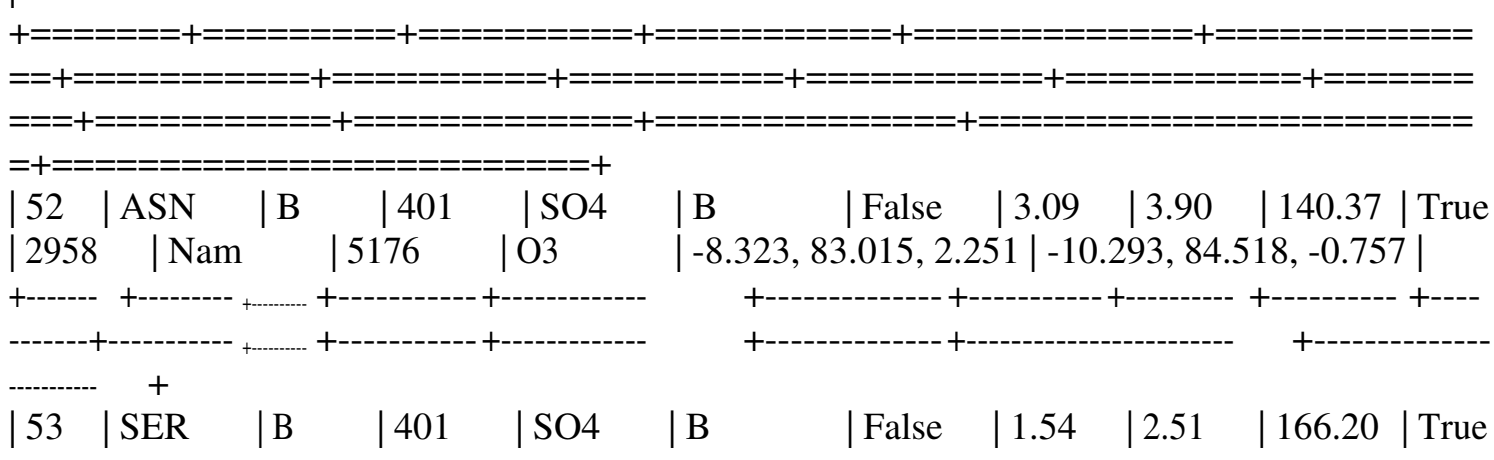




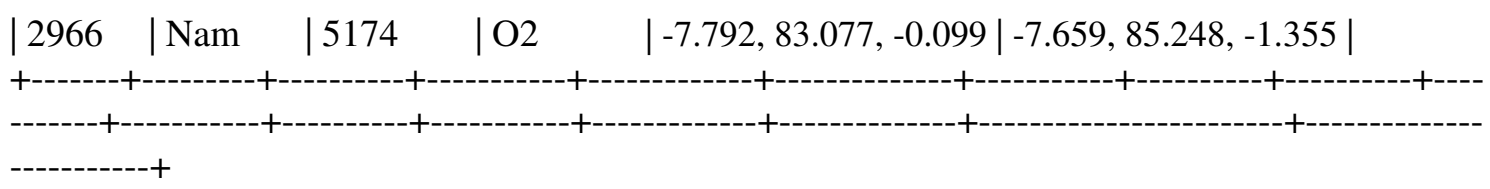

**Water Bridges**

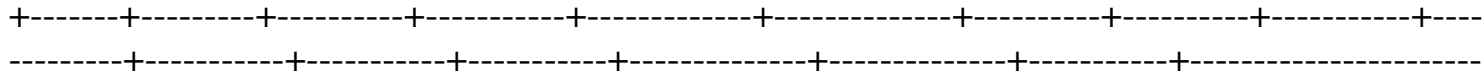

-+---------------------------+-------------------------+

| RESNR | RESTYPE | RESCHAIN | RESNR_LIG | RESTYPE_LIG | RESCHAIN_LIG | DIST_A-W | DIST_D-W | DON_ANGLE | WATER_ANGLE | PROTISDON | DONOR_IDX | DONORTYPE | ACCEPTOR_IDX | ACCEPTORTYPE | WATER_IDX | LIGCOO PROTCOO

$\mid$ WATERCOO

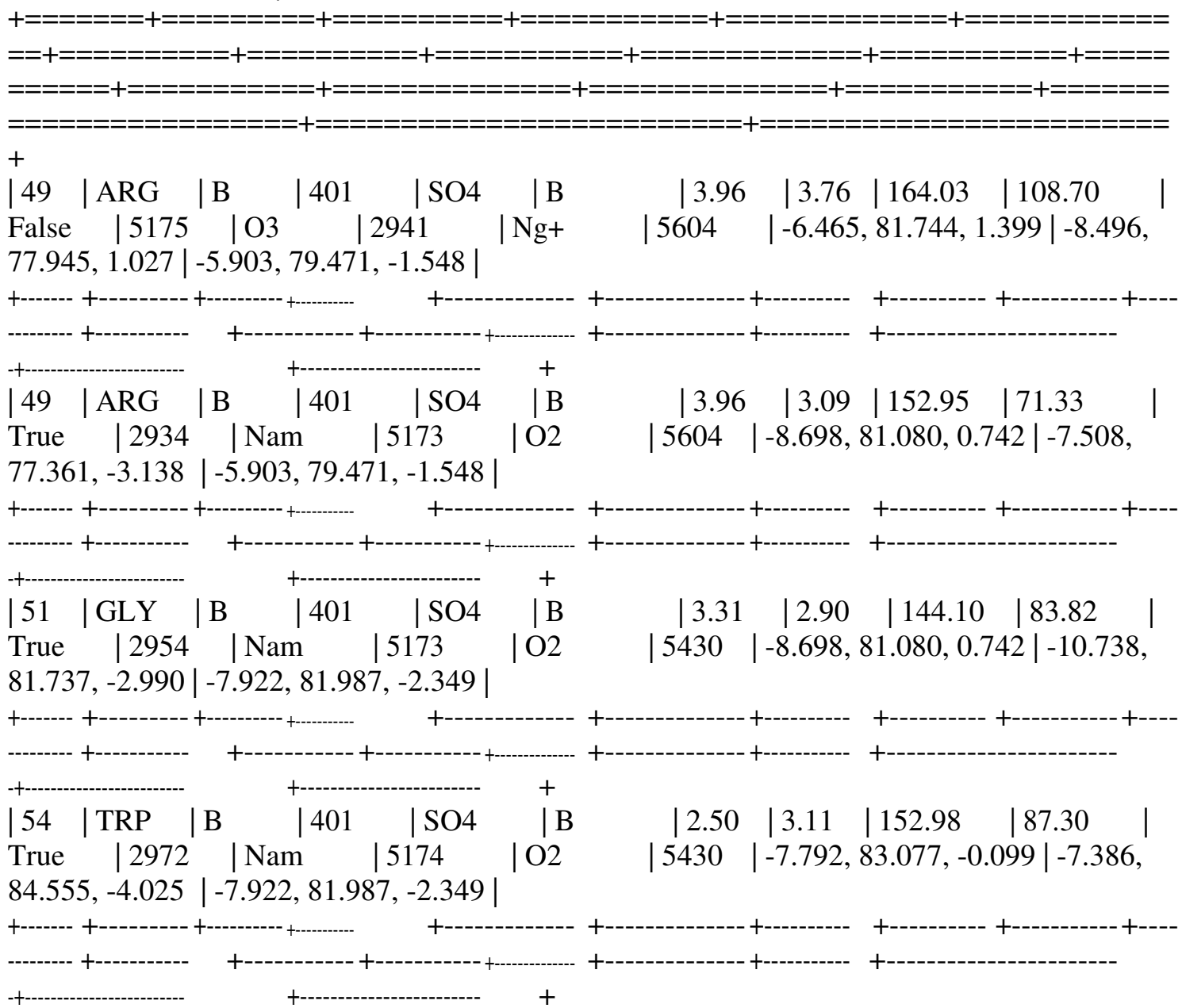

**Salt Bridges**

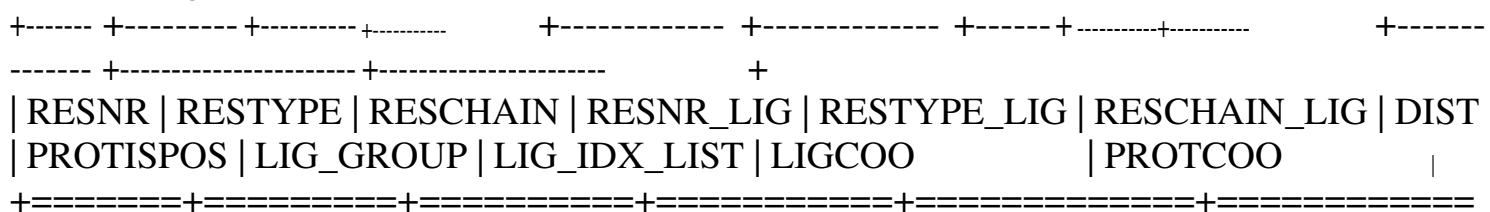

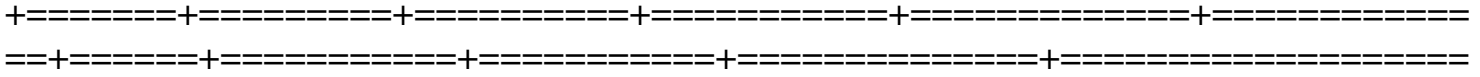

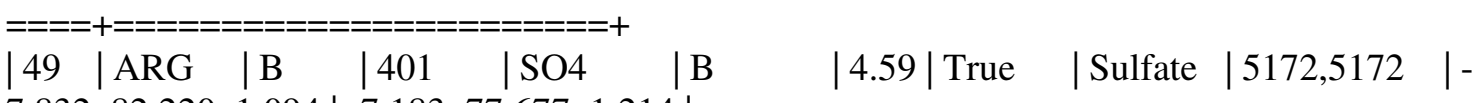
$7.832,82.220,1.094|-7.183,77.677,1.214|$ 
SO4:B:402 (SO4) - SMALLMOLECULE

Interacting chain(s): A,B

**Hydrogen Bonds**

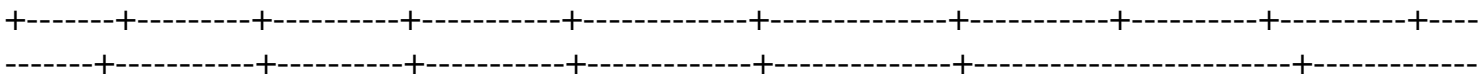

| RESNR | RESTYPE | RESCHAIN | RESNR_LIG | RESTYPE_LIG | RESCHAIN_LIG |

SIDECHAIN | DIST_H-A | DIST_D-A | DON_ANGLE | PROTISDON | DONORIDX |

DONORTYPE | ACCEPTORIDX | ACCEPTORTYPE | LIGCOO | PROTCOO

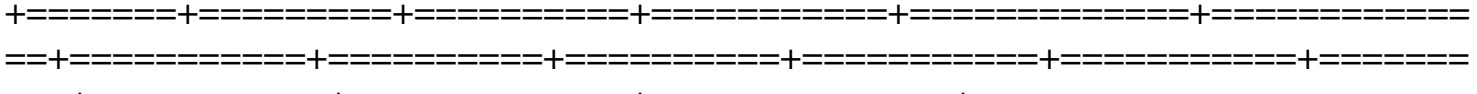

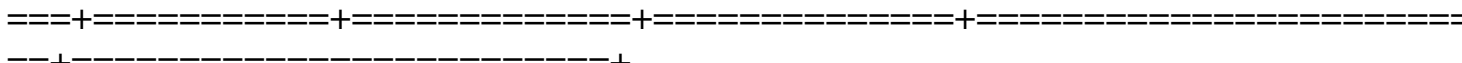

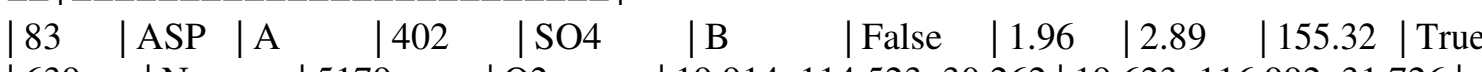
| 639 | Nam | | $5179 \quad$ |O2 $\quad|19.914,114.523,30.262| 19.623,116.992,31.726 \mid$

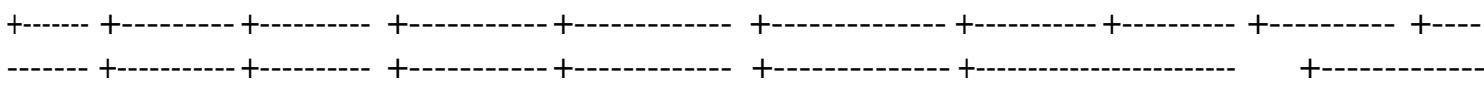

| 252 | SER | B | 402 |SO4 |B $\quad$ | True |3.00 |3.97 | 176.18 |

False $|5180 \quad| \mathrm{O} 3 \quad|4535 \quad| \mathrm{O} 3 \quad|21.169,112.802,31.511| 18.851,110.258$,

29.533

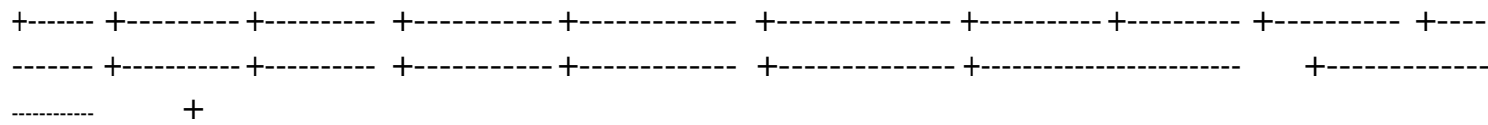

| $253 \mid$ ASP | B | 402 | SO4 | B |False | $1.85|2.80 \quad| 162.98$ | True $|4536|$ Nam $\quad$ 5178 |O2 $|22.059,113.747,29.310| 22.361,110.967$, $29.100 \mid$

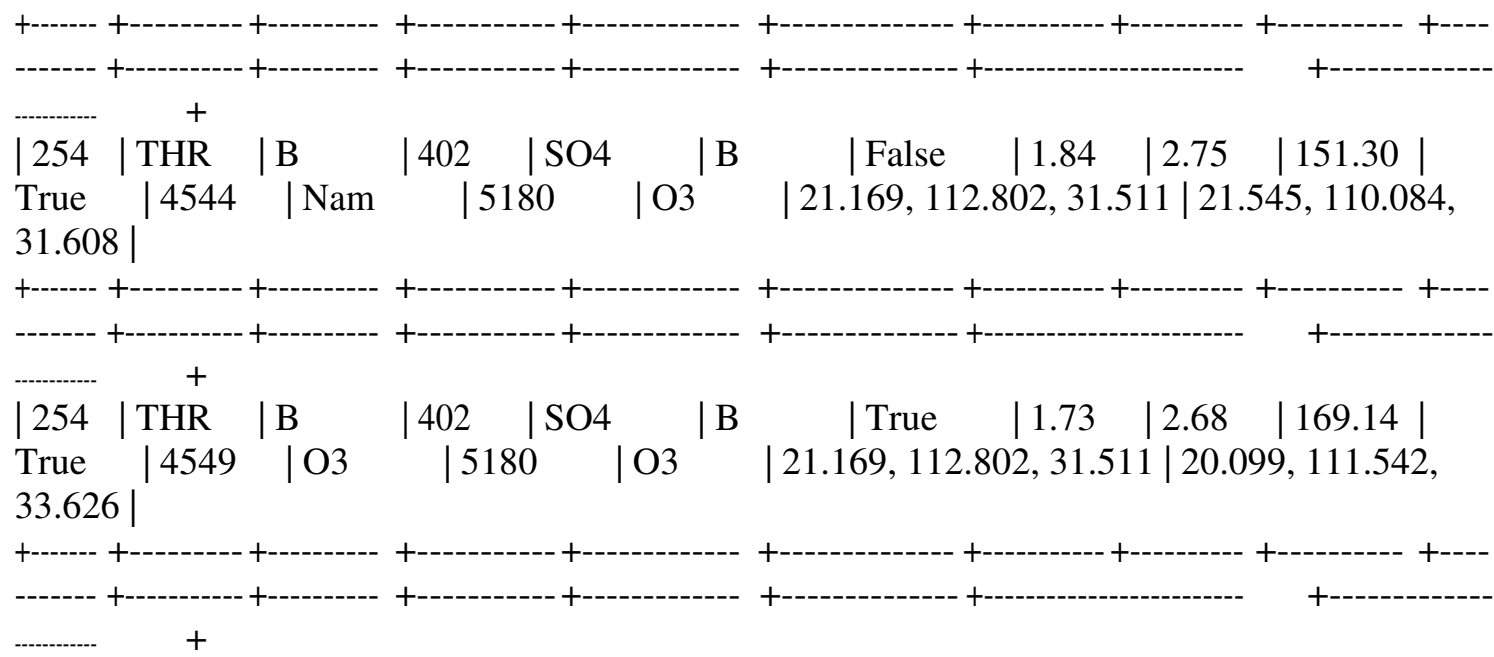

**Water Bridges**

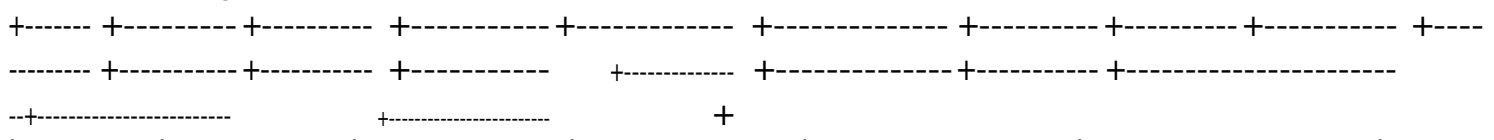

| RESNR | RESTYPE | RESCHAIN | RESNR_LIG | RESTYPE_LIG | RESCHAIN_LIG | DIST_A-W | DIST_D-W | DON_ANGLE | WATER_ANGLE | PROTISDON | DONOR_IDX | DONORTYPE | ACCEPTOR_IDX | ACCEPTORTYPE | WATER_IDX | LIGCOO 


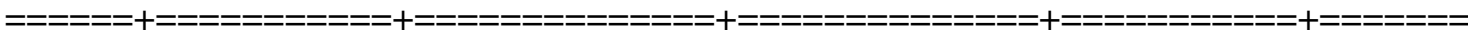

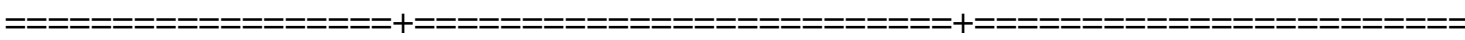

$==+$

\begin{tabular}{ll|l|l|l|l|l|l|}
$\mid 253$ & $\mid$ ASP & $\mid \mathrm{B}$ & $|402| \mathrm{SO} 4$ & $\mid \mathrm{B}$ & $|3.44| 2.82$ & $|136.02| 98.77 \mid$ \\
True & $|4543 \quad| \mathrm{O} 3$ & $|5178 \quad| \mathrm{O} 2$ & $|5468| 22.059,113.747,29.310 \mid 25.813$,
\end{tabular}

$112.960,29.568|24.752,115.246,30.832|$
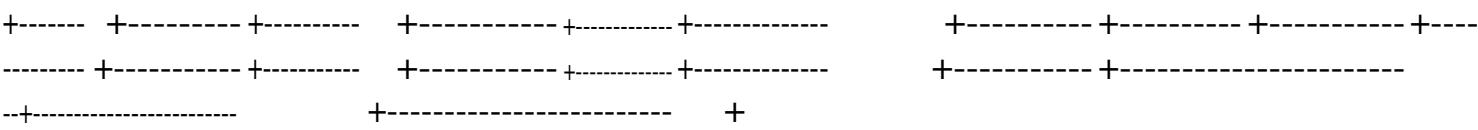

SO4:B:403 (SO4) - SMALLMOLECULE

Interacting chain(s): B

**Hydrogen Bonds**

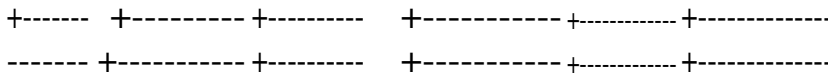

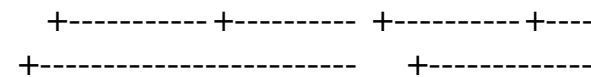

| RESNR | RESTYPE | RESCHAIN | RESNR_LIG | RESTYPE_LIG | RESCHAIN_LIG |

SIDECHAIN | DIST_H-A | DIST_D-A | DON_ANGLE | PROTISDON | DONORIDX | DONORTYPE | ACCEPTORIDX | ACCEPTORTYPE | LIGCOO | PROTCOO

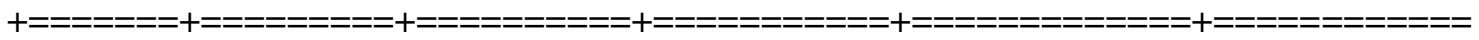

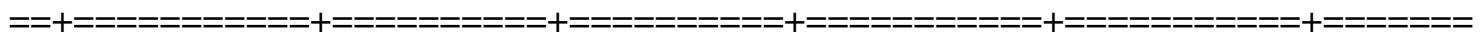

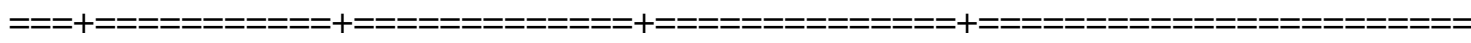

$==+=======================++$

| 10 | VAL | B $\quad$ | $403 \quad$ |SO4 | B $\quad$ | False | $2.57 \quad$ | 3.37 | 139.45 |

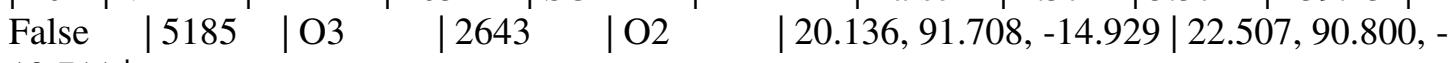

$12.711 \mid$
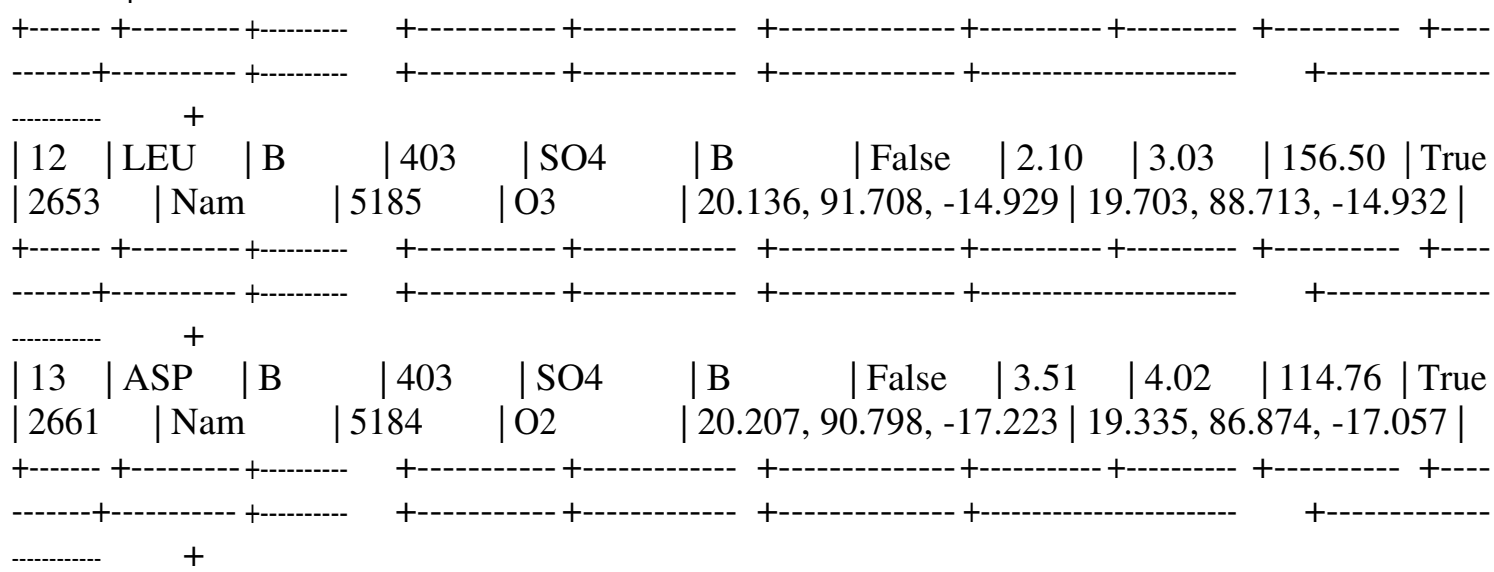

\footnotetext{
**Water Bridges**

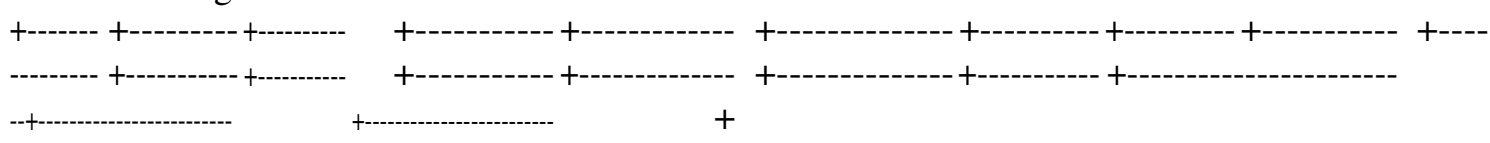

| RESNR | RESTYPE | RESCHAIN | RESNR_LIG | RESTYPE_LIG | RESCHAIN_LIG | DIST_A-W | DIST_D-W | DON_ANGLE | WATER_ANGLE | PROTISDON | DONOR_IDX | DONORTYPE | ACCEPTOR_IDX | ACCEPTORTYPE | WATER_IDX | LIGCOO PROTCOO | WATERCOO 


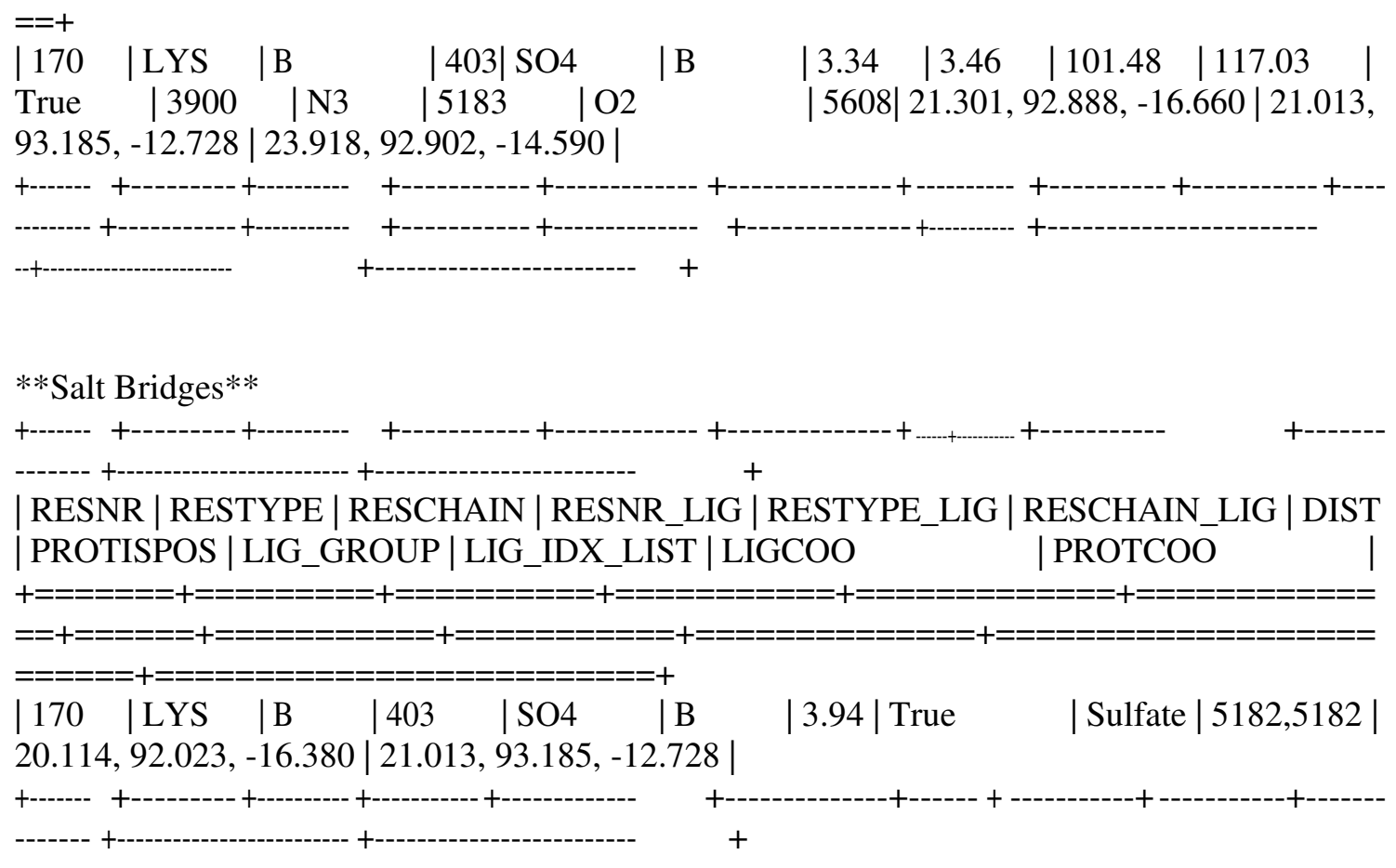




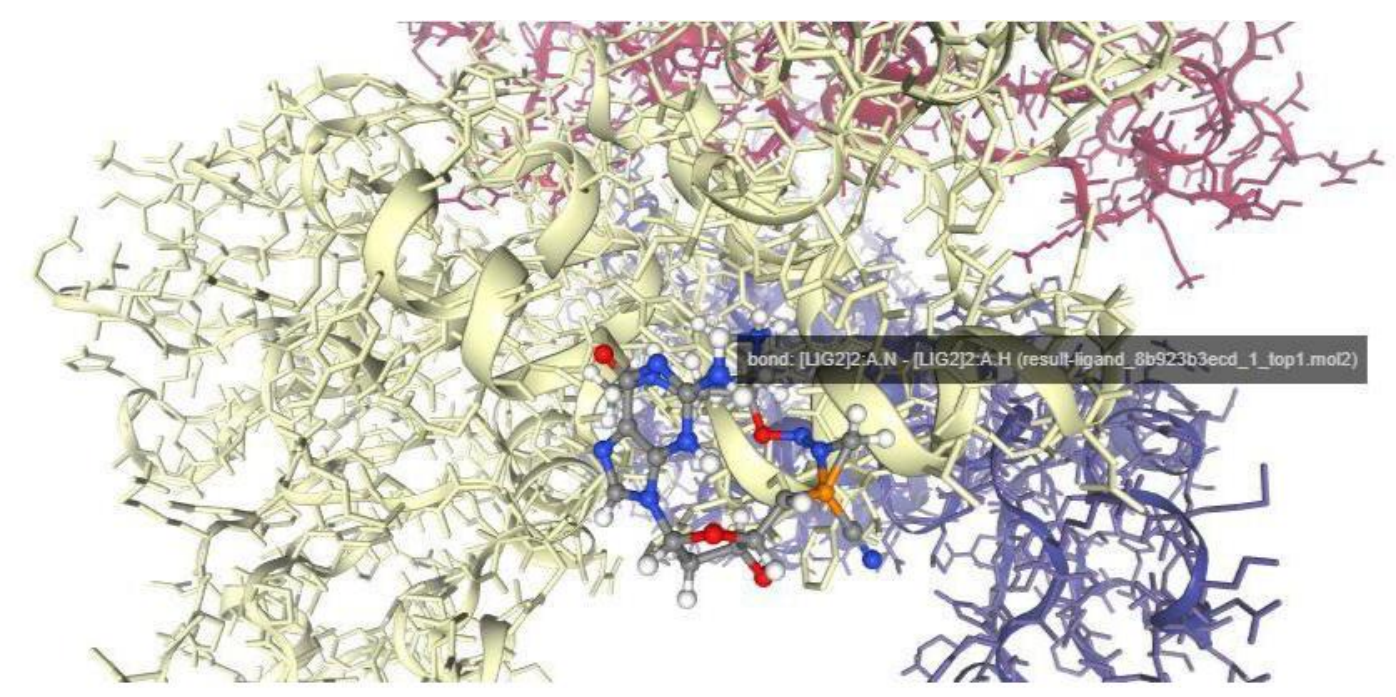

\begin{tabular}{l}
\hline File Model T.Energy I.Energy vdW Coul NumRotors RMSD Score \\
\hline ligand_8b923b3ecd_1_run_2.log 1 -36.678 -55.648 -7.519 -48.129 $70.000-6.762$ \\
ligand_8b923b3ecd_1_run_16.log 1 -36.224 -53.111 -5.988 -47.123 7 2.941 -6.543 \\
ligand_8b923b3ecd_1_run_4.log 1 -32.903 -52.904 -14.237 -38.667 $73.940-7.030$
\end{tabular}

Figure4b. Shows the receptor binding domain and the CoMFA contour map of steric regions (blue, favored; yellow; disfavored) around Roccustyrna; Blue regions are favored by positively charged groups and red regions favored by negatively charged groups inside the SARS-COV-2 protein targets (pdb:6W9C). 02J:C:1 (02J) - SMALLMOLECULE

Interacting chain(s): A

**Hydrophobic Interactions**

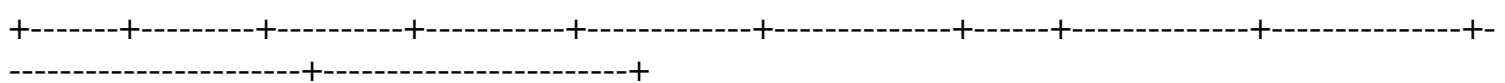

| RESNR | RESTYPE | RESCHAIN | RESNR_LIG | RESTYPE_LIG | RESCHAIN_LIG | DIST | LIGCARBONIDX | PROTCARBONIDX | LIGCOO | PROTCOO |

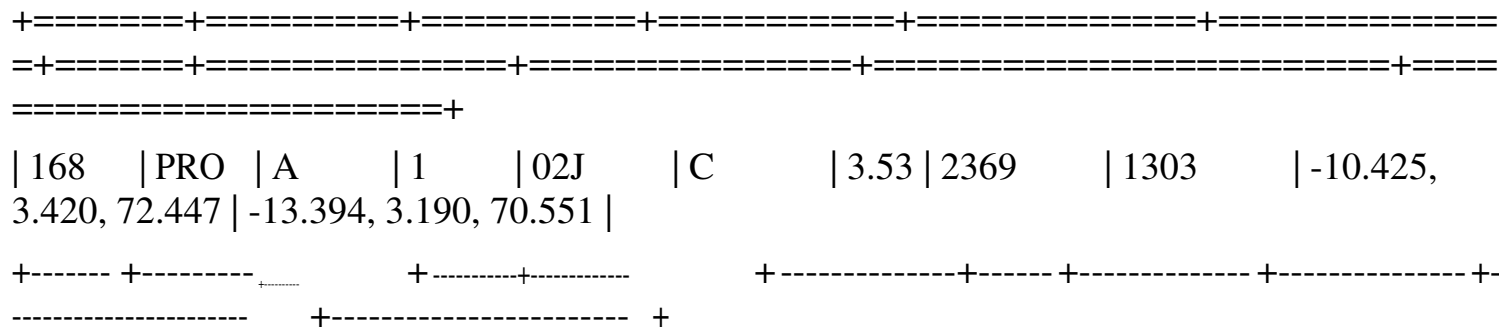

PJE:C:5 (PJE-010) - SMALLMOLECULE

$+010: \mathrm{C}: 6$

Interacting chain(s): A

**Hydrophobic Interactions $* *$ 


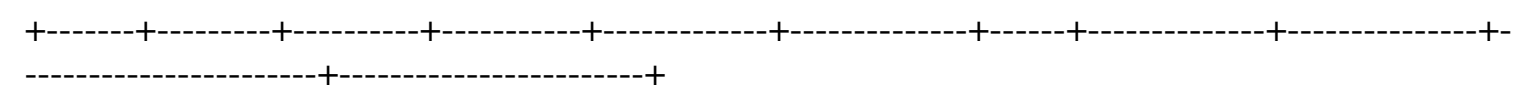

| RESNR | RESTYPE | RESCHAIN | RESNR_LIG | RESTYPE_LIG | RESCHAIN_LIG | DIST | LIGCARBONIDX | PROTCARBONIDX | LIGCOO | PROTCOO |
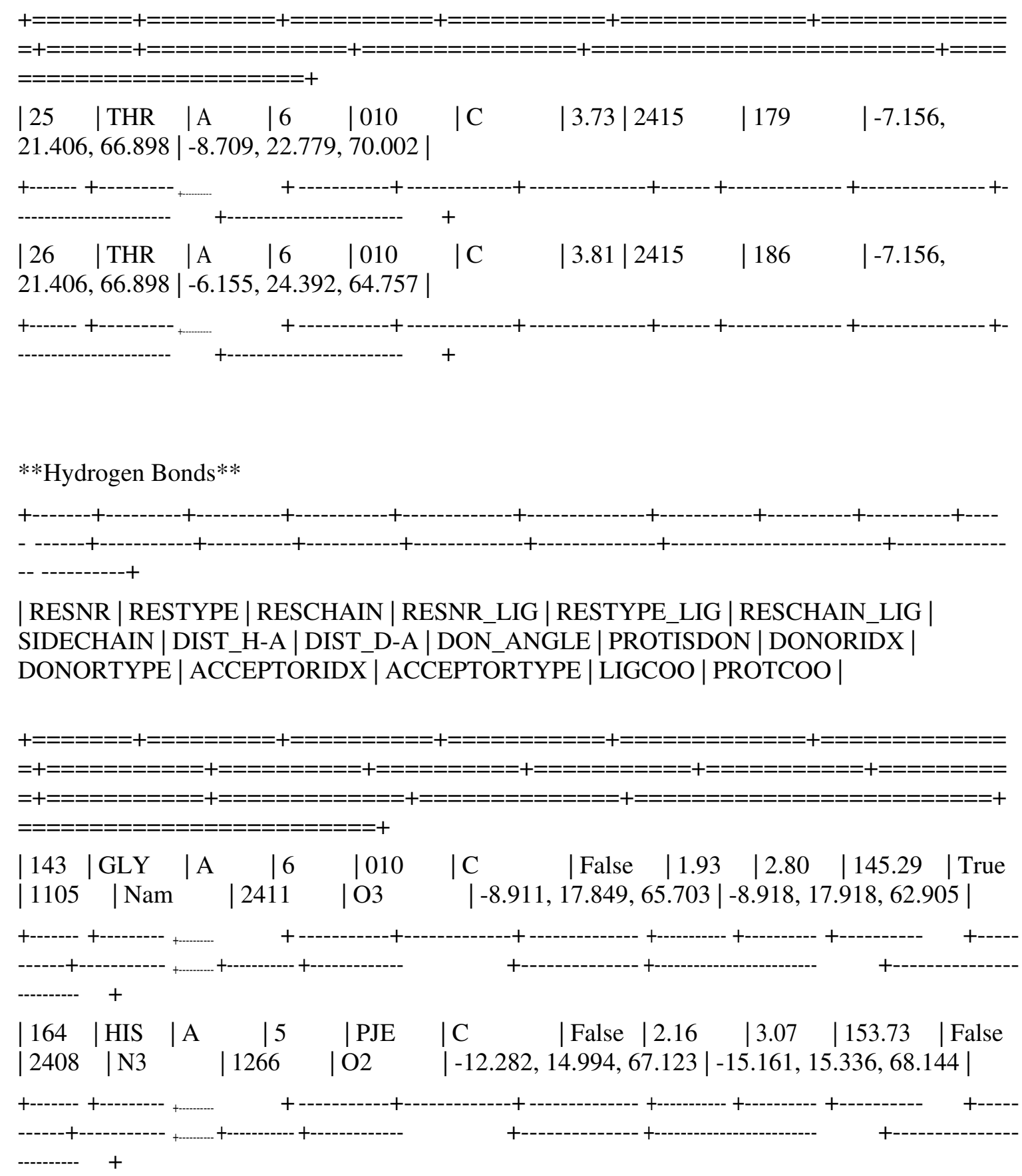


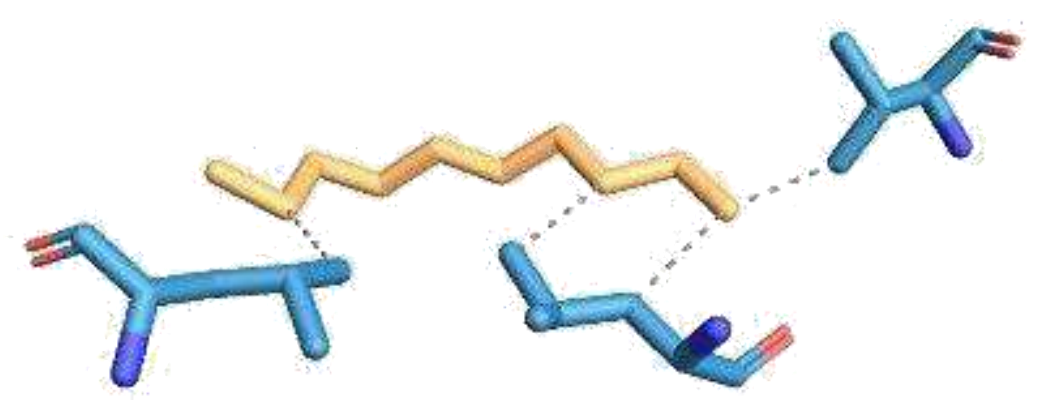

Roccustyrna binding site(s) in $\underline{2 C M E}$ (hypothetical protein 5).

\section{SMALLMOLECULE}

\section{$\underline{\text { D10 (decane) }}$}

\section{D10-B-1099}

Interacting chains: $\mathrm{A}, \mathrm{B}$

Figure5a. Roccustyrna binding site(s) inside the ( pdb:6lu7)binding domains. 02J:C:1 (02J) SMALLMOLECULE

Interacting $\operatorname{chain}(s): A$

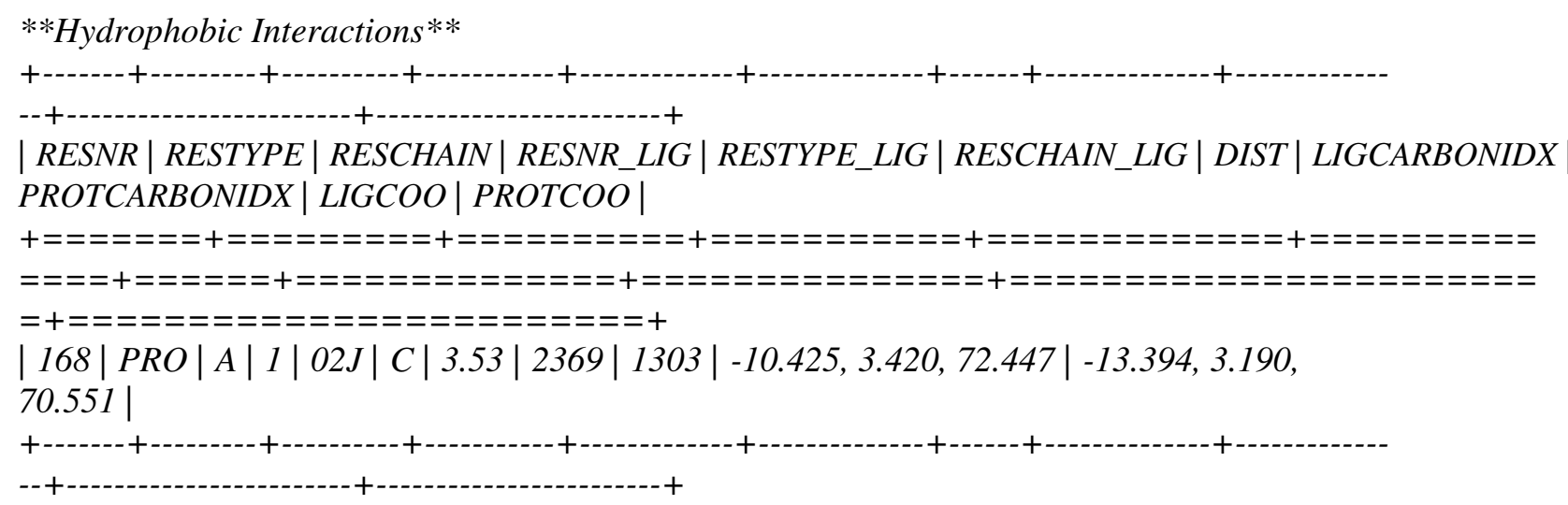

PJE:C:5 (PJE-010) - SMALLMOLECULE

$+010: C: 6$

Interacting chain( $s): A$

**Hydrophobic Interactions $* *$

$\mid$ RESNR $\mid$ RESTYPE $\mid$ RESCHAIN $\mid$ RESNR_LIG $\mid$ RESTYPE_LIG $\mid$ RESCHAIN_LIG $\mid$ DIST $\mid$ LIGCARBONIDX PROTCARBONIDX | LIGCOO $\mid$ PROTCOO $\mid$

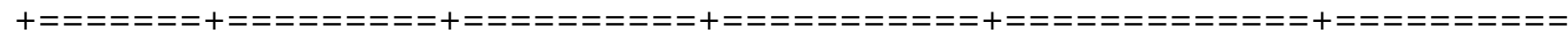

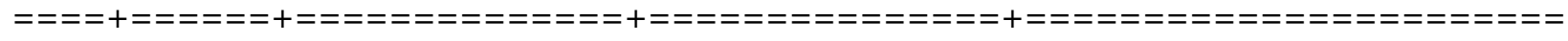

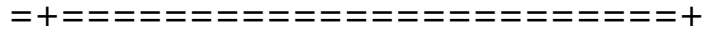

$|25| T H R|A| 6|010| C|3.73| 2415|179|-7.156,21.406,66.898 \mid-8.709,22.779,70.002$ 


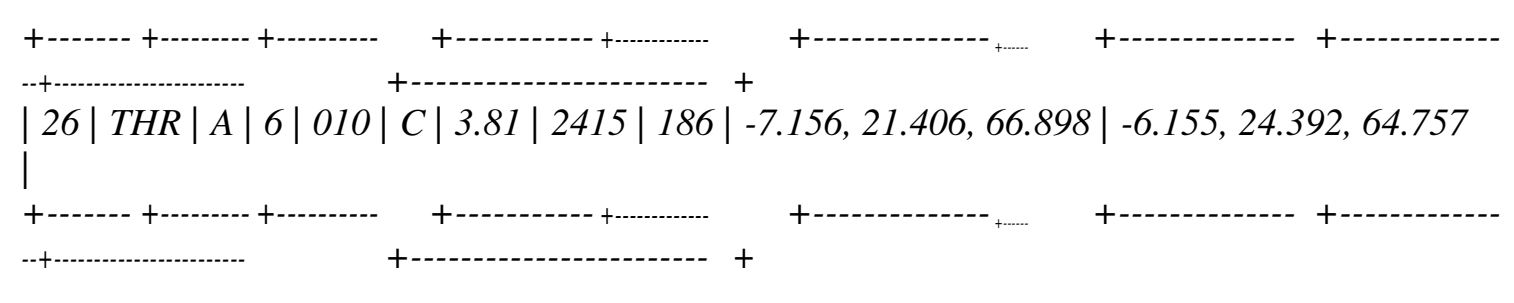

**Hydrogen Bonds**

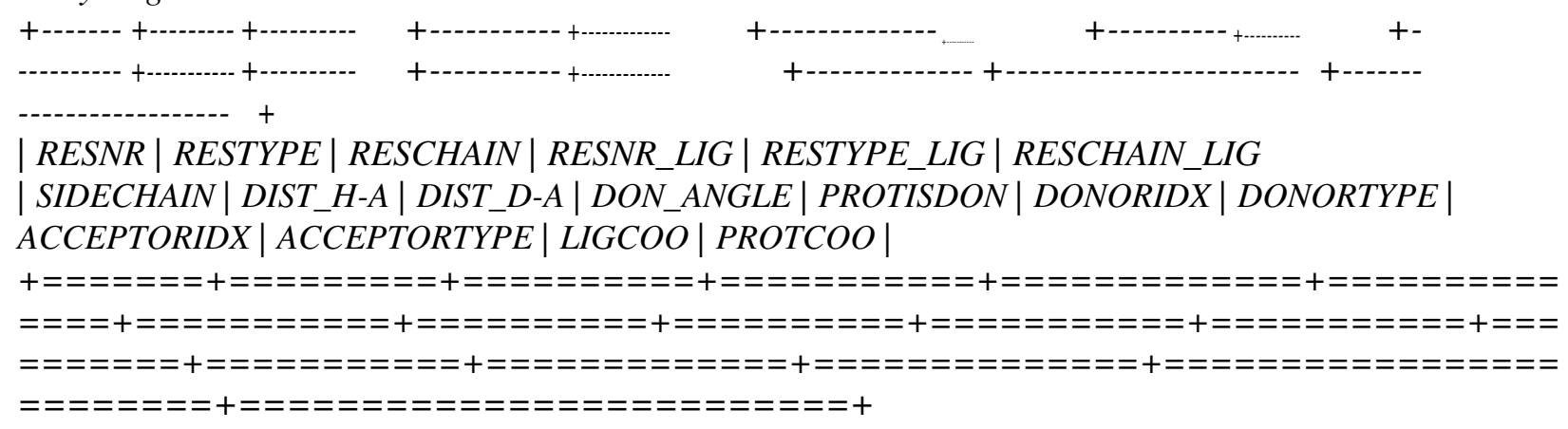

| $143 \mid$ GLY $|A| 6|010| C 1.93|2.80| 145.291105 \mid$ Nam | 2411| O3| -8.911, 17.849, $65.703|-8.918,17.918,62.905|$

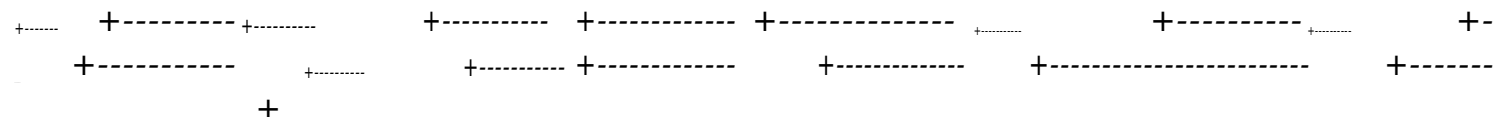

| $164 \mid$ HIS | A | 5 | PJE | C 2.16 | 3.07| 153.732408 |N3 | 1266 | O2 | -12.282, 14.994, $67.123|-15.161,15.336,68.144|$
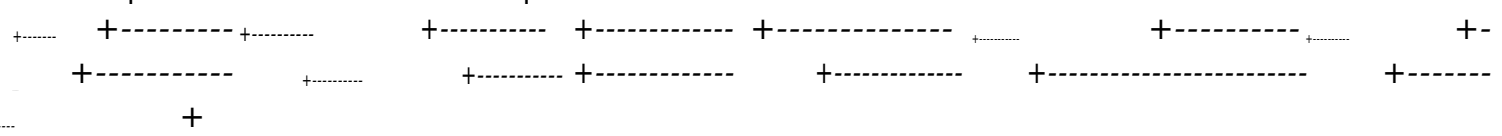

Metal Complexes

\begin{tabular}{|c|c|c|c|c|c|c|}
\hline Index & Residue & AA & Metal & Target & Distance & Location \\
\hline \multicolumn{7}{|c|}{ Complex 1: Zn, tetrahedral (4) } \\
\hline 1 & $50 A$ & CYS & 9316 & 386 & 2.18 & protein.sidechain \\
\hline 2 & $55 \mathrm{~A}$ & CYS & 9316 & 416 & 2.07 & protein.sidechain \\
\hline 3 & $72 \mathrm{~A}$ & CYS & 9316 & 549 & 1.90 & protein.sidechain \\
\hline
\end{tabular}




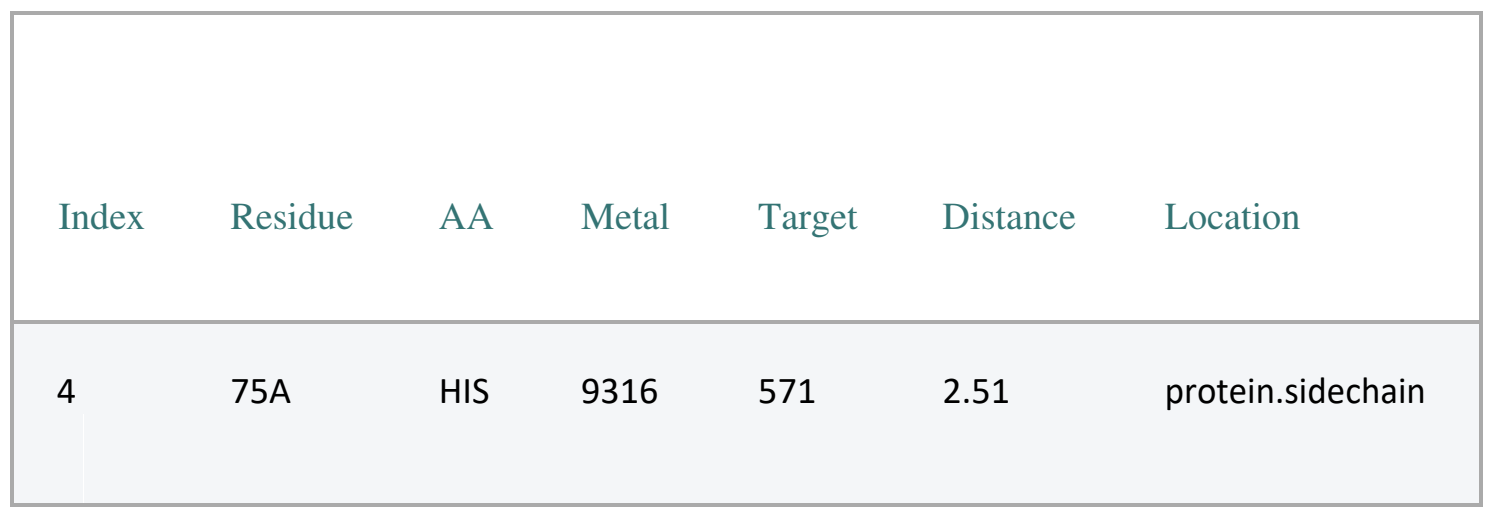

\section{$\underline{\text { Hydrophobic Interactions }}$}

\begin{tabular}{|c|c|c|c|c|c|}
\hline Index & Residue & $\mathrm{AA}$ & Distance & Ligand Atom & Protein Atom \\
\hline 1 & $53 \mathrm{~A}$ & LEU & 3.76 & 4787 & 254 \\
\hline 2 & $53 \mathrm{~B}$ & LEU & 3.87 & 4793 & 869 \\
\hline 3 & $53 \mathrm{~B}$ & LEU & 3.83 & 4795 & 866 \\
\hline 4 & $77 \mathrm{~B}$ & VAL & 3.62 & 4795 & 1054 \\
\hline
\end{tabular}




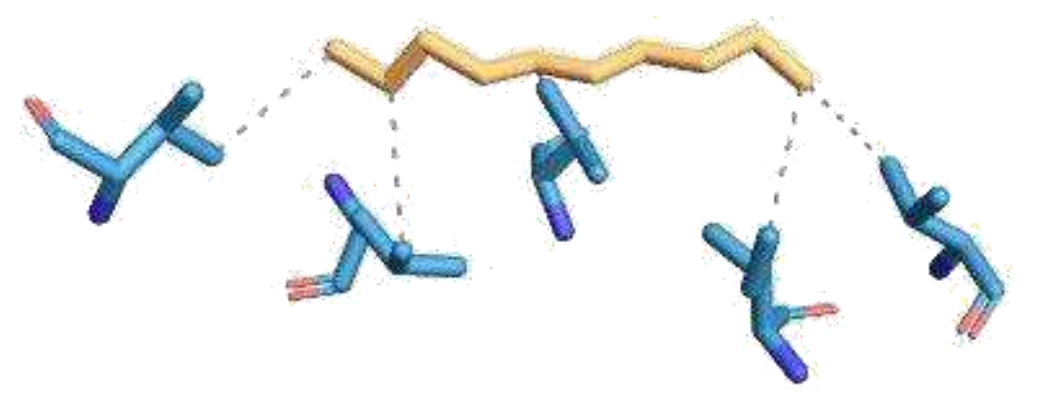

$\underline{\text { D10-C-1099 }}$

Interacting chains: $\mathrm{C}, \mathrm{D}$

Figure5b. Roccustyrna binding site(s) in ( pdb:6lu7)D10-C-1099 DMS:A:402 (DMS) -

SMALLMOLECULE

Interacting chain(s): A

**Hydrogen Bonds $* *$

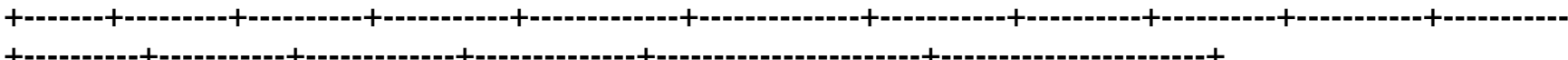

| RESNR | RESTYPE | RESCHAIN | RESNR_LIG | RESTYPE_LIG | RESCHAIN_LIG | SIDECHAIN | DIST_H-A | DIST_D-A | DON_ANGLE | PROTISDON | DONORIDX | DONORTYPE | ACCEPTORIDX | ACCEPTORTYPE | LIGCOO | PROTCOO

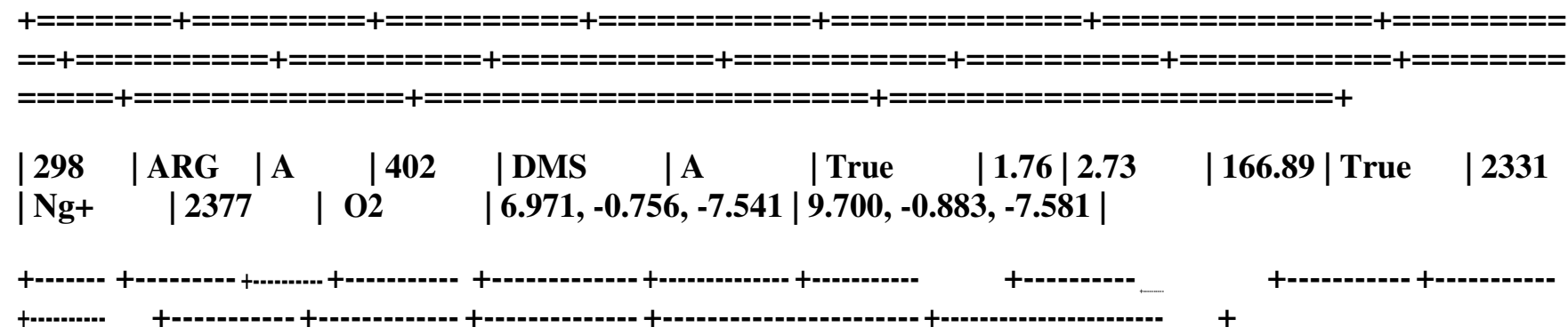

**Salt Bridges**

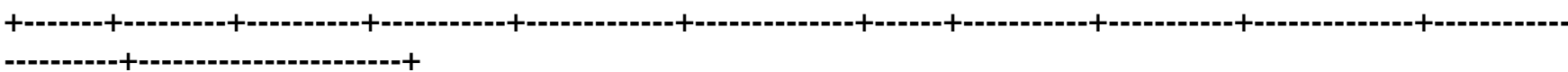

| RESNR | RESTYPE | RESCHAIN | RESNR_LIG | RESTYPE_LIG | RESCHAIN_LIG | DIST |

PROTISPOS | LIG_GROUP | LIG_IDX_LIST | LIGCOO | PROTCOO | 


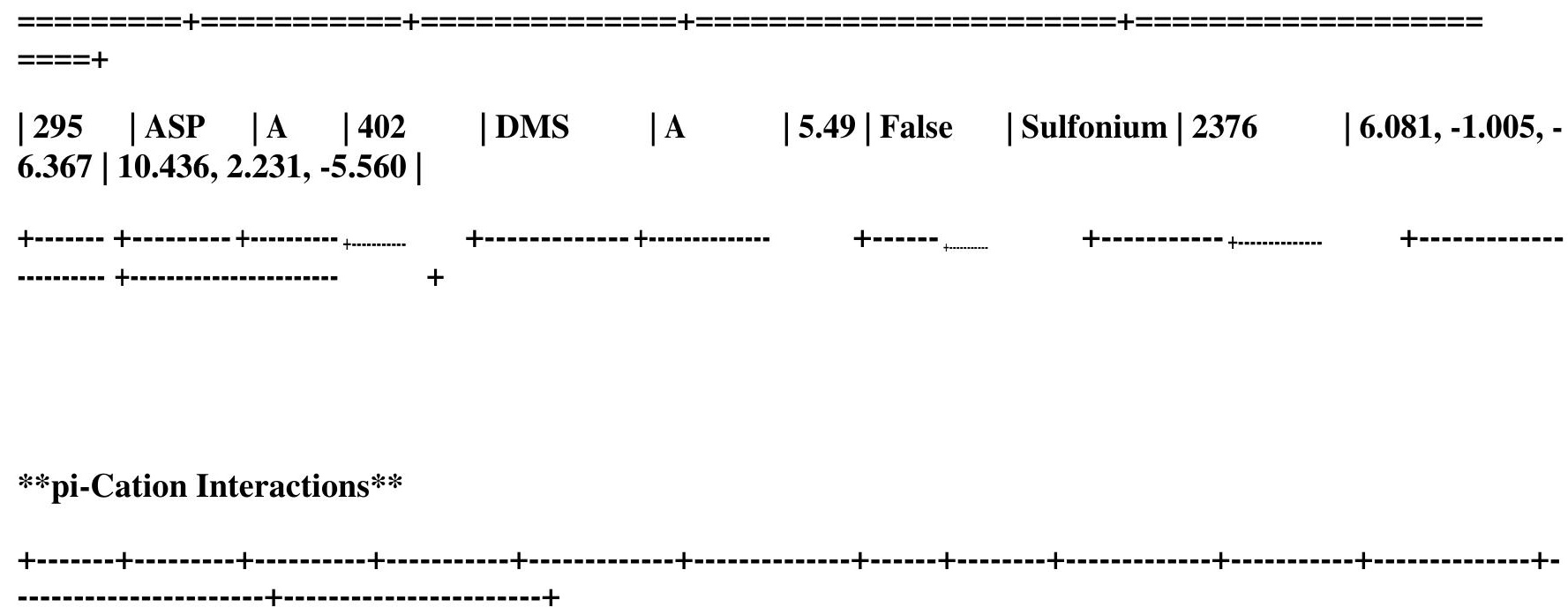

| RESNR | RESTYPE | RESCHAIN | RESNR_LIG | RESTYPE_LIG | RESCHAIN_LIG | DIST | OFFSET | PROTCHARGED | LIG_GROUP | LIG_IDX_LIST | LIGCOO | PROTCOO |

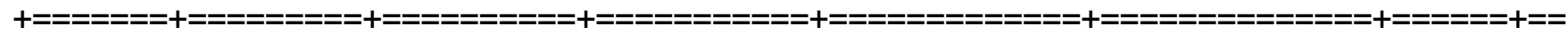

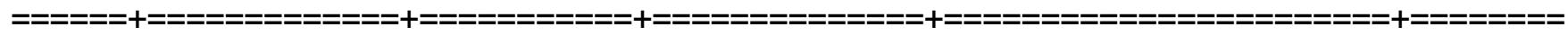

=ニニ=ニ=ニニ=ニ=ニ=ニ+

| 8 | PHE |A | |402 |DMS $\quad$ |A $\quad|4.70| 1.01 \mid$ False $\quad$ sulfonium|2376 $\quad$ 6.081, $1.005,-6.367|8.339,-4.556,-4.264|$
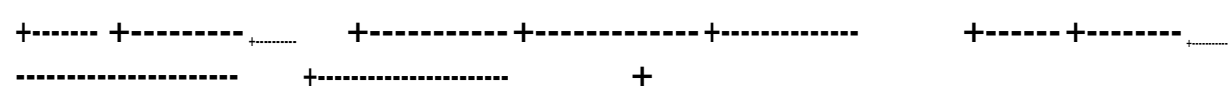

DMS:A:403 (DMS) - SMALLMOLECULE

Interacting chain(s):

RZG:A:404 (RZG) - SMALLMOLECULE

Interacting chain(s): A 


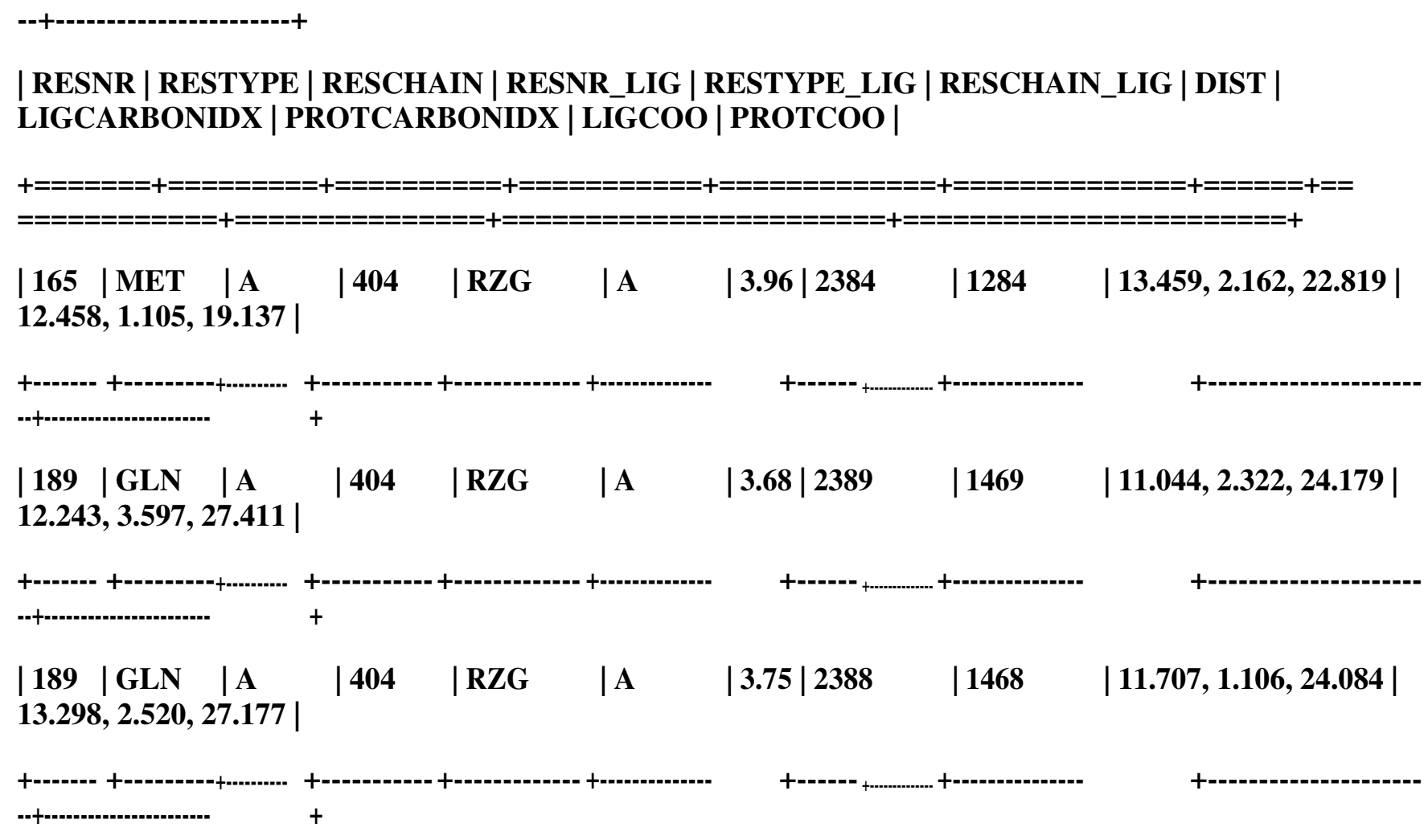

**Hydrogen Bonds**

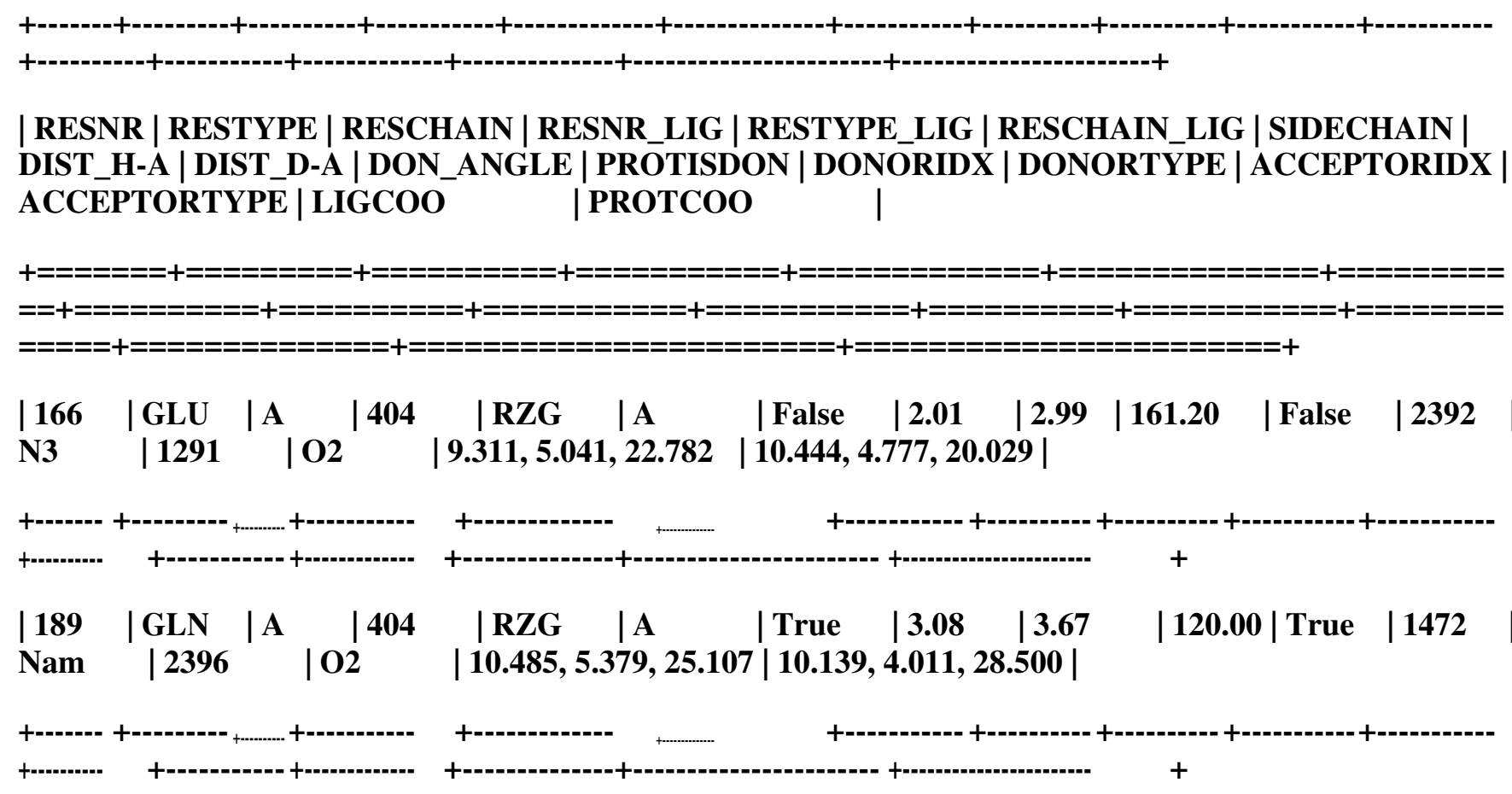


| RESNR | RESTYPE | RESCHAIN | RESNR_LIG | RESTYPE_LIG | RESCHAIN_LIG | DIST_A-W | DIST_D-W | DON_ANGLE | WATER_ANGLE | PROTISDON | DONOR_IDX | DONORTYPE | ACCEPTOR_IDX | ACCEPTORTYPE | WATER_IDX | LIGCOO WATERCOO

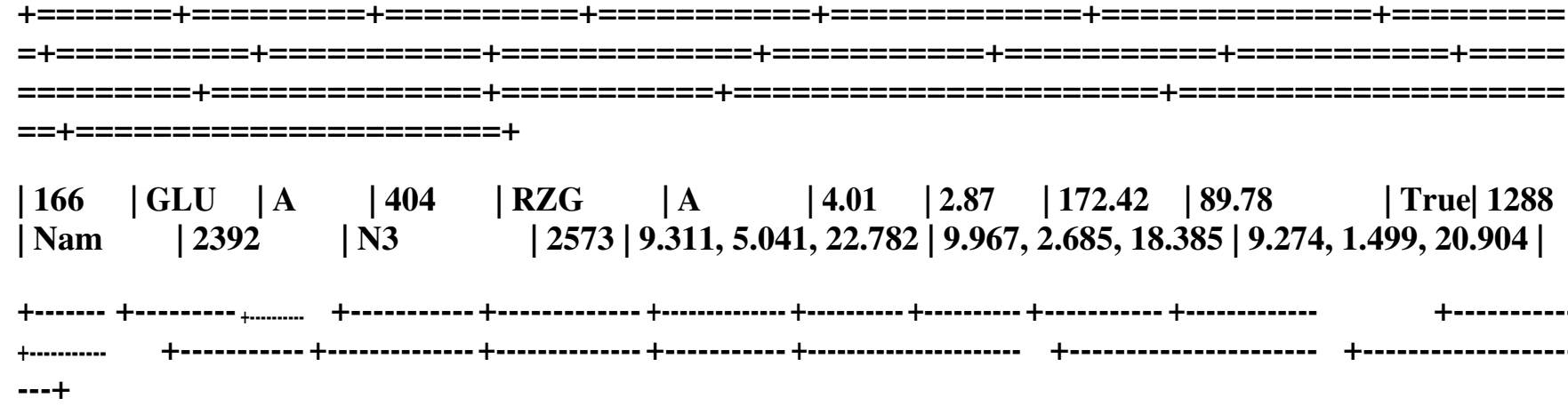

TH $\quad 3.81$

2415 


\begin{tabular}{|c|c|c|c|c|c|c|c|c|c|}
\hline I & $\begin{array}{l}\mathrm{R} \\
\mathrm{e}\end{array}$ & A & Dist & Dist & Don & Prote & Sid & Don & Acce \\
\hline $\mathrm{n}$ & $\mathrm{s}$ & A & ance & ance & or & in & ech & or & ptor \\
\hline d & $\begin{array}{l}\mathrm{i} \\
\mathrm{d}\end{array}$ & & $\mathrm{H}-\mathrm{A}$ & D-A & Ang & dono & ain & Ato & Ato \\
\hline $\mathrm{e}$ & $\begin{array}{l}\mathrm{u} \\
\mathrm{e}\end{array}$ & & & & le & $\mathrm{r} ?$ & & $\mathrm{~m}$ & $\mathrm{~m}$ \\
\hline $\mathrm{x}$ & & & & & & & & & \\
\hline 1 & $\begin{array}{l}14 \\
3 \\
\text { A }\end{array}$ & $\begin{array}{l}\mathrm{G} \\
\mathrm{L} \\
\mathrm{Y}\end{array}$ & 1.93 & 2.80 & $\begin{array}{l}145 . \\
29\end{array}$ & $\checkmark$ & $x$ & $\begin{array}{l}110 \\
5 \\
(\mathrm{Na} \\
\mathrm{m})\end{array}$ & $\begin{array}{l}24 \\
11 \\
(\mathrm{O} \\
3)\end{array}$ \\
\hline
\end{tabular}

\section{Hydrogen Bonds}

\begin{tabular}{|c|c|c|c|c|c|c|c|c|c|}
\hline Inde & Resid & A & Distance & Distance & Donor & Protein & Sidech & Donor & Acceptor \\
\hline $\mathrm{x}$ & ue & A & $\mathrm{H}-\mathrm{A}$ & D-A & Angle & donor? & ain & Atom & Atom \\
\hline 1 & $160 \mathrm{~A}$ & $\begin{array}{l}A R \\
G\end{array}$ & 2.25 & 3.23 & 171.34 & $\checkmark$ & $x$ & $\begin{array}{l}986 \\
{[\text { Nam] }}\end{array}$ & 2475 [03] \\
\hline 2 & $160 B$ & $\begin{array}{l}A R \\
G\end{array}$ & 2.15 & 3.13 & 176.63 & $\checkmark$ & $x$ & $\begin{array}{l}2216 \\
{[\text { Nam] }}\end{array}$ & 2471 [03] \\
\hline 3 & $161 \mathrm{~A}$ & $\begin{array}{l}\text { TY } \\
\mathrm{R}\end{array}$ & 2.32 & 3.19 & 148.19 & $\checkmark$ & $x$ & $\begin{array}{l}997 \\
{[\text { Nam] }}\end{array}$ & 2473 [03] \\
\hline 4 & $161 \mathrm{~A}$ & $\begin{array}{l}\text { TY } \\
\mathrm{R}\end{array}$ & 3.12 & 3.48 & 103.86 & $x$ & $x$ & $\begin{array}{l}2473 \\
{[03]}\end{array}$ & $1000[02]$ \\
\hline
\end{tabular}




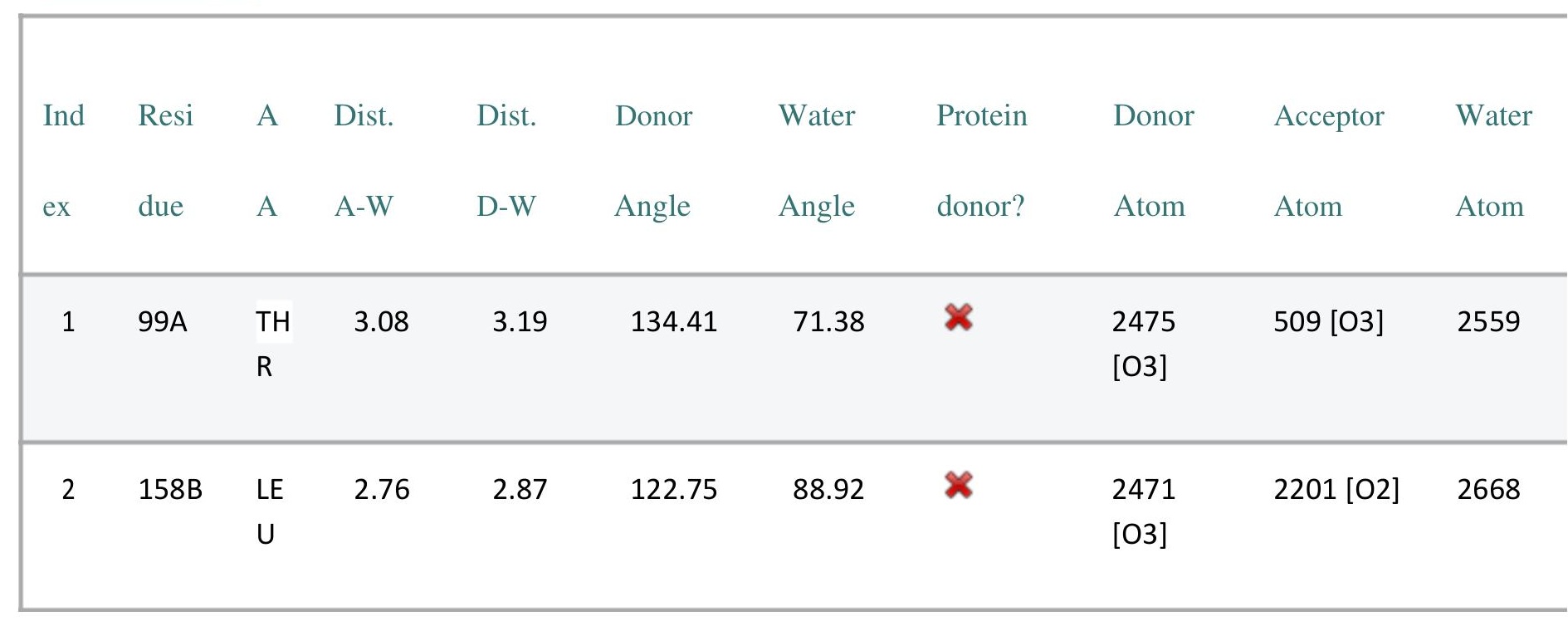


Hydrophobic Interactions

\begin{tabular}{|c|c|c|c|c|c|}
\hline Index & Residue & AA & Distance & Ligand Atom & Protein Atom \\
\hline 1 & $16 \mathrm{C}$ & VAL & 4.00 & 4796 & 1271 \\
\hline 2 & $45 \mathrm{C}$ & ILE & 3.83 & 4796 & 1400 \\
\hline 3 & $55 \mathrm{D}$ & LEU & 3.89 & 4804 & 2067 \\
\hline 4 & $77 \mathrm{D}$ & VAL & 3.74 & 4805 & 2238 \\
\hline
\end{tabular}


Index Residue AA Distance $\quad$ Ligand Atom Protein Atom

$95 \mathrm{C}$

VAL

3.96

4801

1787

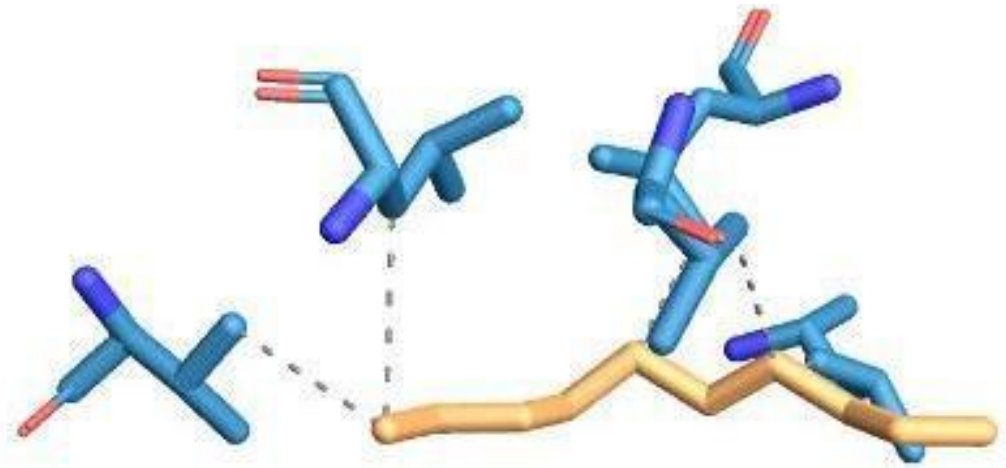

$\underline{\text { D10-F-1099 }}$

Interacting chains: $\mathrm{E}, \mathrm{F}$

Figure5c. Roccustyrna binding site(s) in ( pdb:6lu7)D10-F-1099. 2J:C:1 (02J) -

SMALLMOLECULE

Interacting chain(s): A

**Hydrophobic Interactions**

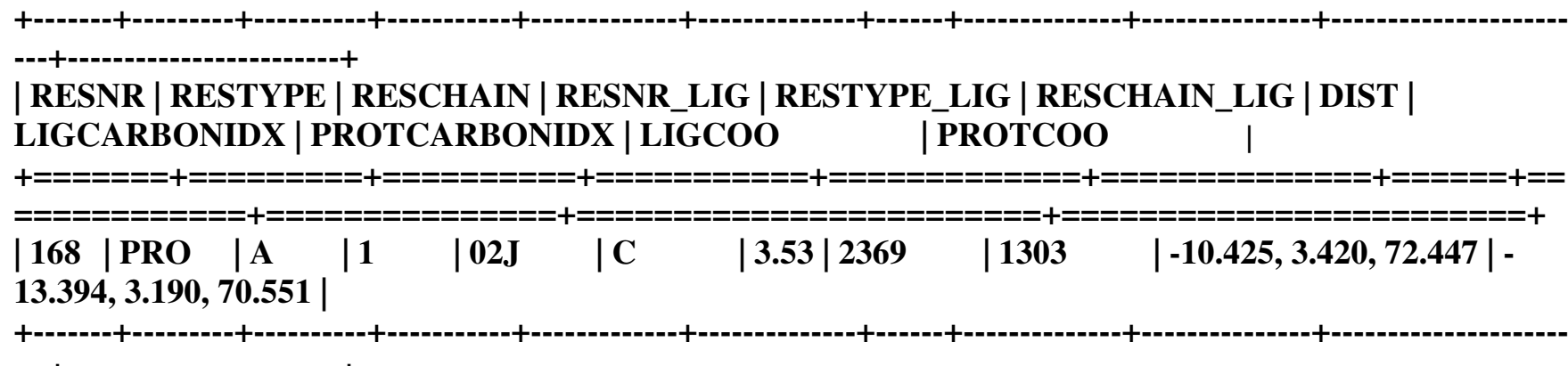


Interacting chain(s): A

**Hydrophobic Interactions**

| RESNR | RESTYPE | RESCHAIN | RESNR_LIG | RESTYPE_LIG | RESCHAIN_LIG | DIST | LIGCARBONIDX | PROTCARBONIDX | LIGCOO | PROTCOO

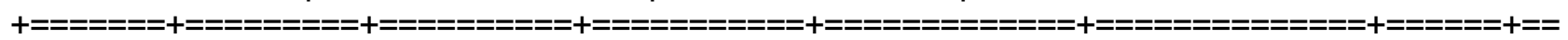

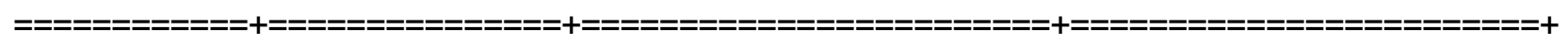
$\mid 25$ | THR $\mid$ A $|6 \quad| 010 \quad \mid$ C $\mid$ 3.73|2415 $|179 \quad|-7.156,21.406,66.898 \mid-$ 8.709, 22.779, 70.002 |

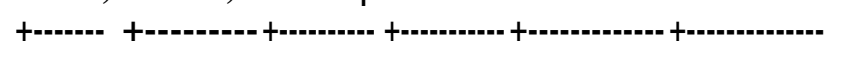

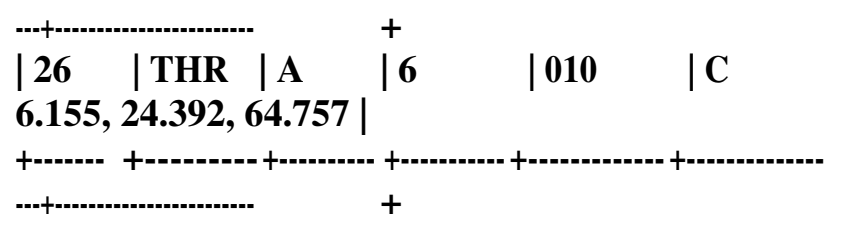

**Hydrogen Bonds**

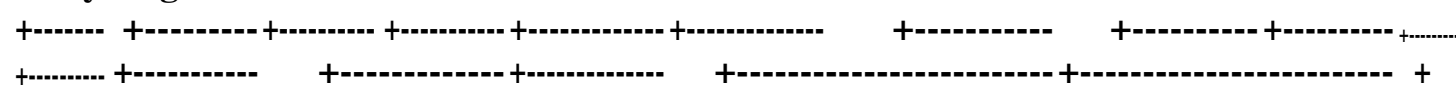

| RESNR | RESTYPE | RESCHAIN | RESNR_LIG | RESTYPE_LIG | RESCHAIN_LIG | SIDECHAIN DIST_H-A | DIST_D-A | DON_ANGLE | PROTISDON | DONORIDX | DONORTYPE | ACCEPTORIDX | ACCEPTORTYPE | LIGCOO | PROTCOO |

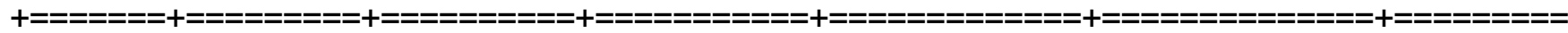

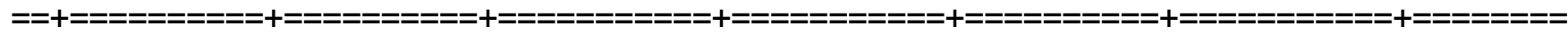

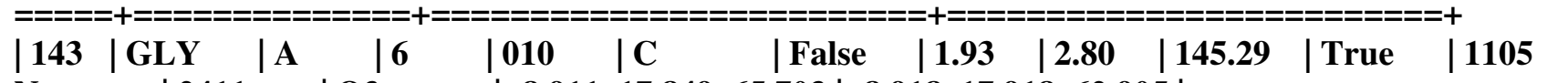

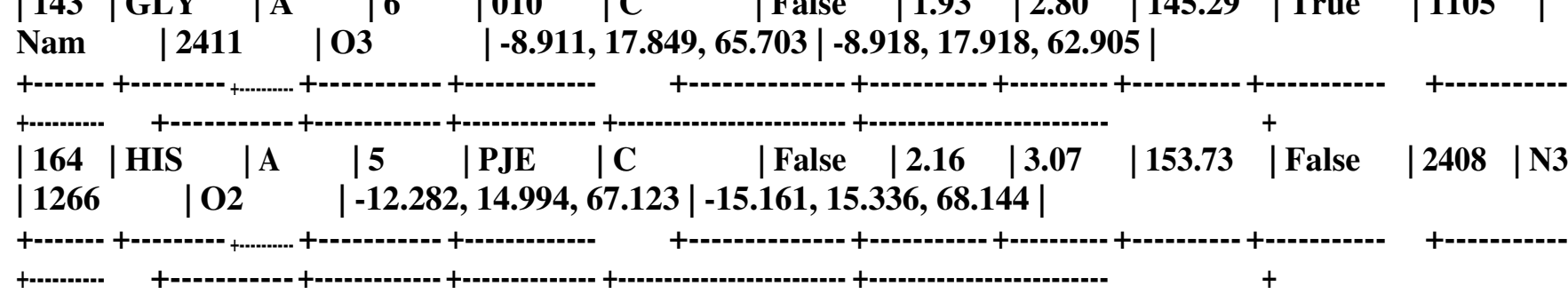




\section{$\underline{\text { Hydrophobic Interactions }}$}

\begin{tabular}{|c|c|c|c|c|c|}
\hline Index & Residue & $\mathrm{AA}$ & Distance & Ligand Atom & Protein Atom \\
\hline 1 & $22 \mathrm{E}$ & LEU & 3.94 & 4811 & 2510 \\
\hline 2 & $22 \mathrm{~F}$ & LEU & 3.98 & 4810 & 3102 \\
\hline 3 & $55 \mathrm{~F}$ & LEU & 3.85 & 4815 & 3255 \\
\hline 4 & $77 \mathrm{~F}$ & VAL & 3.70 & 4815 & 3429 \\
\hline 5 & $95 \mathrm{E}$ & VAL & 3.69 & 4809 & 2979 \\
\hline
\end{tabular}




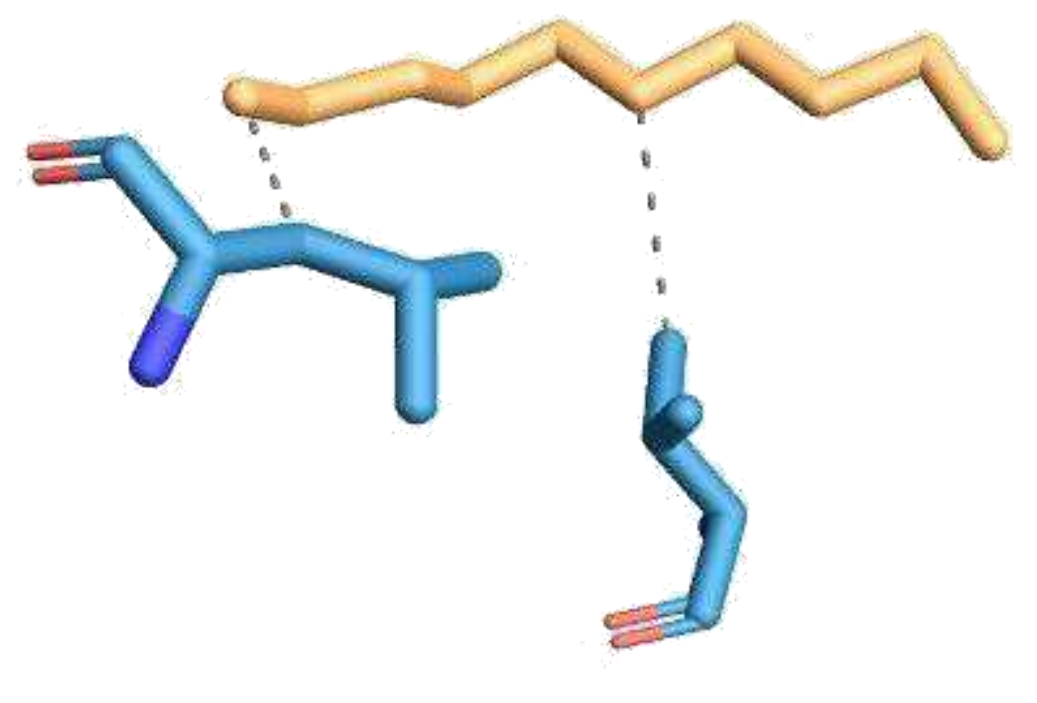

$\underline{\text { D10-H-1099 }}$

Interacting chains: $\mathrm{H}$

Figure5d. Roccustyrna binding site(s) in ( pdb:6lu7)D10-H-1099. X77:A:401 (X77) SMALLMOLECULE

Interacting chain(s): A

**Hydrophobic Interactions**

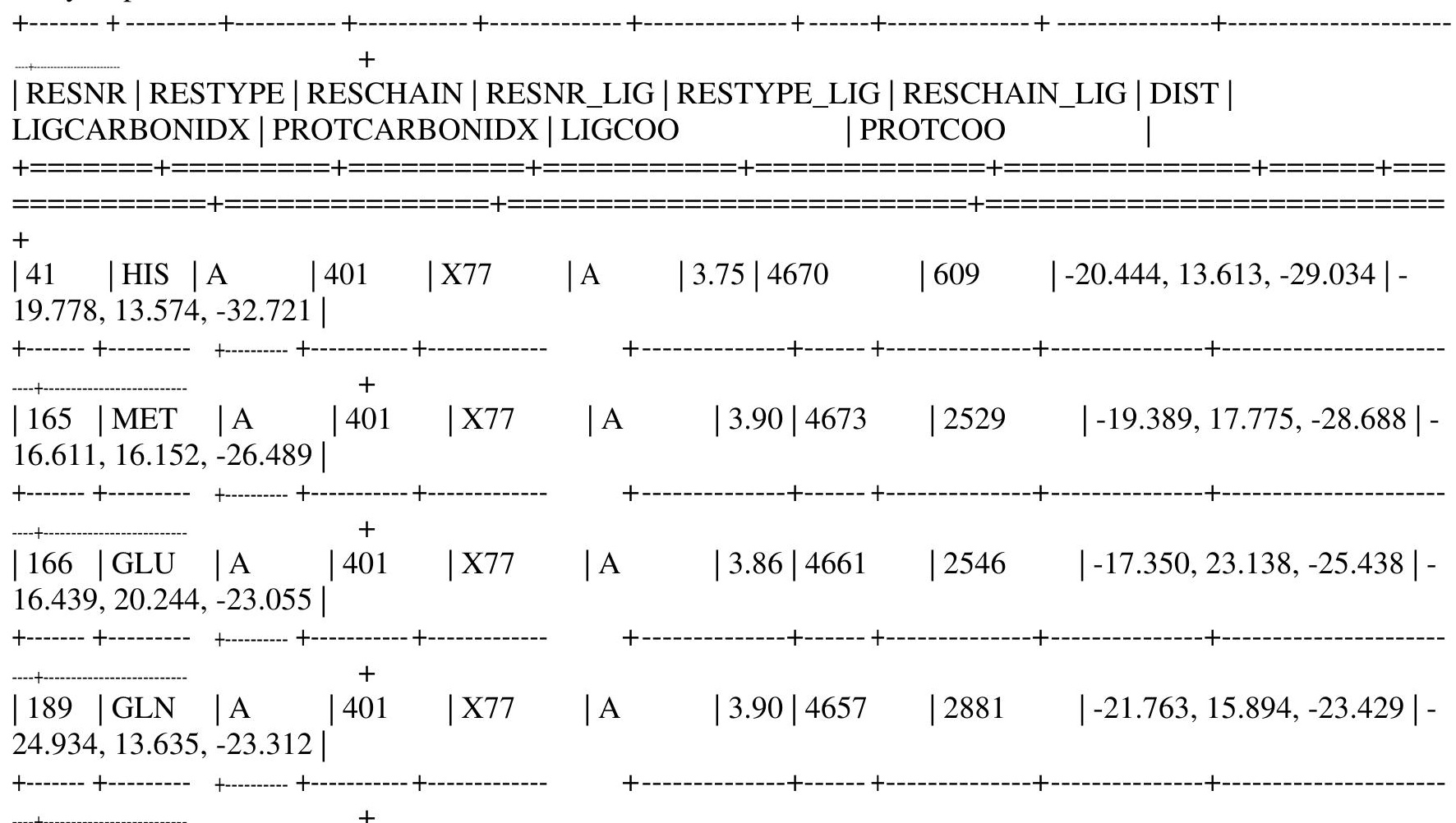




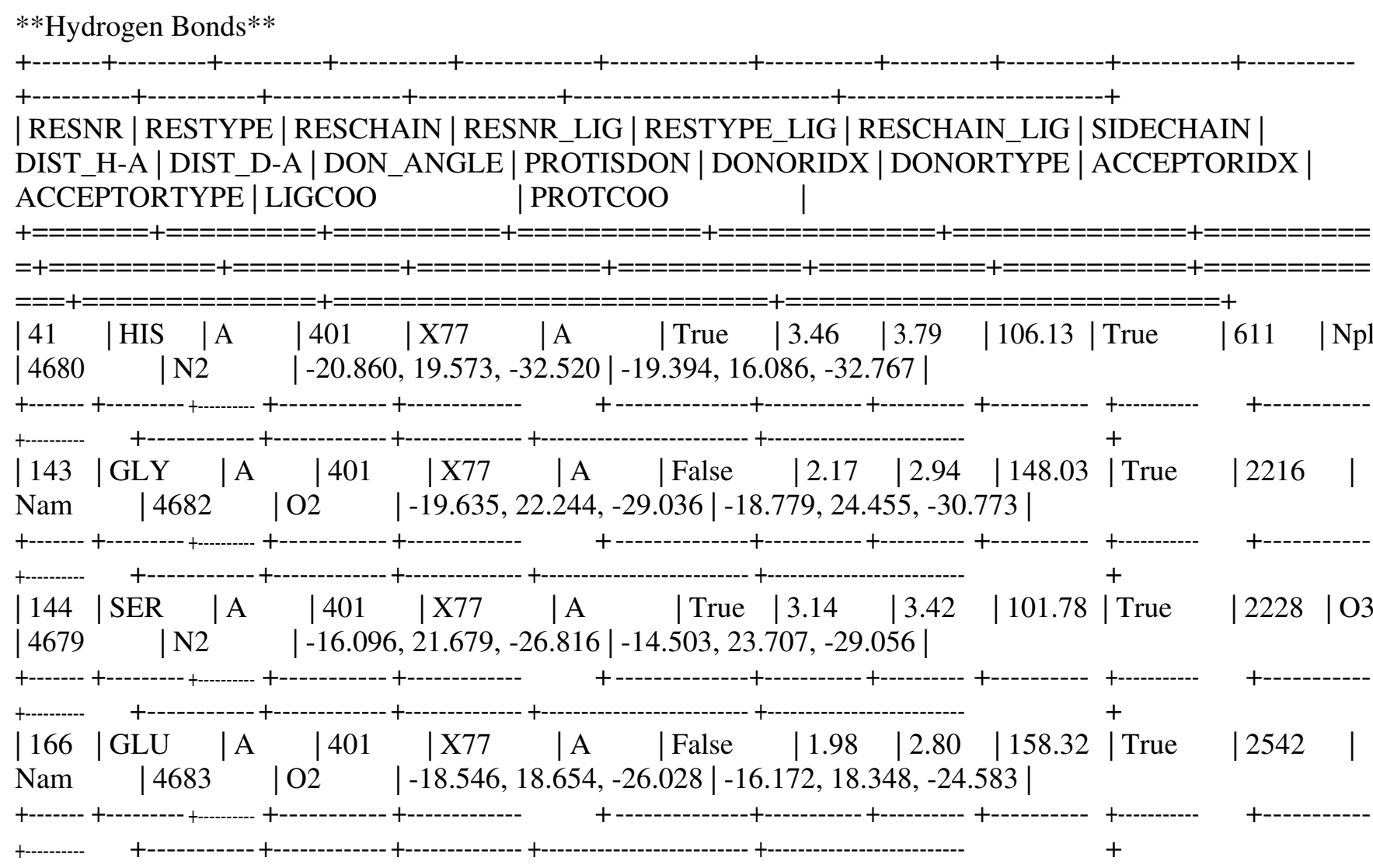




\begin{tabular}{|c|c|c|c|c|c|}
\hline Inde & Residu & AA & Distanc & Ligand & Protein \\
\hline $\mathrm{x}$ & $\mathrm{e}$ & & e & Atom & Atom \\
\hline \multirow[t]{2}{*}{1} & $53 \mathrm{H}$ & $\begin{array}{l}\mathrm{L} \\
\mathrm{E}\end{array}$ & 3.42 & 4825 & 4432 \\
\hline & & $\mathrm{U}$ & & & \\
\hline \multirow[t]{2}{*}{2} & $95 \mathrm{H}$ & $\begin{array}{l}\mathrm{V} \\
\mathrm{A}\end{array}$ & 3.89 & 4820 & 4762 \\
\hline & & $\mathrm{L}$ & & & \\
\hline
\end{tabular}

Prediction of noncovalent interactions for PDB structure 2CME

Created on 2020/11/04 usining PLIP v1.4.4

If you are usining PLIP in your work, please cite:

Salentin,S. et al. PLIP: fully automated protein-ligand interaction profiler. Nucl. Acids Res. (1 July 2015) 43 (W1): W443-W447. doi: 10.1093/nar/gkv315

D10:B:1099 (D10) - SMALLMOLECULE

Interacting chain(s): A,B

**Hydrophobic Interactions**

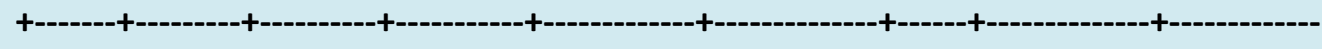

| RESNR | RESTYPE | RESCHAIN | RESNR_LIG | RESTYPE_LIG | RESCHAIN_LIG | DIST | LIGCARBONIDX | PROTCARBONIDX | LIGCOO | PROTCOO |

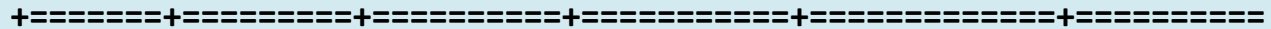

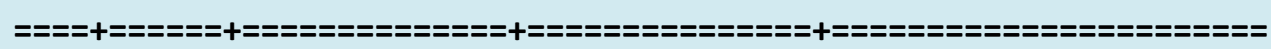

| 53 | LEU | A | 1099 | D10 | B | 3.76 | 4787 | 254 | 48.955, 23.619, 16.401 | 47.080, 24.675, 13.313

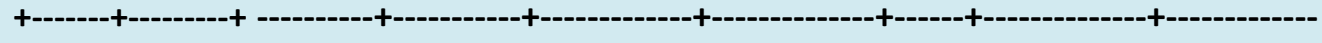

| 53 | LEU | B | 1099 | D10 | B | 3.87 | 4793 | 869 | 52.046, 20.761, 11.577 | 49.032, 23.137, 


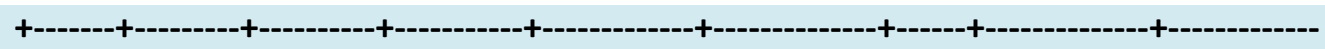

| 53 | LEU | B | 1099 | D10 | B | 3.83 | 4795 | 866 | 52.630, 20.477, 9.083 | 50.000, 23.248,

\section{Metal Complexes}

\begin{tabular}{|c|c|c|c|c|c|c|}
\hline Index & Residue & AA & Metal & Target & Distance & Location \\
\hline \multicolumn{7}{|c|}{ Complex 1: Zn, tetrahedral (4) } \\
\hline 1 & $39 B$ & CYS & 2498 & 1290 & 2.35 & protein.sidechain \\
\hline 2 & $57 \mathrm{~B}$ & CYS & 2498 & 1432 & 2.45 & protein.sidechain \\
\hline 3 & $59 B$ & CYS & 2498 & 1445 & 2.29 & protein.sidechain \\
\hline 4 & $80 B$ & CYS & 2498 & 1595 & 2.18 & protein.sidechain \\
\hline
\end{tabular}




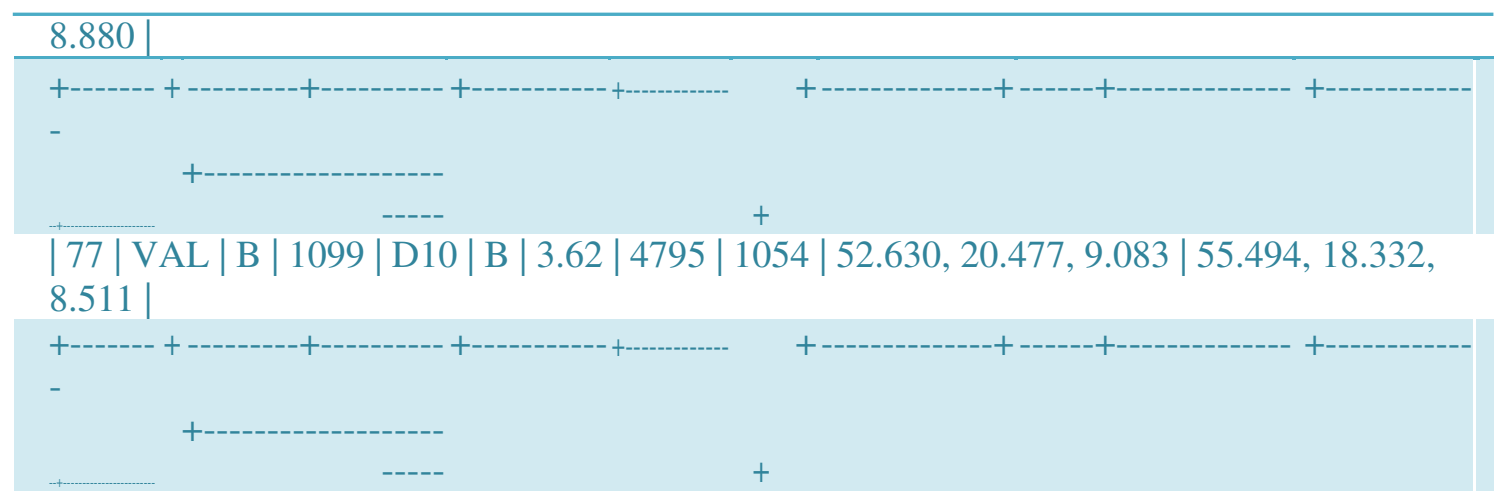

\section{D10:C:1099 (D10) - SMALLMOLECULE}

Interacting chain(s): C,D

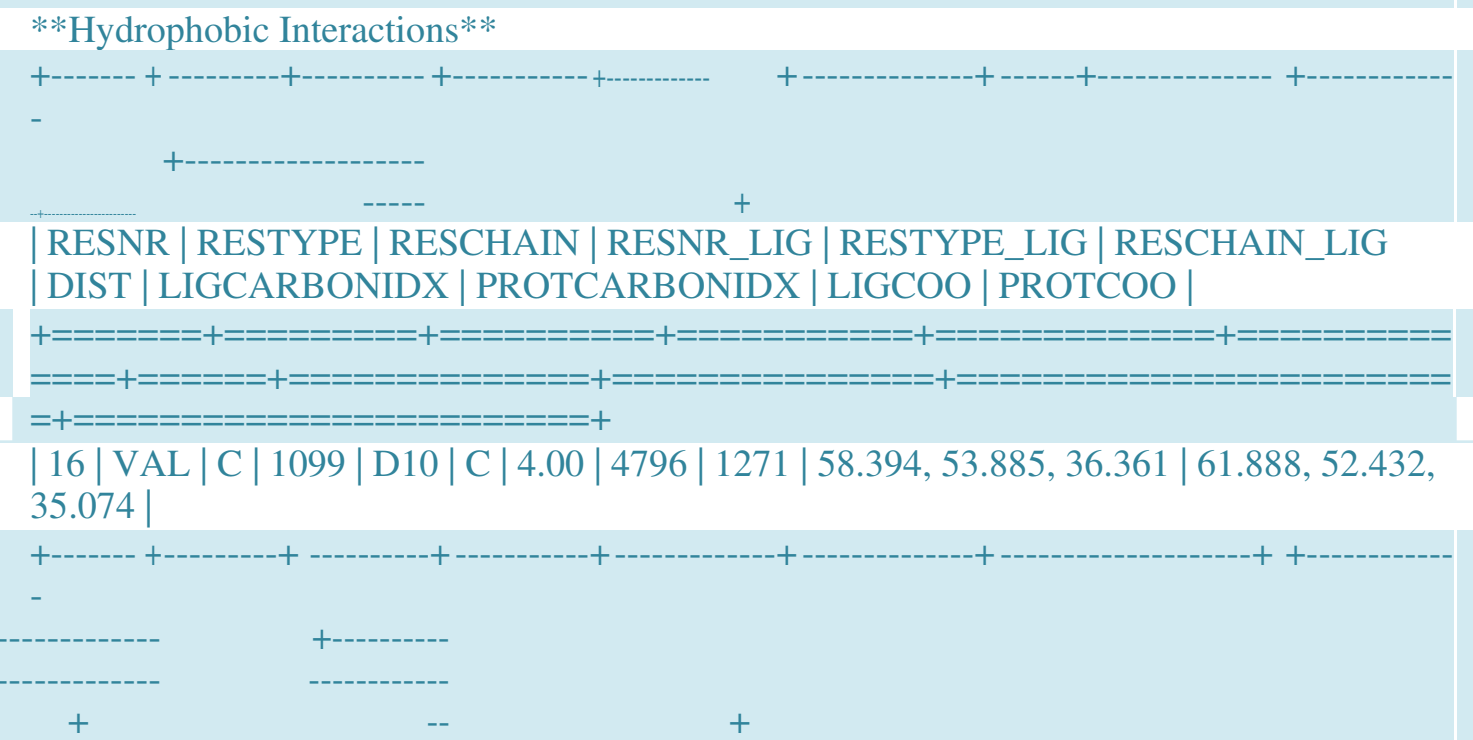

| 45 | ILE | C | 1099 | D10 | C | 3.83 | 4796 | 1400 | 58.394, 53.885, 36.361 | 60.025, 53.415, 39.796

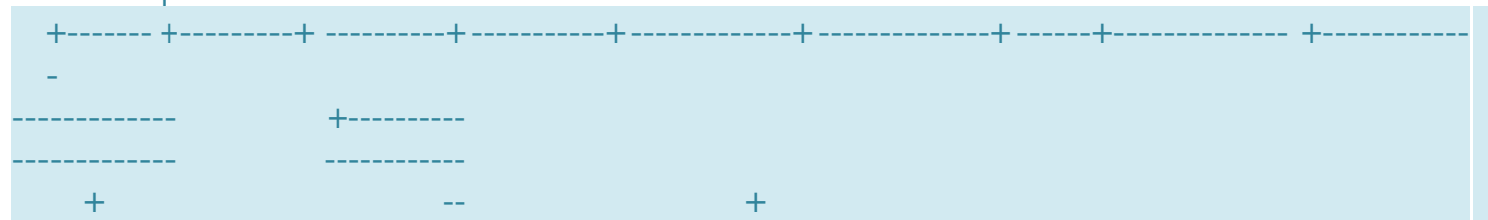

| 55 | LEU | D | 1099 | D10 | C | 3.89 | 4804 | 2067 | 52.052, 48.749, 41.009 | 55.000, 47.466, 43.193

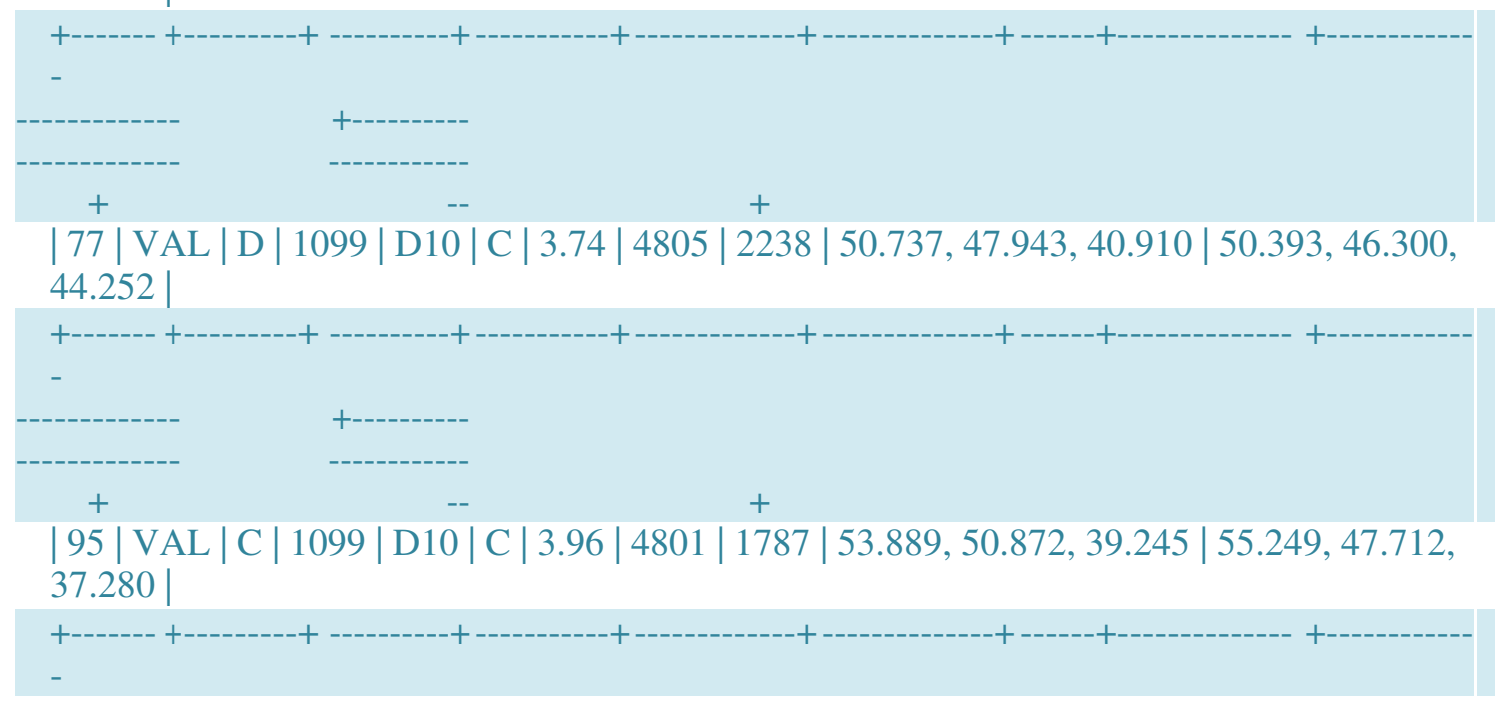




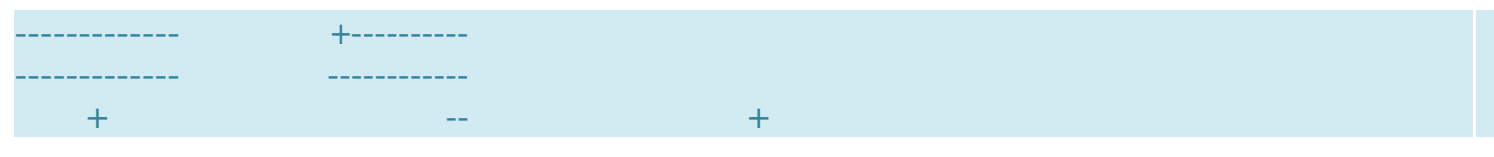

D10:F:1099 (D10) - SMALLMOLECULE

Interacting chain(s): E,F 


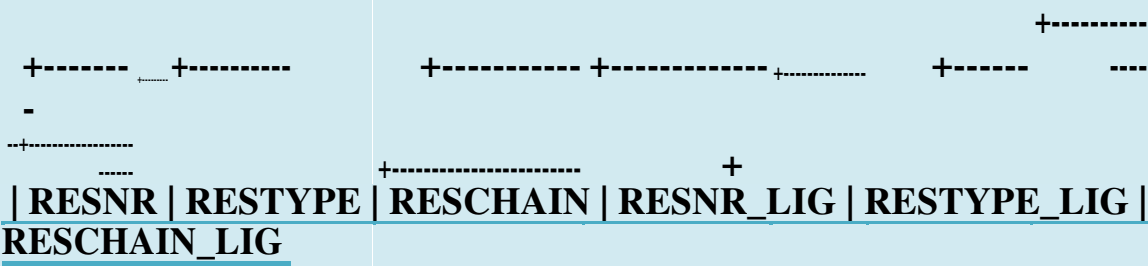

$\mathrm{ZN}: \mathrm{A}: 701(\mathrm{ZN})$ - ION

Interacting chain(s): A

**Metal Complexes**

| RESNR | RESTYPE | RESCHAIN | RESNR_LIG | RESTYPE_LIG | RESCHAIN_LIG | METAL_IDX |

METAL_TYPE | TARGET_IDX | TARGET_TYPE | COORDINATION | DIST | LOCATION

GEOMETRY | COMPLEXNUM | METALCOO | TARGETCOO

\section{$+=$}

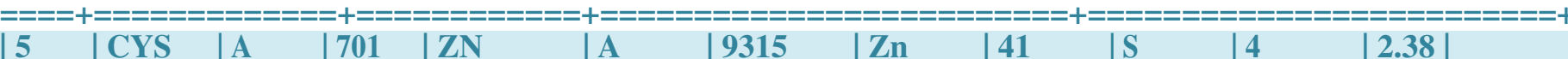

protein.sidechain $\mid 18.83$ | tetrahedral $|1 \quad| 434.311,16.825,92.518|434.537,18.307,90.668|$

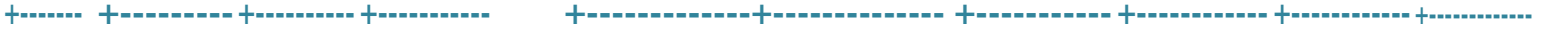

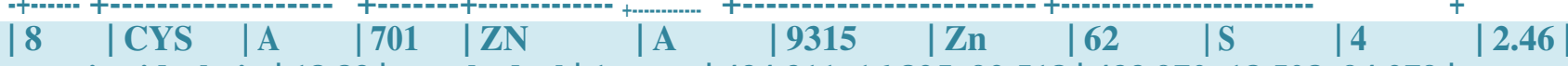

protein.sidechain | 18.83 | tetrahedral | $1 \quad$ | 434.311, 16.825, 92.518|433.970, 18.508, 94.279 |

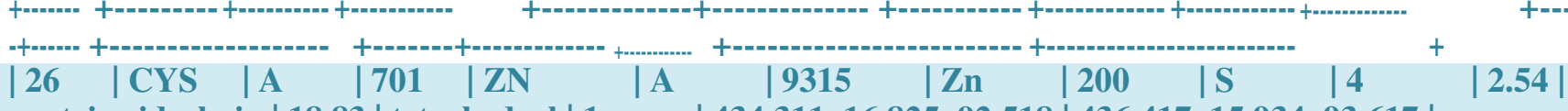

protein.sidechain $|18.83|$ tetrahedral $|1 \quad| 434.311,16.825,92.518|436.417,15.934,93.617|$

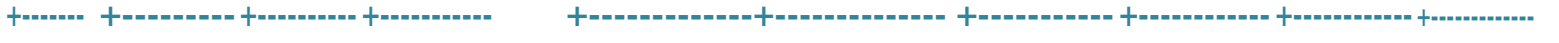

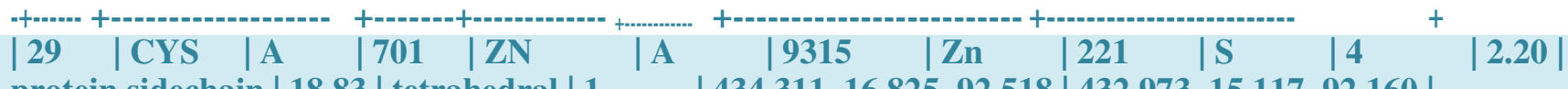

protein.sidechain | 18.83 | tetrahedral | $1 \quad|434.311,16.825,92.518| 432.973,15.117,92.160 \mid$

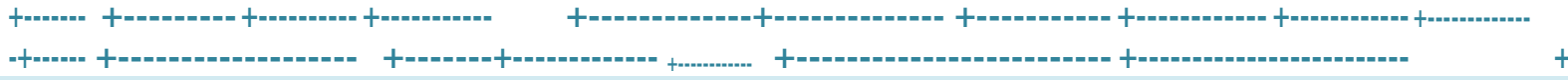

$\mathrm{ZN}: \mathrm{A}: 702(\mathrm{ZN})$ - ION

Interacting chain(s): A

***Metal Complexes**

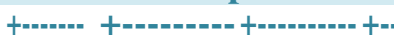

| RESNR | RESTYPE | RESCHAIN | RESNR_LIG | RESTYPE_LIG | RESCHAIN_LIG | METAL_IDX |

METAL_TYPE | TARGET_IDX | TARGET_TYPE | COORDINATION | DIST | LOCATION

|RMS |

GEOMETRY | COMPLEXÑUM | METALCOO

| TARGETCOO

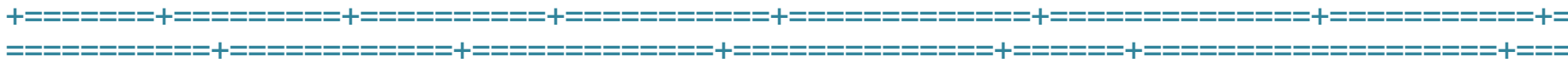

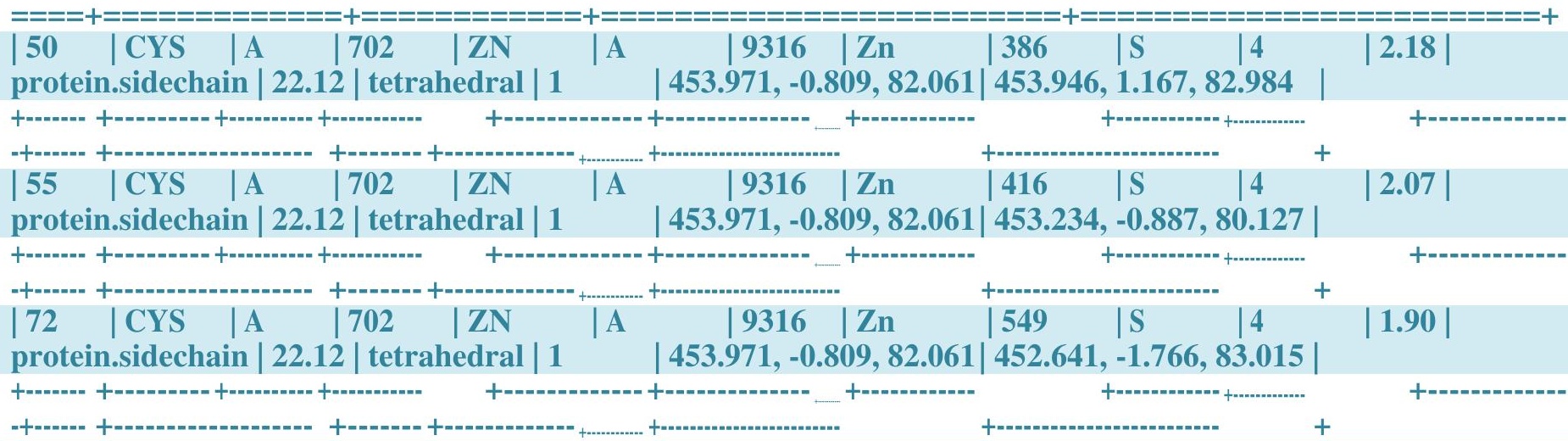




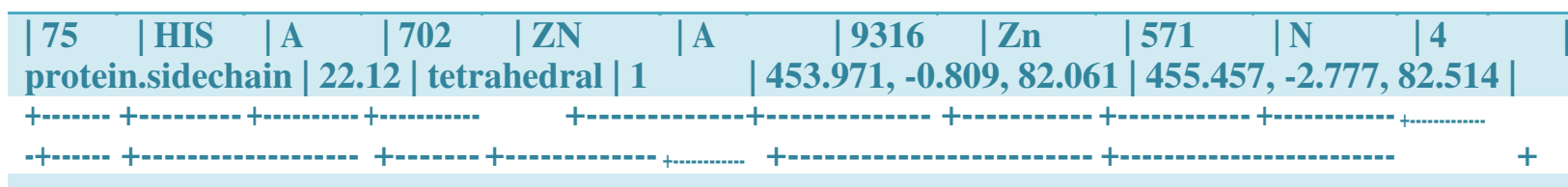

\begin{tabular}{l} 
ZN:A:703 (ZN) - ION \\
\hline Interacting chain(s): A
\end{tabular}

***atal Complexes**

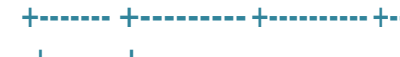

| RESNR | RESTYPE | RESCHAIN | RESNR_LIG | RESTYPE_LIG | RESCHAIN_LIG | METAL_IDX | METAL_TYPE | TARGET_IDX | TARGET_TYPE | COORDINATION | DIST | LOCATION |RMS | GEOMETRY | COMPLEXNUM | METALCOO | TARGETCOO

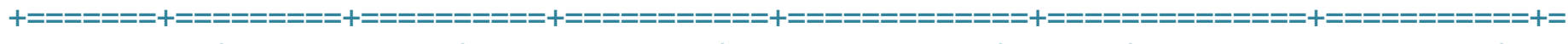

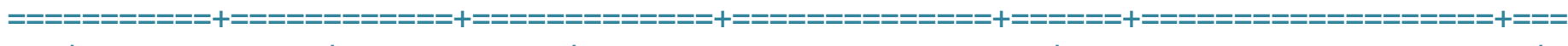

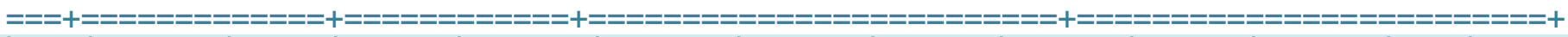

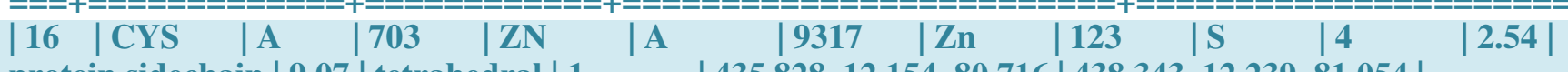
protein.sidechain $|9.07|$ tetrahedral | $1 \quad|435.828,12.154,80.716| 438.343,12.239,81.054 \mid$

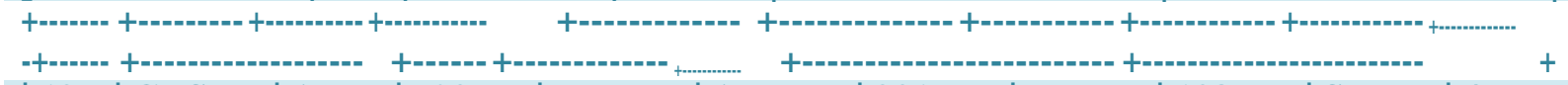

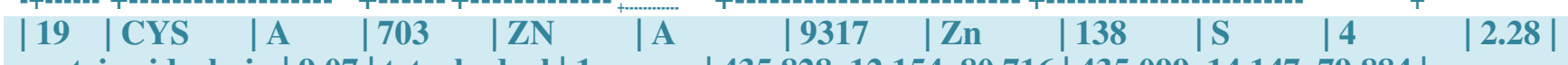
protein.sidechain | 9.07| tetrahedral | $1 \quad$ |435.828, 12.154, 80.716|435.099, 14.147, 79.884|

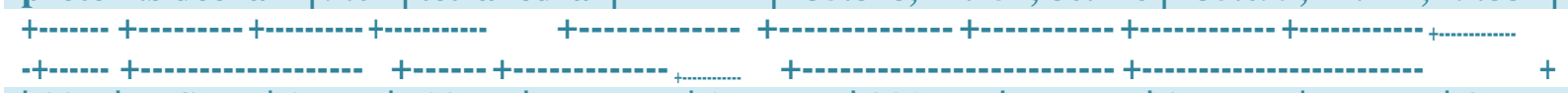

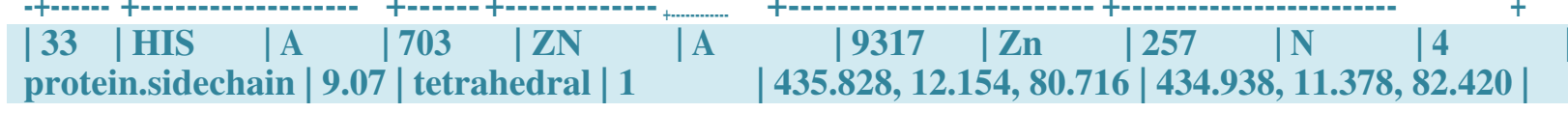

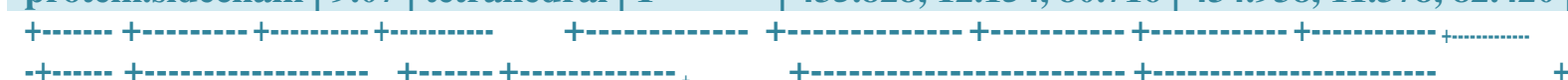

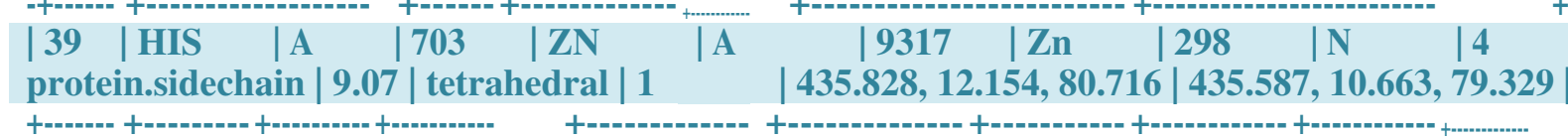
$+-\cdot$ | $2.05 \mid$

ZN:B:701 (ZN) - ION

Interacting chain(s): B

**Metal Complexes**

+------ +--------- +---------.+-

| RESNR | RESTYPE | RESCHAIN | RESNR_LIG | RESTYPE_LIG | RESCHAIN_LIG | METAL_IDX | METAL_TYPE | TARGET_IDX | TARGET_TYPE | COORDINATION | DIST | LOCATION |RMS | GEOMETRY | COMPLEXNUM | METALCOO | TARGETCOO

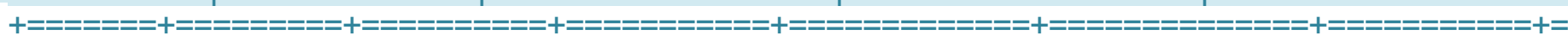

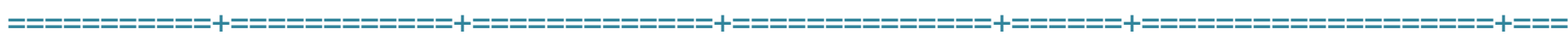

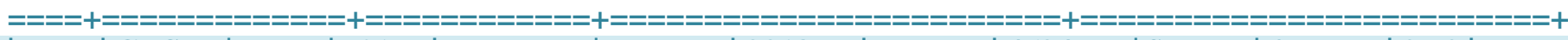
\begin{tabular}{|l|l|l|l|l|l|l|l|l|l|l|l|}
5 & CYS & B & 701 & ZN & B & 9318 & Zn & 4694 & S & |4 & | 2.53 |
\end{tabular} protein.sidechain | 22.85| tetrahedral | $1 \quad$ | 442.651, 0.750, 57.290| 442.223, -0.879, 59.178 |

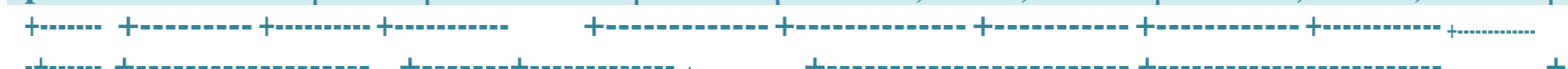
| 8 | CYS | B |701 | ZN $\mid$ B protein.sidechain | 22.85 | tetrahedral | $1 \quad$ | 442.651, 0.750, 57.290| 443.030, -1.102, 55.789|

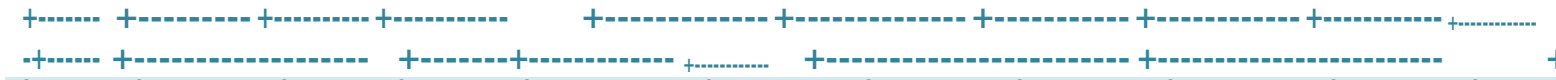

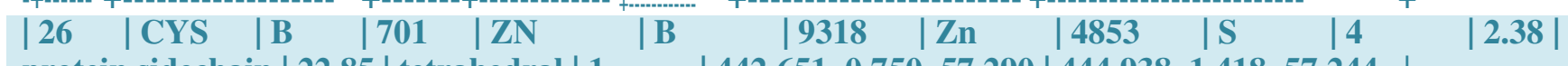
protein.sidechain | 22.85 | tetrahedral | $1 \quad|442.651,0.750,57.290| 444.938,1.418,57.244 \mid$ 


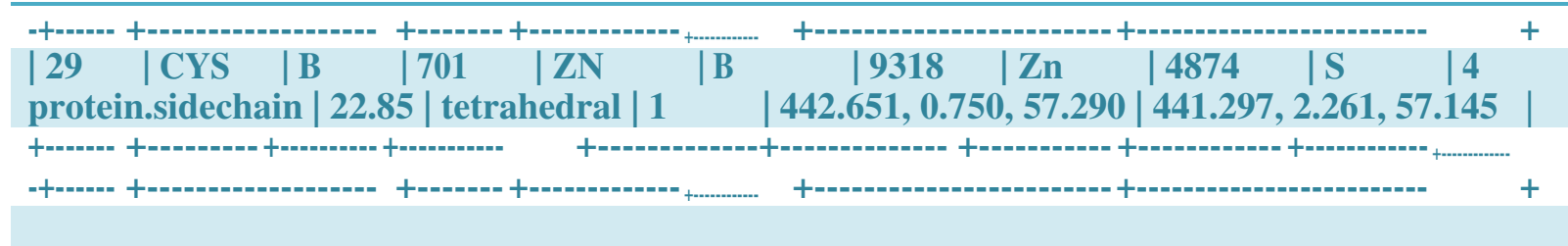

\begin{tabular}{l} 
ZN:B:702 (ZN) - ION \\
\hline Interacting chain(s): B
\end{tabular}

***Metal Complexes**

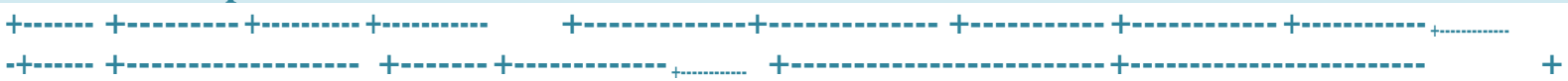

| RESNR | RESTYPE | RESCHAIN | RESNR_LIG | RESTYPE_LIG | RESCHAIN_LIG | METAL_IDX | METAL_TYPE | TARGET_IDX | TARGET_TYPE | COORDINATION | DIST | LOCATION |RMS | GEOMETRY | COMPLEXNUM | METALCOO | TARGETCOO

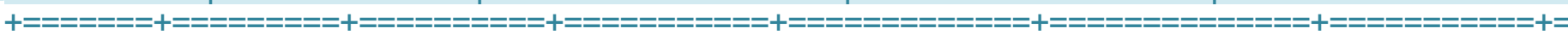

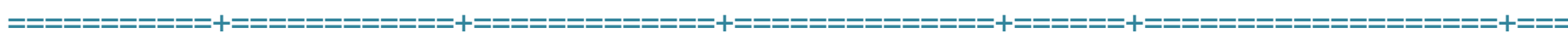

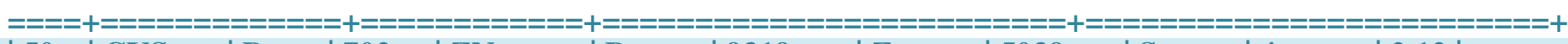

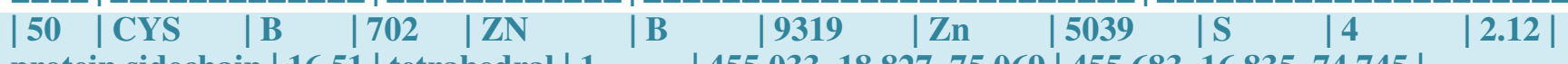
protein.sidechain | 16.51 | tetrahedral | $1 \quad$ | 455.033, 18.827, 75.069| 455.683, 16.835, 74.745 |

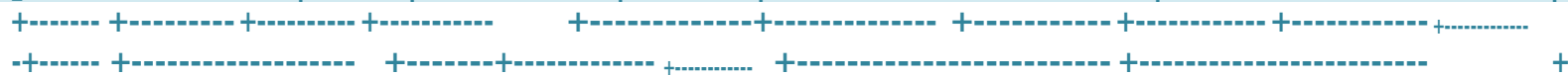

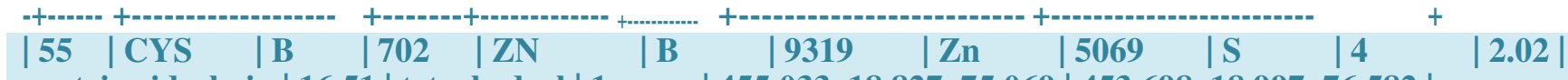
protein.sidechain | 16.51 | tetrahedral | $1 \quad$ | 455.033, 18.827, 75.069|453.698, 18.997, 76.582 |

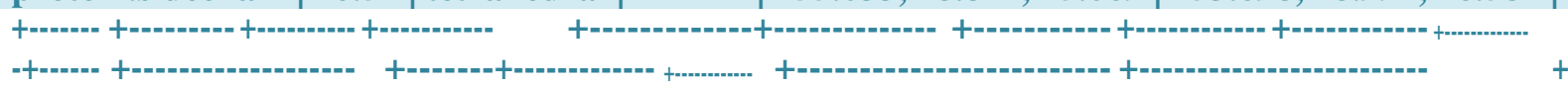

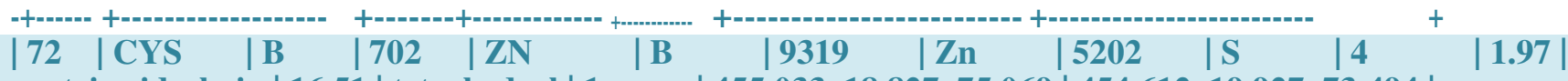
protein.sidechain | 16.51 | tetrahedral | $1 \quad$ | 455.033, 18.827, 75.069|454.612, 19.927, 73.494|

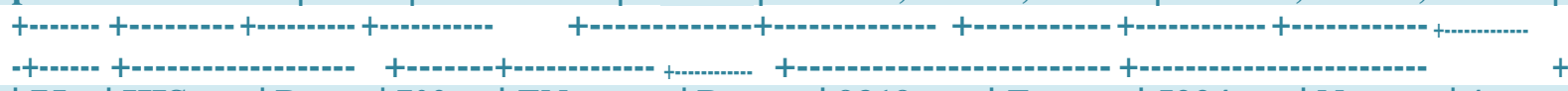

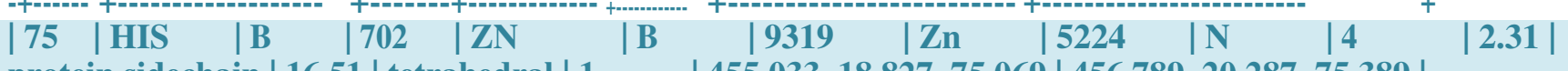
protein.sidechain | 16.51 | tetrahedral | $1 \quad|455.033,18.827,75.069| 456.789,20.287,75.389 \mid$

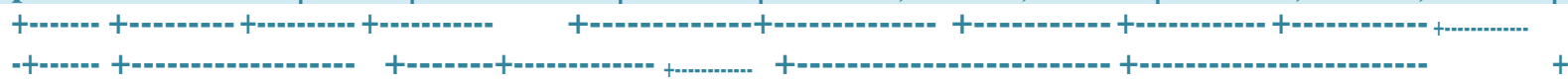

ZN:B:703 (ZN) - ION

Interacting chain(s): B

**Metal Complexes**

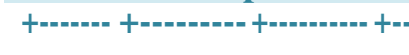

| RESNR | RESTYPE | RESCHAIN | RESNR_LIG | RESTYPE_LIG | RESCHAIN_LIG | METAL_IDX | METAL_TYPE | TARGET_IDX | TARGET_TYPE | COORDINATION | DIST | LOCATION |RMS | GEOMETRY | COMPLEXNUM|METALCOO |TARGETCOO

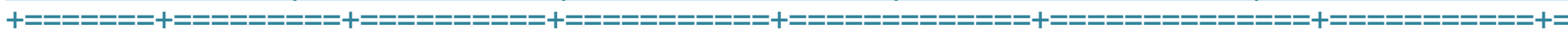

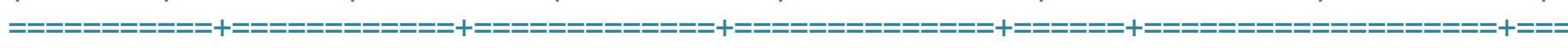

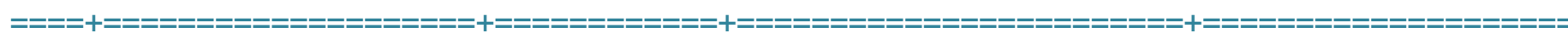
$===+$

\begin{tabular}{|l|l|l|l|l|l|l|l|l|l|l|l|}
10 & CYS & B & 703 & ZN & B & | 9320 & Zn & 4776 & S & $\mid 3$ & $2.39 \mid$
\end{tabular} protein.sidechain | 48.78 | trigonal.pyramidal | $1 \quad$ | 439.355, 6.392, 70.261 | 441.544, 5.678, 69.635 |

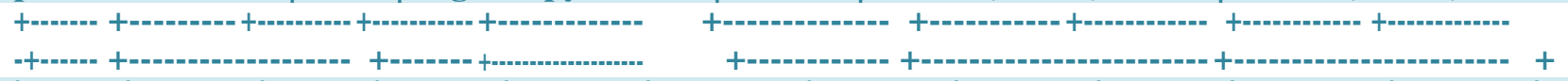

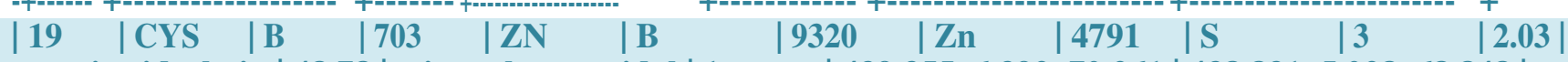
protein.sidechain | 48.78 | trigonal.pyramidal | $1 \quad|439.355,6.392,70.261| 438.831,5.028,68.848 \mid$

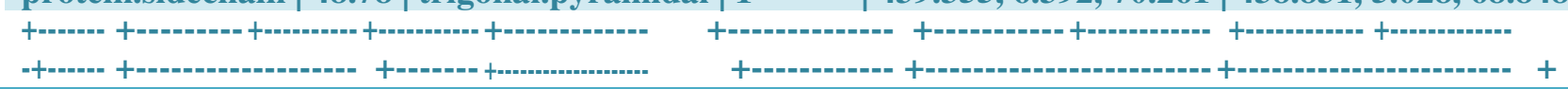




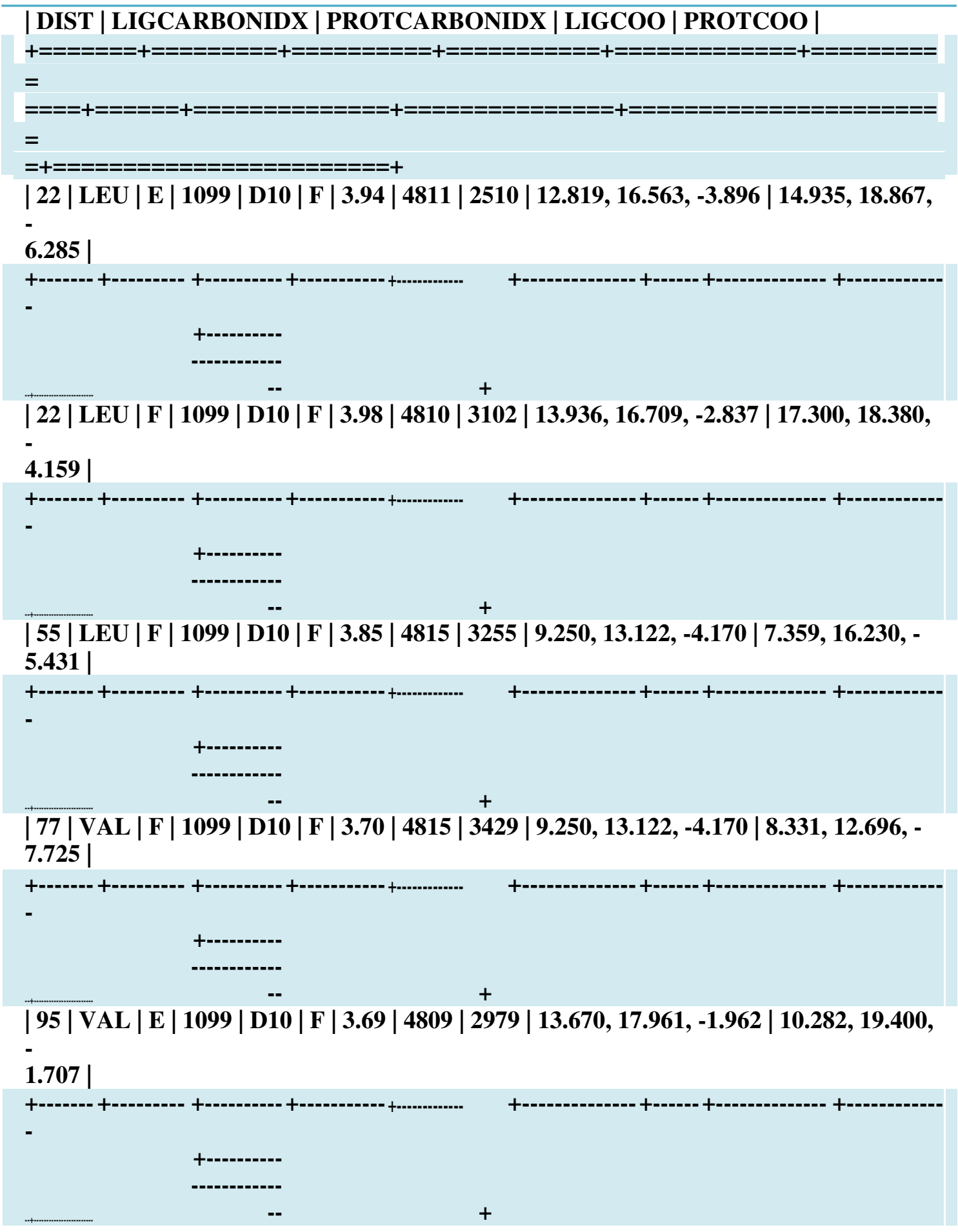

\section{D10:H:1099 (D10) - SMALLMOLECULE}

Interacting chain(s): H

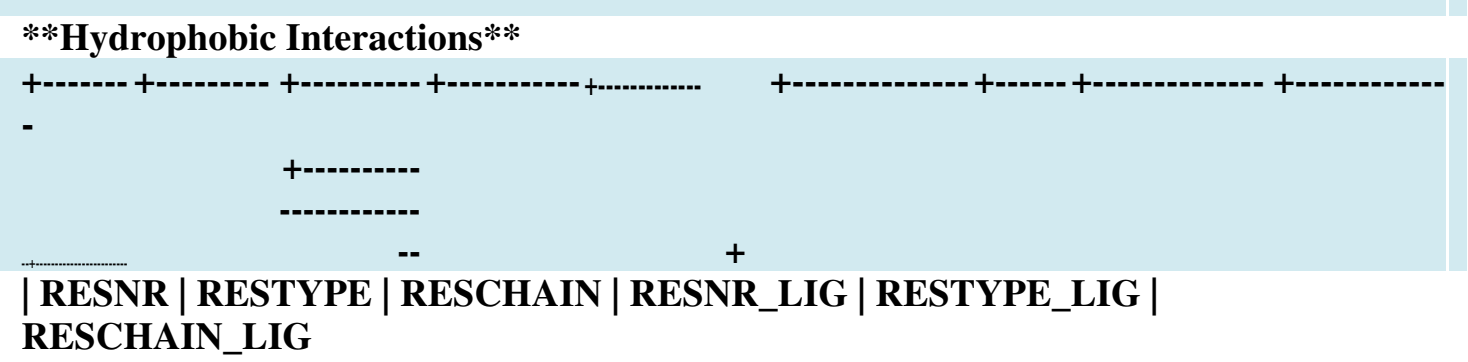




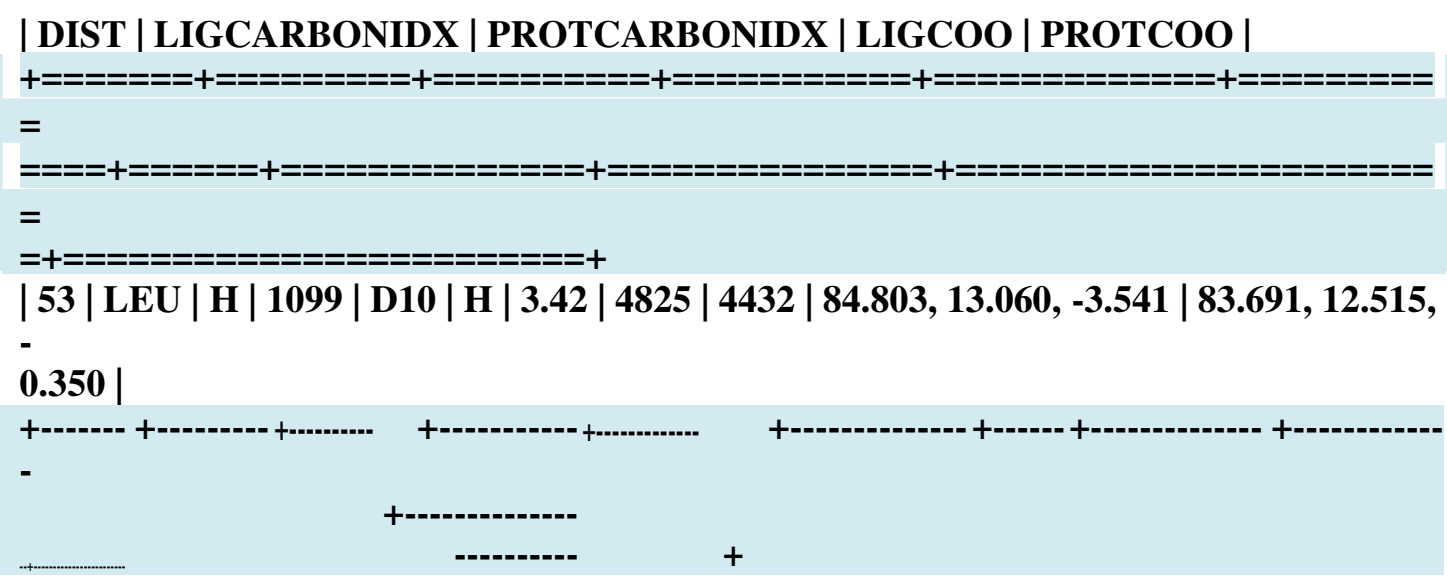



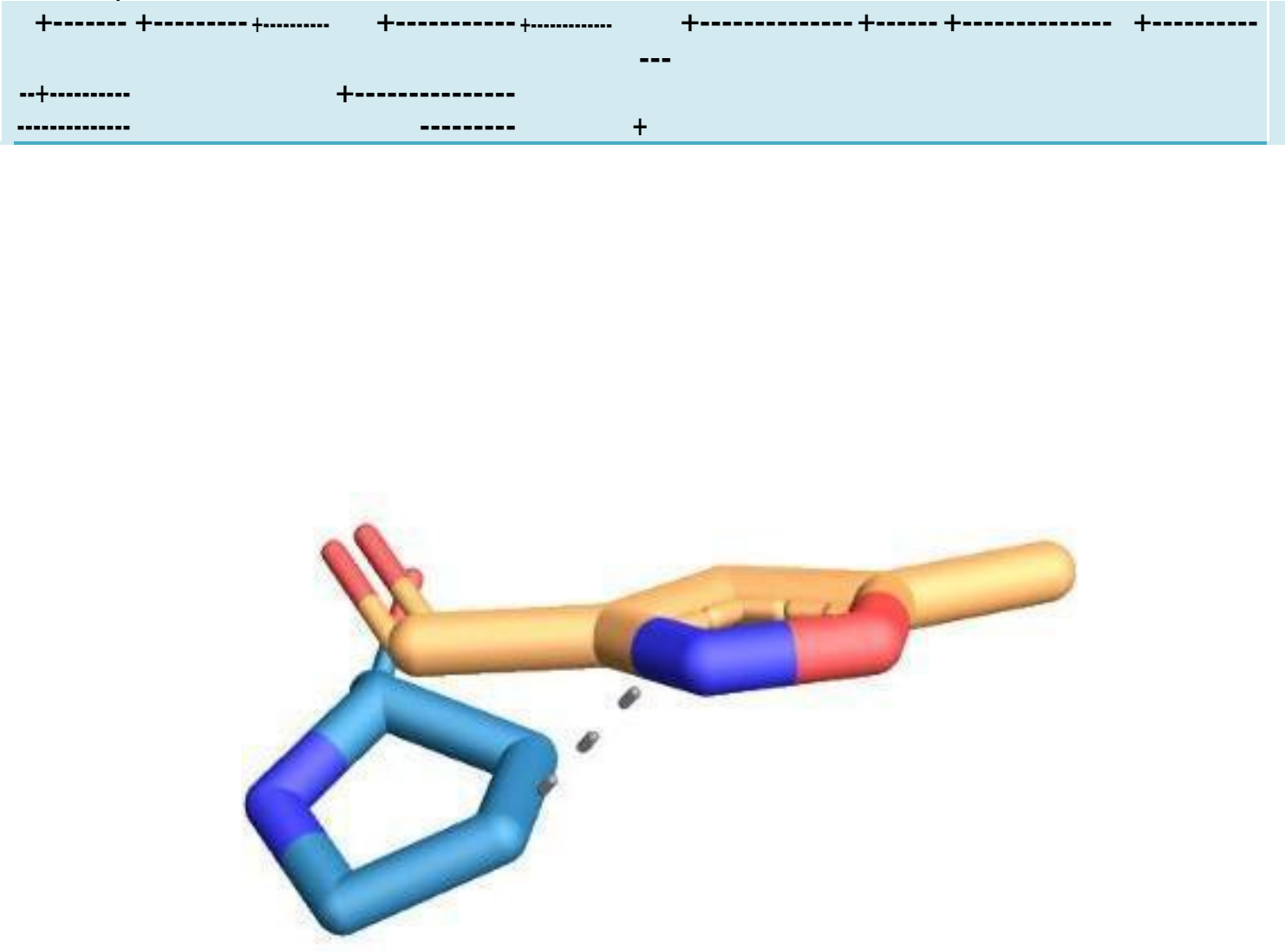

$\underline{\text { 02J (5-Methylisoxazole-3-carboxylic acid) }}$

$\underline{02 \mathrm{~J}-\mathrm{C}-1}$

Interacting chains: A

Figure6a. Roccustyrna binding sites into the 02J (5-Methylisoxazole-3-carboxylic acid) 6LU7 protein targets. DMS:A:402 (DMS) - SMALLMOLECULE

Interacting chain(s): A

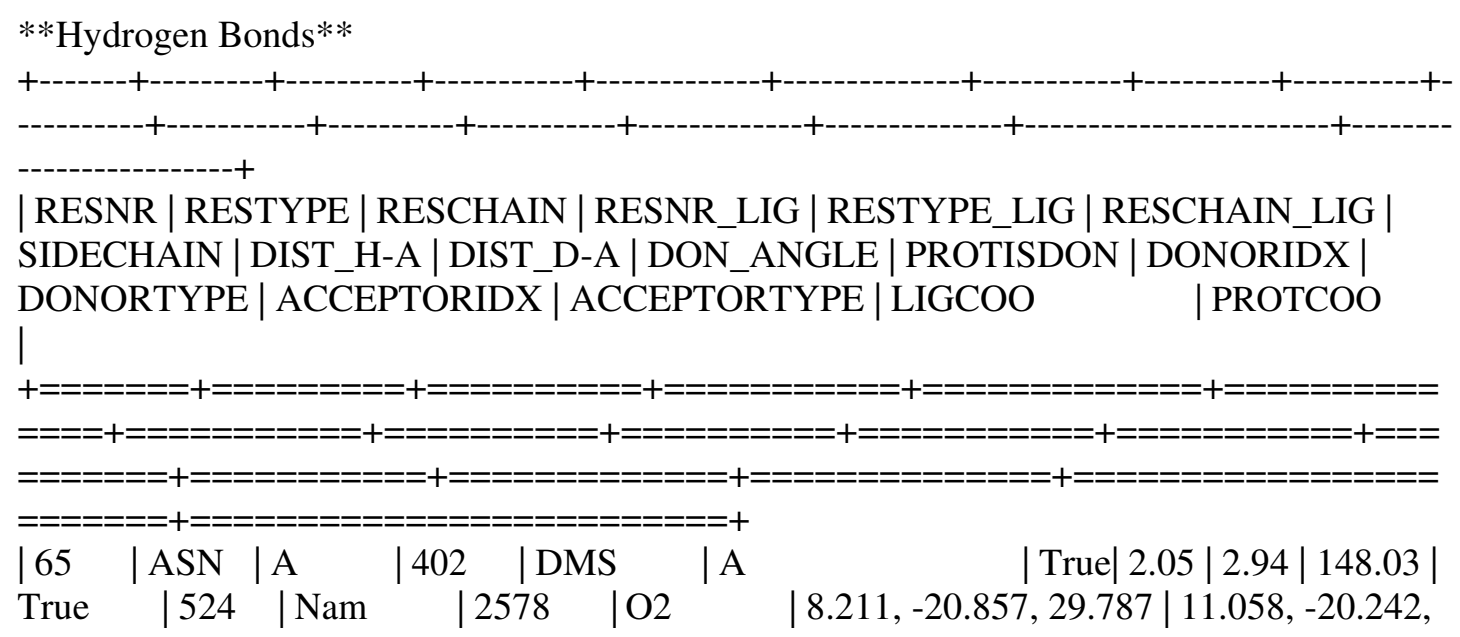


30.160

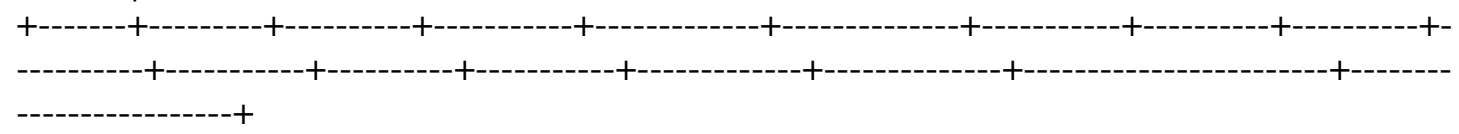

DMS:A:403 (DMS) - SMALLMOLECULE

Interacting chain(s): A

**Hydrogen Bonds**

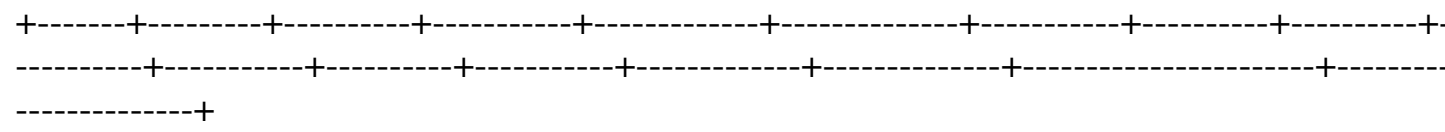

| RESNR | RESTYPE | RESCHAIN | RESNR_LIG | RESTYPE_LIG | RESCHAIN_LIG | SIDECHAIN | DIST_H-A | DIST_D-A | DON_ANGLE | PROTISDON | DONORIDX | DONORTYPE $\mid$ ACCEPTORIDX | ACCEPTORTYPE | LIGCOO | PROTCOO

+ニニニニニニニ+ニニニニニニニニニ+ニニニニニニニニニニ+ニニニニニニニニニニニ+ニニニニニニニニニニニニニ+ニニニニニニニニニニ

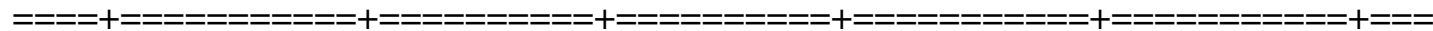

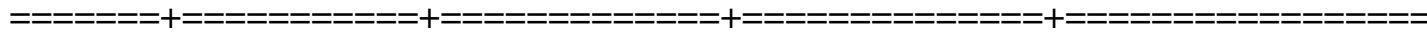
ニニニニニニニニニニニニニニニニニニニニニニニニニニニ+

\begin{tabular}{|l|l|l|l|l|l|l|l|l|l|}
298 & ARG & A & 403 & DMS & A & True & 1.93 & 2.87 & 160.38
\end{tabular} True $|2512| \mathrm{Ng}+\quad|2582 \quad| \mathrm{O} 2 \quad|7.044,-0.753,-7.469| 9.865,-1.270,-$ 7.327

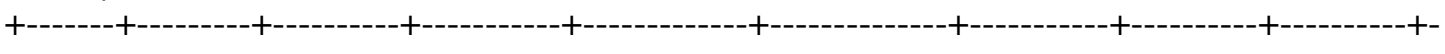

**Salt Bridges**

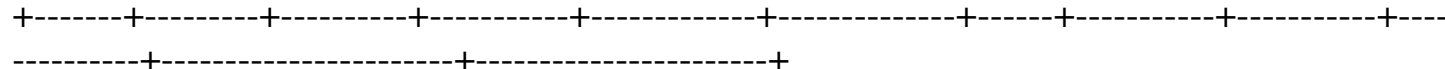

| RESNR | RESTYPE | RESCHAIN | RESNR_LIG | RESTYPE_LIG | RESCHAIN_LIG | DIST | PROTISPOS | LIG_GROUP | LIG_IDX_LIST | LIGCOO | PROTCOO

|

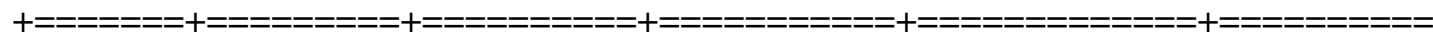

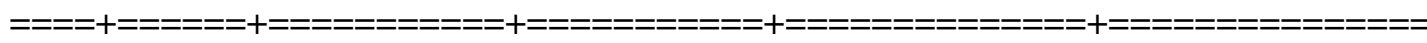
$=======+=======================+$

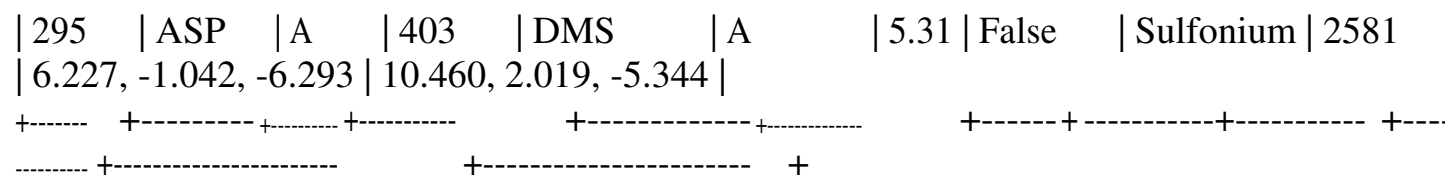

**pi-Cation Interactions**

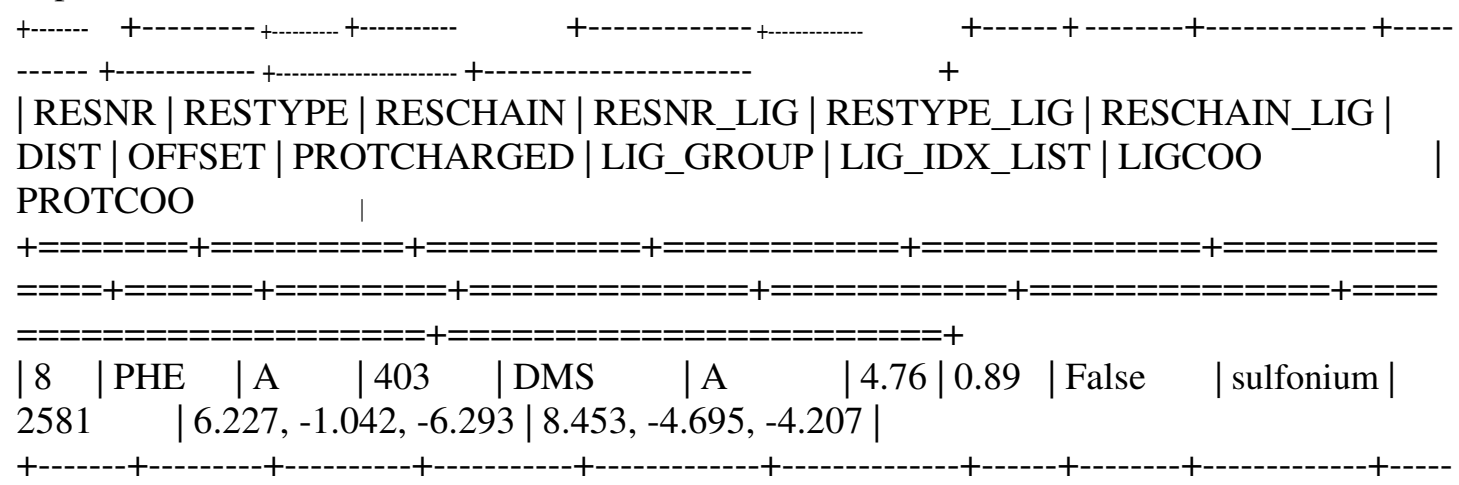


DMS:A:404 (DMS) - SMALLMOLECULE

Interacting chain(s):

DMS:A:405 (DMS) - SMALLMOLECULE

Interacting chain(s): A
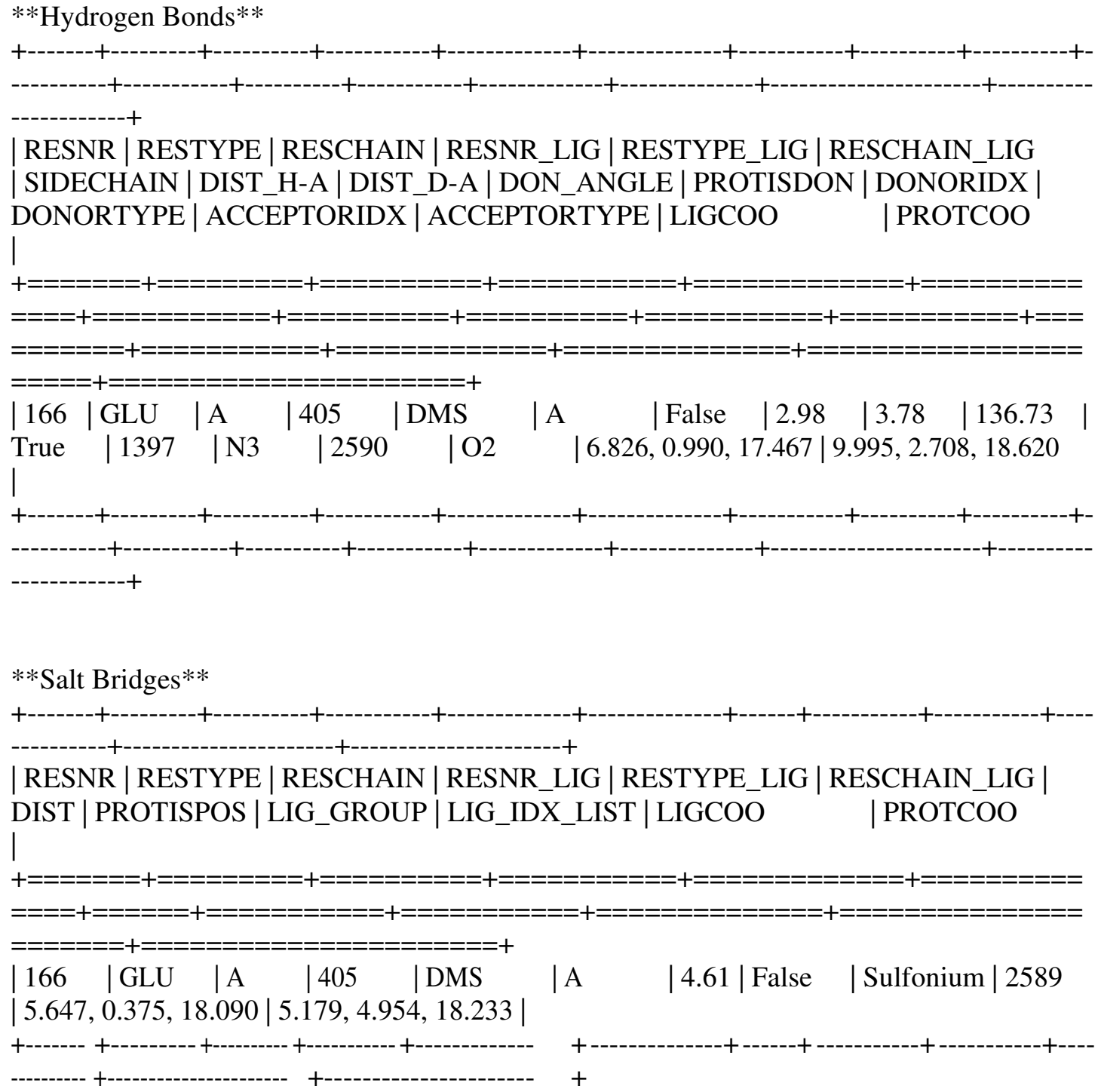

DMS:A:406 (DMS) - SMALLMOLECULE

Interacting chain(s): 
DMS:A:407 (DMS) - SMALLMOLECULE

Interacting chain(s): A

**Hydrogen Bonds**

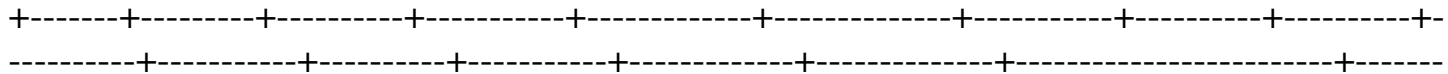

| RESNR | RESTYPE | RESCHAIN | RESNR_LIG | RESTYPE_LIG | RESCHAIN_LIG | SIDECHAIN | DIST_H-A | DIST_D-A | DON_ANGLE | PROTISDON | DONORIDX | DONORTYPE | ACCEPTORIDX | ACCEPTORTYPE | LIGCOO

$\mid$ PROTCOO

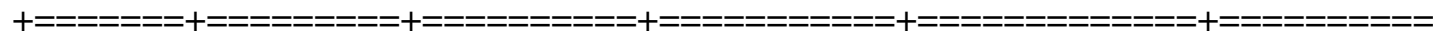

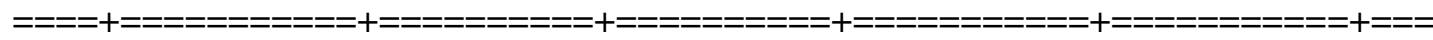

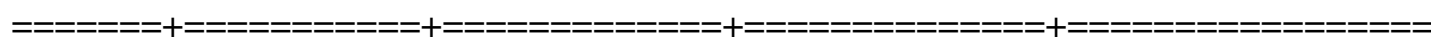
$========+=========================+$

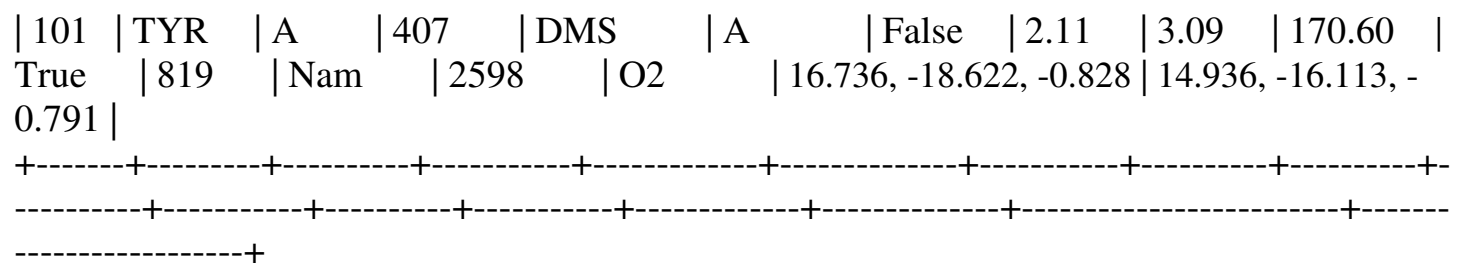

**pi-Cation Interactions**

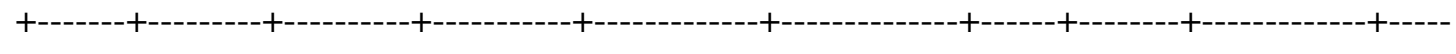

| RESNR | RESTYPE | RESCHAIN | RESNR_LIG | RESTYPE_LIG | RESCHAIN_LIG | DIST | OFFSET | PROTCHARGED | LIG_GROUP | LIG_IDX_LIST | LIGCOO PROTCOO

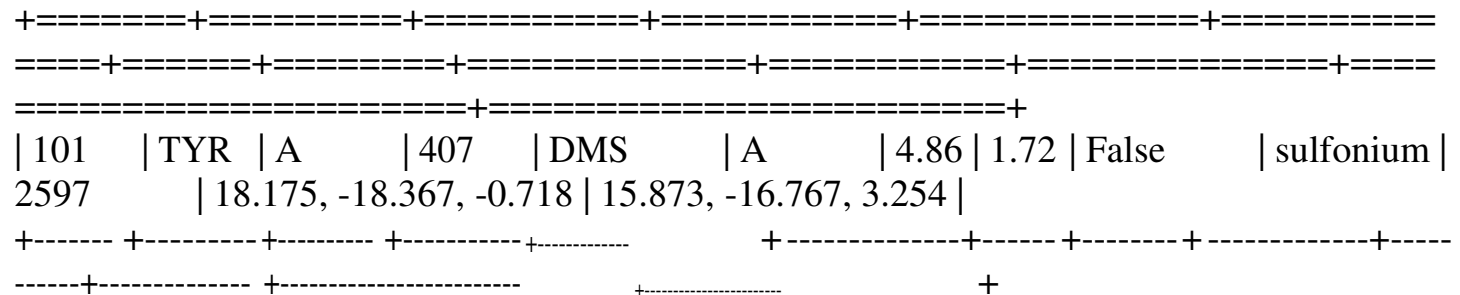

DMS:A:408 (DMS) - SMALLMOLECULE

Interacting chain(s): A

**Water Bridges**

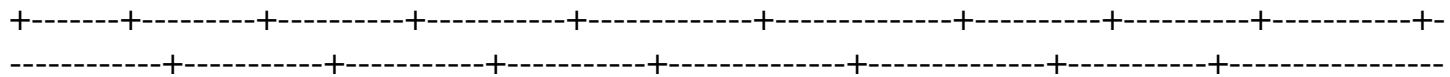

------+---------------------+---------------------+

| RESNR | RESTYPE | RESCHAIN | RESNR_LIG | RESTYPE_LIG | RESCHAIN_LIG | DIST_A-W | DIST_D-W | DON_ANGLE | WATER_ANGLE | PROTISDON |

DONOR_IDX | DONORTYPE | ACCEPTOR_IDX | ACCEPTORTYPE | WATER_IDX |

LIGCOO | PROTCOO |WATERCOO

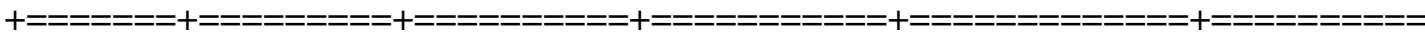

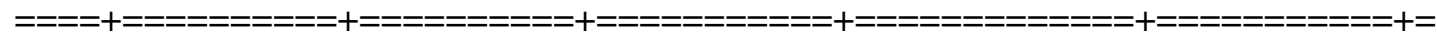

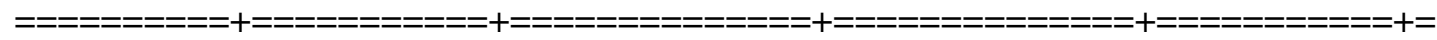

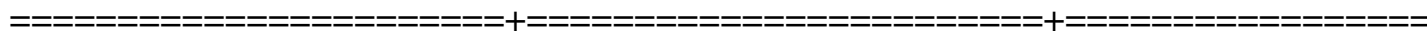



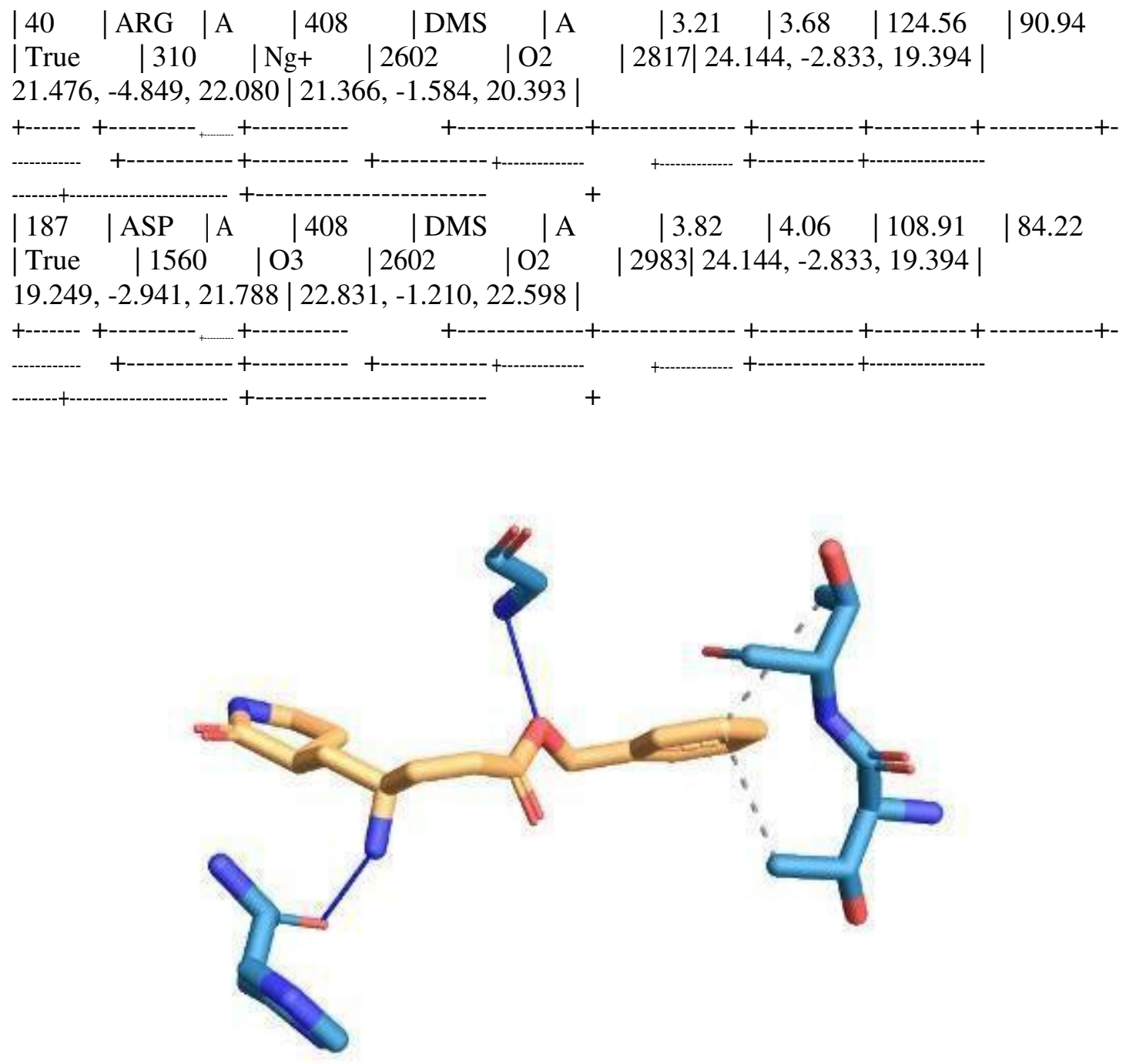

$\underline{\text { Hydrophobic Interactions }}$

\begin{tabular}{|c|c|c|c|c|c|}
\hline Index & $\begin{array}{l}\text { Residu } \\
\mathrm{e}\end{array}$ & $\mathrm{AA}$ & $\begin{array}{l}\text { Distanc } \\
\mathrm{e}\end{array}$ & $\begin{array}{l}\text { Ligand } \\
\text { Atom }\end{array}$ & $\begin{array}{l}\text { Protein } \\
\text { Atom }\end{array}$ \\
\hline 1 & $168 \mathrm{~A}$ & PR & 3.53 & 2369 & 1303 \\
\hline
\end{tabular}

$\underline{\text { PJE (composite ligand) }}$

PJE-C-5

Composite ligand consists of PJE:C:5, 010:C:6.

Interacting chains: A

Figure6b. Roccustyrna binding sites into the 02J (5-Methylisoxazole-3-carboxylic acid) PJE-C-56LU7 protein targets. ZN:A:998 (ZN) - ION 
Interacting chain(s): A

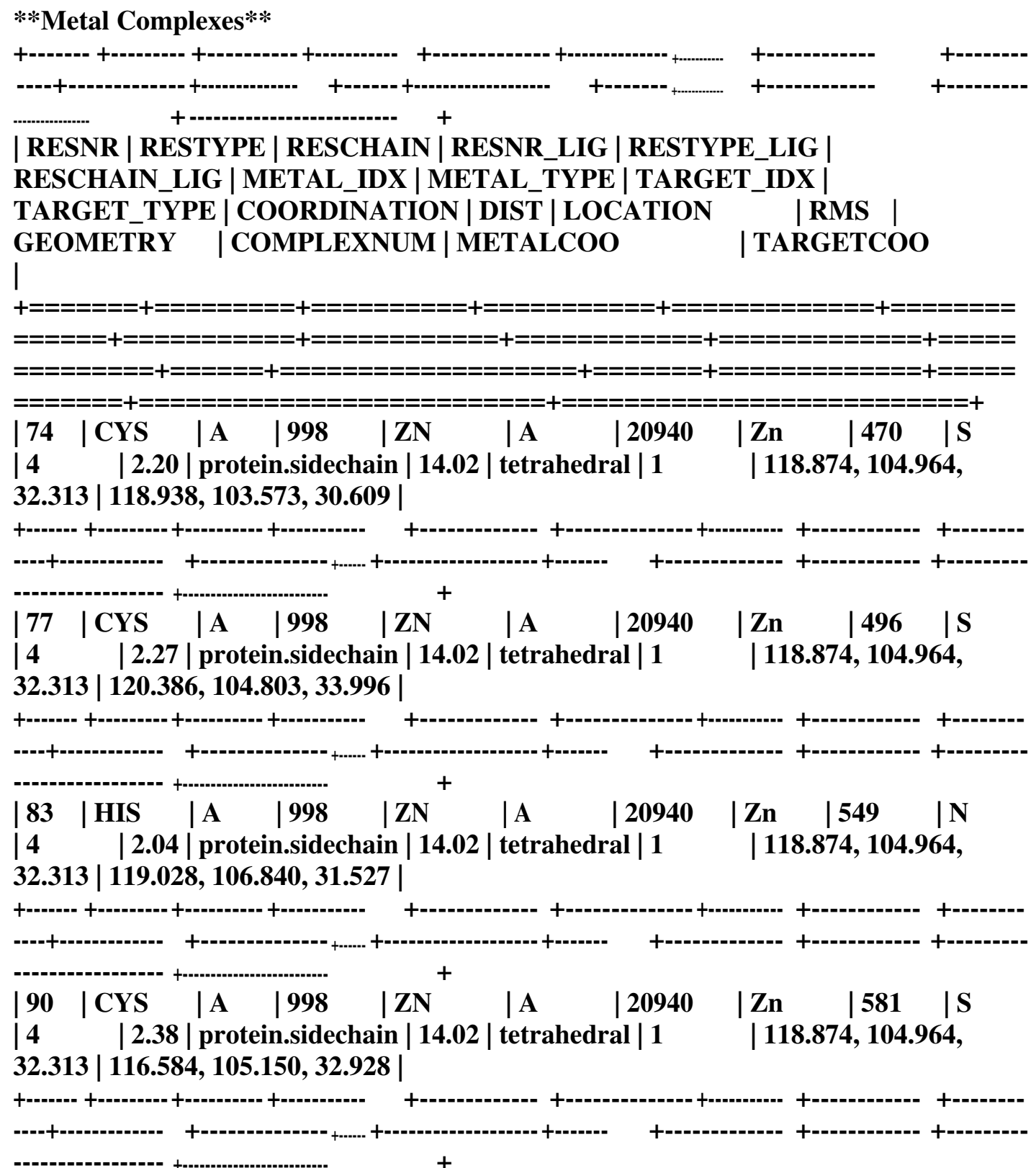

ZN:A:999 (ZN) - ION

Interacting chain(s): A

**Metal Complexes**

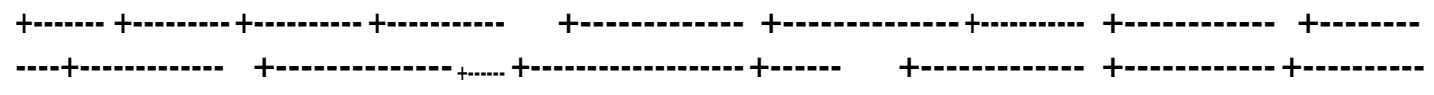




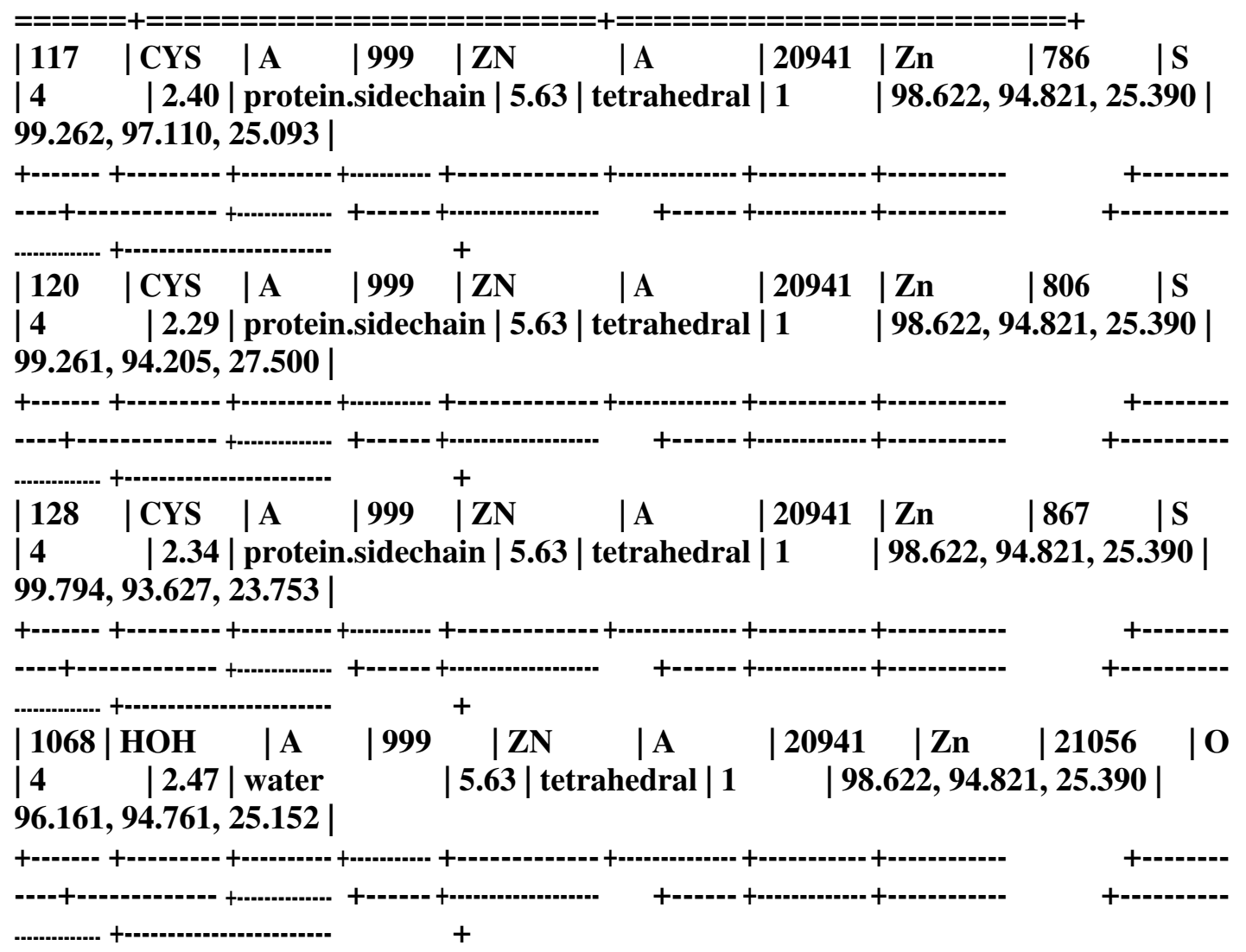

ZN:B:998 (ZN) - ION

Interacting chain(s): B

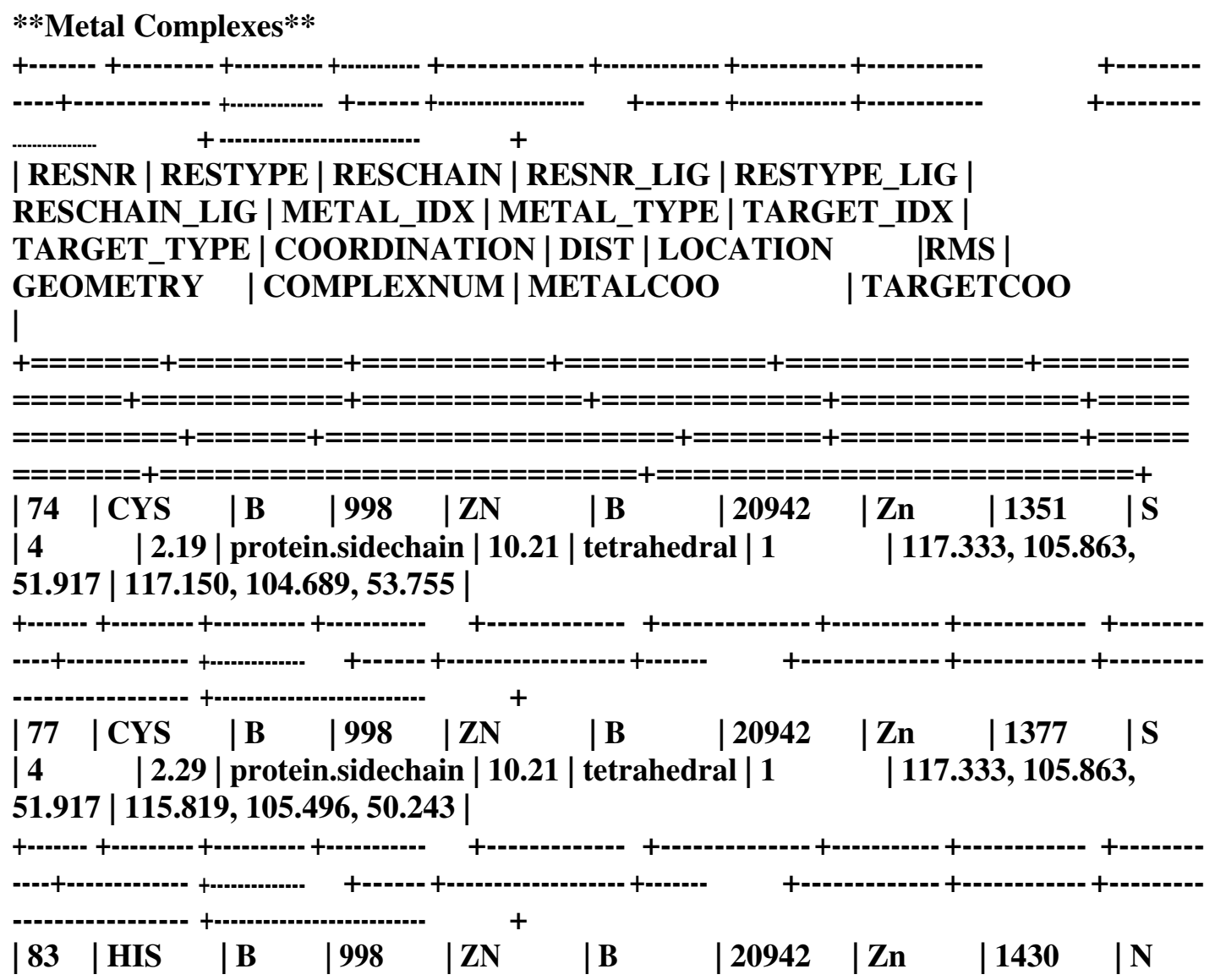


| 4 | 2.07| protein.sidechain | 10.21 | tetrahedral | $1 \quad$ | 117.333, 105.863, 51.917| 116.930, 107.835, 52.389|

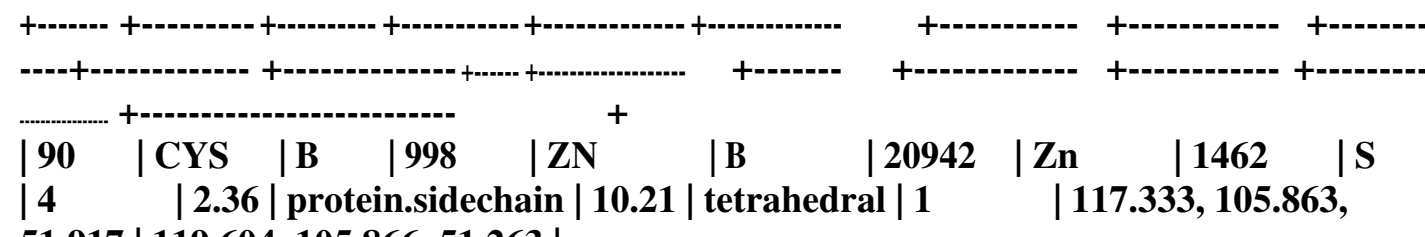

51.917| 119.604, 105.866, 51.263 |

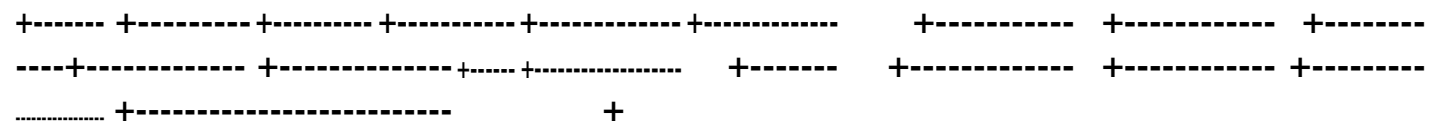

ZN:B:999 (ZN) - ION

Interacting chain(s): B

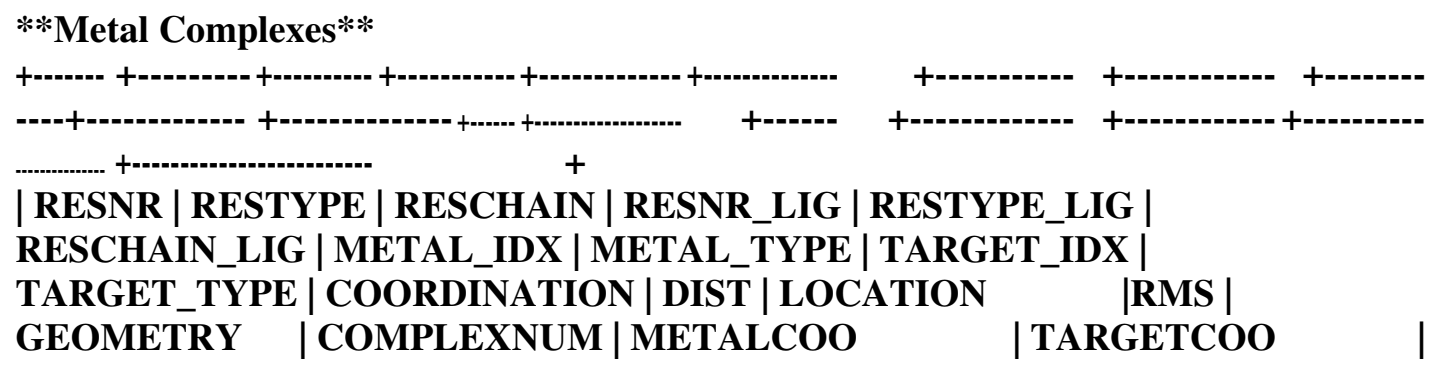

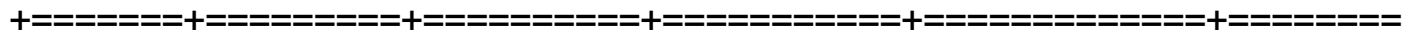

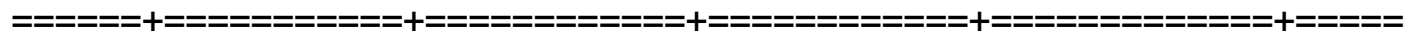

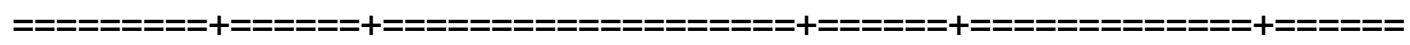

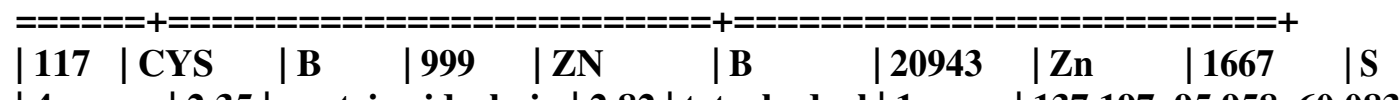

| 4 | 2.35 | protein.sidechain $|2.82|$ tetrahedral $|1 \quad| 137.197,95.958,60.083 \mid$ 136.561, 98.220, 60.179 |

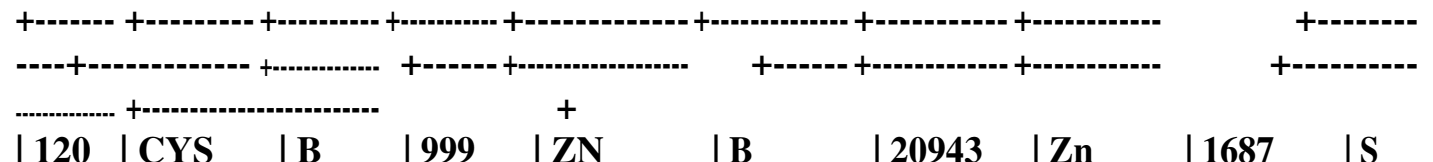

| $4|2.48|$ protein.sidechain $|2.82|$ tetrahedral $|1 \quad| 137.197,95.958,60.083 \mid$ 136.438, 95.012, 57.924 |

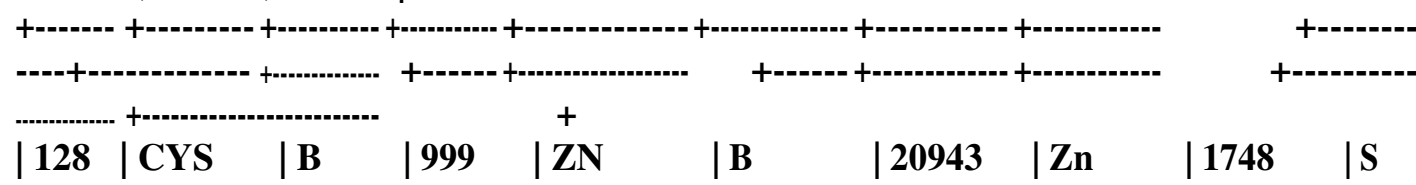

| $4 \quad$ 2.47| protein.sidechain $|2.82|$ tetrahedral $|1 \quad| 137.197,95.958,60.083 \mid$ 136.060, 94.833, 61.960 |

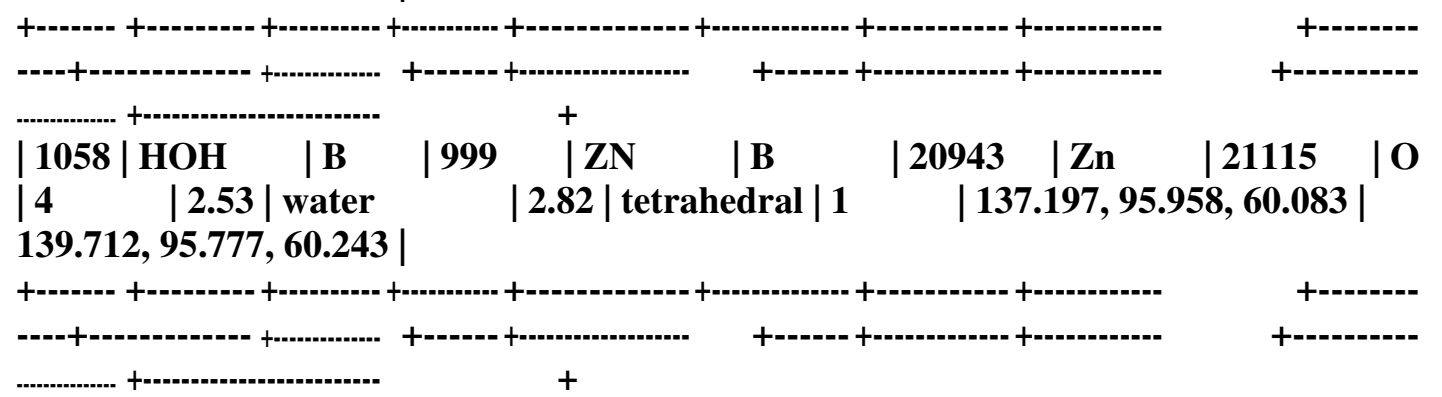


Interacting chain(s): C

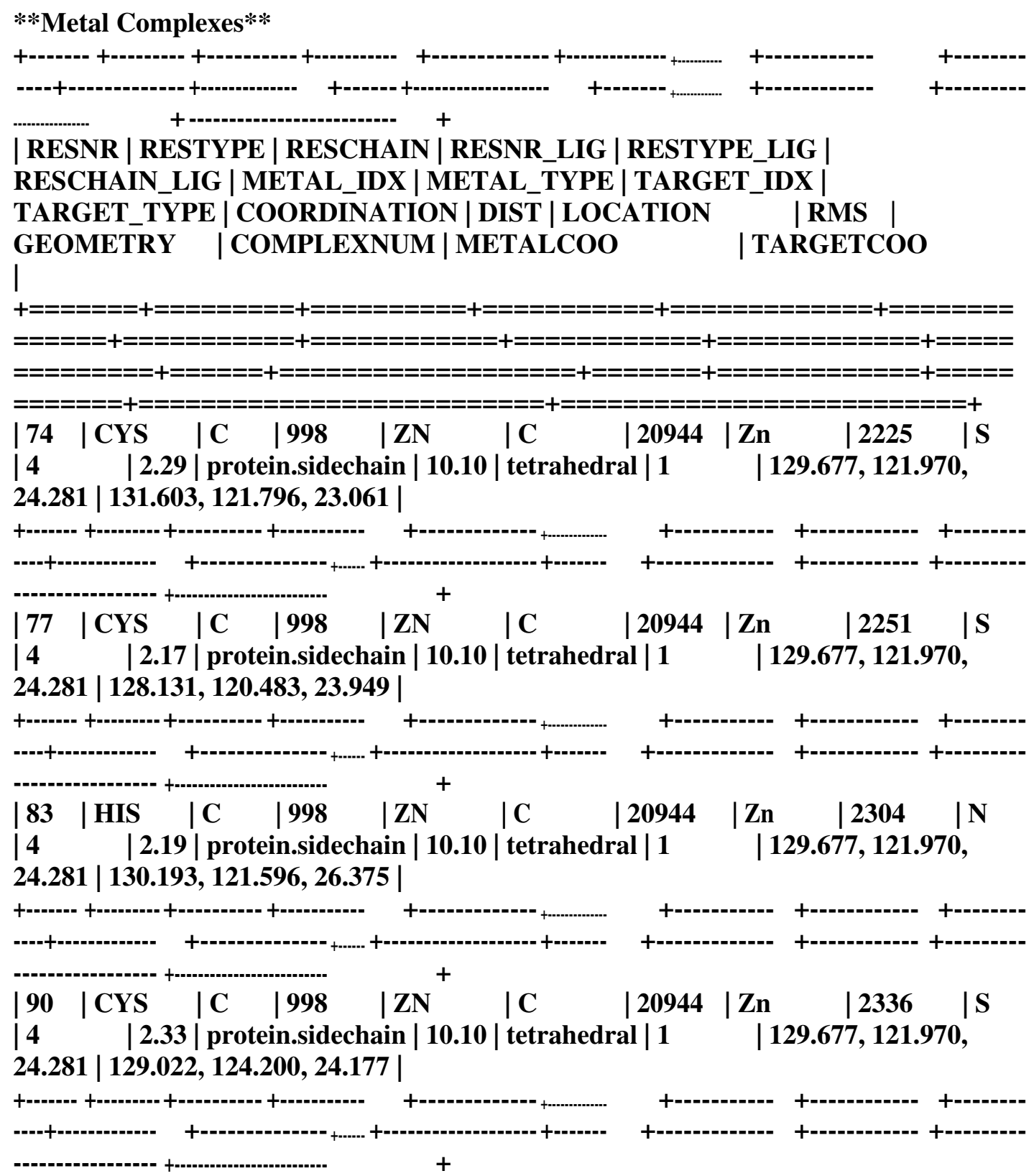

ZN:C:999 (ZN) - ION

Interacting chain(s): C

**Metal Complexes**

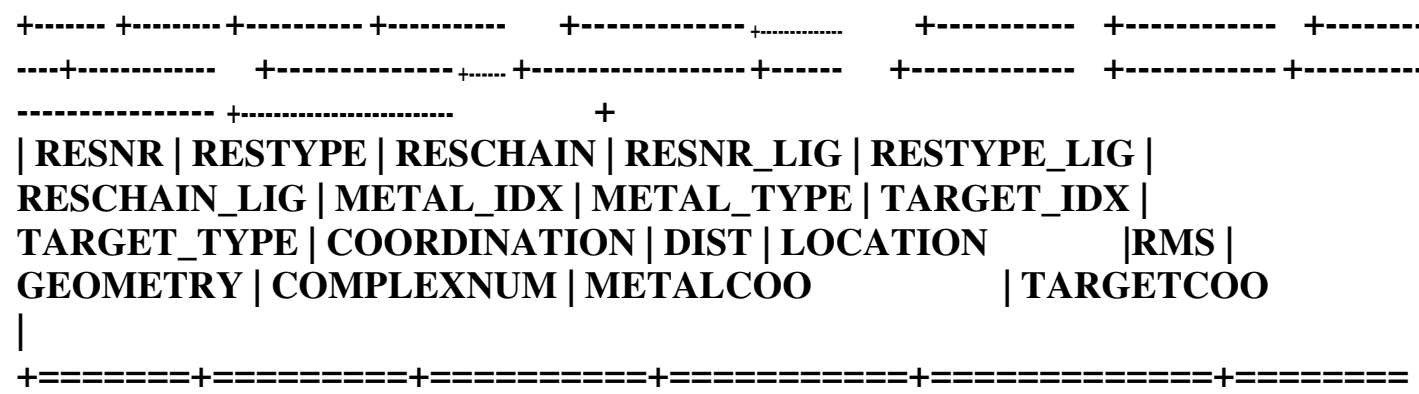




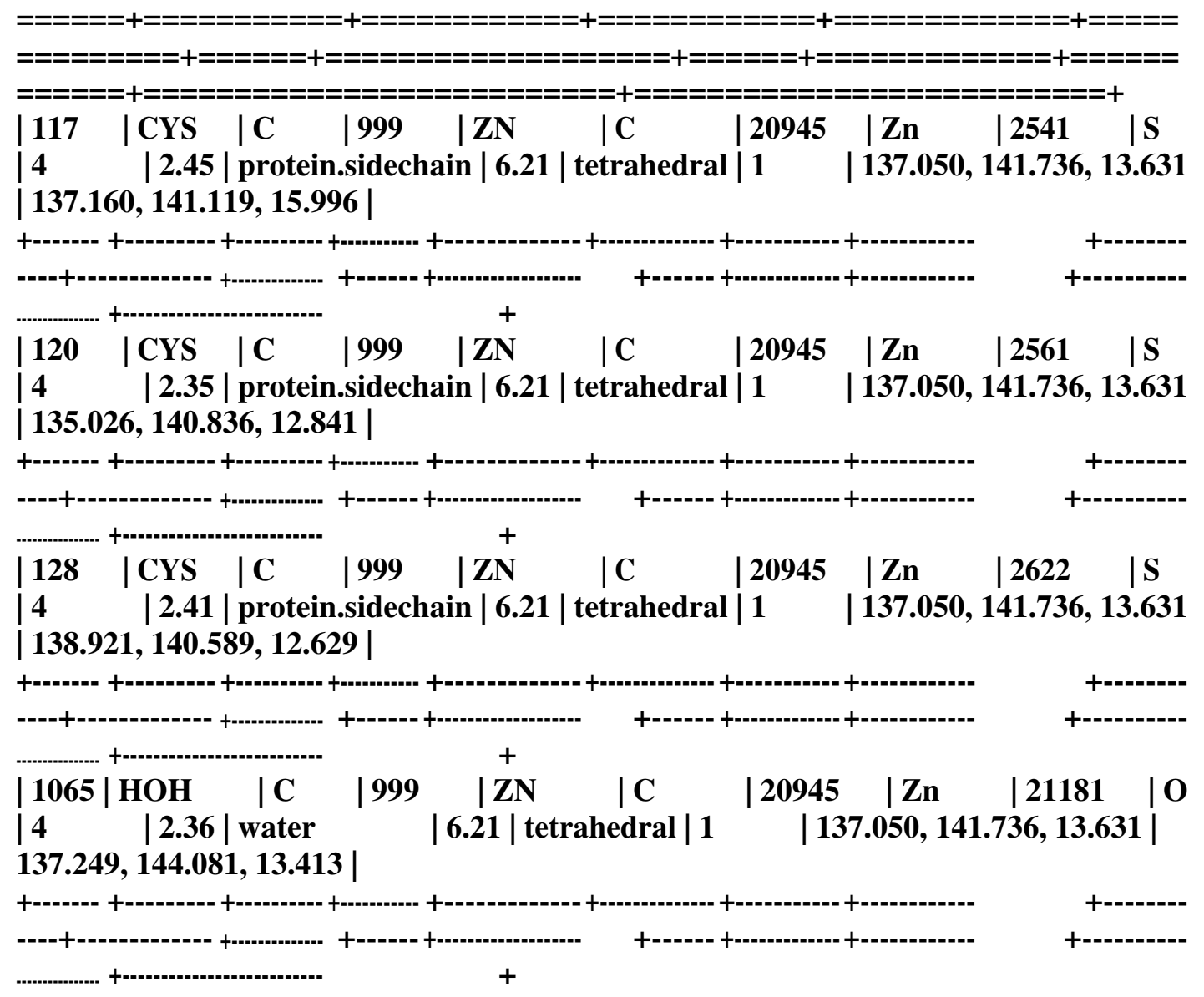

ZN:D:998 (ZN) - ION

Interacting chain(s): D

**Metal Complexes**

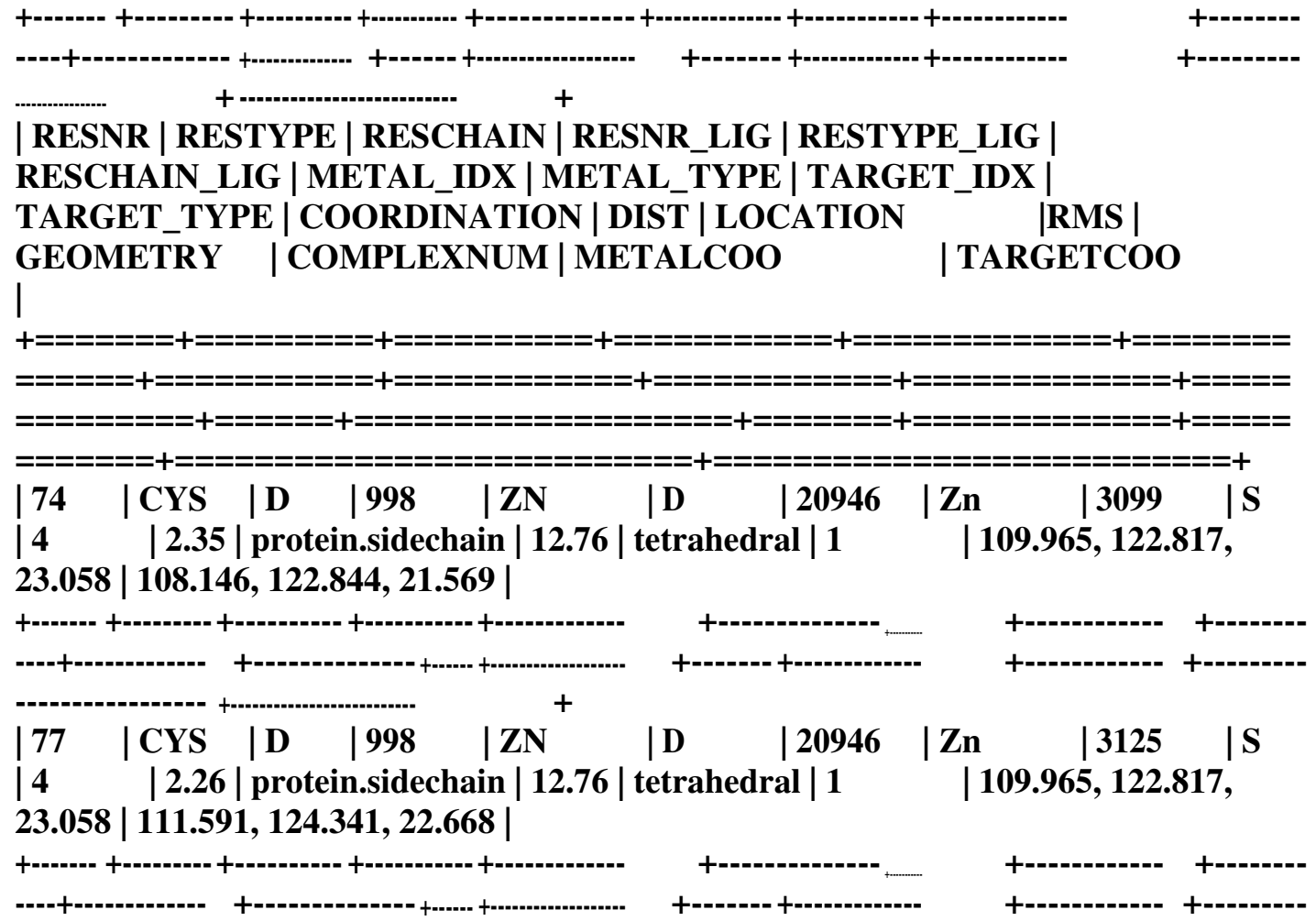




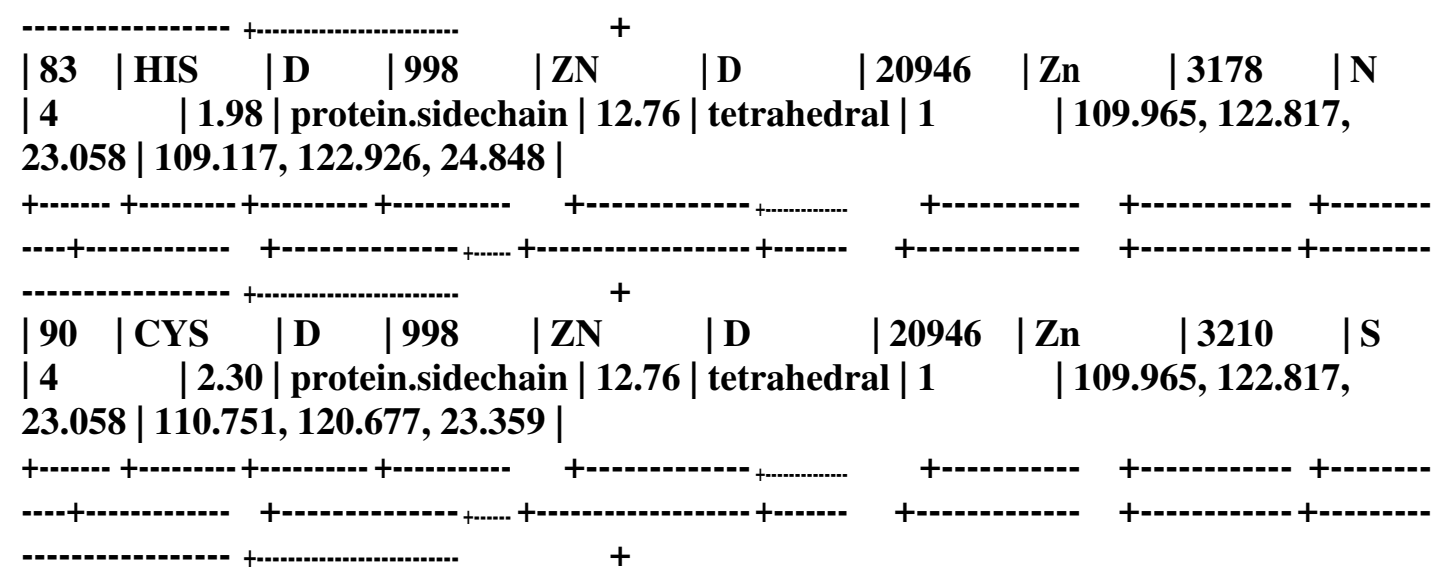

ZN:D:999 (ZN) - ION

Interacting chain(s): D

**Metal Complexes**

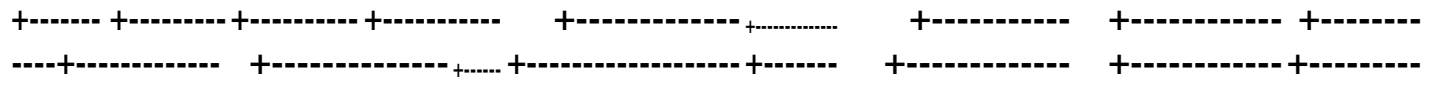

| RESNR | RESTYPE | RESCHAIN | RESNR_LIG | RESTYPE_LIG |

RESCHAIN_LIG | METAL_IDX | METAL_TYPE | TARGET_IDX |

TARGET_TYPE | COORDINATION | DIST | LOCATION |RMS |

GEOMETRY | COMPLEXNUM | METALCOO | TARGETCOO

1

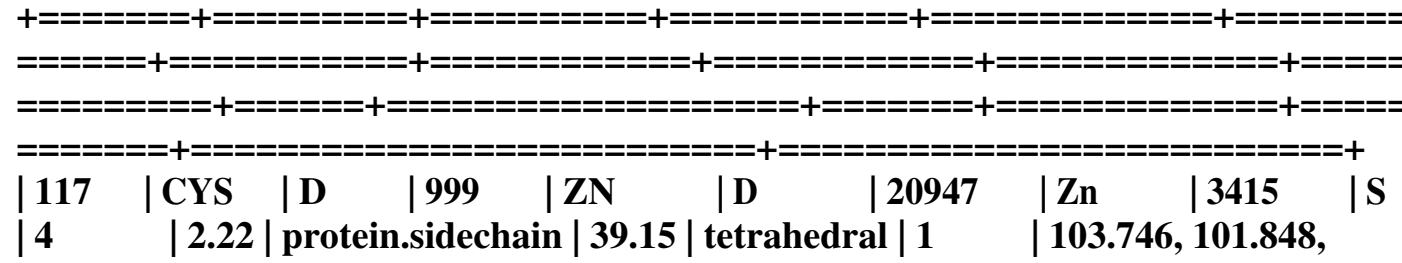

13.968 | 103.306, 102.613, $16.001 \mid$

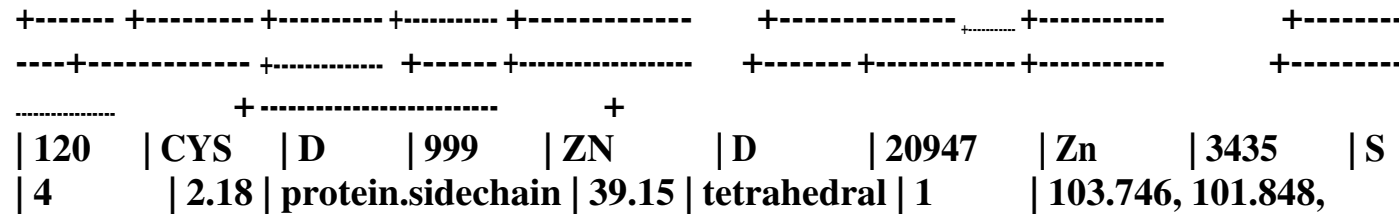

| 4 2.18 | protein.sidechain $|39.15|$ tetrahedral $|1 \quad| 103.746,101.848$,

13.968 | 105.355, 103.254, 13.535|

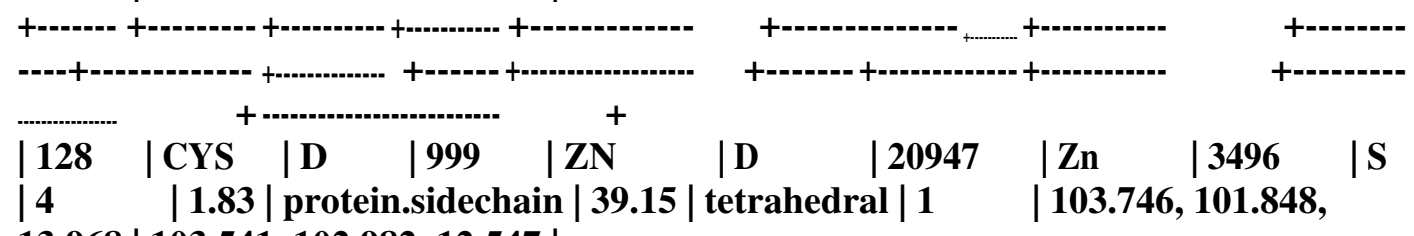

13.968 | 103.541, 102.982, $12.547 \mid$

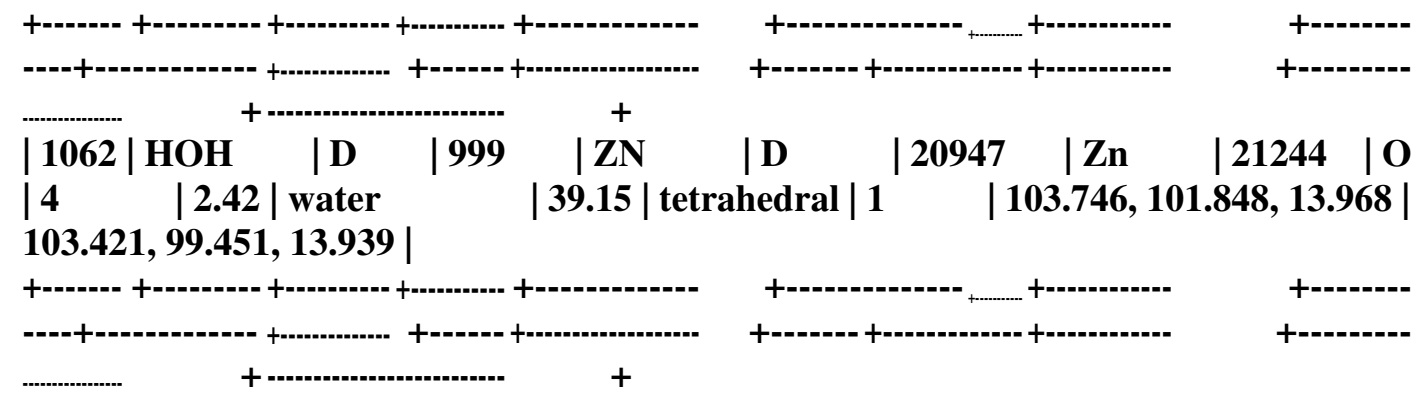


ZN:E:998 (ZN) - ION

Interacting chain(s): E

**Metal Complexes**

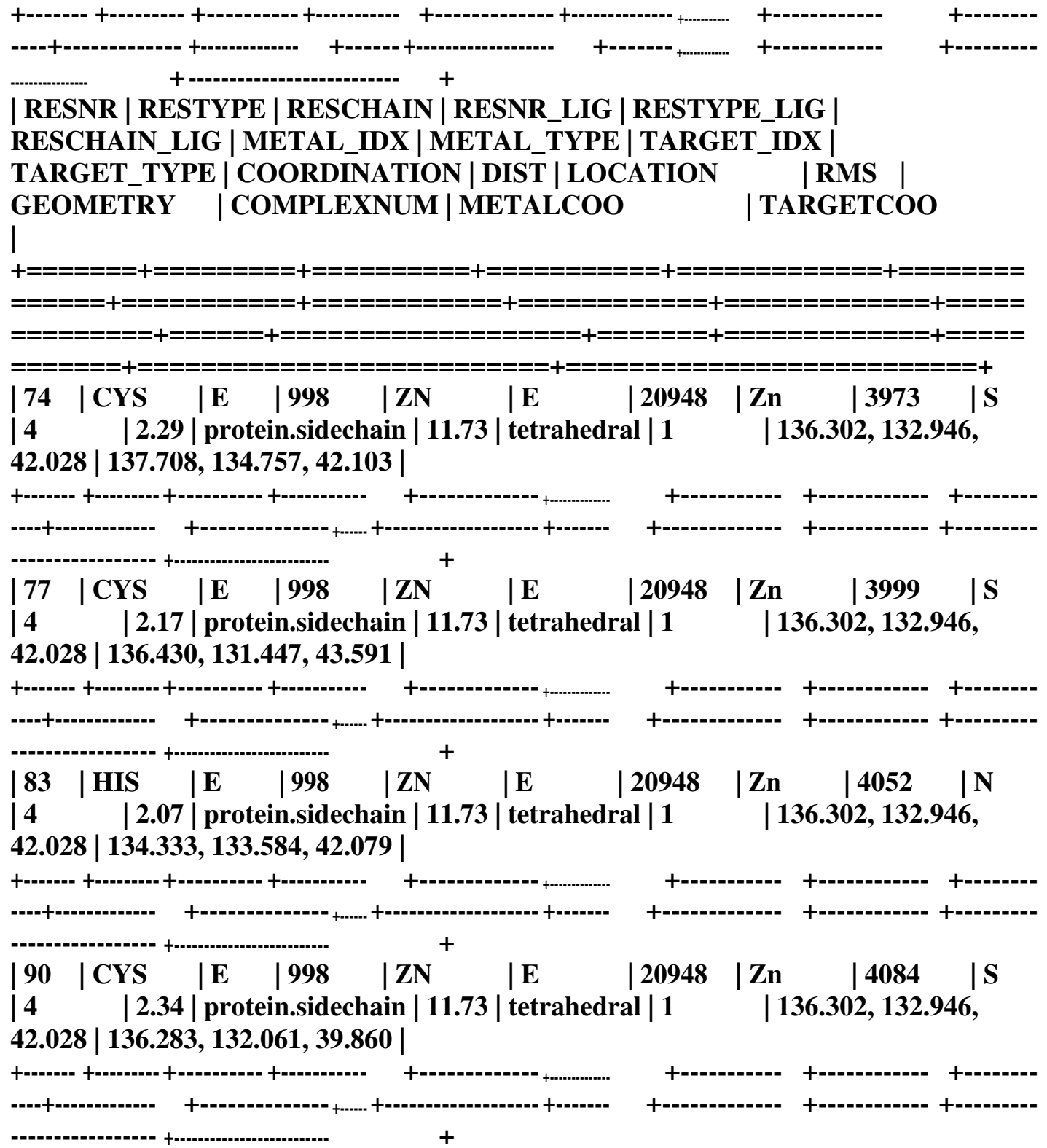

ZN:E:999 (ZN) - ION

Interacting chain(s): E

**Metal Complexes**

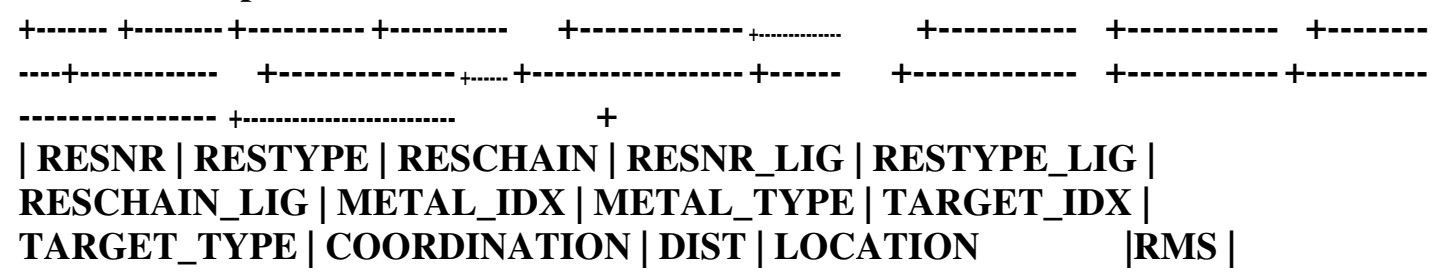




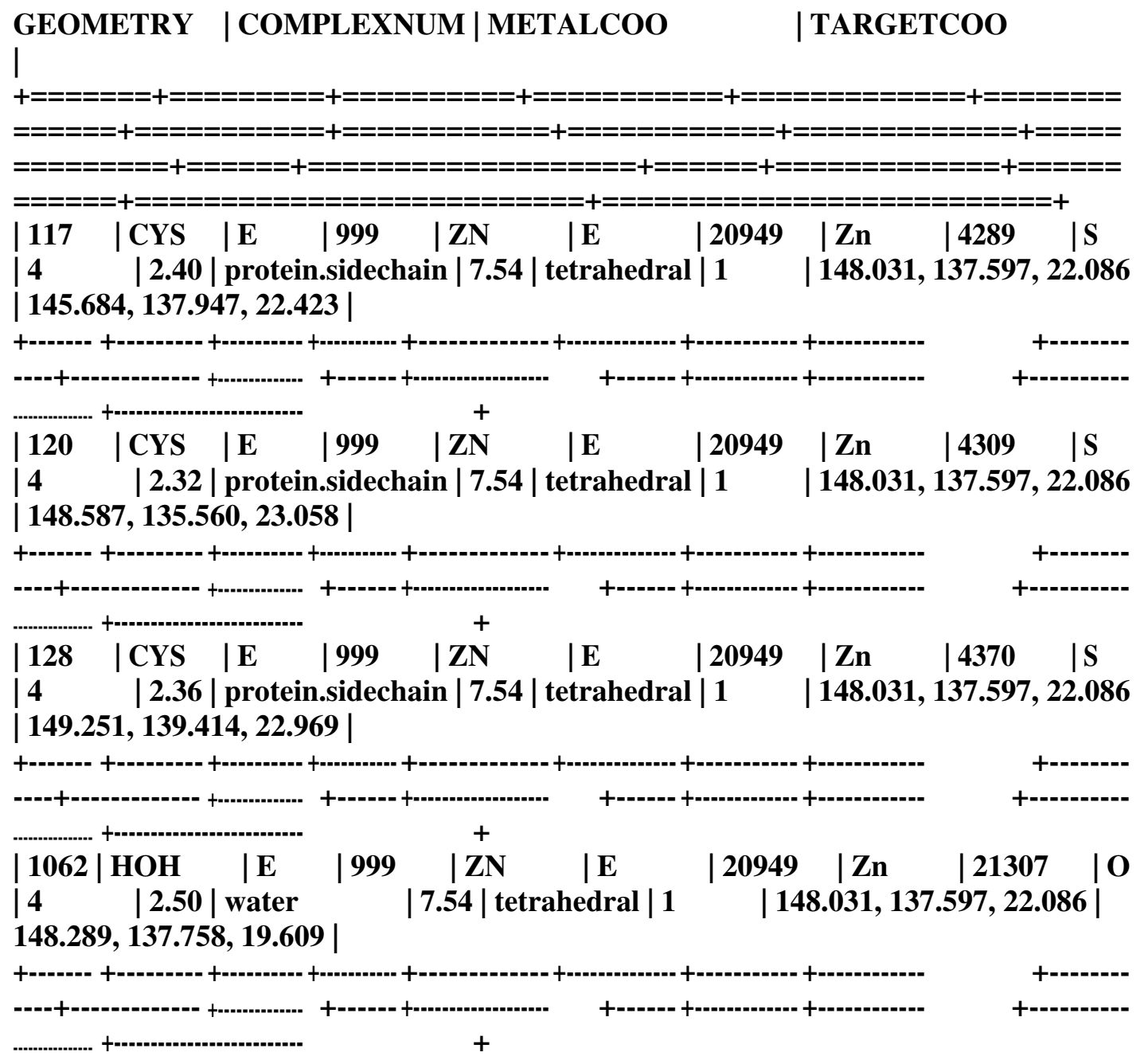

ZN:F:998 (ZN) - ION

Interacting chain(s): F

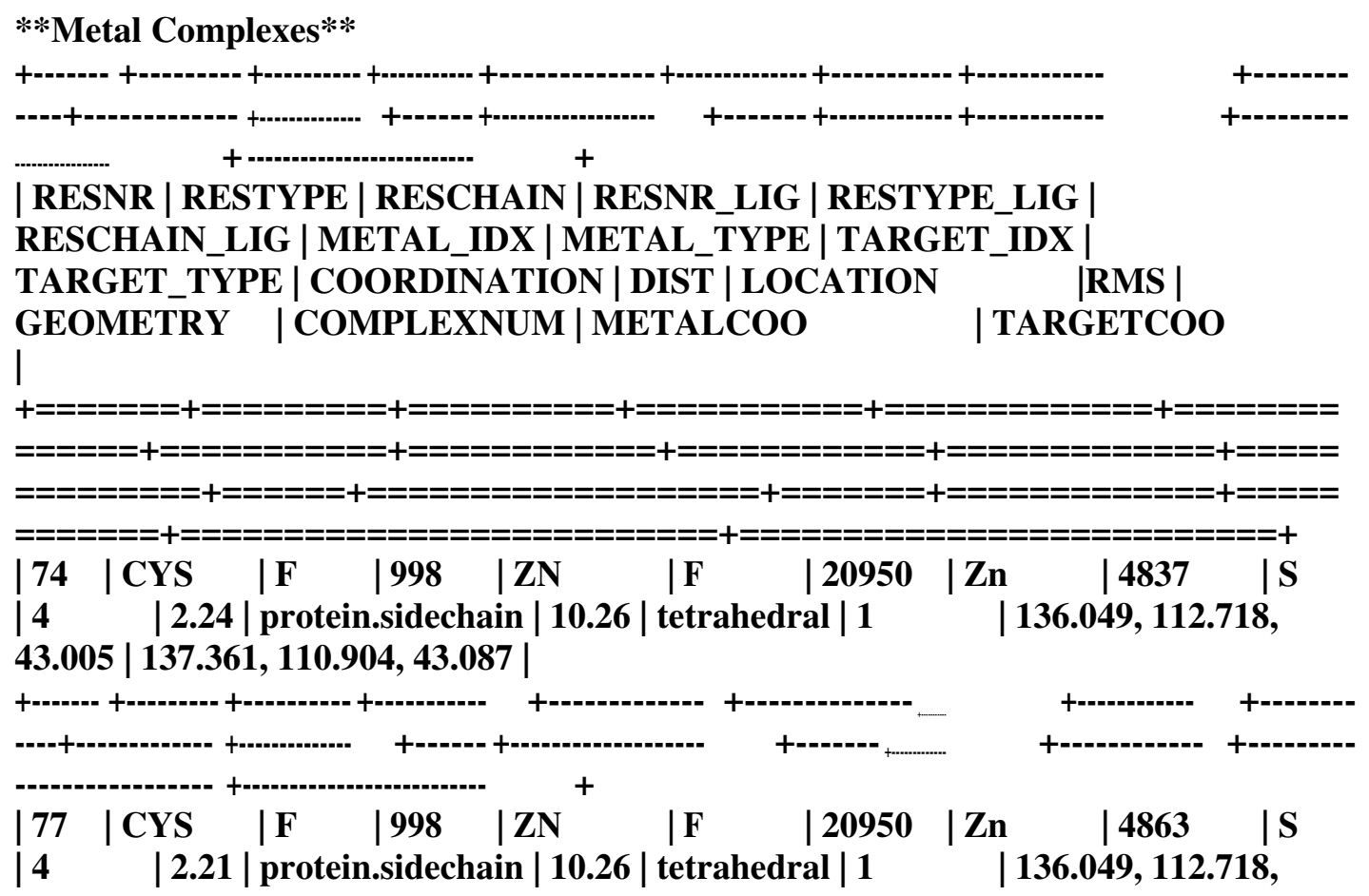




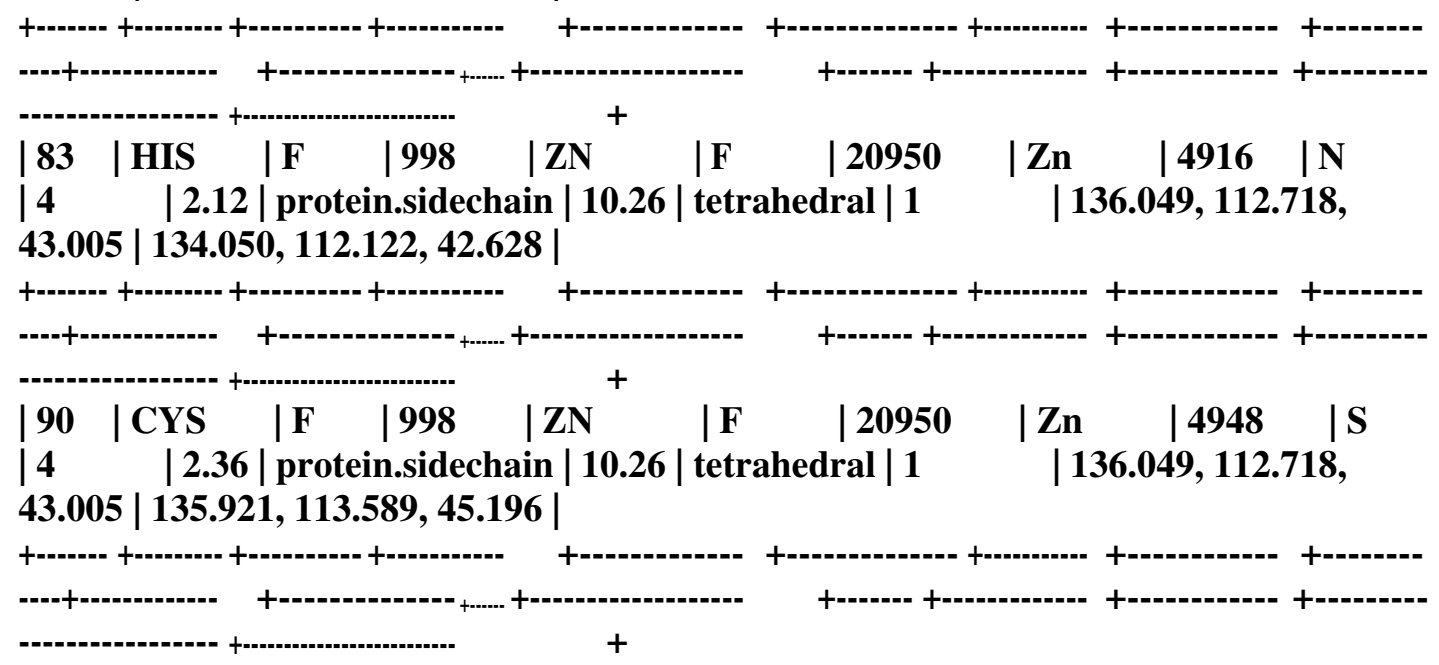

ZN:F:999 (ZN) - ION

Interacting chain(s): F

***Metal Complexes**

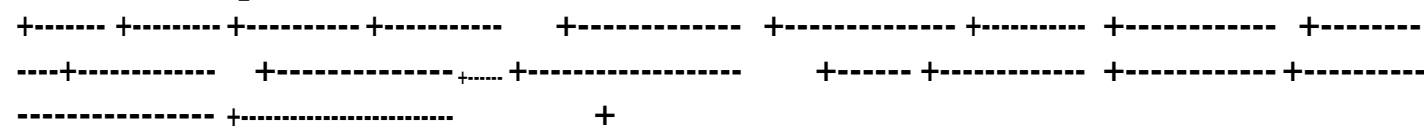

| RESNR | RESTYPE | RESCHAIN | RESNR_LIG | RESTYPE_LIG |

RESCHAIN_LIG | METAL_IDX | METAL_TYPE | TARGET_IDX |

TARGET_TYPE | COORDINATION | DIST | LOCATION_ |RMS |

GEOMETRY | COMPLEXNUM | METALCOO | TARGETCOO

I

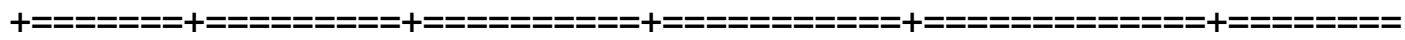

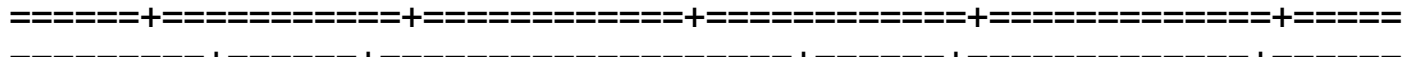

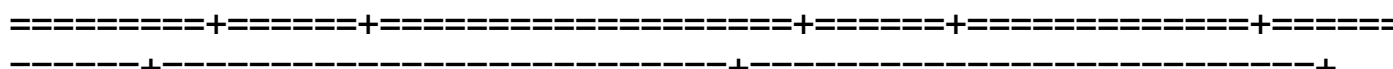

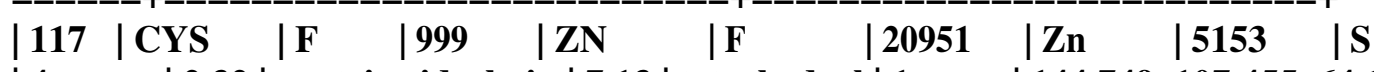

| $4 \quad|2.32|$ protein.sidechain $|7.13|$ tetrahedral $|1 \quad| 144.749,107.455,64.104$ | 142.581, 107.152, 63.330|

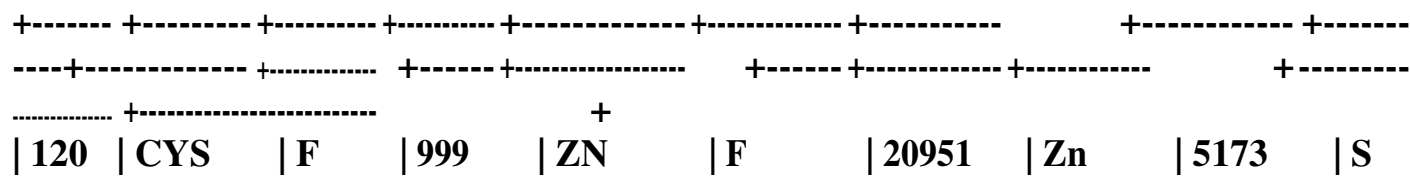

| $4 \quad|2.31|$ protein.sidechain $|7.13|$ tetrahedral $|1 \quad| 144.749,107.455,64.104$ | 145.599, 109.413, 63.216 |

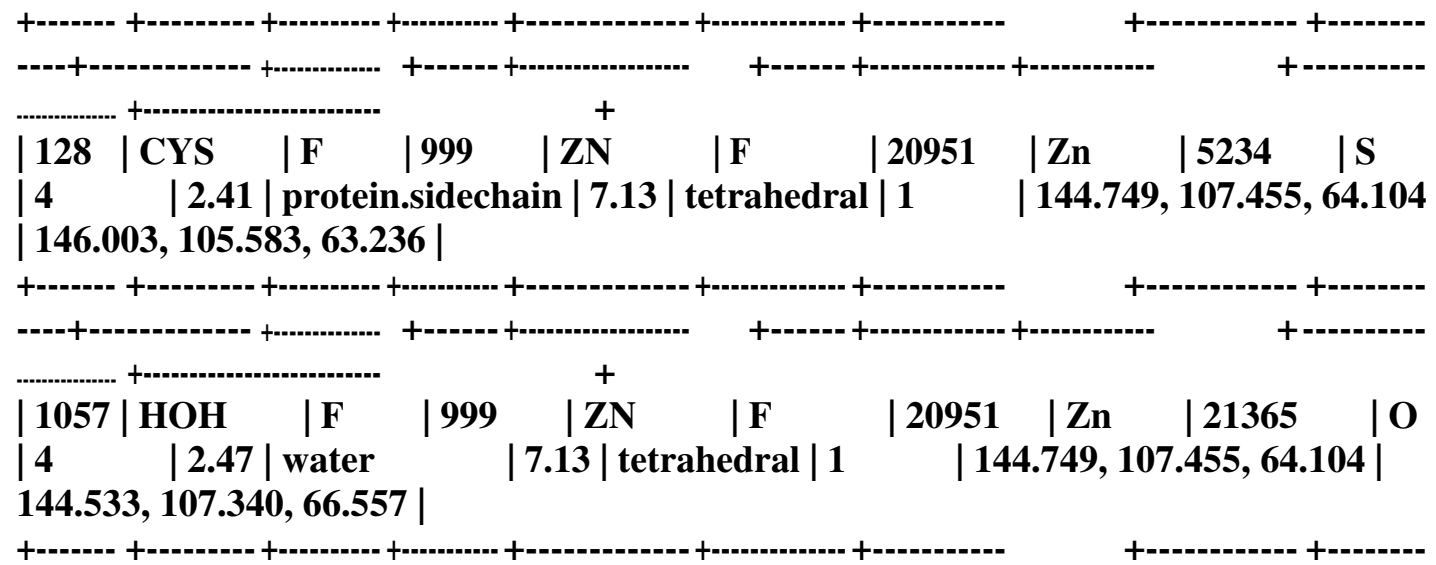



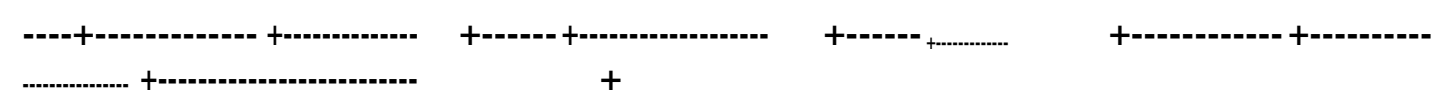

ZN:G:998 (ZN) - ION

Interacting chain(s): G

**Metal Complexes**

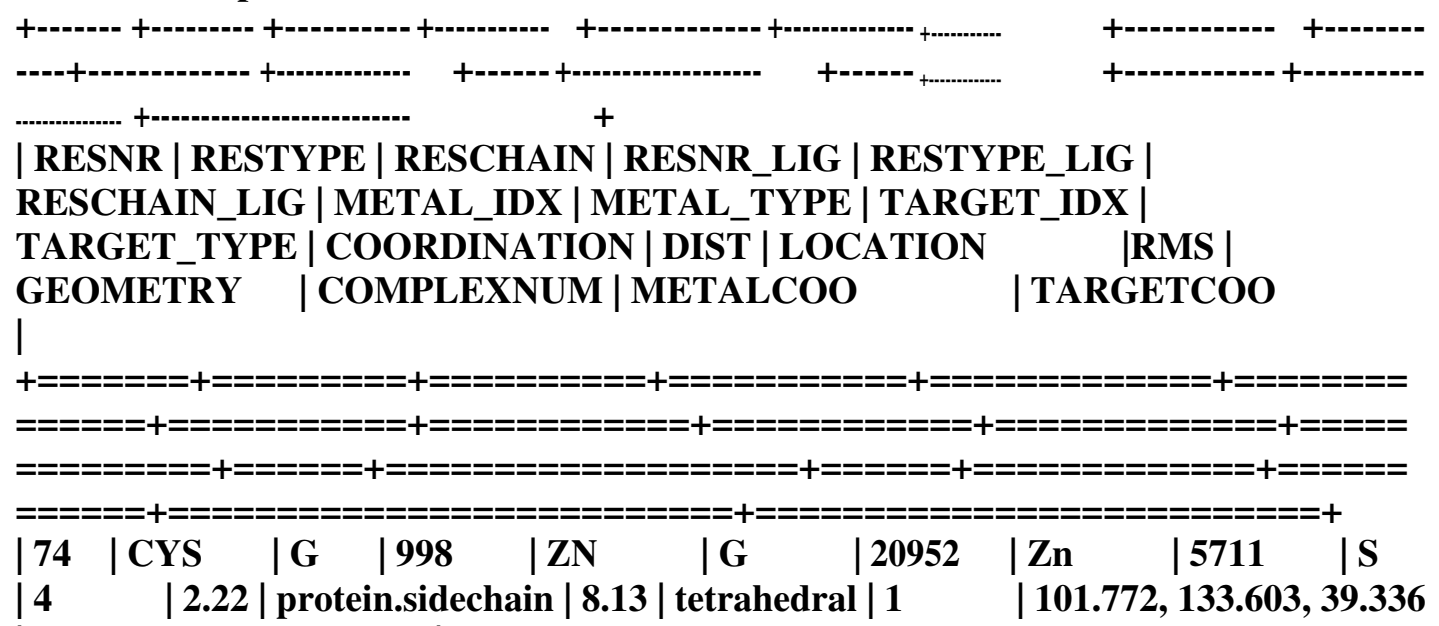
| 100.475, 135.398, 39.149|

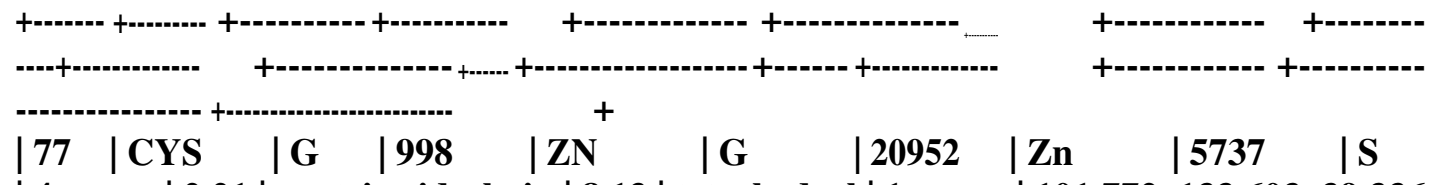

| $4|2.21|$ protein.sidechain $|8.13|$ tetrahedral | $1 \quad \mid 101.772,133.603,39.336$ | 101.451, 132.007, 37.848 |

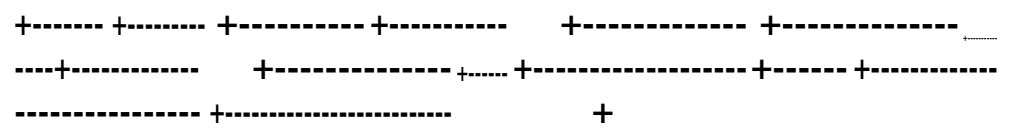

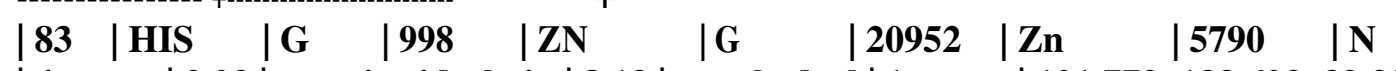

| $4|2.03|$ protein.sidechain $|8.13|$ tetrahedral $|1 \quad| 101.772,133.603,39.336$ | 103.711, 134.136, 39.084 |

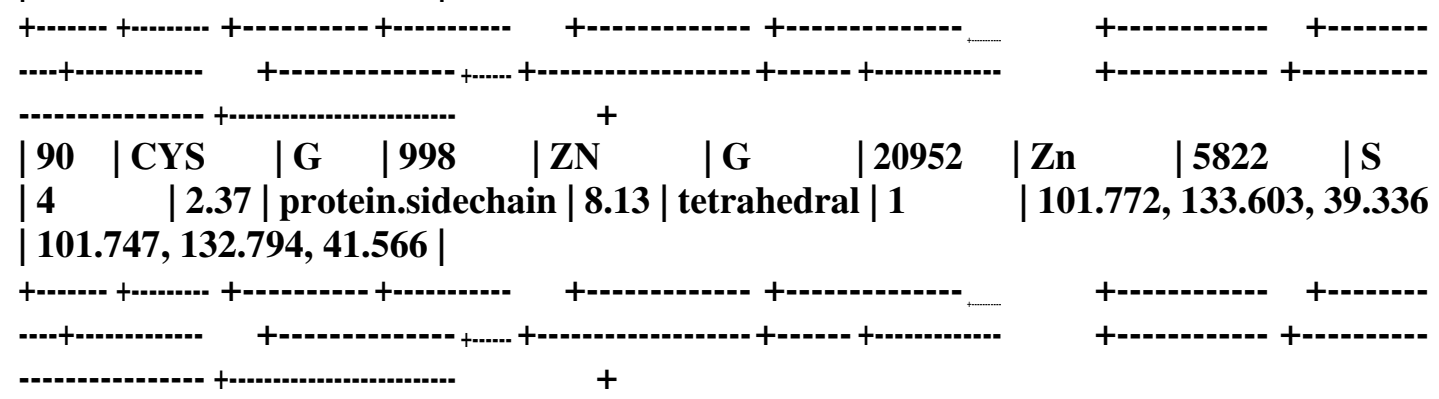

ZN:G:999 (ZN) - ION

Interacting chain(s): G

**Metal Complexes**
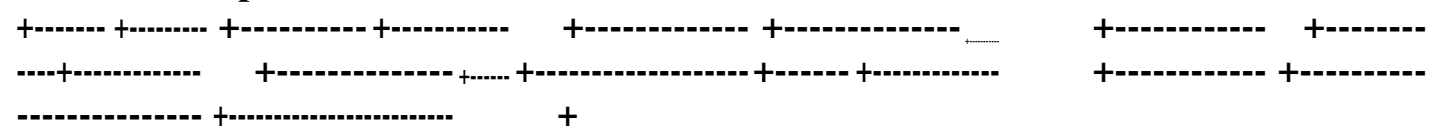
| RESNR | RESTYPE | RESCHAIN | RESNR_LIG | RESTYPE_LIG | RESCHAIN_LIG | METAL_IDX | METAL_TYPE | TARGET_IDX | TARGET_TYPE | COORDINATION | DIST | LOCATION | RMS | GEOMETRY | COMPLEXNUM | METALCOO | TARGETCOO |

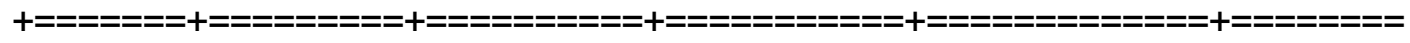

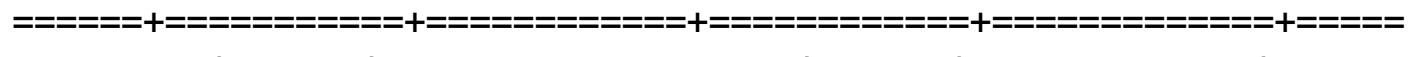

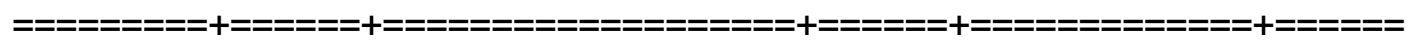

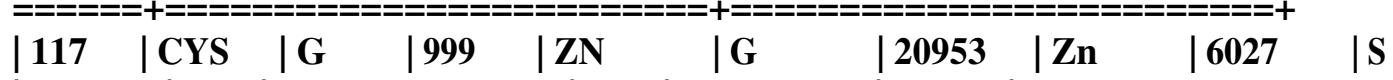

| 4 | 2.36 | protein.sidechain | 4.74 | tetrahedral | $1 \quad \mid$ 90.358, 141.040, 58.747 | 92.674, 141.224, 58.353

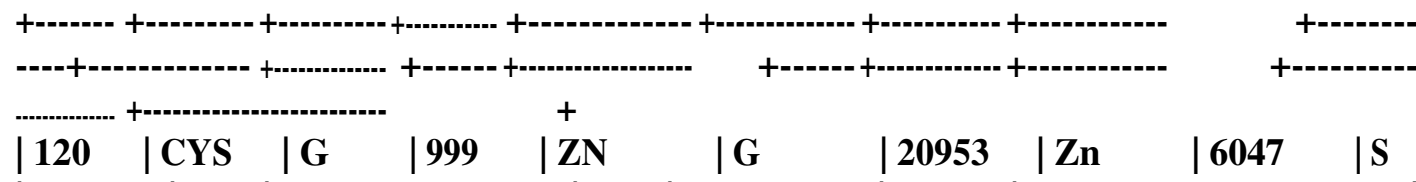

| 4 | 2.39| protein.sidechain | 4.74| tetrahedral | $1 \quad \mid$ 90.358, 141.040, 58.747 | 89.631, 138.849, 58.124 |

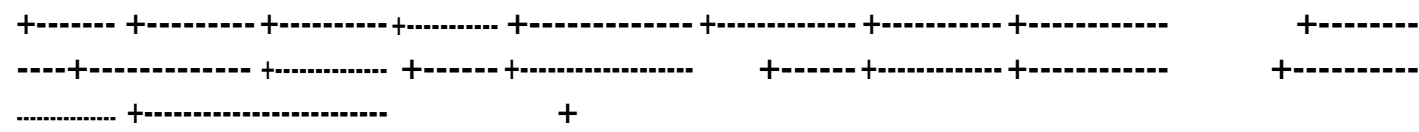

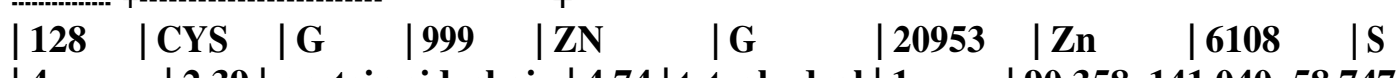
| 4 | 2.39| protein.sidechain $|4.74|$ tetrahedral | $1 \quad \mid$ 90.358, 141.040, 58.747 | 89.253, 142.741, 57.484

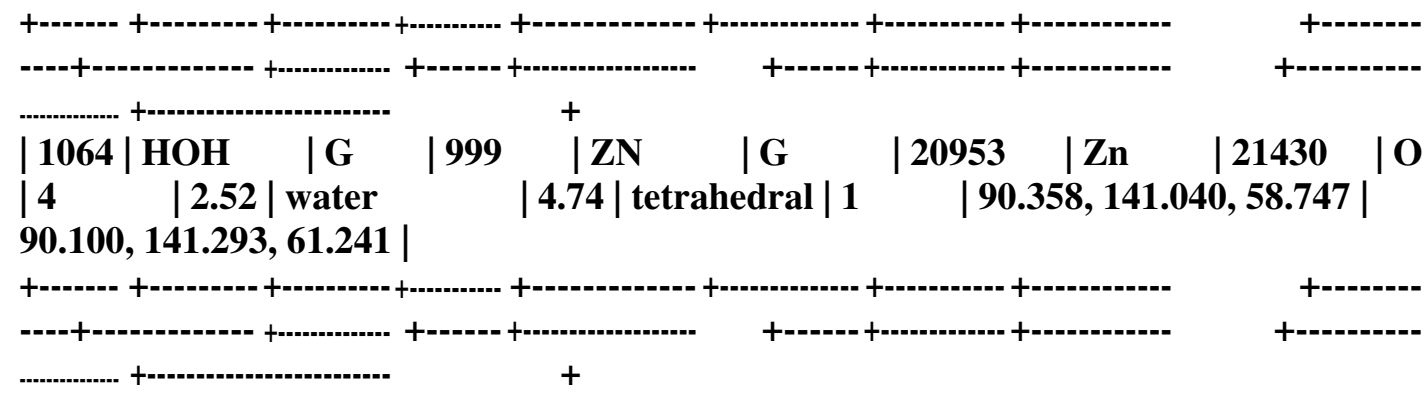

ZN:H:998 (ZN) - ION

Interacting chain(s): H

**Metal Complexes**

+-

| RESNR | RESTYPE | RESCHAIN | RESNR_LIG | RESTYPE_LIG |

RESCHAIN_LIG | METAL_IDX | METAL_TYPE | TARGET_IDX |

TARGET_TYPE | COORDINATION | DIST | LOCATION |RMS |

GEOMETRY | COMPLEXNUM|METALCOO | TARGETCOO

|

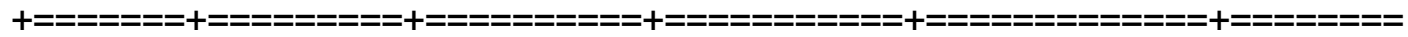

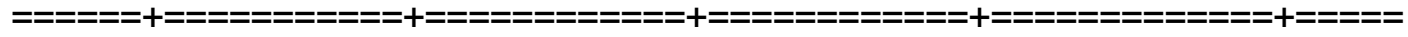

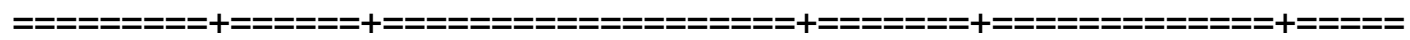

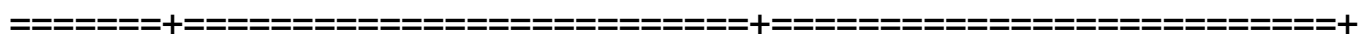

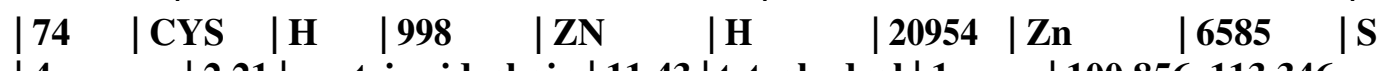

| 4 | 2.21 | protein.sidechain | $11.43 \mid$ tetrahedral | $1 \quad \mid$ 100.856, 113.346,

40.447 | 99.446, 111.645, 40.544

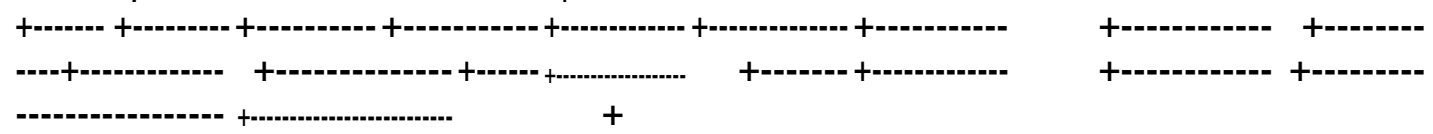




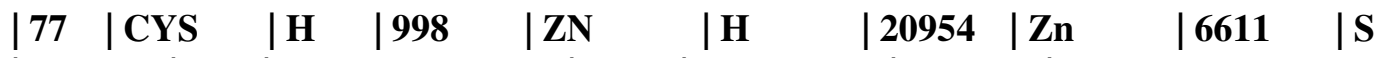

| $4 \quad$ 2.26 | protein.sidechain | 11.43 | tetrahedral | $1 \quad$ | 100.856, 113.346,

40.447| 100.513, 115.048, 41.899|

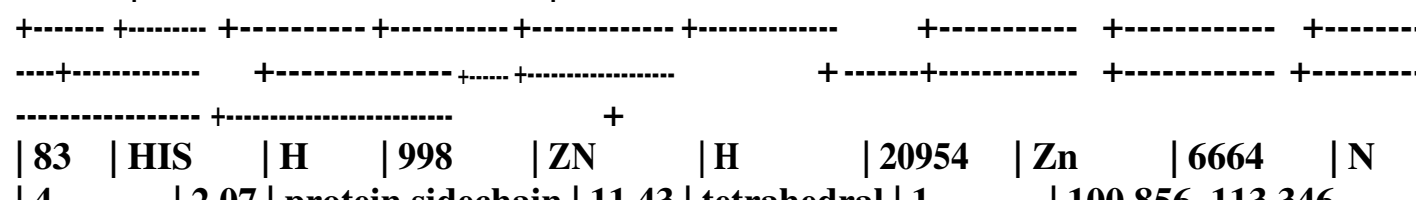

| 4 | 2.07| protein.sidechain | $11.43 \mid$ tetrahedral | $1 \quad$ | 100.856, 113.346, 40.447 | 102.751, 112.566, 40.765 |

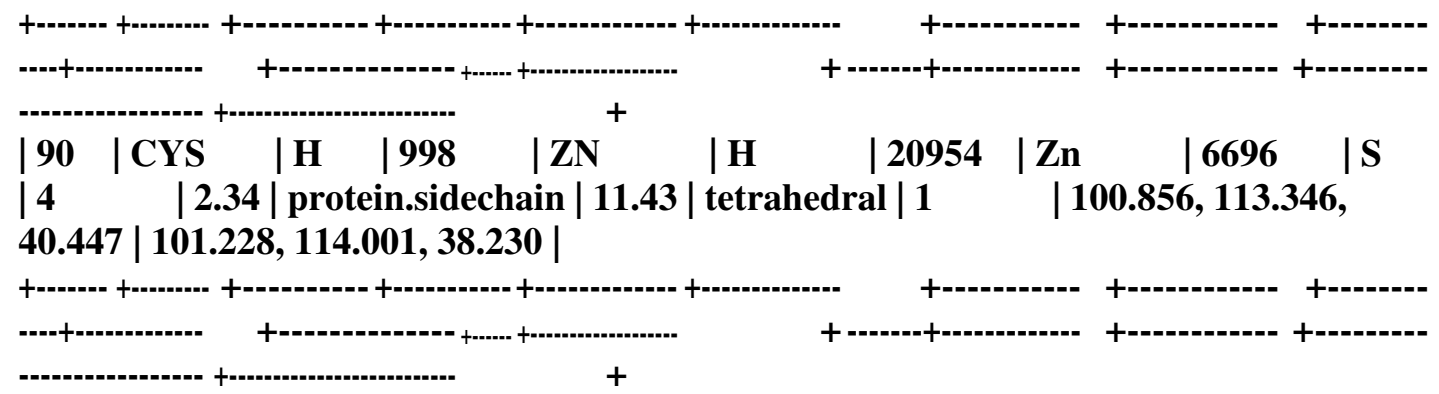

\section{ZN:H:999 (ZN) - ION}

Interacting chain(s): H

**Metal Complexes**

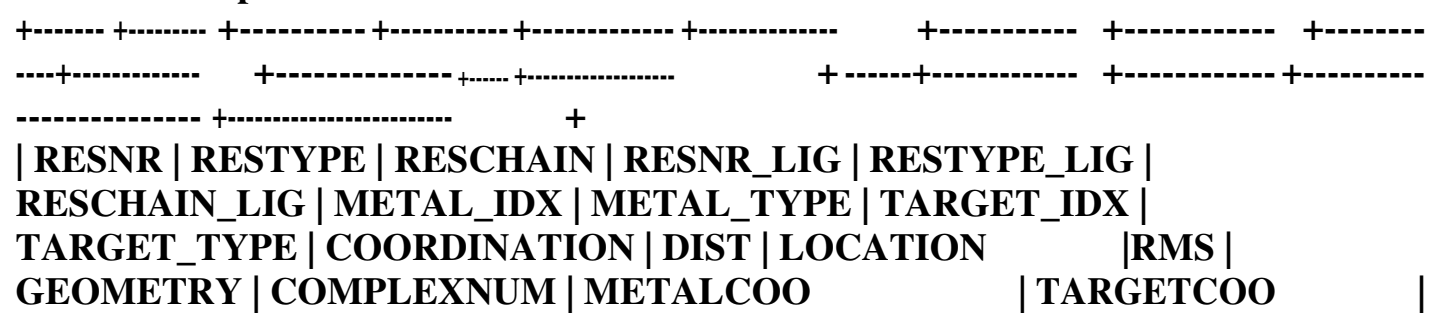

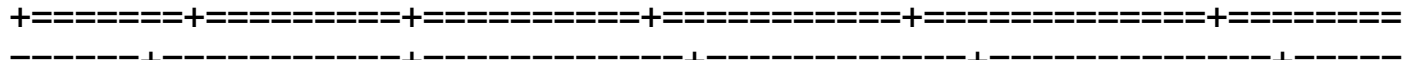

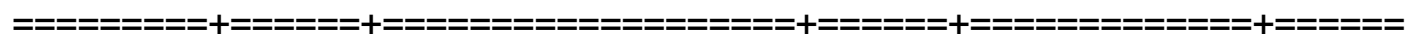

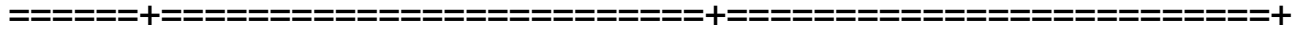

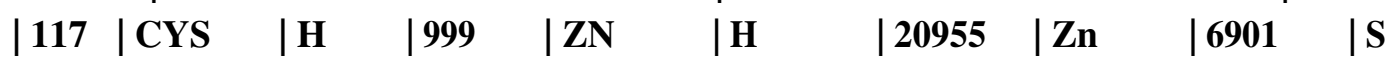

| 4 | 2.32 | protein.sidechain | 6.69| tetrahedral | $1 \quad|91.822,106.447,19.838|$ 93.984, 106.182, 20.634

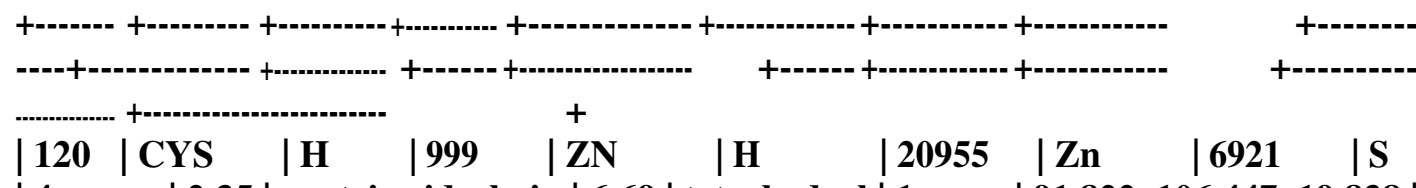

| 4 | 2.35 | protein.sidechain | $6.69 \mid$ tetrahedral | $1 \quad|91.822,106.447,19.838|$ 91.103, 108.567, 20.555 |

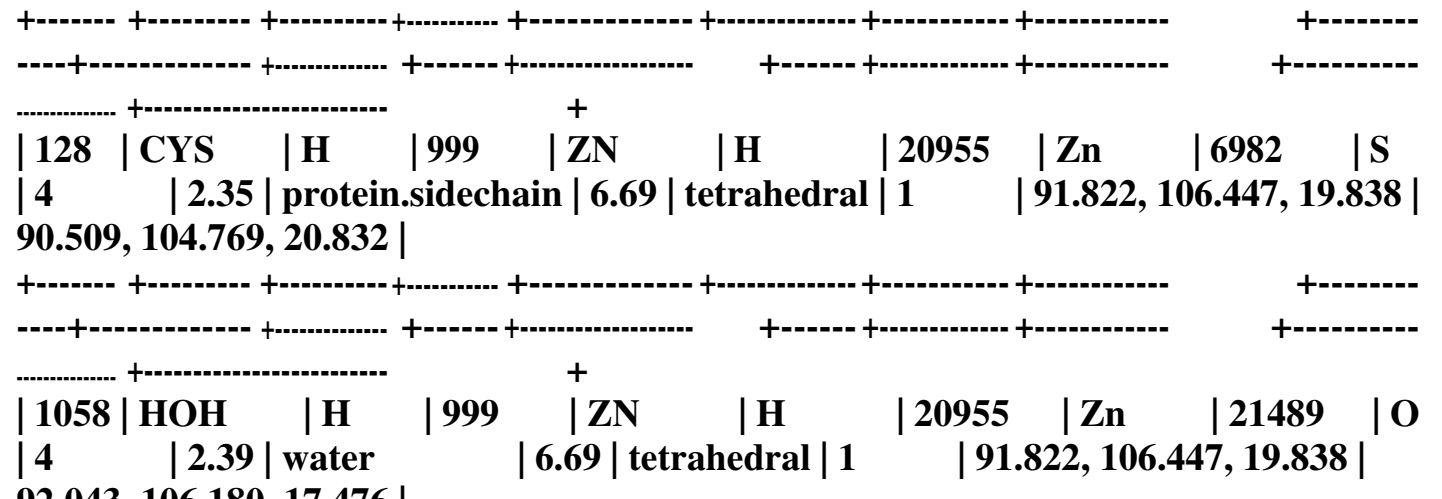




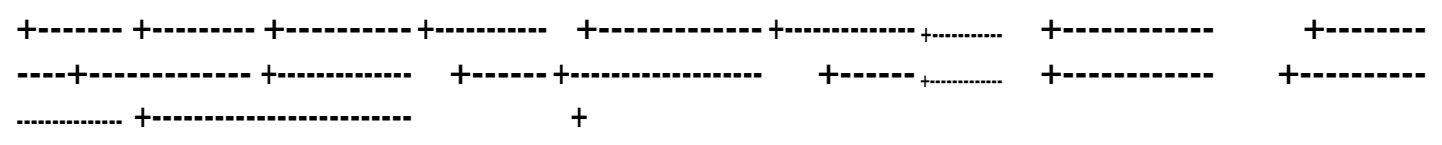

ZN:I:998 (ZN) - ION

Interacting chain(s): I

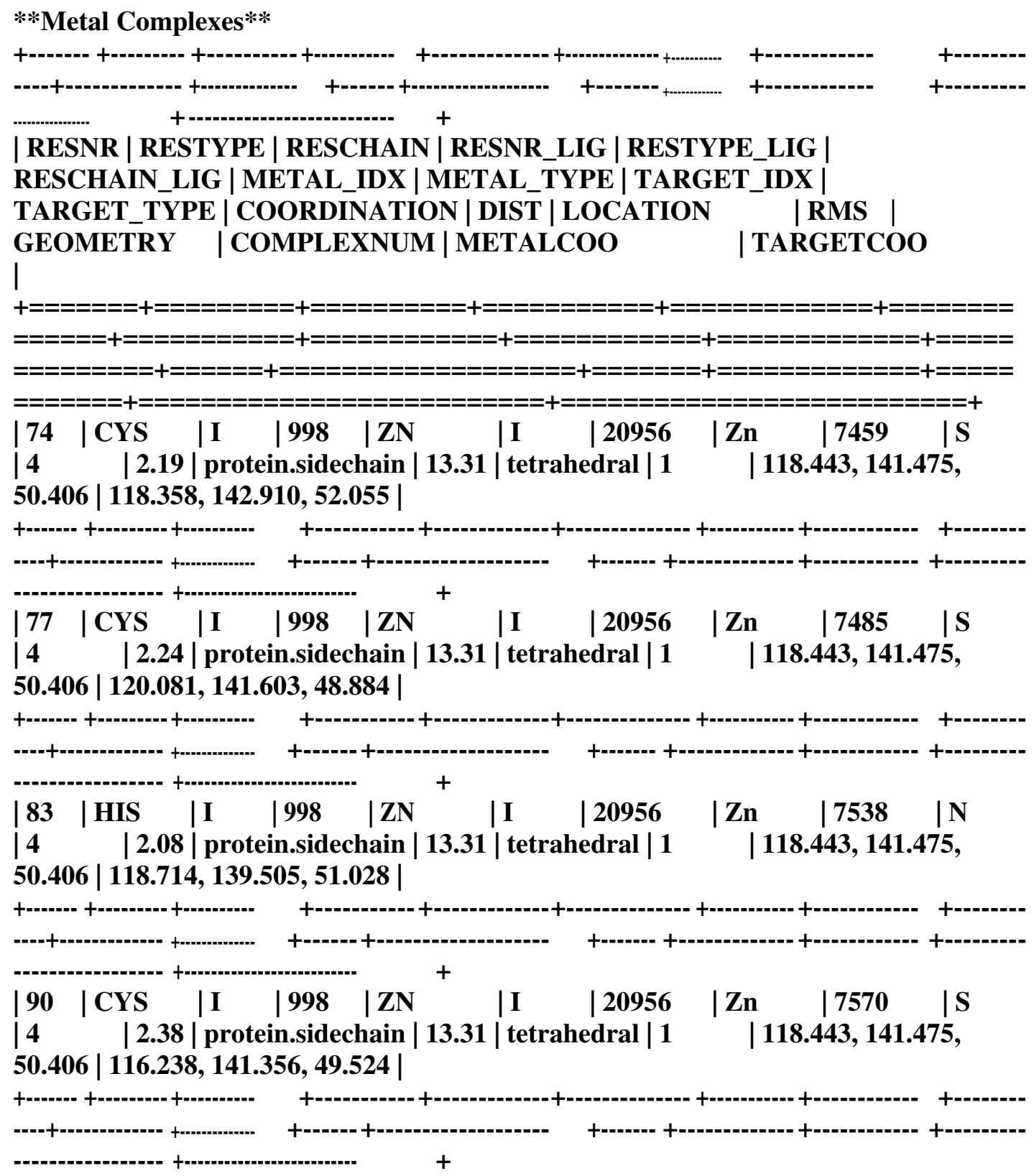

ZN:I:999 (ZN) - ION

Interacting chain(s): I

**Metal Complexes**

+-----. +---------+------.-.

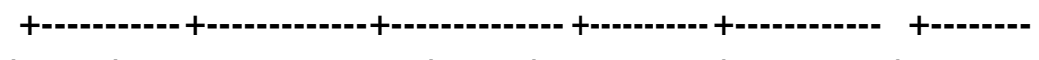


| RESNR | RESTYPE | RESCHAIN | RESNR_LIG | RESTYPE_LIG | RESCHAIN_LIG | METAL_IDX | METAL_TYPE | TARGET_IDX |

TARGET_TYPE | COORDINATION | DIST | LOCATION | RMS | GEOMETRY | COMPLEXNUM | METALCOO

| TARGETCOO

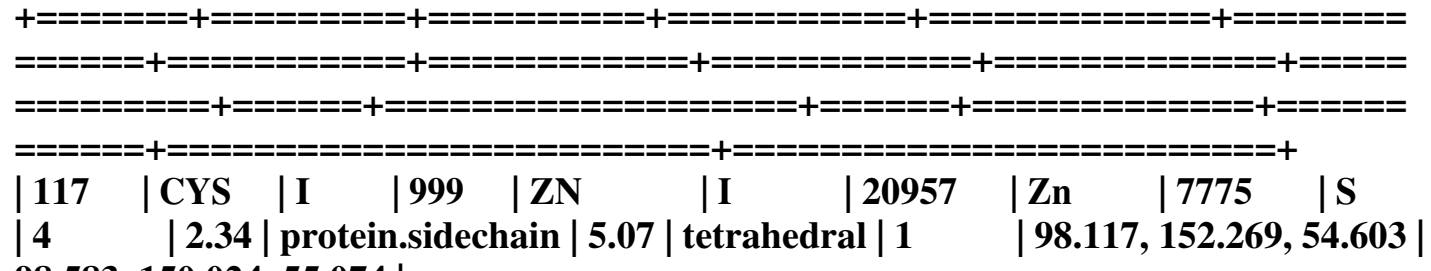
98.583, 150.024, 55.074 |

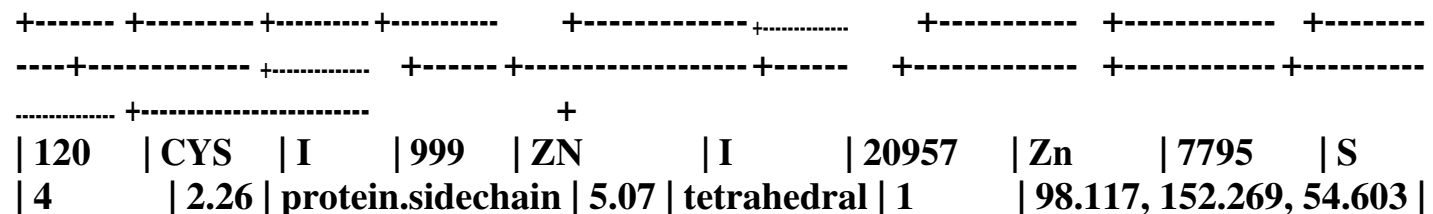
98.982, 152.902, 52.616

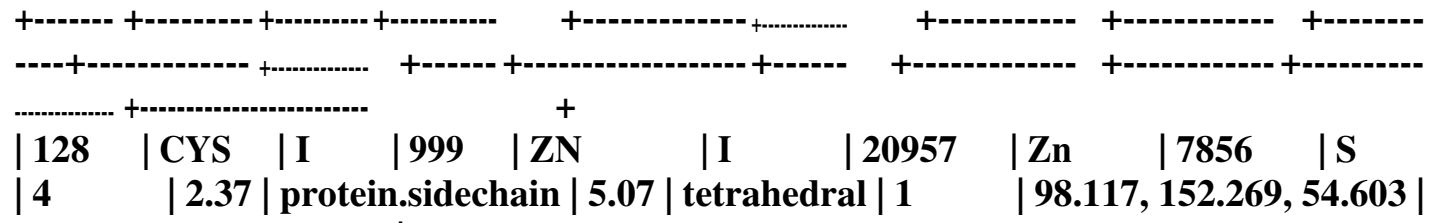
99.036, 153.495, 56.409 |

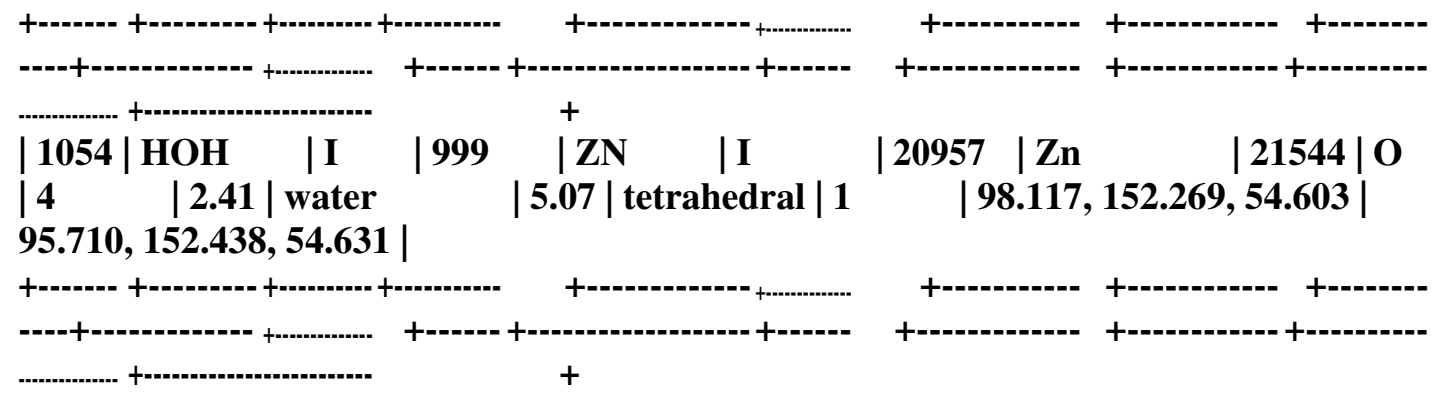

ZN:J:998 (ZN) - ION

Interacting chain(s): J

**Metal Complexes**

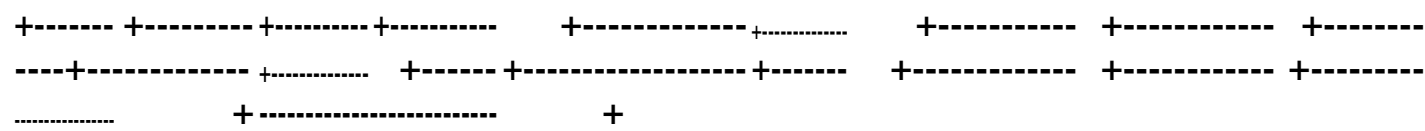

| RESNR | RESTYPE | RESCHAIN | RESNR_LIG | RESTYPE_LIG |

RESCHAIN_LIG | METAL_IDX | METAL_TYPE | TARGET_IDX |

TARGET_TYPE | COORDINATION | DIST | LOCATION_ |RMS |

GEOMETRY | COMPLEXNUM | METALCOO | TARGETCOO

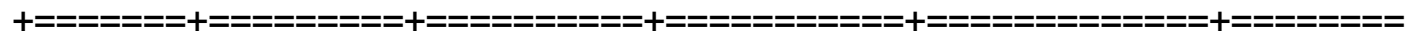

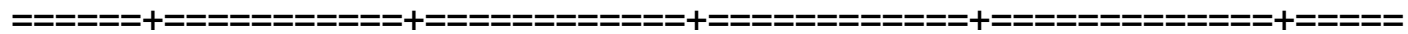

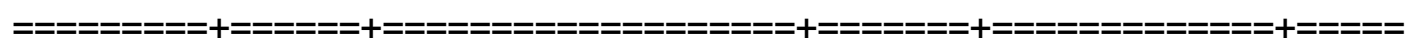

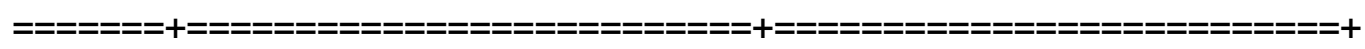

\begin{tabular}{|c|c|}
\hline | CYS | J & | 998 \\
\hline
\end{tabular}

| 4 | $2.21 \mid$ protein.sidechain | 10.46| tetrahedral | $1 \quad \mid 119.837,140.554$,

30.186 | 119.929, 141.789, 28.358 |

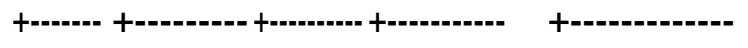

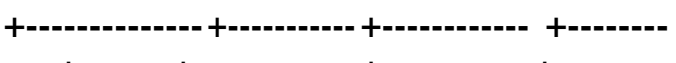




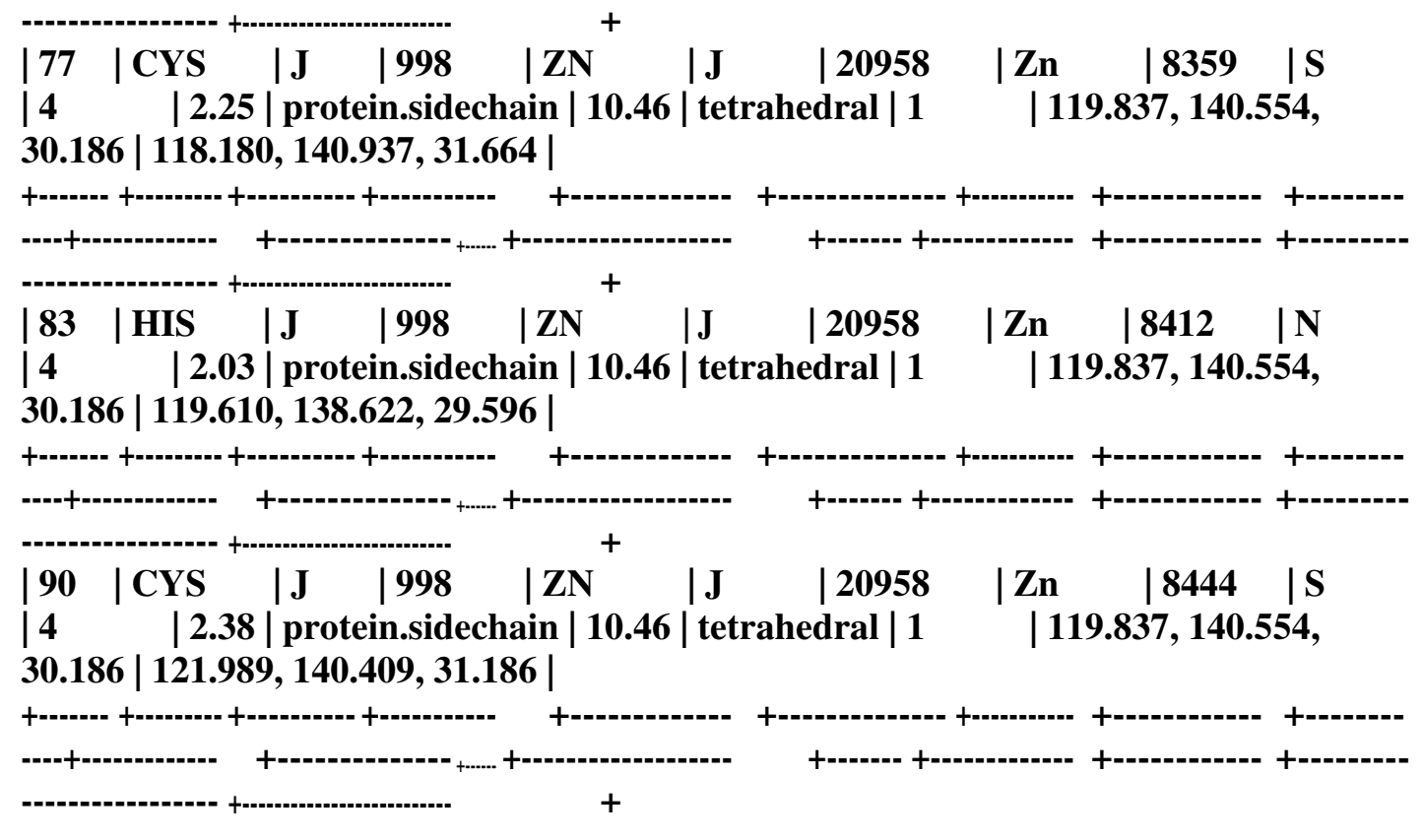

ZN:J:999 (ZN) - ION

Interacting chain(s): J

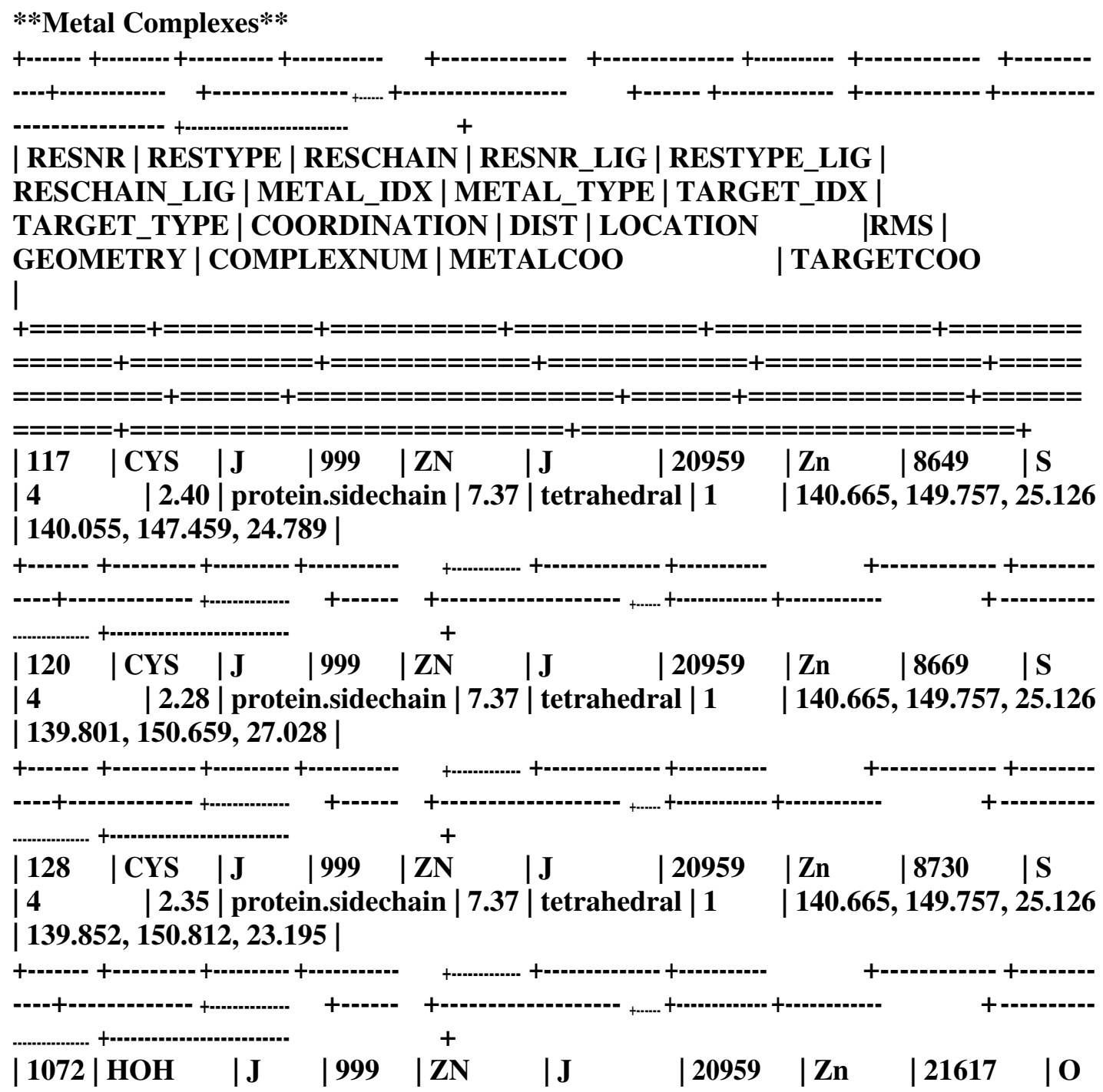




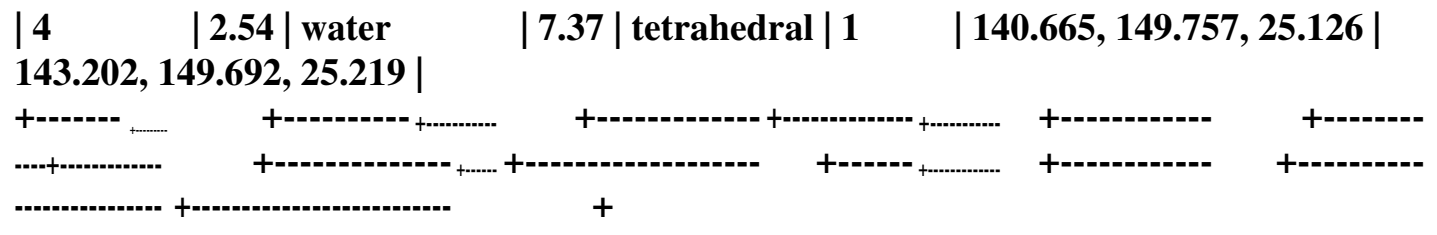

ZN:K:998 (ZN) - ION

Interacting chain(s): K

**Metal Complexes**

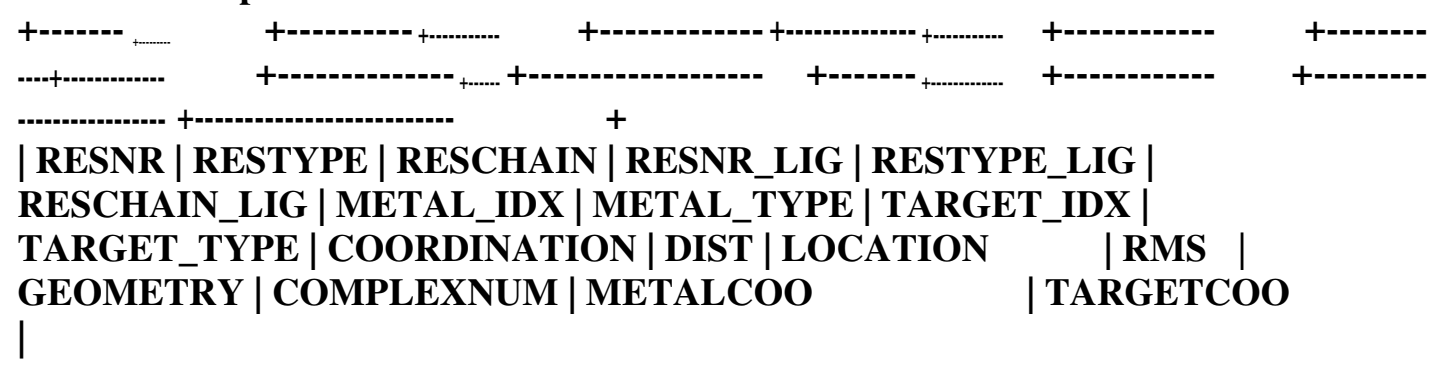

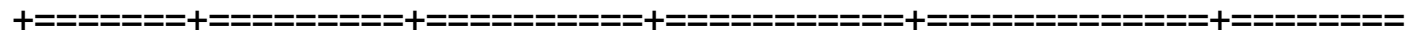

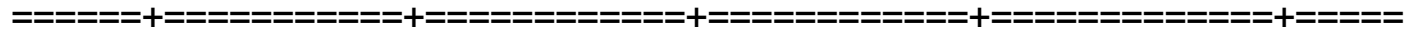

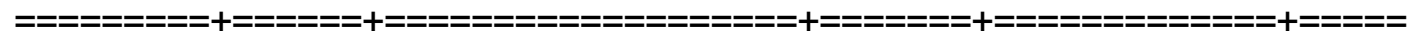

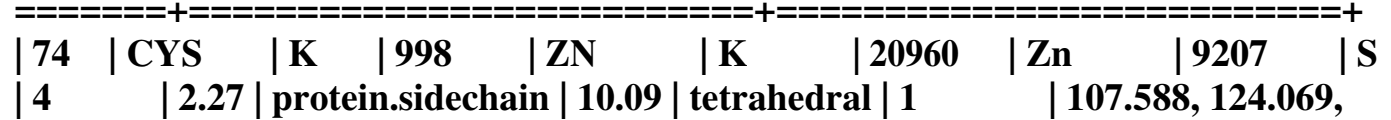

$58.141 \mid$ 105.700, 124.145, 59.403 |

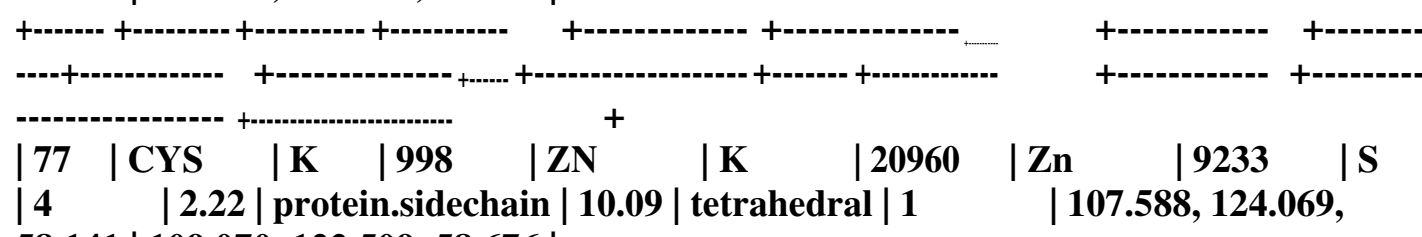

$58.141 \mid$ 109.070, 122.509, 58.676 |

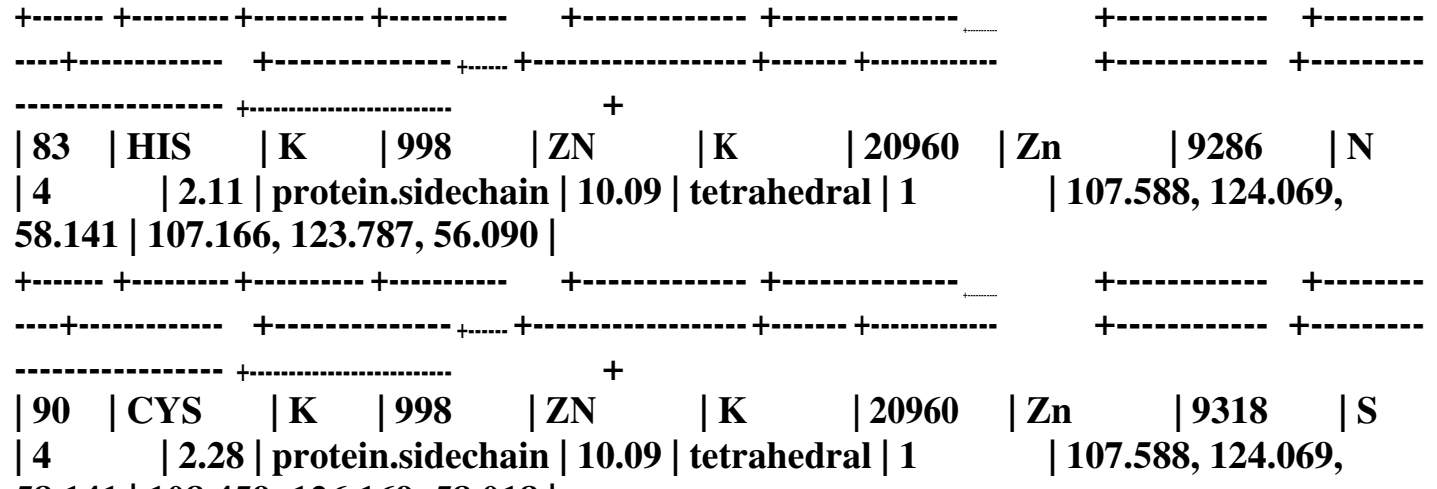

58.141 | 108.459, 126.169, 58.018 |

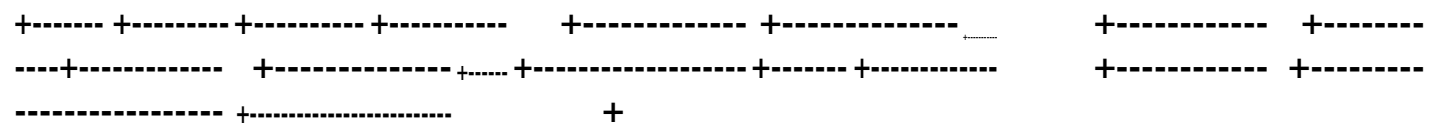

ZN:K:999 (ZN) - ION

Interacting chain(s): K 


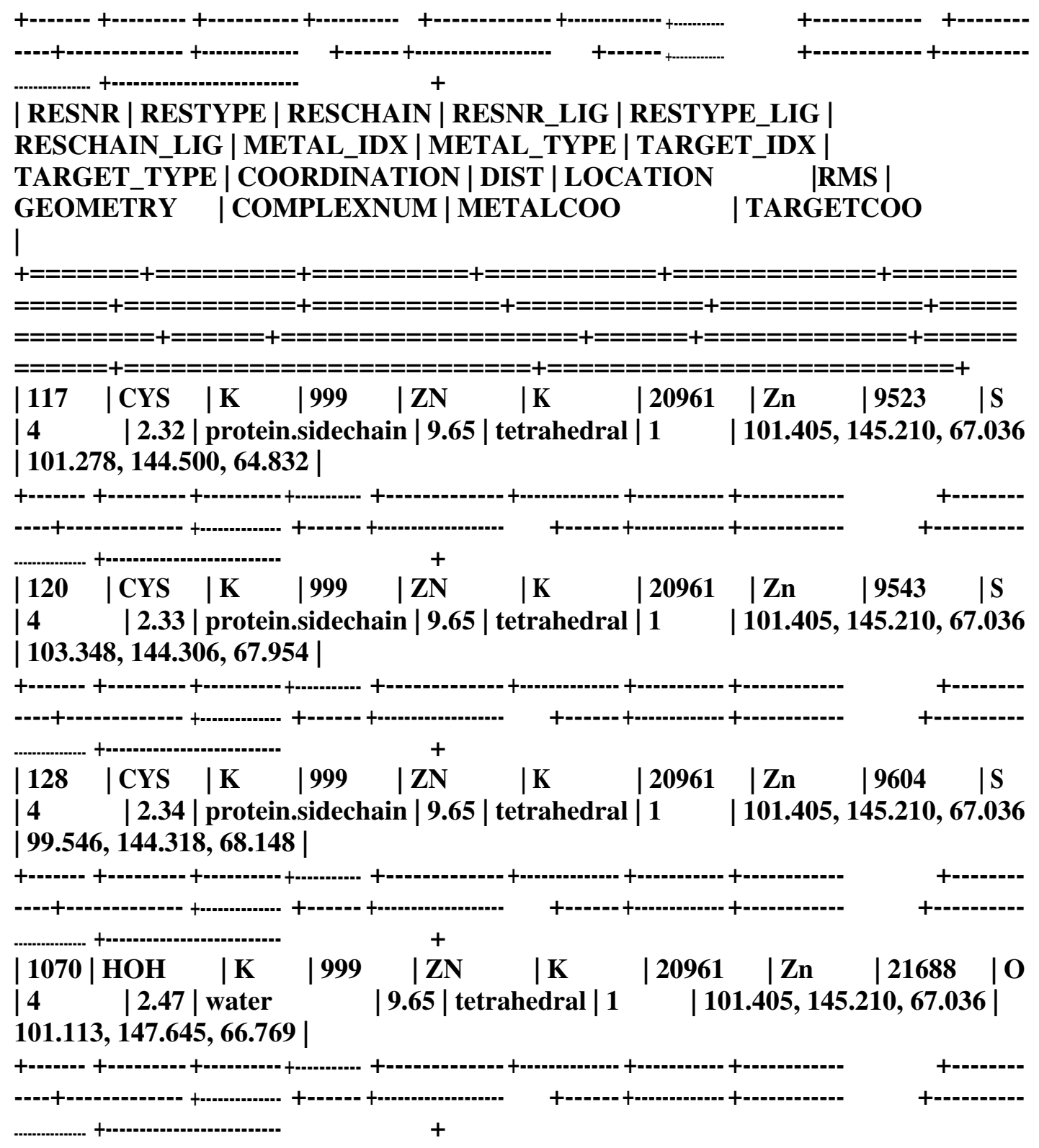

ZN:L:998 (ZN) - ION

Interacting chain(s): L

**Metal Complexes**

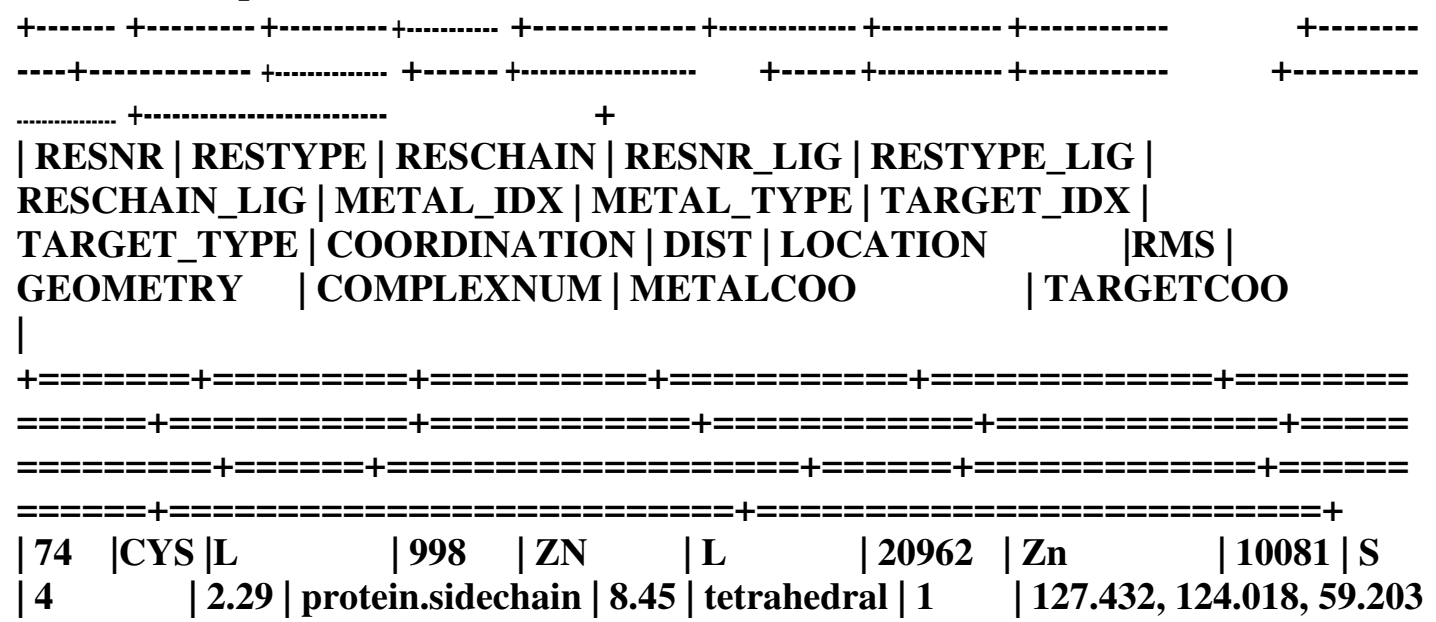




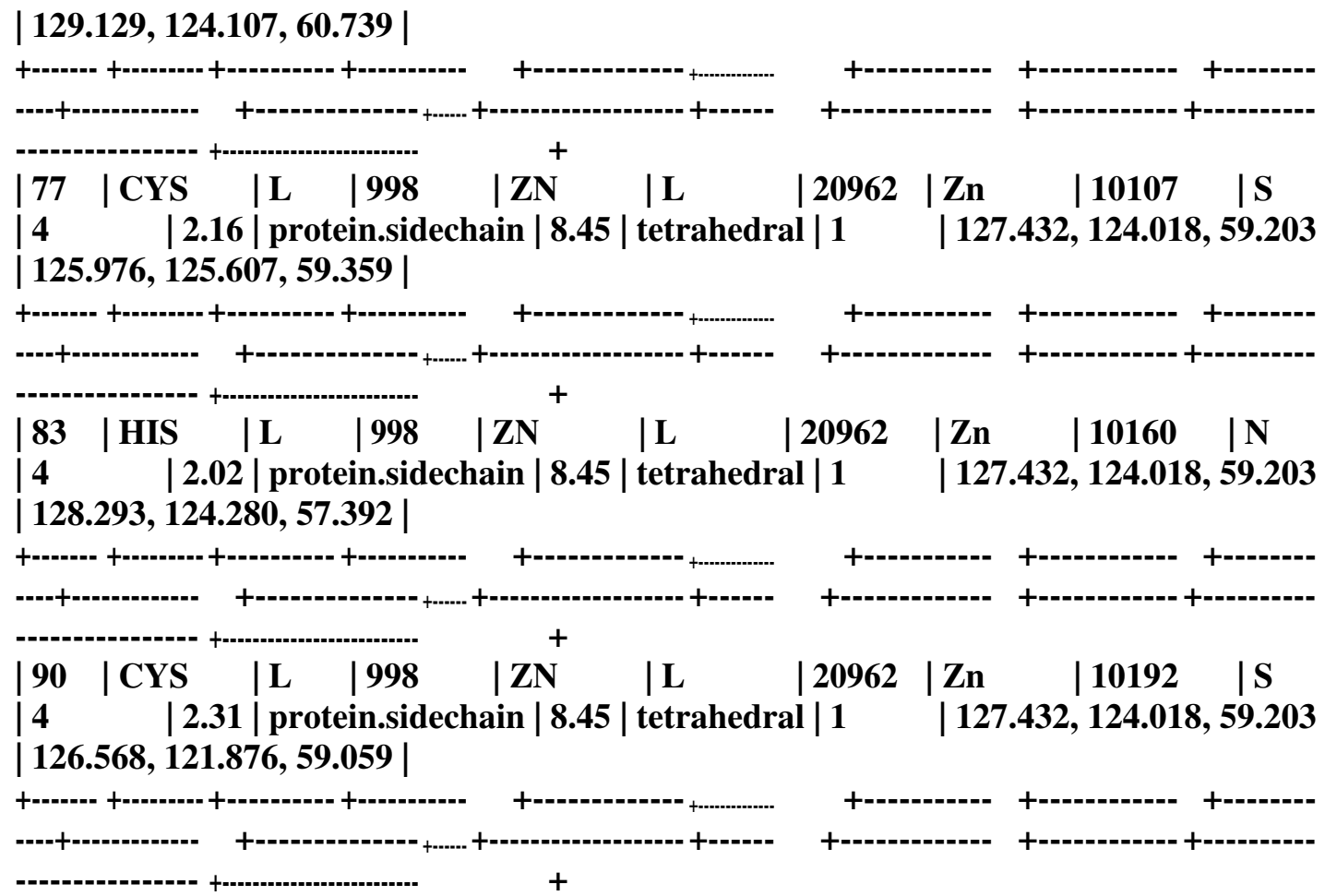

ZN:L:999 (ZN) - ION

Interacting chain(s): L

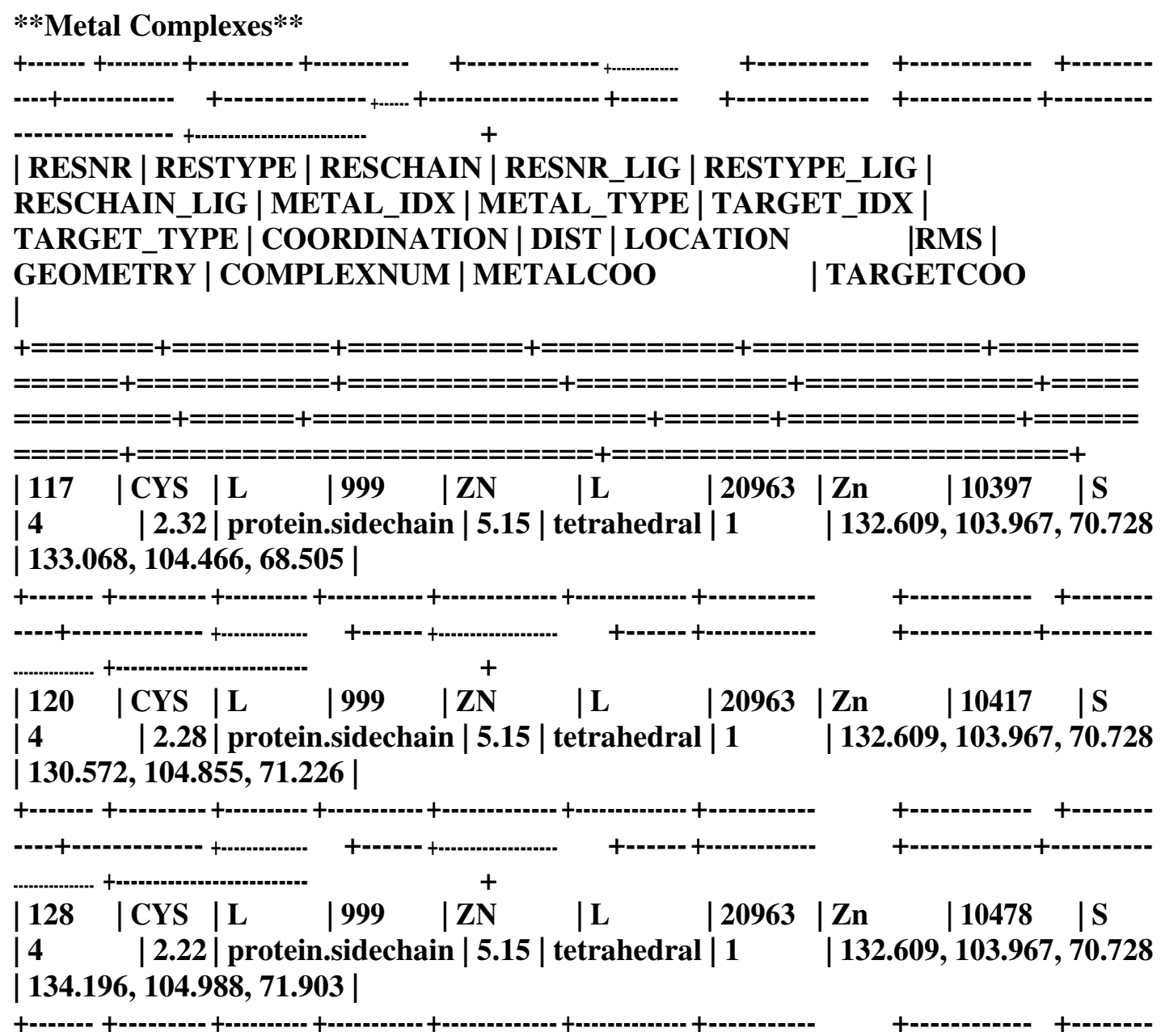




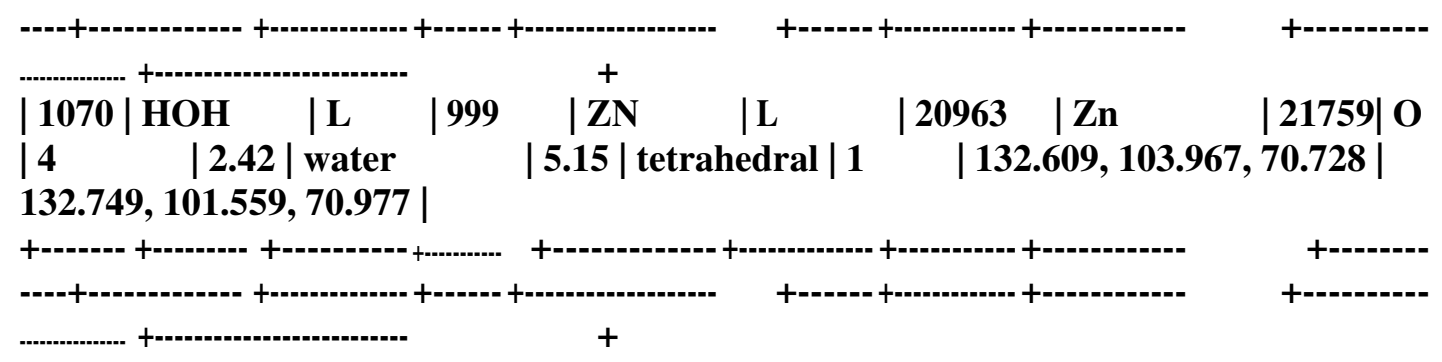

ZN:M:998 (ZN) - ION

Interacting chain(s): $\mathbf{M}$

***Metal Complexes**

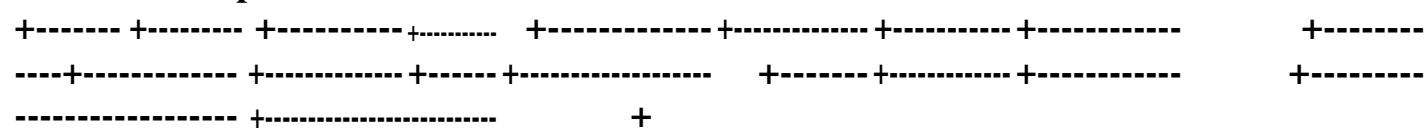

| RESNR | RESTYPE | RESCHAIN | RESNR_LIG | RESTYPE_LIG |

RESCHAIN_LIG | METAL_IDX | METAL_TYPE | TARGET_IDX |

TARGET_TYPE | COORDINATION | DIST | LOCATION |RMS |

GEOMETRY | COMPLEXNUM|METALCOO | TARGETCOO

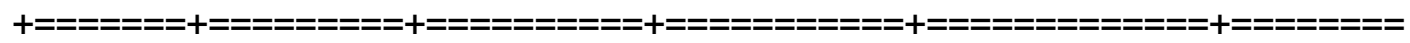

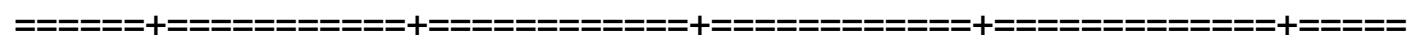

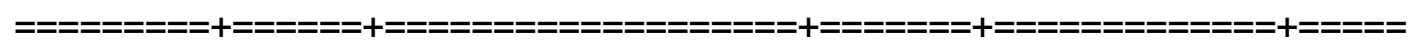

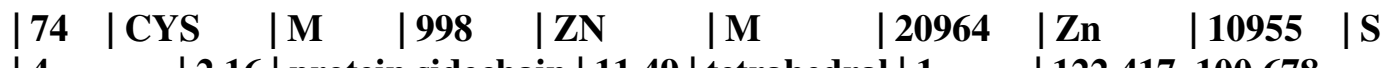

| $4 \quad|2.16|$ protein.sidechain $|11.49|$ tetrahedral $|1 \quad| 122.417,100.678$,

$112.594|122.622,99.428,110.842|$

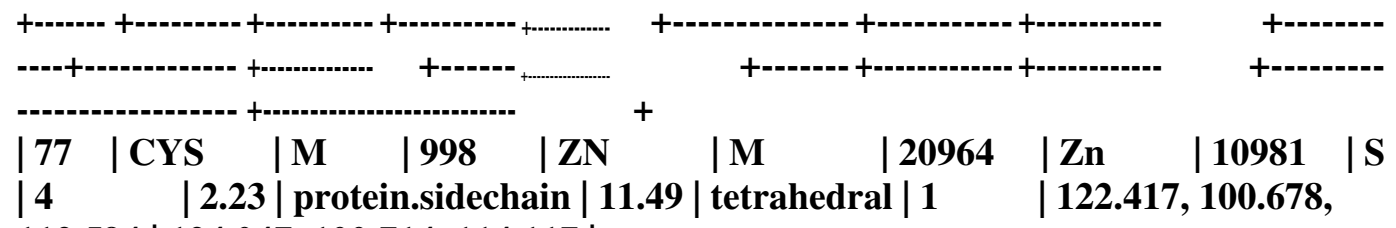

112.594 | 124.047, 100.714, 114.117|

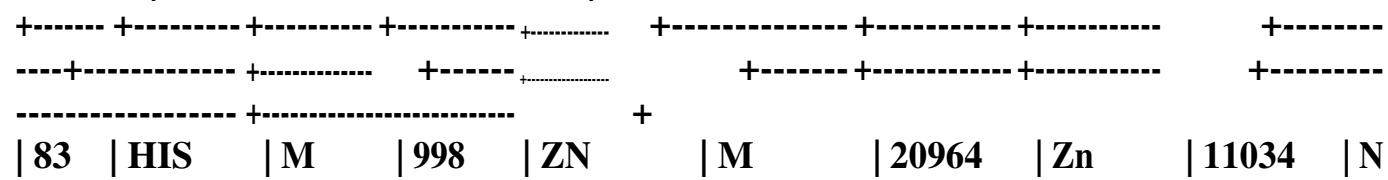

| $4 \quad|2.09|$ protein.sidechain $|11.49|$ tetrahedral $|1 \quad| 122.417,100.678$,

$112.594|122.219,102.604,111.816|$

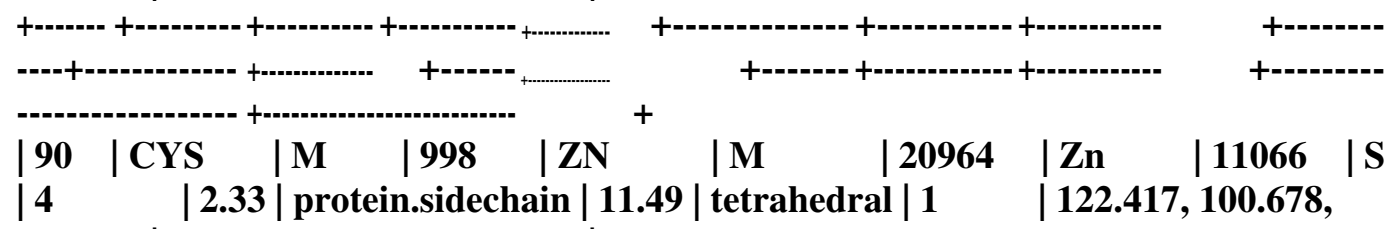

$112.594 \mid$ 120.265, 100.339, 113.422 |

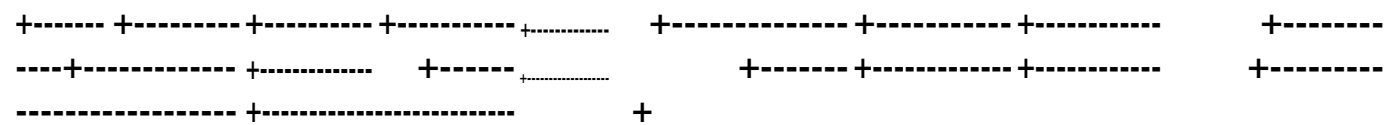

ZN:M:999 (ZN) - ION

Interacting chain(s): $M$ 
**Metal Complexes**

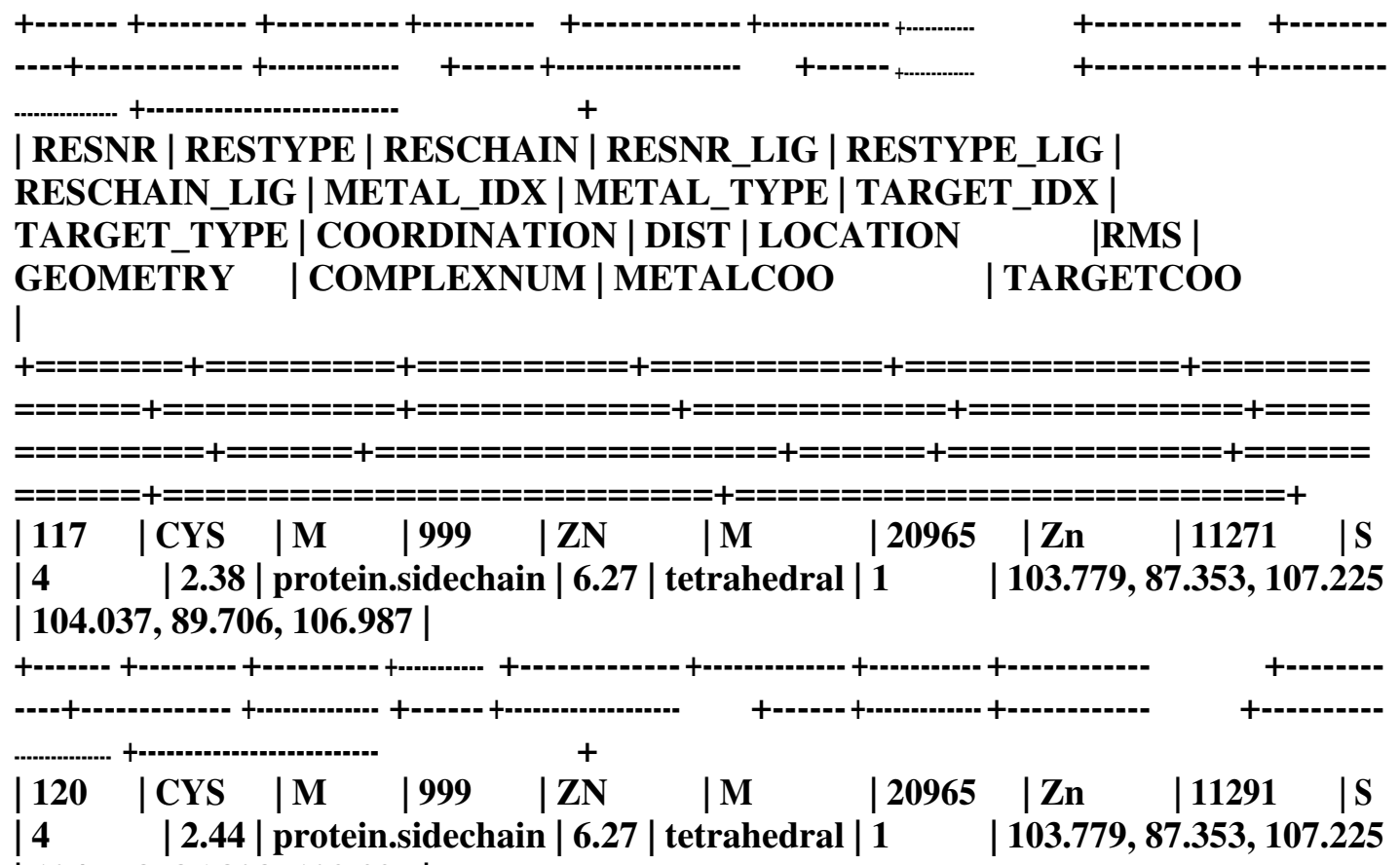

| 104.758, 86.808, 109.395 |

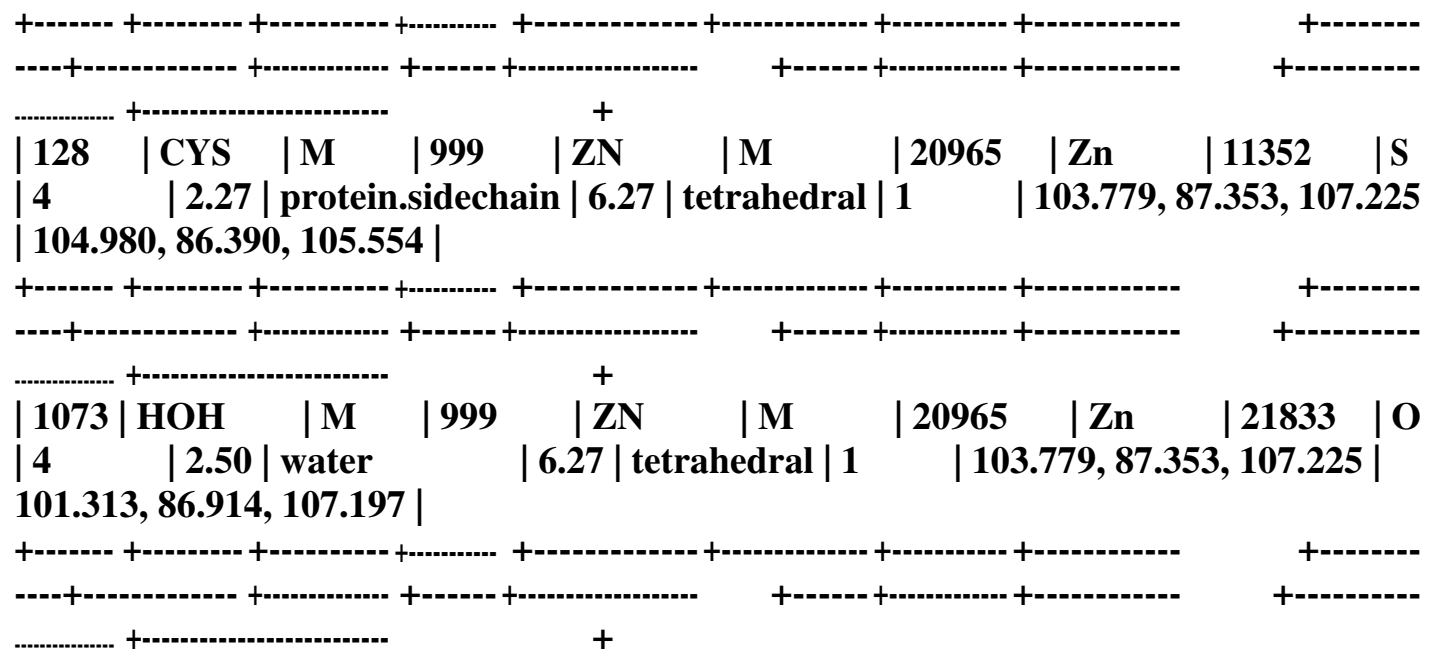

ZN:N:998 (ZN) - ION

Interacting chain(s): $\mathbf{N}$

**Metal Complexes**

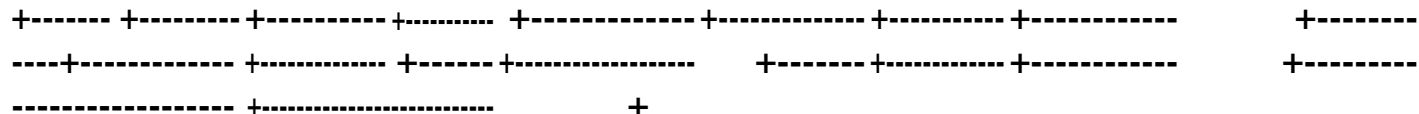

| RESNR | RESTYPE | RESCHAIN | RESNR_LIG | RESTYPE_LIG |

RESCHAIN_LIG | METAL_IDX | METAL_TYPE | TARGET_IDX |

TARGET_TYPE | COORDINATION | DIST $\mid$ LOCATION

GEOMETRY | COMPLEXNUM | METALCOO

|RMS |

|

| TARGETCOO

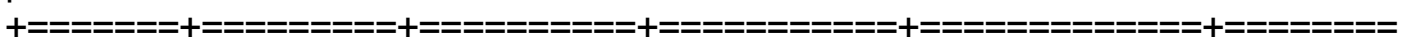

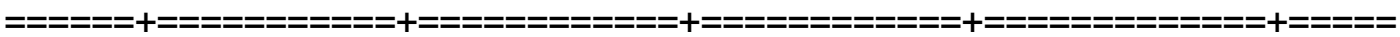




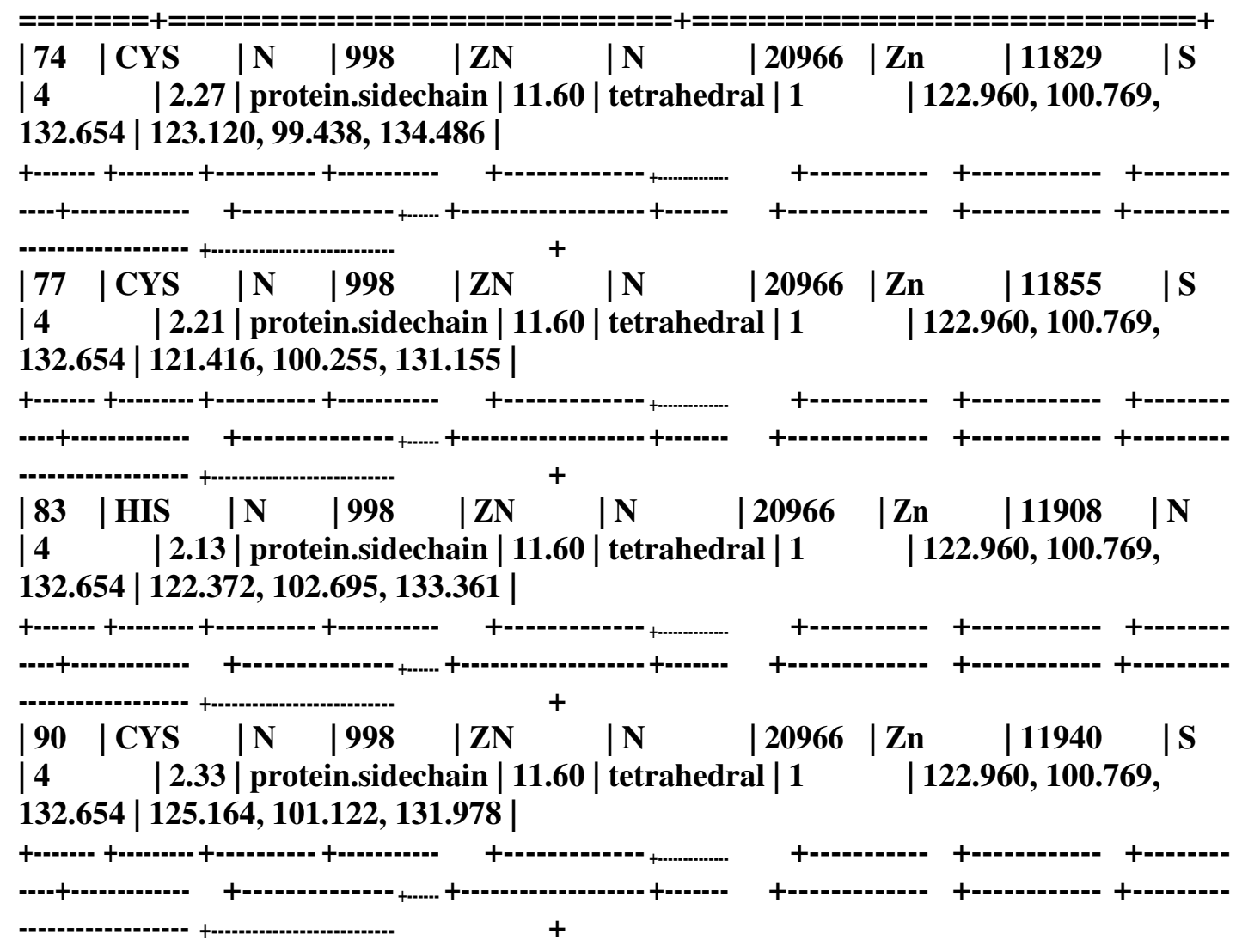

ZN:N:999 (ZN) - ION

Interacting chain(s): $\mathbf{N}$

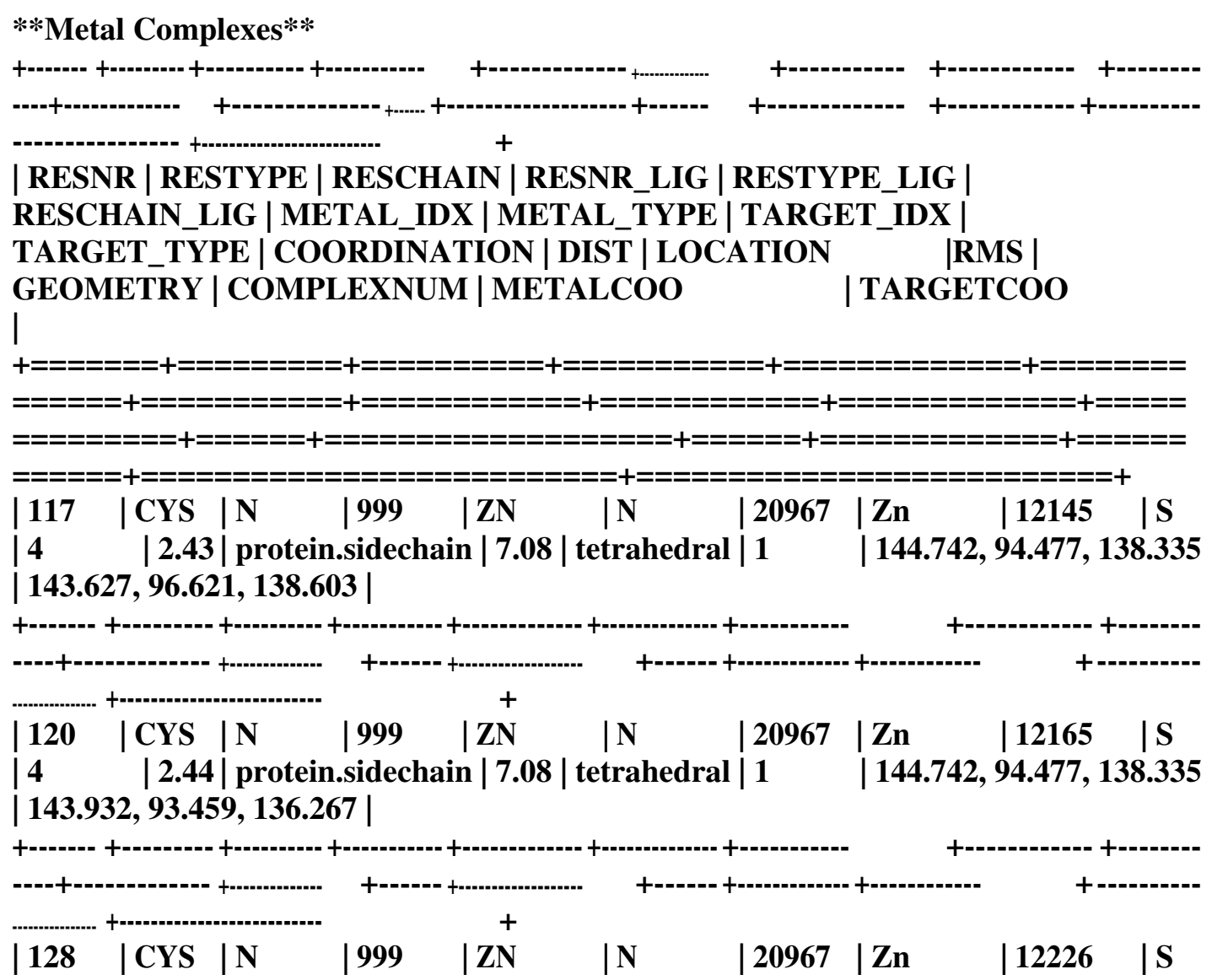




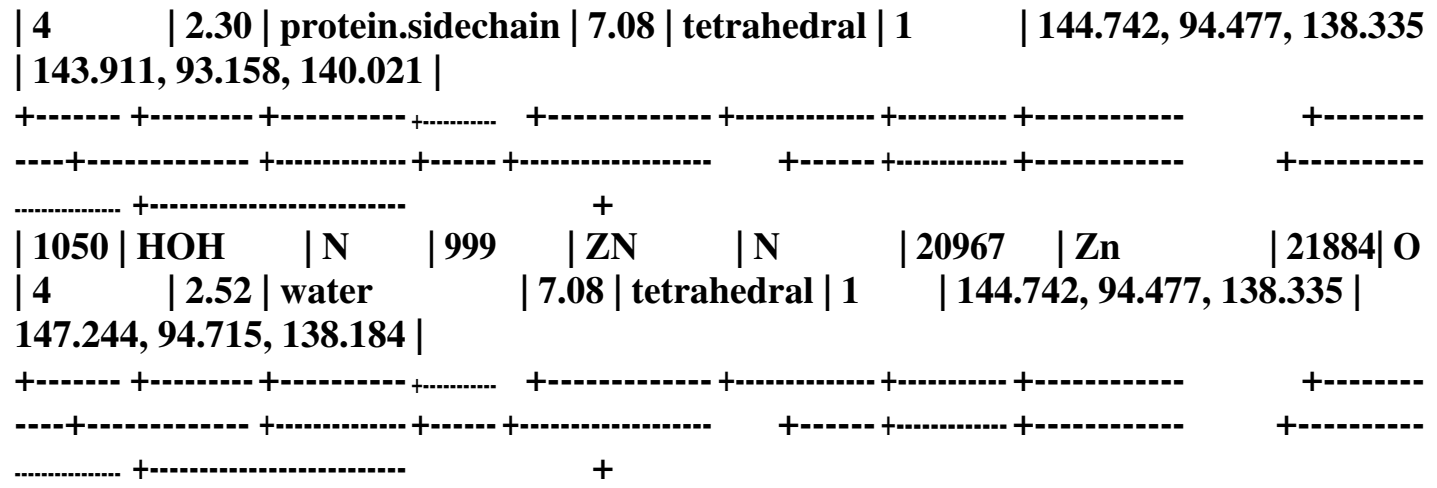

ZN:O:998 (ZN) - ION

Interacting chain(s): O

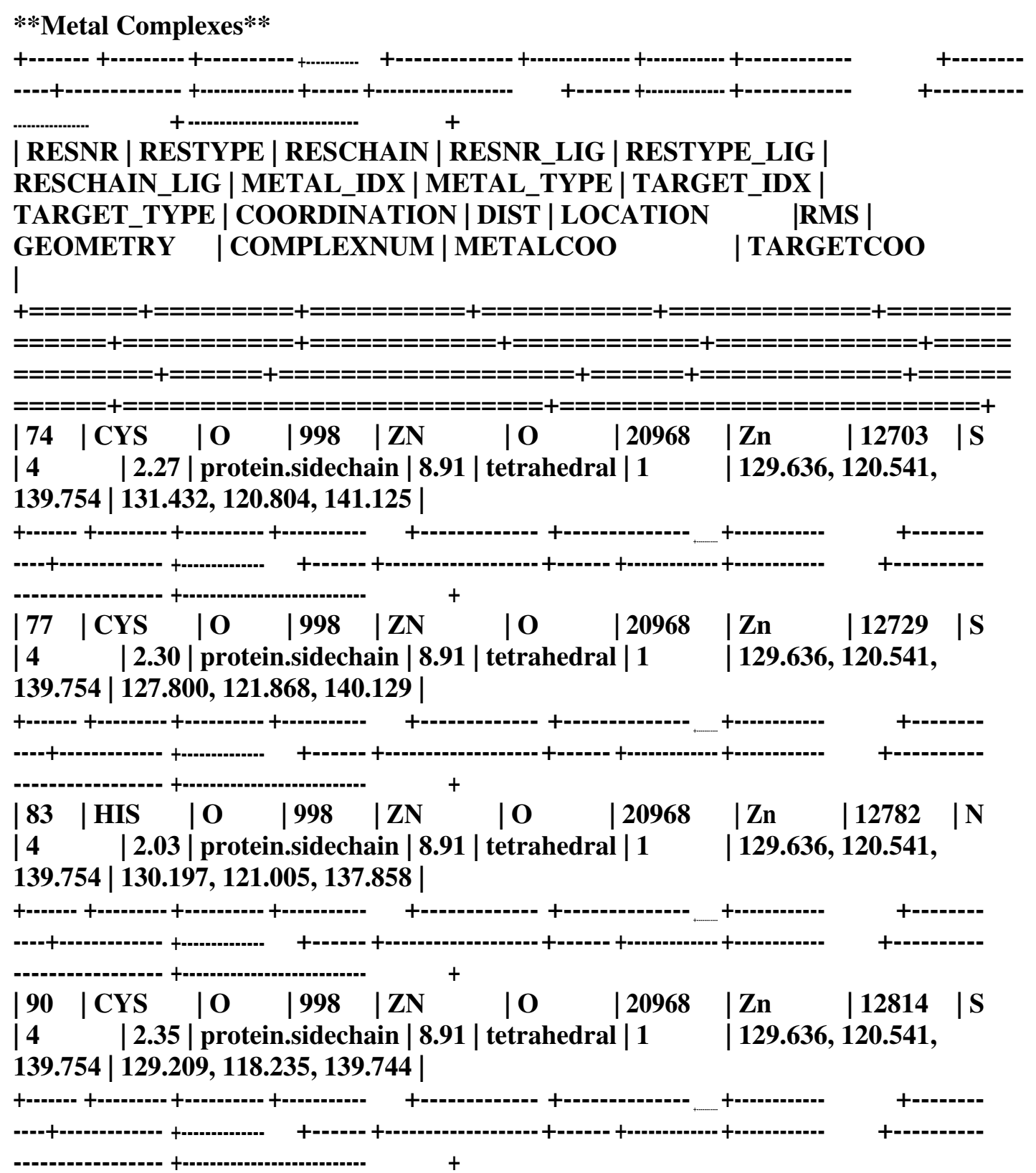


ZN:O:999 (ZN) - ION

Interacting chain(s): O

**Metal Complexes**

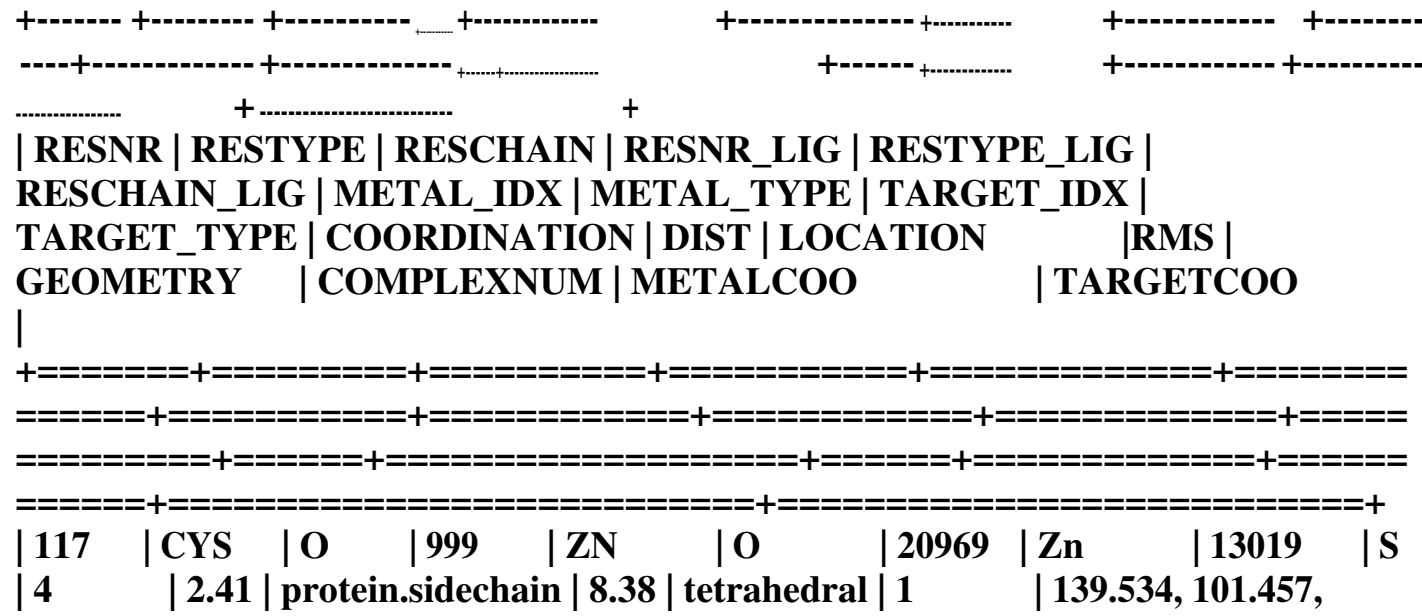

$149.706|139.644,102.162,147.409|$
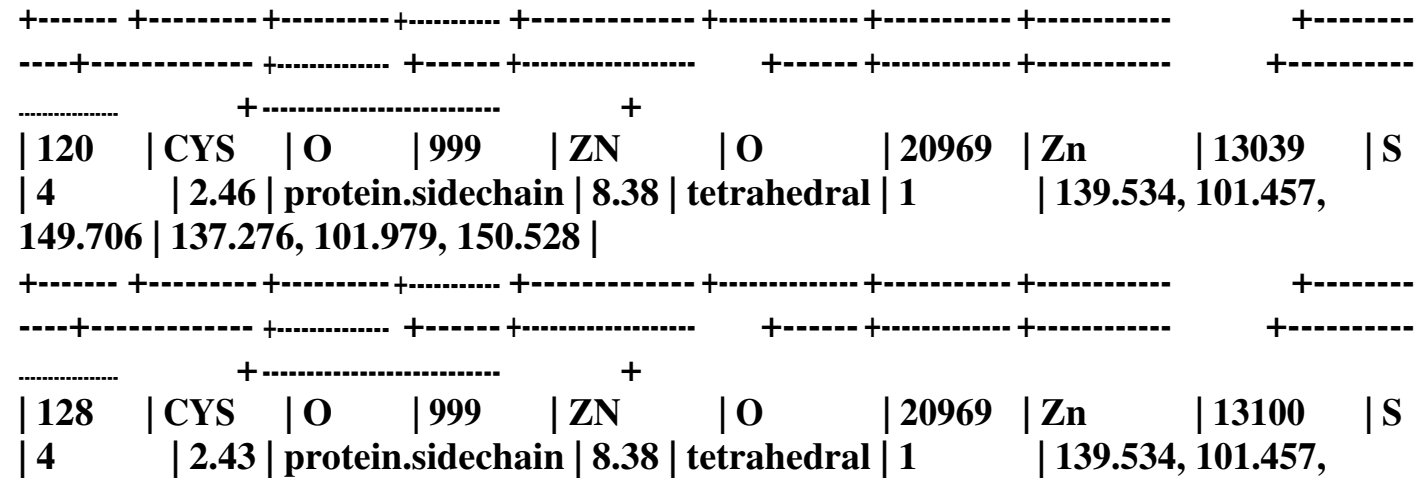

| $4|2.43|$ protein.sidechain $|8.38|$ tetrahedral $|1 \quad| 139.534,101.457$, $149.706|141.310,102.732,150.775|$

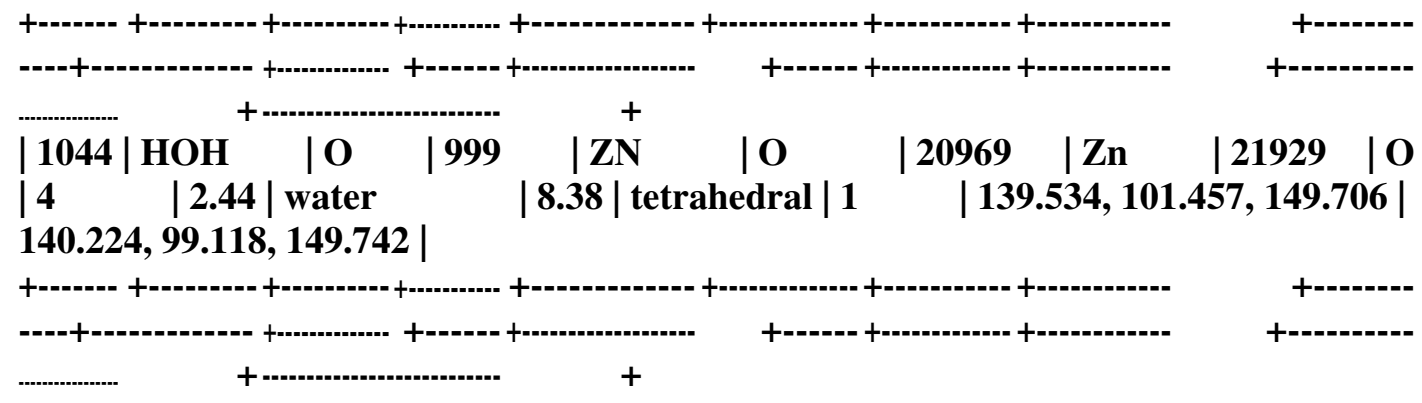

ZN:P:998 (ZN) - ION

Interacting chain(s): $\mathbf{P}$

**Metal Complexes**

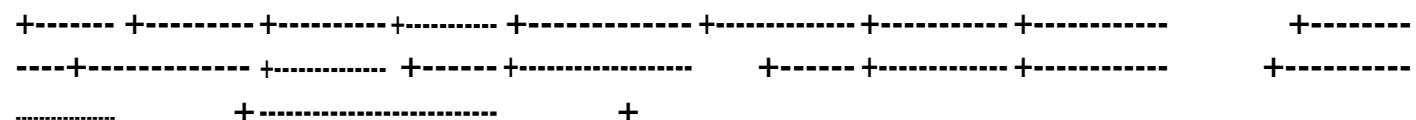

| RESNR | RESTYPE | RESCHAIN | RESNR_LIG | RESTYPE_LIG | RESCHAIN_LIG | METAL_IDX | METAL_TYPE | TARGET_IDX |

TARGET_TYPE | COORDINATION | DIST | LOCATION_ |RMS |

GEOMETRY | COMPLEXNUM|METALCOO | TARGETCOO 


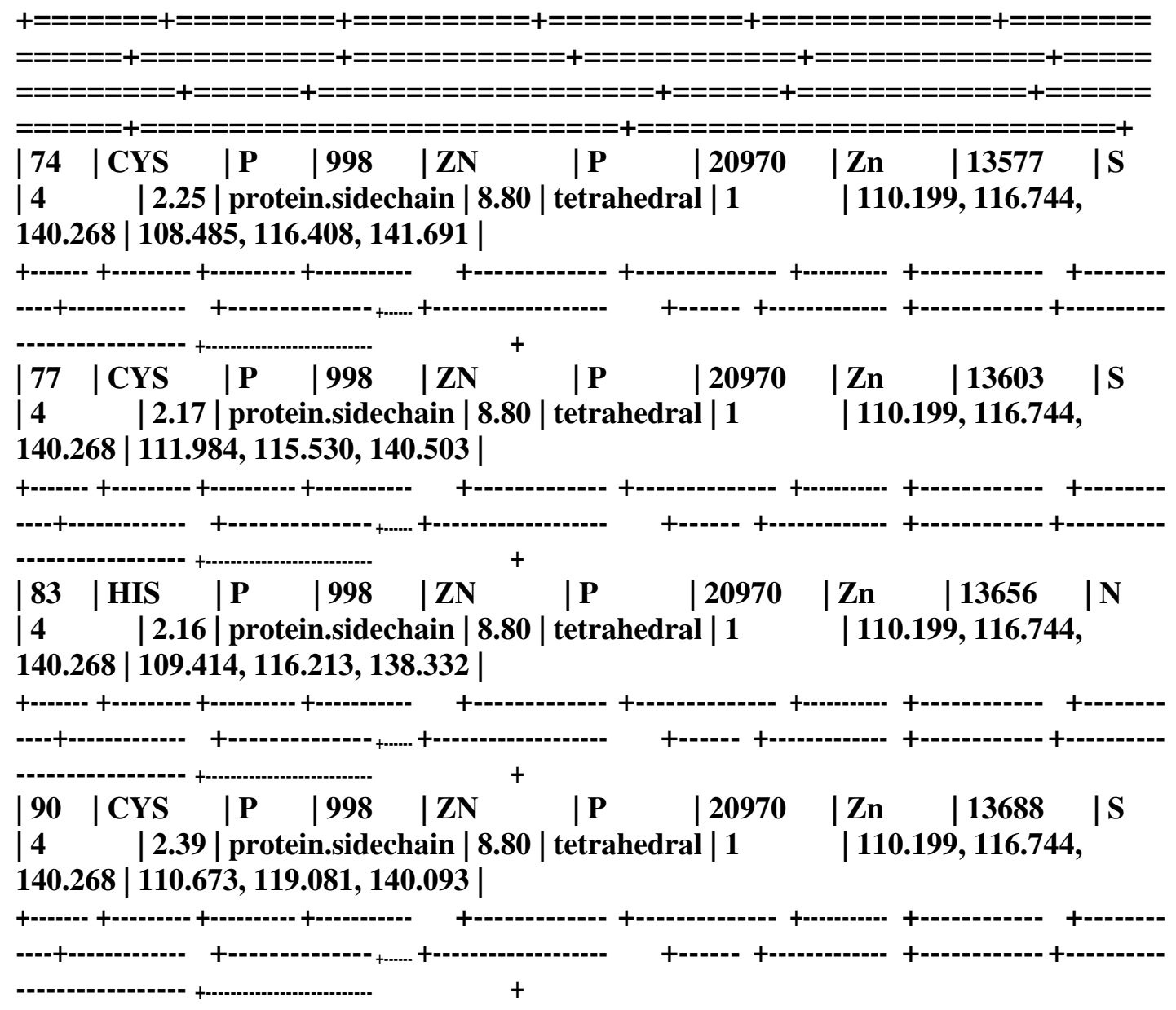

ZN:P:999 (ZN) - ION

Interacting chain(s): P

**Metal Complexes**

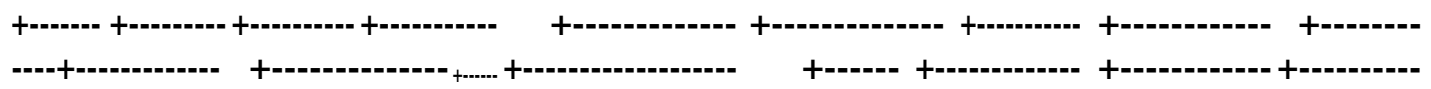

| RESNR | RESTYPE | RESCHAIN | RESNR_LIG | RESTYPE_LIG

RESCHAIN_LIG | METAL_IDX | METAL_TYPE | TARGET_IDX |

TARGET_TYPE | COORDINATION | DIST | LOCATION |RMS |

GEOMETRY | COMPLEXNUM | METALCOO | TARGETCOO

|

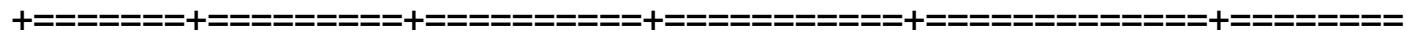

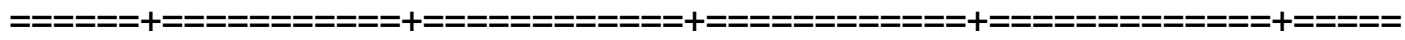

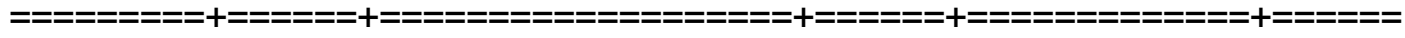

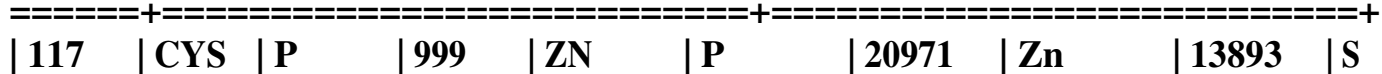

| 4 | 2.35 $\mid$ protein.sidechain $|5.77|$ tetrahedral $|1 \quad| 100.772,135.856$, $149.996|100.614,135.152,147.762|$

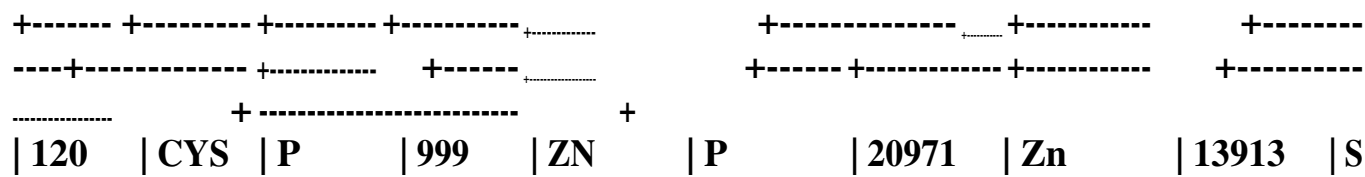

| $4 \quad|2.42|$ protein.sidechain $|5.77|$ tetrahedral | $1 \quad$ | 100.772, 135.856, $149.996|103.054,135.433,150.678|$ 


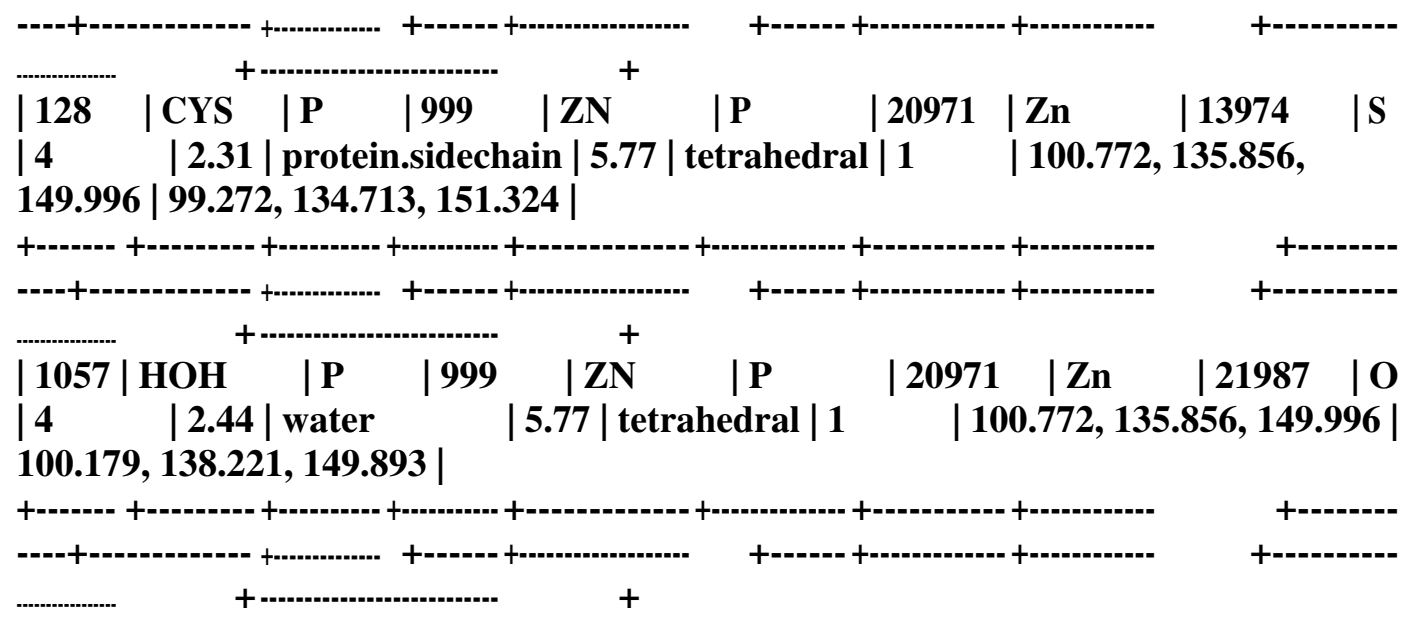

ZN:Q:998 (ZN) - ION

Interacting chain(s): Q

**Metal Complexes**

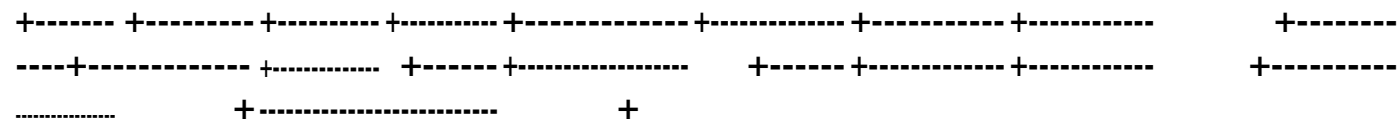

| RESNR | RESTYPE | RESCHAIN | RESNR_LIG | RESTYPE_LIG |

RESCHAIN_LIG | METAL_IDX | METAL_TYPE | TARGET_IDX |

TARGET_TYPE | COORDINATION | DIST | LOCATION |RMS |

GEOMETRY | COMPLEXNUM | METALCOO | TARGETCOO

1

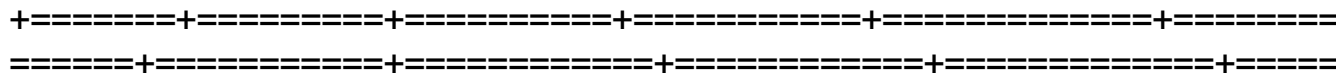

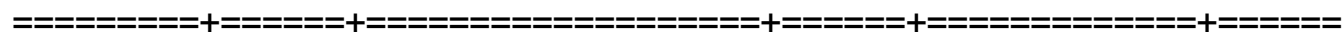

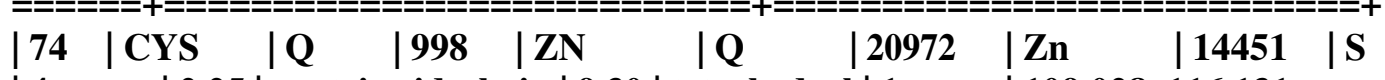

| $4 \quad|2.25|$ protein.sidechain $|9.30|$ tetrahedral $|1 \quad| 109.028,116.131$, 105.044| 107.224, 115.856, 103.725 |

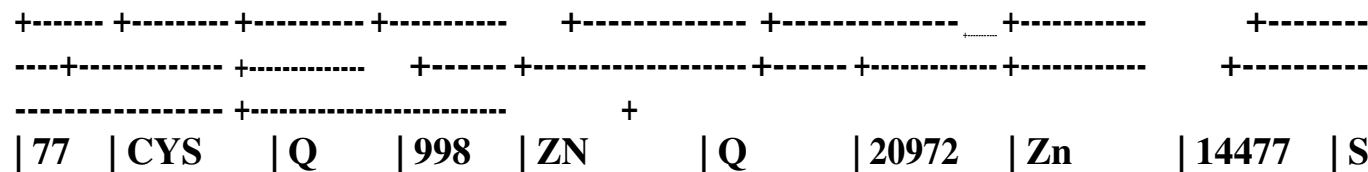

| $4 \quad|2.15|$ protein.sidechain $|9.30|$ tetrahedral $|1 \quad| 109.028,116.131$, $105.044|110.294,117.827,104.678|$

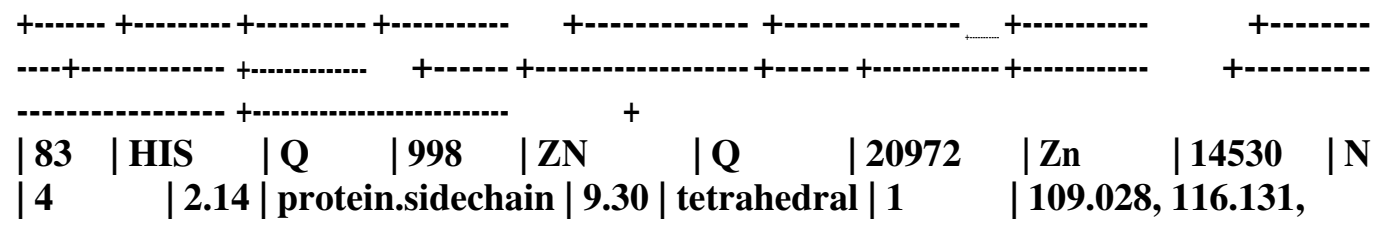
105.044 | 108.402, 116.258, 107.084 |

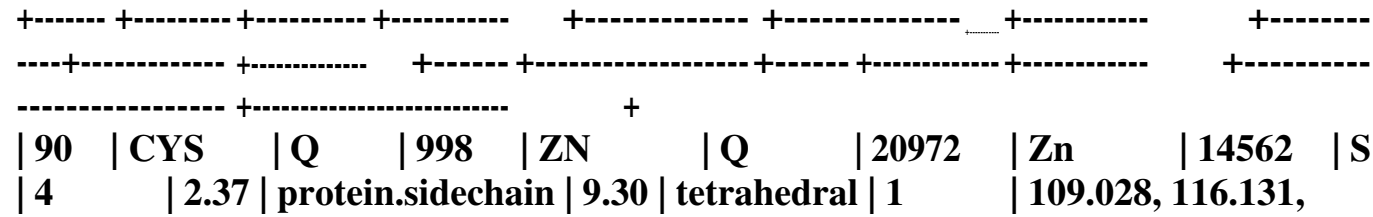

$105.044|110.235,114.096,105.097|$

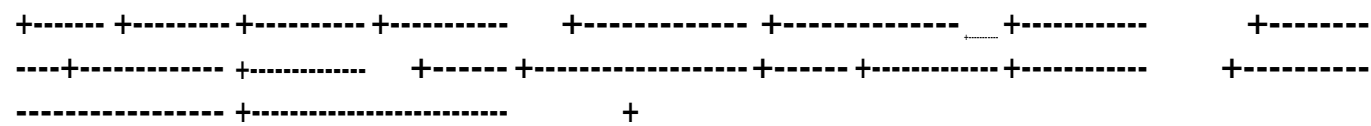


ZN:Q:999 (ZN) - ION

Interacting chain(s): Q

**Metal Complexes**

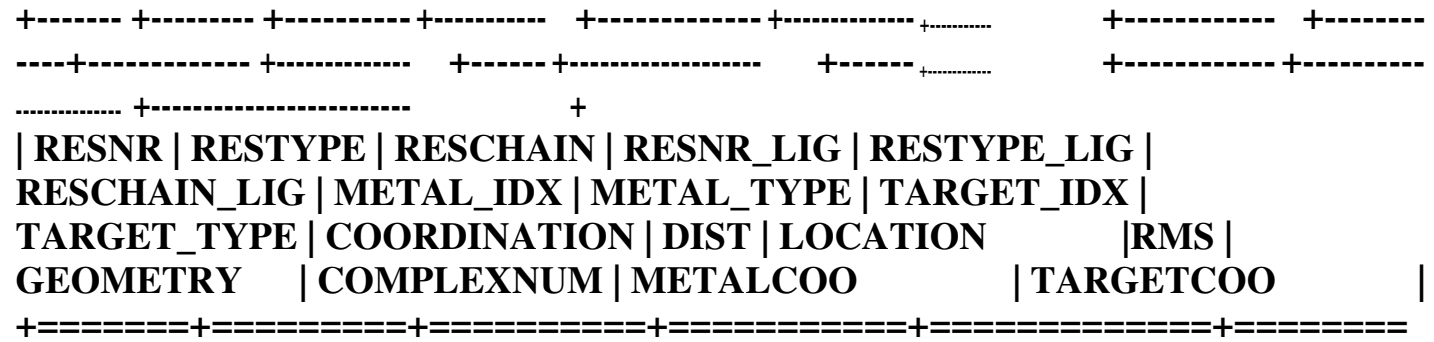

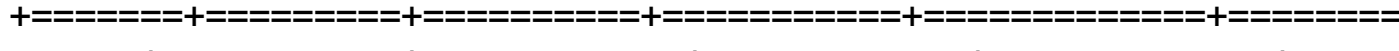

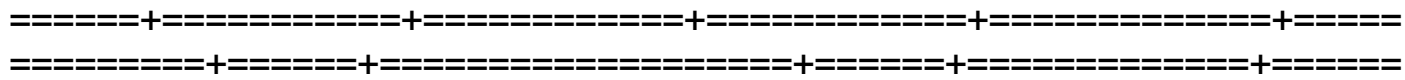

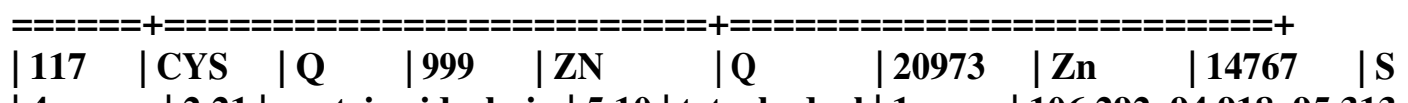

| 4 | 2.21 | protein.sidechain $|5.10|$ tetrahedral | $1 \quad|106.292,94.918,95.313|$ 105.957, 95.482, 97.423 |

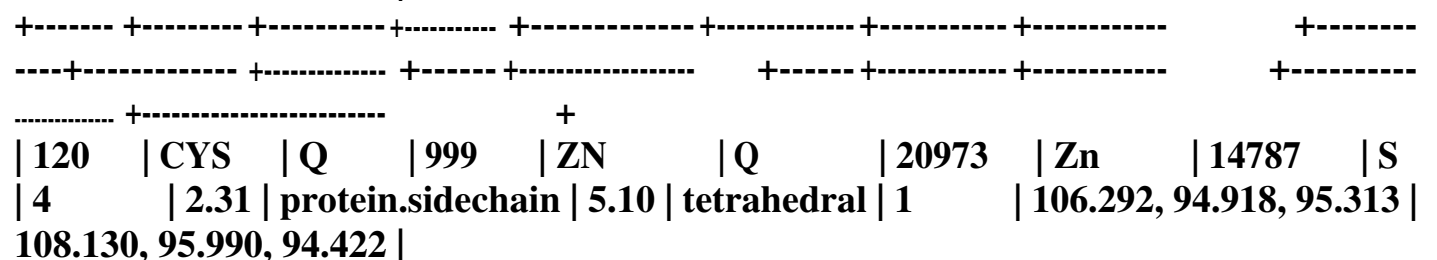
108.130, 95.990, 94.422 |
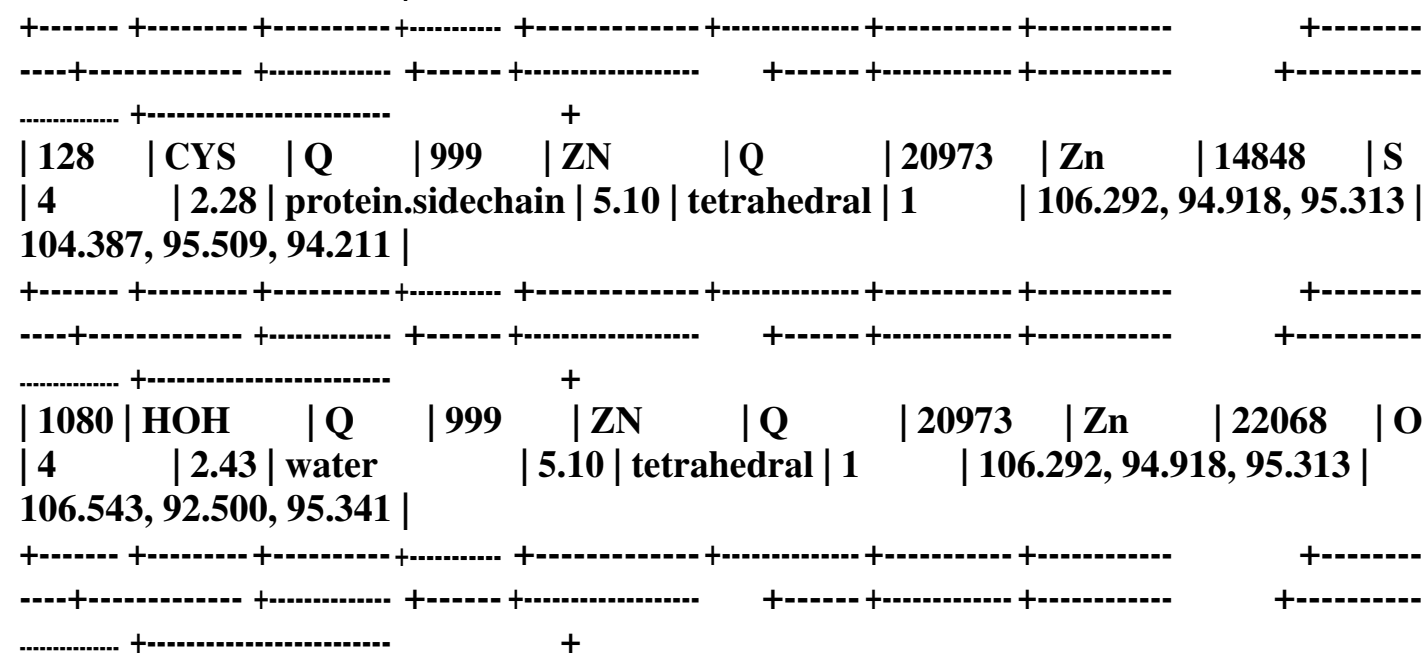

ZN:R:998 (ZN) - ION

Interacting chain(s): $\mathbf{R}$

**Metal Complexes**

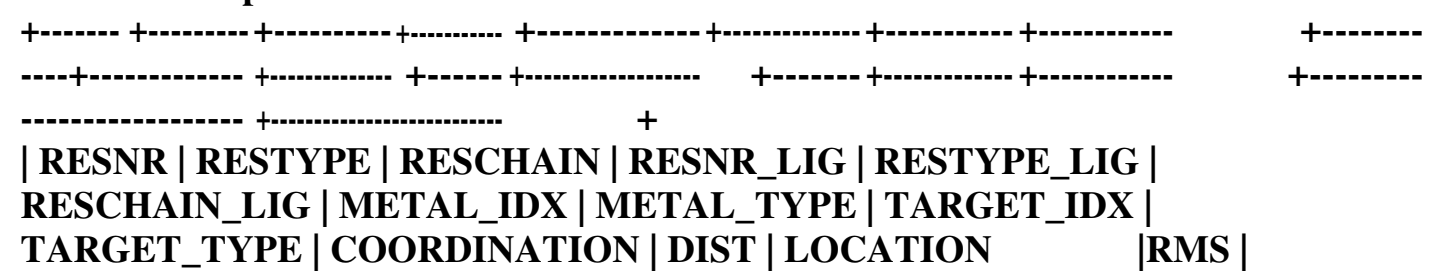




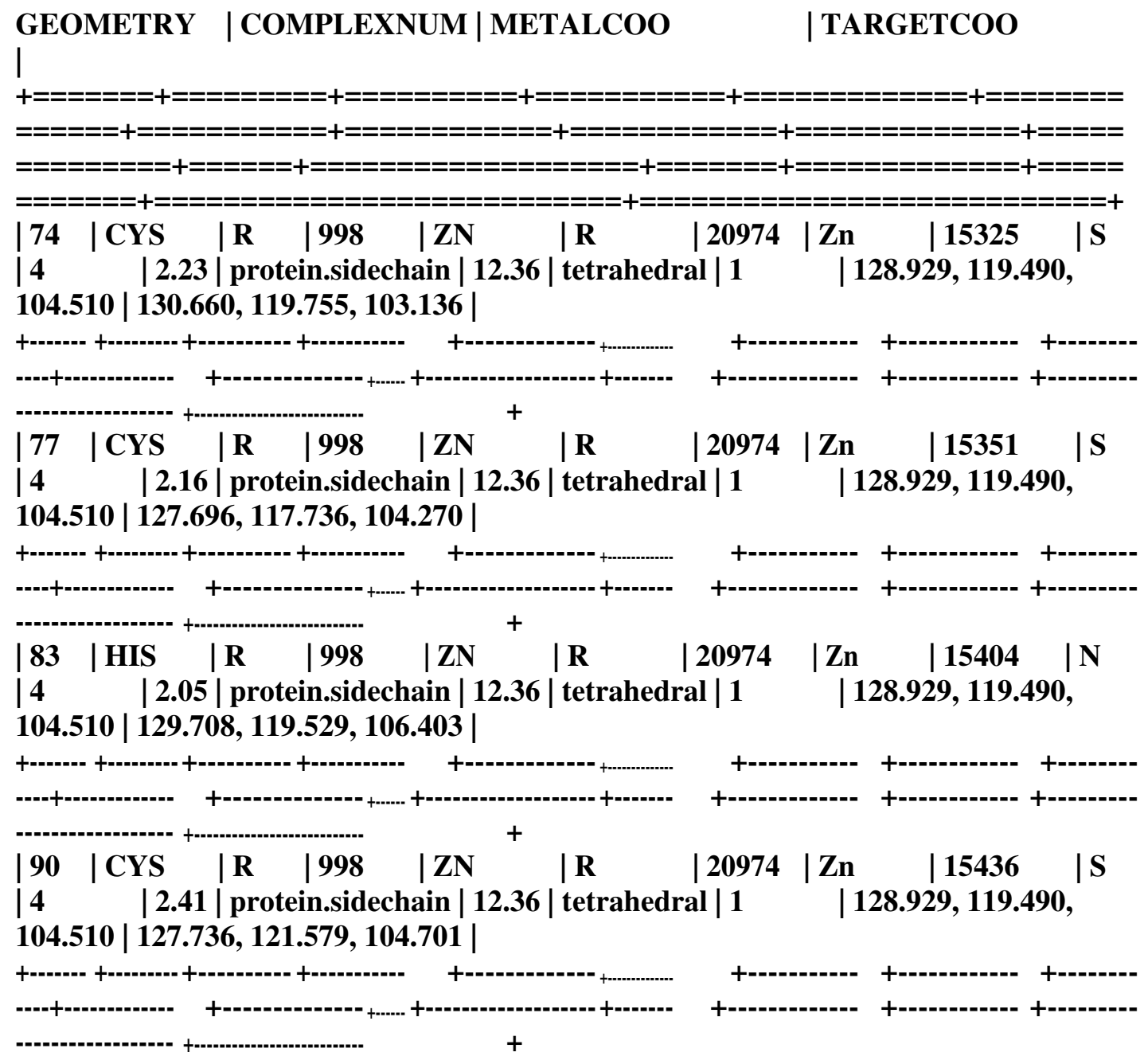

ZN:R:999 (ZN) - ION

Interacting chain(s): $\mathbf{R}$

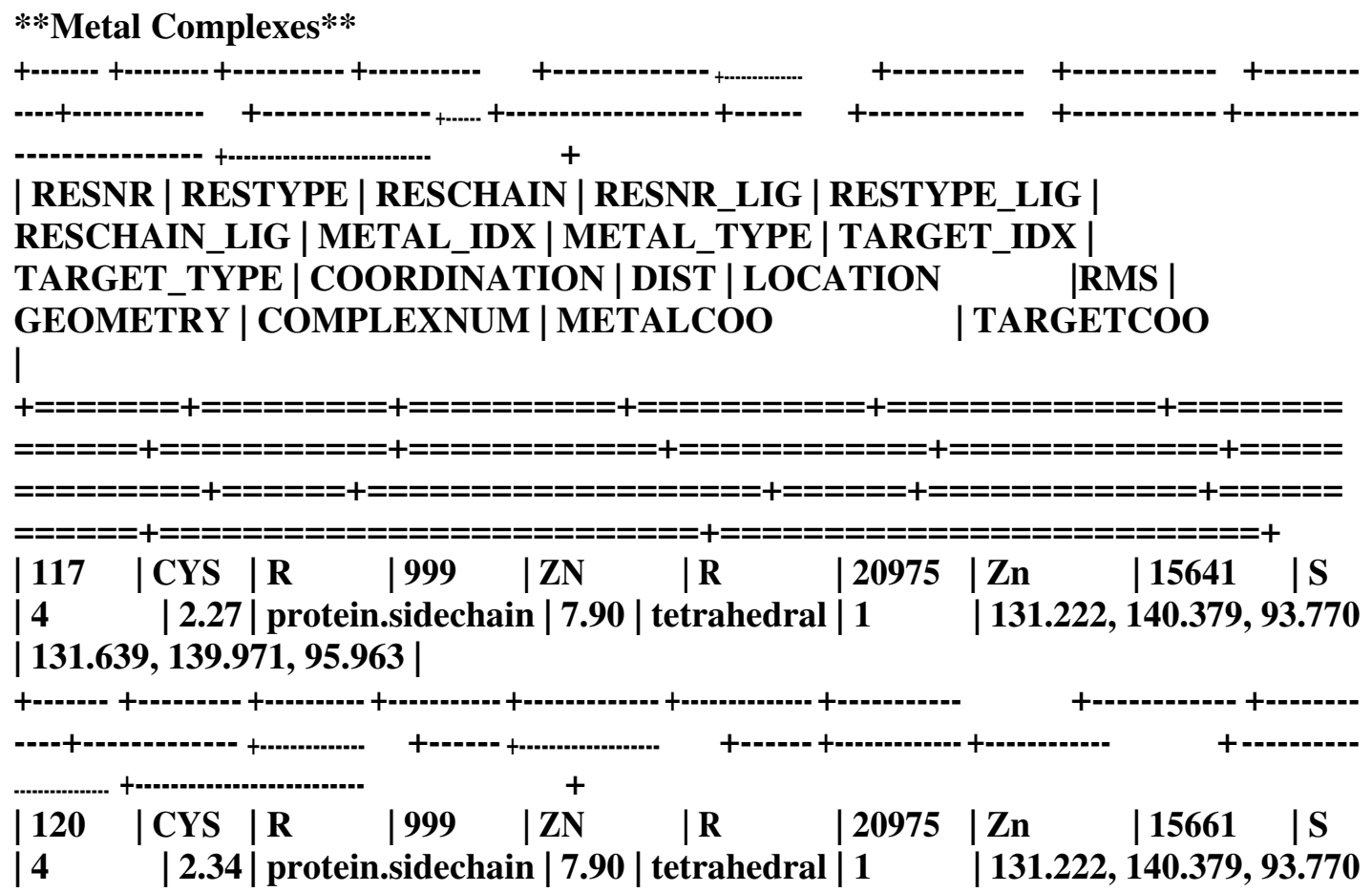




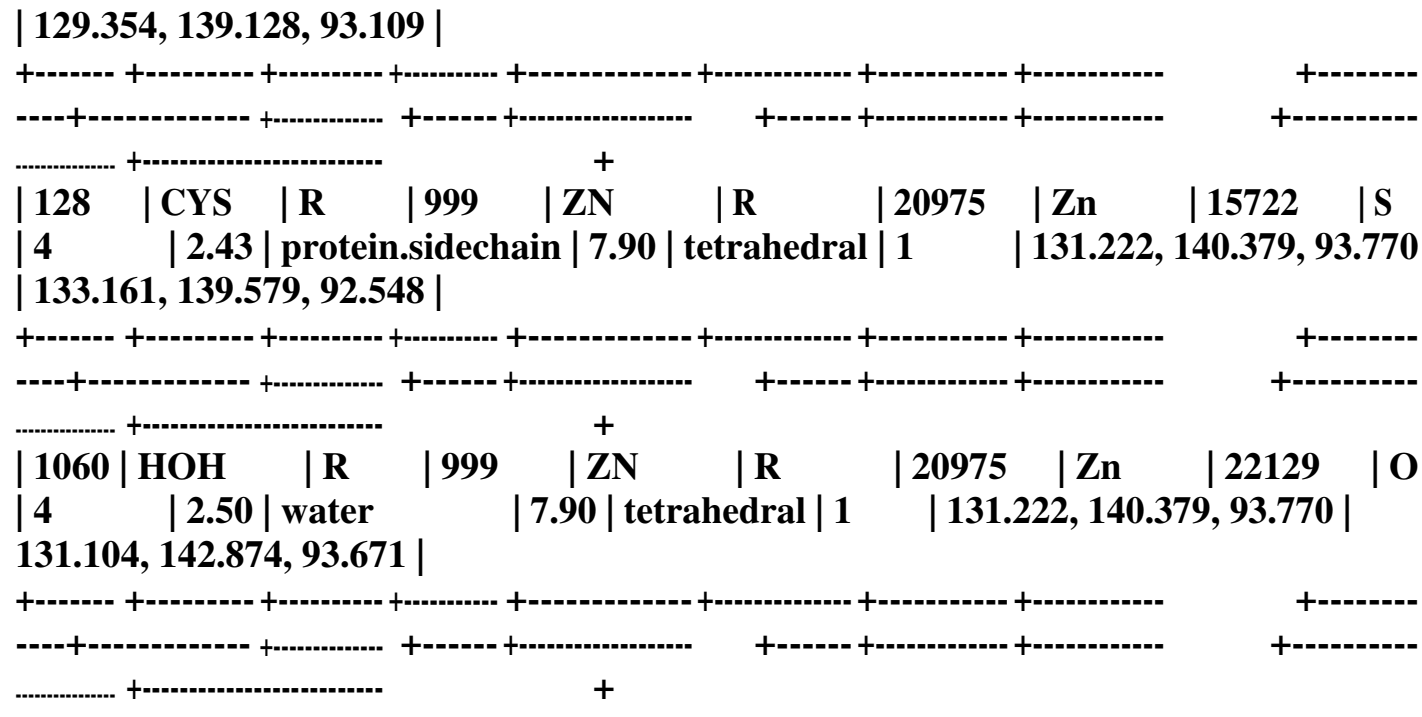

ZN:S:998 (ZN) - ION

Interacting chain(s): S

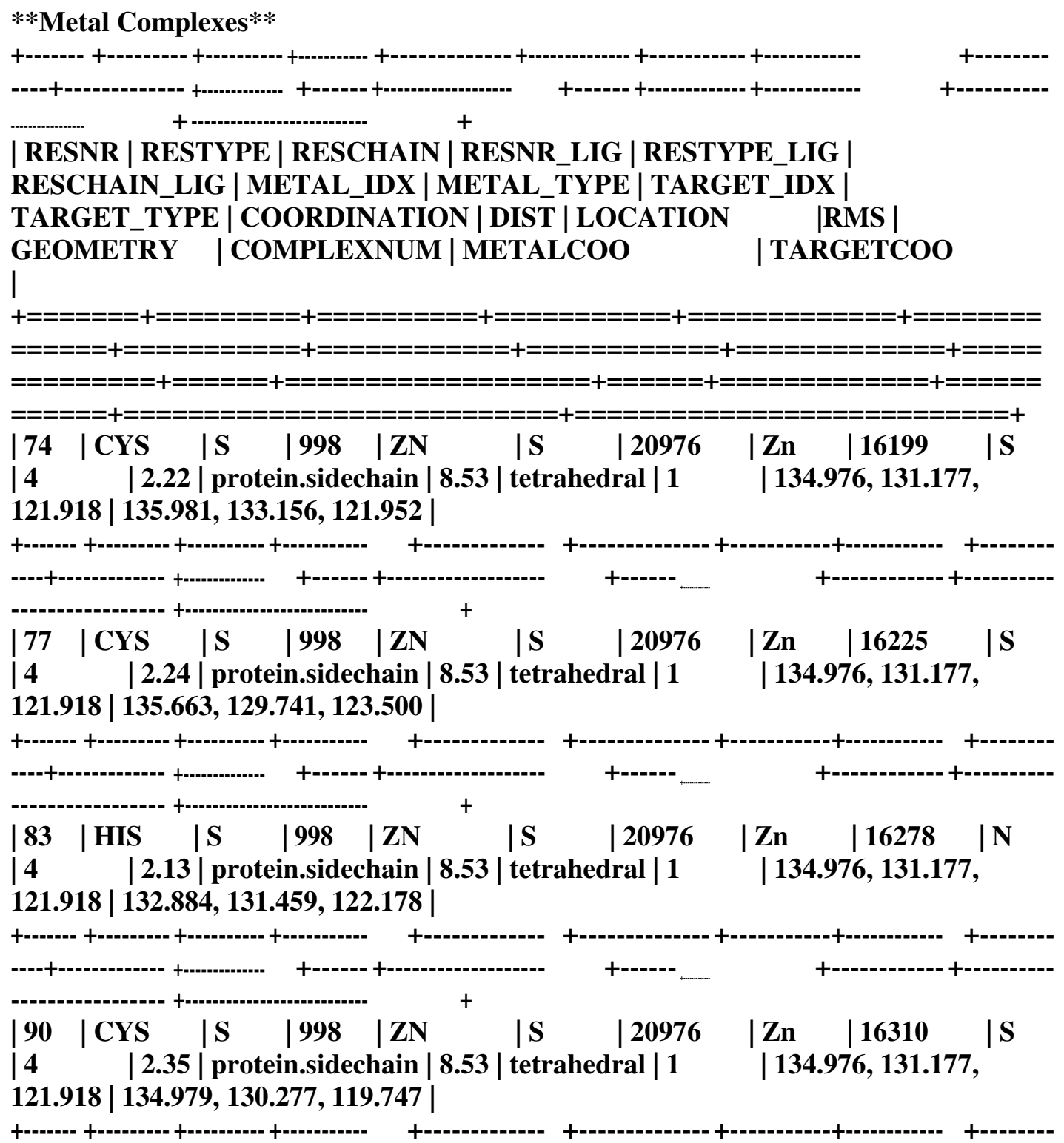



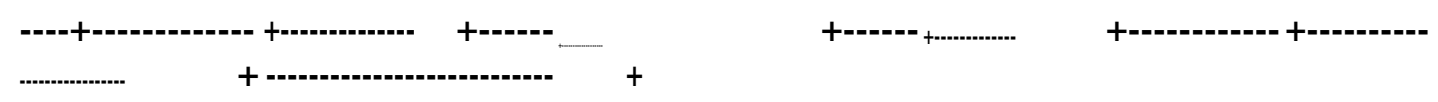

ZN:S:999 (ZN) - ION

Interacting chain(s): S

**Metal Complexes**

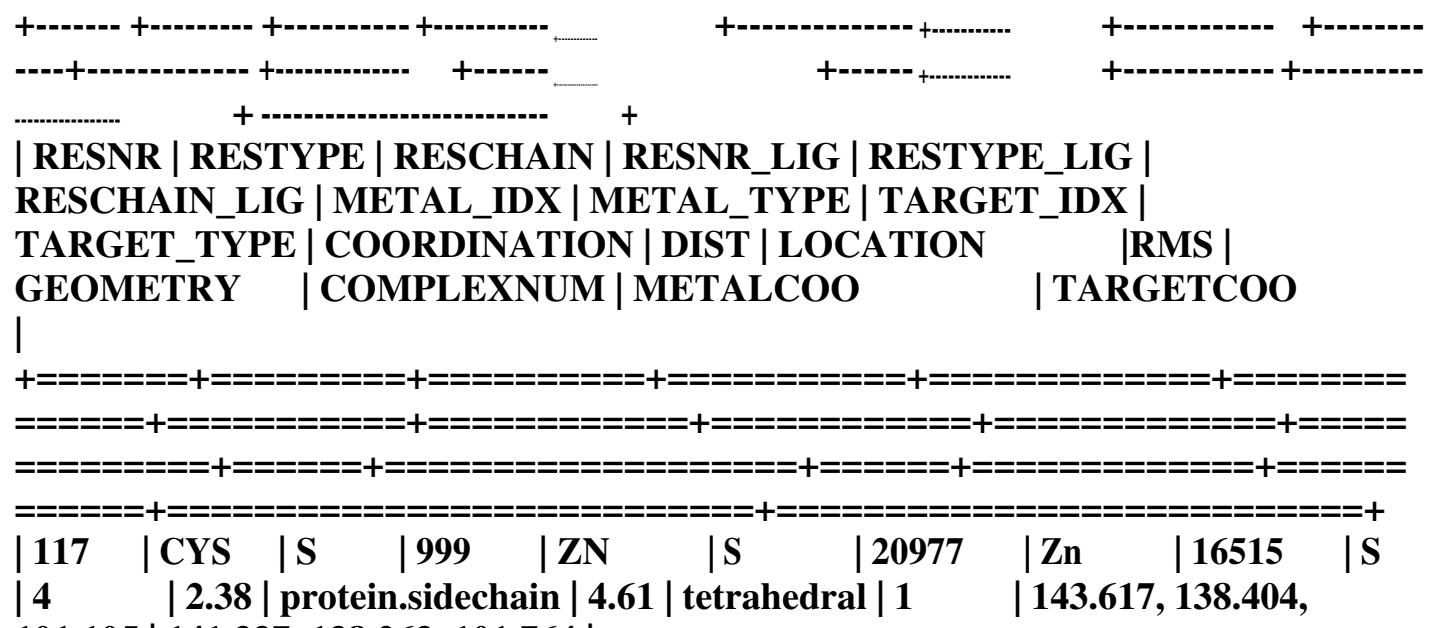

$101.105 \mid 141.337,138.263,101.764$ |

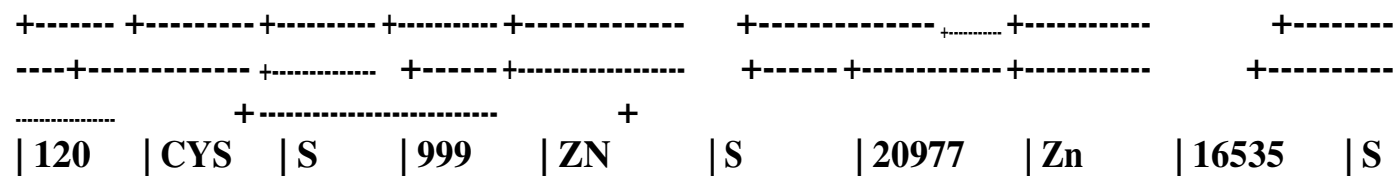

| 4 | $2.28 \mid$ protein.sidechain | 4.61| tetrahedral | $1 \quad \mid 143.617,138.404$,

101.105 | 144.634, 136.496, 101.837|

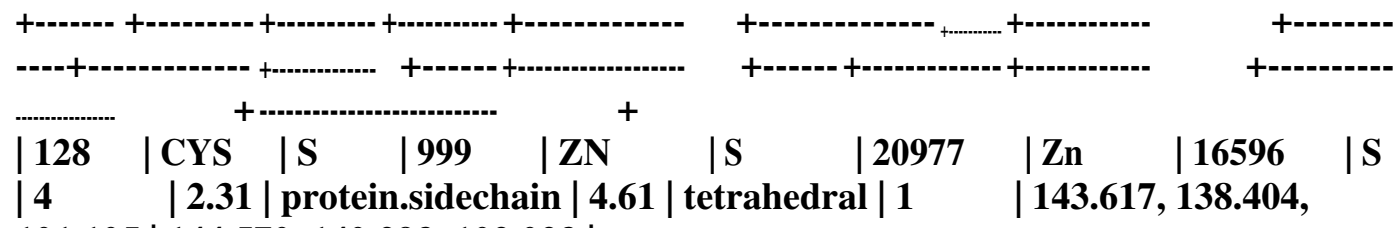

$101.105|144.570,140.293,102.023|$

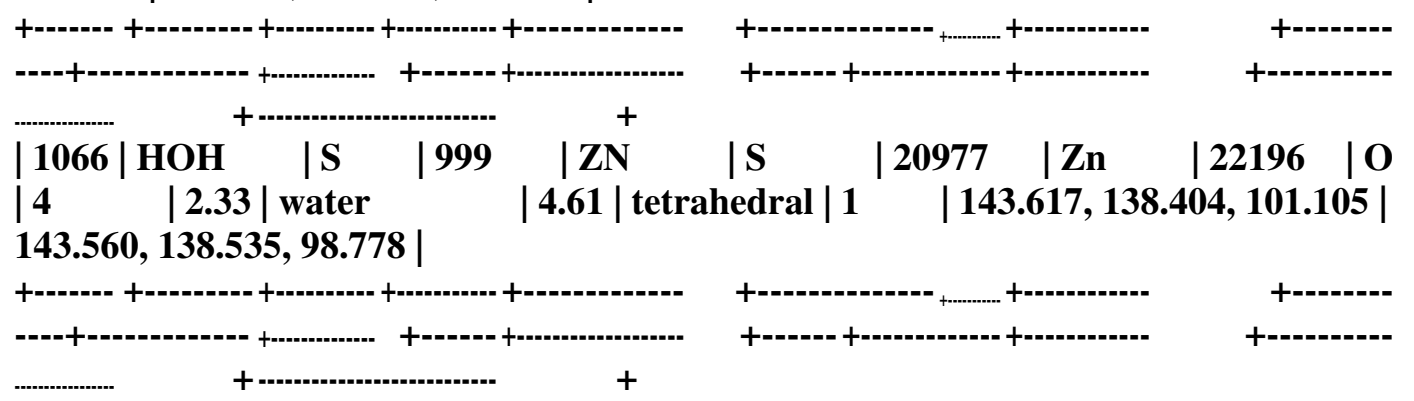

ZN:T:998 (ZN) - ION

Interacting chain(s): T

**Metal Complexes**
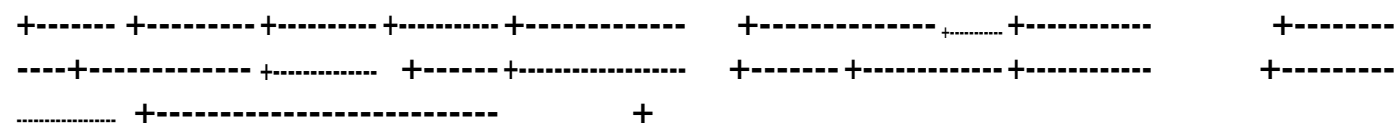
| RESNR | RESTYPE | RESCHAIN | RESNR_LIG | RESTYPE_LIG | RESCHAIN_LIG | METAL_IDX | METAL_TYPE | TARGET_IDX | TARGET_TYPE $\mid$ COORDINATION | DIST $\mid$ LOCATION $\quad$ RMS | GEOMETRY | COMPLEXNUM | METALCOO

| TARGETCOO

1

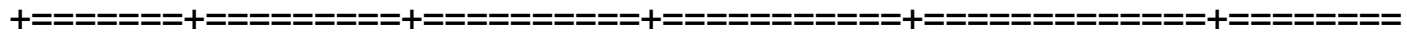

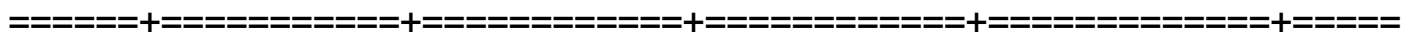

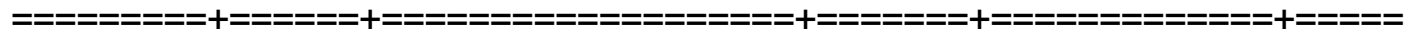

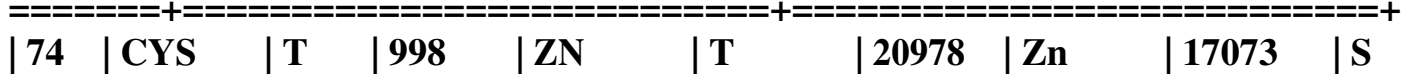

| 4 | 2.30| protein.sidechain $|\mathbf{1 0 . 4 4}|$ tetrahedral $|1 \quad| 138.235,111.330$, $121.970 \mid$ 139.950, 109.793, 121.934 |

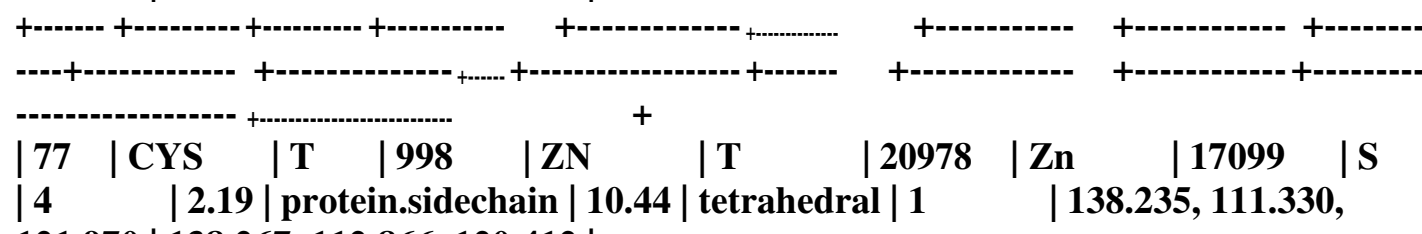

$121.970|138.267,112.866,120.412|$

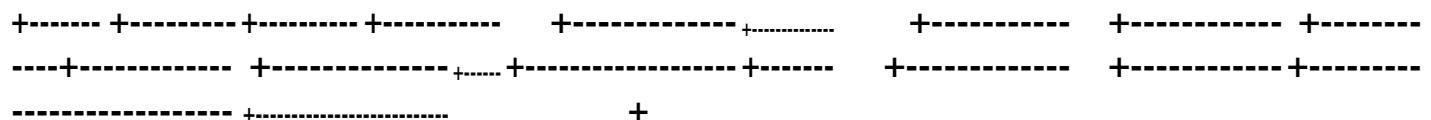

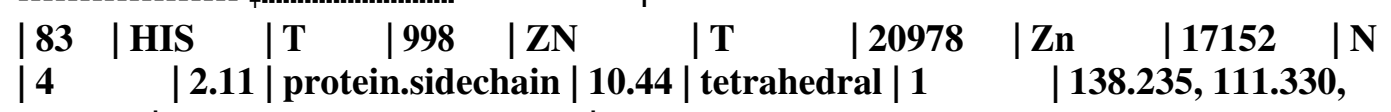
$121.970|136.338,110.441,121.708|$

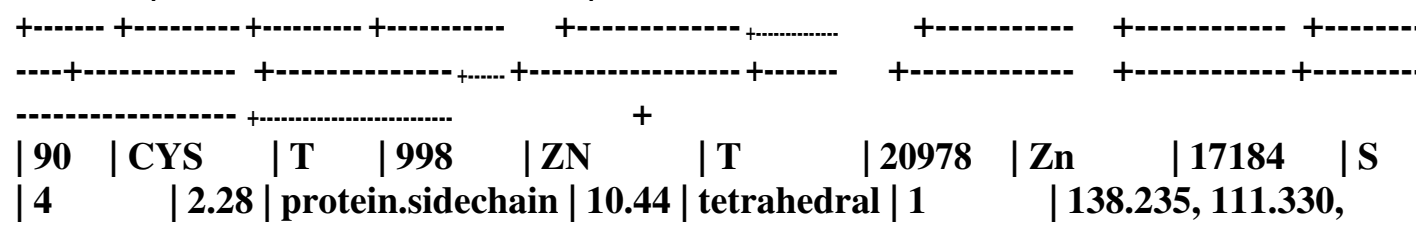

$121.970 \mid$ 138.004, 112.132, 124.088 |
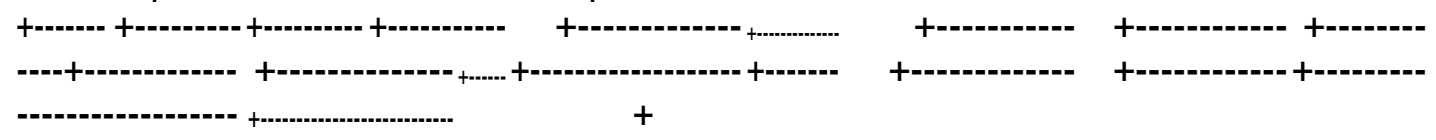

ZN:T:999 (ZN) - ION

Interacting chain(s): $\mathbf{T}$

**Metal Complexes**
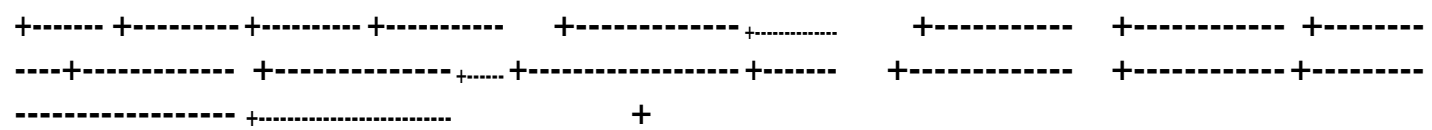

| RESNR | RESTYPE | RESCHAIN | RESNR_LIG | RESTYPE_LIG |

RESCHAIN_LIG | METAL_IDX | METAL_TYPE | TARGET_IDX |

TARGET_TYPE | COORDINATION | DIST | LOCATION

GEOMETRY | COMPLEXNUM | METALCOO

|RMS |

| TARGETCOO

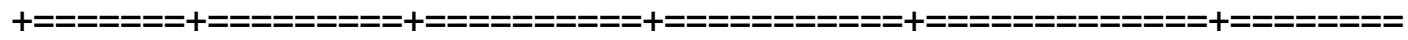

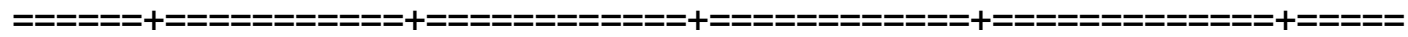

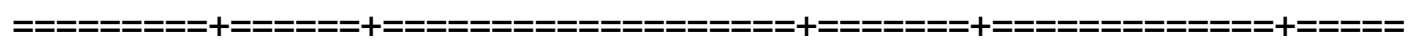

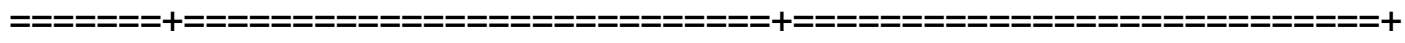

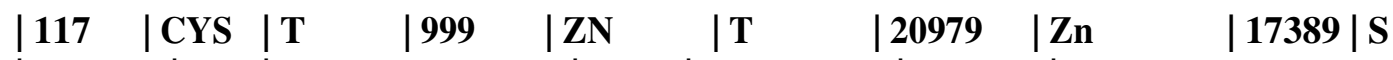

| $4 \quad|2.90|$ protein.sidechain $|\mathbf{4 0 . 4 3}|$ tetrahedral $|1 \quad| 149.868,107.463$,

$142.255|149.152,105.577,140.172|$

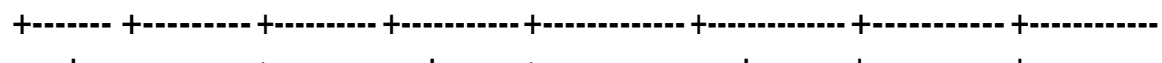

+-1--1-- 


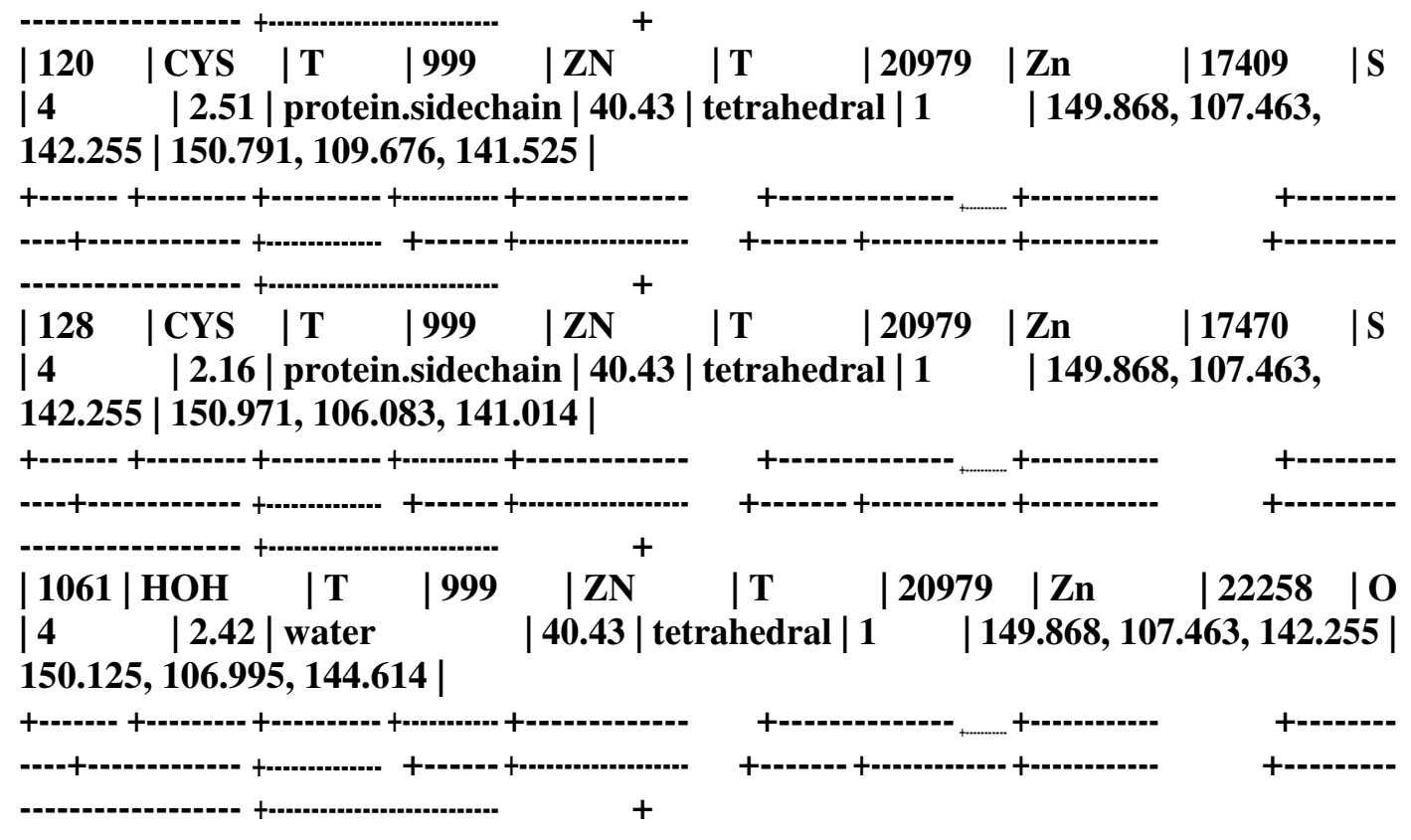

ZN:U:998 (ZN) - ION

Interacting chain(s): U

**Metal Complexes**

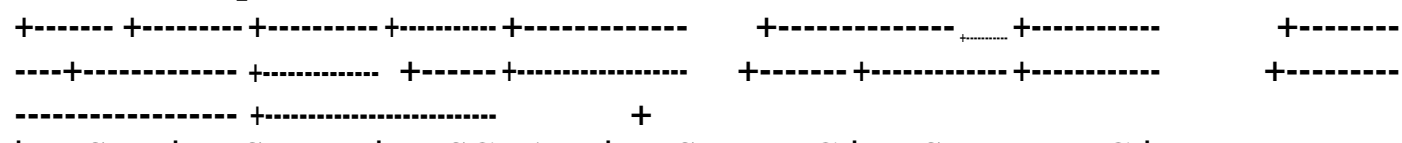

| RESNR | RESTYPE | RESCHAIN | RESNR_LIG | RESTYPE_LIG

RESCHAIN_LIG | METAL_IDX | METAL_TYPE | TARGET_IDX |

TARGET_TYPE | COORDINATION | DIST | LOCATION |RMS |

GEOMETRY | COMPLEXNUM | METALCOO

| TARGETCOO

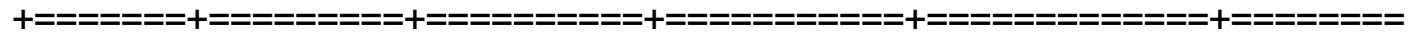

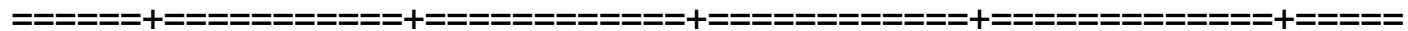

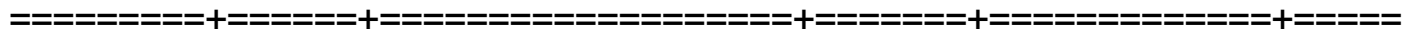

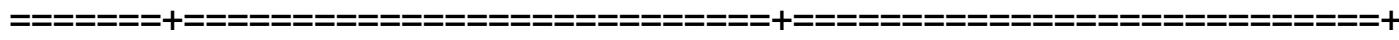

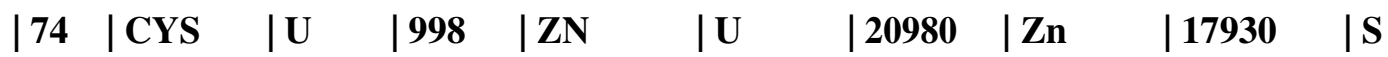

| $4 \quad|2.30|$ protein.sidechain $|10.30|$ tetrahedral $|1 \quad| 100.420,124.979$,

$122.666|98.761,126.573,122.609|$

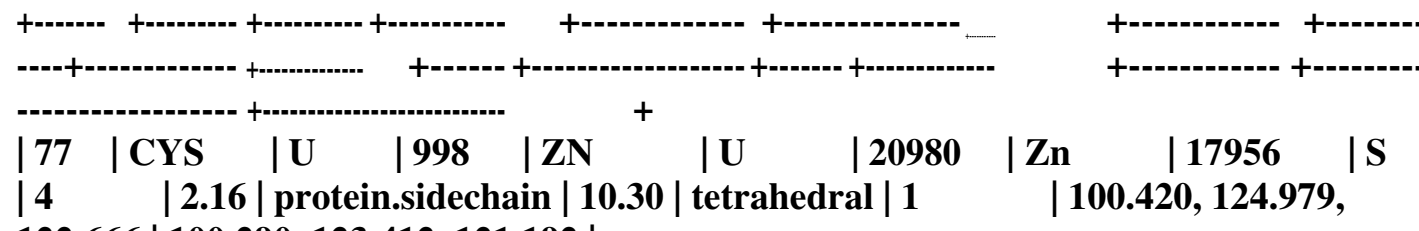

$122.666|100.290,123.412,121.192|$

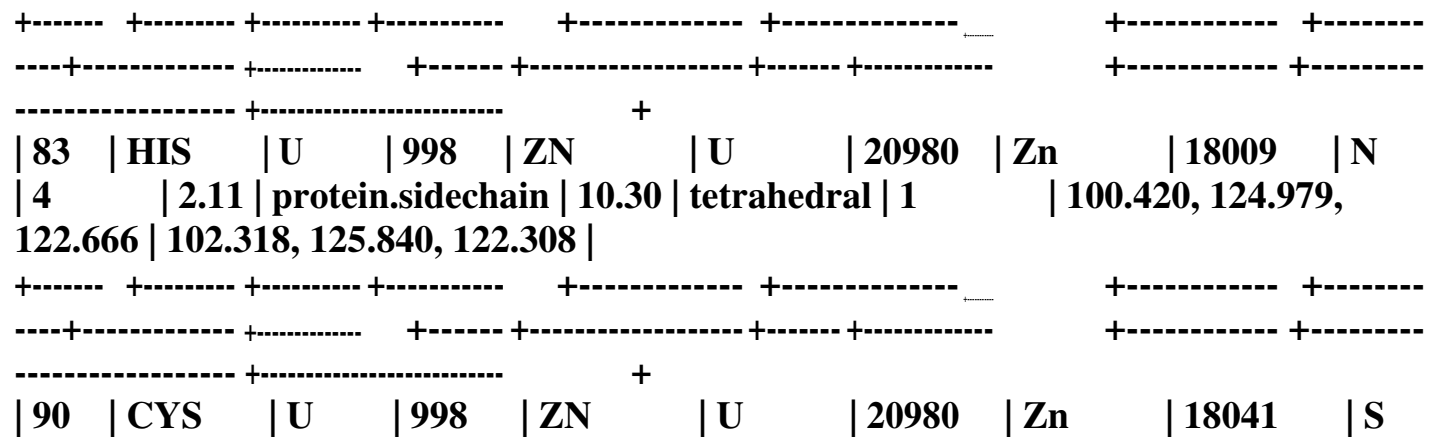



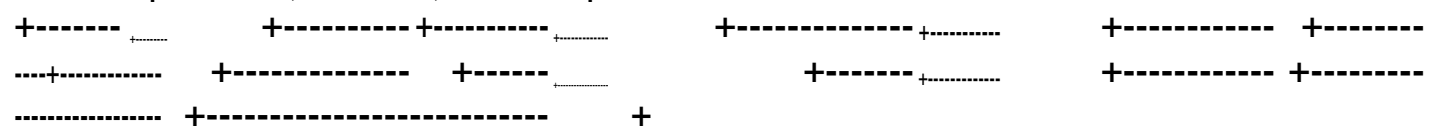

ZN:U:999 (ZN) - ION

Interacting chain(s): $\mathbf{U}$

**Metal Complexes**

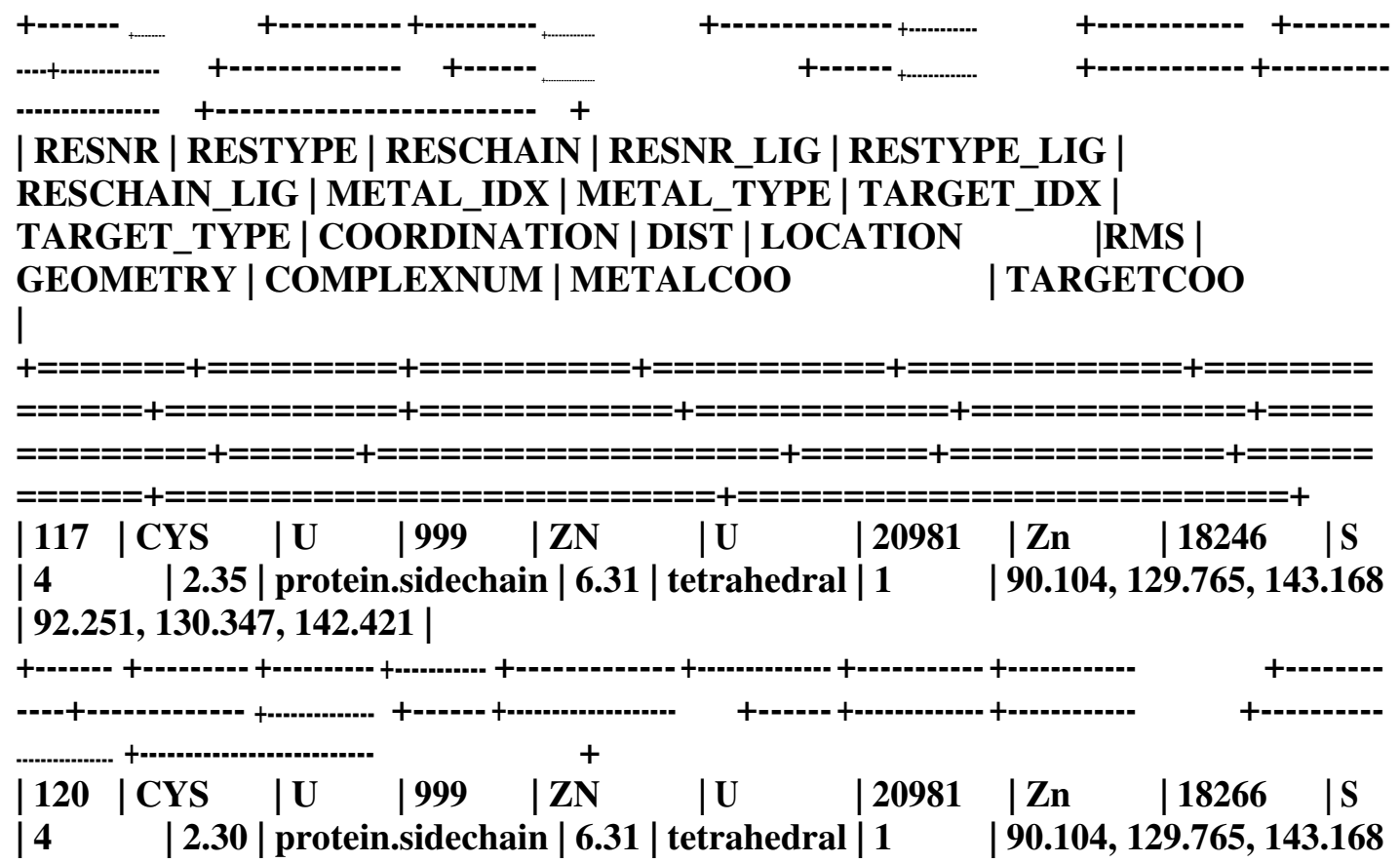
| 89.646, 127.637, 142.437 |
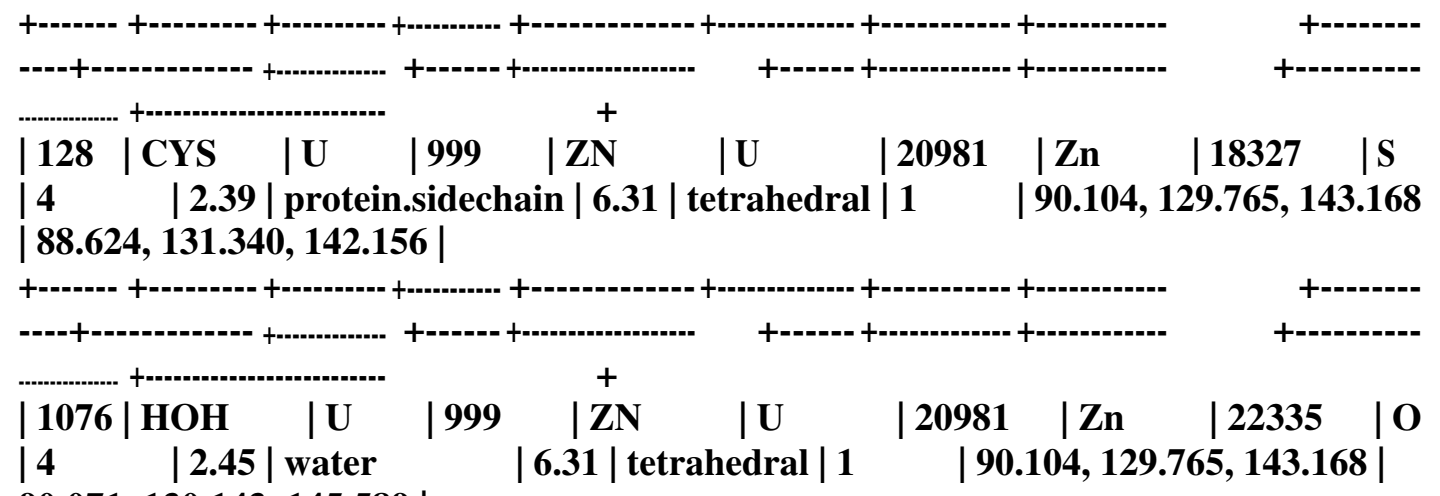

90.071, 130.143, 145.589 |

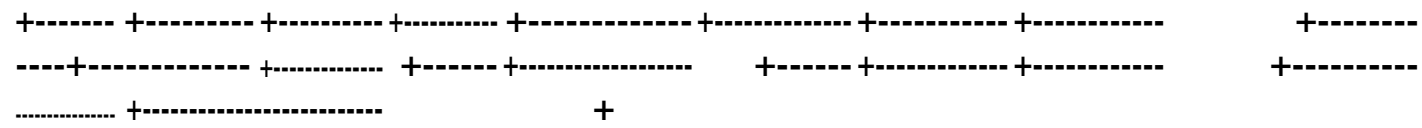

ZN:V:998 (ZN) - ION

Interacting chain(s): V 


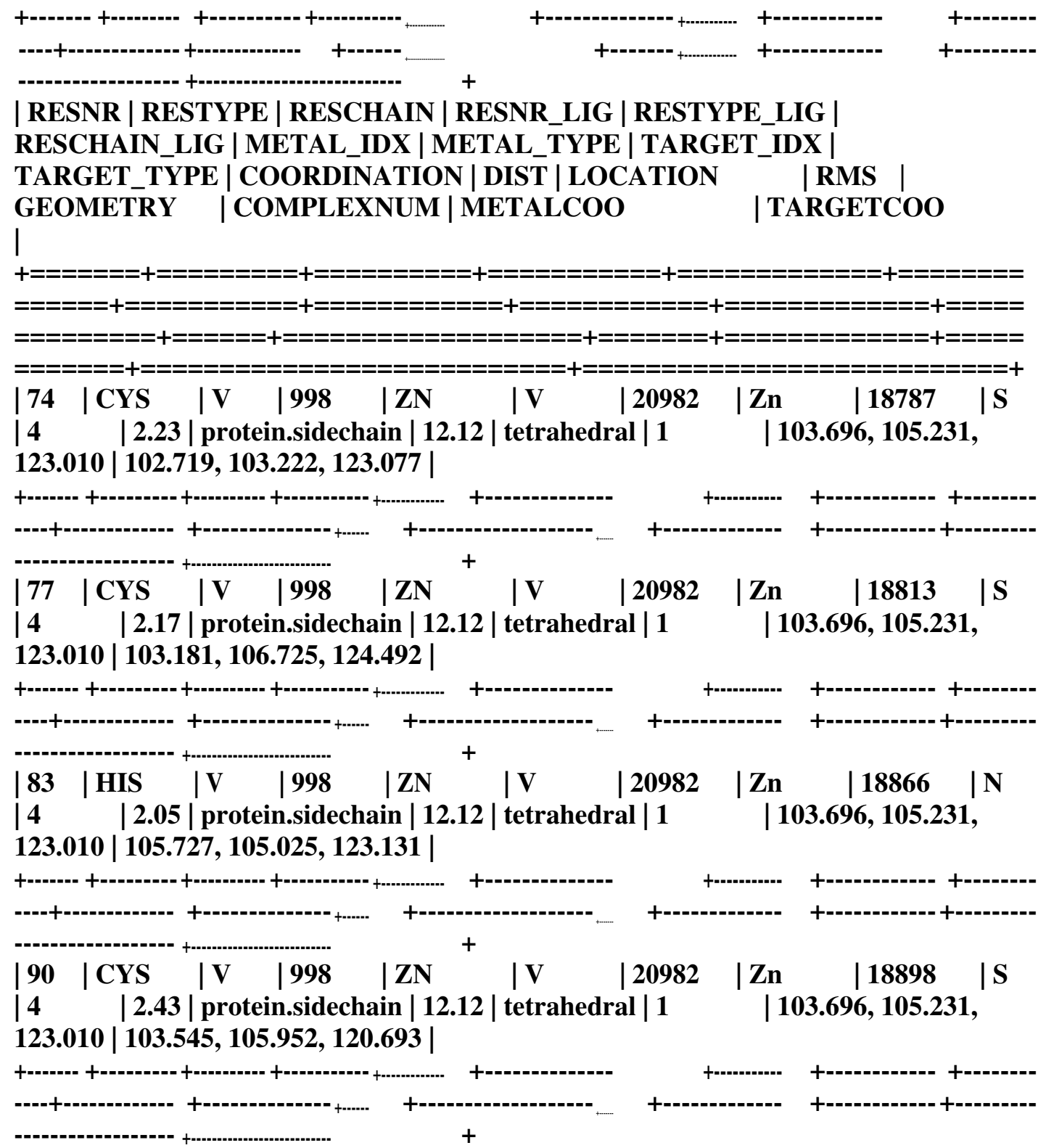

ZN:V:999 (ZN) - ION

Interacting chain(s): V

**Metal Complexes**

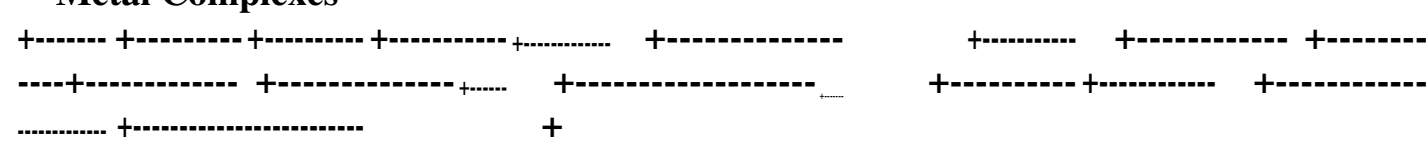

| RESNR | RESTYPE | RESCHAIN | RESNR_LIG | RESTYPE_LIG |

RESCHAIN_LIG | METAL_IDX | METAL_TYPE | TARGET_IDX |

TARGET_TYPE | COORDINATION | DIST | LOCATION | RMS

GEOMETRY | COMPLEXNUM | METALCOO

| TARGETCOO

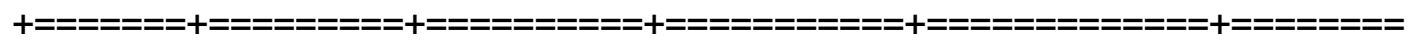

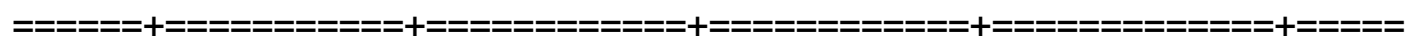

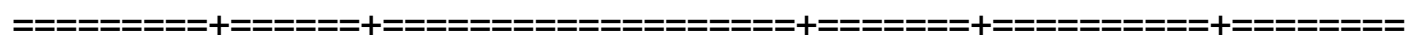

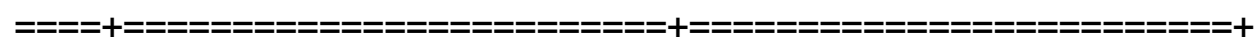

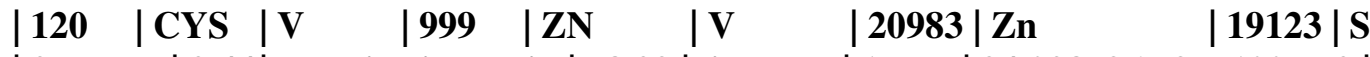

| 2 | 2.44| protein.sidechain $|78.29|$ linear $\quad|1 \quad| 94.028,96.597,100.772 \mid$

94.153, 97.353, 103.087 | 


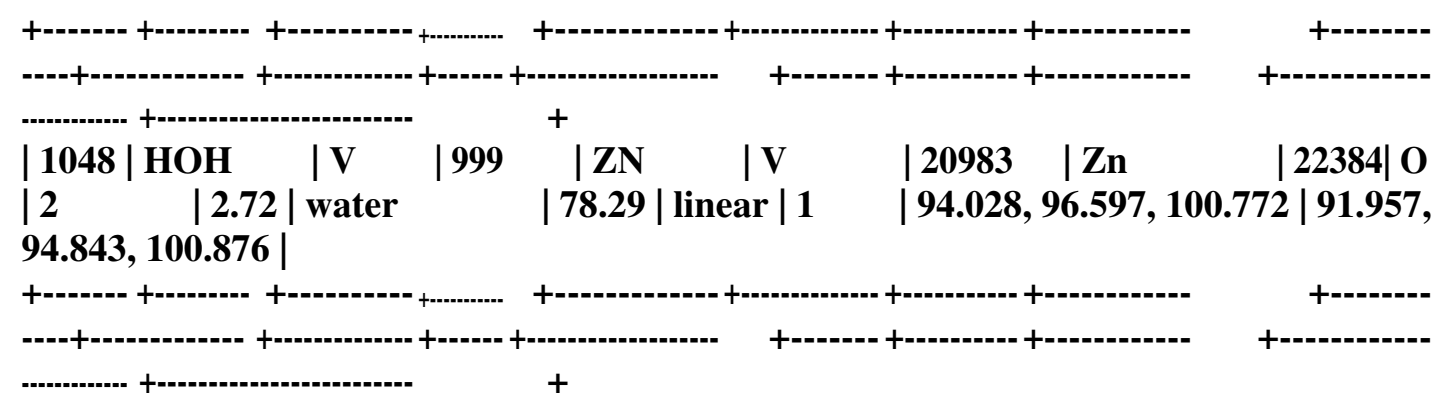

ZN:W:998 (ZN) - ION

Interacting chain(s): W

**Metal Complexes**

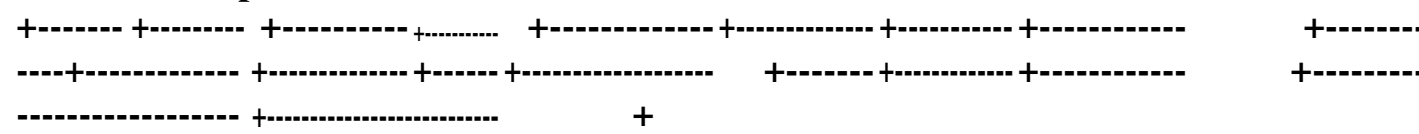

| RESNR | RESTYPE | RESCHAIN | RESNR_LIG | RESTYPE_LIG |

RESCHAIN_LIG | METAL_IDX | METAL_TYPE | TARGET_IDX |

TARGET_TYPE | COORDINATION | DIST | LOCATION | RMS

GEOMETRY | COMPLEXNUM|METALCOO | TARGETCOO

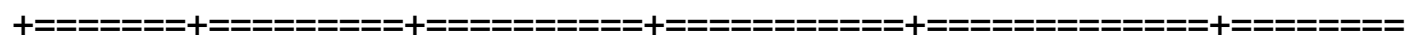

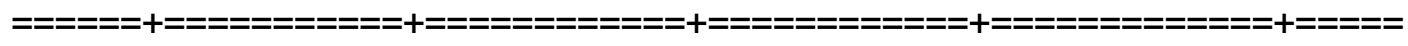

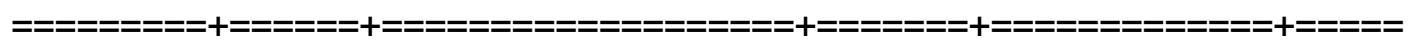

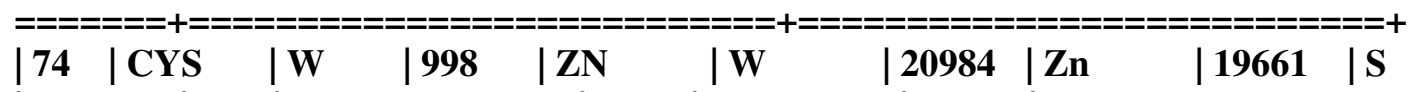

| 4 | 2.25 | protein.sidechain | 11.48 | tetrahedral | 1 | 116.892, 135.879,

132.151 | 116.772, 137.229, 133.942 |

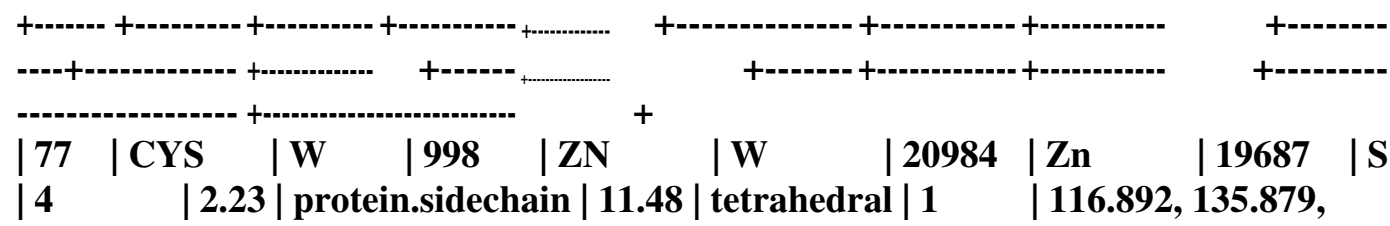

132.151 | 118.371, 136.362, 130.555 |

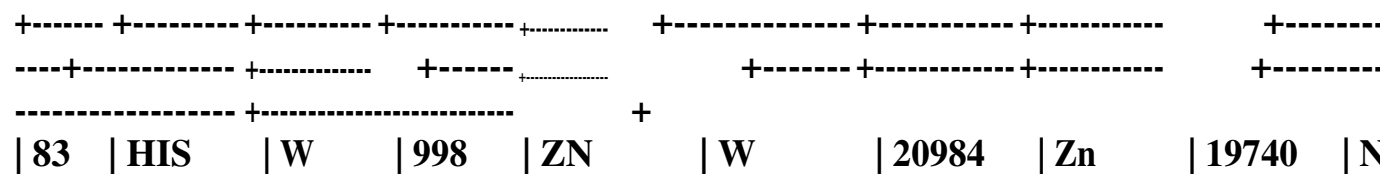

| 4 | 2.05 | protein.sidechain | 11.48 | tetrahedral | 1 |116.892, 135.879,

132.151 | 117.342, 134.011, 132.879|

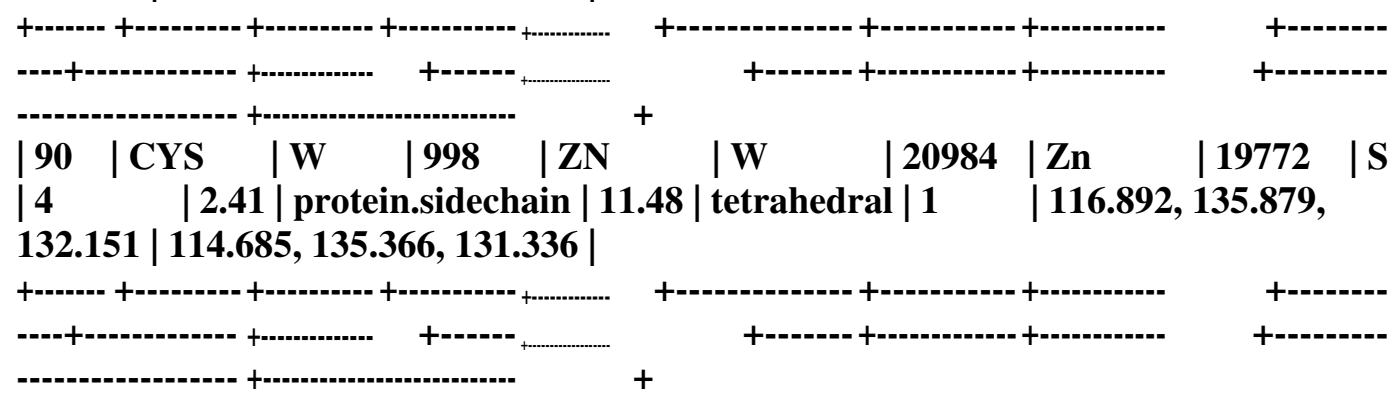


Interacting chain(s): W

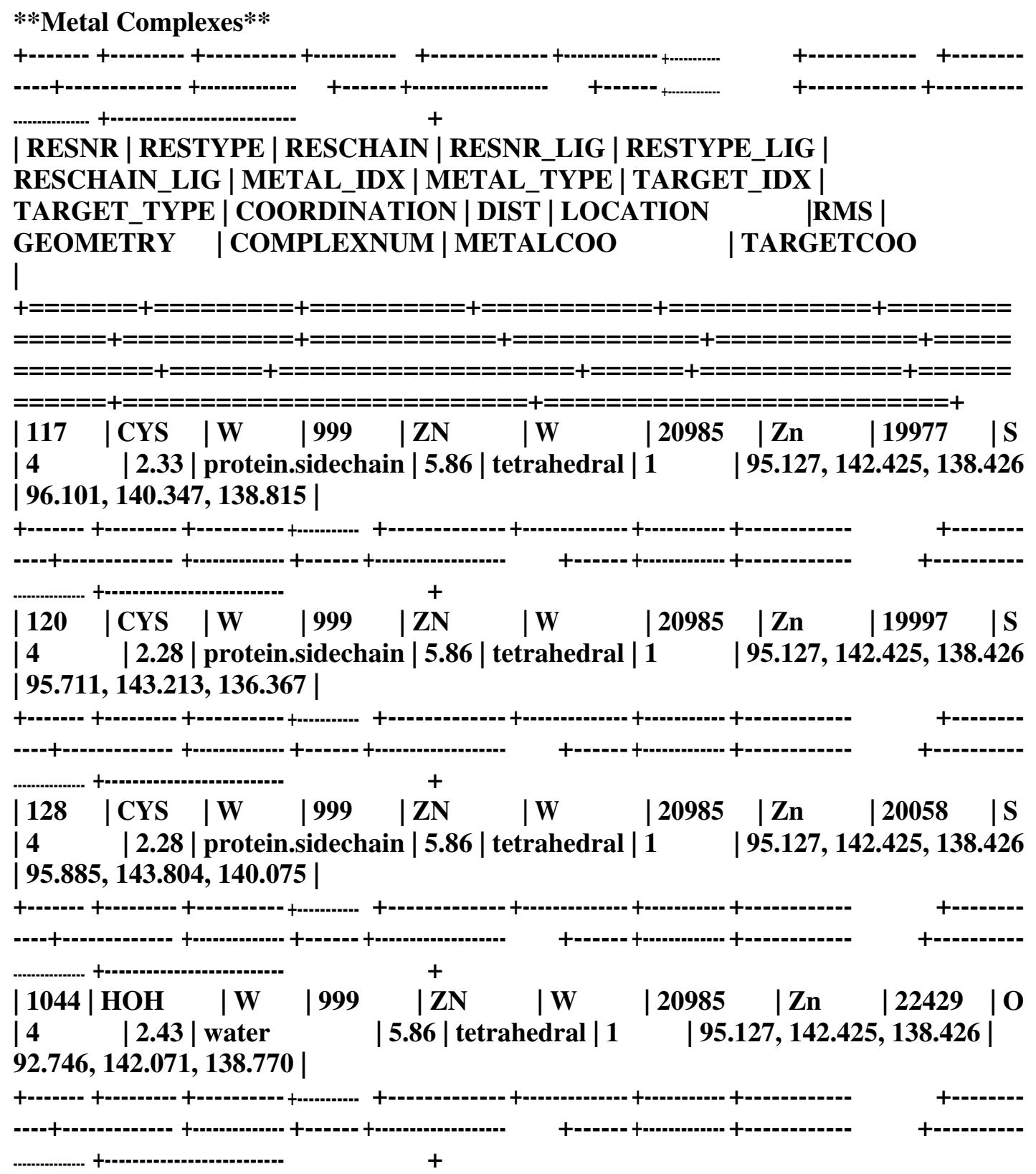

ZN:X:998 (ZN) - ION

Interacting chain(s): $\mathbf{X}$

**Metal Complexes**

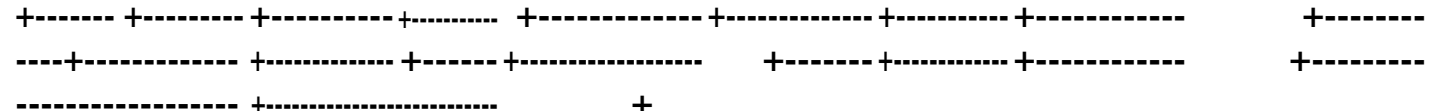

| RESNR | RESTYPE | RESCHAIN | RESNR_LIG | RESTYPE_LIG |

RESCHAIN_LIG | METAL_IDX | METAL_TYPE | TARGET_IDX |

TARGET_TYPE | COORDINATION | DIST | LOCATION

GEOMETRY | COMPLEXNUM | METALCOO

|RMS |

I

| TARGETCOO

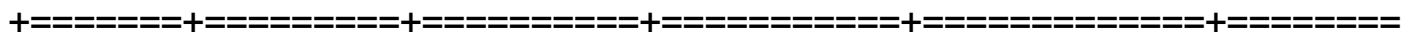

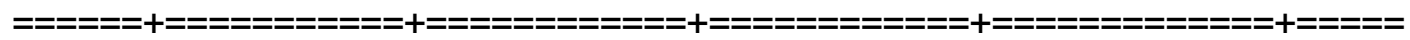




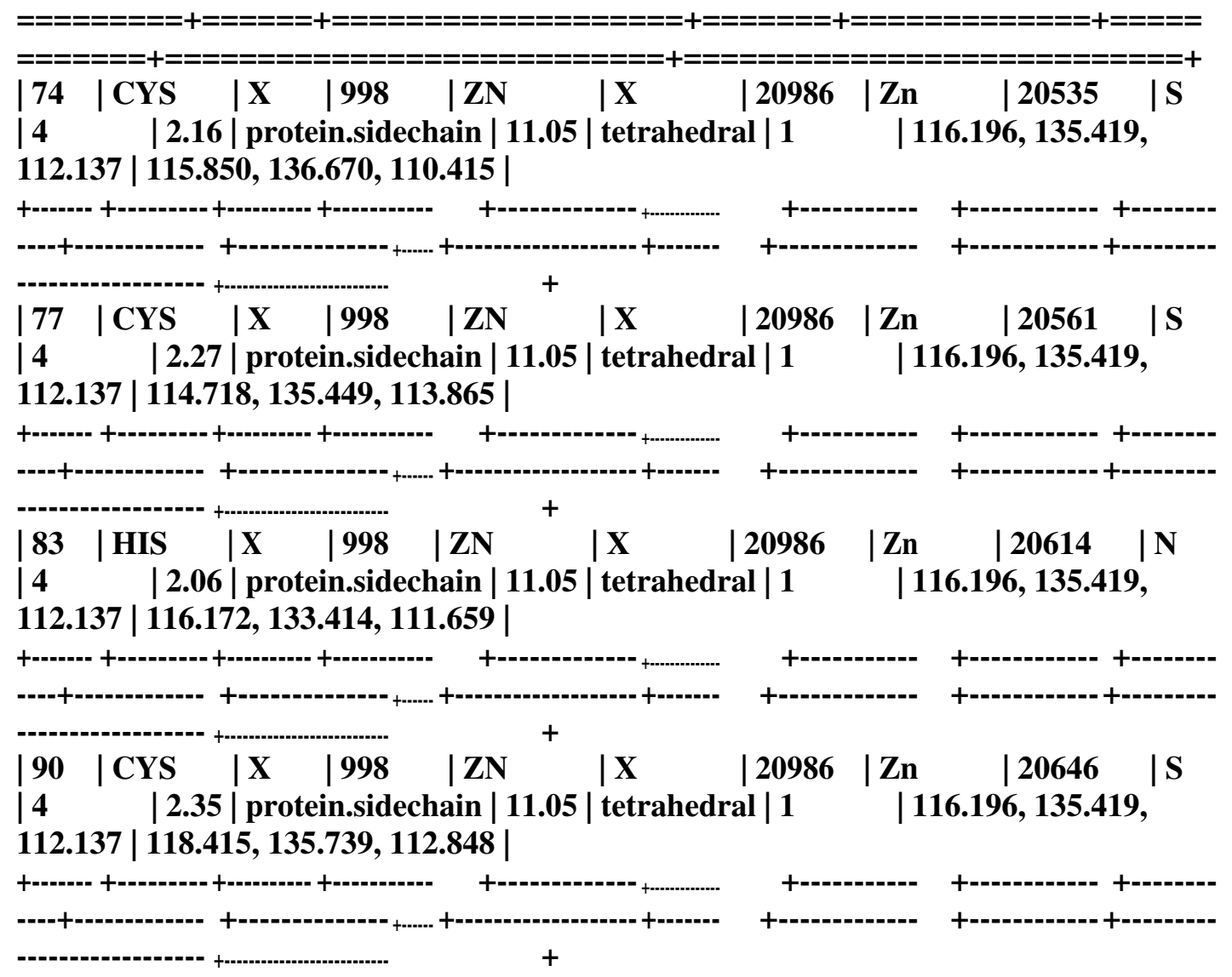

ZN:X:999 (ZN) - ION

Interacting chain(s): $\mathbf{X}$

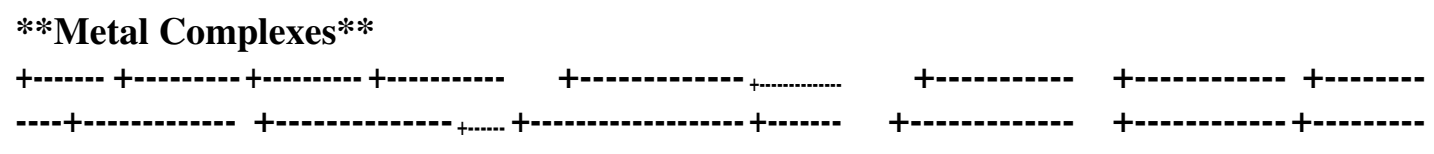

| RESNR | RESTYPE | RESCHAIN | RESNR_LIG | RESTYPE_LIG |

RESCHAIN_LIG | METAL_IDX | METAL_TYPE | TARGET_IDX |

TARGET_TYPE | COORDINATION | DIST | LOCATION

GEOMETRY | COMPLEXNUM | METALCOO

|RMS |

I

| TARGETCOO

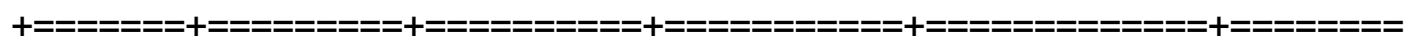

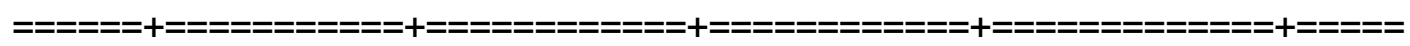

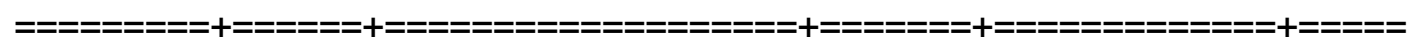

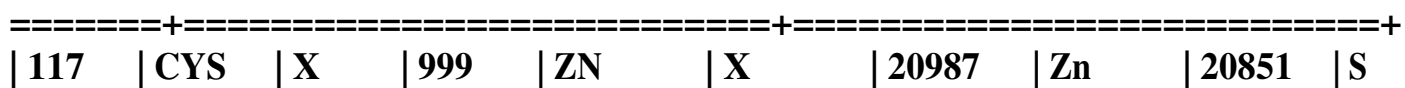
| $4 \quad|1.99|$ protein.sidechain $|68.10|$ tetrahedral $|1 \quad| 134.607,148.465$, 105.131 | 133.246, 147.084, 104.692 |

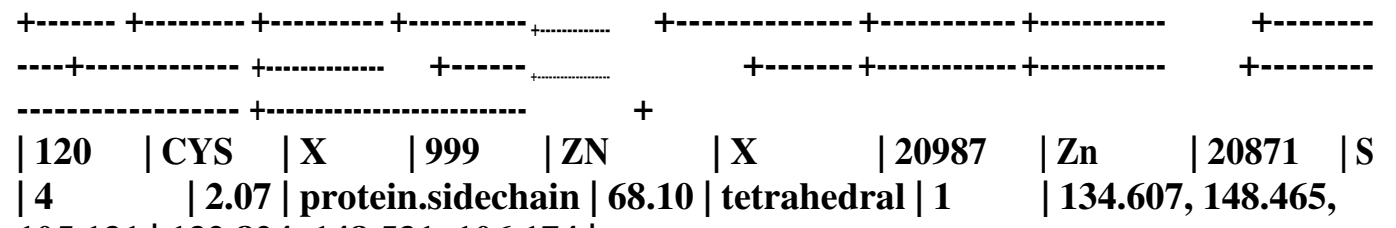
105.131 | 132.824, 148.531, 106.174 |

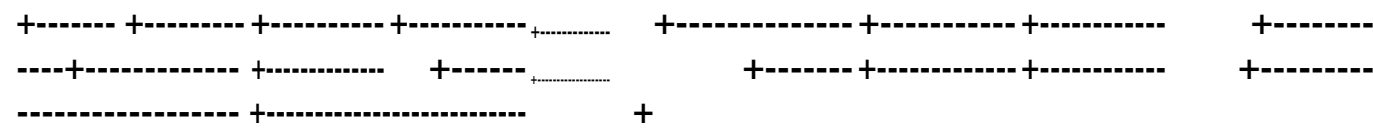




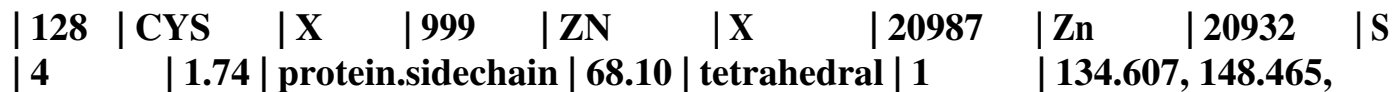
105.131 | 133.246, 149.141, 104.288 |
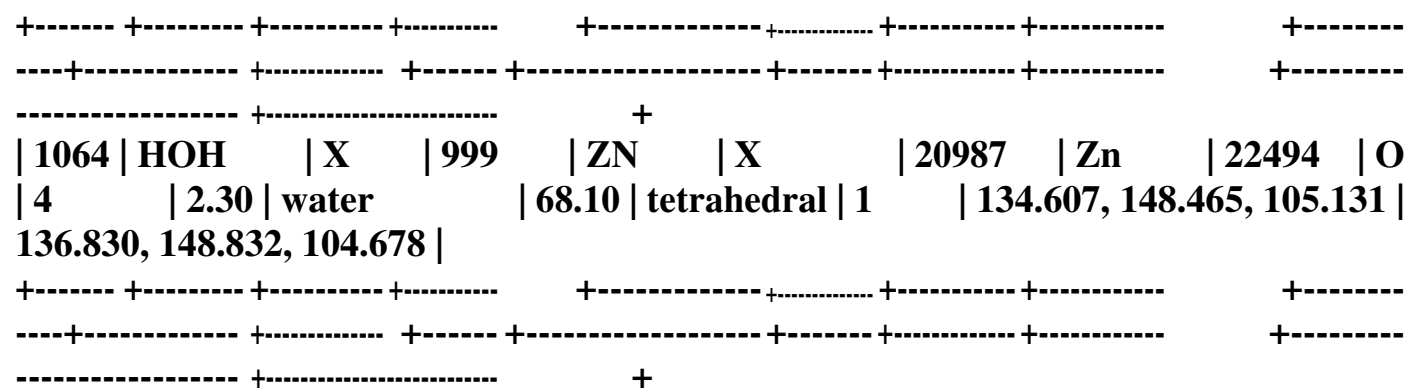


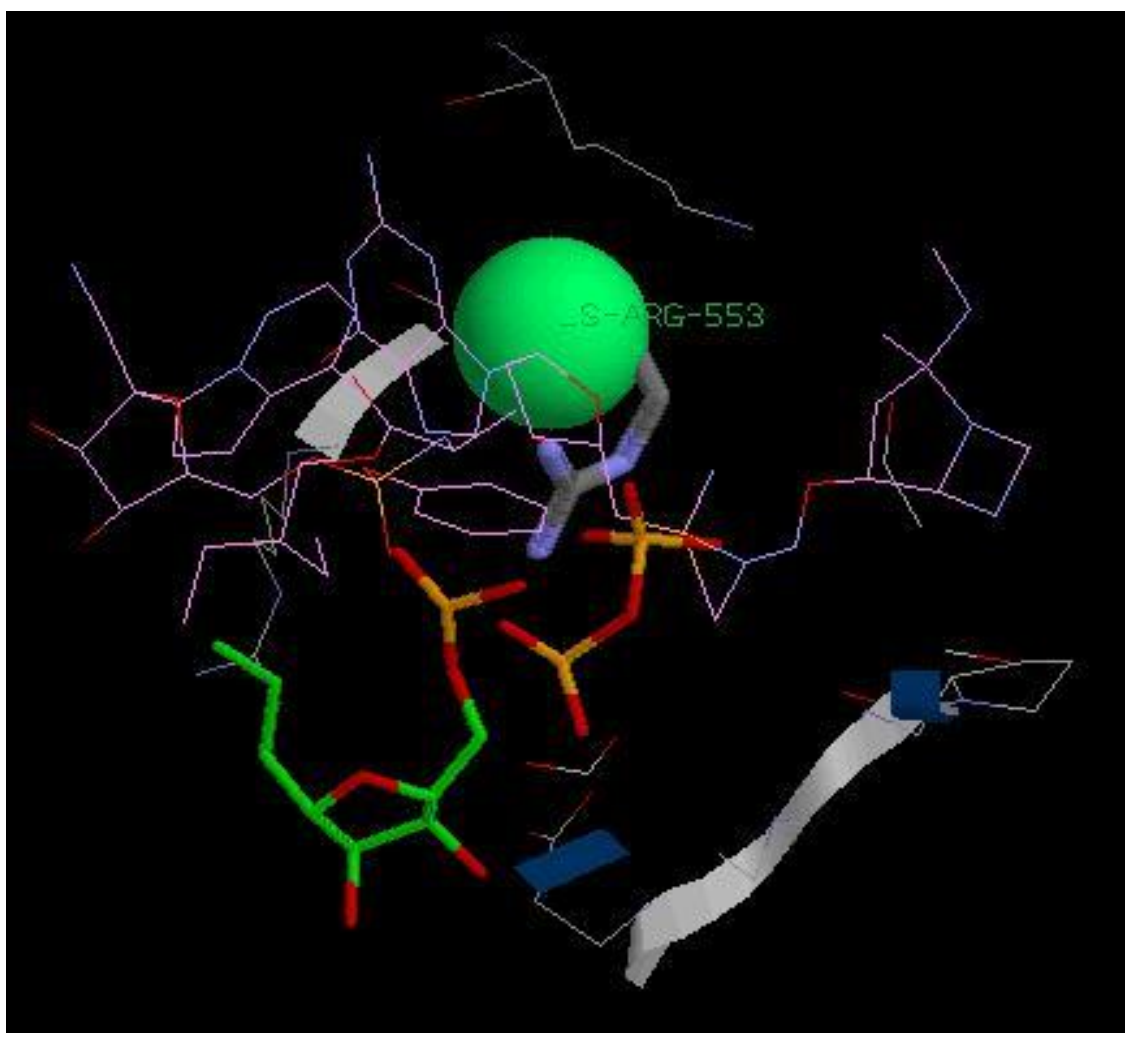

Figure6c. CoMFA contour map of electrostatic regions around Roccustyrna chemical structure. Contact residues of the Roccustyrna small molecule when docked onto the SARS-COV-2 protein targets, (pdb:7bv2). (green, favored; yellow; disfavored) around the Roccustyrna chemical structure. Blue regions are favored by positively charged groups and red regions favored by negatively charged groups.

GID Compound NAME GWEIGHT H-S-ARG-553 H-S-ASP-623 H-M-F86- $101 \mathrm{H}-$ M-F86-101 V-M-LYS-551 V-S-LYS-551 V-S-ARG-553 V-S-ARG-555 V- S-ASP-618 VM-TYR-619 V-M-PRO-620 V-M-CYS-622

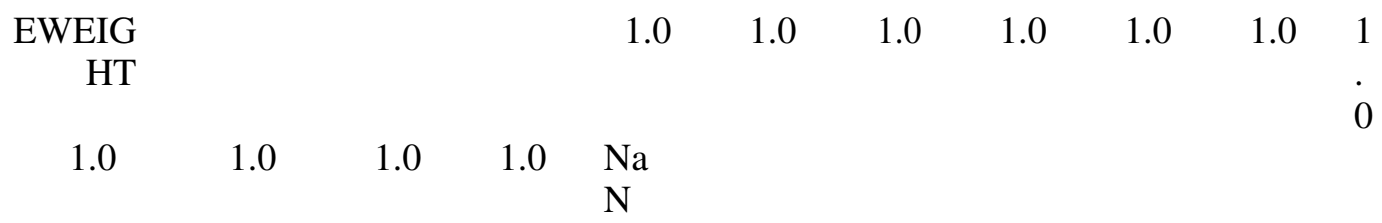

GENE0X cav7bv2_POP-Roccustyrna-0.pdb cav7bv2_POP-Roccustyrna-0.pdb

$\begin{array}{llllllll}1.0 & 0.0 & 0.0 & 0.0 & 0.0 & -4.71516 & -10.4842 & -4.7999-\end{array}$

2.78895-6.65538 $-5.1339-6.28532 \quad-0.923983$

\$Number_of_Clusters $=10$

$\$$ Seed $=-1985$

\$Leader_Info 1 \{

Num_Members $=105$

Total_Energy $=-1.004$

$\mathrm{vdW}=-6.072$

Coulomb $=-7.135$

Internal $=12.203$

rmsd $=0.000$ 
\$Leader_Info 2 \{

Num_Members $=47$

Total_Energy $=0.920$

$\mathrm{vdW}=-2.068$

Coulomb $=-9.214$

Internal $=12.203$

$\mathrm{rmsd}=10.703$

\}

\$Leader_Info 3 \{

Num_Members $=47$

Total_Energy $=1.118$

$\mathrm{vdW}=-6.320$

Coulomb $=-4.765$

Internal $=12.203$

$\mathrm{rmsd}=2.300$

\}

\$Leader_Info 4 \{

Num_Members $=41$

Total_Energy $=1.190$ $\mathrm{vdW}=-6.775$

Coulomb $=-4.238$

Internal $=12.203$

$\mathrm{rmsd}=3.039$

\}

\$Leader_Info 5 \{

Num_Members $=44$

Total_Energy $=1.613$

$\mathrm{vdW}=-3.033$

Coulomb $=-7.556$

Internal $=12.203$

$\mathrm{rmsd}=9.734$

\}

\$Leader_Info 6 \{

Num_Members $=40$

Total_Energy $=1.653$

$\mathrm{vdW}=-3.268$

Coulomb $=-7.281$

Internal $=12.203$

$\mathrm{rmsd}=9.324$

\}

\$Leader_Info 7 \{

Num_Members $=46$

Total_Energy $=1.675$ 


$$
\mathrm{vdW}=-6.062
$$

Coulomb $=-4.466$

Internal $=12.203$

$\mathrm{rmsd}=2.382$

\}

\$Leader_Info 8 \{

Num_Members $=42$

Total_Energy $=1.935$

$\mathrm{vdW}=-5.434$

Coulomb $=-4.834$

Internal $=12.203$

$\mathrm{rmsd}=1.449$

\}

\$Leader_Info 9 \{

Num_Members $=54$

Total_Energy $=2.091$

$\mathrm{vdW}=-3.880$

Coulomb $=-6.231$

Internal $=12.203$

$\mathrm{rmsd}=3.179$

\}

\$Leader_Info 10 \{

Num_Members $=37$

Total_Energy $=2.237$

$\mathrm{vdW}=-5.380$

Coulomb $=-4.586$

Internal $=12.203$

$\mathrm{rmsd}=3.188$

\}

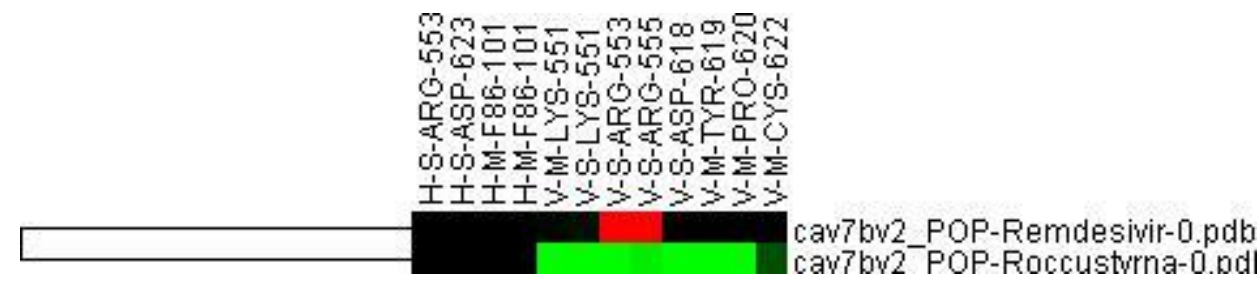

Figure6d. Comparative Docking Cluster Analysis between the Remdesivir and the Roccustyrna small molecules when docked onto the SARS-COV-2 protein targets, (pdb:7bv2). \$Number_of_Clusters $=10$

$\$$ Seed $=-1985$

\$Leader_Info 1 \{

Num_Members $=77$

Total_Energy $=-0.940$ 


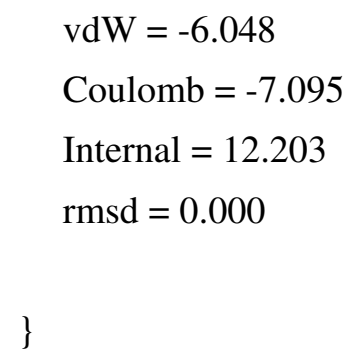

\$Leader_Info 5 \{

Num_Members $=33$

Total_Energy $=1.326$ 


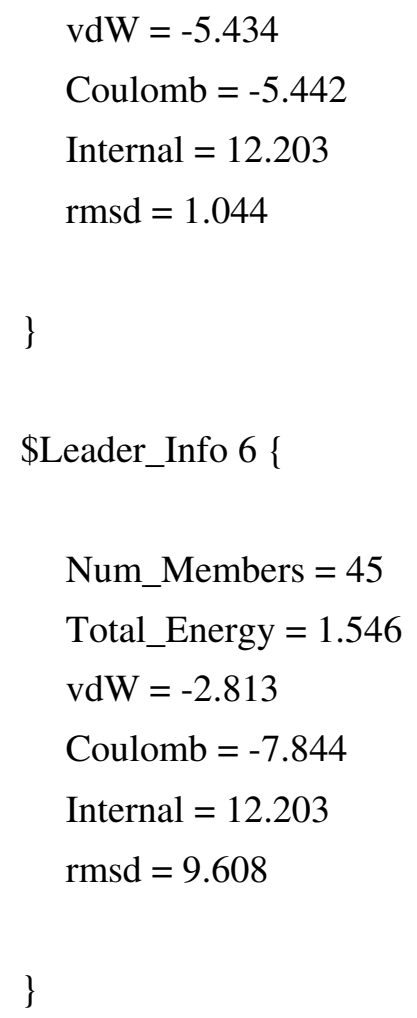

\$Leader_Info 9 \{

Num_Members $=47$

Total_Energy $=2.034$ 

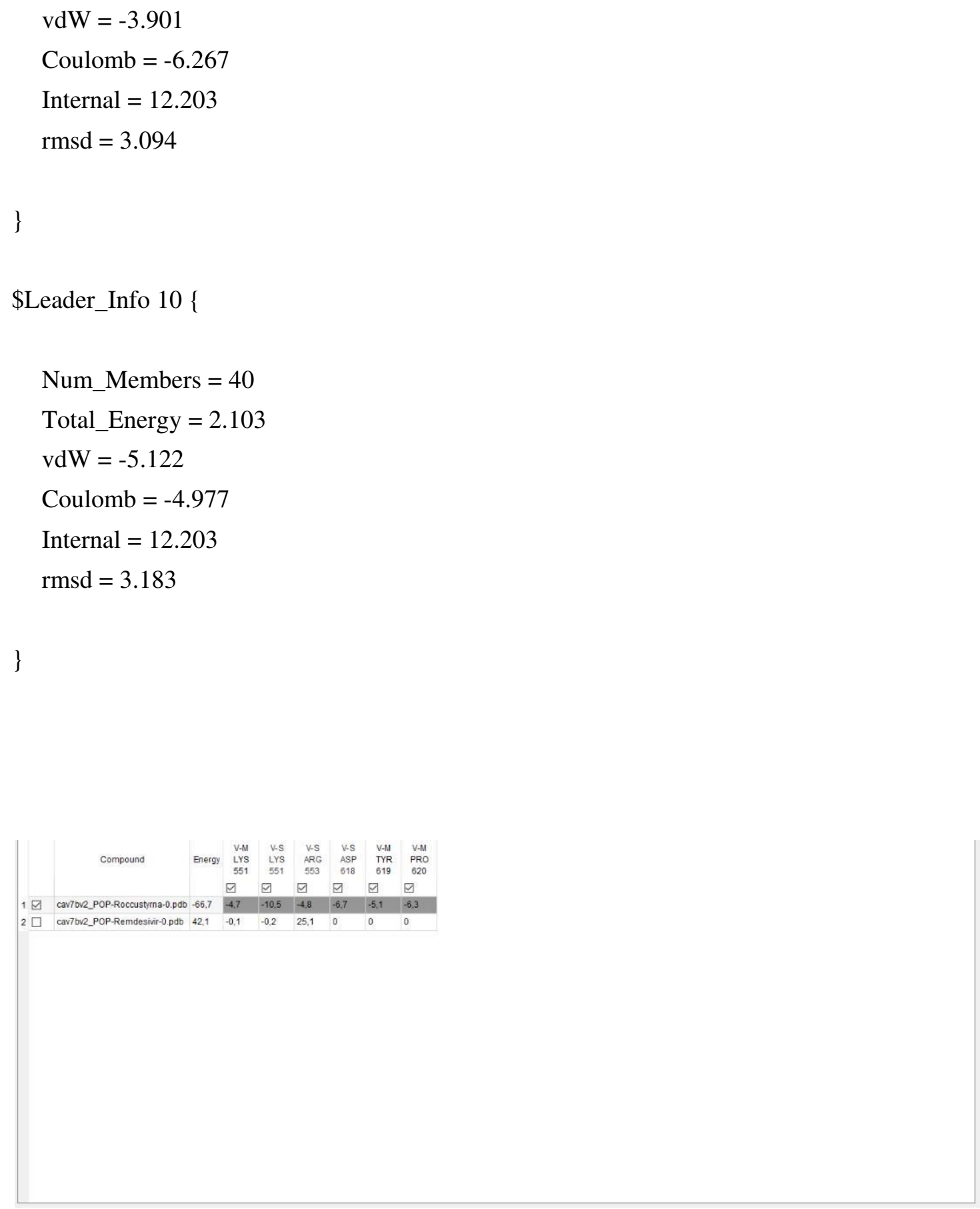

Figure6e. Comparative Docking Enegy Analysis between the Remdesivir and the Roccustyrna small molecules when docked onto the SARS-COV-2 protein targets, (pdb:7bv2). \$Number_of_Clusters $=10$

$\$$ Seed $=-1985$

\section{\$Leader_Info 1\{}

Num_Members $=63$

Total_Energy $=-0.883$

$\mathrm{vdW}=-6.041$

Coulomb $=-7.045$ 


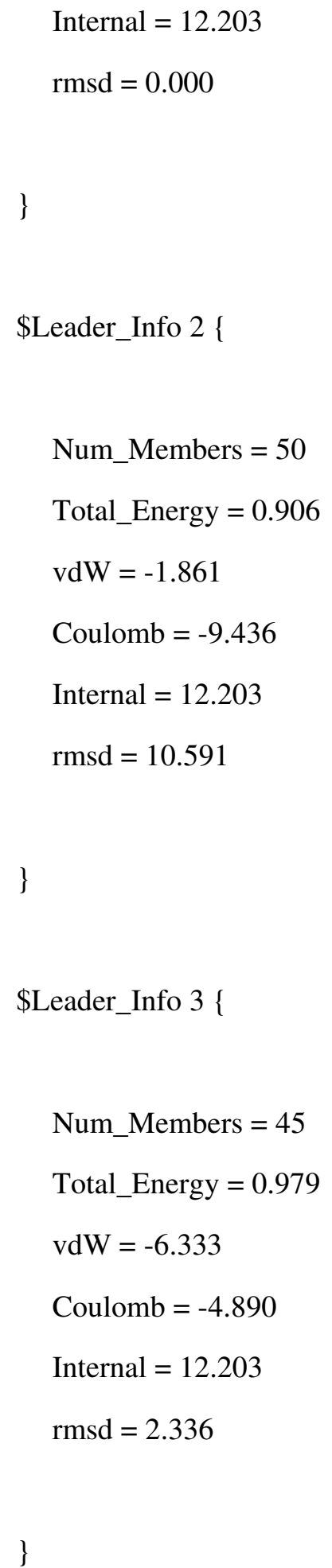




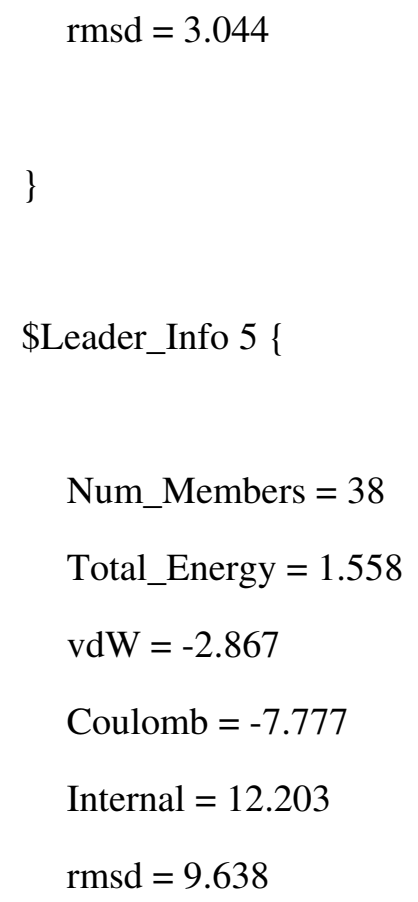

\$Leader_Info 7 \{

Num_Members $=48$

Total_Energy $=1.708$

$\mathrm{vdW}=-5.906$

Coulomb $=-4.588$

Internal $=12.203$

$\mathrm{rmsd}=2.351$ 
\$Leader_Info 8 \{

Num_Members $=28$

Total_Energy $=2.054$

$\mathrm{vdW}=-5.236$

Coulomb $=-4.912$

Internal $=12.203$

$\mathrm{rmsd}=1.304$

\}

\$Leader_Info 9 \{

Num_Members $=52$

Total_Energy $=2.059$

$\mathrm{vdW}=-4.013$

Coulomb $=-6.131$

Internal $=12.203$

$\mathrm{rmsd}=3.035$

\}

\$Leader_Info 10 \{

Num_Members $=43$

Total_Energy $=2.242$

$\mathrm{vdW}=-5.048$

Coulomb $=-4.913$

Internal $=12.203$

$\mathrm{rmsd}=3.188$ 


\begin{tabular}{|c|c|c|c|c|c|c|c|c|c|}
\hline $\begin{array}{l}\text { cav7bv2_POP. } \\
\text { pd } \\
\text { b:ClusterID }\end{array}$ & $\begin{array}{l}\text { T } \\
\text { op } \\
\text { of } \\
\text { cl } \\
\text { us } \\
\text { t } \\
\text { er }\end{array}$ & $\begin{array}{l}\text { Comp } \\
\text { ou nd }\end{array}$ & $\begin{array}{l}\mathbf{E} \\
\mathbf{n} \\
\mathbf{e} \\
\mathbf{r} \\
\mathbf{g} \\
\mathbf{y}\end{array}$ & $\begin{array}{l}\text { V- } \\
\text { M- } \\
\text { L } \\
\text { YS } \\
- \\
55 \\
1\end{array}$ & $\begin{array}{l}\text { V } \\
- \\
\text { S- } \\
\text { L } \\
\text { Y } \\
\text { S } \\
- \\
55 \\
1\end{array}$ & $\begin{array}{l}\text { V } \\
- \\
\text { S- } \\
\text { A } \\
\text { R } \\
\text { G } \\
- \\
5 \\
5 \\
3\end{array}$ & $\begin{array}{l}\text { V } \\
- \\
\text { S- } \\
A \\
\text { S } \\
\text { P } \\
- \\
61 \\
8\end{array}$ & $\begin{array}{l}\text { V- } \\
\mathbf{M} \\
- \\
\mathbf{T} \\
\mathbf{Y}\end{array}$ & $\begin{array}{l}\text { V- } \\
M \\
- \\
P \\
R \\
\\
O \\
- \\
620\end{array}$ \\
\hline 1 & 1 & $\begin{array}{l}\text { cav7bv } \\
2 \\
\text { _POP- } \\
\text { Roccust } \\
\text { y } \\
\text { rna } \\
- \\
0 . p \\
\text { db }\end{array}$ & $\begin{array}{l}- \\
6 \\
6 . \\
7\end{array}$ & $\begin{array}{l}- \\
4.7 \\
15 \\
16\end{array}$ & $\begin{array}{l}- \\
1 \\
0 . \\
4 \\
84 \\
2\end{array}$ & $\begin{array}{l}- \\
4 . \\
7 \\
9 \\
99\end{array}$ & $\begin{array}{l}- \\
6 . \\
6 \\
5 \\
53 \\
8\end{array}$ & $\begin{array}{l}- \\
5 . \\
1 \\
33 \\
9\end{array}$ & $\begin{array}{l}- \\
6 . \\
2 \\
8 \\
532\end{array}$ \\
\hline 1 & $\begin{array}{l}0.00 \\
0 \\
000\end{array}$ & $\begin{array}{l}\text { cav7bv } \\
2 \\
\text { _POP- } \\
\text { Remd } \\
\text { esi } \\
\text { vir- } \\
0 . p d b \\
\end{array}$ & $\begin{array}{l}42 . \\
1\end{array}$ & $\begin{array}{l}- \\
0.1 \\
04 \\
885\end{array}$ & $\begin{array}{l}- \\
0 . \\
1 \\
9 \\
98 \\
6\end{array}$ & $\begin{array}{l}25 . \\
0 \\
57 \\
5\end{array}$ & 0 & 0 & 0 \\
\hline
\end{tabular}

Table2b. Comparative Docking Enegy Numerical Score Analysis between the Remdesivir and the Roccustyrna small molecules when docked onto the SARS-COV-2 protein targets, (pdb:7bv2).

This small molecule prototype was obtained directly from the solution of the $D\left[-\left(k^{\wedge}\left(-4-k^{\wedge} 4 n^{\wedge} 33835 N^{\wedge} 2\right) n^{\wedge} 33834\right.\right.$

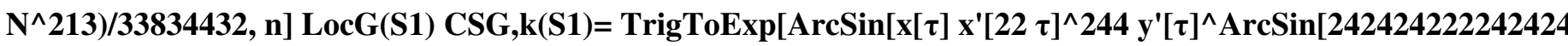
$\left.\left.\left.\operatorname{Sin}[X] \times[\tau]^{\wedge} 22\right]\right]\right]_{V e c t k G}[G]=\operatorname{Rep}(\operatorname{Dk}(C[G]))=Z(\operatorname{Vectk}[G])$ equation $(1)$ into the $\left(k^{\wedge}\left(-8-k^{\wedge} 4 n^{\wedge} 33835 N^{\wedge} 2\right)\right.$ $\left.\mathbf{N}^{\wedge} 211\right) /(1144788006720 \log [3 d \mathrm{~N}=2 \mathrm{CS}$ k]) (equation $2,3,4,14)$ by a procedure akin to geometric quantization as derived by a first phase solution was taken as the $\mathbf{1 3}(\mathbf{a}+\mathfrak{\ell}) \mathbf{3 d} \mathbf{N}=\mathbf{2} \mathbf{C S} \operatorname{ta}+\boldsymbol{\ell}-\mathbf{1} \mathrm{A} \wedge[\mathrm{A} \wedge \mathrm{A}] \mathbf{2 z E G} \mathbf{k}(\bmod 2 \pi)$ equation (5) determined fragmentation. $(36,37,39)$ and larger groups are prioritized over smaller chemical patterns with potential antiviral properties of the RoccustyrnaTM 's small molecule (1S,2R,3S)-2-(\{[(1S,2S,4S,5R)-4- ethenyl-4- sulfonyl bicyclo[3.2.0] heptan-2-yl]oxy\}amino)-3-[(2R,5R)-5- (2-methyl-6- methylidene-6,9-dihydro-3H-purin-9yl)-3-methylideneoxolan-2-yl]phosphirane-1-carbonitrile PDB generated (Figure1b) patterns.

\section{Roccustyrna ligand Protein targets}

The docking engine employed in this computer-aided drug design effort is the DockThor program, which generates preparations of the acceptable topology files for the Roccustyrna ligand for the protein (.in) and ligand cofactors (.top) and a specific input .pdb file containing our prototype 's ligand atoms and Roccustyrna partial charges from the MMFF94S49 force field. $(19,21,37,42)$ The .pdbqt file of the Roccustyrna new ligand was generated by the MMFFLigand software, which is based on the utilities of the OpenBabel chemical toolbox for extracting atom types and partial charges with MMFF94S applied force field, and for the identification of the rotatable bonds, and calculating the properties necessary for computing the intramolecular interactions. $(16,17,41)$ In the MMFFLigand, all hydrogen atoms were removed and the PdbThorBox software was utilized to set the protein atomic types, and the partial ionization charges from the MMFF94S force field analysis considering the nonpolar atomic groups as implicit to rebuild missing residue side chain atoms. (3-9,31) Thus, in this KNIME based GEMDOCK-DockThor-Virtual Screening platform, both the Roccustyrna small molecule, SARS-COV-2 protein targets, and cofactors were treated again with the MMFF94S force field by keeping the same set of equations and parameters that define the new 
molecule's molecular force field parameterizations. (2,31-40) The preparations of the steps to be used for diagonal force field for modeling such as modifying the protonation state of all the keeping amino acid residues, to parameterize a simple group of knots and atoms by adding metal complexes, hydrogen atoms and freezing rotatable bonds was done interactively for a variety of all-Roccustyrna atoms in the publically available web servers and performed automatically by the programs cited without the need for intervention. $(3,15,16,17)$ The search docking space to rapidly simulate the Roccustyrna's molecular systems and the configuration of the new molecule's grid box were interactively set in the KNIME designed BiogenetoligandorolTM pipeline which were represented as a grid box and the docking potentials are stored at the best grid points for the description of the Roccustyrna's molecular energetic and structures through the parameters of the center of coordinates, size of the grid and discretization (i.e., the spacing between the grid points). $(5,13,29,31)$ The initial population for the rotational, and translational, was randomly generated within the conformational degrees and grid box using random values of freedom of the Roccustyrna ligand. $(15,16,17,38)$ For each SARS-CoV-2 therapeutic target DockThor-VS default parameters were uploaded as a recommended set of parameters for the grid box (i.e., center and grid sizes) which can be used or modified according to the objectives of this docking experiment which was specially designed to deal with highly flexible ligands such as the Roccustyrna small molecule. $(15,29,31)$ In this strategy, a replacement ligand based method was introduced by using a low mass phase phenotypic steady-state and crowding-based protocol and a multiple genetic parental algorithm as a Dynamic Solution Modified Restricted Tournament Selection (DSMRTS) approach, which provided us a better machine learning exploration of the energetic hypersurface for the identification of multiple quantum phase minima solutions in a single Hadamard run, preserving the population diversity of the generated structures. The default parameters of this parallel docking algorithm (named BiogenetoligandorolTM) is set in the KNIME-web server as follows: (i) 24 inverse docking runs, (ii) 1.000 .000 evaluations per parallel docking run, (iii) population of the Roccustyrna individuals, (iv) maximum of 20 cluster small molecule top leaders on each parallel inverse docking run. For this sequential screening experiment, we also provided an alternative dataset of geometric parameters to improve the Euclidean space between the Roccustyrna and protein interacting chains without significantly losing binding site accuracy (named EuTHTS Euclidean Topology Virtual Screening): (i) 120 docking runs, (ii) 200.000 evaluations per docking run, (iii) population of Roccustyrna individuals, (iv) maximum of 20 cluster leaders on each docking run. The docking experiments were performed on DockThor CPU nodes of the SDumont supercomputer, each one containing two processors Intel Xeon E5-2695v2 Ivy Bridge (12c @2,4 GHz) and 64 Gb of RAM memory. We validated the docking experiments through the redocking of the non-covalent Roccustyrna ligand present in the complexes 6W63 (Mpro) using the standard configuration, successfully predicting the co-crystallized conformation of each complex. In the crystallographic structure, this moiety is exposed to the solvent and has insufficient electronic density data. The free energy scoring function applied to score the best docked poses of the same Roccustyrna ligand was based on the sum of the following terms from the MMFF94S force field and is named - Total Energy (Etotal)\|: (i) intermolecular interaction energy calculated as the sum of the van der Waals between the hydroxyl and cyno groups (buffering constant $=0.35$ ) and electrostatic potentials between the protein-ligand atom pairs, (ii) intramolecular interaction energy of the van der Waals and electrostatic potentials calculated as the sum between the 1-4 atom pairs, and (iii) torsional term of the ligand. All best docking poses generated during all the docking steps in this project were then low mass weight categorized and clustered by our in-house tool BiogenetoligandorolTM. The top docking energy-poses of each Roccustyrna-Protein complex were selected as top hit representatives of cluster energetic representatives to be made available in the homogenicity results analysis and Chern-Simons pharmacophoric fragmentation section $(27,28)$. The binding affinity prediction and total energy ranking with the linear protein model and untailored for specific ligand interacted protein classes, of the Roccustyrna small molecule was generated by utilizing the DockTScoreGenLscoring function as a set of empirical scoring functions. Biogenetoligandorol cluster of DockTScore, PLIP, DockThor and GEMDOCK-AUTODOCK-VINA current docking scoring functions for protein and small molecule preparation take into account important terms, multiple protein-ligand binding, such as intermolecular interactions, binding affinity predictions, Roccustyrna's ligand entropy and desolvation of the specific target classes such as SARS-COV-2 6W63 (Mpro) proteases using protein-protein interactions (PPIs) trained with PdbThorBox and MMFFLigand sophisticated machine-learning derived topology algorithms totalizing 66892 contacts between a carbon and a halogen, carbon or sulfur atom. The docking visualization of the SARS-COV-2 protein, cofactors and the Roccustyrna compound, the grid space location superposed with the protein targets of the (PDB codes of the PDB:6xs6,1 xak,2g9t,3fqq, 2ghv,6yb7) $(3,4,35,39)$ and the docking outputs were generated with NGL, a WebGL-based library for intra-molecular visualization. 


\section{In silico Bioactivity Prediction and ADMET Analysis of the Roccustyrna small molecule.}

The drug likeliness and bioactivity of the Roccustyrna small molecule were initially analyzed utilizing the Molinspiration server (http://www.molinspiration.com). (30,31) By using the Molinspiration web-tool, a cheminformatics multi-tasking software we calculated the Roccustyrna 's molecular modeling docking energy properties as well as drug likeness and bioactivity prediction of our prototype ligand (Mabkhot et al., 2016). (40,41) In this section, the Molinspiration-based drug-likeness analysis web platform was incorporated to predict the Roccustyrna 's two important factors, including the polar surface area (PSA), and lipophilicity level (log P) directly associated with the pharmacokinetic properties (PK) of the same lead structure (Beetge et al., 2000). (31,40) Then, by uploading the Roccustyrna 's smiles in the Molinspiration-based bioactivity analysis web server, we calculated the bioactivity score of this ligand through the systemic examinationion channel modulators, GPCR ligands, nuclear receptor ligands, kinase inhibitors, protease inhibitors, and other enzyme targets which were analyzed by sophisticated Bayesian statistics (Mabkhot et al., 2016). (5,7,40) The PK properties, such as adverse effect predictive modeling, Absorption, Distribution, Metabolism, Excretion, and Toxicity (ADMET), of the Roccustyrna pharmacophoric scaffold were predicted by utilizing the admerSAR v2.0 server (http://lmmd.ecust.edu.cn/admetsar2/) for the prediction of our novel Roccustyrna 's ADMET properties on factors such as membrane permeability [designated by colon cancer cell line (Caco-2)], human intestinal absorption (HIA), and the status of either P-glycoprotein substrate or inhibitor. Finally, knowledge of these processes and more specifically the ability of the Roccustyrna 's small molecule to penetrate the blood-brain barrier and its metabolism is of crucial importance to evaluate the risk of exposure to toxins and was evaluated by the MATE1, CYP, and OATP1B3 -OATP1B1testing models. The Excretion of the Roccustyrna ligand was estimated by applying the advanced matched molecular pair analysis (MMPA), based on the renal OCT substrate and the toxicity which was then predicted accordingly on the Human Ether-a-go-go-related gene inhibition, mutagenic status, carcinogenic status, and acute oral toxicity default parameters $(30,31,40,41)$.

\section{Results}

\section{In silico Prediction of the Roccustyrna ADMET Properties and Bioactivity Score}

To predict important molecular properties such as $\log$, polar surface area, drug-likeness and bioactivity of our new prototype and small sized Roccustyrna ligand (1S,2R,3S)- 2-([[(1S,2S,4S,5R)-4- ethenyl-4-sulfonylbicyclo $(1 S, 2 R, 3 S)-2-([[(1 S, 2 S, 4 S, 5 R)-4$ - ethenyl-4- sulfonylbicyclo[3.2.0]heptan-2-yl]oxy\}amino)- 3-[(2R,5R)-

5-(2-methyl-6-methylidene-6,9-dihydro-3H-purin-9-yl)-3-methylideneoxolan-2-yl]phosphirane-1-carbonitrile[3.2.0]he ptan-2-yl]oxy\}amino)-3-[(2R,5R)-5-(2-methyl-6-methylidene-6,9-dihydro-3H-purin-9-yl)-3-methylideneoxolan-2-yl]p hosphirane-1-carbonitrile, the Molinspiration tool was employed as customized on the basis of this rational anti-viral drug design study.The milogP (Octanol-water partition coefficient $\log \mathrm{P}$ ) and TPSA (Topological polar surface area) values were calculated by utilizing the same online tool using Bayesian statistics. These In-Silico results indicated that the milogP value of the Roccustyrna small molecule was predicted as having optimum lipophilicity properties $(\log P<5)(H a n$ et al., 2019$)$ in the aspect of dermal absorption and parallel artificial permeation (Table1c).

\section{Screening of the Roccustyrna Inhibitor for Spike Protein-RBD-ACE2 Interaction}

In this study we have shown that the QMMM designed Roccustyrna small molecule which was designed in silico by using Topology Euclidean Geometric and Artificial Intelligence-Driven Predictive Neural Networks was engaged in the binding domains of the protein targets of the (pdb:1xak) (Figure4a) with the docking energy values of the (T.Energy, I.Energy, vdW, Coul, NumRotors, RMSD, Score), (-19.625, -35.483, 7.633, -43.116, 7, -5.813)Kcal/mol, (Tables1a,1b,1c,2a,2b) The Roccustyrna chemical structure interacted into the binding sites of the protein targets (pdb:6w9c), (Figure4b),(Figure7e) with the negative docking energies of the (T.Energy, I.Energy, vdW, Coul, NumRotors, RMSD, Score), (-36.678,-55.648, -7.519, -48.129, 7, -6.762) Kcal/Mol. It also generated hydrophobic interactions when docked onto the binding cavities of the amino acid of the $168 \mathrm{PRO}, \mathrm{A} 1,02 \mathrm{~J} \mathrm{C}$ with the docking energy values of the $3.53,2369,1303,-10.425,3.42,72.447,-13.394,3.19,70.551 \mathrm{Kcal} / \mathrm{mol}$. Our new QMMM designed small molecule named Roccustyrna involved in the generation of the hydrogen bonding within the PJE:C:5 (PJE-010) 010:C:6 Interacting chain(s) while generating hydrophobic interactions when docked into the binding domains of the amino acid of the 25THR, A6, $010 \mathrm{C}$ domains with the docking energy values of the $3.73,2415,179$, 7.156, 21.406, $66.898-8.709,22.779,70.002 \mathrm{Kcal} / \mathrm{mol}$. 
The Roccustyrna 's active pharmacophoric site of the (methylamino)-1,6- diazabicyclo(3.2.0) heptan-4-yl)oxy\}imino) interacted into the binding cavities of the amino acid of the 26 THR, A6 010C with the docking energy values of the $3.81,2415,186,-7.156,21.406,66.898,-6.155,24.392,64.757 \mathrm{Kcal} / \mathrm{mol}$. The Roccustyrna 's active pharmacophoric site of the dihydro-3H-purin-9-yl)-3- hydroxyoxolan generated a docking effect which was involved in the generation of hydrogen bonds when docked into the binding cavities of the amino acid of the 143 GLY A $6010 \mathrm{C}$ with the docking energy values of the 1.93, 2.8, 145.29, 1105, 2411, -8.911, 17.849, 65.703-8.918, 17.918, 62.905

$\mathrm{Kcal} / \mathrm{mol}$. The same prototype pharmacophoric elements named Roccustyrna when docked into the binding sites of the amino acid of the 164HIS, A5, PJE C2.generated hydrogen interactions with the binding energy values of the 16 3.07, $153.73,2408$, in the coupled atoms of the $\mathrm{N} 3$ and $\mathrm{O} 2$ with the docking energy values of the $-12.282,14.994,67.123$ 15.161, 15.336, 68.144 Kcal.Mol. The binding patterns of the 02J:C:1 (02J) active sites of the amino acid 168 PRO, A1, 02J C binding domains generated hydrophobic interactions with docking energy values of the 3.53, 2369, 1303, 10.425, 3.42, 72.447, -13.394, 3.19, 70.551 inside the PJE:C:5 (PJE-010) + 010:C:6 interacting chain(s): A C of the amino acid of the 164HIS, A5, PJE C2. (Figure5b). D10-C-1099 DMS:A:402 (DMS) binding sites were also constructed when RoccustyrnaTM's pharmacophoric elements docked inside the ( pdb:6lu7)protein targets. Hydrogen Bonds were then identified when the RoccustyrnaTM's chemical coupled atoms interacted within the 298 ARG A amino acid's 402 DMS A Ng+ $2377 \mathrm{O} 2$ binding cavities with the docking energy values of the $(-1.76,-$ $2.73,-166.89,-2331,6.971,-0.756,-7.541-9.700,-0.883,-7.581) \mathrm{Kcal} / \mathrm{mol} / \mathrm{A}$. Salt Bridges were also shown to be involved in the generation of the Sulfonium bondings when docked inside the DMS A 5.49 binding cavities of the amino acid of the 295 ASP A with the docking energy values of the $(-402,-2376,-6.081,-1.005,-6.367-10.436$, $-2.231,-5.560) \mathrm{Kcal} / \mathrm{mol} / \mathrm{A}$. pi-Cation Interactions of sulfonium bondings within our small molecule whole residue subsurface were also constructed within the 8 PHE A amino acid 402 DMS A pharmacophoric sites with the docking energy values of the $(-4.70,-1.01,-2376,-6.081,-1.005,-6.367,-8.339,-4.556,-4.264) \mathrm{Kcal} / \mathrm{mo} / \mathrm{A}$. (Figure5d). Hydrophobic Interactions were simultaneously generated by the Roccustyrna chemical residues when docked in the ( $p d b: 6 l u 7$ )inside the D10-H-1099. X77:A:401 (X77) protein domains within the active sites of the amino acids of the $41 \mathrm{HIS}$ A 401 X77 A, 165 MET A 401 X77 A, and 166 GLU A 401 X77 A with the docking energy values of the $(-3.75,-4670,-609,-20.444,-13.613,-29.034,-19.778,-13.574,-32.721-3.90,-4673,-2529,-$ $19.389,-17.775,-28.688,-16.611,16.152,-26.489,-3.86,-4661,-2546,-17.350,-23.138,-25.438-16.439,-$ 20.244, -23.055, -189 GLN A 401 X77 A -3.90, -4657,- 2881, -21.763, -15.894, -23.429-24.934, -13.635, $23.312) \mathrm{Kcal} / \mathrm{mol} / \mathrm{A}$ showing that my Ai-quantum thinking chemical structure named Roccustyrna is capable of generating Hydrogen Bonds when docked onto the 41 HIS A 401 X77 A, 143 GLY A 401 X77 A, 144 SER A 401 X77 A, and 166 GLU A 401 X77 A, sequence of amino acids while targeting the Npl 4680 N2, O3 4679 N2 Nam $4682 \mathrm{O} 2$, and Nam $4683 \mathrm{O} 2$ binding sites with the binding free energy values of the $(-3.46,-3.79,-106.13,-611,-$ $20.860,-19.573,-32.52,-19.394,-16.086,-32.767,-2.17,-2.94,-148.03,-2216-19.635,-22.244,-29.036-18.779$, $-24.455,-30.773,-3.14,-3.42,-101.78,-2228-16.096,-21.679,-26.816,-14.503,-23.707,-29.056,-1.98,-2.80,-$ 158.32, $-2542-18.546,-18.654,-26.028-16.172,-18.348,-24.583) \mathrm{Kcal} / \mathrm{molA}$ respectively. (Figure 7f). The (1Z)-2-\{((2S,3S,5R)-5-(2-amino-6-oxo-6,9-dihydro-1H-purin-9-yl)-3-hydroxyoxolan-2-yl)methylidene\}-2-cyano1 -(\{((2S,4R,5R)-2-methyl-2-(methylamino)-1,6-diazabicyclo(3.2.0)heptan-4-yl)oxy\}imino)-1lambda5,2lambda5-azap hosphiridin-1-ylium. druggable scaffold of the Roccustyrna small molecule therefore competes with endogenous SARS-CoV2 PLpro for binding to Lys711 and Arg355 targeting into the binding domains of the critical SARSCoV2 PLpro residues onto the SARS- COV-2 protein targets (pdb:2zu5) within the binding sites of the amino acid of the V-M-THR-25, V-S-THR-25, V-M-THR-26, V-S-HIS-41, V-M-LEU-141, V-M-ASN-142, V-S-ASN-142, $\mathrm{V}-\mathrm{M}-\mathrm{GLY}-143, \mathrm{~V}-\mathrm{S}-\mathrm{CYS}-145, \mathrm{~V}-\mathrm{M}-\mathrm{MET}-165$ with the binding energy values of the -97.2 and $-5.16512,-4.15949$, $-9.8487,-4.77062,-4.72901,-6.7295,-5.82428,-5.35883,-4.2588,-5.37491$ respectively. (Figure7d). CoMFA contour map analysis of electrostatic regions around the Roccustyrna chemical structure indicated to us that Hydrogen bonds, Salt bridges and Metal complexes containing Diphosphate, dihydrogen and ION binding sites were generated into the contact residues of the Roccustyrna small molecule when docked onto the SARS- COV-2 protein targets of the (pdb:2zu5) within the sequence of the amino acid of the V-M-THR-25, V-S-THR-25, V-MTHR-26, V-S-HIS-41, V-M-LEU-141, V-M-ASN-142, V-S-ASN-142, V-M-GLY-143, V-S-CYS-145, V-M-MET- 
165 with the binding energy values of the negative docking values of the $(-97.2$ and $-5.16512,-4.15949,-9.8487,-$ $4.77062,-4.72901,-6.7295,-5.82428,-5.35883,-4.2588,-5.37491) \mathrm{Kcal} / \mathrm{mol} / \mathrm{A}$ respectively. (Figure6a)

Roccustyrna DMS:A:402 (DMS) binding sites into the 524 Nam 2578 O2 02J (5-Methylisoxazole-3-carboxylic acid) domains inside the 65 ASN A 402 DMS A cavities of the pdb:6LU7 protein targets generated Hydrogen Bonds of the docking energy values of the $(-2.05,-2.94,-148.0,-8.211,-20.857,-29.787-11.058,-20.242,-30.160298)$

) $\mathrm{Kcal} / \mathrm{mol} / \mathrm{A}$. Salt Bridges were also contructed when our prototype's surface sites docked inside the DMS -A, $\mathrm{Ng}+2582 \mathrm{O} 2$ binding pocket cavities of the amino acid of the ARG A 403 with the docking energy values of the ($1.93,-2.87,-160.38,-2512-7.044,-0.753,-7.4699 .865,-1.270,-7.327) \mathrm{Kcal} / \mathrm{mol} / \mathrm{A}$. Sulfonium bondings were also constructed when our small molecule 403 DMS A contact residues of the binding sites of the 295 ASP amino acid with the docking energy values of the $(-5.31,-2581,-6.227,-1.042,-6.293-10.460,-2.019,-5.344) \mathrm{Kcal} / \mathrm{mol} / \mathrm{A}$. (Figure6b). 999 ZN D 20947 Zn , ZN:A:998 (ZN) and 998 ZN A 20940 Zn 470 S Metal Complexes were constructed into the 02J (5-Methylisoxazole-3-carboxylic acid) PJE-C-56LU7 Roccustyrna's

(1Z)-2-\{((2S,3S,5R)-5-(2-amino- 6-oxo-6,9-dihydro-1H-purin-9-yl

-4-yl)oxylimino)-1lambda5,2lambda5-azaphosphiridin-1-ylium. binding sites inside the 117 CYS D, 74 CYS A amino acids tetrahedral side chains with the docking energy values of the $(-1103.746,-101.848,-13.968,-103.306,-$ $102.613,-16.001-1118.874,-104.964,-32.313-118.938,-103.573,-30.6090 \mathrm{Kcal} / \mathrm{mol} / \mathrm{A}$ generated indicating to us that our multi-targeted drug design could of generating a self-assembled monolayer inside the 1: Mg, NA (1), 1, 10P, G Metal Complexes when docked onto the 1,553A binding cavities of the amino acid of the ARG into the pdb:7bv2 protein targets. The Roccustyrna 's pharmacophoric

(1Z)-2-\{((2S,3S,5R)-5-(2-amino-6-2-yl)methylidene $\}-2$ - cyano-1 - (\{((2S,4R,5R)-2-methyl-2-(methylamino)-1,6-dia zabicyclo(3.2.0)heptan-4-yl)oxy\}imino)-1lambda5,2lambda5-azaphosphiridin-1-ylium. active site of the 2-lambda5azaphosphiridin-1-ylium was engaged in hydrogen bonding interactions with the formation of hydrogen bonds inside the N3 $1266 \mathrm{O} 2$ binding cavities of the amino acid of the $143 \mathrm{GLY}, \mathrm{A} 6,010 \mathrm{C}$ with the docking energy values of the ($1.93,-2.80,-145.29,-1105,-3.81,-2415,-186,-7.156,-21.406,-66.898-6.155,-24.392,-64.757,-2411,-8.911,-$ $17.849,-65.703-8.918,-17.918,-62.905,-2.16,-3.07,-153.73,-2408,-12.282,-14.994,-67.123,-15.161,-15.336,-68.144)$ $\mathrm{Kcal} / \mathrm{mol}$. The Roccustyrna small molecule involved also in the generation of the hydrophobic interactions within the binding domains of the amino acid of the 25 THR A $6010 \mathrm{C}$ with the docking energy values of the 3.73, 2415, 179, -7.156, 21.406, 66.898 $-8.709,22.779,70.002 \mathrm{Kcal} / \mathrm{mol}$ as illustrated in the (Figure5c). (Figure6c). In this drug designing project the electrostatic regions around the RoccustyrnaTM's 3-hydroxyoxolan-2-yl)methylidene $\}-2-c y a n o-1-(\{((2 \mathrm{~S}, 4 \mathrm{R}, 5 \mathrm{R})-2-m e t h y l-2-$ (methylamino)-1,6diazabicyclo(3.2.0)heptan-4-yl)oxy \}imino)-1lambda5,2lambda5-azaphosphiridin-1-ylium. substrate targets the SARS-COV-2 protein targets, (pdb:7bv2) with the docking values if the (Total Docking Energy $=-1.004$, vdW $=-6.072$, Coulomb $=-7.135$, Internal $=12.203) \mathrm{Kcal} / \mathrm{mol} / \mathrm{A}$, (Figure5a) showing that RoccustyrnaTM's binding site(s) inside the (pdb:6lu7) binding domains of the 02J:C:1 (02J) regions while co-generating Hydrophobic Interactions and Hydrogen Bonds against the coupled atoms of the Nam 2411 O3 inside the cavities of the crucial entering amino acids of the 25 THR A 6010 C and 143 GLY A $6010 \mathrm{C}$ with the docking energy values of the $(-3.73,-2415,-179,-7.156,21.406,-66.898,-8.709,-22.779,-70.002,-26,-81,-2415,-186,-7.156$, 21.406, -66.898, -6.155, -24.392,- 64.757,- 1.93, -2.80, -145,.-29, -1105, -8.911, 17.849, -65.703, -8.918, - 17.918, -

62.905.) Kcal/mol/A respectively. In this project, we implemented Quantum Heuristic Fragmentation Algorithms for the merging and recoring of the hit selected Drug Pair interactions by using Quantum Hamiltonians for the $=\gamma \mathrm{B} \cdot\left(\mathrm{S}^{\wedge} 1+\mathrm{S}^{\wedge} 2\right)+\mathrm{I}^{\wedge} \cdot \mathrm{A} \cdot \mathrm{S}^{\wedge} 2$,

$\mathrm{S}^{\wedge} \mathrm{i}=\left(\sigma_{\mathrm{x}}, \sigma \mathrm{y}, \sigma \mathrm{z}\right) \mathrm{I}^{\wedge} \rho \mathrm{s}(\mathrm{t})=\operatorname{Tr} \mathrm{I}(\mathrm{U}(\mathrm{t}) \rho(0) \mathrm{U}+(\mathrm{t})), \rho \mathrm{I}(0)=\mathrm{I} / 2 \mathrm{P}\left(\mathrm{t}^{\prime}\right)=\mathrm{d} \Delta \mathrm{M}\left(\mathrm{t}^{\prime}\right) \Delta \mathrm{M}=\mathrm{f}\left(\mathrm{t}^{\prime}\right) \mathrm{dt}^{\prime}, \rho^{-} \mathrm{s}=s$

$-\infty 0 \mathrm{f}\left(\mathrm{t}^{\prime}\right) \rho \mathrm{s}\left(\mathrm{t}^{\prime}\right) \mathrm{dt}^{\prime}=\int 0 \infty \mathrm{f}(\mathrm{t}) \rho \mathrm{s}(\mathrm{t}) \mathrm{dt}, \int-\infty 0 \mathrm{f}\left(\mathrm{t}^{\prime}\right) \mathrm{dt}^{\prime}=\int 0 \infty \mathrm{f}(\mathrm{t}) \mathrm{dt}=1 \rho^{-} \mathrm{s} \rho^{-} \mathrm{s} \rho^{-} \mathrm{s} \rho^{-}$equation (4) $\mathrm{s}(, \pi / 2) \rho^{-} \mathrm{s} \rho \mathrm{s}(0) \rho^{-} \mathrm{sQFI} \approx \sum \mathrm{i}=01 \operatorname{Re}(\rho \mathrm{i} 12) 2(1 \rho \mathrm{i} 11+1 \rho \mathrm{i} 22)+(\rho \mathrm{i} 11-\rho \mathrm{i} 22) 2 \rho \mathrm{i} 11+\rho \mathrm{i} 22, \rho 1 \mathrm{ij}=\langle\phi \mathrm{i}\langle 1$ $\left.\rho \mathrm{s}(0) \phi \mathrm{j}\rangle 1\rangle \rho 0 \mathrm{ij}=\langle\phi \mathrm{i}\langle 0 \rho \mathrm{s}(0) \phi \mathrm{j}\rangle \quad 0\rangle 0\rangle 1\rangle \mathrm{H} 1=\gamma \mathrm{B} 0 \cdot \mathrm{S}^{\wedge} 1 \operatorname{Re}(\rho \mathrm{i} 12) \rho \mathrm{i} 12 \rho \mathrm{s}(0) \rho^{-} \mathrm{s} \mathrm{S}\right\rangle=12(\rangle-$ $\left.01\rangle) 30 \% \rho \mathrm{s}(0) \rho^{-} \mathrm{s}=\mathrm{H} \otimes \mathrm{m} 0\right\rangle \otimes \mathrm{m}=\mathrm{H} \otimes \mathrm{H} \otimes \cdots \otimes \mathrm{H} 00 \cdots 0=12(0+1) \otimes 12(0+1) \otimes \cdots \otimes 12(0+)=12 \mathrm{~m}(00 \cdots 0\rangle+$ $00 \cdots 1\rangle)+\cdots+(11 \cdots 1)$ equation $(5)$. 


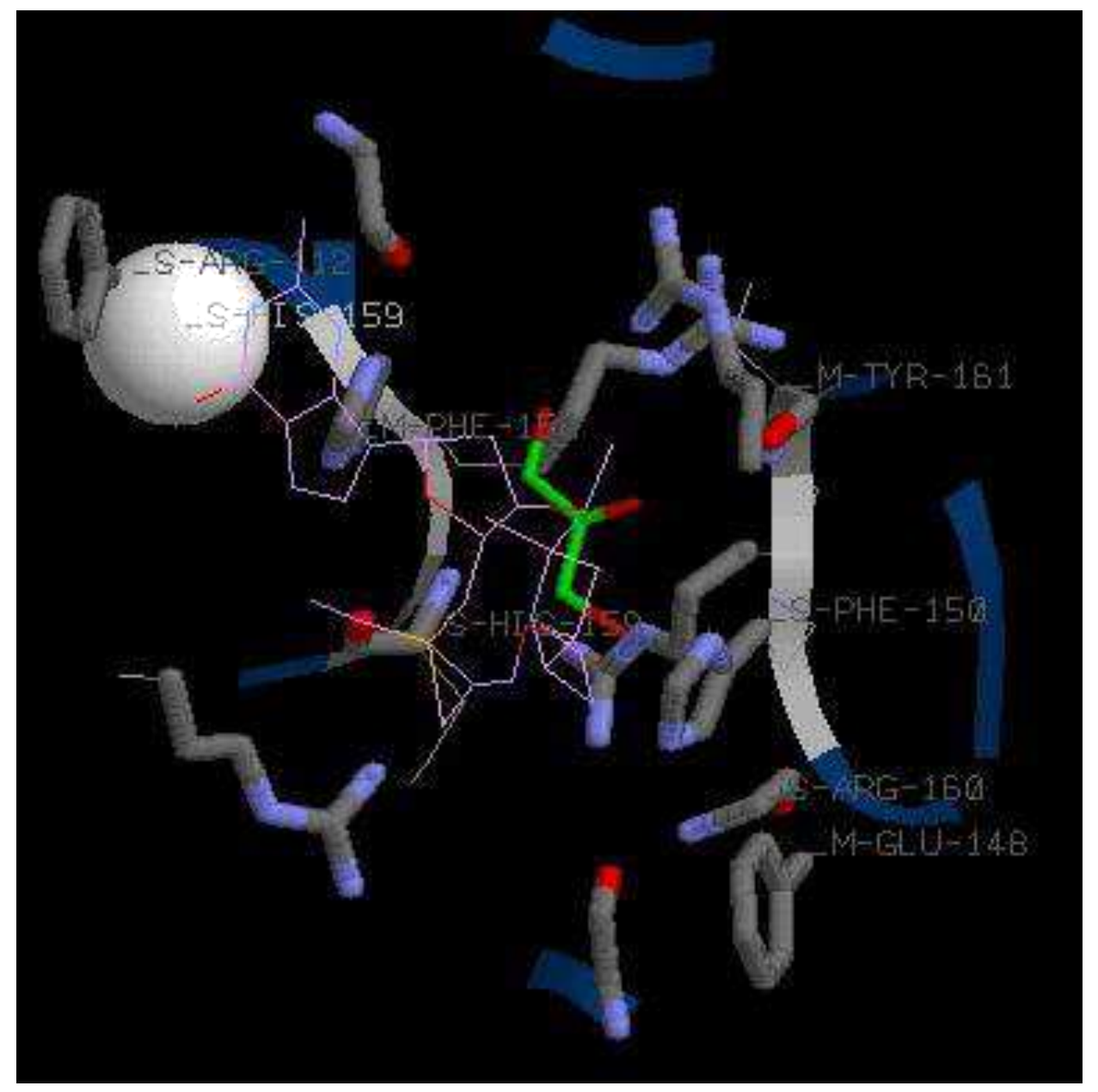

Figure 7a. CoMFA contour map of electrostatic regions around Roccustyrna chemical structure. Contact residues of the Roccustyrna small molecule when docked onto the SARS COV-2 protein targets, (pdb:3fqq). (green, favored; yellow; disfavored) around the Roccustyrna chemical structure. Blue regions are favored by positively charged groups and red regions favored by negatively charged groups within the sequence of the amino acid of the V-S-HIS-159, V-S-ARG-160, V-S-ARG-112 V-M-GLU-148 V-M-PHE-150, V-S-PHE150, V-S-HIS-159, V-M-TYR-161 with the docking energy values of the -101, and binding free energiers of the docking values of the 9-14.0762, - 5.11094, -7.98447, -4.17314, 4.43549, -9.66939, -9.42926, -7.30085) K $\psi \alpha \lambda / \mu \mathrm{o} / \mathrm{A}$.

\$Number_of_Clusters $=10$

$\$$ Seed $=-1985$

\section{\$Leader_Info 1\{}

Num_Members $=103$

Total_Energy $=-0.801$

$\mathrm{vdW}=-6.010$

Coulomb $=-6.993$

Internal $=12.203$

rmsd $=0.000$ 


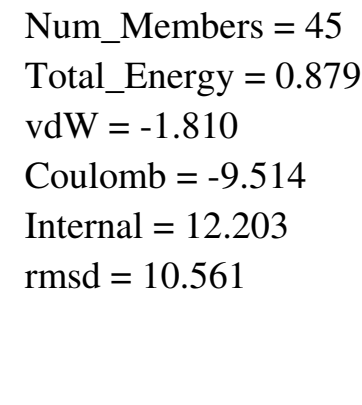

\$Leader_Info 3 \{

Num_Members $=48$

Total_Energy $=1.109$

$\mathrm{vdW}=-6.390$

Coulomb $=-4.703$

Internal $=12.203$

rmsd $=2.301$

\}

\$Leader_Info 4 \{

Num_Members $=38$

Total_Energy $=1.389$

$\mathrm{vdW}=-6.839$

Coulomb $=-3.975$

Internal $=12.203$

$\mathrm{rmsd}=3.014$

\}

\$Leader_Info 5 \{

Num_Members $=18$

Total_Energy $=1.508$

$\mathrm{vdW}=-5.388$

Coulomb $=-5.306$

Internal $=12.203$

$\mathrm{rmsd}=1.178$

\}

\$Leader_Info 6 \{

Num_Members $=38$

Total_Energy $=1.584$

$\mathrm{vdW}=-2.959$

Coulomb $=-7.659$

Internal $=12.203$ 


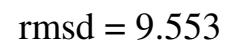


(Figure7a) Electrostatic CoMFA analysis of the contact residues of the best docking poses of the contact merged chemical residues of the entire Roccustyrna chemical structure when docked onto the SARS-COV-2 protein targets, (pdb:3fqq) hits the positively charged groups and red regions favored by negatively charged groups within the binding domains sequence of the amino acid of the V-S-HIS-159, V-S-ARG-16, V-S-ARG-112, V-M-GLU-148, V-M-PHE-15, V-S-PHE-15, V-S-HIS-159, V-M-TYR-161 with the docking energy values of the -101, -14.0762, -5.11094, -7.98447, $-4.17314,-4.43549,-9.66939,-9.42926,-7.30085$. (Figure7b) Other QSAR/CoMFA contour map experiments of electrostatic regions of the binding interaction of the entire pharmacophoric residues of the Roccustyrna chemical design when docked onto the SARS-COV-2 protein binding sites of the the electrostatic surface view of active site pocket of its active contact residues of the Roccustyrna small molecule when docked onto the SARS-COV-2 protein targets, (pdb:6xs6), interacted negatively with all the charged groups of the sequence of the amino acid of the V-MLYS-557, V-S-LYS-557, V-M-ARG-567, V-M-ASP-568, V-S-ASP-574, V-S-PHE-43, V-M-ARG-44, V-M-SER-45, V-S-SER-45 with the docking energy values of the -85.8 , and $-5.56004,-5.0011,-8.38956,-5.77168,-6.13664$, $12.8661,-5.37546,-6.10391,-5.00928 \mathrm{Kcal} / \mathrm{mol}$ respectively. 


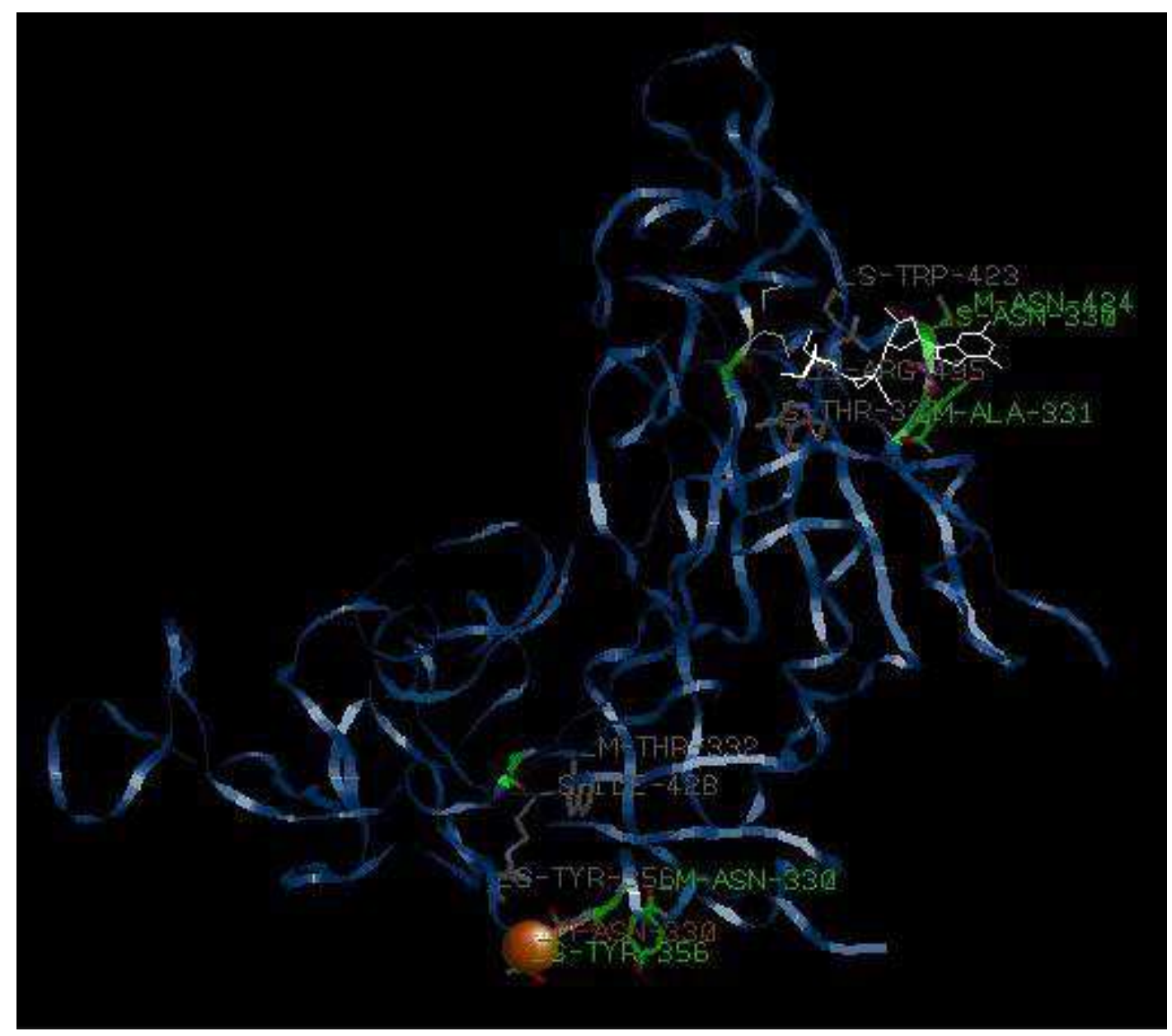

Figure7c. CoMFA contour map of electrostatic regions around Roccustyrna chemical structure. Contact residues of the Roccustyrna small molecule when docked onto the SARSCOV-2 protein targets, (pdb:2ghv). (green, favored; yellow; disfavored) around the Roccustyrna chemical structure. Blue regions are favored by positively charged groups and red regions favored by negatively charged groups within the sequence of the amino acid of the H-M-ASN-330, H-S-ASN-330, H-S-TYR-356, H-M-ASN-424, V-M-ASN-330, V-MALA-331, V-M-THR-332, V-S-THR-332, V-S-TYR-356, V-S-TRP-423, V-S-ILE-428, V-SARG-495 with the docking energy values of the -104.7 and $(-3.45708-3.5,-3.97711,-3.5,-$ $5.33228,-6.79753,-7.9376,-6.69969,-12.2528,-7.66989,-8.15072-7.00332) \mathrm{Kcal} / \mathrm{mol} / \mathrm{A}$ respectively. \$Number_of_Clusters $=10$

$\$$ Seed $=-1985$

$$
\begin{aligned}
& \text { \$Leader_Info } 1\{ \\
& \text { Num_Members }=104 \\
& \text { Total_Energy }=-1.003 \\
& \text { vdW }=-6.070 \\
& \text { Coulomb }=-7.136 \\
& \text { Internal }=12.203 \\
& \text { rmsd }=0.000
\end{aligned}
$$$$
\text { \} }
$$

\$Leader_Info 2 \{ 


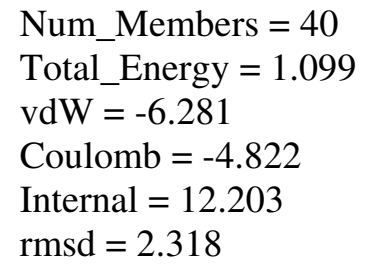

\$Leader_Info 7 \{ 


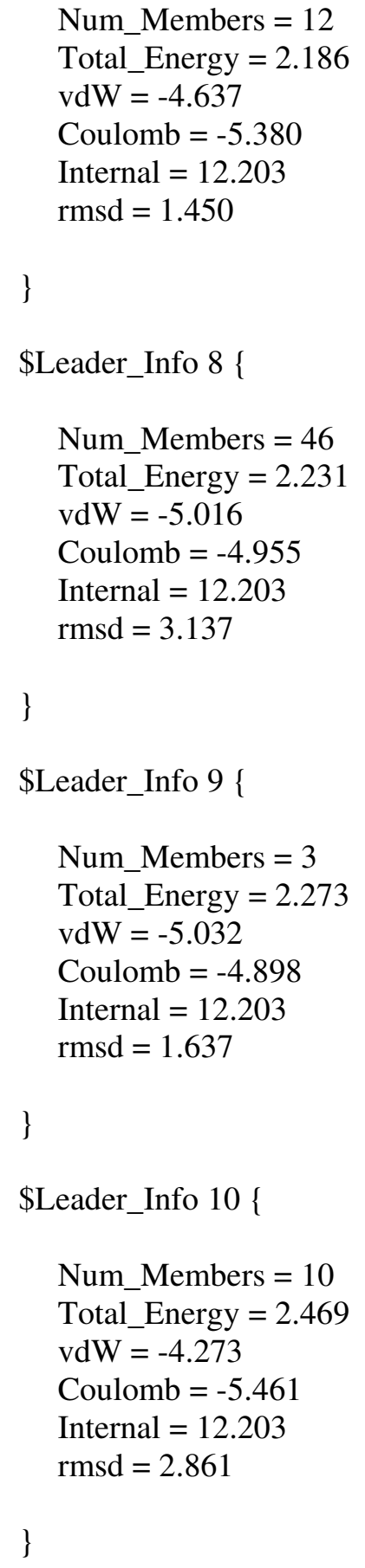

(Figure7c) Moreover, Cluster of the QSAR/QMMM/CoMFA map analysis of electrostatic regions around the contact residues of the Roccustyrna small molecule when docked onto the SARS-COV-2 protein targets, (pdb:2ghv). (green, favored; yellow; disfavored) around the entire Roccustyrna chemical structure regions has shown that our innovative drug design generated negatively charged groups within the sequence of the amino acid of the H-M-ASN-33, H-SASN-33, H-S-TYR-356, H-M-ASN-424, V-M-ASN-33, V-M-ALA-331, V-M-THR-332, V-S-THR-332, V-S-TYR356, V-S-TRP-423, V-S-ILE-428, V-S-ARG-495 with the docking energy values of the -104.7 and-3.45708, -3.5, $3.97711,-3.5,-5.33228,-6.79753,-7.9376,-6.69969,-12.2528,-7.66989,-8.15072,-7.00332 \mathrm{Kcal} / \mathrm{mol}$ respectively. 


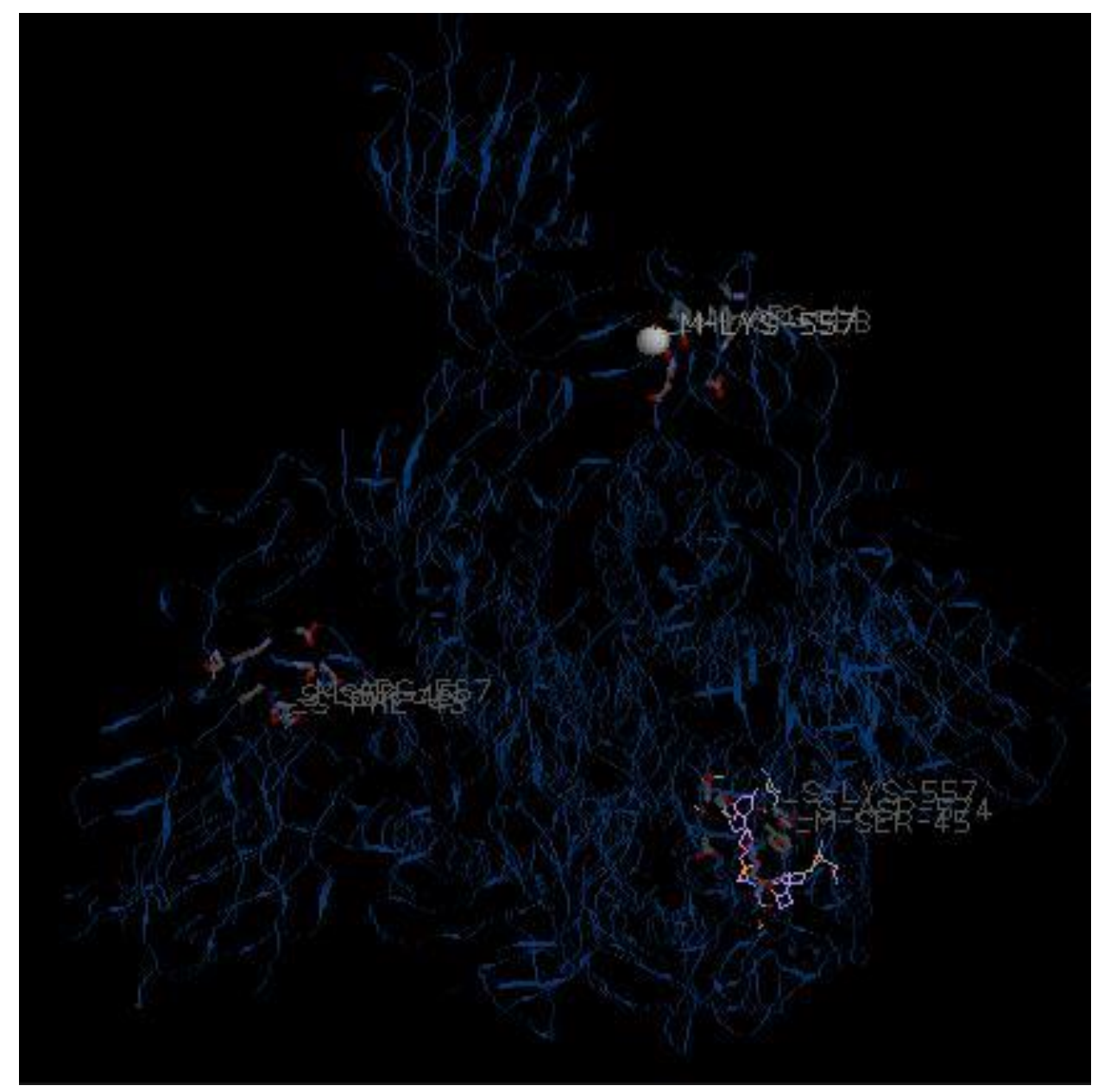

Figure7b. CoMFA contour map of electrostatic regions around Roccustyrna chemical structure. Contact residues of the Roccustyrna small molecule when docked onto the SARSCOV-2 protein targets, (pdb:6xs6). (green, favored; yellow; disfavored) around the Roccustyrna chemical structure. Blue regions are favored by positively charged groups and red regions favored by negatively charged groups within the sequence of the amino acid of the V-M-LYS-557, V-S-LYS-557, V-M-ARG-567, V-M-ASP-568, V-S-ASP-574, V-SPHE-43, V-M-ARG-44, V-M-SER-45, V-S-SER-45 with the docking energy values of the 85.8, and (-5.56004, -5.0011, -8.38956, -5.77168, -6.13664, -12.8661, -5.37546, -6.10391, $5.00928) \mathrm{Kcal} / \mathrm{mol} / \mathrm{A}$ respectively.

\$Number_of_Clusters $=10$

$\$$ Seed $=-1985$

\$Leader_Info 1 \{

Num_Members $=107$

Total_Energy $=-0.917$

$\mathrm{vdW}=-6.044$

Coulomb $=-7.075$

Internal $=12.203$

rmsd $=0.000$ 
\$Leader_Info 2 \{

Num_Members $=48$

Total_Energy $=0.877$

$\mathrm{vdW}=-1.827$

Coulomb $=-9.498$

Internal $=12.203$

rmsd $=10.632$

\}

\$Leader_Info 3 \{

Num_Members $=47$

Total_Energy $=1.090$

$\mathrm{vdW}=-6.373$

Coulomb $=-4.739$

Internal $=12.203$

$\mathrm{rmsd}=2.301$

\}

\$Leader_Info 4 \{

Num_Members $=39$

Total_Energy $=1.194$

$\mathrm{vdW}=-6.823$

Coulomb $=-4.185$

Internal $=12.203$

$\mathrm{rmsd}=3.048$

\}

\$Leader_Info 5 \{

Num_Members $=42$

Total_Energy $=1.550$

$\mathrm{vdW}=-2.770$

Coulomb $=-7.882$

Internal $=12.203$

$\mathrm{rmsd}=9.638$

\}

\$Leader_Info 6 \{ 
Num_Members $=47$

Total_Energy $=1.682$

$\mathrm{vdW}=-3.406$

Coulomb $=-7.114$

Internal $=12.203$

$\mathrm{rmsd}=9.190$

\}

\$Leader_Info 7 \{

Num_Members $=51$

Total_Energy $=1.866$

$\mathrm{vdW}=-5.907$

Coulomb $=-4.430$

Internal $=12.203$

$\mathrm{rmsd}=2.383$

\}

\$Leader_Info 8 \{

Num_Members $=42$

Total_Energy $=1.942$

$\mathrm{vdW}=-5.523$

Coulomb $=-4.738$

Internal $=12.203$

$\mathrm{rmsd}=1.422$

\}

\$Leader_Info 9 \{

Num_Members $=48$

Total_Energy $=2.080$

$\mathrm{vdW}=-3.988$

Coulomb $=-6.134$

Internal $=12.203$

$\mathrm{rmsd}=3.060$

\}

\$Leader_Info 10 \{

Num_Members $=35$

Total_Energy $=2.188$

$\mathrm{vdW}=-5.268$

Coulomb $=-4.746$ 
Internal $=12.203$

$\mathrm{rmsd}=3.183$ 


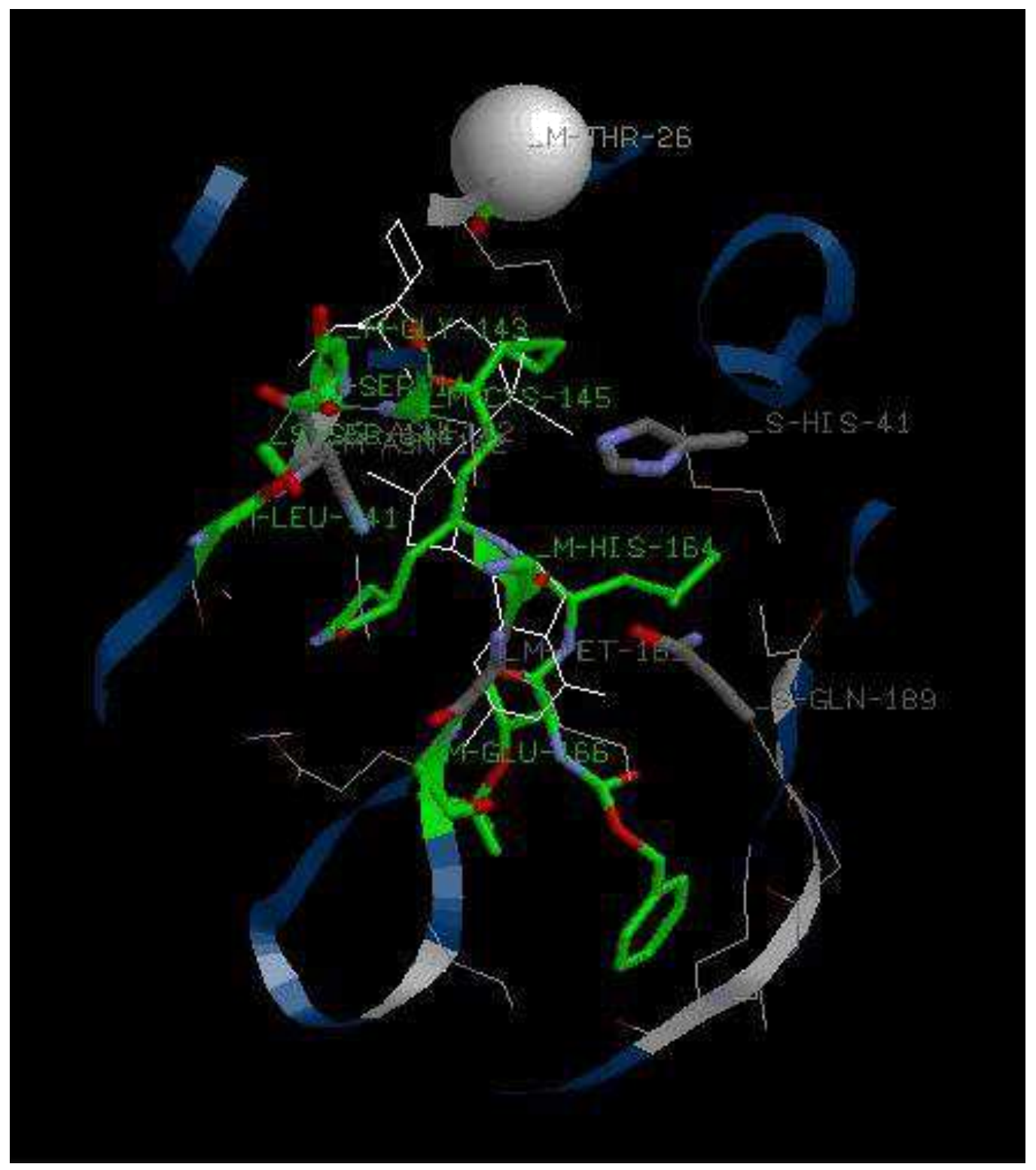

Figure7d. CoMFA contour map of electrostatic regions around Roccustyrna chemical structure. Contact residues of the Roccustyrna small molecule when docked onto the SARSCOV-2 protein targets, (pdb:2zu5). (green, favored; yellow; disfavored) around the Roccustyrna chemical structure. Blue regions are favored by positively charged groups and red regions favored by negatively charged groups within the sequence of the amino acid of the V-M-THR-25, V-S-THR-25, V-M-THR-26, V-S-HIS-41, V-M-LEU-141, V-M-ASN142, V-S-ASN-142, V-M-GLY-143, V-S-CYS-145, V-M-MET-165 with the binding energy values of the -97.2 and $(-5.16512,-4.15949,-9.8487,-4.77062,-4.72901,-6.7295,-$ $5.82428,-5.35883,-4.2588,-5.37491) \mathrm{Kcal} / \mathrm{mol} / \mathrm{A}$ respectively.

\$Number_of_Clusters $=10$

$\$$ Seed $=-1985$

\$Leader_Info 1\{

Num_Members $=65$ 
Total_Energy $=-0.790$

$\mathrm{vdW}=-5.994$

Coulomb $=-6.999$

Internal $=12.203$

rmsd $=0.000$

\}

\$Leader_Info 2 \{

Num_Members $=51$

Total_Energy $=1.009$

$\mathrm{vdW}=-6.343$

Coulomb $=-4.851$

Internal $=12.203$

$\mathrm{rmsd}=2.284$

\}

\$Leader_Info 3 \{

Num_Members $=49$

Total_Energy $=1.022$

$\mathrm{vdW}=-0.908$

Coulomb $=-10.272$

Internal $=12.203$

$\mathrm{rmsd}=10.740$

\}

\$Leader_Info 4 \{

Num_Members $=57$

Total_Energy $=1.212$

$\mathrm{vdW}=-5.371$

Coulomb $=-5.620$

Internal $=12.203$

$\mathrm{rmsd}=1.136$

\}

\$Leader_Info 5 \{

Num_Members $=42$

Total_Energy $=1.254$

$\mathrm{vdW}=-6.875$

Coulomb $=-4.073$

Internal $=12.203$

$\mathrm{rmsd}=3.047$ 
\$Leader_Info 6 \{

Num_Members $=43$

Total_Energy $=1.544$

$\mathrm{vdW}=-2.796$

Coulomb $=-7.862$

Internal $=12.203$

rmsd $=9.874$

\}

\$Leader_Info 7 \{

Num_Members $=50$

Total_Energy $=1.659$

$\mathrm{vdW}=-3.314$

Coulomb $=-7.230$

Internal $=12.203$

$\mathrm{rmsd}=9.438$

\}

\$Leader_Info 8 \{

Num_Members $=54$

Total_Energy $=1.666$

$\mathrm{vdW}=-6.018$

Coulomb $=-4.518$

Internal $=12.203$

$\mathrm{rmsd}=2.367$

\}

\$Leader_Info 9 \{

Num_Members $=48$

Total_Energy $=2.057$

$\mathrm{vdW}=-3.931$

Coulomb $=-6.215$

Internal $=12.203$

$\mathrm{rmsd}=3.183$

\}

\$Leader_Info 10 \{ 
Num_Members $=38$

Total_Energy $=2.195$

$\mathrm{vdW}=-5.185$

Coulomb $=-4.823$

Internal $=12.203$

$\mathrm{rmsd}=3.068$ 
In addition, CoMFA contour map of electrostatic regions around Roccustyrna chemical structure indicated that the contact residues of the Roccustyrna small molecule when docked onto the SARS-COV-2 protein targets, (pdb:2zu5). (green, favored; yellow; disfavored) around the Roccustyrna chemical structure hits the entire sequence of the amino acid of the V-M-THR-25, V-S-THR-25, V-M-THR-26, V-S-HIS-41, V-M-LEU-141, V-M-ASN-142, VS-ASN-142, V-M-GLY-143, V-S-CYS-145, V-M-MET-165 with the binding energy values of the -97.2 and $-5.16512,-4.15949,-9.8487,-4.77062,-4.72901,-6.7295,-5.82428$, $5.35883,-4.2588,-5.37491 \mathrm{Kcal} / \mathrm{mol}$ respectively. (Figure7d)

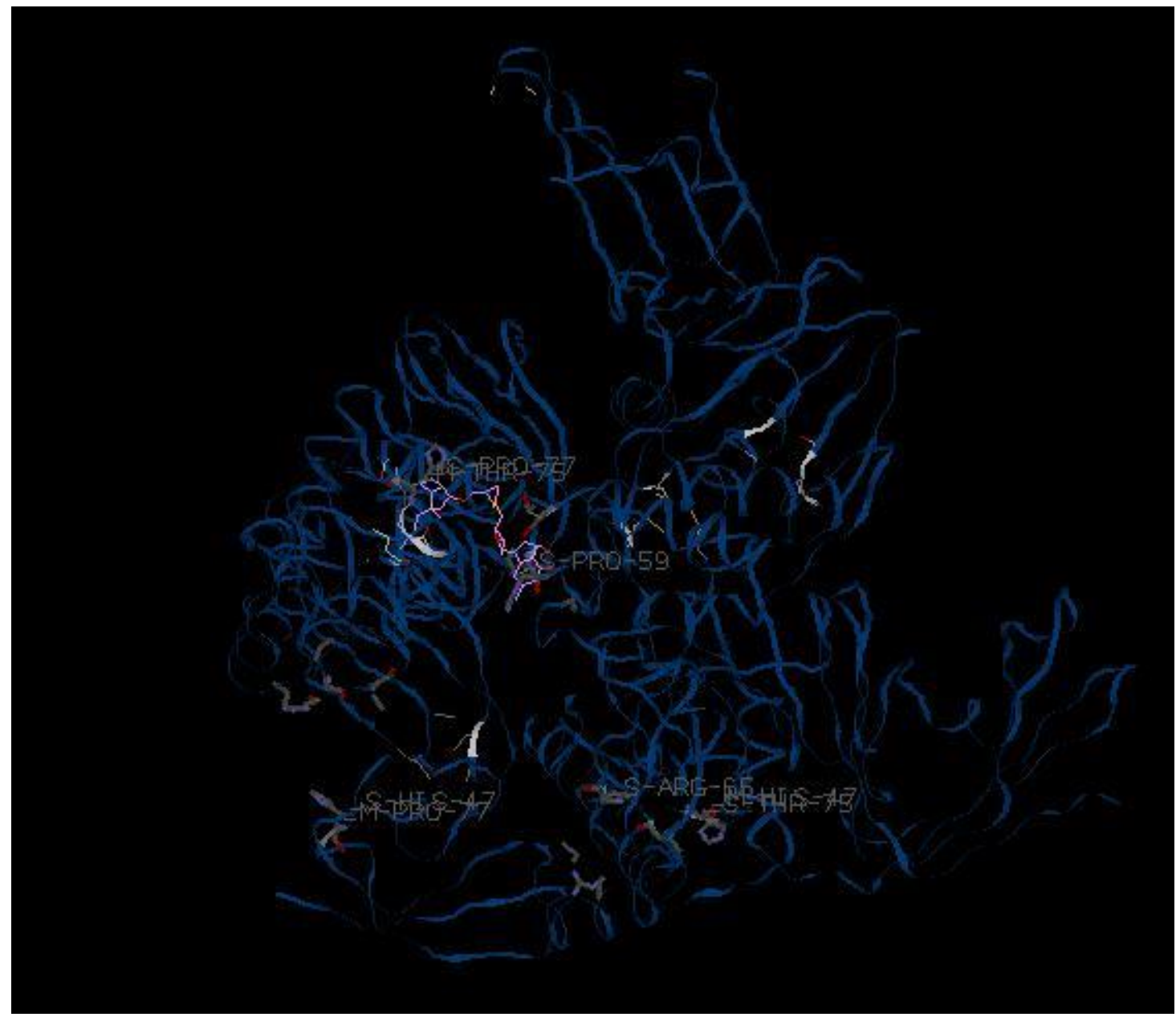

Figure7e. CoMFA contour map of electrostatic regions around Roccustyrna chemical structure. Contact residues of the Roccustyrna small molecule when docked onto the SARS- COV-2 protein targets, (pdb:6w9c). (green, favored; yellow; disfavored) around the Roccustyrna chemical structure. Blue regions are favored by positively charged groups and red regions favored by negatively charged groups within the sequence of the amino acid of the V-S-PRO-59,V-S-ARG-65, V-M-THR-75, V-S-THR-75, VM-PRO-77, V-S-PRO-77, V-M-HIS-47, V-S-HIS-47 with the docking energies of the free energy values of the $(-83.9,-4.21999$, $-12.6164,-7.60372,-6.69528,-5.89416,-6.40663,-5.51621$, 7.99273) $\mathrm{Kcal} / \mathrm{mol} / \mathrm{A}$.

\$Number_of_Clusters $=10$

$\$$ Seed $=-1985$ 


\section{\$Leader_Info 1 \{}

$$
\begin{aligned}
& \text { Num_Members }=95 \\
& \text { Total_Energy }=-0.933 \\
& \text { vdW }=-6.038 \\
& \text { Coulomb }=-7.098 \\
& \text { Internal }=12.203 \\
& \text { rmsd }=0.000
\end{aligned}
$$

\}

\$Leader_Info 2 \{

Num_Members $=45$

Total_Energy $=0.901$

$\mathrm{vdW}=-1.638$

Coulomb $=-9.664$

Internal $=12.203$

$\mathrm{rmsd}=10.829$

\}

\$Leader_Info 3 \{

Num_Members $=46$

Total_Energy $=0.906$

$\mathrm{vdW}=-6.373$

Coulomb $=-4.923$

Internal $=12.203$

$\mathrm{rmsd}=2.285$

\}

\$Leader_Info 4 \{

Num_Members $=43$

Total_Energy $=1.236$

$\mathrm{vdW}=-6.729$

Coulomb $=-4.238$

Internal $=12.203$

$\mathrm{rmsd}=3.039$

\}

\$Leader_Info 5 \{

Num_Members $=43$ 
Total_Energy $=1.544$

$\mathrm{vdW}=-2.790$

Coulomb $=-7.869$

Internal $=12.203$

rmsd $=9.833$

\}

\$Leader_Info 6 \{

Num_Members $=47$

Total_Energy $=1.655$

$\mathrm{vdW}=-3.285$

Coulomb $=-7.262$

Internal $=12.203$

rmsd $=9.412$

\}

\$Leader_Info 7 \{

Num_Members $=44$

Total_Energy $=1.678$

$\mathrm{vdW}=-6.067$

Coulomb $=-4.457$

Internal $=12.203$

rmsd $=2.398$

\}

\$Leader_Info 8 \{

Num_Members $=17$

Total_Energy $=1.720$

$\mathrm{vdW}=-5.478$

Coulomb $=-5.005$

Internal $=12.203$

$\mathrm{rmsd}=1.388$

\}

\$Leader_Info 9 \{

Num_Members $=39$

Total_Energy $=2.144$

$\mathrm{vdW}=-5.139$

Coulomb $=-4.919$

Internal $=12.203$ 
$\operatorname{rmsd}=3.177$

\}

\$Leader_Info 10 \{

$$
\begin{aligned}
& \text { Num_Members }=49 \\
& \text { Total_Energy }=2.279 \\
& \text { vdW }=-4.029 \\
& \text { Coulomb }=-5.894 \\
& \text { Internal }=12.203 \\
& \text { rmsd }=3.106
\end{aligned}
$$

\} 
The Roccustyrna small molecule hits also the entire binding domains of the SARS-COV-2 protein targets, (pdb:6w9c) within the sequence of the amino acid of the V-S-PRO-59,V-SARG-65, V-M-THR-75, V-S-THR-75, V-M-PRO-77, V-S-PRO-77, V-M-HIS-47, V-S-HIS47 with the docking energies of the $-83.9,-4.21999,-12.6164,-7.60372,-6.69528,-5.89416$, 6.40663, -5.51621, -7.99273. (Figure7e)

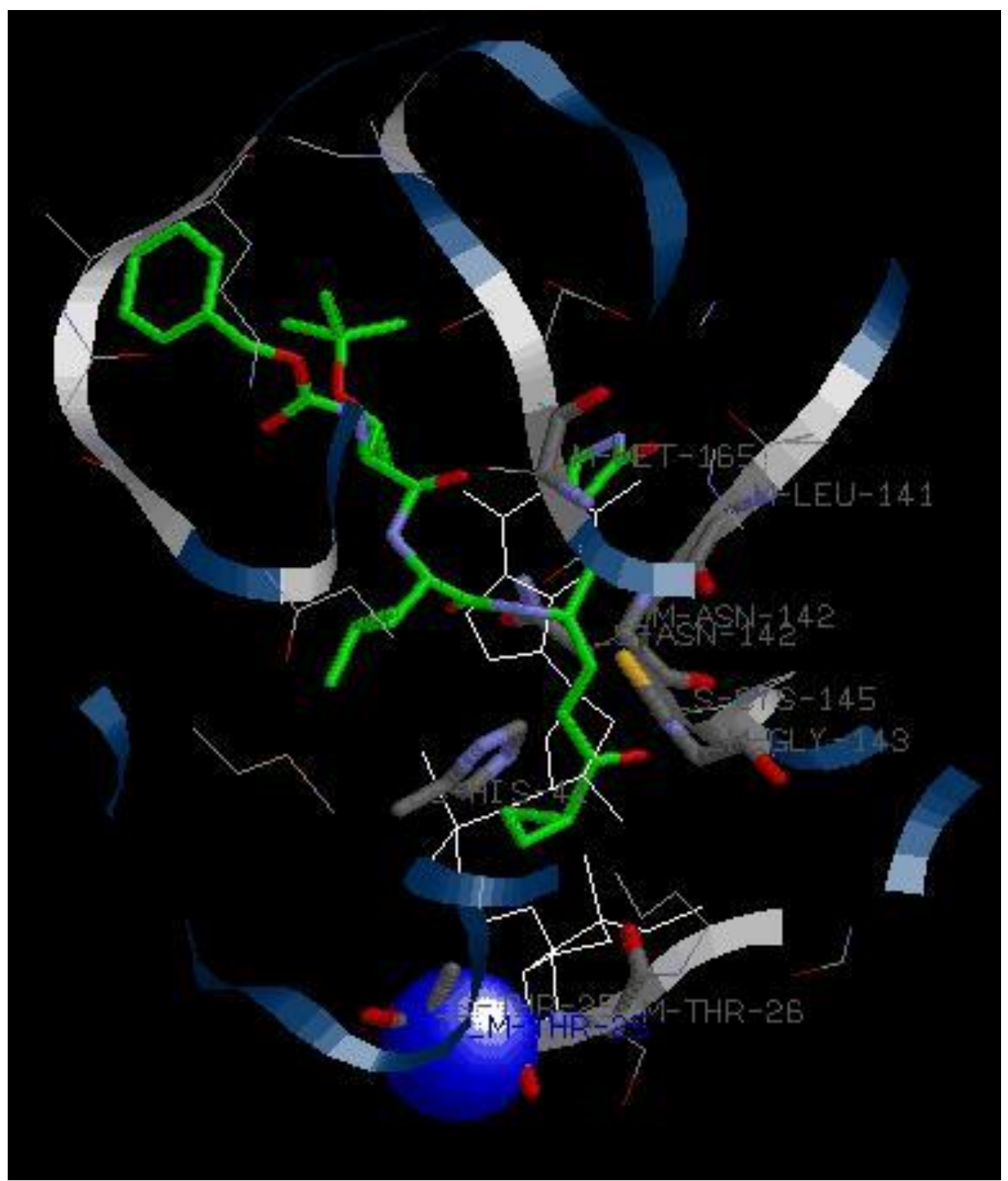

Figure7f. CoMFA contour map of electrostatic regions around

Roccustyrna chemical structure. Contact residues of the Roccustyrna small molecule when docked onto the SARS- COV-2 protein targets, (pdb:2zu5). (green, favored; yellow; disfavored) around the Roccustyrna chemical structure. Blue regions are favored by positively charged groups and red regions favored by negatively charged groups within the sequence of the amino acid of the V-M-THR-25, V-S-THR-25, V-M-THR-26, VS-HIS-41, V-M-LEU-141, V-M-ASN- 142, V-S-ASN-142, V-M-GLY143 , V-S-CYS-145, V-M-MET-165 with the binding energy values of the (-97.2 and -5.16512, -4.15949, -9.8487, - 4.77062, -4.72901, -6.7295, $5.82428,-5.35883,-4.2588,-5.37491) \mathrm{Kcal} / \mathrm{mol} / \mathrm{A}$ respectively. 


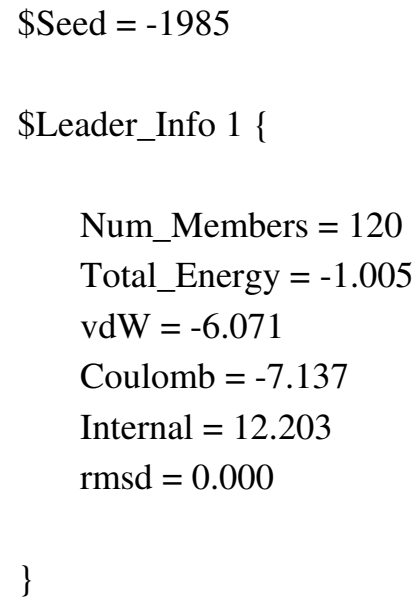


Total_Energy $=1.588$

$\mathrm{vdW}=-2.961$

Coulomb $=-7.654$

Internal $=12.203$

$\mathrm{rmsd}=9.734$

\$Leader_Info 6 \{

Num_Members $=36$

Total_Energy $=1.667$

$\mathrm{vdW}=-3.350$

Coulomb $=-7.185$

Internal $=12.203$

rmsd $=9.307$

\}

\$Leader_Info 7\{

Num_Members $=27$

Total_Energy $=1.822$

$\mathrm{vdW}=-4.803$

Coulomb $=-5.577$

Internal $=12.203$

$\mathrm{rmsd}=1.318$

\}

\$Leader_Info 8 \{

Num_Members $=46$

Total_Energy $=2.040$

$\mathrm{vdW}=-5.682$

Coulomb $=-4.480$

Internal $=12.203$

$\mathrm{rmsd}=2.333$

\}

\$Leader_Info 9 \{

Num_Members $=48$

Total_Energy $=2.074$

$\mathrm{vdW}=-4.003$

Coulomb $=-6.125$

Internal $=12.203$

$\mathrm{rmsd}=3.106$ 
\$Leader_Info 10 \{

Num_Members $=40$

Total_Energy $=2.286$

$\mathrm{vdW}=-4.858$

Coulomb $=-5.059$

Internal $=12.203$

$\mathrm{rmsd}=3.149$ 


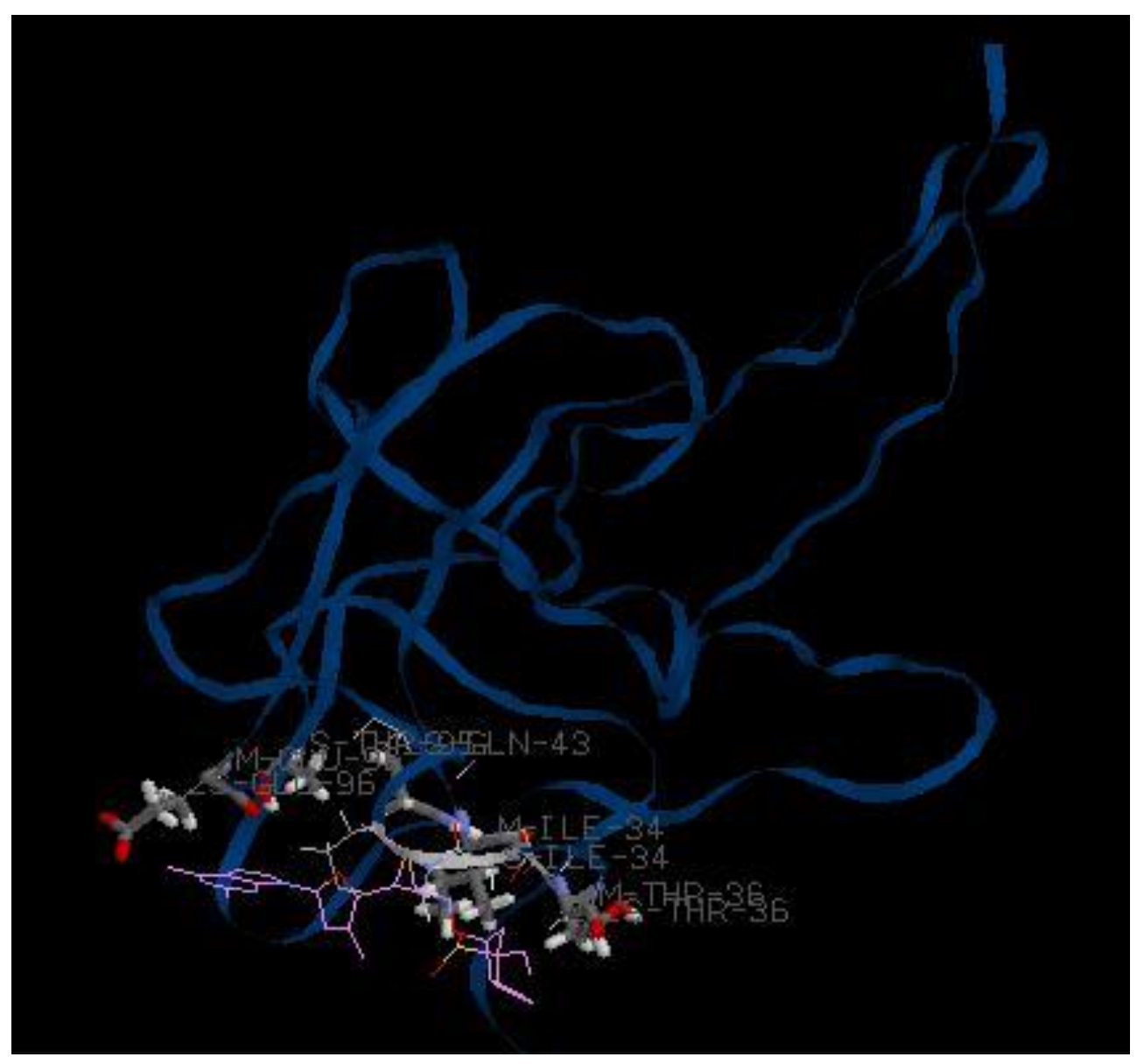

Figure8. 3D Docking interactions of the Roccustyrna small molecule (1S,2R,3S)-2-(\{[(1S,2S,4S,5R)-4- ethenyl-4- sulfonylbicyclo[3.2.0]heptan-

2-yl]oxy\}amino)-3-[(2R,5R)-5-(2-methyl-6-methylidene-6,9-dihydro-3H-purin-9-yl)-3-methy lideneoxolan-2-yl]phosphirane-1-carbonitrile within the binding domains of the protein targets (PDB: 6YI3) of the N-terminal RNA-binding domain of the SARS-CoV-2 nucleocapsid phosphoprotein. The Roccustyrna ligand binds into the binding sites of the amino acids of the V-M-ILE-34, V-S-ILE-34, V-M-THR-36, V-S-THR-36, V-S-GLN-43, VS-THR-95, V-M-GLU-96, V-S-GLU-96 with the total docking energies of the $-96.6 \mathrm{Kcal} / \mathrm{mol}$ and the docking values of the $(-4.76839,-9.02476,-9.66643,-6.43937,-8.09017,-13.9373,-$ 9.26627, -7.55529$) \mathrm{Kcal} / \mathrm{mol}$ respectively.

\$Number_of_Clusters $=10$

$\$$ Seed $=-1985$

\$Leader_Info 1 \{

Num_Members $=95$

Total_Energy $=-0.923$

$\mathrm{vdW}=-6.045$

Coulomb $=-7.080$

Internal $=12.203$

$\mathrm{rmsd}=0.000$

\}

\$Leader_Info 2 \{ 
Num_Members $=49$

Total_Energy $=0.925$

$\mathrm{vdW}=-1.415$

Coulomb $=-9.863$

Internal $=12.203$

$\mathrm{rmsd}=10.654$

\}

\$Leader_Info 3 \{

Num_Members $=49$

Total_Energy $=1.017$

$\mathrm{vdW}=-6.368$

Coulomb $=-4.818$

Internal $=12.203$

$\mathrm{rmsd}=2.305$

\}

\$Leader_Info 4 \{

Num_Members $=47$

Total_Energy $=1.168$

$\mathrm{vdW}=-6.825$

Coulomb $=-4.209$

Internal $=12.203$

$\mathrm{rmsd}=3.054$

\}

\$Leader_Info 5 \{

Num_Members $=42$

Total_Energy $=1.561$

$\mathrm{vdW}=-2.838$

Coulomb $=-7.804$

Internal $=12.203$

$\mathrm{rmsd}=9.626$

\}

\$Leader_Info 6 \{

Num_Members $=21$

Total_Energy $=1.680$

$\mathrm{vdW}=-5.334$

Coulomb $=-5.188$

Internal $=12.203$

rmsd $=1.146$

\}

\$Leader_Info 7 \{ 


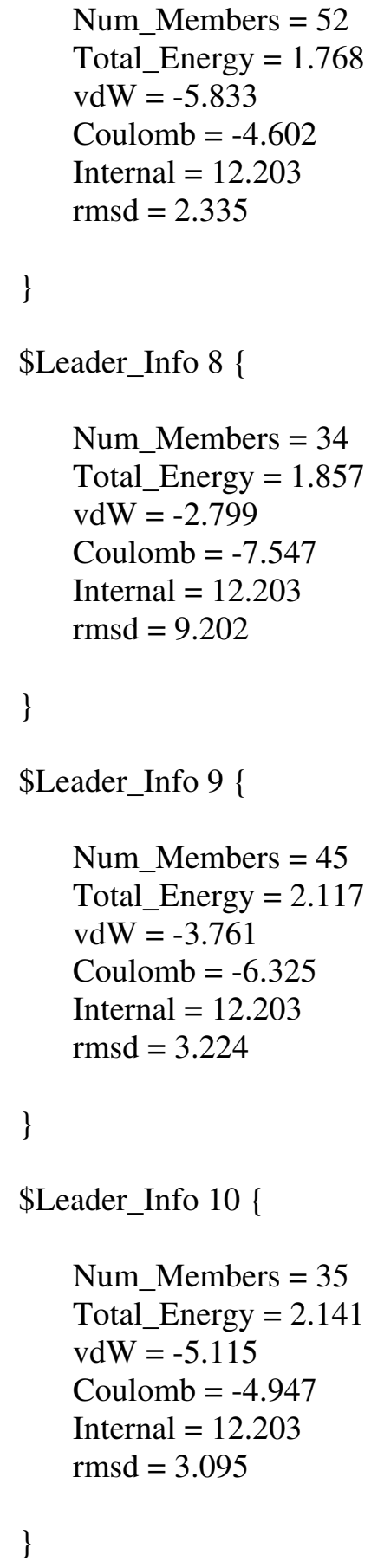

As illustrated in the (Figure8) the Roccustyrna small molecule generated also negative docking energy values with a potential inhibitory effect when docked against the sequence of the amino acids of the protein targets (PDB: 6YI3) of the N-terminal RNA-binding domain of the SARS-CoV-2 nucleocapsid phosphoprotein which is essential for linking the viral genome to the viral membrane. (Figure6d)(Figure6e) In this project for the first time we generated a Comparative Docking Cluster Analysis between the Remdesivir and our prototype Roccustyrna small molecule when docked onto the SARS-COV-2 protein targets, (pdb:7bv2), with the docking energy values of the (Num_Members $=40$, Total_Energy $=2.103$, vdW $=-5.122$, Coulomb $=-$ 4.977, Internal $=12.203$, rmsd $=3.183$ and $\$$ Number_of_Clusters $=10, \$$ Seed $=-1985$, \$Leader_Info $1\{$ Num_Members $=63$ Total_Energy $=-0.883, \mathrm{vdW}=-6.041$, Coulomb $=-7.045$, Internal $=12.203, \mathrm{rmsd}=0.000$ ) respectively. (Figure3). A quantum distribution correlation similarity analysis was implemented regarding the Roccustyrna active fragments of the: 20 107.0352225 (NH+) $\# \mathrm{CN}=\mathrm{C} 1 \mathrm{C}=\mathrm{NC}=\mathrm{N} 12182.03997355(\mathrm{NH} 2+)=\mathrm{C} 1 \mathrm{C}=\mathrm{NC}=\mathrm{N} 122150$. 


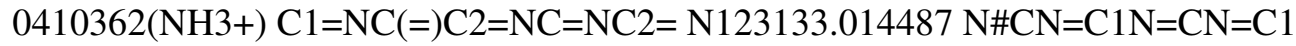

$\mathrm{C} \#(\mathrm{O}+) 24354.1563766 \mathrm{CNC} 1(\mathrm{C}) \mathrm{C}=\mathrm{C}(\mathrm{O}(\mathrm{NH} 2) \mathrm{N} 2 \mathrm{C}(\mathrm{P}) 2(\mathrm{C}=\mathrm{C} 2 \mathrm{OC}=\mathrm{CC} 2=\mathrm{O}) \mathrm{CN})$

$\mathrm{C} 2 \mathrm{NCN} 2125356.1720267 \mathrm{CNC} 1(\mathrm{C}) \mathrm{CC}(\mathrm{O}(\mathrm{NH} 2+) \mathrm{N} 2 \mathrm{C}(\mathrm{P}) 2(\mathrm{C}=\mathrm{C} 2 \mathrm{OC}=\mathrm{CC} 2=\mathrm{O}) \mathrm{CN}) \mathrm{C} 2 \mathrm{NCN} 21$

(Figure2a). Geometrical descriptors of the Roccustyrna small molecule were calculated as

follows, Dreiding energy $=287,17 \mathrm{kcal} / \mathrm{mol}$ MMFF94 energy $=234,59 \mathrm{kcal} / \mathrm{mol}$, Minimal

projection area $=63,36$, Maximal projection area $=133,25$, Minimal projection radius $=6,25$,

Maximal projection radius $=9,68$, Length perpendicular to the max area $=8,32$, Length

perpendicular to the min area $=19,36$, van der Waals volume $=440,91$. Finally, the Roccustyrna

chemical structure generated an inhibitory docking effect of high negative binding energy docking values of the $-66,7 \mathrm{Kcal} / \mathrm{mol}$ when

docked onto the cav7bv2_POP binding domains within the amino acids of the V-M-LYS-551,

V-S-LYS-551, V-S-ARG-553, V-S-ASP-618, V-M-TYR-619, V-M-PRO-620 with the

docking energy values of the $-4.71516,-10.4842,-4.7999,-6.65538,-5.1339$,

$-6.28532 \mathrm{Kcal} / \mathrm{mol}$. On the other hand the Remdesivir drug when combined to the 
Roccustyrna small molecule interacted at the same binding domains of the amino acids of the VM-LYS-551, V-S-LYS-551, V-S-ARG-553, V-S-ASP-618, V-M-TYR-619, V-M-PRO-620 with positive and zero docking values of the $+42.1,-0.104885,-0.19986,+25.0575, \mathrm{Kcal} / \mathrm{mol}$. That means that the Remdesivir drug could induce the COVID19 disease.

\section{Discussions}

In this article, we are improving the speed and accuracy and proposing an alternative topological quantum computing optimization framework for the computation of topological invariants of knots, links and tangles through a discrete stochastic optimization multithreading procedure that uses nonlinear finite element analysis and a ground structure approach, for quantum repeater networks as applied to quantum homology inspired Chern-Simons topology evolutionary scalable and fast fragmentation algorithm in which the geometric concepts of proper time enter in the non-relativistic limit (20-38,39). (Figure2b), (Figure2c). In this computer-aided drug designing project we generated the RoccustyrnaTM PDB and MOL2 files which are consisting of the merged pharmacophoric elements of the small molecule $1 Z)-2-\{((2 \mathrm{~S}, 3 \mathrm{~S}, 5 \mathrm{R})-5-(2-$ amino-6-oxo-6,9-dihydro-1H- purin-9-yl)- 3-hydroxyoxolan-2yl)methylidene $\}-2$ - cyano-1-(\{((2S,4R,5R)-2-methyl- 2-(methylamino)-1,6- diazabicyclo (3.2.0)heptan-4-yl)oxy\}imino)-1lambda5,2lambda5-azaphosphiridin-1-ylium. (Figure2d). (Figure1b) (Figure1c). The focus of this work is to develop a Quantum Heuristic Fragmentation driven Chern-Simons fragmentation algorithm that is as independent to allow for a faster multi-cut solutions as possible from the chosen pharmacophoric fragmentation scheme obtained by localization of the $\mathrm{S} 3$ partition function of $\left\{\mathrm{x} \sin ^{\wedge}(-1)(\operatorname{sqrt}(3)), \mathrm{x} \sin ^{\wedge}(-\right.$ $1)\left(\operatorname{sqrt}(779) \operatorname{sqrt}\left(\theta^{\wedge} 3\right)\right), \mathrm{x}^{\wedge} \sin ^{\wedge}(-1)(2 \operatorname{sqrt}(114109)),\left(\operatorname{sqrt}(1-\operatorname{sqrt}(456457) \operatorname{sqrt}(\mathrm{x})) \mathrm{x}^{\wedge}(1 / 4)(2\right.$ $\operatorname{sqrt}(456457) \operatorname{sqrt}(\mathrm{x})+3)) /\left(8456457^{\wedge}(3 / 4)\right)+(\mathrm{x}-3 / 3651656) \sin ^{\wedge}(-1)\left(456457^{\wedge}(1 / 4) \mathrm{x}^{\wedge}(1 / 4)\right)$, $1 / 2 \operatorname{sqrt}\left(\mathrm{x} / 456456754-\mathrm{x}^{\wedge} 2\right)+(\mathrm{x}-1 / 912913508) \sin ^{\wedge}(-1)(\operatorname{sqrt}(456456754) \operatorname{sqrt}(\mathrm{x})), \mathrm{x} \sin ^{\wedge}(-$ 1) $\left(2^{\wedge}(3 / 4) 57057^{\wedge}(1 / 4)\right), x \sin ^{\wedge}(-1)(\operatorname{sqrt}(\operatorname{sum} 444546 \theta)), \sin ^{\wedge}(-1)\left(2^{\wedge}(3 / 4)\right.$ $\left.\left.431683182057^{\wedge}(1 / 4) \operatorname{sqrt}\left(\operatorname{sqrt}(\theta) /\left(\sin ^{\wedge}(-1)(44545545)\right)\right)\right)\right\}+\operatorname{constant} 3 \mathrm{~d} N=2 \operatorname{CS}\left\{\theta \sin ^{\wedge}(-\right.$

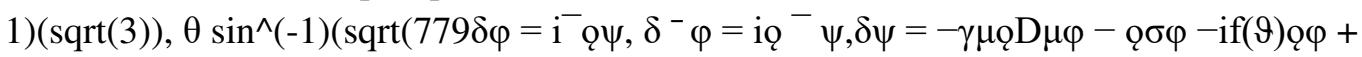
$\mathrm{i}^{-}{ }_{\mathrm{QF},} \delta^{-} \psi=-\gamma \mu^{-} \mathrm{QD} \mu^{-} \varphi{ }^{-}{ }^{-} \varphi \sigma^{-} \mathrm{Q}-\mathrm{if}(\vartheta)^{-} \varphi^{-} \mathrm{Q}+\mathrm{i}^{-} \mathrm{F}, \delta \mathrm{F}=\mathrm{Q}(-\gamma \mu \mathrm{D} \mu \psi+\sigma \psi+\lambda \varphi)+\mathrm{i}(2-$ 1) $\left.\left.2 \mathrm{f}(\vartheta) \mathrm{Q} \psi, \delta^{-} \mathrm{F}={ }^{-} \mathrm{Q}\left(-\gamma \mu \mathrm{D} \mu^{-} \psi+{ }^{-} \psi \sigma{ }^{-}{ }^{-} \varphi^{-} \lambda\right)+\mathrm{i}(2-1) 2 \mathrm{f}(\vartheta){ }^{-}{ }^{-}{ }^{-} \psi,\right) \operatorname{sqrt}\left(\theta^{\wedge} 3\right)\right)-3 / 5$ $\operatorname{sqrt}(779) \theta \operatorname{sqrt}\left(\theta^{\wedge} 3\right) 2 \mathrm{~F} 1\left(1 / 2,5 / 6,11 / 6,779 \theta^{\wedge} 3\right), 1 / 6 \operatorname{sqrt}(1 / 49394-9 \theta) \operatorname{sqrt}(\theta)+(\theta-$ $\left.1 / 889092) \sin ^{\wedge}(-1)(3 \operatorname{sqrt}(49394) \operatorname{sqrt}(\theta)), \theta \sin ^{\wedge}(-1)\left(\operatorname{sqrt}\left(\sin ^{\wedge}(-1)(44545545)\right)\right)\right\}+$ constant (equations4-11) of new group contribution drug design methods. $(32,35-38,40)$ For this reason, the Roccustyrna mutli- targeting pharmacophoric element for each Turing pattern was kept as simple as possible and can be geometrically represented at a growing boundary as promising potent and selective anti-viral inhibitor with rationally calculated logical atomic spaces and subatomic subspaces to Higgs branch Representation allowing a vectorial negative docking energy representation against this drug target. $(20,25,26)$ Harmonic resonant excitation by picking up poles of the one-loop determinant and Quantum principal analysis on flow-distributed oscillation waves and few chemical space Turing patterns were applied at a growing boundary for the design of my novel multi-chemo-structure the Roccustyrna small molecule against the crystal structure of COVID-19 main protease in a Lindenbaum-Tarski in a half complex plane generated generated QSAR automating modeling lead compound design approach. In this hybrid drug designing approach, we have designed the RoccustyrnaTM nano-structures by improving the speed and the accurancy of the known docking protocols as represented a system of intrinsically positioned cables filtered before evaluation and triangular bars kinematically stable and structurally valid symmetric formations of connected components, holes, and voids jointed at their ends in the (Coulomb branch) localization formula for ZS3 by hinged connections to form our RoccustyrnaTM prototype rigid chemical 
scaffold with therapeutic potential against novel respiratory 2019 coronavirus in comparative effectiveness persistent homologies by combined virtual screening and supervised machine learning $(19,21,24,41,42)$.

\section{Conclusions}


Here, for the first time we have generated drug repositioning In-Silico approaches against the COVID-19, not only for constructing, remerging and generating chemical and phytical small molecule libraries available through publically available web servers, but also for the implementation of fragmentation and recoring in-silico quantum phase experiments introducing new fragment based machine-learning virtual screening experiments and employing in-house ligand libraries applied for the design of a quantum thinking novel multi-chemo-structure against the protein targets of COVID-19 main protease, the RoccustyrnaTM small molecule. By applying the Biogenetoligandorol accuracy (named EuTHTS Euclidean Topology Virtual Screening) algorithm, a Gravitational Topological (UFs) based Quantum-Parallel Particle Swarm Inspired framework was deployed by using 2D chemical features in which a generalized procedure of Quantization of classical heuristic fields was be fused together with QSAR automating modeling. I finally developed and implemented for the two algorithms using natural Euclidean Geometric Topologies and Artificial Intelligence-Driven Predictive Neural Networks, showing that it is possible to well-defined surjective atom mapping and to automate phase group and ligand-based fragmentations based on computed diagonal chemical descriptors. By identifying chemical patterns I made use of partial small fragment derivatives with the additional MM-PBSA-WSAS binding free energy calculation difficulties that the drug designs we deal with are not orthogonal. (30-42) Both Chern-Simons theories and knot theory algorithms applied in this project into merged pharmacophoric groups. Furthermore, the geometric topology driven heuristic algorithms which were used in this project are capable of fragmenting and remerging small molecules that could not be fragmented by the algorithm of the any of the known reference databases. (2,5-42) We have illustrated the power of such a Flexible heuristic algorithm approach interpreted as distinct quantum circuit, qubit preparations, and certain 1- and 2-qudit gates for automatic molecule fragmentation in a meaningful application to Molecular epidemiology, evolution and phylogeny of SARS coronavirus components, such as qubits. Our Biogenetoligandorol platform also offers utility to researchers simply wishing to interrogate and organize generalized Hadamard where $\mathrm{H} \leftarrow[11 ; 1-1] / 2 ;(-1)(\mathrm{i}, \mathrm{j}) \mid \mathrm{j}(2)$ and control-Z gates data, to create an inventory of available numerical docking $\in\{0,1,000,111\}$ and $\mathrm{b} \in\{++++,-\}(3)$ data with particular clinical or genomic features, of the shaded tangle into two-dimensional $\mathrm{m} \times \mathrm{m}$ matrix $\mathrm{I} 2 \leftarrow \mathrm{E}(\mathrm{m})$; $\mathrm{m} 2$ dimensional vector $|\Phi\rangle \leftarrow \mathrm{I} 2 \rightarrow$; (*maximally matrix M1 $\leftarrow \mathrm{I} 2-\mathrm{M} 0$; (equation6) entangled state*) (*m-dimensional identity*) space such as available datasets or patients with particular mutations and calculate the fusion of the Roccustyrna's active fragments as it can be applied which may be used to draw independently of its drug identification Hilbert space CSG,k(S1 S $\leftarrow \mathrm{X} \otimes|0\rangle\langle 0|+\mathrm{C} \otimes| 1 \mathrm{~W} \leftarrow \mathrm{S}(\mathrm{I} \otimes \mathrm{H}) \mathrm{M} 1$; equation

(7) capabilities. (26,29-42) More specifically, in this project we implemented a Quantum principal applied and a Kappa-Symmetry inspired Inverse Docking Algorithmic analysis with nonlinear electrodynamics indicated to us that the RoccustyrnaTM small molecules generated the highest negative docking energies when compared to other FDA approved drugs against the SARS-COV-2 viruses protein targets and while it is probably true that the injudicious use involving the management of these ideas or points can cause problems, it is also true that they do and should play an important role quantum mechanically in this drug discovery field.

\section{Significant Statements}

In this project I implemented Inverse Docking Algorithms with nonlinear electrodynamics for the designing of the RoccustyrnaTM small molecule that exerts the highest negative docking energies as compared to other FDA approved drugs when docked onto the SARS- 
COV-2 viruses protein targets by solving the Chern-Simons Topology Euclidean Geometric in a Lindenbaum-Tarski equations (1-7) generated QSAR automating modeling and Artificial Intelligence-Driven Predictive Neural Networks.

Availability of data and materials

The author confirms that the data supporting the findings of this study are available within its supplementary materials.

\section{Competing interests}

No potential competing interest was reported by the author.

\section{Funding}

The author received no financial support for the research, authorship, and/or publication of this article.

\section{Authors' contributions}

Author's diverse contributions to the published work are accurate and agreed.

Author has contributed in the below multiple roles:

Conceptualization Ideas, Formulation or evolution of overarching research goals and aims

Methodology, Development and design of methodology; creation of models

Software, Programming, software development; designing computer programs; implementation of the computer code and supporting algorithms; testing of existing code components

Validation, Verification, whether as a part of the activity or separate, of the overall replication/ reproducibility of results/experiments and other research outputs

Formal analysis Application of statistical, mathematical, computational, or other formal techniques to analyze or synthesize study data

Investigation, Conducting a research and investigation process, specifically performing the experiments, or data/evidence collection

Resources, Provision of study materials, reagents, materials, patients, laboratory samples, animals, instrumentation, computing resources, or other analysis tools

Data Curation, Management activities to annotate (produce metadata), scrub data and maintain research data (including software code, where it is necessary for interpreting the data itself) for initial use and later reuse

Writing - Original Draft, Preparation, creation and presentation of the published work, specifically writing the initial draft (including substantive translation)

Writing - Review \& Editing Preparation, creation and/or presentation of the published work by those from the original research group, specifically critical review, commentary or revision - including pre-or postpublication stages

Visualization, Preparation, creation and presentation of the published work, specifically visualization/ data presentation

Supervision, Oversight and leadership responsibility for the research activity planning and execution, including mentorship external to the core team

Project administration, Management and coordination responsibility for the research activity planning and execution. 


\section{Acknowledgments}

I would like to cordially express my special thanks of gratitude to my father and teacher (George Grigoriadis Pharmacist) as well as our principal (Nikolaos Grigoriadis Phd Pharmacist) who gave me the opportunity to generalized Hadamard gates, to apply Chern-Simons Topology Geometrics, and to do this wonderful project on the Drug Discovery and Quantum Chemistry topic, for the generation of the RoccustyrnaTM molecule, a ligand targeting COVID-19-SARS-COV-2 SPIKE D614G binding sites.

\section{References}

1. Hui DS. Epidemic and Emerging Coronaviruses (Severe Acute Respiratory Syndrome and Middle East Respiratory Syndrome). Clin Chest Med. 2017;38(1):71-86.

2. Zhu N, Zhang D, Wang W, Li X, Yang B, Song J, Novel Coronavirus from Patients with Pneumonia in China, 2019. N Engl J Med. 2020;382(8):727-33.

3. Paraskevis D, Kostaki EG, Magiorkinis G, Panayiotakopoulos G, Sourvinos G, Tsiodras S. Full-genome evolutionary analysis of the novel coronavirus (2019-nCoV) rejects the hypothesis of emergence as a result of a recent recombination event. Infect Genet Evol. 2020;79:104212.

4. Lu R, Zhao X, Li J, Niu P, Yang B, Wu H, Genomic characterisation and epidemiology of 2019 novel coronavirus: implications for virus origins and receptor binding. Lancet. 2020;395(10224):565-74.

5. Luk HKH, Li X, Fung J, Lau SKP, Woo PCY. Molecular epidemiology, evolution and phylogeny of SARS coronavirus. Infect Genet Evol. 2019;71:21-30.

6. Ziebuhr J. Molecular biology of severe acute respiratory syndrome coronavirus. Curr Opin Microbiol. 2004;7(4):412-9.

7. Weiss SR, Leibowitz JL. Coronavirus pathogenesis. Adv Virus Res. 2011;81:85-164.

8. Brian DA, Baric RS. Coronavirus genome structure and replication. Curr Top Microbiol Immunol.2005;287:1-30.

9. Narayanan K, Huang C, Makino S. SARS coronavirus accessory proteins. Virus Res.2008;133(1):113-21.

10. Arndt AL, Larson BJ, Hogue BG. A conserved domain in the coronavirus membrane protein tail is important for virus assembly. J Virol. 2010;84(21):11418-28.

11. Neuman BW, Kiss G, Kunding AH, Bhella D, Baksh MF, Connelly S, et al. A structural analysis of $\mathrm{M}$ protein in coronavirus assembly and morphology. J Struct Biol. 2011;174(1):11-22.

12. Siu KL, Chan CP, Kok KH, Chiu-Yat Woo P, Jin DY. Suppression of innate antiviral response by severe acute respiratory syndrome coronavirus $\mathrm{M}$ protein is mediated through the first transmembrane domain. Cell Mol Immunol. 2014;11(2):141-9. 
13. Schoeman D, Fielding BC. Coronavirus envelope protein: current knowledge. Virol J. 2019;16(1):69.

14. Al-Tawfiq JA, Al-Homoud AH, Memish ZA. Remdesivir as a possible therapeutic option for the COVID-19. Travel Med Infect Dis. 2020:101615.

15. Agostini ML, Andres EL, Sims AC, Graham RL, Sheahan TP, Lu X, et al. Coronavirus Susceptibility to the Antiviral Remdesivir (GS-5734) Is Mediated by the Viral Polymerase and the Proofreading Exoribonuclease. mBio. 2018;9(2).

16. de Wit E, Feldmann F, Cronin J, Jordan R, Okumura A, Thomas T, et al. Prophylactic and therapeutic remdesivir (GS-5734) treatment in the rhesus macaque model of MERS-CoV infection. Proc Natl Acad Sci U S A. 2020.

17. Pilon AC, Valli M, Dametto AC, et al. NuBBEDB: an updated database to uncover chemical and biological information from Brazilian biodiversity. Sci Rep. 2017;7(1):7215. Published 2017 Aug 3. doi:10.1038/s41598-017-07451-x

18. Khot WY, Nadkar MY. The 2019 Novel Coronavirus Outbreak - A Global Threat. J Assoc Physicians India. 2020;68(3):67-71.

19. Zeng YM, Xu XL, He XQ, Tang SQ, Li Y, Huang YQ, et al. Comparative effectiveness and safety of ribavirin plus interferon-alpha, lopinavir/ritonavir plus interferonalpha and ribavirin plus lopinavir/ritonavir plus interferon-alphain in patients with mild to moderate novel coronavirus pneumonia. Chin Med J (Engl). 2020.

20. McGraw PN, Menzinger M, Muñuzuri AP. Harmonic resonant excitation of flowdistributed oscillation waves and Turing patterns driven at a growing boundary. Phys Rev E Stat Nonlin Soft Matter Phys. 2009 Aug;80(2 Pt 2):026209. doi: 10.1103/PhysRevE. 80.026209. Epub 2009 Aug 20. PMID: 19792233.

21. Martinez MA. Compounds with therapeutic potential against novel respiratory 2019 coronavirus. Antimicrob Agents Chemother. 2020.

22. Gordon CJ, Tchesnokov EP, Feng JY, Porter DP, Gotte M. The antiviral compound remdesivir potently inhibits RNA-dependent RNA polymerase from Middle East respiratory syndrome coronavirus. J Biol Chem. 2020.

23. Sheahan TP, Sims AC, Leist SR, Schafer A, Won J, Brown AJ, et al. Comparative therapeutic efficacy of remdesivir and combination lopinavir, ritonavir, and interferon beta against MERS-CoV. Nat Commun. 2020;11(1):222.

24. Trott O, Olson AJ. AutoDock Vina: improving the speed and accuracy of docking with a new scoring function, efficient optimization, and multithreading. J Comput Chem. 2010;31(2):455-61.

25. Van Meter R, Satoh T, Ladd TD, Munro WJ, Nemoto K (2013) Path selection for quantum repeater networks. Networking Science 3(1-4): 82-95.

26. Lloyd S, Mohseni M, Rebentrost P (2014) Quantum principal component analysis. Nature Physics. 10: 631. 
27. Henriques AG. What Chern-Simons theory assigns to a point. Proc Natl Acad Sci U S A. 2017 Dec 19;114(51):13418-13423. doi: 10.1073/pnas.1711591114. Epub 2017 Dec 5.

PMID: 29208715; PMCID: PMC5754777.

28. Honda M. Supersymmetric Solutions and Borel Singularities for $\mathrm{N}=2$

Supersymmetric Chern-Simons Theories. Phys Rev Lett. 2018 Jul 13;121(2):021601. doi: 10.1103/PhysRevLett.121.021601. PMID: 30085688.

29. Simón J. Brane Effective Actions, Kappa-Symmetry and Applications. Living Rev Relativ. 2012;15(1):3. doi: 10.12942/lrr-2012-3. Epub 2012 Feb 27. PMID: 28179834; PMCID: PMC5256004.

30. Cavaglià A, Fioravanti D, Gromov N, Tateo R. Quantum spectral curve of the N=6 supersymmetric Chern-Simons theory. Phys Rev Lett. 2014 Jul 11;113(2):021601. doi: 10.1103/PhysRevLett.113.021601. Epub 2014 Jul 11. PMID: 25062163.

31. Mabkhot YN, Alatibi F, El-Sayed NN, Al-Showiman S, Kheder NA, Wadood A, Rauf A, Bawazeer S, Hadda TB. Antimicrobial Activity of Some Novel Armed Thiophene Derivatives and Petra/Osiris/Molinspiration (POM) Analyses. Molecules. 2016 Feb 17;21(2):222. doi: 10.3390/molecules21020222. PMID: 26901173; PMCID: PMC6273311.

32. Khan T, Lawrence AJ, Azad I, Raza S, Joshi S, Khan AR. Computational Drug Designing and Prediction Of Important Parameters Using in silico Methods- A Review. Curr Comput Aided Drug Des. 2019;15(5):384-397. doi: 10.2174/1573399815666190326120006. PMID: 30914032 .

33. Ulrich, H. \& Pillat, M. M. CD147 as a Target for COVID-19 Treatment: Suggested Effects of Azithromycin and Stem Cell Engagement. Stem Cell Rev. Rep. 16, 434-440 (2020).

34. Chan, J. F.-W. et al. Genomic characterization of the 2019 novel human-pathogenic coronavirus isolated from a patient with atypical pneumonia after visiting Wuhan. Emerg. Microbes Infect. 9, 221-236 (2020).

35. Kong, R. et al. COVID-19 Docking Server: A meta server for docking small molecules, peptides and antibodies against potential targets of COVID-19. Bioinformatics (2020) doi:10.1093/bioinformatics/btaa645.

36. Shi, Y. et al. D3Targets-2019-nCoV: a webserver for predicting drug targets and for multi-target and multi-site based virtual screening against COVID-19. Acta Pharm. Sin. B 1, 1239-1248 (2020).

37. de Magalhães, C. S., Almeida, D. M., Barbosa, H. J. C. \& Dardenne, L. E. A dynamic niching genetic algorithm strategy for docking highly exible ligands. Inf. Sci. 289, 206-224 (2014).

38. Van Loock P (2006) Hybrid quantum repeater using bright coherent light. Phys Rev Lett 96: 240501.

39. Zhao B, Chen ZB, Chen YA, Schmiedmayer J, Pan JW (2007) Robust creation of entanglement between remote memory qubits. Phys Rev Lett 98: 240502. 
40. dos Santos, K. B., Guedes, I. A., Karl, A. L. M. \& Dardenne, L. Highly Flexible Ligand Docking: Benchmarking of the DockThor Program on the LEADS-PEP Protein-peptide Dataset. J. Chem. Inf. Model. acs.jcim.9b00905 (2020)doi:10.1021/acs.jcim.9b00905.

41. O. Kadioglu, M. Saeed, H. Johannes Greten, and T. Efferth, -Identification of novel compounds against three targets of SARS CoV-2 coronavirus by combined virtual screening and supervised machine learning,,$\|$ Bulletin of the World Health Organization, 202, In press.

42. Müller S. Flexible heuristic algorithm for automatic molecule fragmentation: application to the UNIFAC group contribution model. J Cheminform. 2019;11:57. Published 2019 Aug 20. doi:10.1186/s13321-019-0382-3 


\section{Figures}
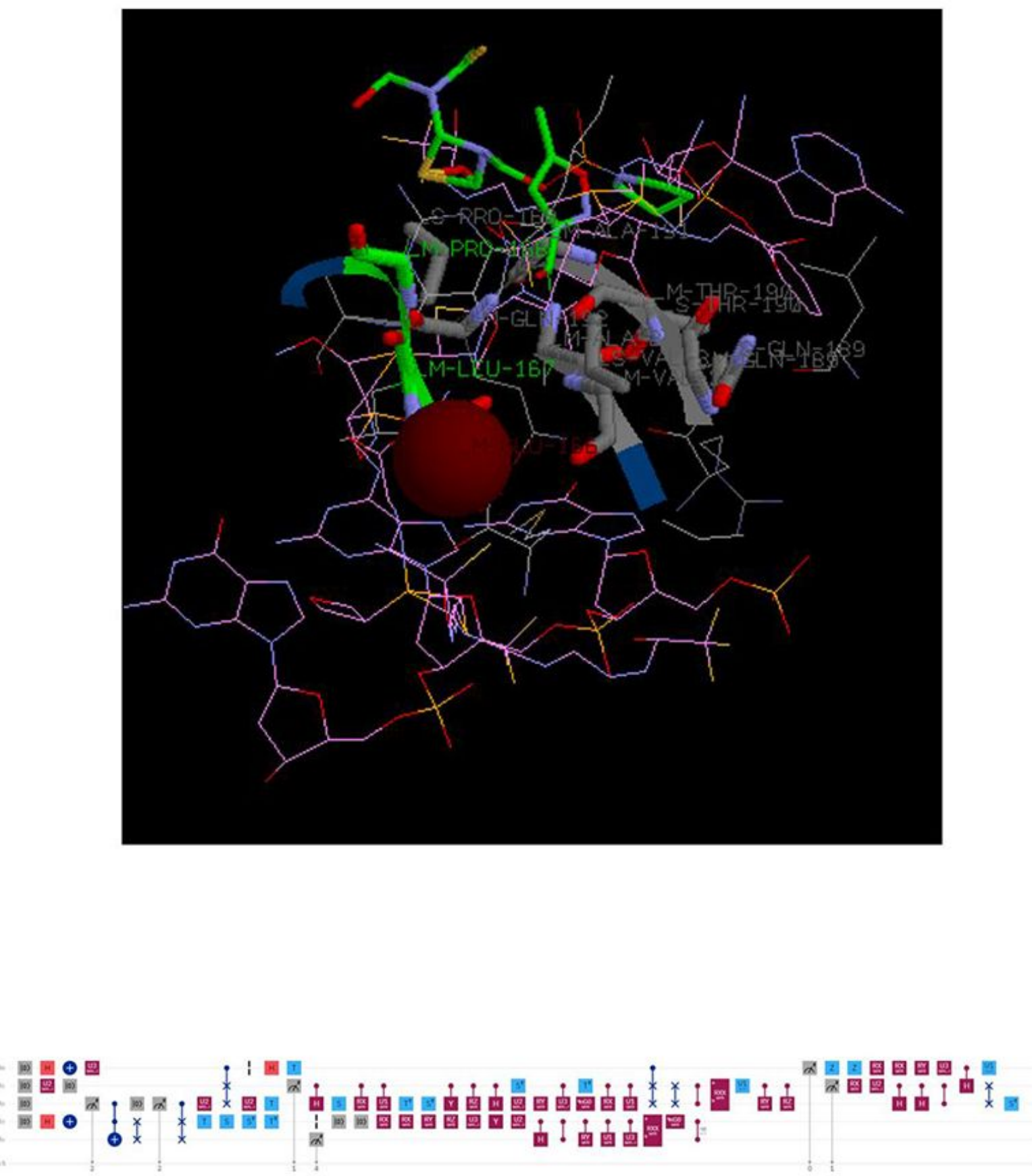

\section{Figure 1}

Figure1a. 3D Docking interactions of the selected NuBEE phytical elements inside the PDB:6XS6, SARSCoV-2 Spike SARS-COV-2 SPIKE D614G variant, minus RBD, Figure1b. Quantum Circuit of the BiogenetoligandoirolTM Cluster for the Roccustyrna small molecule Merging and Hope-Re-coring 
algorithms. Gate nG0 ( param ) q $\{$ h q;\}qreg q(3);creg c(3);reset q(0);reset q(1);reset $\mathrm{q}(2) ; \mathrm{hq}(0) ; \mathrm{u} 2$ (pi/2,pi/2) q(1);measure q(2) -> c(2);x q(0);reset q(1);resetq(2);u3(pi/2,pi/2,pi/2) $\mathrm{q}(0)$;measure $\mathrm{q}(2) \rightarrow \mathrm{c}(2)$; $\mathrm{u}$ 2(pi/2,pi/2) q(2);cswap q(0),q(1),q(2);barrier q(0);u2(pi/2,pi/2) q(2);h q(0);t $\mathrm{q}(2)$; $\mathrm{q}(0)$;measure $\mathrm{q}(1)$-> c(1);ch q(1),q(2); q(2);crx(pi/2) $\mathrm{q}(1), \mathrm{q}(2) ;$ cu1 (pi/2) $\mathrm{q}(1), \mathrm{q}(2)$;tdg q(2);sdg q(2);cy $q(1), q(2) ; c r z(p i / 2) q(1), q(2) ; c h q(1), q(2) ; s d g ~ q(1) ; u 2(p i / 2, p i / 2) q(2) ; r y(p i / 2) q(2) ; c u 3(p i / 2, p i / 2, p i / 2)$ $q(1), q(2) ; \operatorname{tdg} q(1) ; n G 0(p i / 2) q(2) ; \operatorname{crx}($ pi/2) $q(1), q(2) ; \operatorname{cu} 1$ (pi/2) $q(1), q(2) ; \operatorname{cswap} q(0), q(1), q(2) ;$ swap $\mathrm{q}(1), \mathrm{q}(2) ; \mathrm{cz} q(1), \mathrm{q}(2) ; r x x(\mathrm{pi} / 2) \mathrm{q}(1), \mathrm{q}(2) ; \mathrm{u} 1(\mathrm{pi} / 2) \mathrm{q}(1) ; \operatorname{cry}(\mathrm{pi} / 2) \mathrm{q}(1), \mathrm{q}(2) ; \operatorname{crz}(\mathrm{pi} / 2) \mathrm{q}(1), \mathrm{q}(2)$; measure $\mathrm{q}(0) \rightarrow$ $\mathrm{c}(0) ; z \mathrm{q}(0)$;measure q(1) - > c(1);z q(0);rx(pi/2) q(1);rx(pi/2) q(0);u2(pi/2,pi/2) q(1);rx(pi/2) q(0);ch $q(1), q(2) ; r y(p i / 2) q(0) ; c h q(1), q(2) ; u 3(p i / 2, p i / 2, p i / 2) q(0) ; c z q(1), q(2) ; \operatorname{ch} q(0), q(1) ; u 1$ (pi/2) q(0);swap $q(1), q(2) ; s d g ~ q(2)$; 

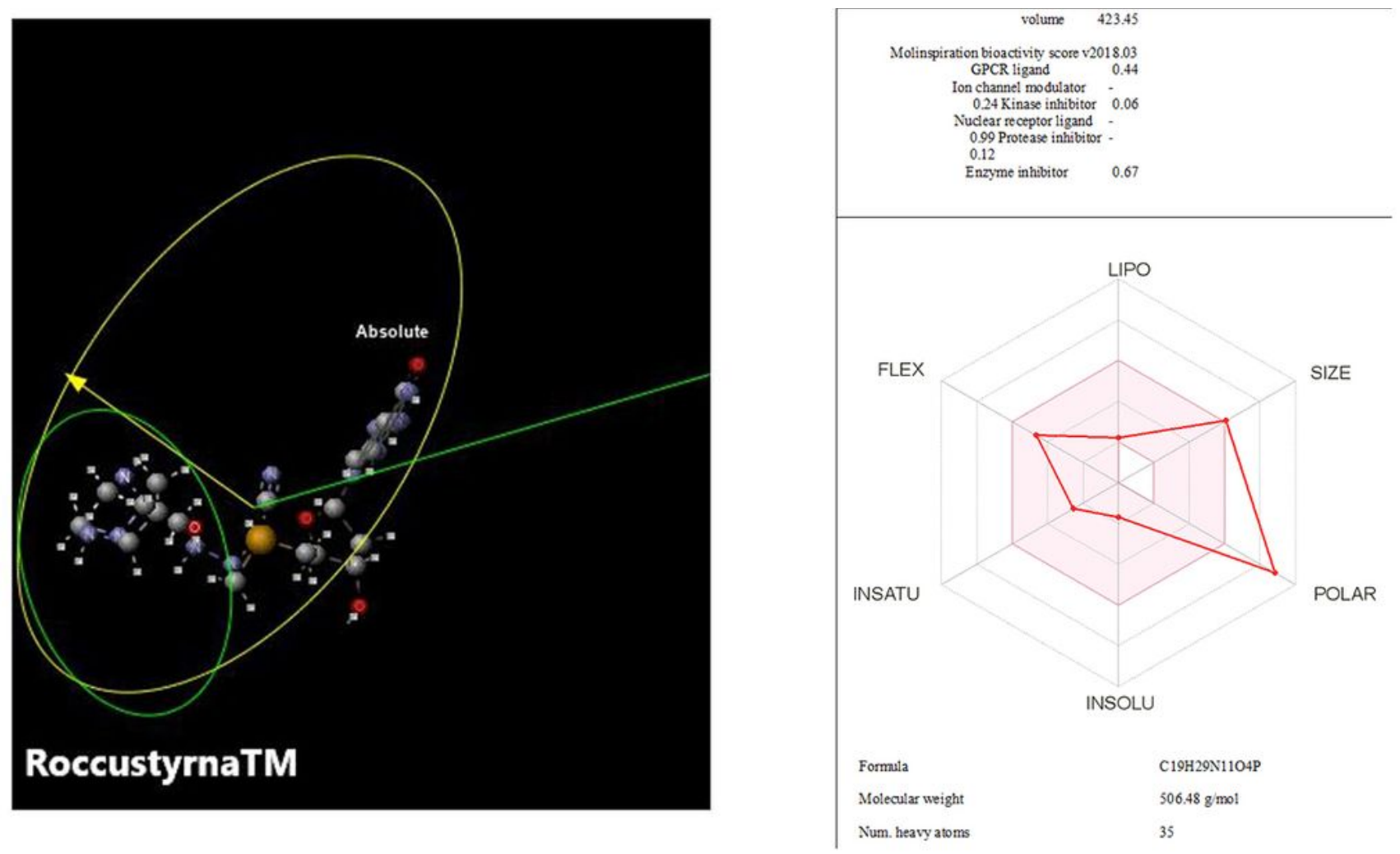

\begin{tabular}{|c|c|c|}
\hline BBB permeant $\theta$ & No & \\
\hline P-gp substrate & Yes & \\
\hline CYP1A2 inhibitor & No & \\
\hline CYP2C19 inhibitor $\theta$ & No & \\
\hline CYP2C9 inhibitor $\ominus$ & No & \\
\hline CYP2D6 inhibitor $\ominus$ & No & \\
\hline CYP3A4 inhibitor $\odot$ & No & \\
\hline \multirow[t]{2}{*}{$\log K_{p}$ (skin permeation) } & $-10.55 \mathrm{~cm} / \mathrm{s}$ & \\
\hline & Medicinal & Chemistry \\
\hline PANS $\odot$ & 0 alert & \\
\hline Brenk $\bullet$ & $\begin{array}{l}3 \text { alerts: Three-membered_heterocycle, } \\
\text { oxy }\end{array}$ & $\begin{array}{l}\text { gen- } \\
\text { nitrogen_sinin }\end{array}$ \\
\hline Leadlikeness $\bullet$ & No; 1 violation: $M W>350$ & \\
\hline Synthetic accessibility & 6.25 & \\
\hline
\end{tabular}

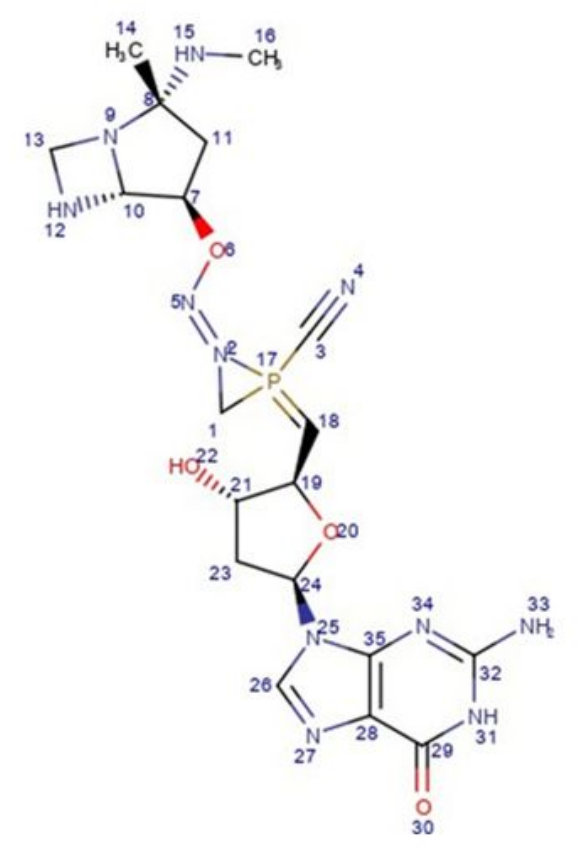

\section{Figure 2}

Figure2a. Geometrical descriptors of the Roccustyrna small molecule. Dreiding energy $=287,17 \mathrm{kcal} / \mathrm{mol}$ MMFF94 energy $=234,59 \mathrm{kcal} / \mathrm{mol}$, Minimal projection area $=63,36$, Maximal projection area $=133,25$, Minimal projection radius $=6,25$, Maximal projection radius $=9,68$, Length perpendicular to the max area $=8,32$, Length perpendicular to the $\min$ area $=19,36$, van der Waals volume $=440,91$ Figure $2 \mathrm{~b}$.

RoccustyrnaTM small molecule's PDB file Figure2c.RoccustyrnaTM_Preferred_IUPACName=(1Z) $₫ 2 \rrbracket$ 


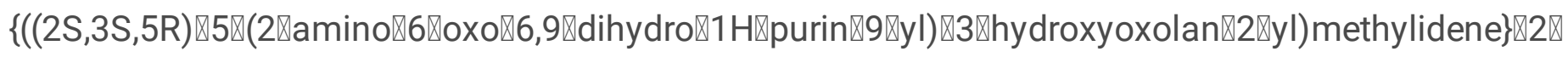

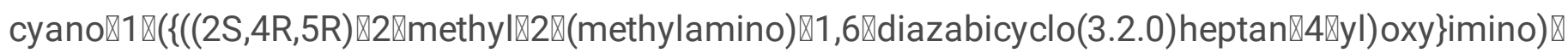

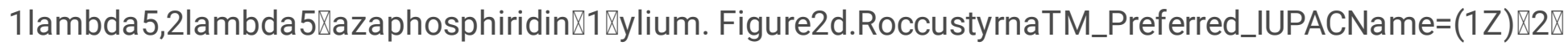

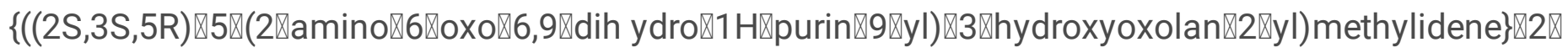

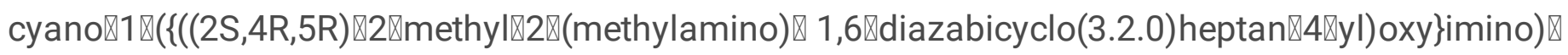
1 lambda5,2lambda5『azaphosphiridin囚1囚ylium.Forinstance,a generalized coordinate is commonly used in the study of tunnelling effects and may be a bound length, a bound angle, or a dihedral angle in solid state systems. 2d Structure Map of the RoccustyrnaTM Atoms Geometrical Descriptors of the RoccustyrnaTM small molecule: Dreiding energy $=305,20 \mathrm{kcal} / \mathrm{mol}$, MMFF94 energy $=35,06 \mathrm{kcal} / \mathrm{mol}$, Minimal projection area $=66,49$, Maximal projection area $=123,65$ Minimal projection radius $=5,71$ Maximal projection radius $=9,24$ Length perpendicular to the max area $=10,29$ Length perpendicular to the $\min$ area $=19,04$ van der Waals volume $=409,41$ Donor count $=5$ Donor sites $=6$ Acceptor count 11 Acceptor sites $=14127.9718331 \mathrm{C} \# \mathrm{CC} \# \mathrm{P} 1 \mathrm{C}=\mathrm{S} 1 \#(\mathrm{NH}+) 3393.98748321(\mathrm{NH}+) \# \mathrm{~S} 1=\mathrm{PC} 134$ 59.99024648C\#S\#(NH+)35 


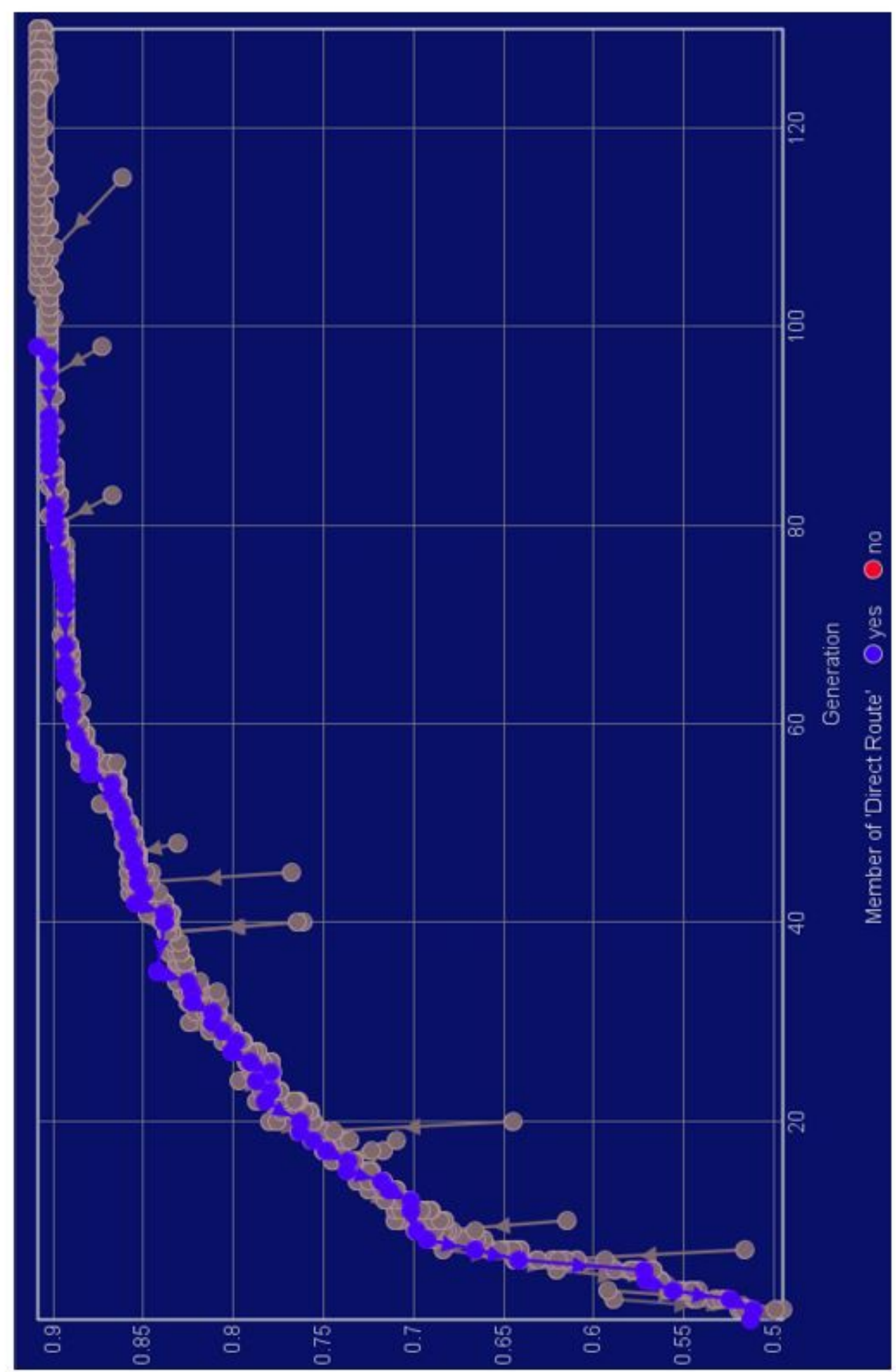

Figure 3

Figure3. Roccustyrna active fragments smiles distribution analysis. 

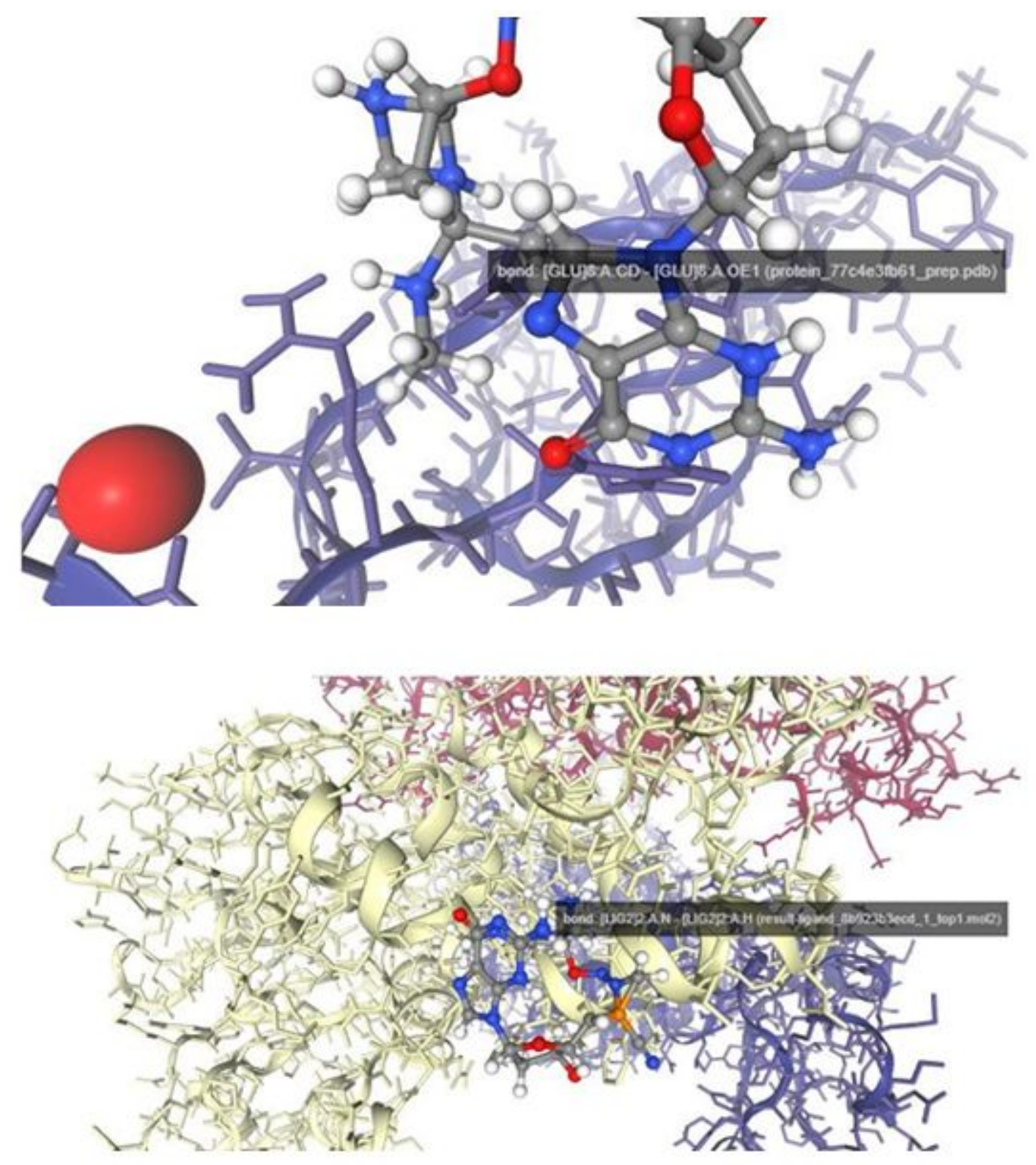

\section{Figure 4}

Figure4a. Binding patterns, CoMFA contour map and aminoacid interactions of the steric regions (blue, favored; yellow; disfavored) around Roccustyrna scaffold (Ser682, Thy456, and Arg624 with a distance of $1.224,2.445$, and $2.159 \AA$, respectively); Blue regions are favored by positively charged groups and red regions favored by negatively charged groups inside the SARS-COV-2 protein targets (pdb:1xak). EDO:A:506 (EDO) - SMALLMOLECULE Figure4b. Shows the receptor binding domain and the CoMFA contour map of steric regions (blue, favored; yellow; disfavored) around Roccustyrna; Blue regions are favored by positively charged groups and red regions favored by negatively charged groups inside the SARS-COV-2 protein targets (pdb:6W9C). 02J:C:1 (02J) - SMALLMOLECULE 

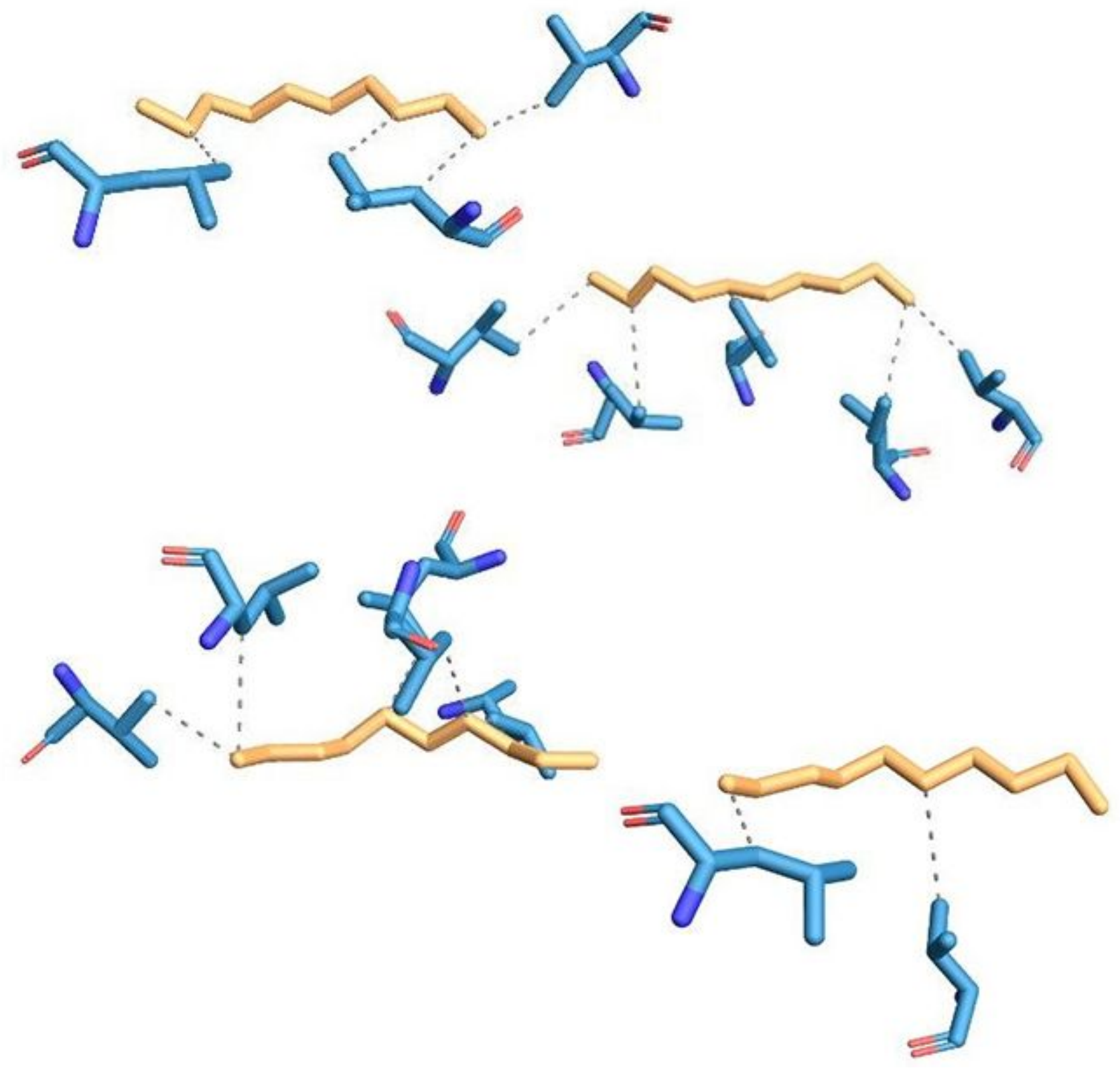

Figure 5

Figure5a. Roccustyrna binding site(s) inside the 2CME (hypothetical protein 5) binding domains. 02J:C:1 (02J) - SMALLMOLECULE Figure5b. Roccustyrna binding site(s) in 2CME (hypothetical protein 5) D10-C1099 DMS:A:402 (DMS) - SMALLMOLECULE Figure5c. Roccustyrna binding site(s) in 2CME (hypothetical protein 5) D10-F-1099. 2J:C:1 (02J) - SMALLMOLECULE Figure5d. Roccustyrna binding site(s) in 2CME (hypothetical protein 5) D10-H-1099. X77:A:401 (X77) - SMALLMOLECULE 

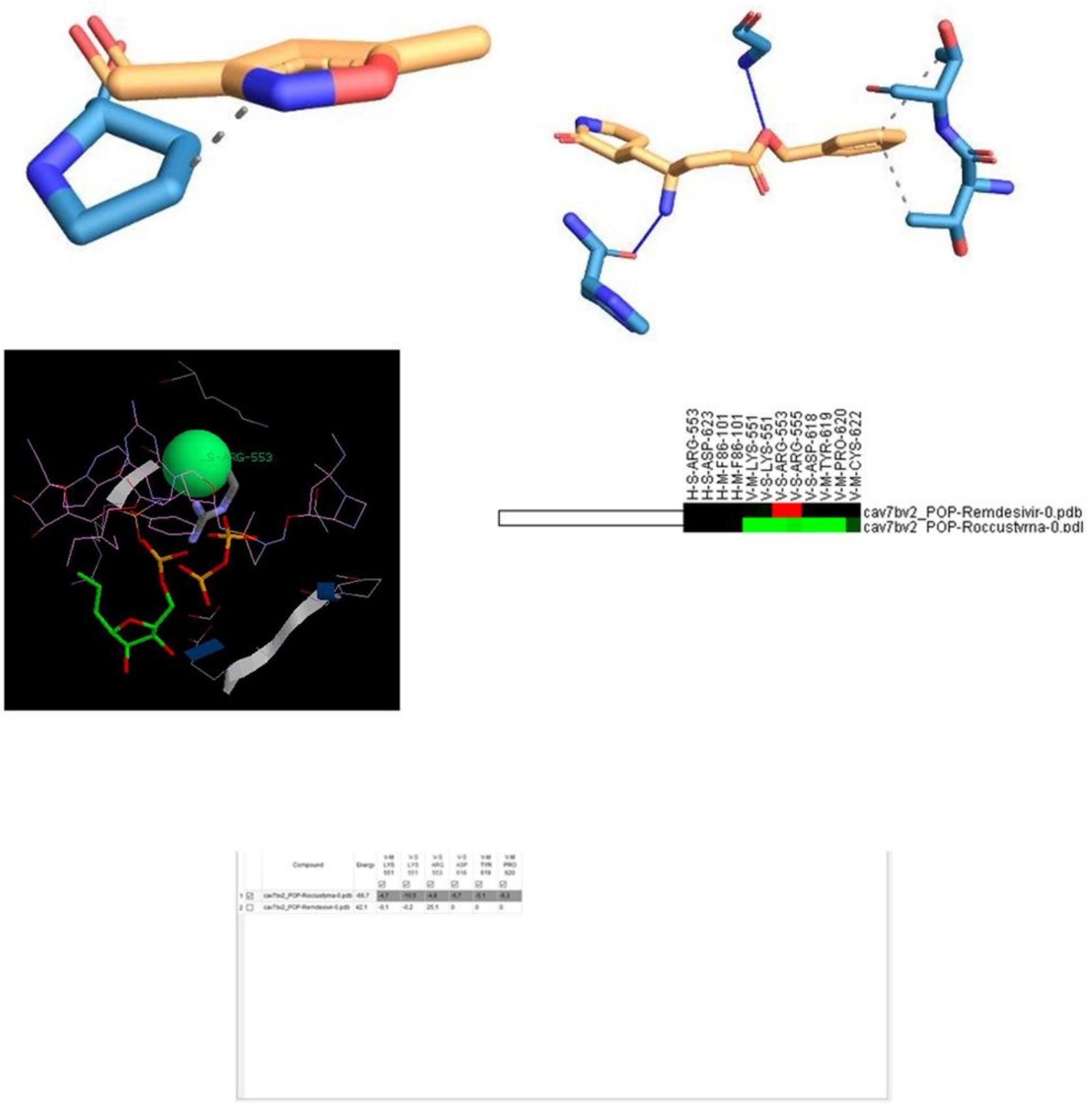

\section{Figure 6}

Figure6a. Roccustyrna binding sites into the 02J (5-Methylisoxazole-3-carboxylic acid) 6LU7 protein targets. DMS:A:402 (DMS) - SMALLMOLECULE Figure6b. Roccustyrna binding sites into the 02J (5Methylisoxazole-3-carboxylic acid) PJE-C-56LU7 protein targets. ZN:A:998 (ZN) - ION Figure6c. CoMFA contour map of electrostatic regions around Roccustyrna chemical structure. Contact residues of the Roccustyrna small molecule when docked onto the SARS-COV-2 protein targets, (pdb:7bv2). (green, 
favored; yellow; disfavored) around the Roccustyrna chemical structure. Blue regions are favored by positively charged groups and red regions favored by negatively charged groups. Figure6d. Comparative Docking Cluster Analysis between the Remdesivir and the Roccustyrna small molecules when docked onto the SARS-COV-2 protein targets, (pdb:7bv2). Figure6e. Comparative Docking Enegy Analysis between the Remdesivir and the Roccustyrna small molecules when docked onto the SARS-COV-2 protein targets, (pdb:7bv2). \$Number_of_Clusters = 10
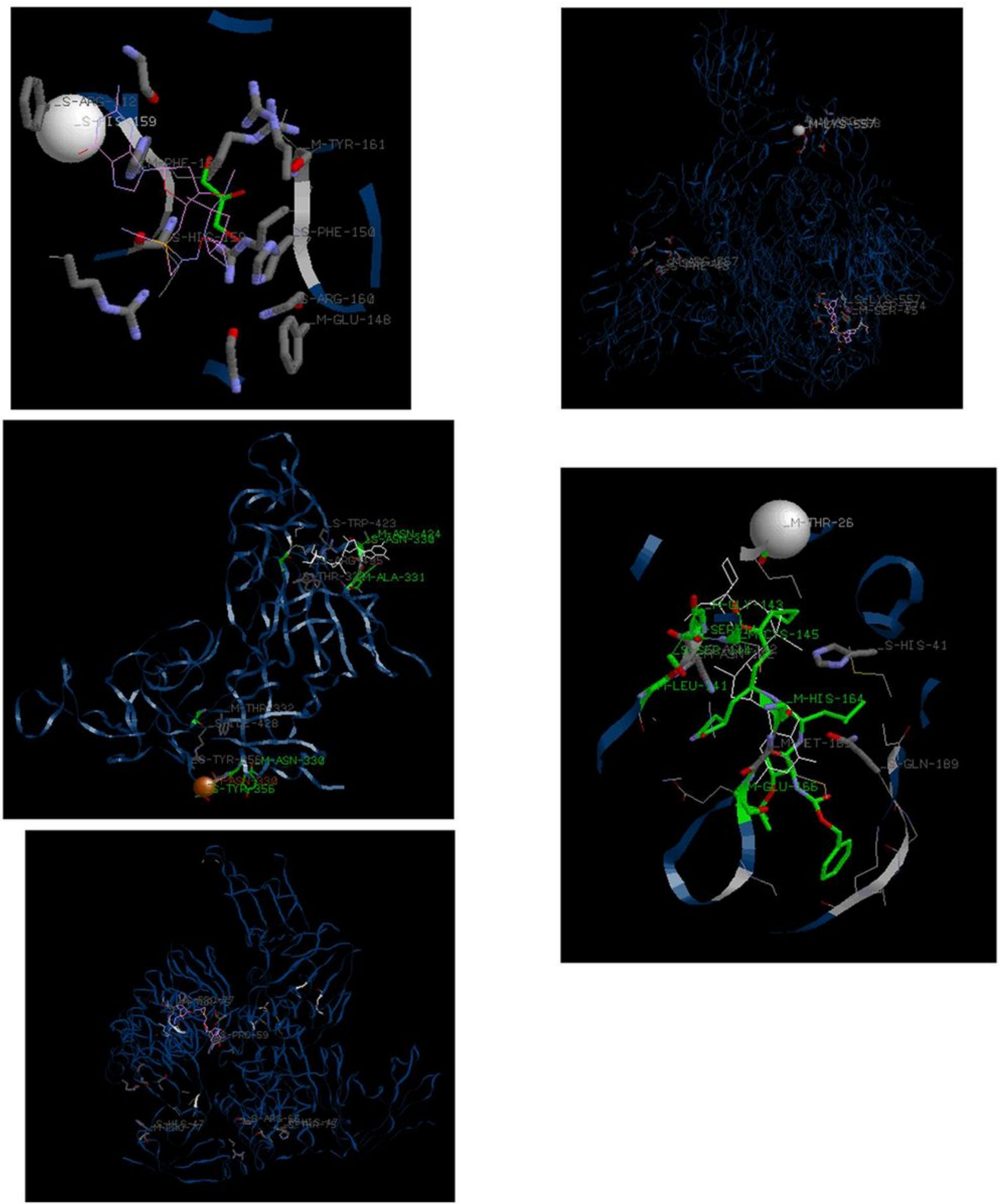

Figure 7 
Figure7a. CoMFA contour map of electrostatic regions around Roccustyrna chemical structure. Contact residues of the Roccustyrna small molecule when docked onto the SARS COV-2 protein targets, (pdb:3fqq). (green, favored; yellow; disfavored) around the Roccustyrna chemical structure. Blue regions are favored by positively charged groups and red regions favored by negatively charged groups within the sequence of the amino acid of the V-S-HIS-159, V-S-ARG-160, V-S-ARG-112 V-M-GLU-148 V-M-PHE-150, VS-PHE-150, V-S-HIS-159, V-M-TYR-161 with the docking energy values of the-101, -14.0762, - 5.11094, $-7.98447,-4.17314,-4.43549,-9.66939,-9.42926,-7.30085$ Figure7b. CoMFA contour map of electrostatic regions around Roccustyrna chemical structure. Contact residues of the Roccustyrna small molecule when docked onto the SARS- COV-2 protein targets, (pdb:6xs6). (green, favored; yellow; disfavored) around the Roccustyrna chemical structure. Blue regions are favored by positively charged groups and red regions favored by negatively charged groups within the sequence of the amino acid of the V-M-LYS557, V-S-LYS-557, V-M-ARG-567, V-M-ASP-568, V-S-ASP-574, V-S-PHE-43, V-M-ARG-44, V-M-SER-45, V-SSER-45 with the docking energy values of the -85.8 , and $-5.56004,-5.0011,-8.38956,-5.77168,-6.13664$, $-12.8661,-5.37546,-6.10391,-5.00928$ respectively. Figure7c. CoMFA contour map of electrostatic regions around Roccustyrna chemical structure. Contact residues of the Roccustyrna small molecule when docked onto the SARS- COV-2 protein targets, (pdb:2ghv). (green, favored; yellow; disfavored) around the Roccustyrna chemical structure. Blue regions are favored by positively charged groups and red regions favored by negatively charged groups within the sequence of the amino acid of the H-M-ASN-330, H-SASN-330, H-S-TYR-356, H-M-ASN-424, V-M-ASN-330, V-M- ALA-331, V-M-THR-332, V-S-THR-332, V-S-TYR356, V-S-TRP-423, V-S-ILE-428, V-S-ARG-495 with the docking energy values of the -104.7 and-3.45708 $-3.5,-3.97711,-3.5,-5.33228,-6.79753,-7.9376,-6.69969,-12.2528,-7.66989,-8.15072-7.00332$ respectively. Figure7d. CoMFA contour map of electrostatic regions around Roccustyrna chemical structure. Contact residues of the Roccustyrna small molecule when docked onto the SARS- COV-2 protein targets, (pdb:2zu5). (green, favored; yellow; disfavored) around the Roccustyrna chemical structure. Blue regions are favored by positively charged groups and red regions favored by negatively charged groups within the sequence of the amino acid of the V-M-THR-25, V-S-THR-25, V-M-THR-26, V-SHIS-41, V-M-LEU-141, V-M-ASN-142, V-S-ASN-142, V-M-GLY-143, V-S-CYS-145, V-M-MET-165 with the binding energy values of the -97.2 and $-5.16512,-4.15949,-9.8487,-4.77062,-4.72901,-6.7295,-5.82428$, $-5.35883,-4.2588,-5.37491$ respectively. Figure7e. CoMFA contour map of electrostatic regions around Roccustyrna chemical structure. Contact residues of the Roccustyrna small molecule when docked onto the SARS- COV-2 protein targets, (pdb:6w9c). (green, favored; yellow; disfavored) around the Roccustyrna chemical structure. Blue regions are favored by positively charged groups and red regions favored by negatively charged groups within the sequence of the amino acid of the V-S-PRO-59,V-S-ARG-65, V-MTHR-75, V-S-THR-75, V-M-PRO-77, V-S-PRO-77, V-M-HIS-47, V-S-HIS-47 with the docking energies of the $-83.9,-4.21999,-12.6164,-7.60372,-6.69528,-5.89416,-6.40663,-5.51621,-7.99273$. 


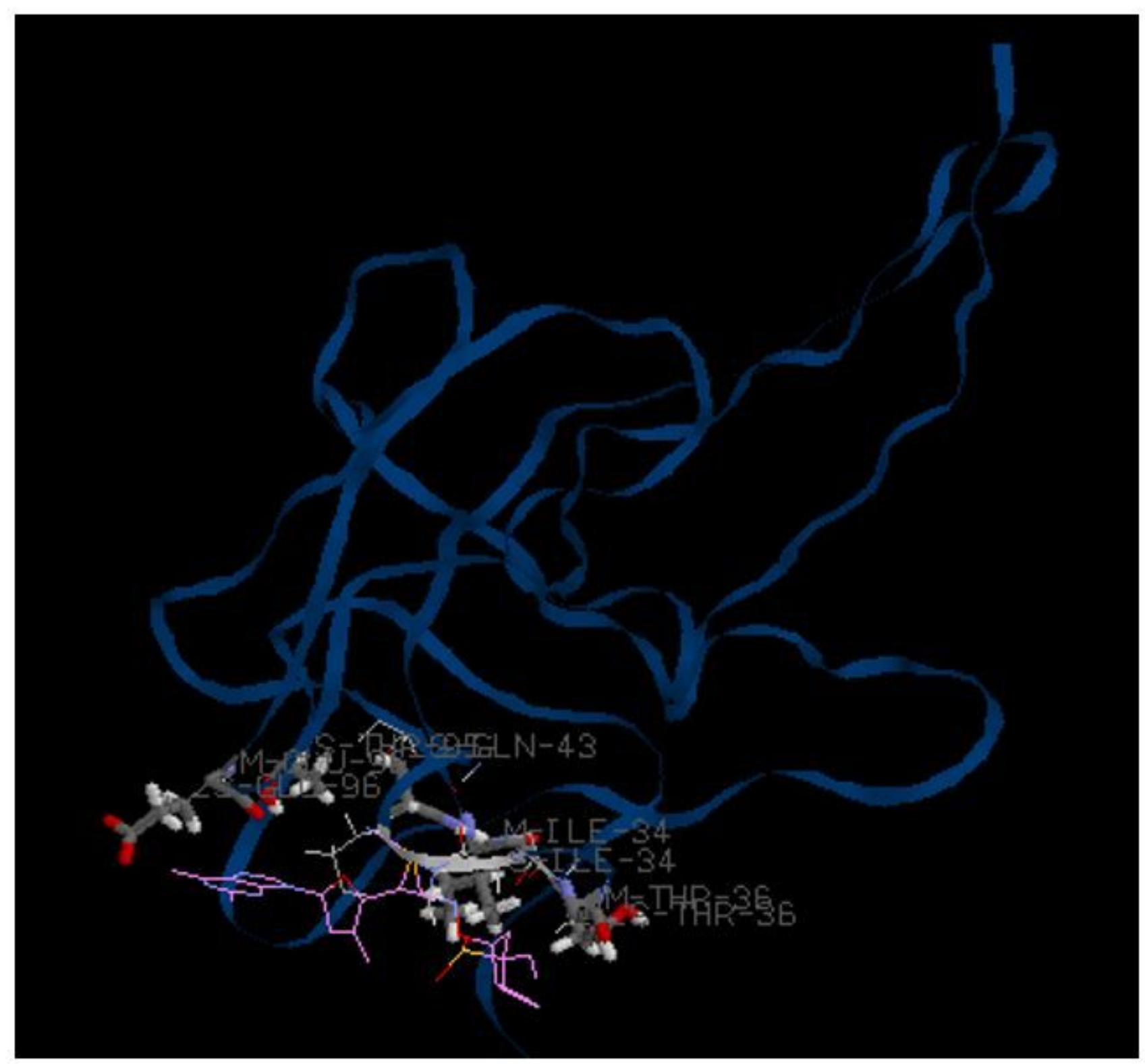

\section{Figure 8}

$3 D$ Docking interactions of the Roccustyrna small molecule $(1 S, 2 R, 3 S) \rrbracket 2 \rrbracket(\{[(1 S, 2 S, 4 S, 5 R) \llbracket 4 \rrbracket e t h e n y l \rrbracket 4 \rrbracket$

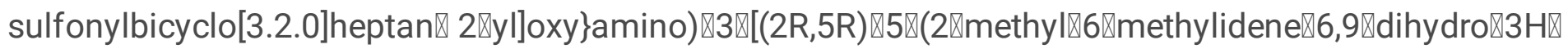

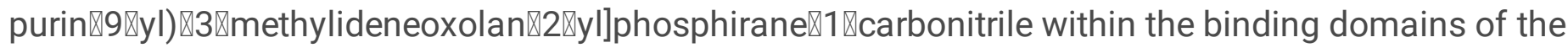
protein targets (PDB: $6 \mathrm{YI3}$ ) of the N-terminal RNA-binding domain of the SARS-CoV-2 nucleocapsid phosphoprotein. The Roccustyrna ligand binds into the binding sites of the amino acids of the V-M-ILE34, V-S-ILE-34, V-M-THR-36, V-S-THR-36, V-S-GLN-43, V-S-THR-95, V-M-GLU-96, V-S-GLU-96 with the total docking energies of the $-96.6 \mathrm{Kcal} / \mathrm{mol}$ and the docking values of the $-4.76839,-9.02476,-9.66643$, $-6.43937,-8.09017,-13.9373,-9.26627,-7.55529 \mathrm{Kcal} / \mathrm{mol}$ respectively. 